\title{
Synthesis of Difluoromethylated Pyrazoles by [3 + 2] \\ Cycloaddition Reaction of Difluoroacetohydrazonoyl Bromides
}

Tongyu Han, $\uparrow$ Ke-Hu Wang, $\uparrow$ Ming Yang, $\uparrow$ Pengfei Zhao,,$\uparrow$ Feng Wang, $\uparrow$ Junjiao Wang, $\uparrow$ Danfeng Huang, $\dagger$ Yulai $\mathrm{Hu}^{*} \uparrow \dagger$

$†$ College of Chemistry and Chemical Engineering, Northwest Normal University, 967 Anning East Road, Lanzhou 730070, P. R. China

†State Key Laboratory of Applied Organic Chemistry, Lanzhou University, Lanzhou 730000, P. R.

China

E-mail: huyl@nwnu.edu.cn.

\section{Table of Contents}

Synthesis of Difluoroacetaldehyde Ethyl Hemiacetal......................................................S2

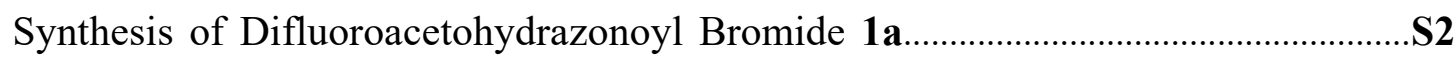

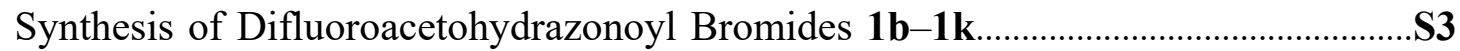

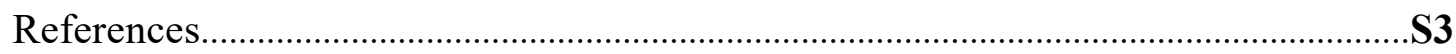

Copies of NMR and HRMS Spectra for Compounds 3a-3ac....................................S4

Copies of NMR and HRMS Spectra for Compounds 5a-5k.................................S101

Copies of NMR and HRMS Spectra for Compounds 7a-7k ..................................S140

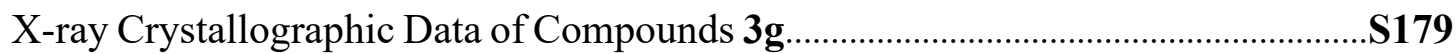




\section{Synthesis of Difluoroacetaldehyde Ethyl Hemiacetal ${ }^{1}$}<smiles>CCOC(=O)C(F)(F)F</smiles><smiles>CCOC(O)C(F)(F)F</smiles>

Under an argon atmosphere, a solution of ethyl difluoroacetate $(40.0 \mathrm{~g}, 322.6 \mathrm{mmol}$, 1.0 equiv.) in $\mathrm{Et}_{2} \mathrm{O}(200 \mathrm{~mL})$ was added a every 15 minutes slowly at $-78{ }^{\circ} \mathrm{C}$ to a solid of $\mathrm{LiAlH}_{4}$ (4.3 g, 112.9 mmol, 0.35 equiv.), a total of four times. The reaction mixture was stirred at this temperature for $3 \mathrm{~h}$ and then treated slowly with cooled $\mathrm{H}_{2} \mathrm{SO}_{4}(1.0$ M, $200 \mathrm{~mL}$ ). The upper layer was separated and the aqueous phase was extracted by $\mathrm{Et}_{2} \mathrm{O}(200 \mathrm{~mL} \times 3)$. The combined organic phases were dried over sodium sulfate, evaporated, and distilled under normal pressure. Ethyl difluoroacetaldehyde hemiacetal was obtained at $84-90^{\circ} \mathrm{C}(31.3 \mathrm{~g}, 77 \%)$.

\section{Synthesis of Difluoroacetohydrazonoyl Bromide $1 a^{2}$}

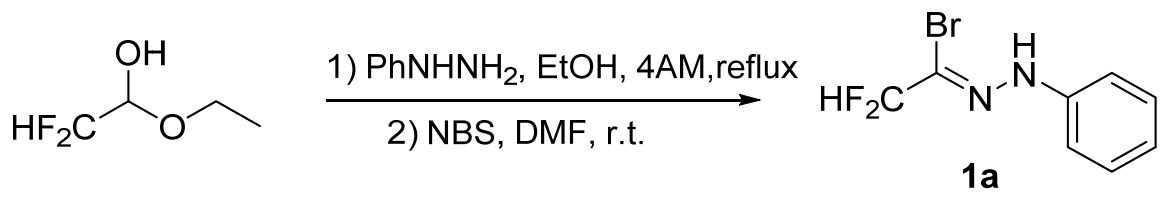

Step 1: A mixture of hydrazines (1.0 mmol, 1.0 equiv.), difluoroacetaldehyde ethyl hemiacetal (1.5 mmol, 1.5 equiv.), and freshly activated molecular sieves $4 \AA$ in EtOH $(8 \mathrm{~mL})$ was stirred at $75{ }^{\circ} \mathrm{C}$ in a round-bottom in an oil bath, and the reaction was monitored by TLC. After the reaction was completed, the solvent was removed in vacuo to afford intermediate product, which was used directly for the next step.

Step 2: To a solution of crude mixture from step 1 in DMF ( $8 \mathrm{~mL})$ was added NBS. The resulting solution was stirred at room temperature, and the reaction was monitored by TLC. After the reaction was completed, the reaction was quenched with sat. $\mathrm{NaCl}$ aq., and the mixture was extracted with ethyl acetate $(3 \times 15 \mathrm{~mL})$, dried over $\mathrm{MgSO}_{4}$, filtered, and concentrated in vacuo. The resulting products was purified by column chromatography on silica gel (EA)/petroleum ether (PE) (1:10-1:20) to afford difluoroacetohydrazonoyl bromide ( $76 \%$ yields). 


\section{Synthesis of Difluoroacetohydrazonoyl Bromide $1 \mathrm{~b}-1 \mathrm{k}^{2}$}

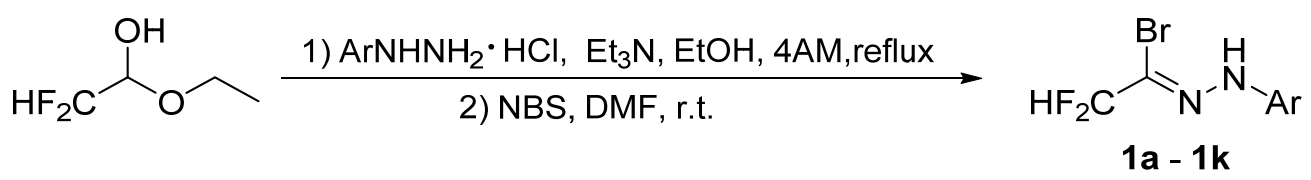

Step 1: A mixture of hydrazine hydrochlorides (1.0 mmol, 1.0 equiv.), triethylamine (1.0 mmol, 1.0 equiv.), difluoroacetaldehyde ethyl hemiacetal (1.5 mmol, 1.5 equiv.), and freshly activated molecular sieves $4 \AA$ in $\mathrm{EtOH}(8 \mathrm{~mL})$ was stirred at $75^{\circ} \mathrm{C}$ in a round-bottom in an oil bath, and the reaction was monitored by TLC. After the reaction was completed, the solvent was removed in vacuo to afford intermediate products, which was used directly for the next step.

Step 2: To a solution of crude mixture from step 1 in DMF ( $8 \mathrm{~mL})$ was added NBS. The resulting solution was stirred at room temperature, and the reaction was monitored by TLC. After the reaction was completed, the reaction was quenched with sat. $\mathrm{NaCl}$ aq., and the mixture was extracted with ethyl acetate $(3 \times 15 \mathrm{~mL})$, dried over $\mathrm{MgSO}_{4}$, filtered, and concentrated in vacuo. The resulting products was purified by column chromatography on silica gel (EA)/petroleum ether (PE) (1:10-1:20) to afford difluoroacetohydrazonoyl bromides.

\section{References}

[1] Kaneko, S.; Yamazaki, T.; Kitazume, T. A remarkably simple route to versatile difluoromethylated molecules. J. Org. Chem. 1993, 58, 2302.

[2] Tanaka, K.; Maeno, S.; Mitsuhashi, K. Cycloadditions of $N$-Aryl-C (Trifluoromethyl)nitrilimines with Dimethyl Fumarate and Maleate. J. Heterocyclic. Chem. 1985, 22, 565 . 
Copies of NMR and HRMS Spectra for Compounds 3a-3ac

NMR copies of compound 3a:

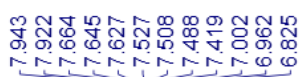

rinaningen
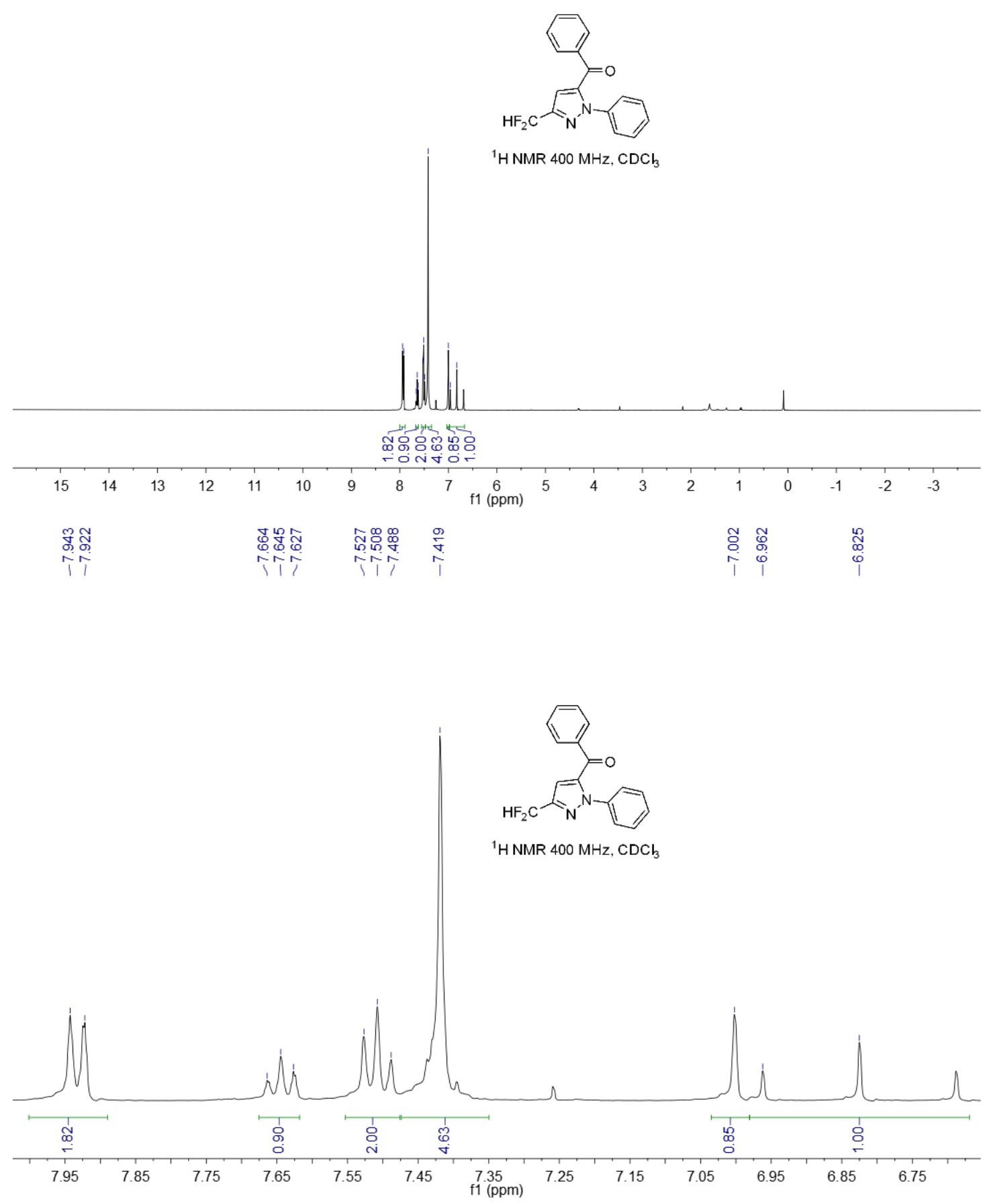

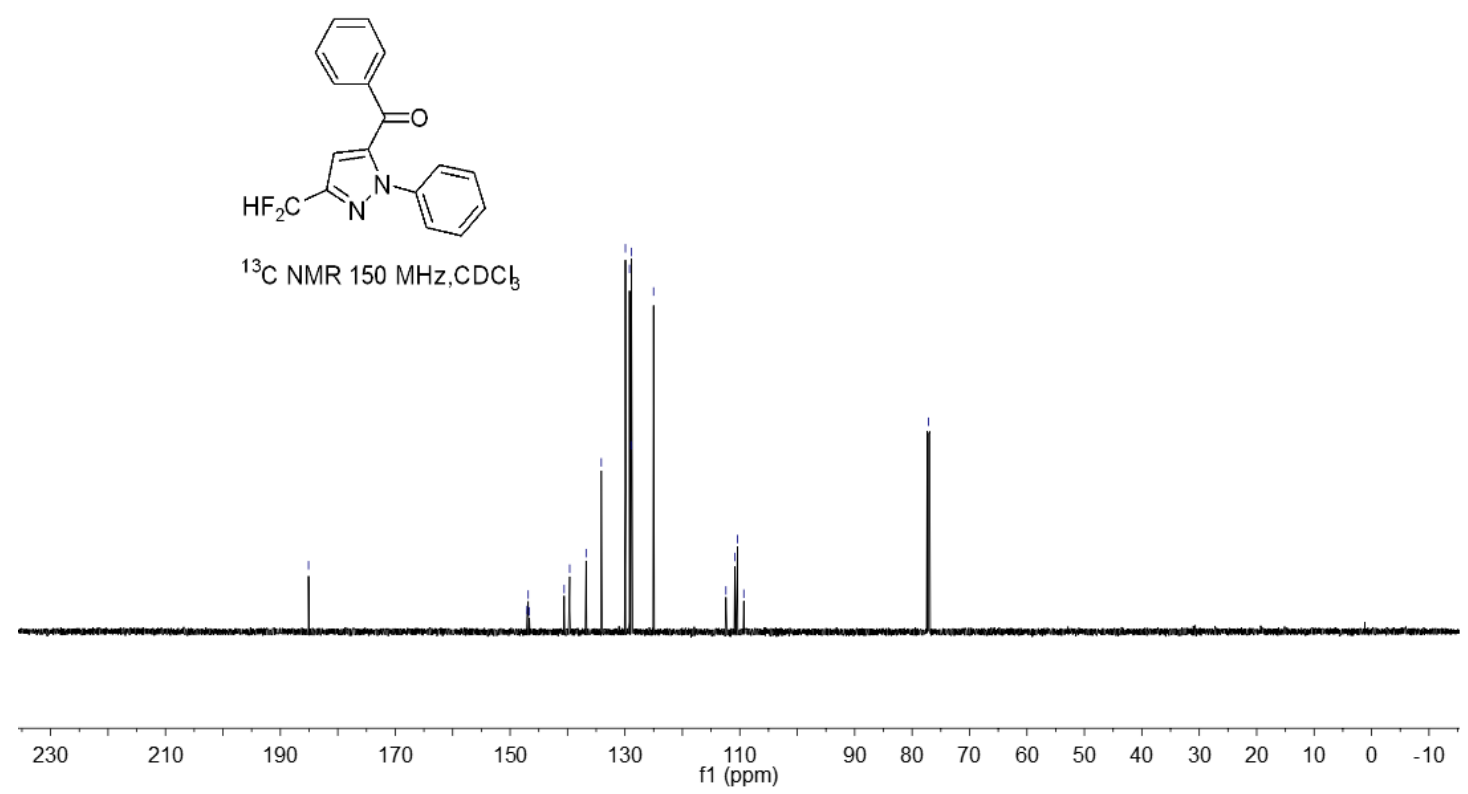

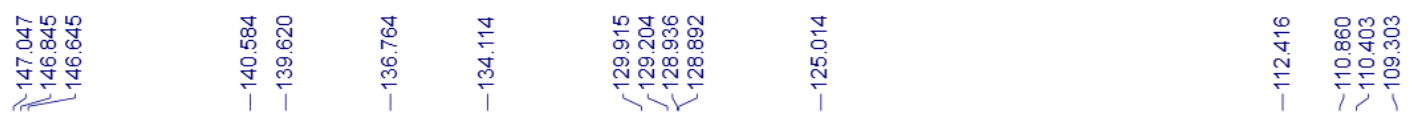

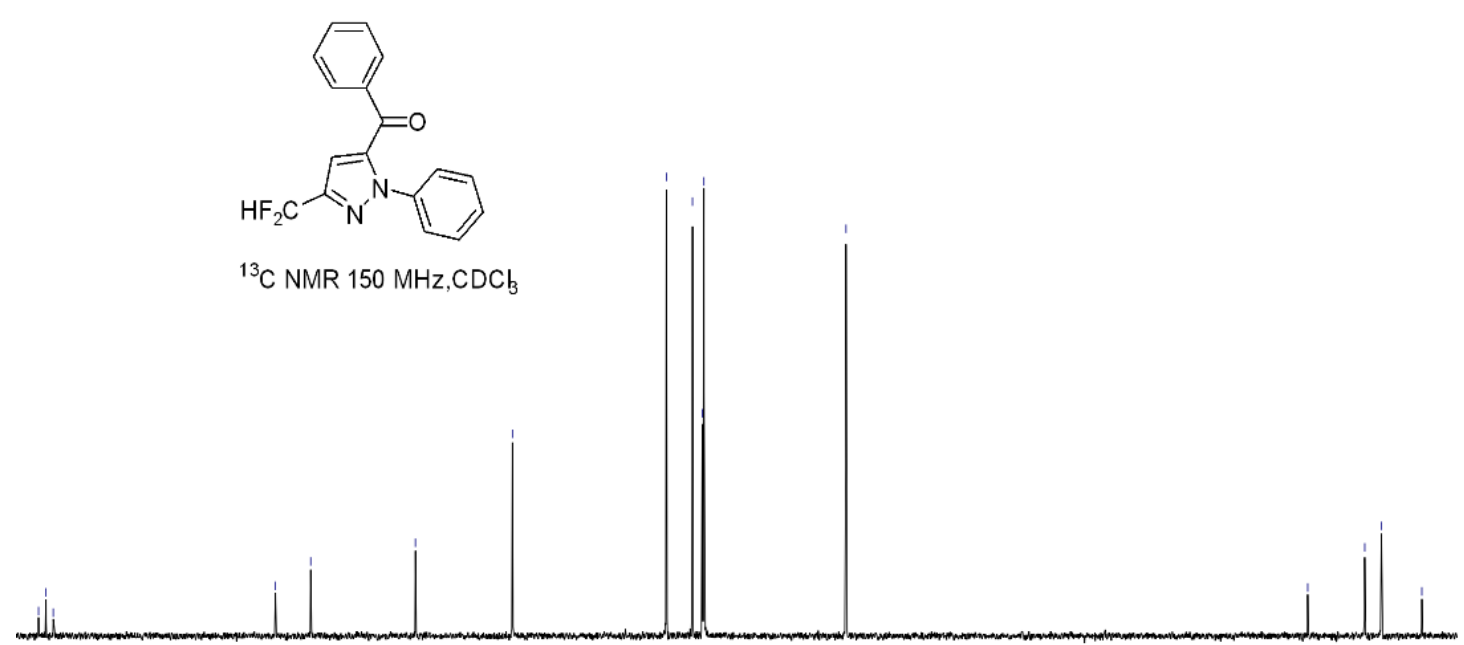

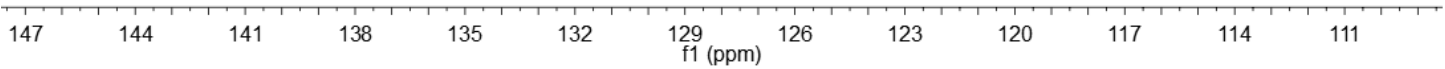




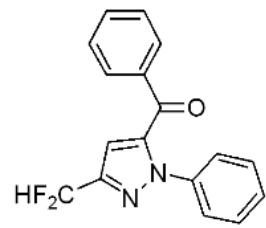

${ }^{19} \mathrm{~F} \mathrm{NMR} 376 \mathrm{MHz}, \mathrm{CDC}_{3}$
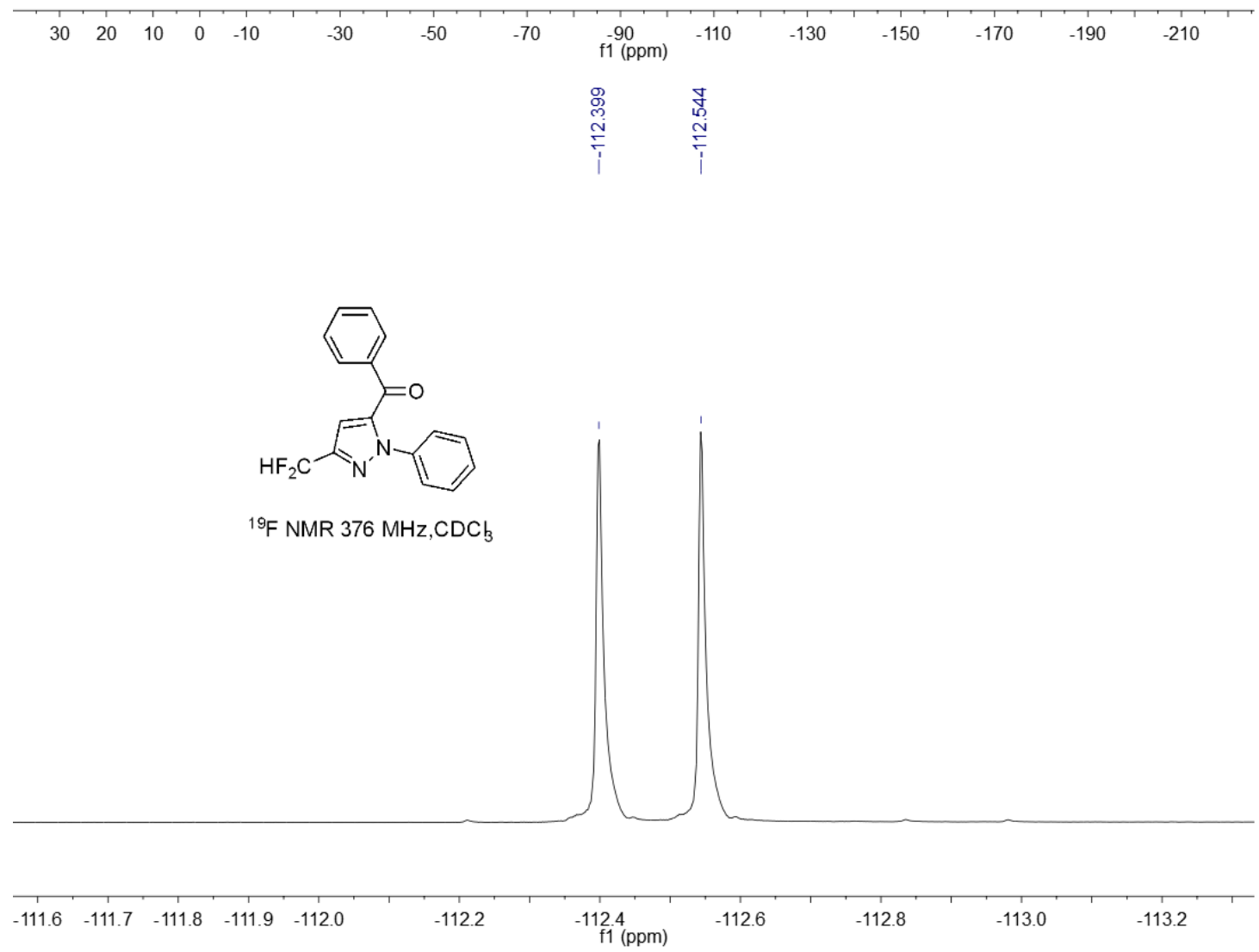
HRMS (ESI) copy of compound 3a:

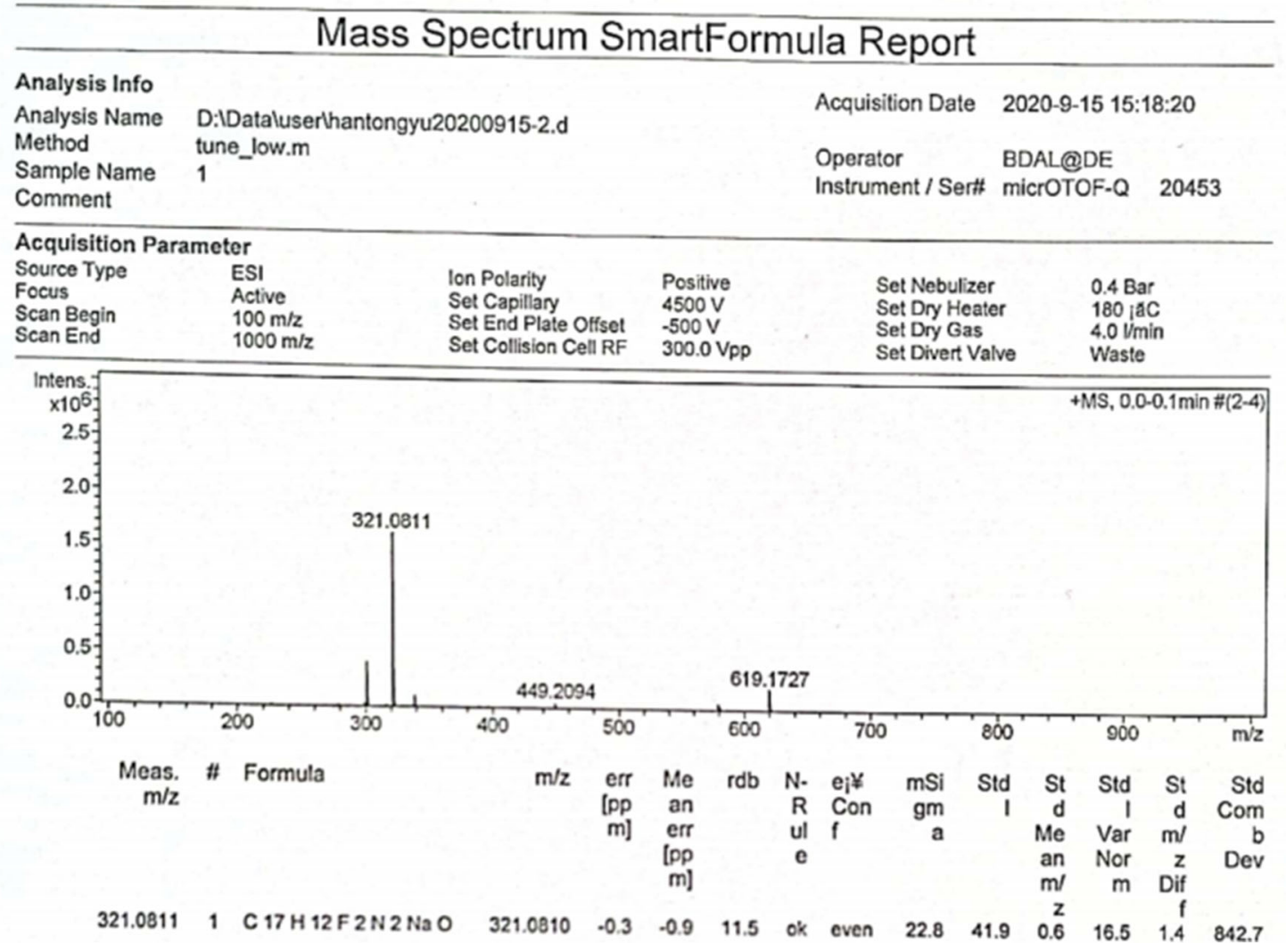

NMR copies of compound $\mathbf{3 b}$ :

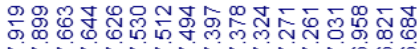

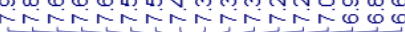

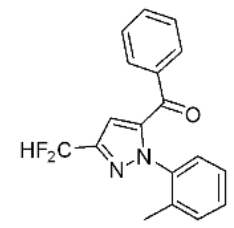

${ }^{1} \mathrm{H}$ NMR $400 \mathrm{MHz}, \mathrm{CDCl}$

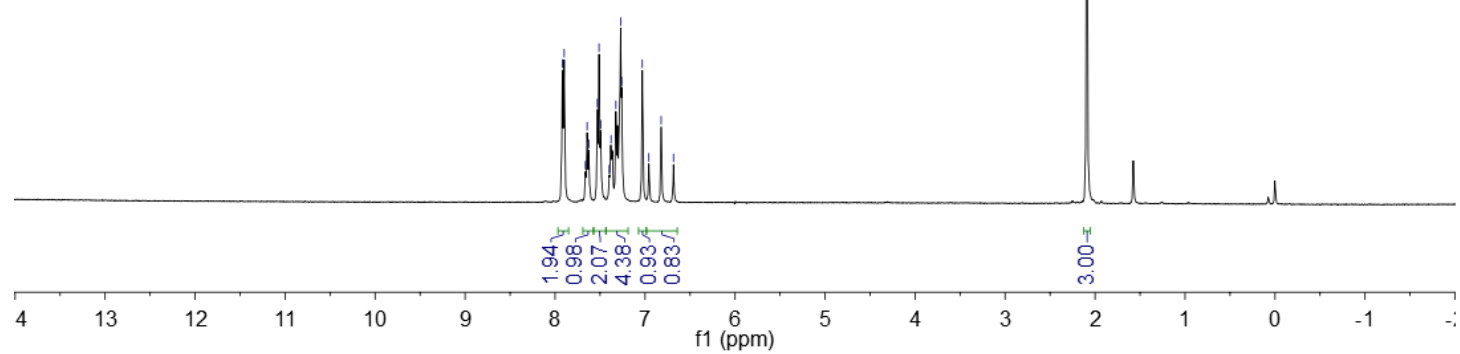




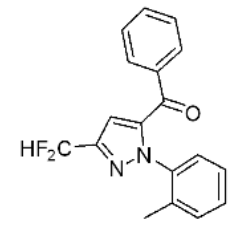

${ }^{1} \mathrm{H}$ NMR $400 \mathrm{MHz}, \mathrm{CDCl}$

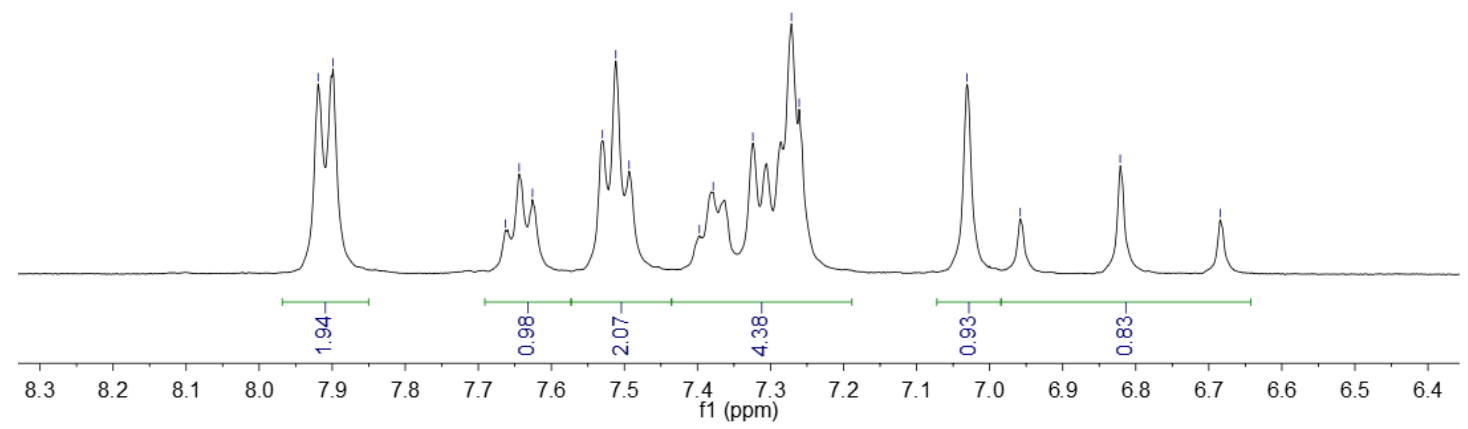

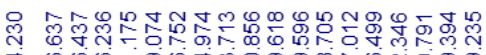

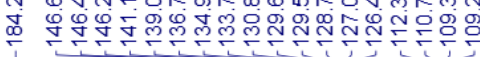

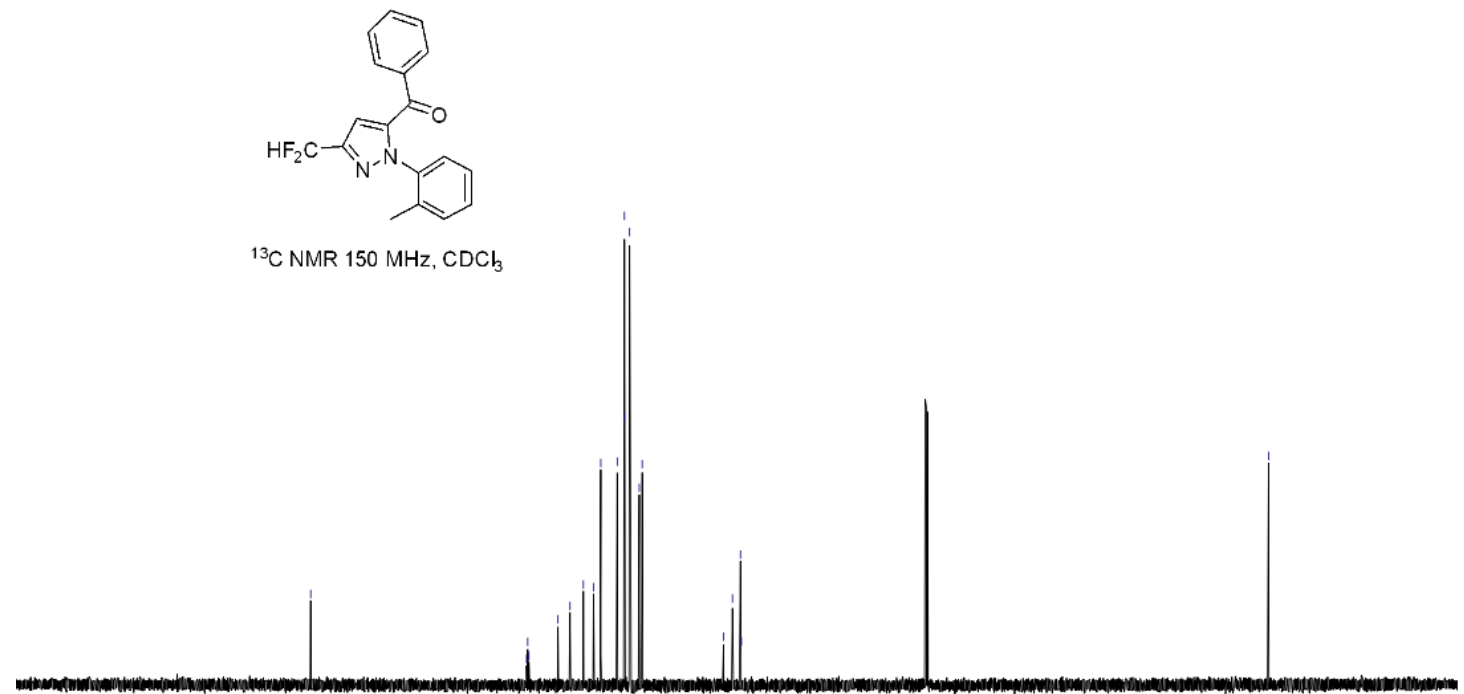

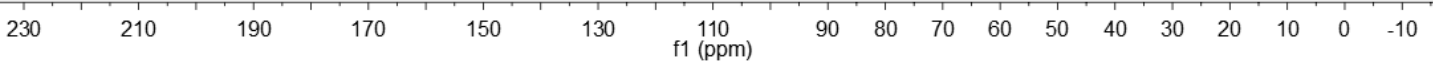




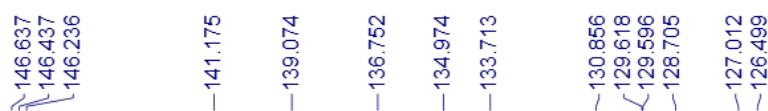

禺

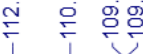
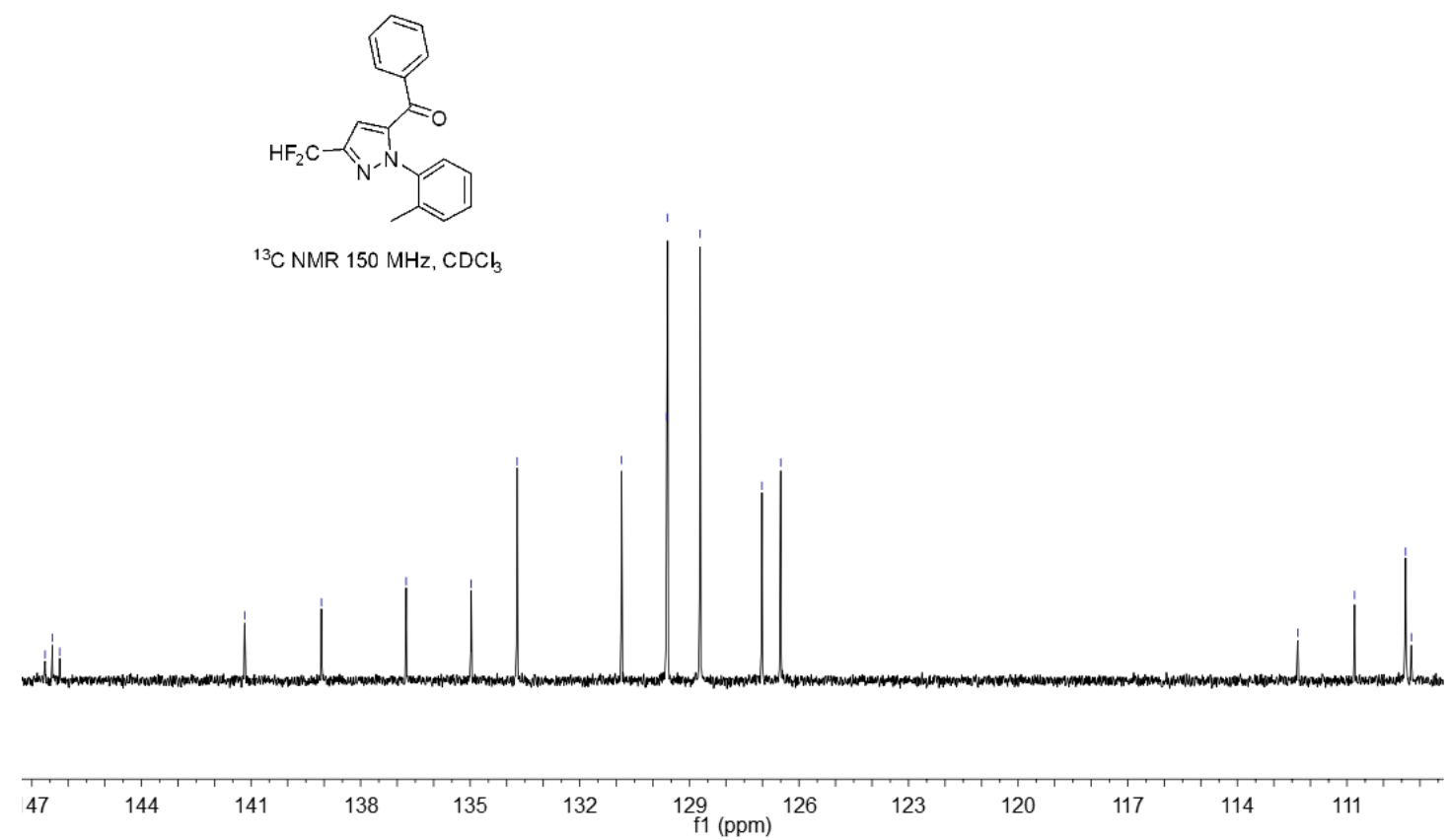

ํㅗㄴ을

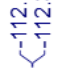

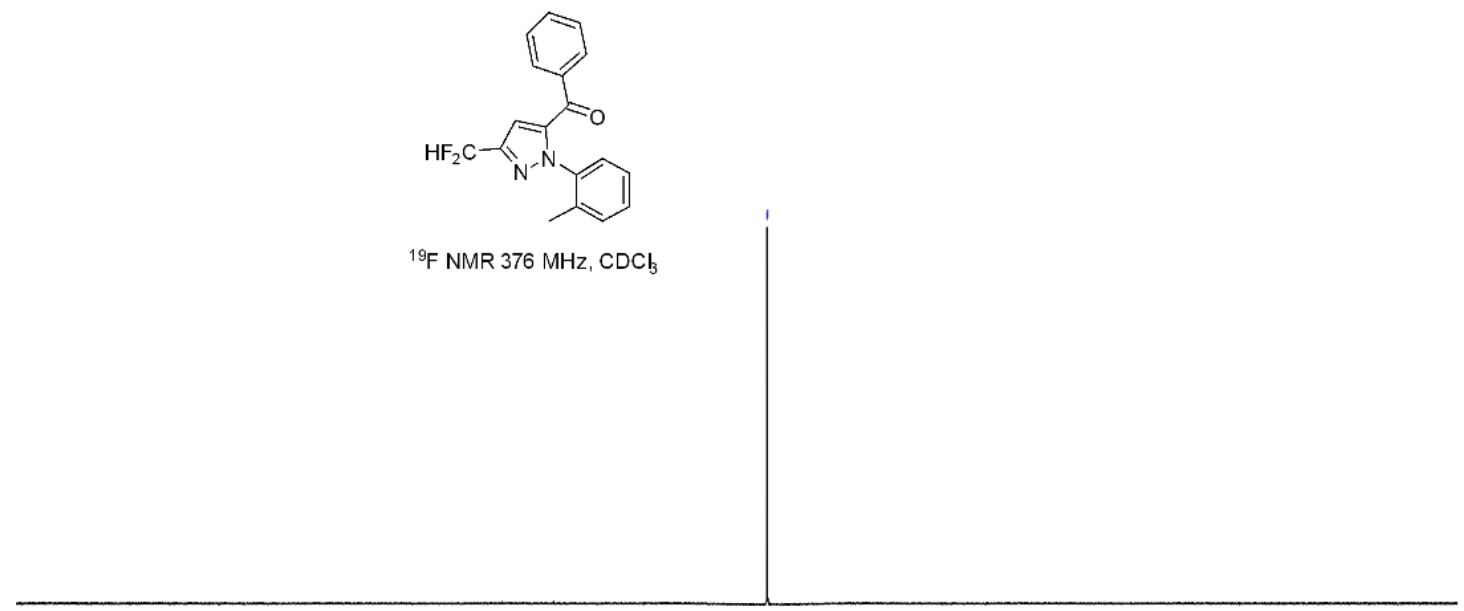

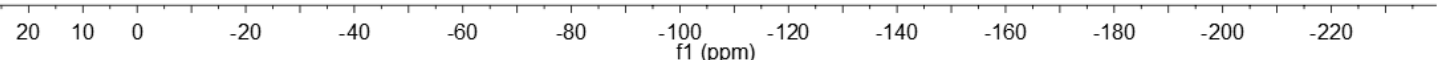




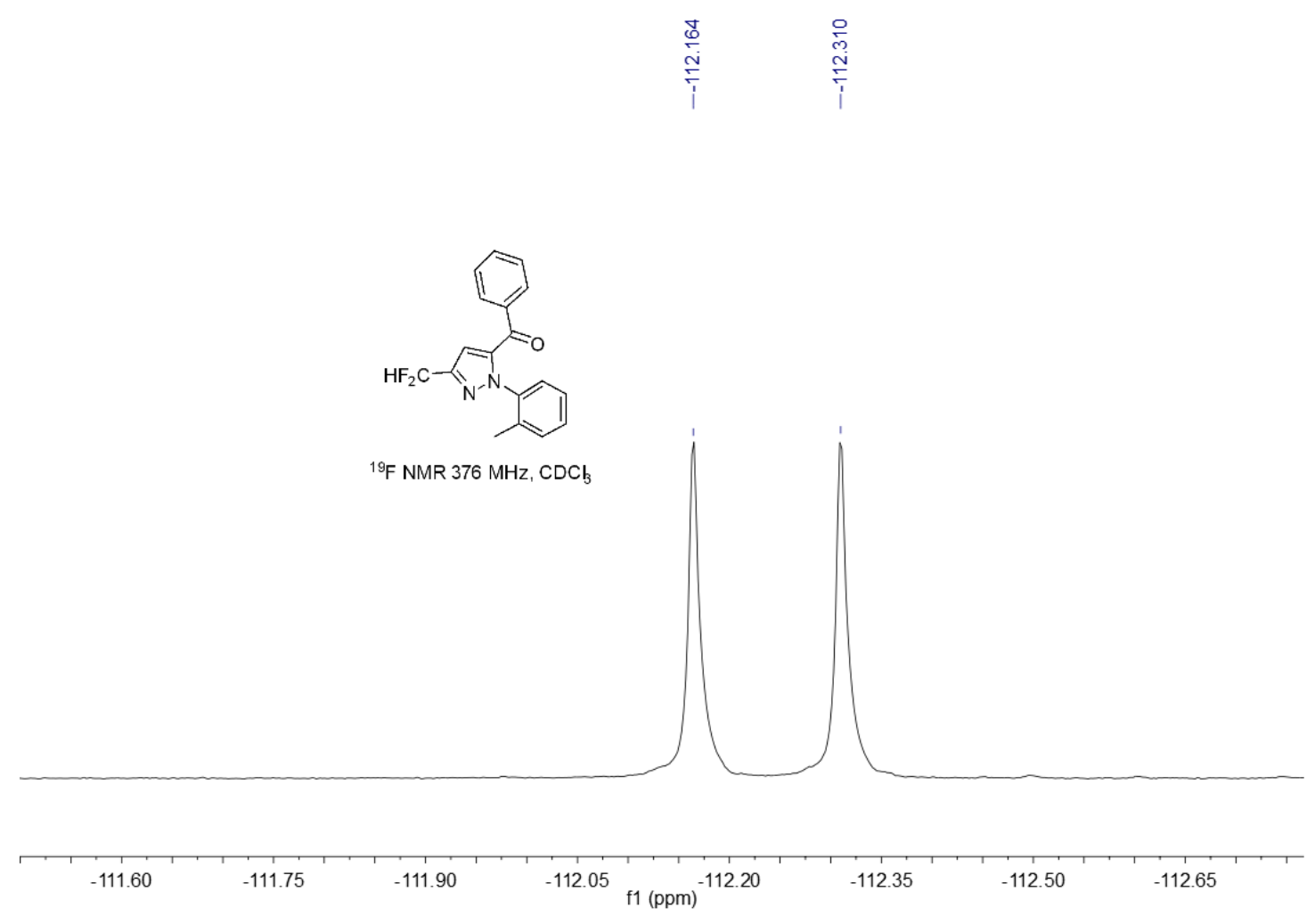

HRMS (ESI) copy of compound $\mathbf{3 b}$ :

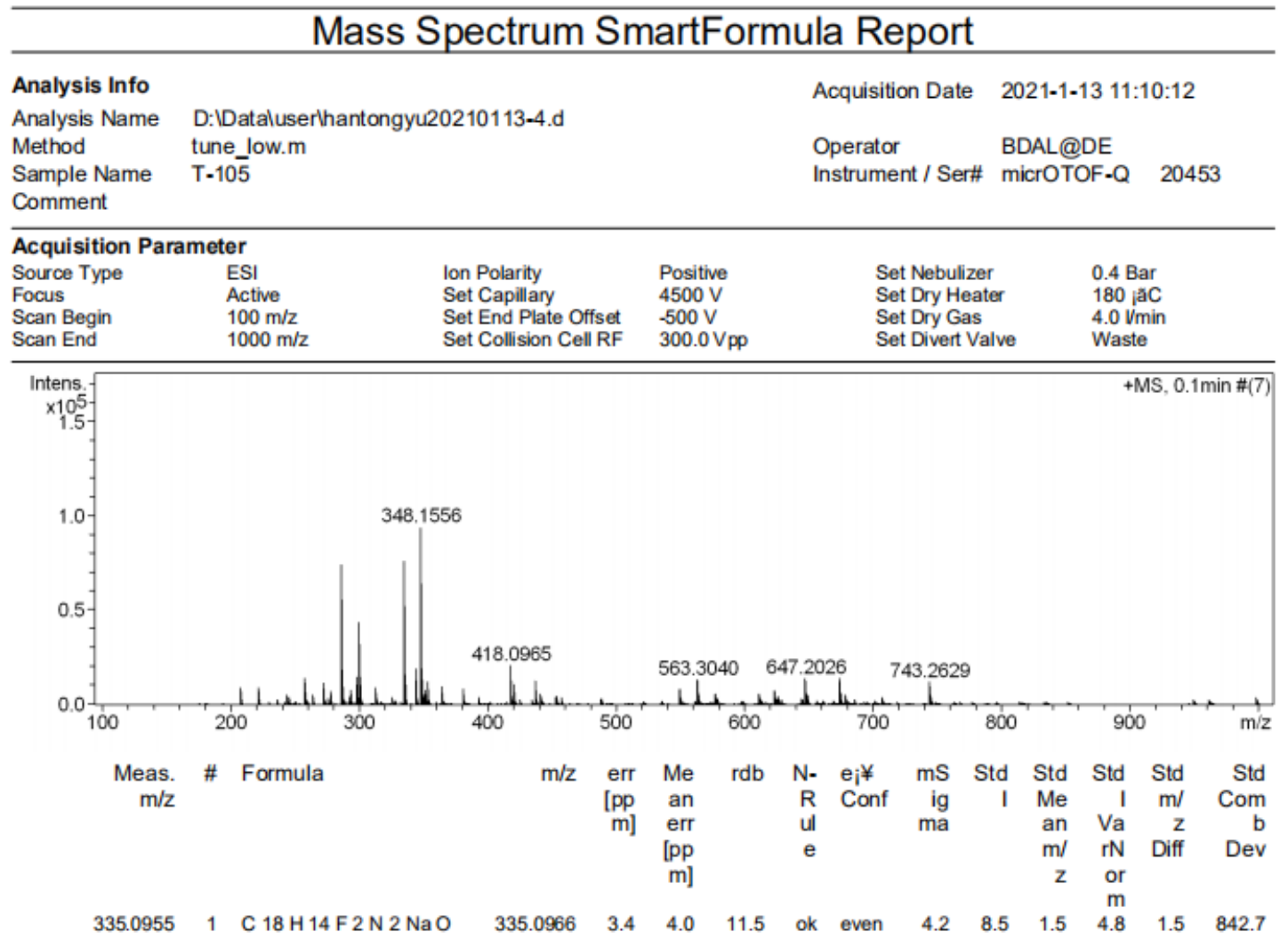


NMR copies of compound 3c:

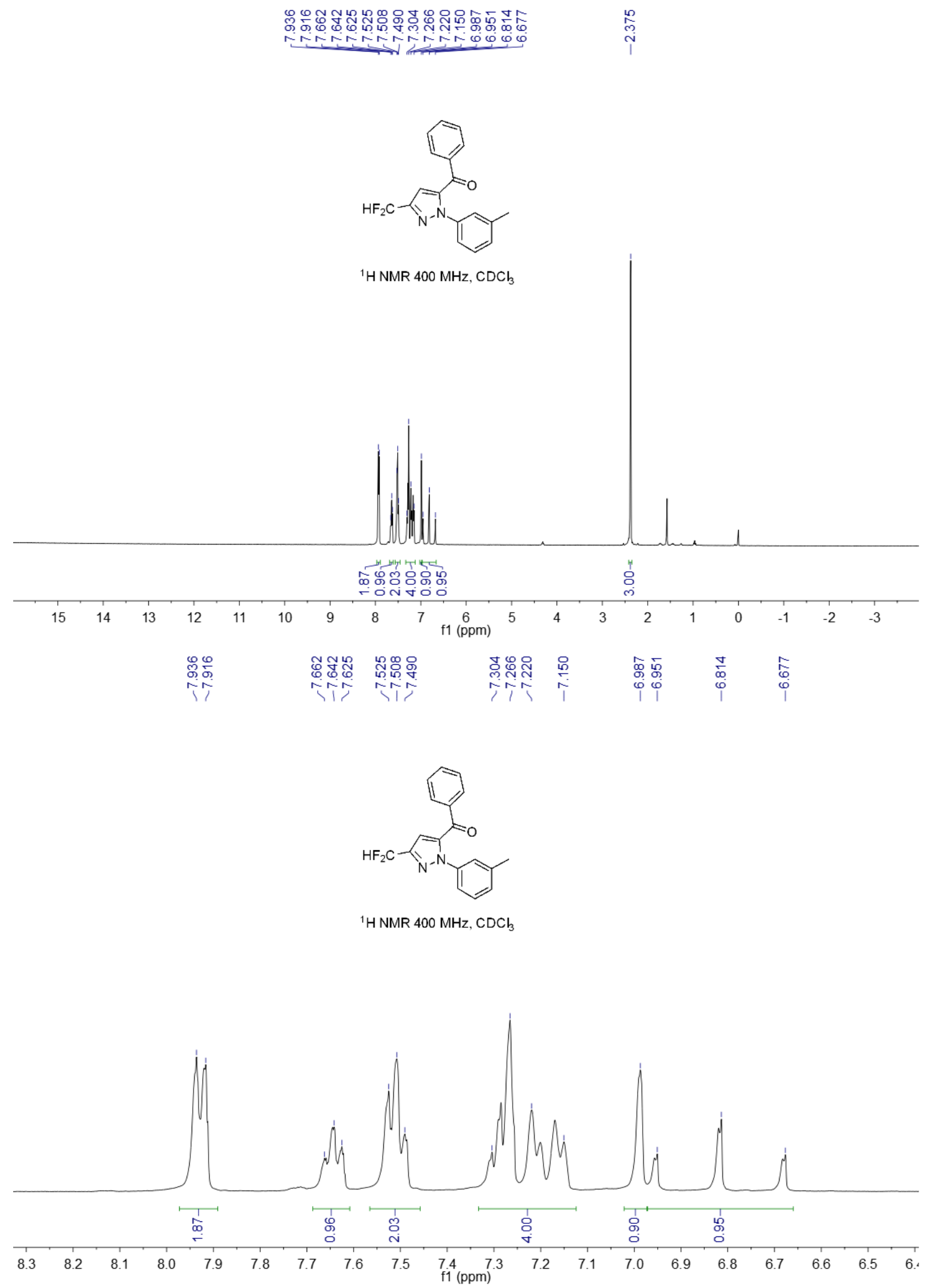



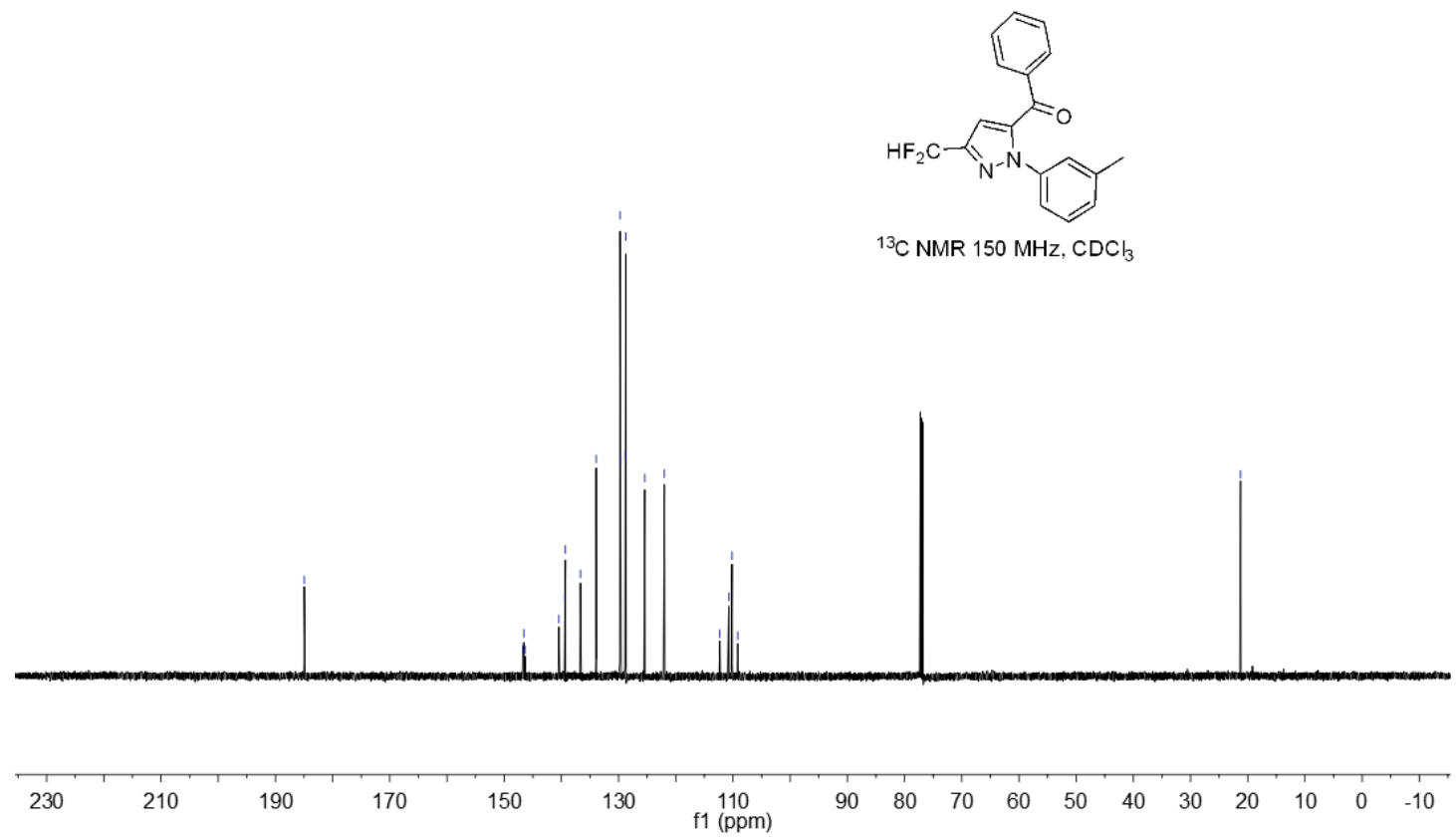

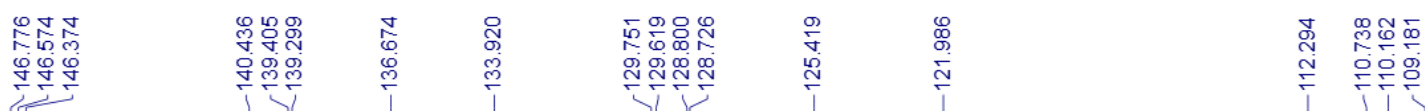

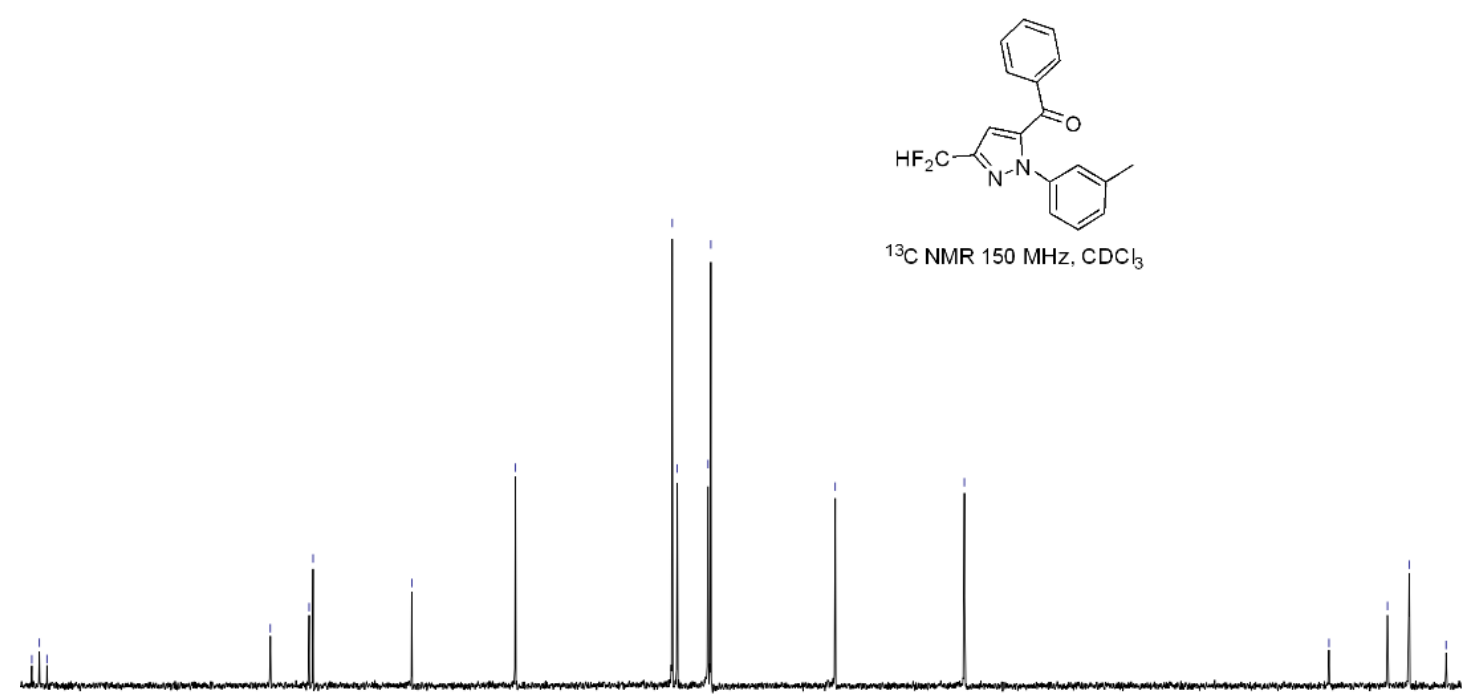

\begin{tabular}{lllllllllllll}
\hline 47 & 144 & 141 & 138 & 135 & 132 & 129 & 126 & 123 & 120 & 117 & 114 & 111
\end{tabular} 
ํํำ

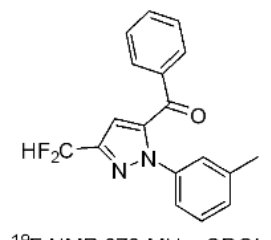

${ }^{19} \mathrm{~F} \mathrm{NMR} 376 \mathrm{MHz}, \mathrm{CDCl}_{3}$
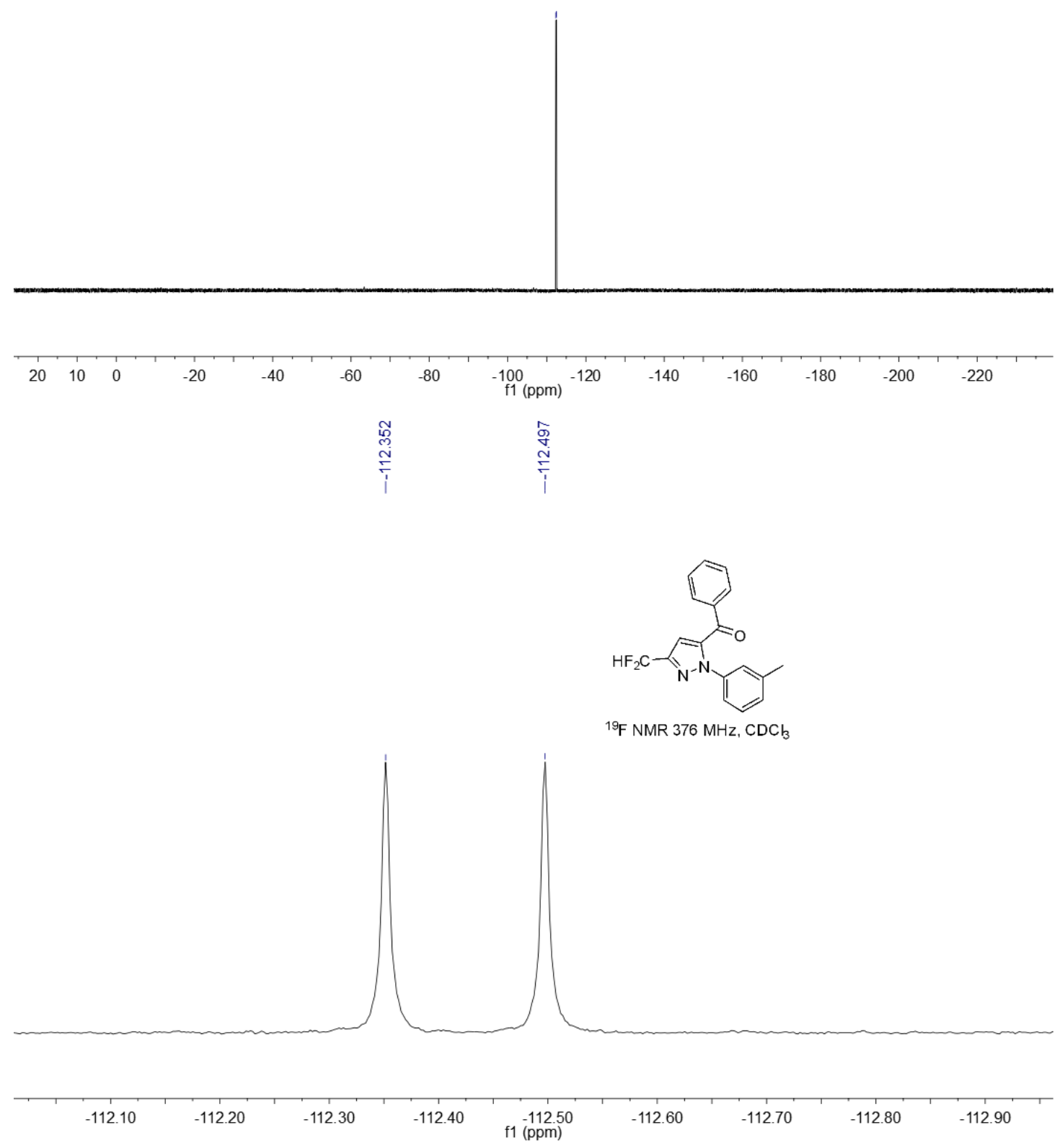
HRMS (ESI) copy of compound 3c:

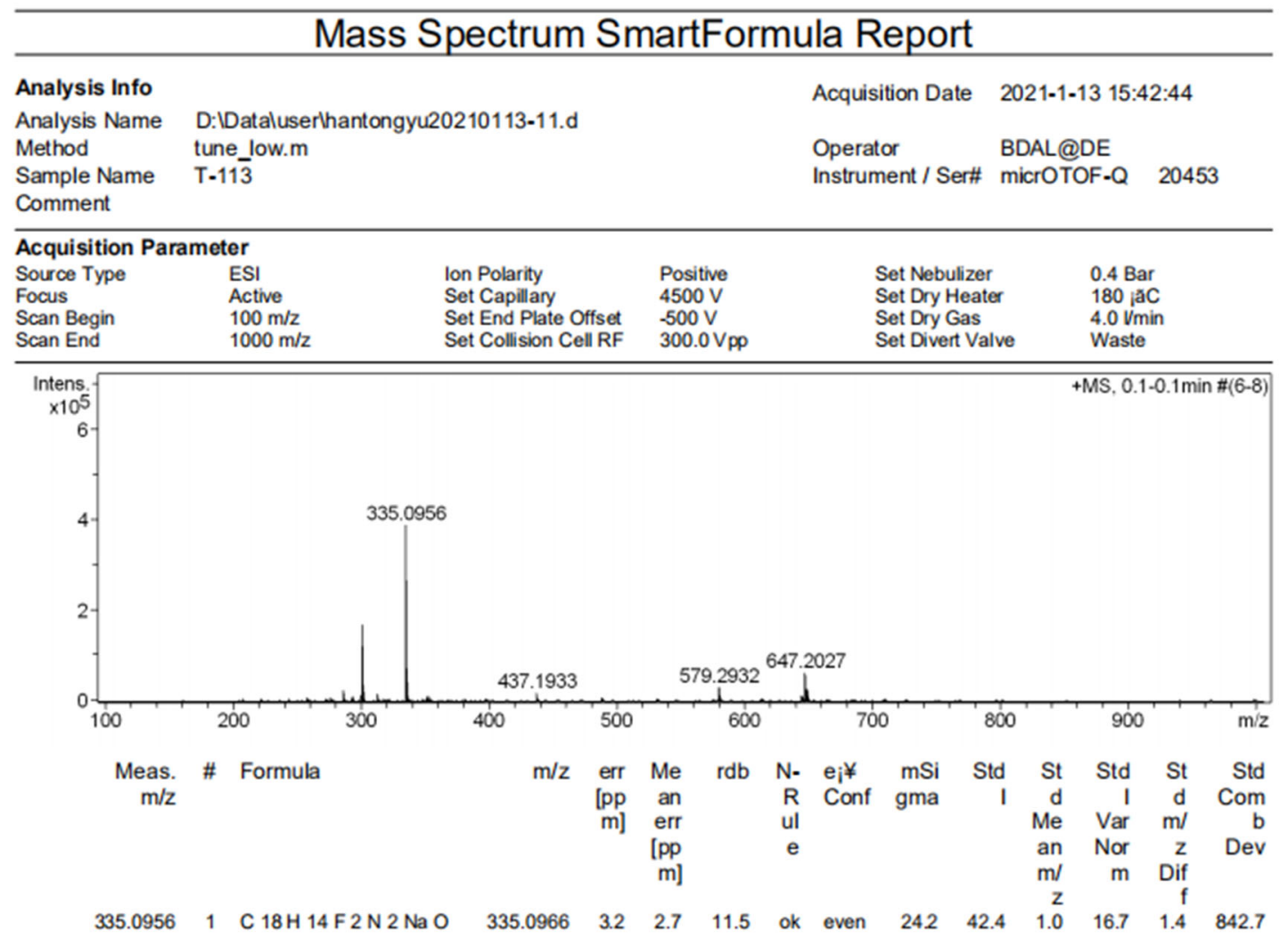

NMR copies of compound $\mathbf{3 d}$ :

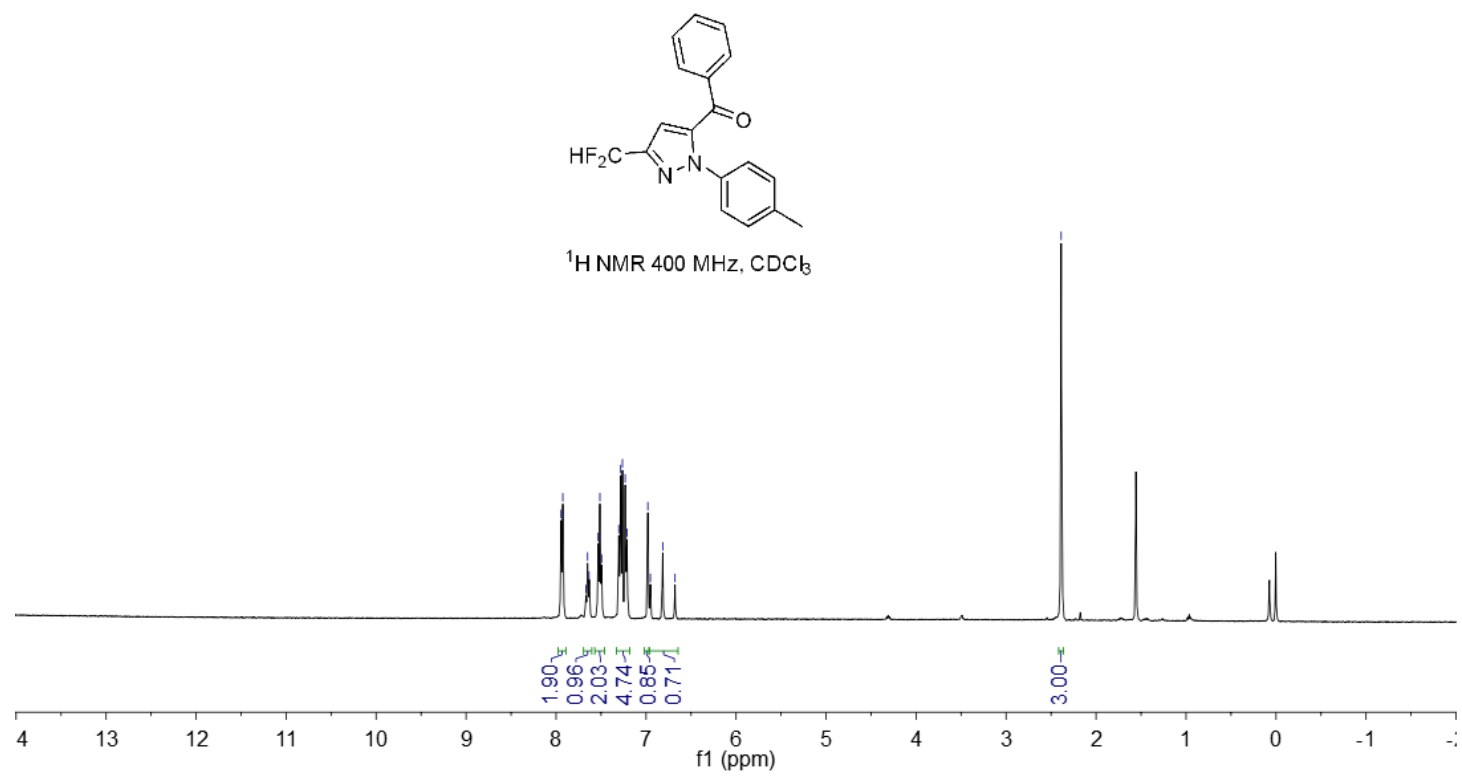




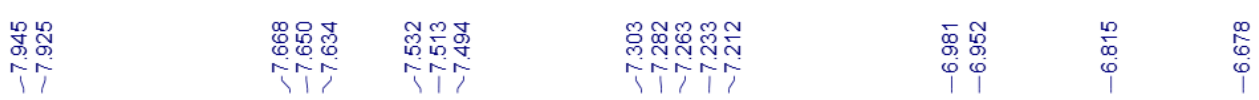

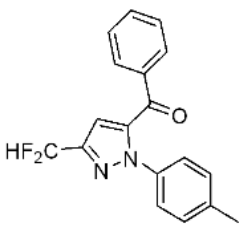

${ }^{1} \mathrm{HNMR} 400 \mathrm{MHz}, \mathrm{CDCl}_{3}$

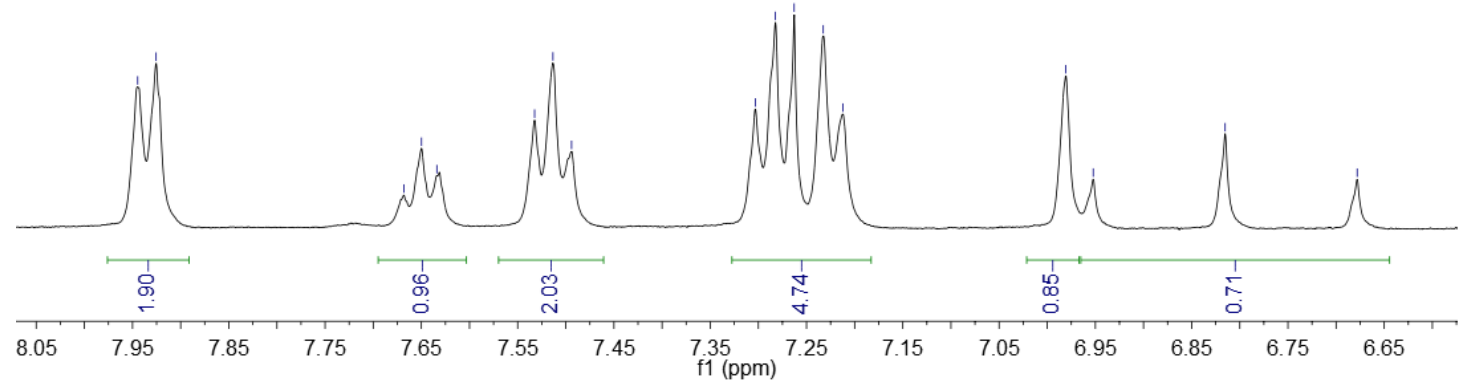

品

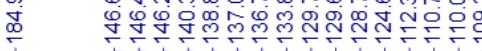

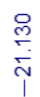

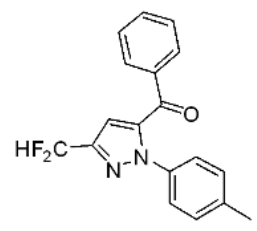

${ }^{13} \mathrm{C}$ NMR $150 \mathrm{MHz}, \mathrm{CDCl}$

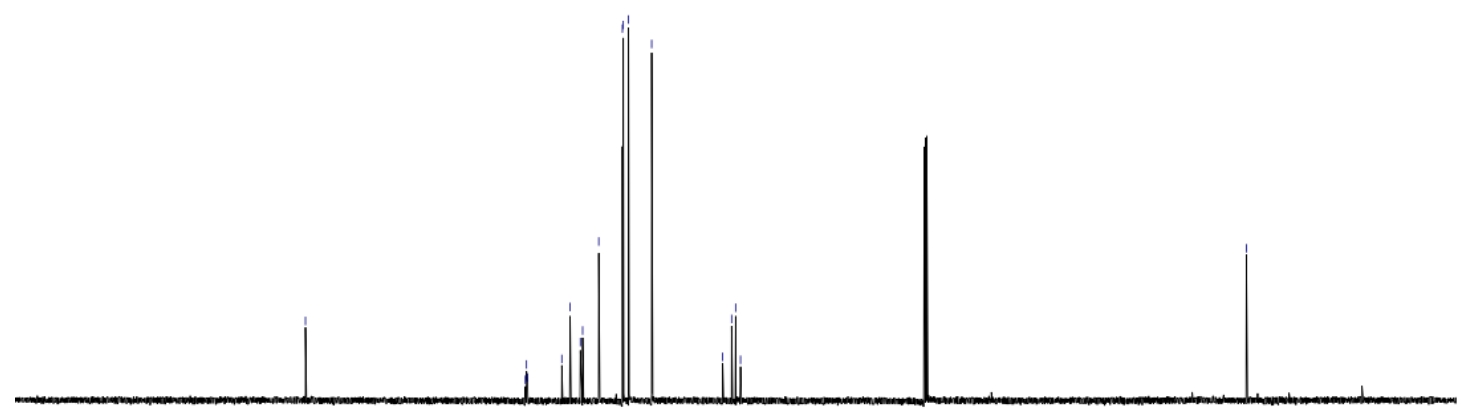

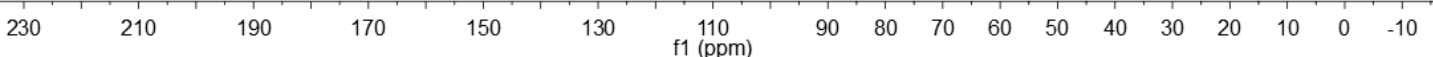



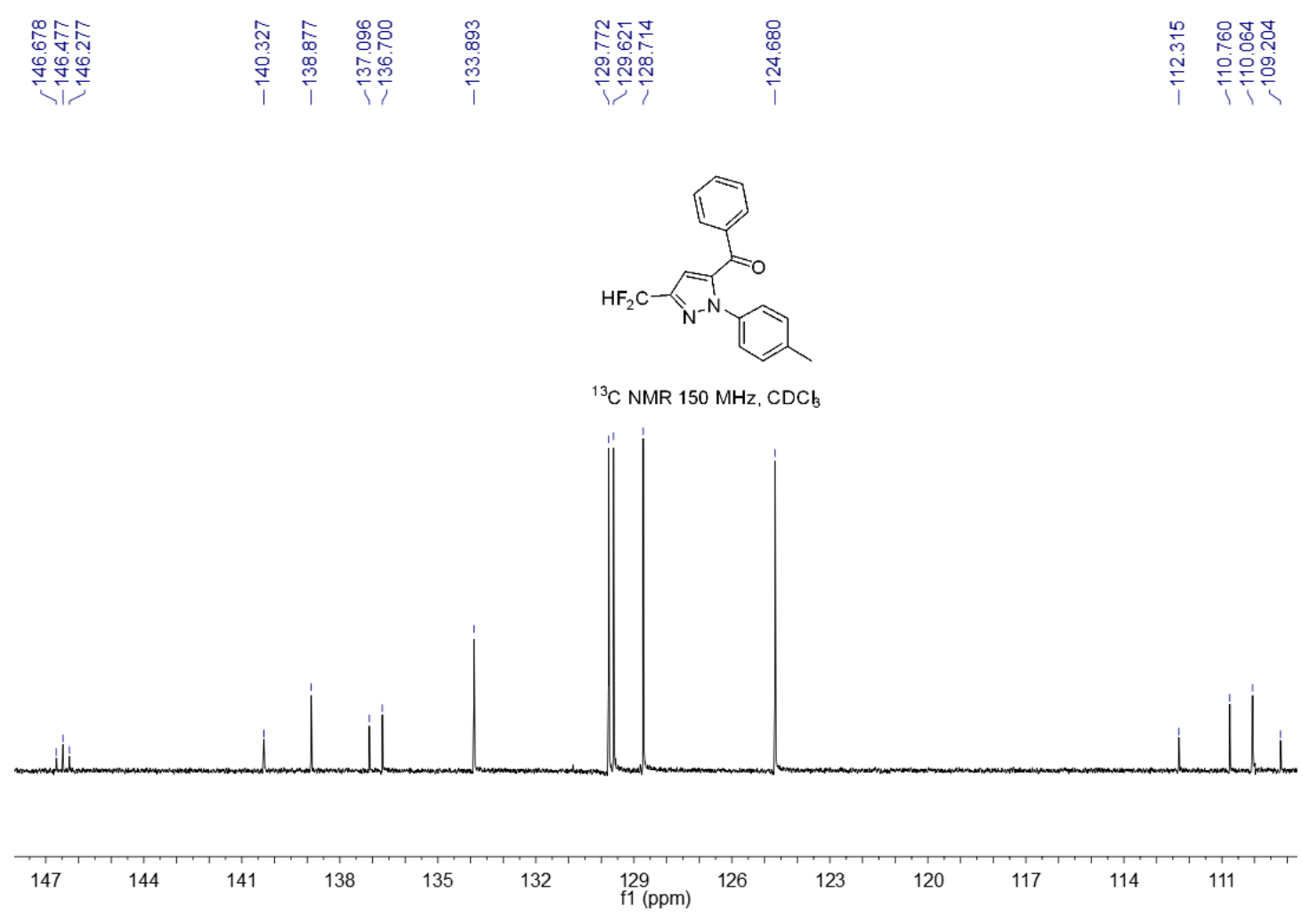

腐

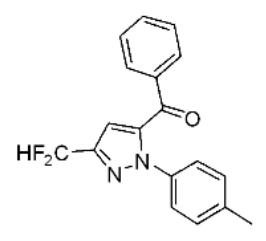

${ }^{19} \mathrm{~F}$ NMR $376 \mathrm{MHz}, \mathrm{CDCl}_{3}$

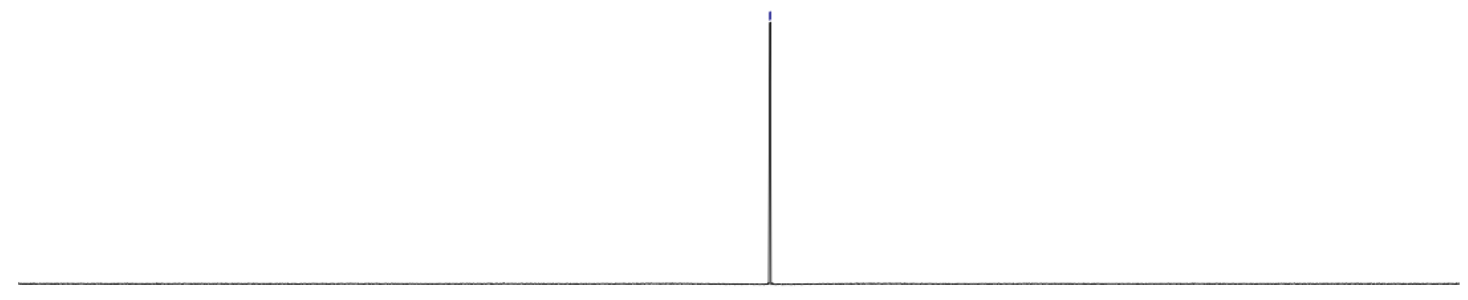

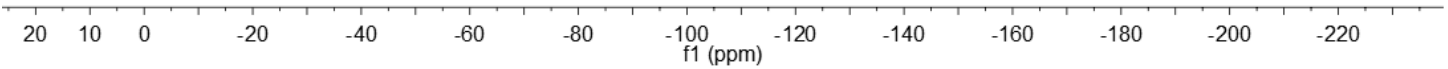




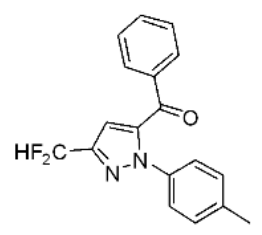

${ }^{19} \mathrm{~F}$ NMR $376 \mathrm{MHz}, \mathrm{CDCl}_{3}$

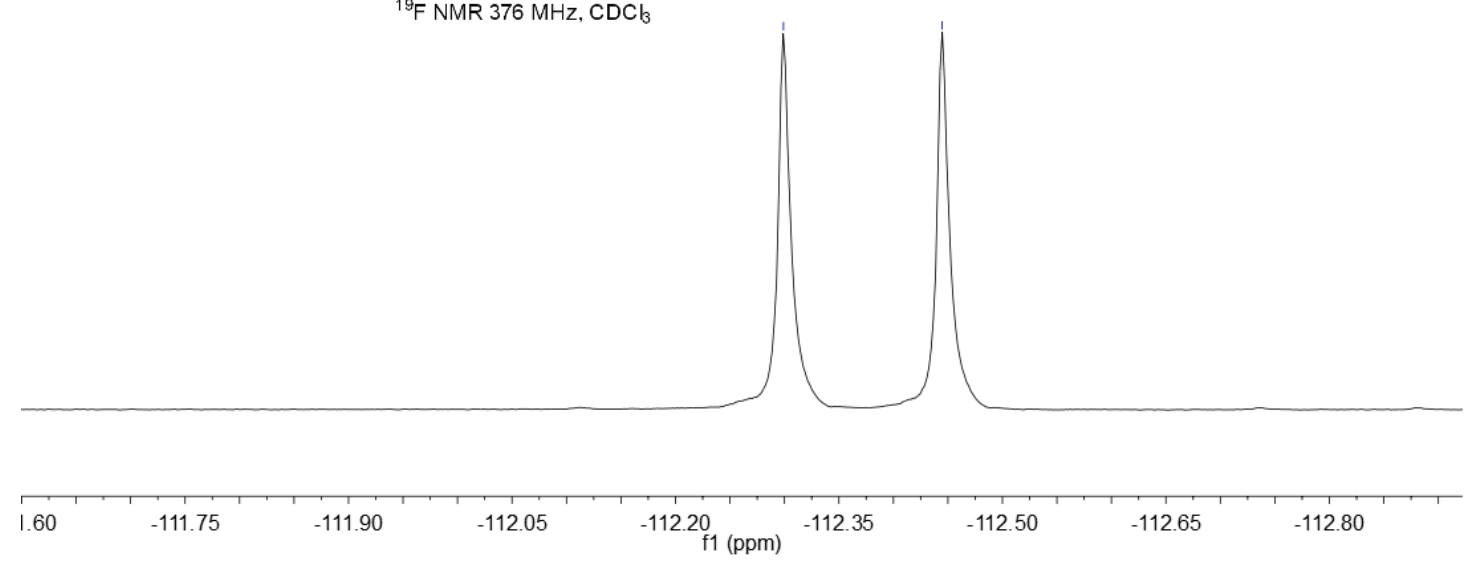

HRMS (ESI) copy of compound 3d:

\section{Mass Spectrum SmartFormula Report}

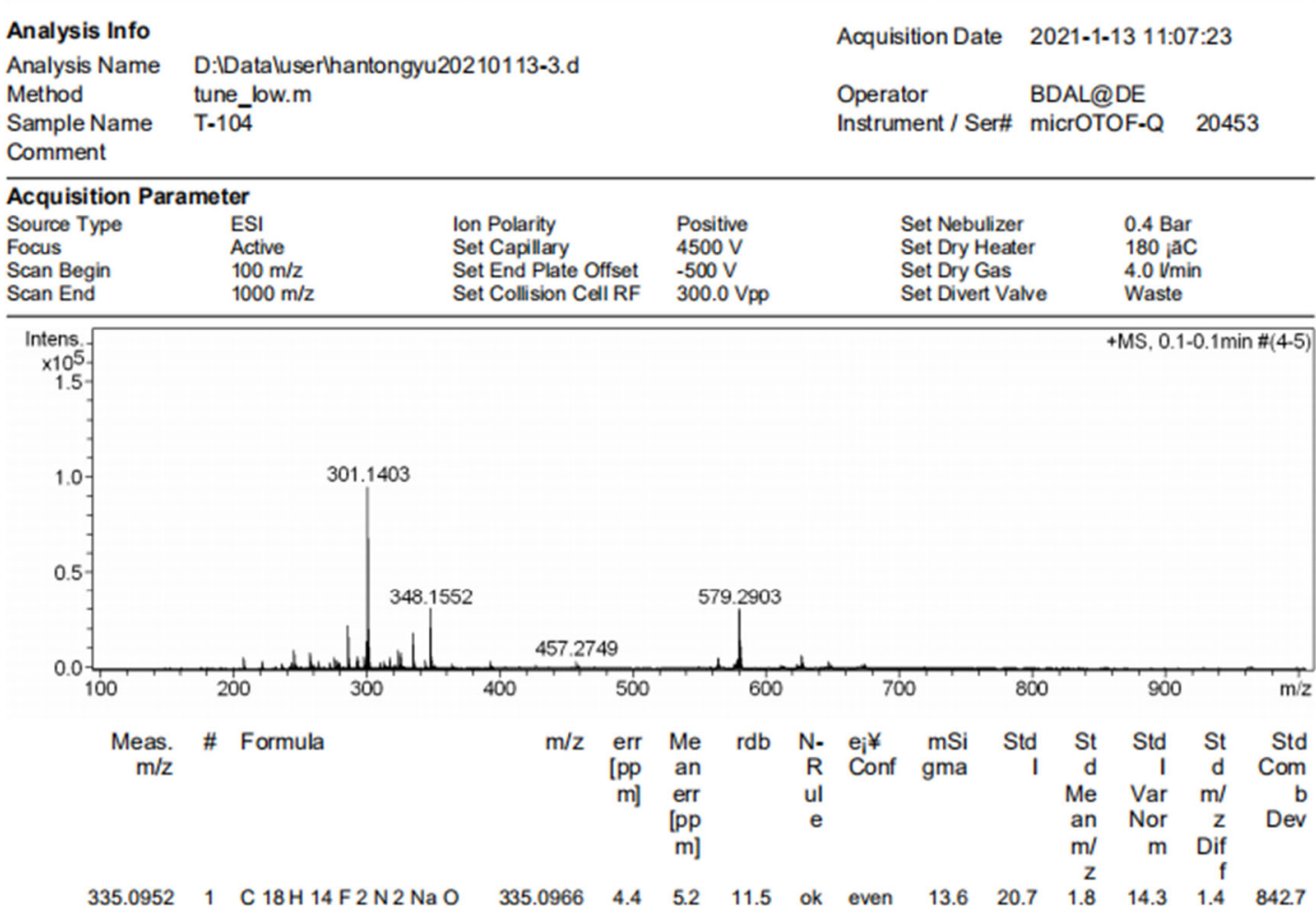


NMR copies of compound 3e:

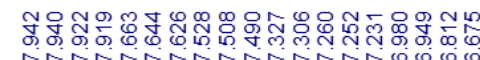

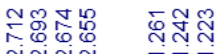

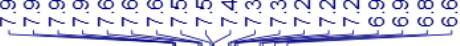

ำ

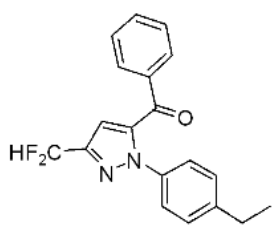

${ }^{1} \mathrm{H} \mathrm{NMR} 400 \mathrm{MHz}, \mathrm{CDCl}_{3}$

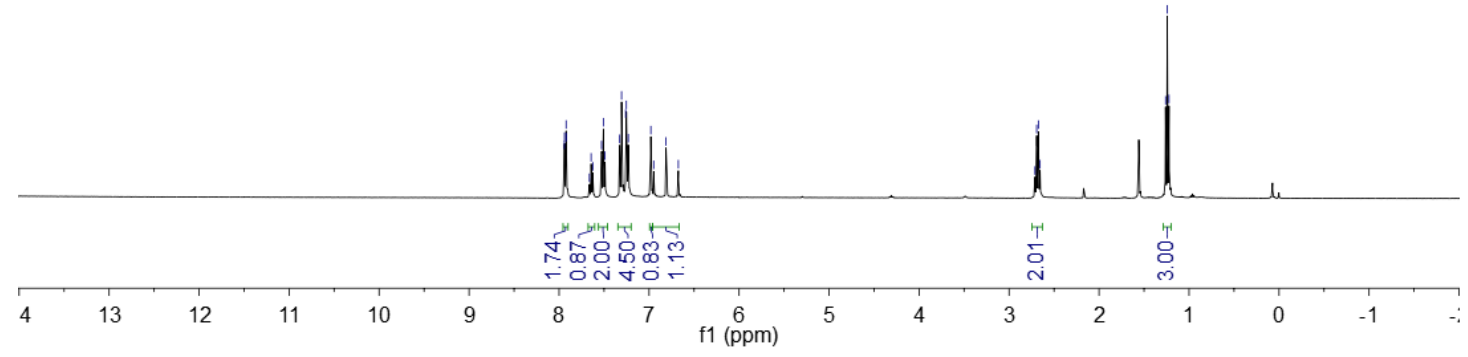

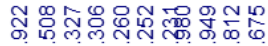

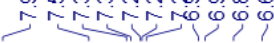

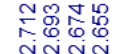

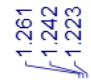

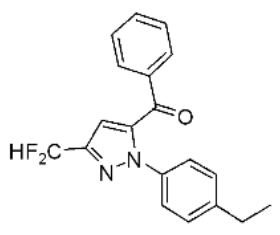

${ }^{1} \mathrm{H}$ NMR $400 \mathrm{MHz}, \mathrm{CDCl}$

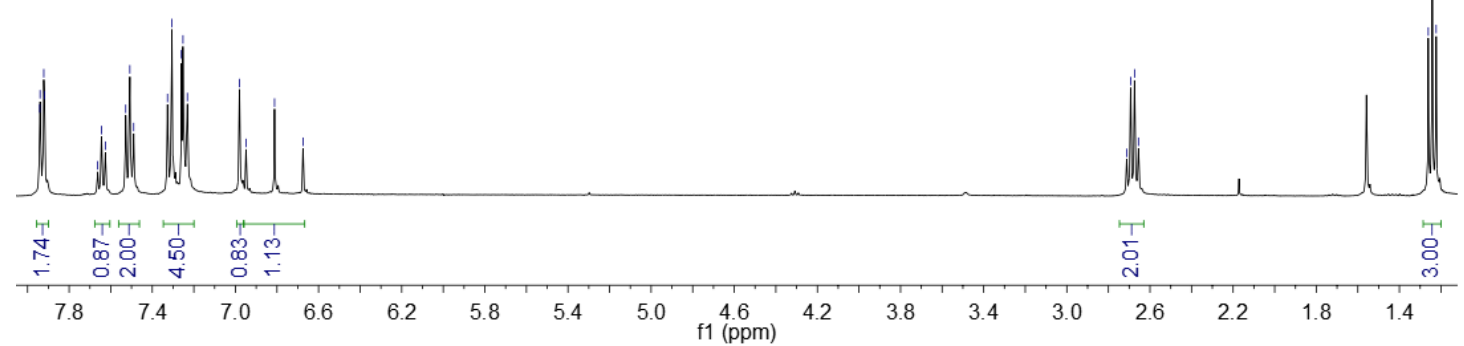




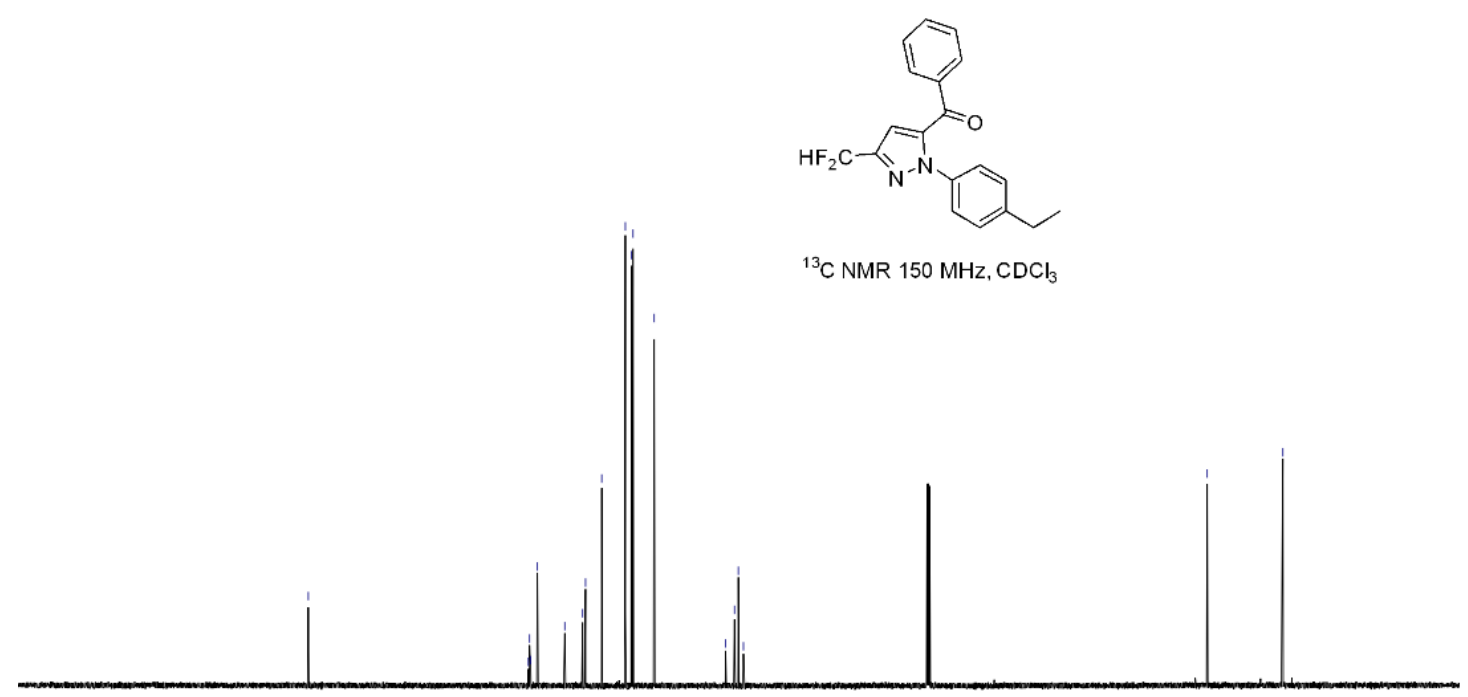

\begin{tabular}{|c|c|c|c|c|c|c|c|c|c|c|c|c|c|c|c|c|}
\hline 230 & 210 & 190 & 170 & 150 & 130 & $\begin{array}{l}110 \\
\mathrm{f} 1(\mathrm{ppm})\end{array}$ & 90 & 80 & 70 & 60 & 50 & 40 & 30 & 20 & 10 & $\begin{array}{ll}0 & -10\end{array}$ \\
\hline 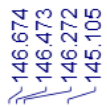 & & 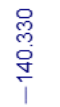 & 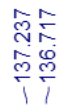 & 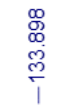 & & 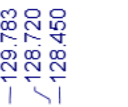 & 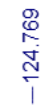 & & & & & & & & 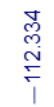 & 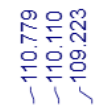 \\
\hline
\end{tabular}
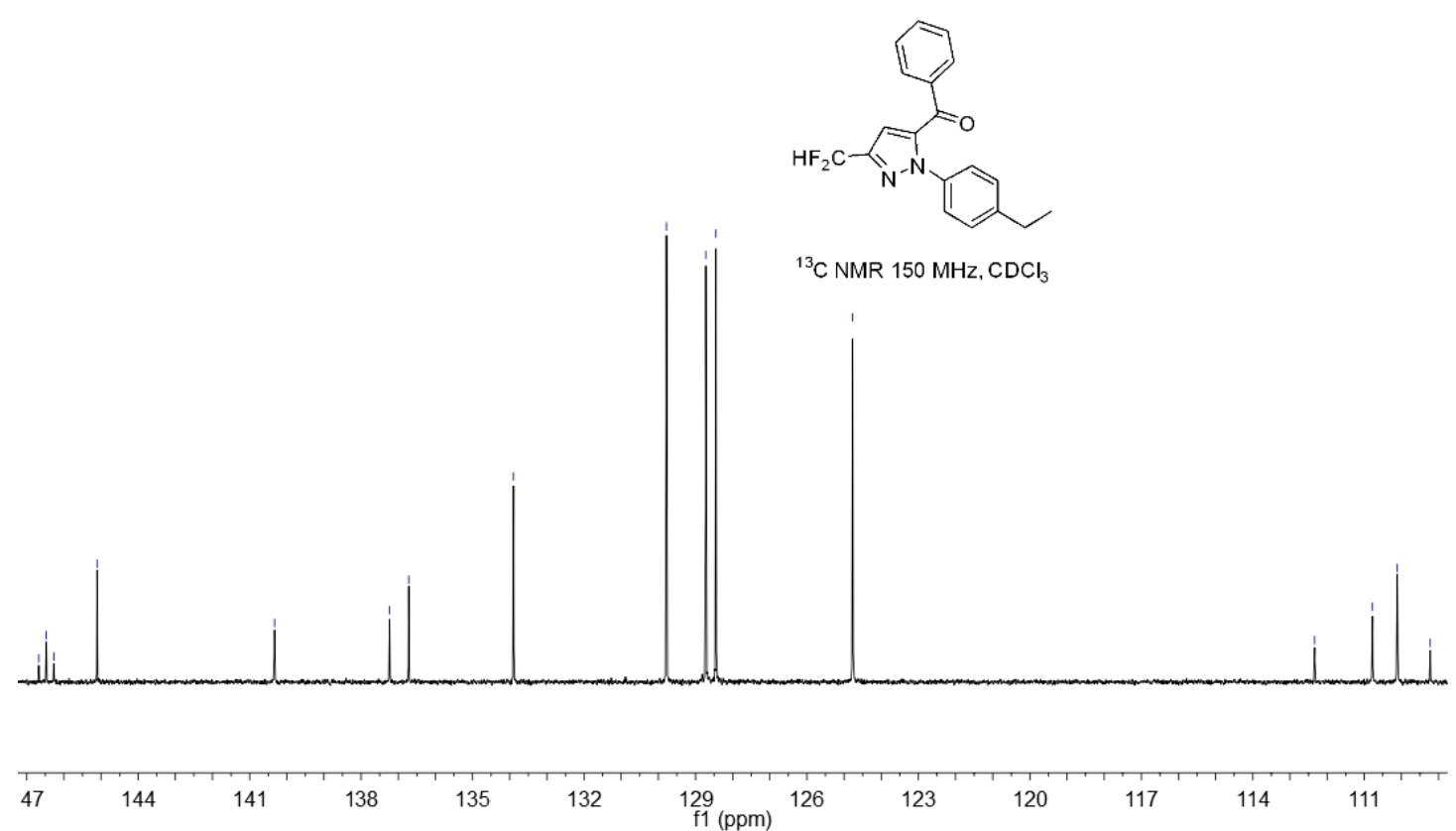


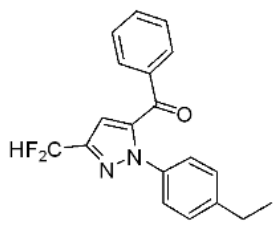

${ }^{19} \mathrm{~F} \mathrm{NMR} 376 \mathrm{MHz}, \mathrm{CDCl}_{3}$
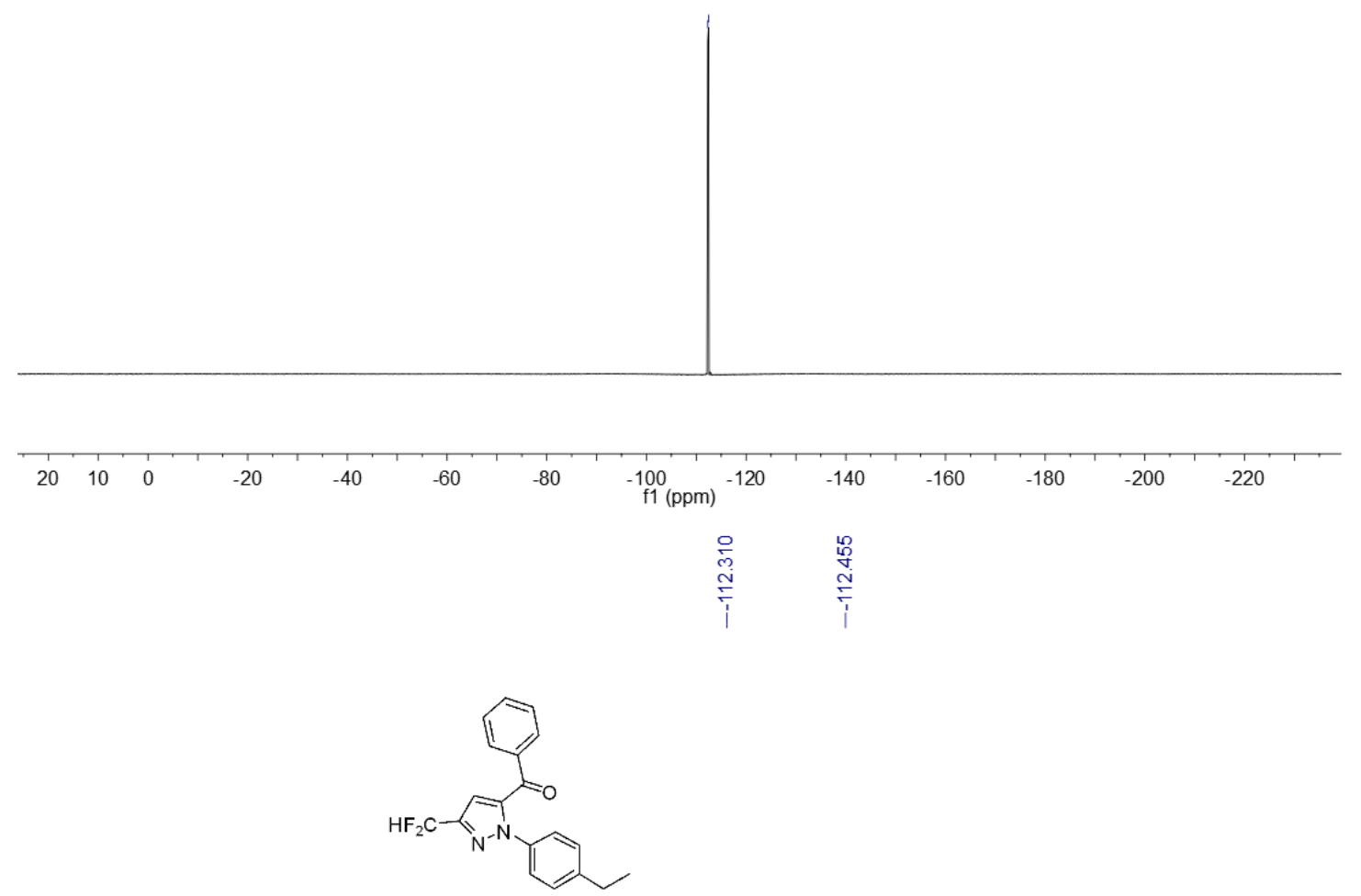

${ }^{19} \mathrm{~F}$ NMR $376 \mathrm{MHz}, \mathrm{CDCl}_{3}$

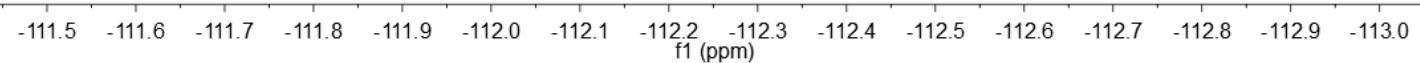


HRMS (ESI) copy of compound 3e:

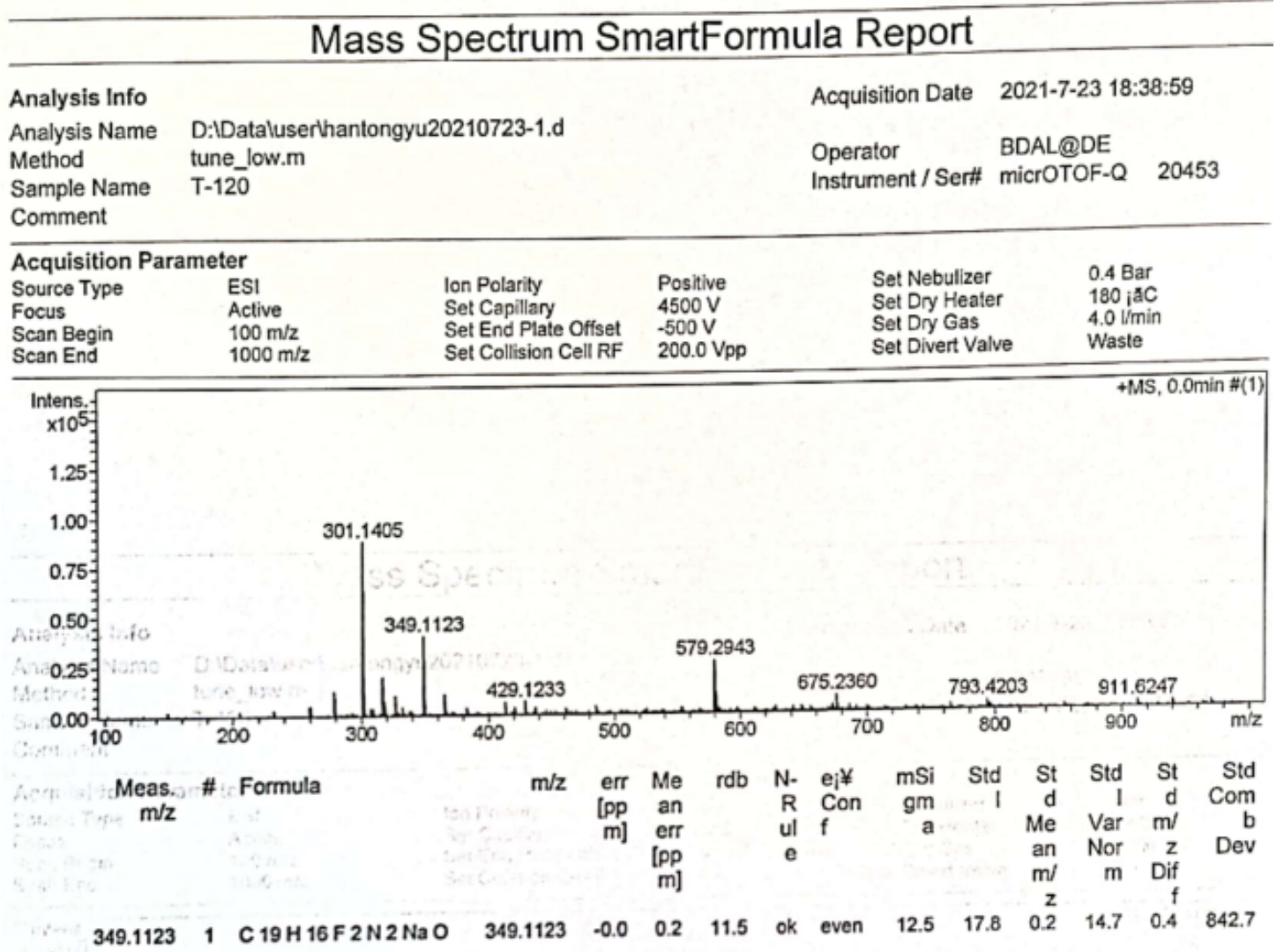

NMR copies of compound $\mathbf{3 f}$ :

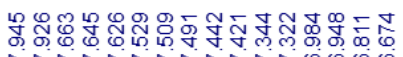

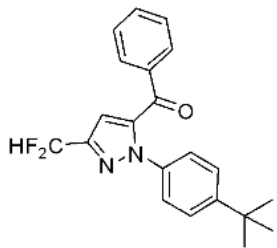

${ }^{1} \mathrm{H}$ NMR $400 \mathrm{MHz}, \mathrm{CDCl}_{3}$

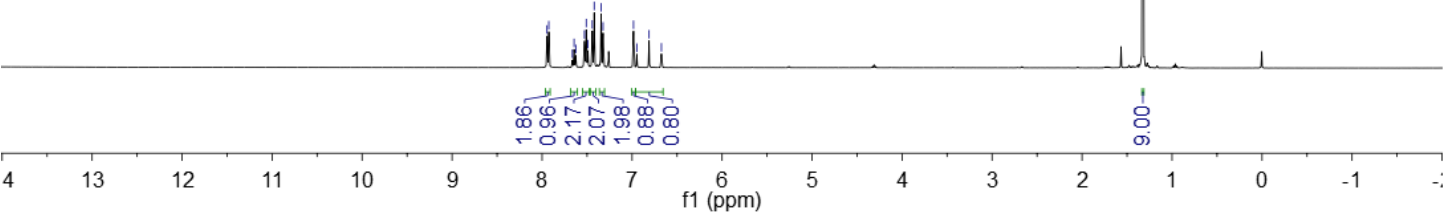




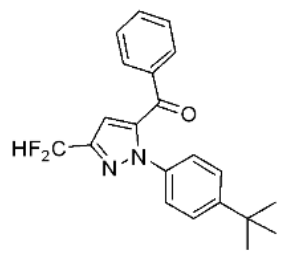

${ }^{1} \mathrm{H}$ NMR $400 \mathrm{MHz}, \mathrm{CDCl}_{3}$
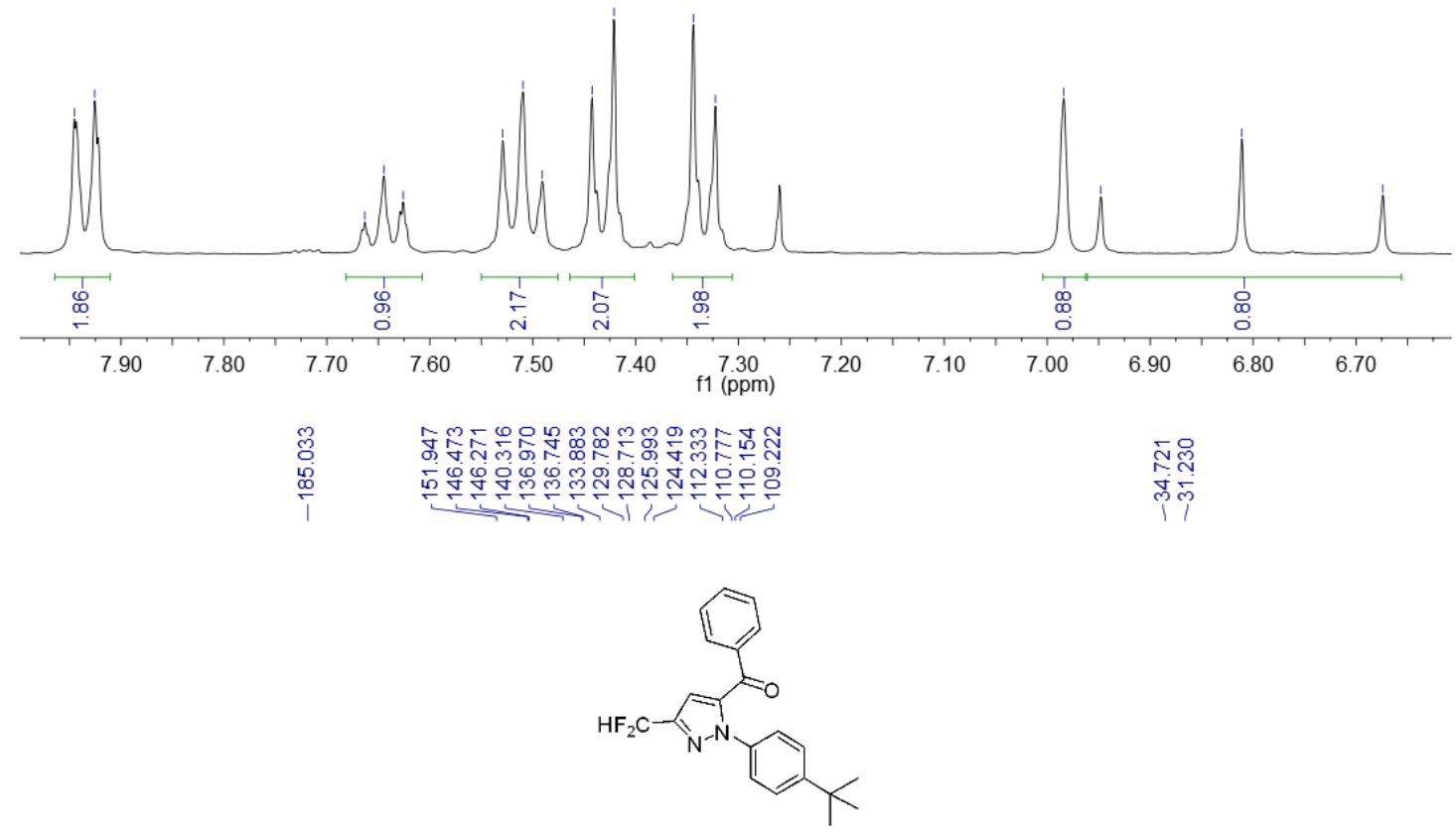

${ }^{13} \mathrm{C}$ NMR $150 \mathrm{MHz}, \mathrm{CDCl}_{3}$
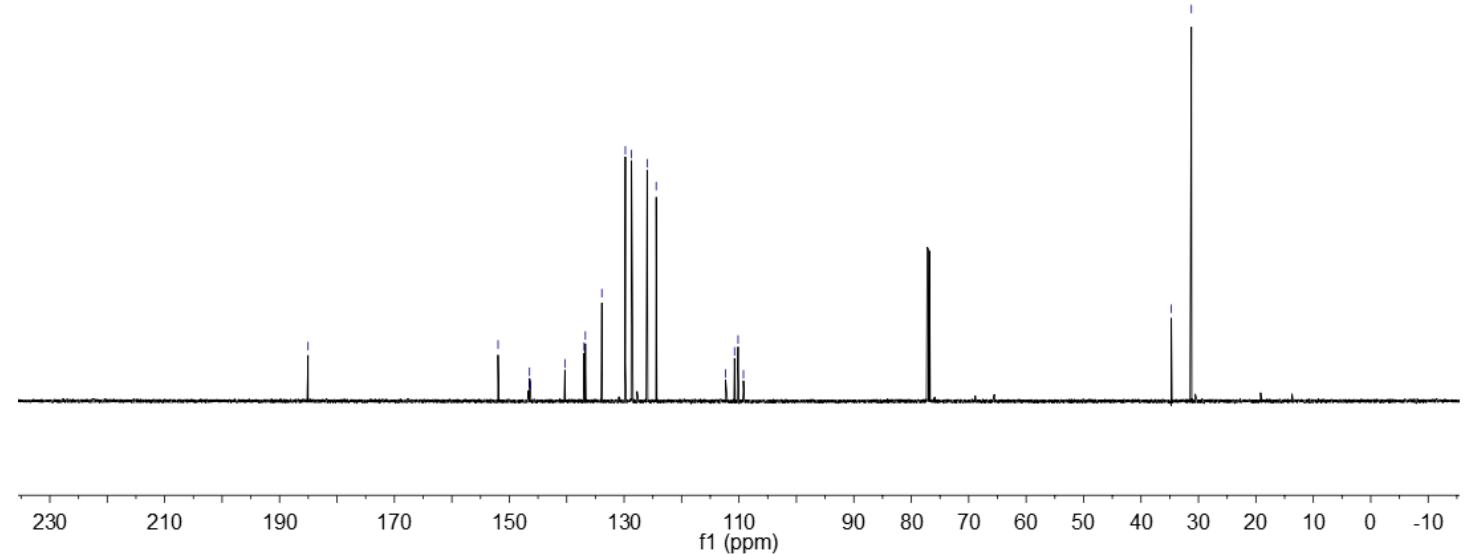


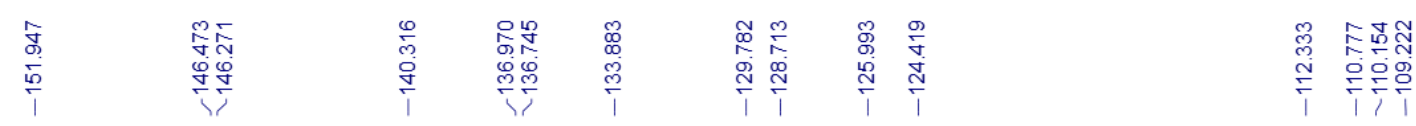

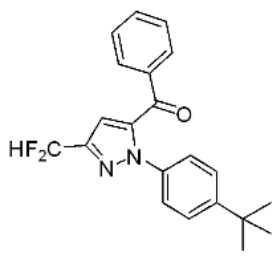

${ }^{13} \mathrm{C}$ NMR $150 \mathrm{MHz}, \mathrm{CDCl}_{3}$

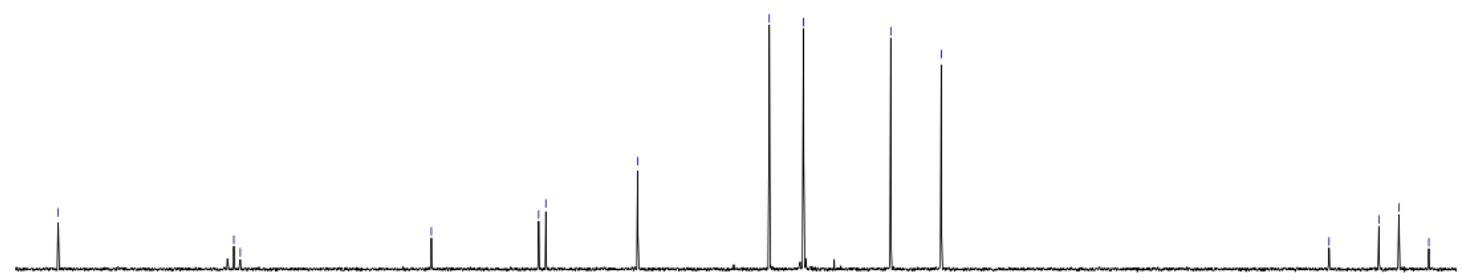

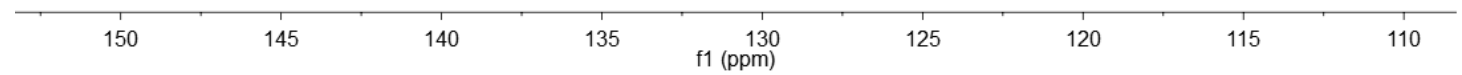

寅哭

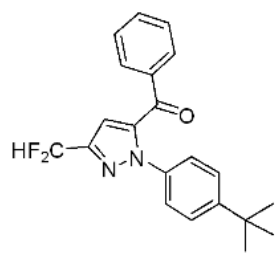

${ }^{19} \mathrm{~F} \mathrm{NMR} 376 \mathrm{MHz}, \mathrm{CDCl}_{3}$

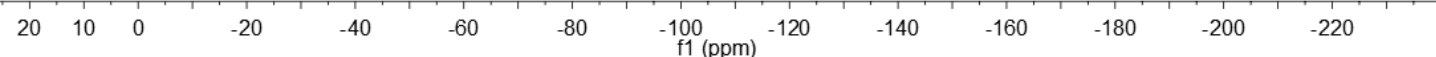




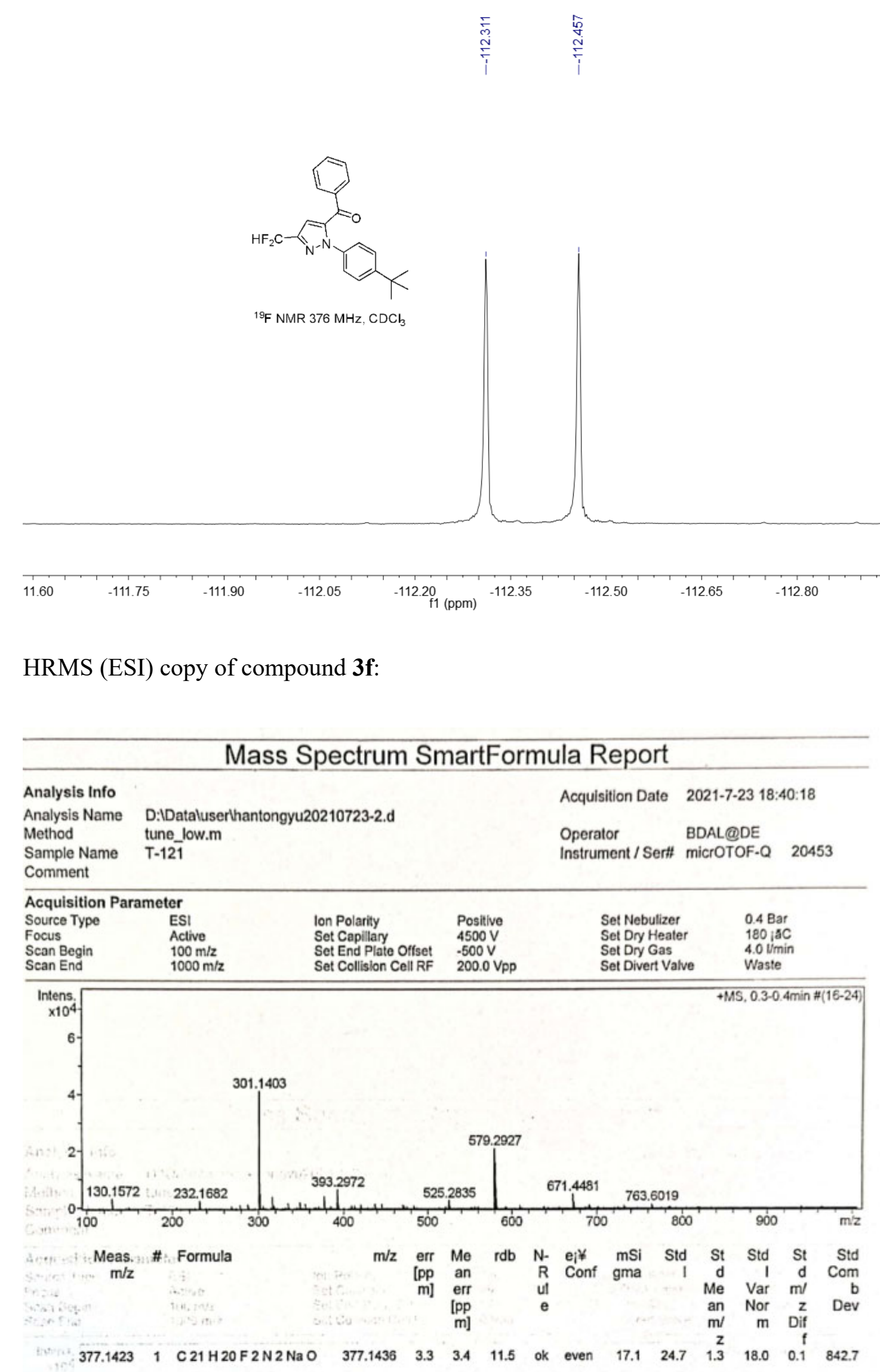




\section{NMR copies of compound 3g:}

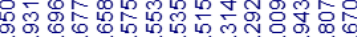

.

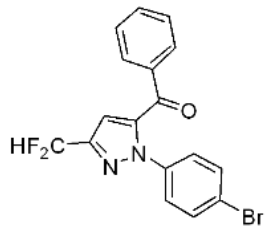

${ }^{1} \mathrm{H}$ NMR $400 \mathrm{MHz}, \mathrm{CDCl}_{3}$
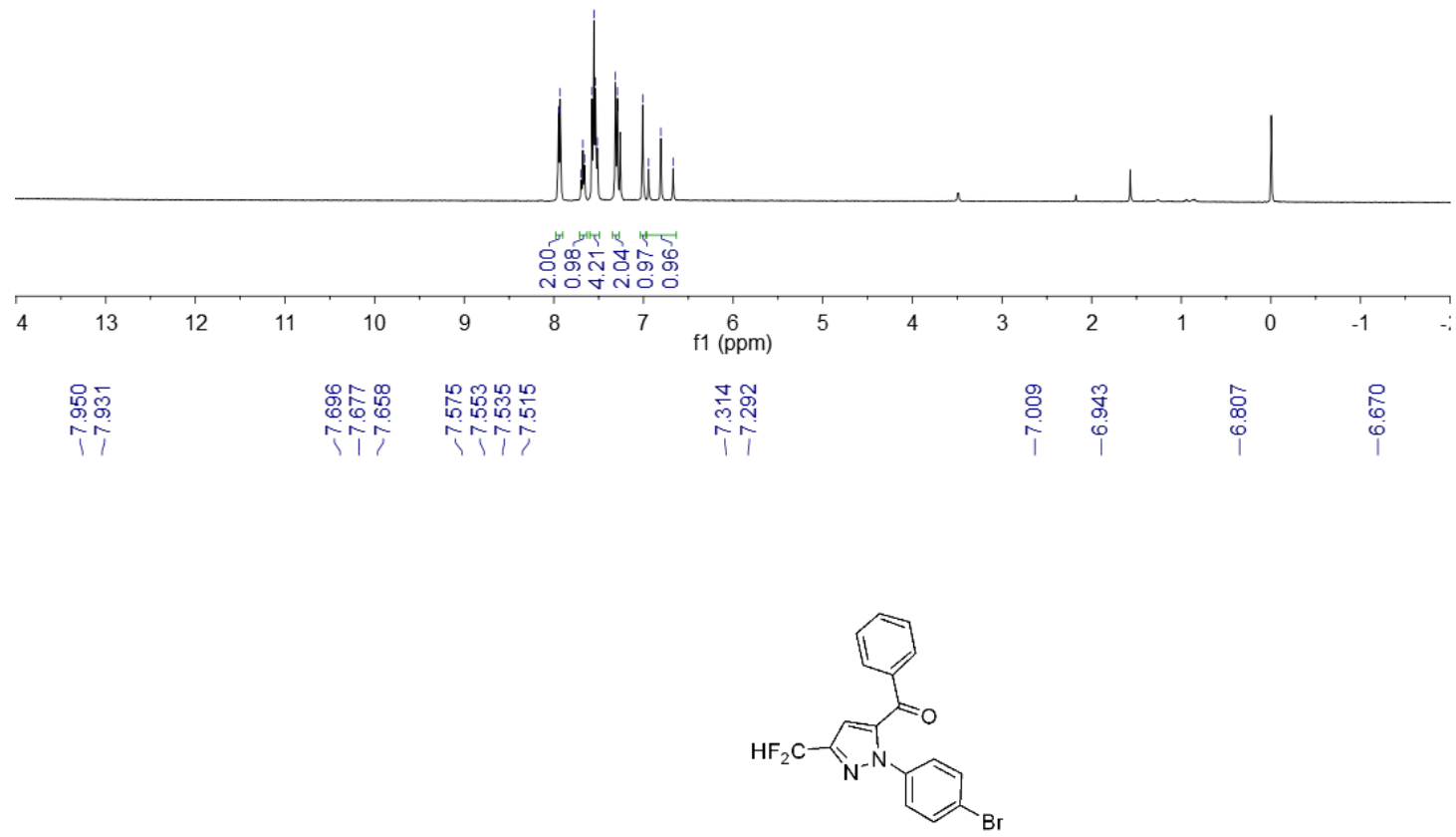

${ }^{1} \mathrm{H} \mathrm{NMR} 400 \mathrm{MHz}, \mathrm{CDCl}_{3}$

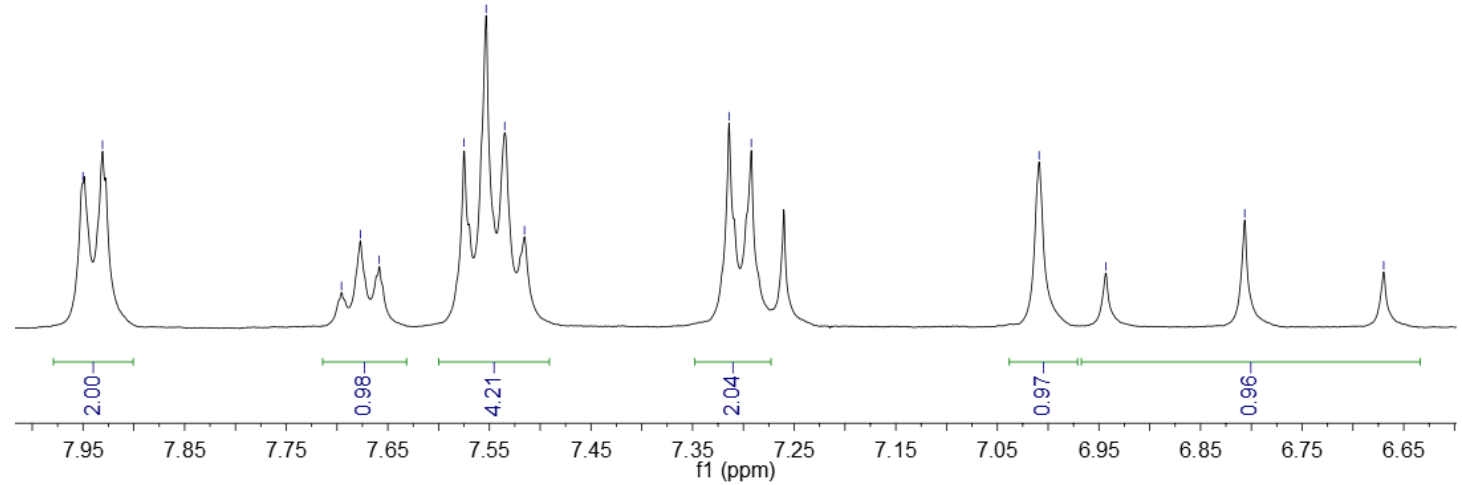



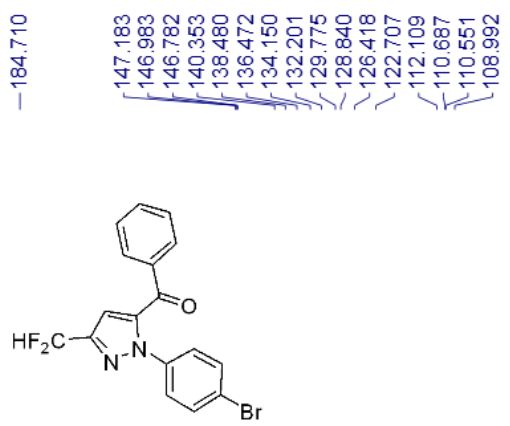

${ }^{13} \mathrm{C}$ NMR $150 \mathrm{MHz}, \mathrm{CDCl}$

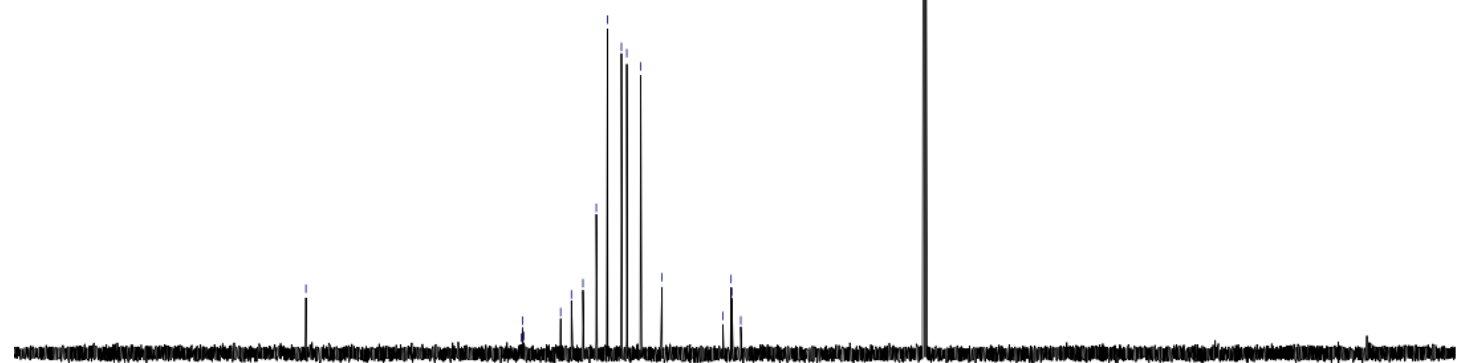

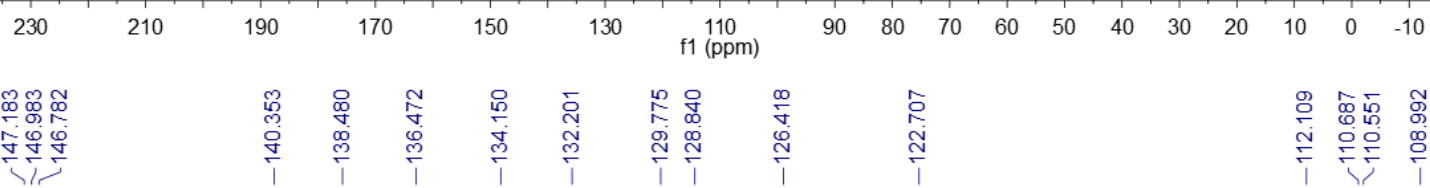

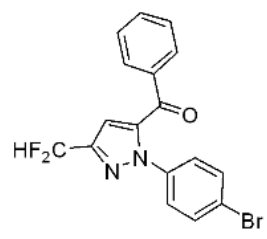

${ }^{13} \mathrm{C} \mathrm{NMR} 150 \mathrm{MHz}, \mathrm{CDCl}$

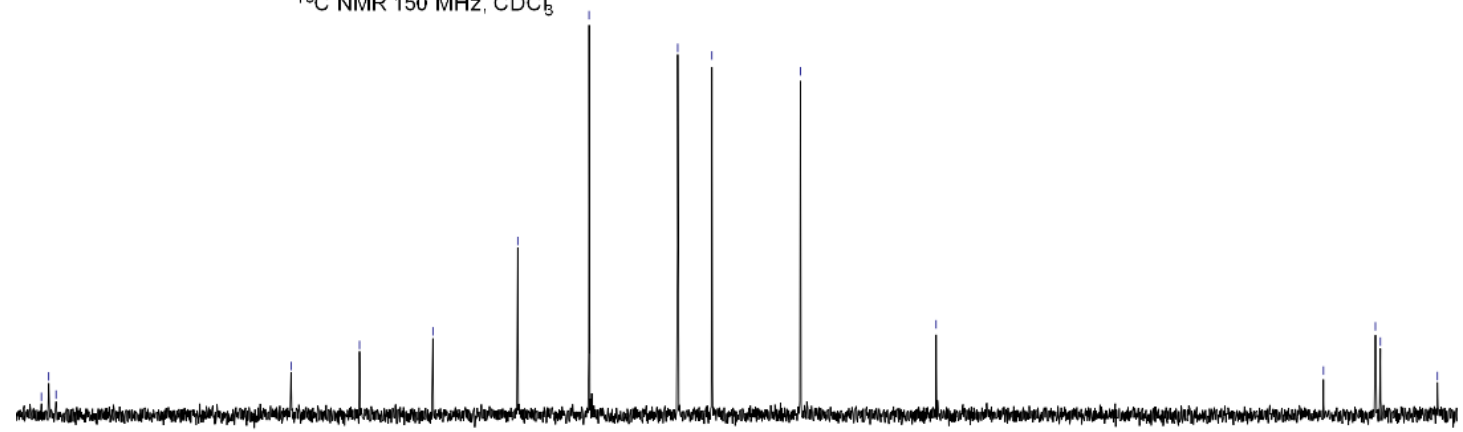

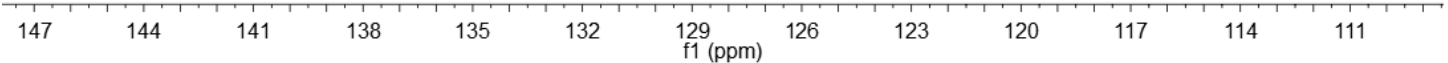



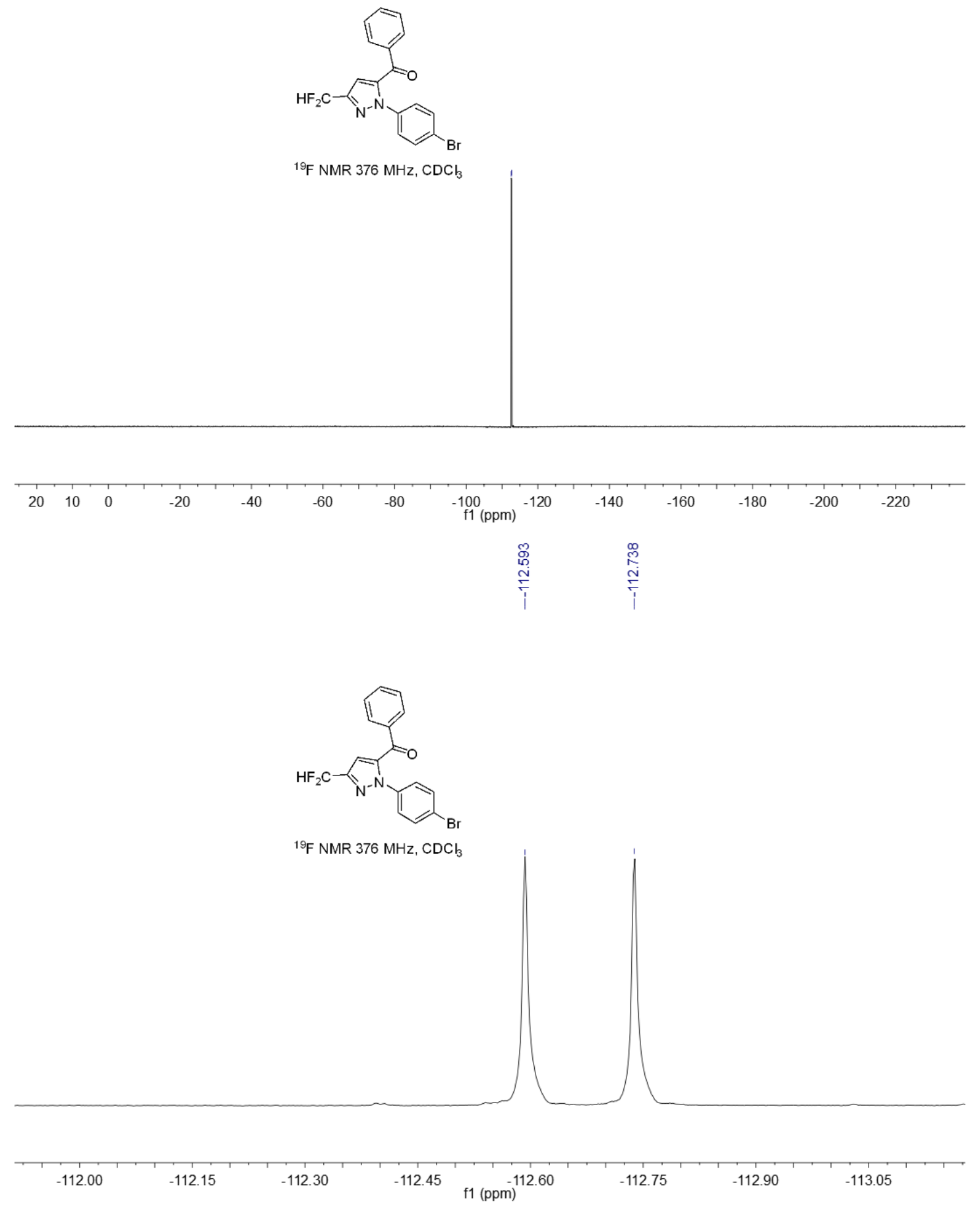
HRMS (ESI) copy of compound $\mathbf{3 g}$ :

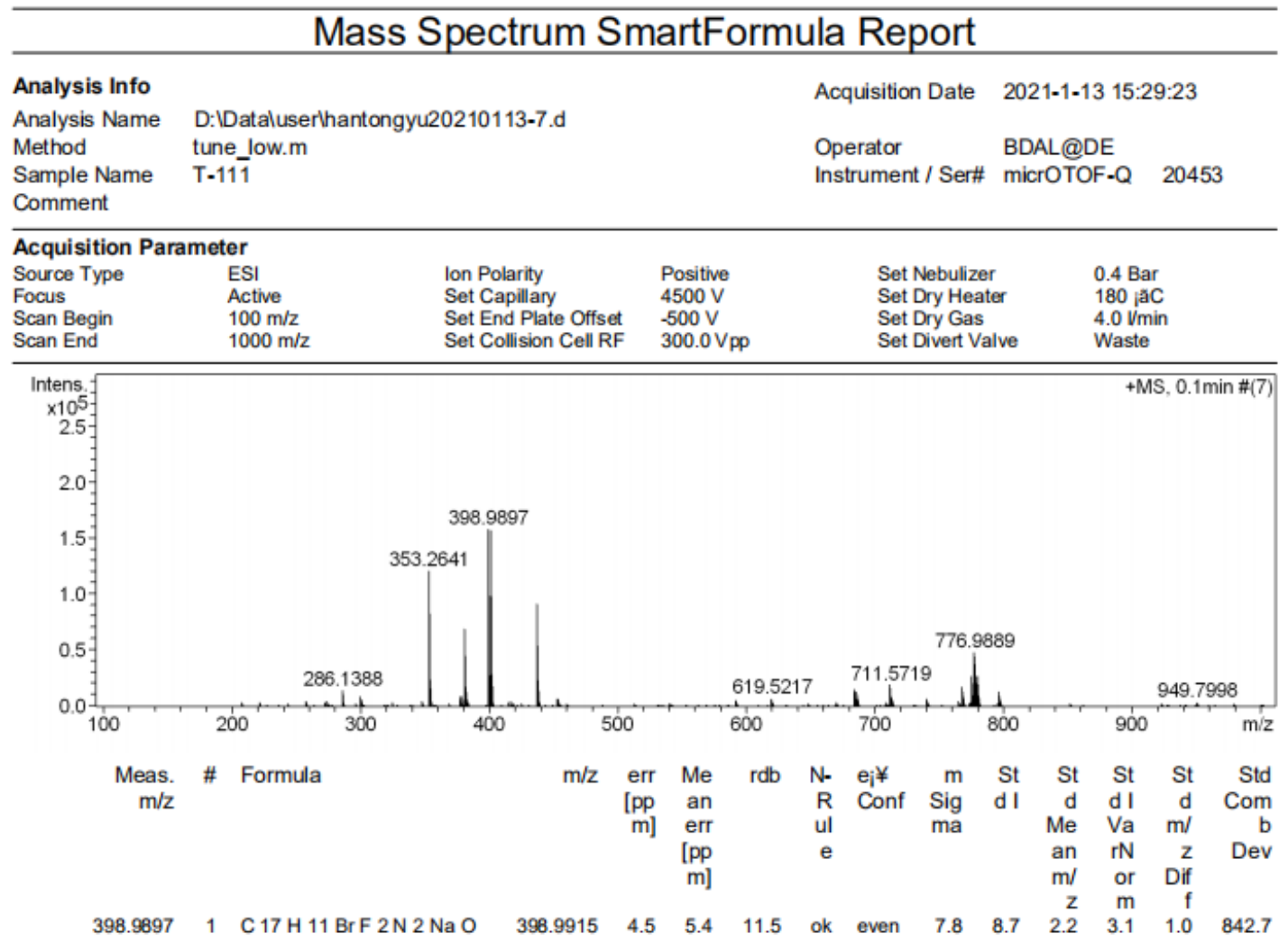

NMR copies of compound $\mathbf{3 h}$ :

\section{幽占

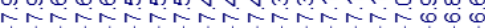

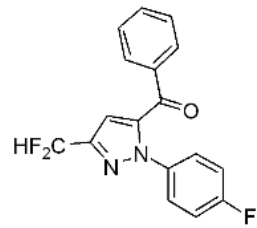

${ }^{1} \mathrm{H}$ NMR $400 \mathrm{MHz}, \mathrm{CDCl}$

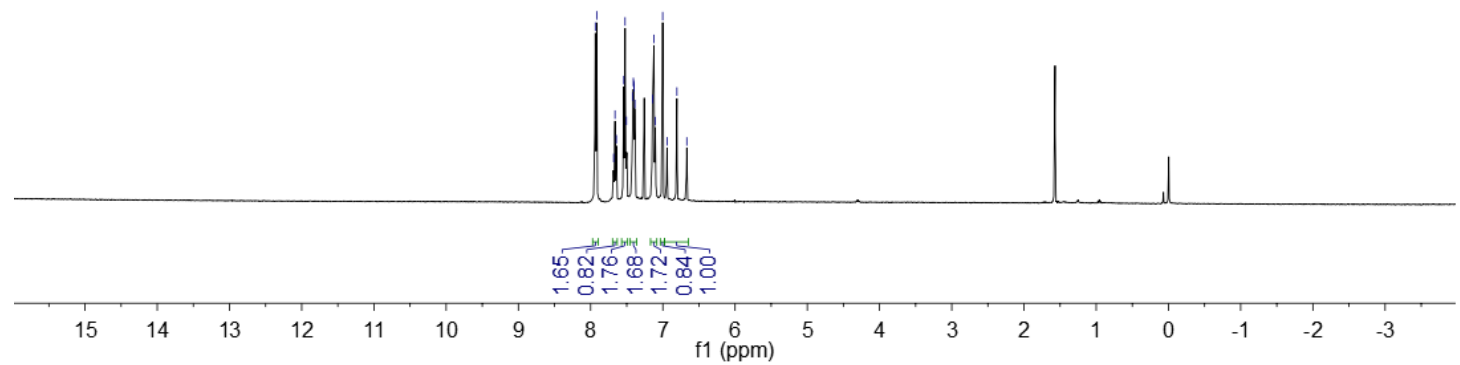




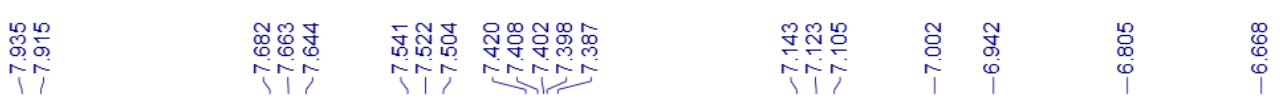<smiles>Cc1cc(-c2ccccc2)n(-c2ccc(F)cc2)n1</smiles>

${ }^{1} \mathrm{H} \mathrm{NMR} 400 \mathrm{MHz}, \mathrm{CDCl}_{3}$

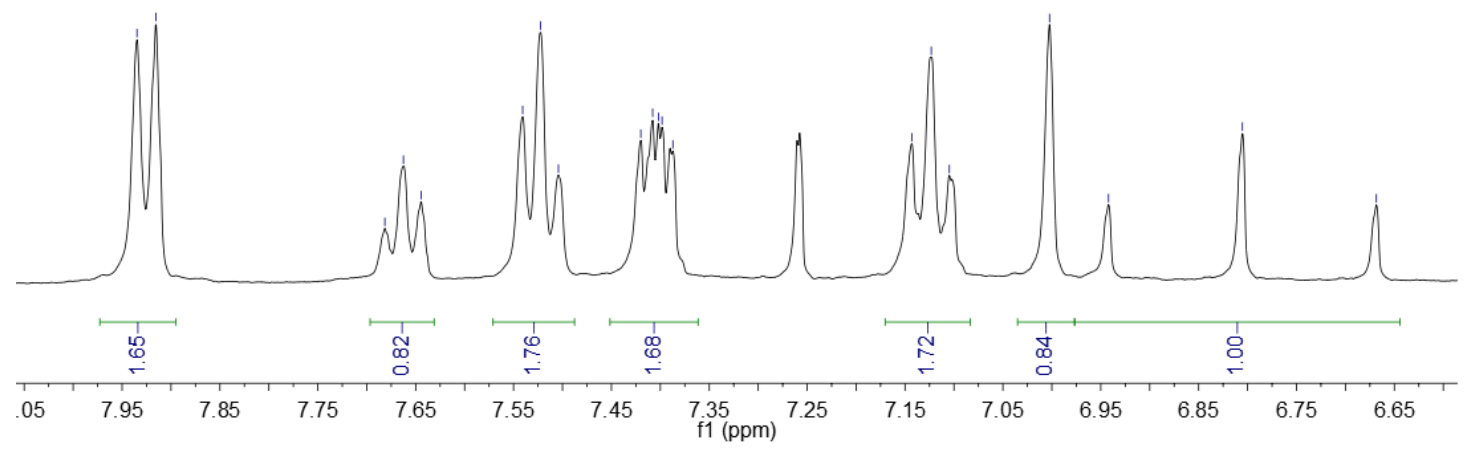

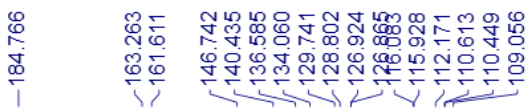

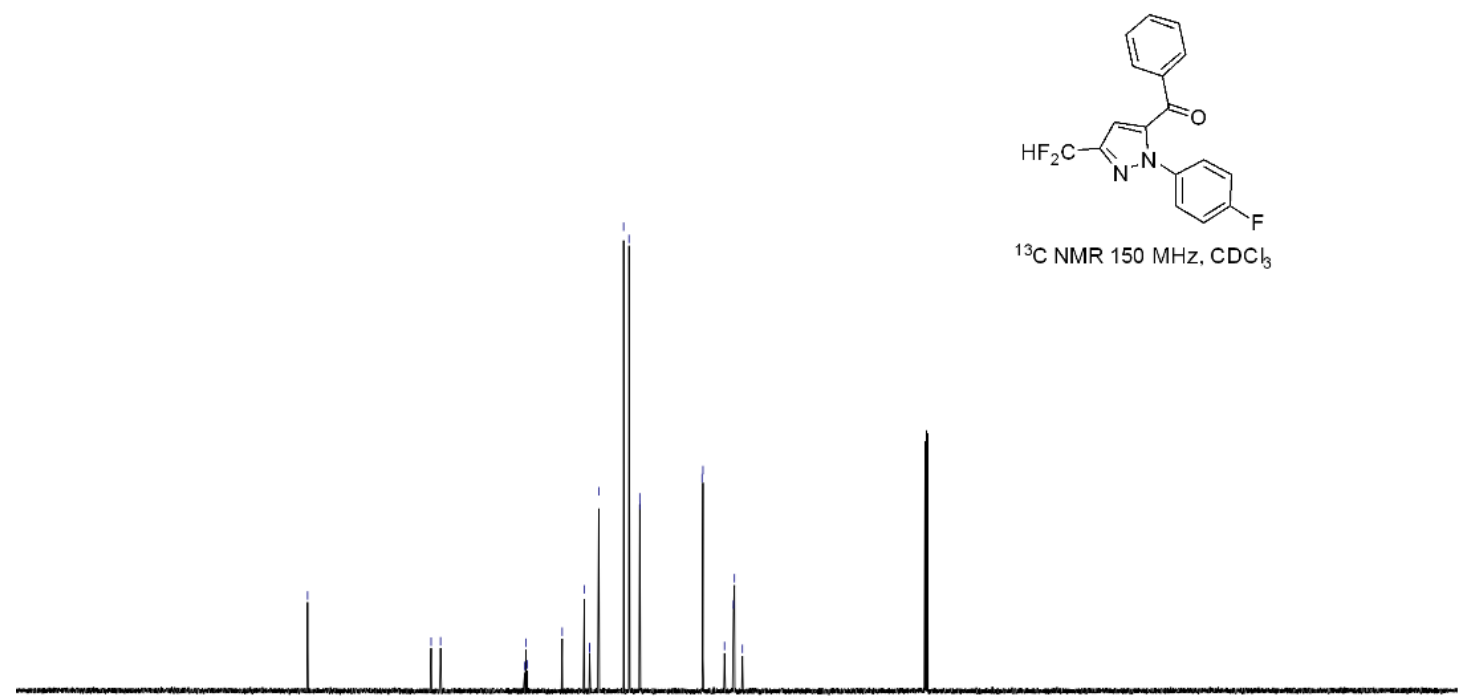

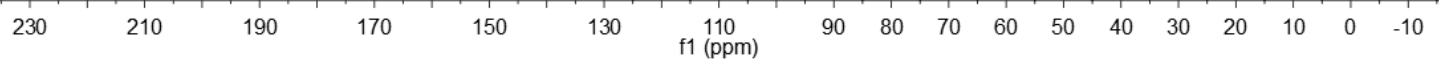



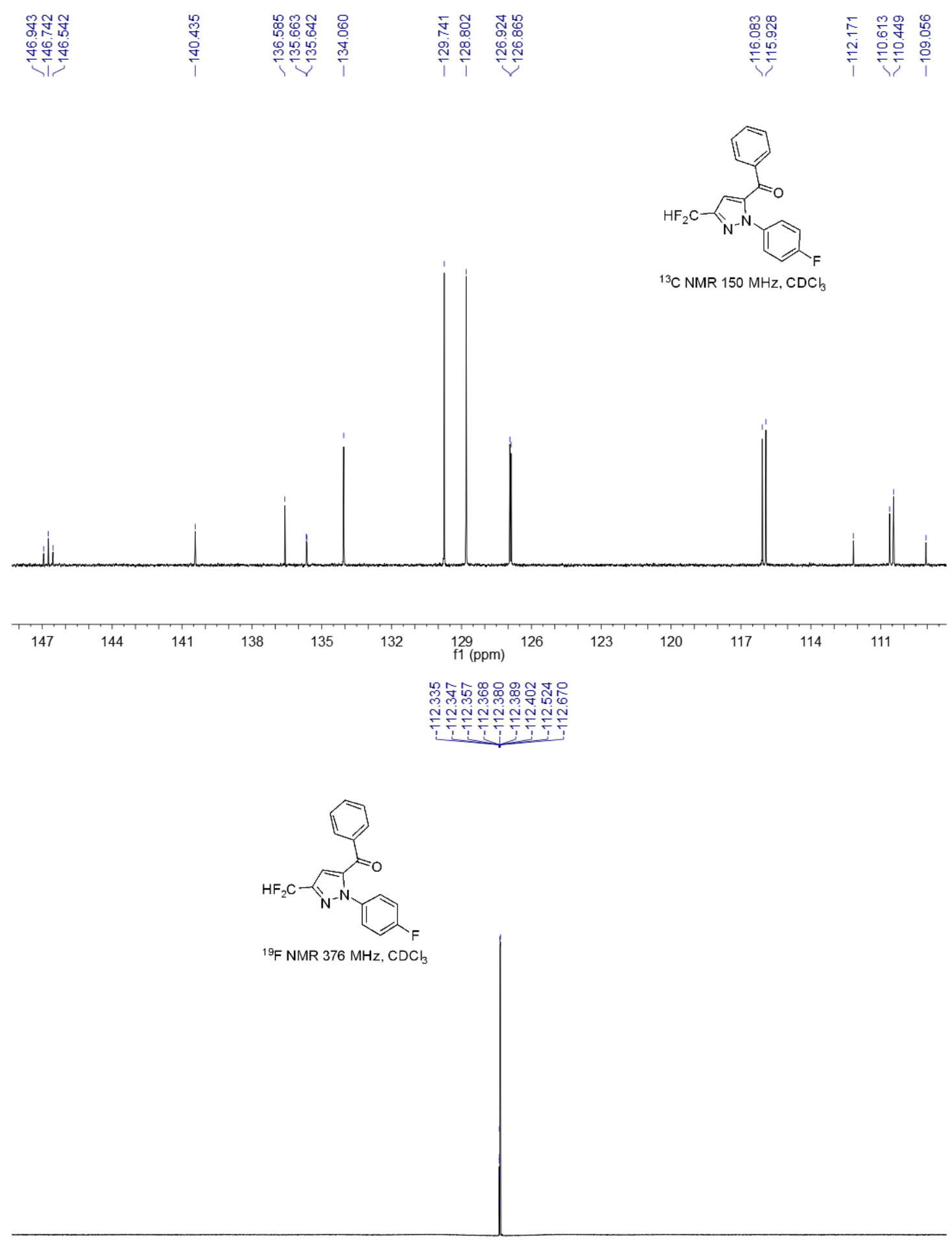

\begin{tabular}{|c|c|c|c|c|c|c|c|c|c|c|c|c|c|}
\hline 20 & 10 & 0 & -20 & -40 & -60 & -80 & -100 & -120 & -140 & -160 & -180 & -200 & -220 \\
\hline
\end{tabular}




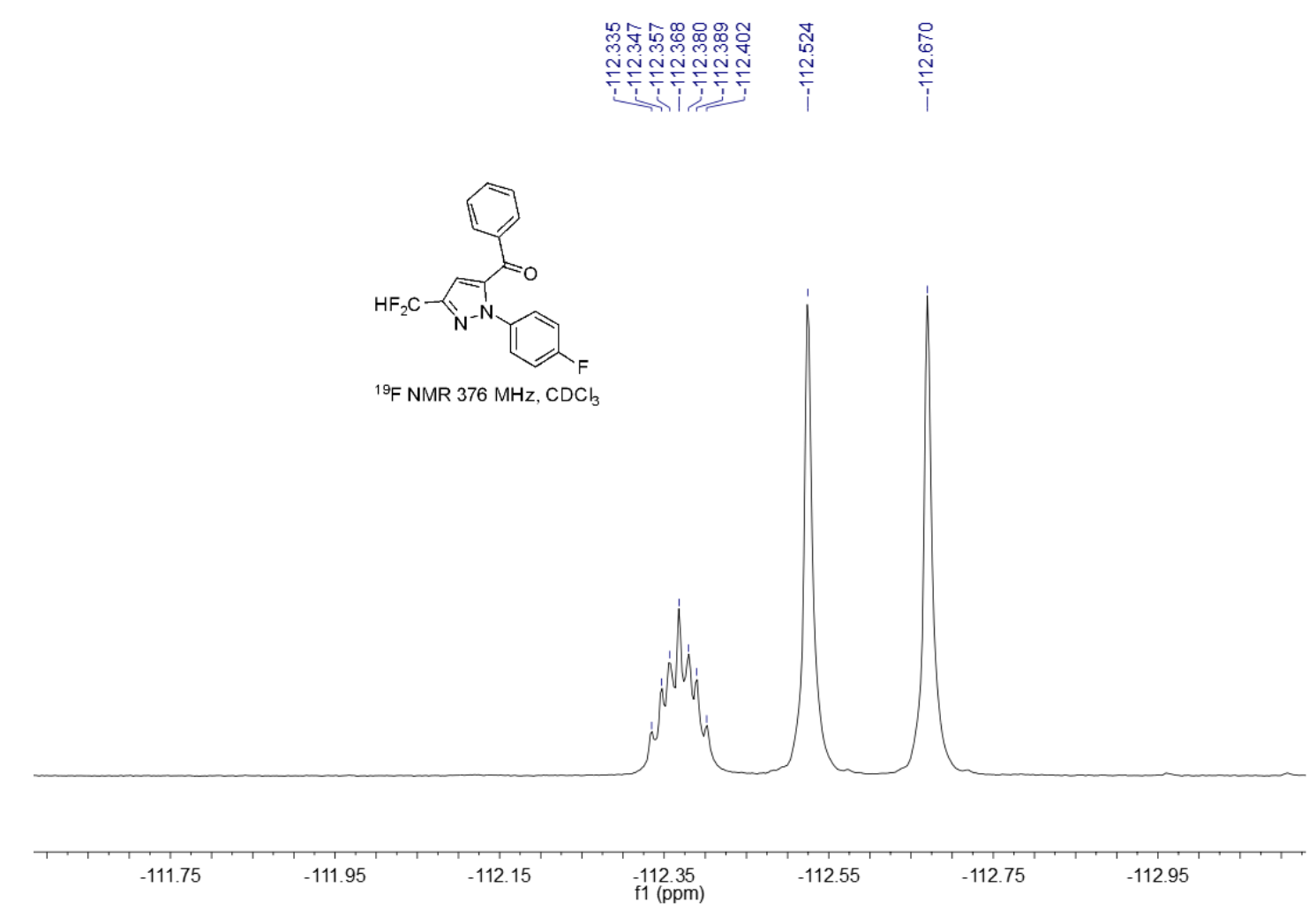

HRMS (ESI) copy of compound $\mathbf{3 h}$ :

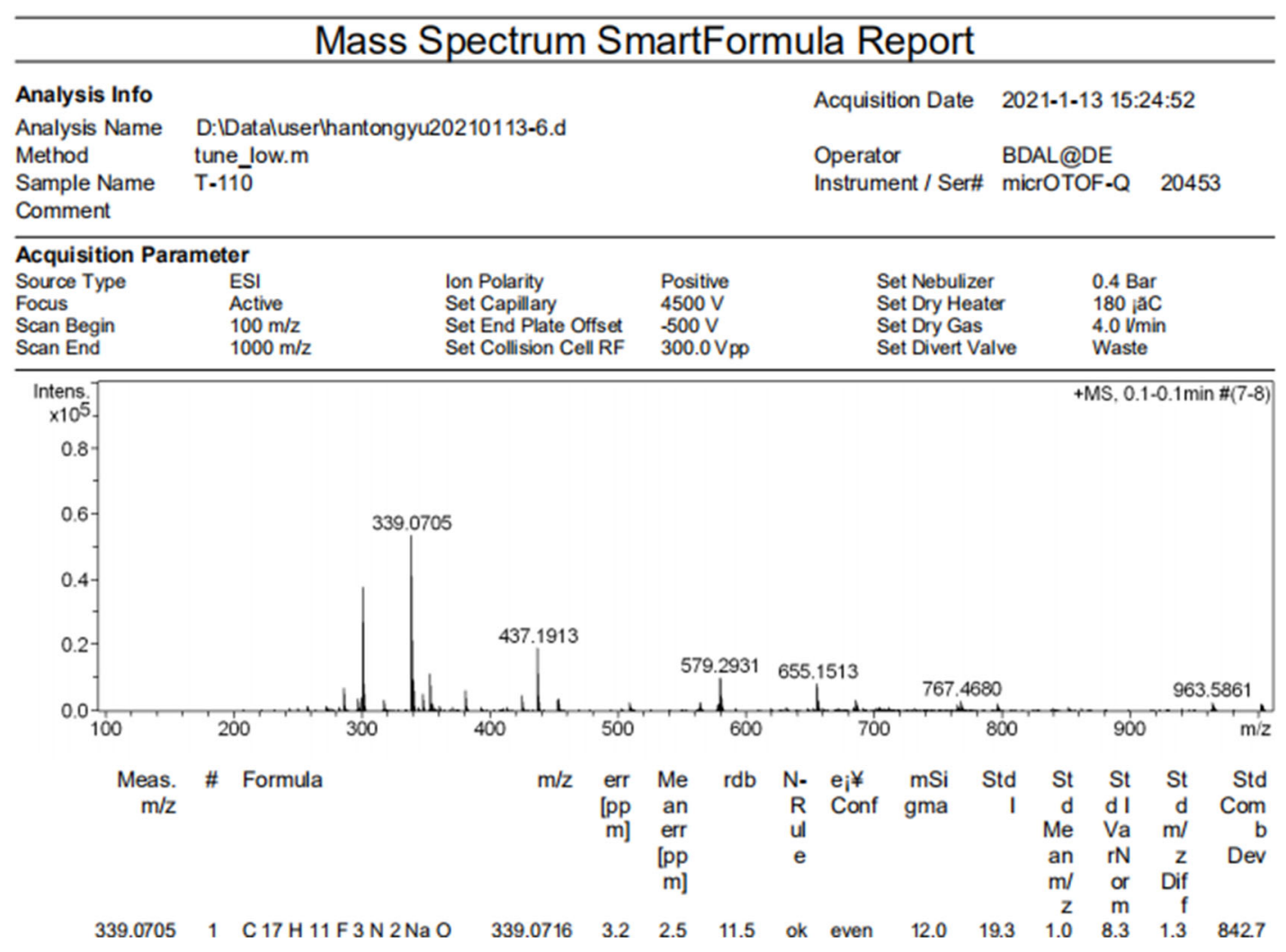


NMR copies of compound 3i:

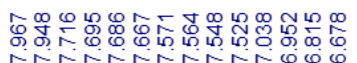

rananange0

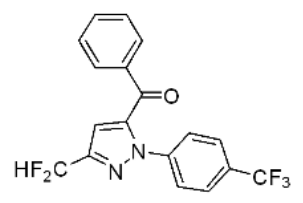

${ }^{1} \mathrm{H} \mathrm{NMR} 400 \mathrm{MHz}, \mathrm{CDCl}_{3}$

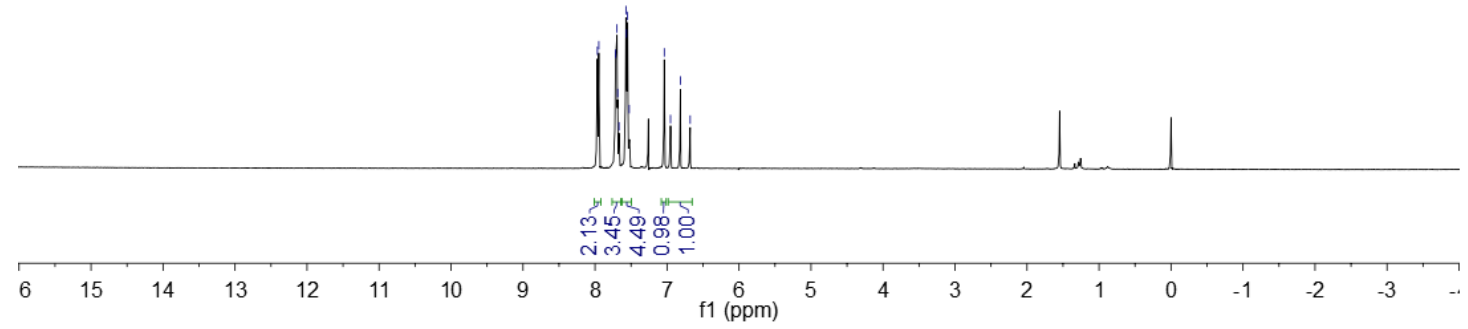

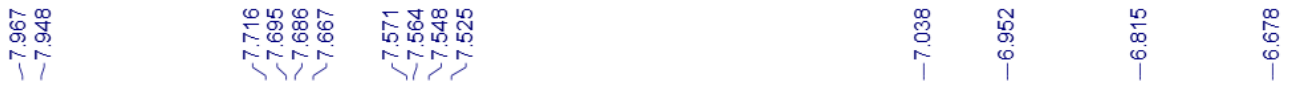

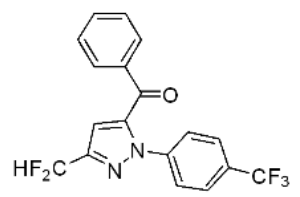

${ }^{1} \mathrm{HNMR} 400 \mathrm{MHz}, \mathrm{CDCl}_{3}$

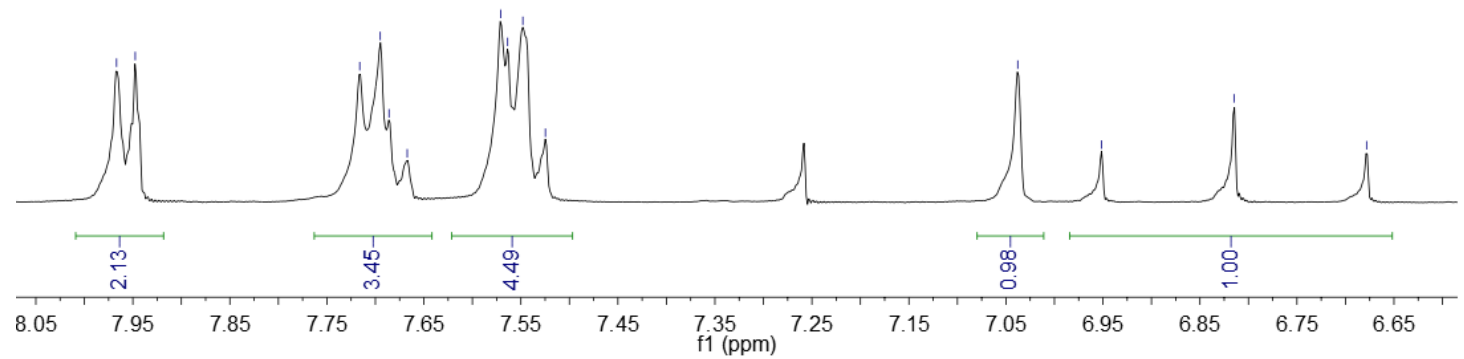




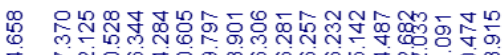

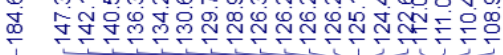

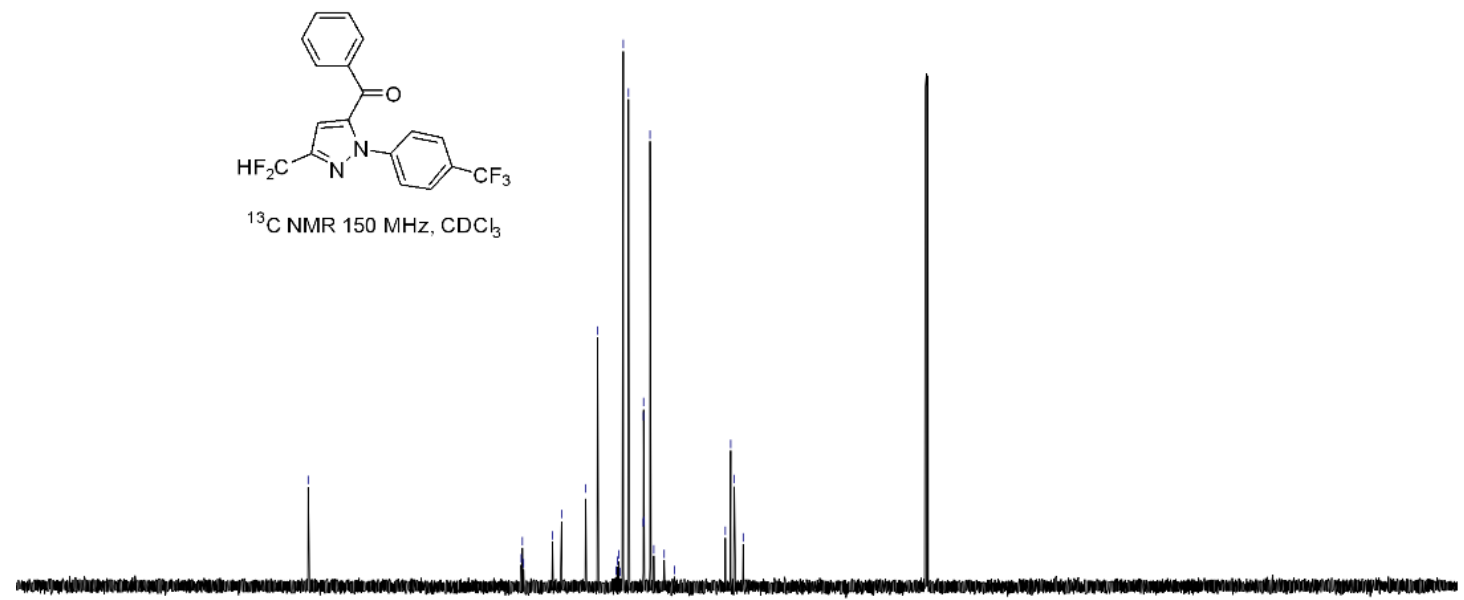

${ }^{13} \mathrm{C} \mathrm{NMR} 150 \mathrm{MHz}, \mathrm{CDCl}_{3}$

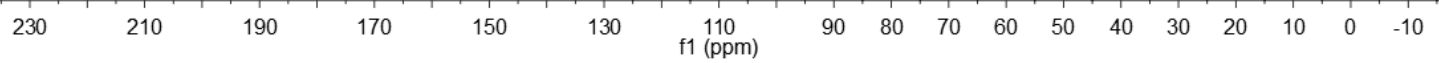

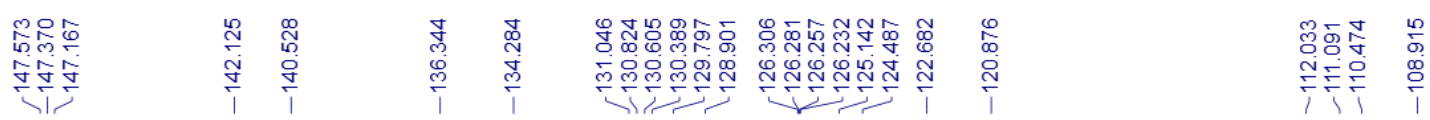

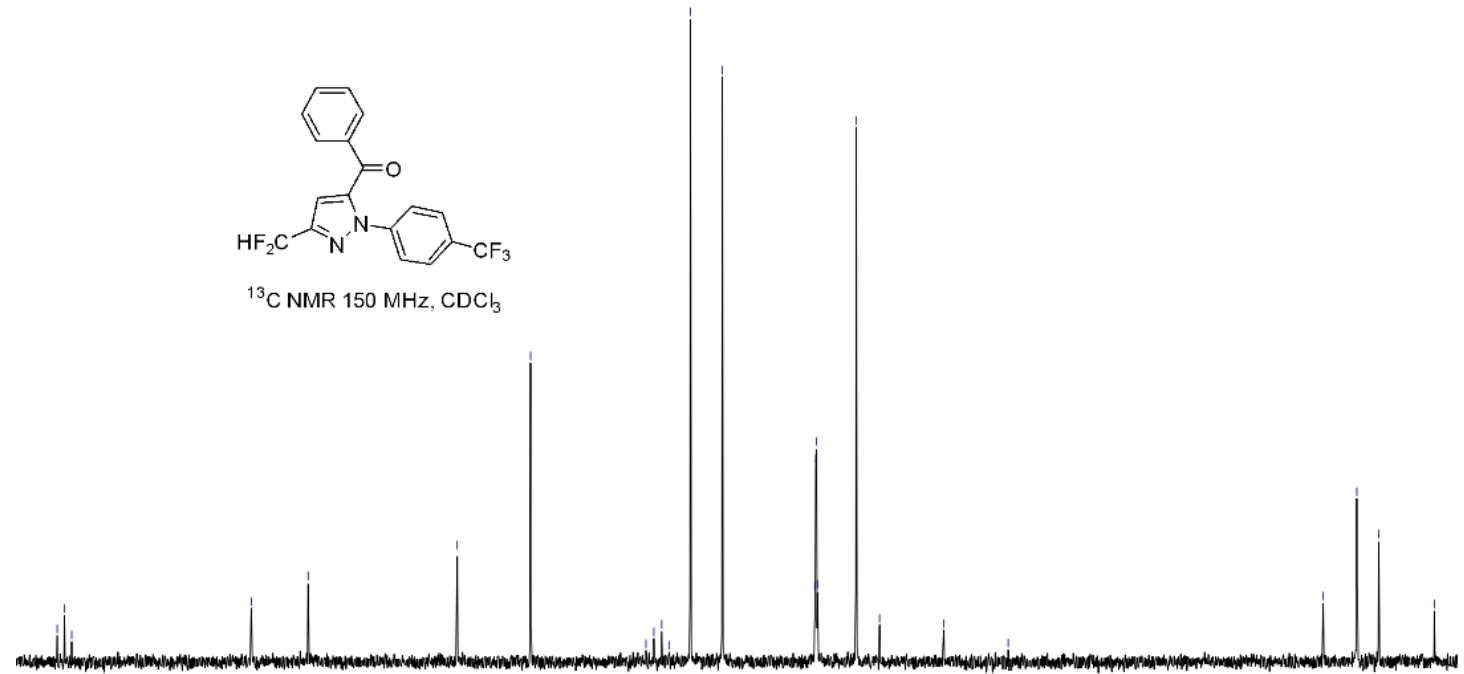

\begin{tabular}{lllllllllllll}
\hline 147 & 144 & 141 & 138 & 135 & 132 & 129 & 126 & 123 & 120 & 117 & 114 & 111
\end{tabular}




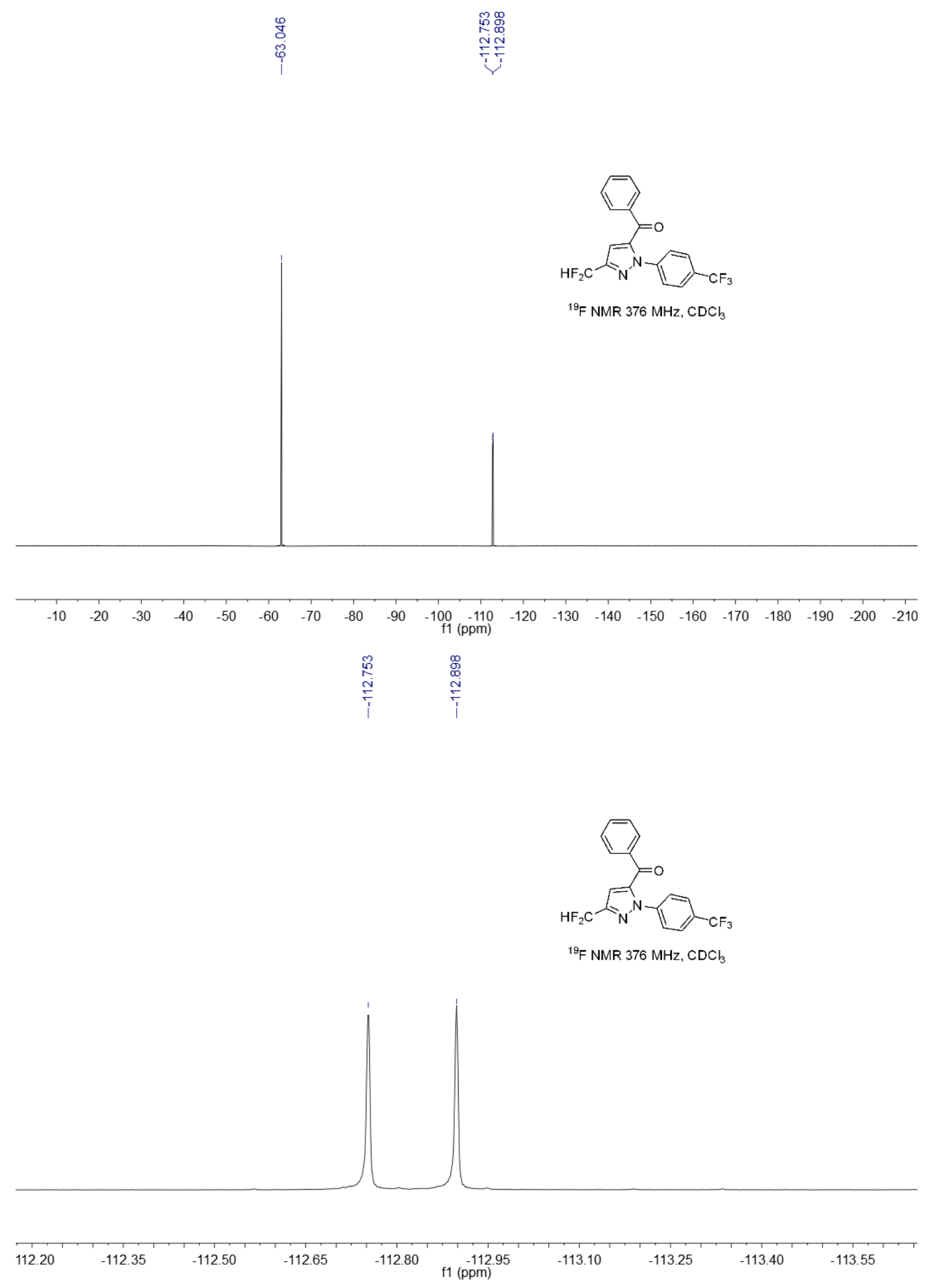


HRMS (ESI) copy of compound $\mathbf{3 i}$ :

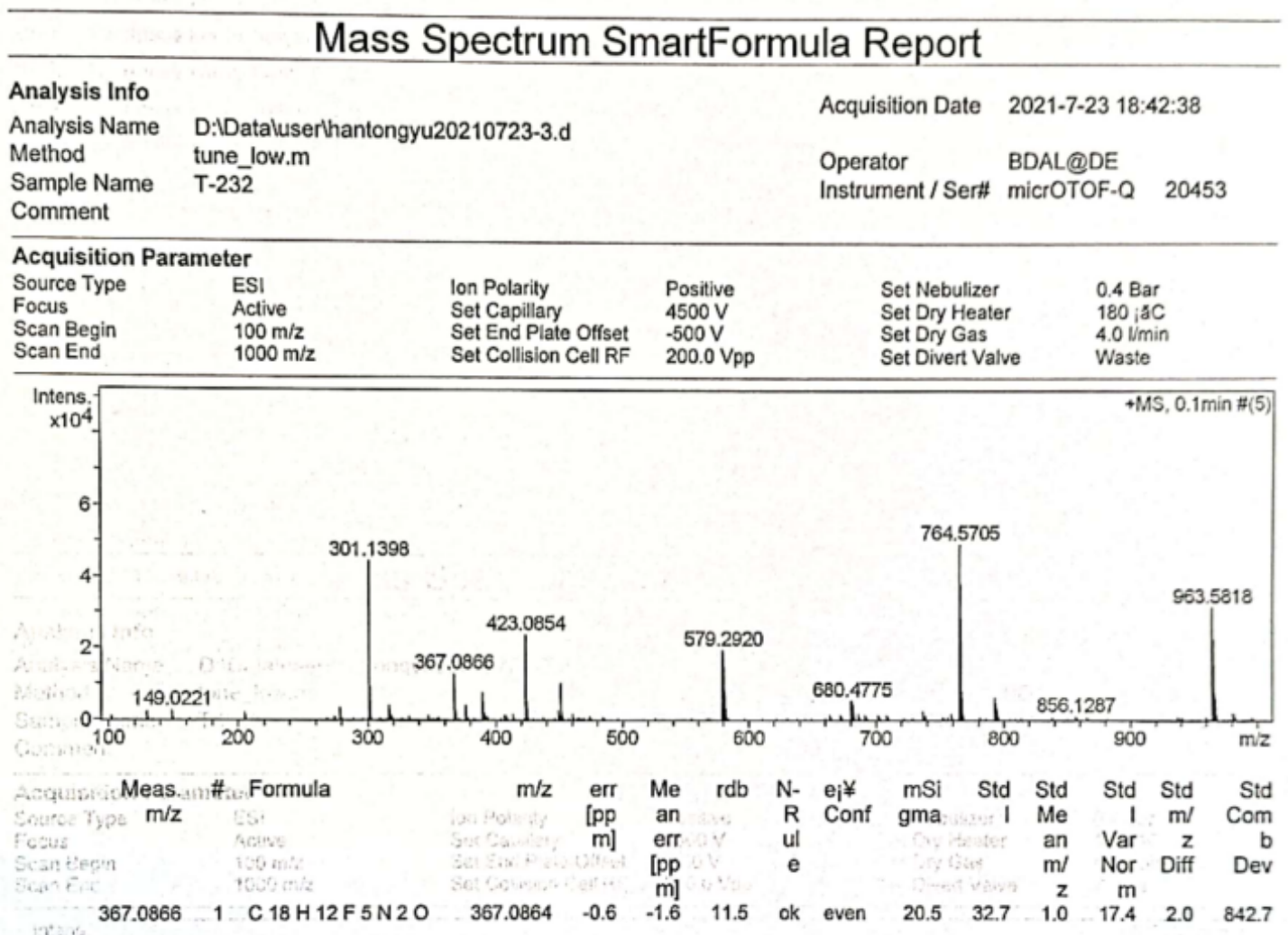

NMR copies of compound $\mathbf{3 k}$ :

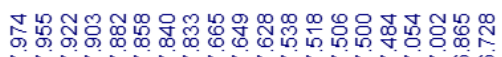

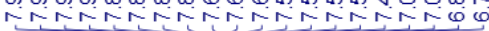

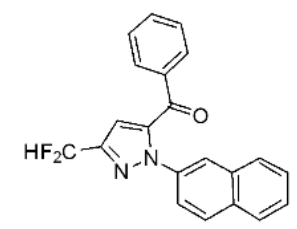

${ }^{1} \mathrm{H}$ NMR $400 \mathrm{MHz}, \mathrm{CDCl}_{3}$

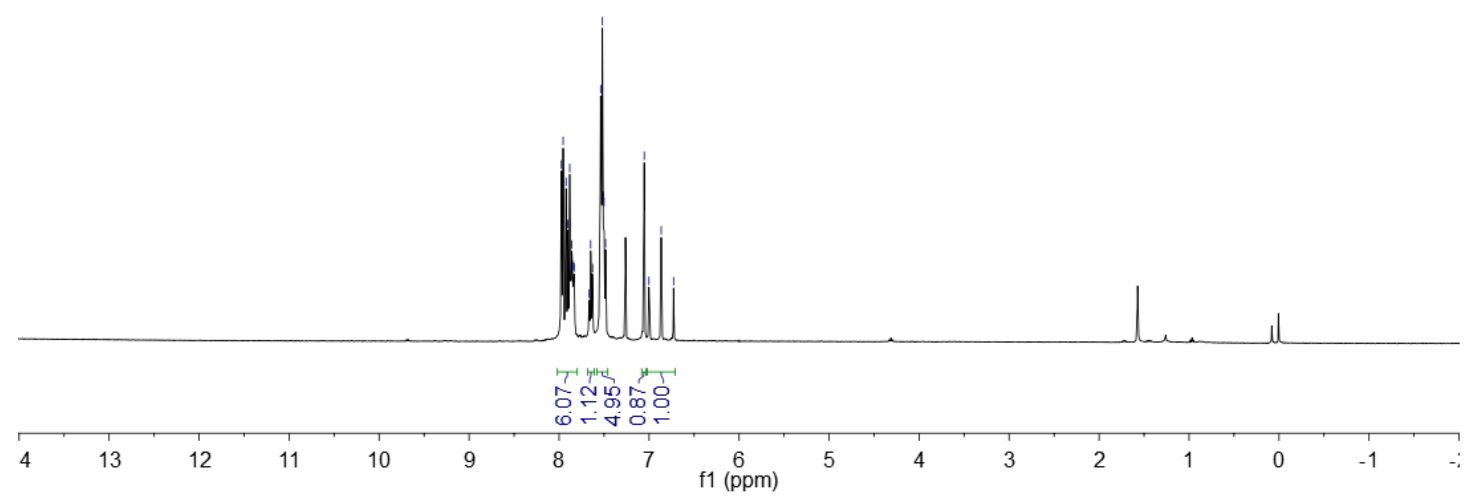




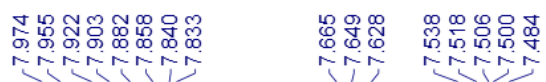

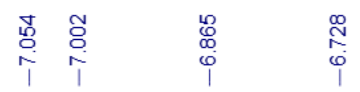

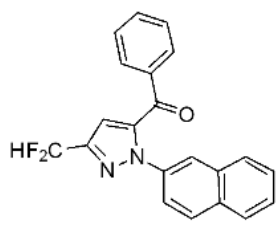

${ }^{1} \mathrm{H} \mathrm{NMR} 400 \mathrm{MHz}, \mathrm{CDCl}_{3}$

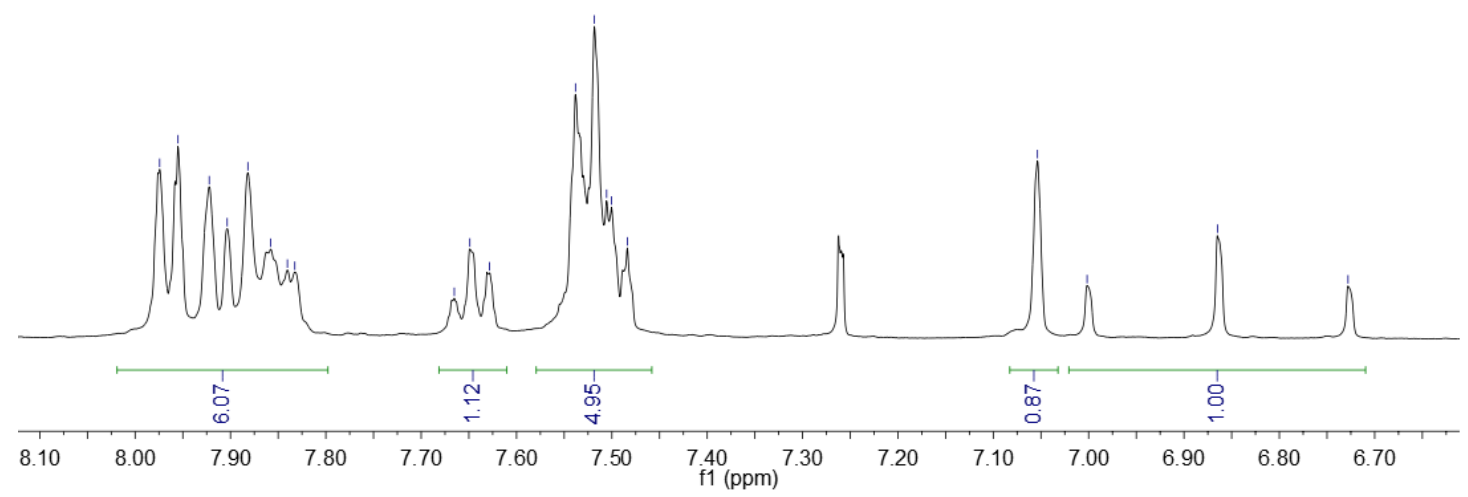

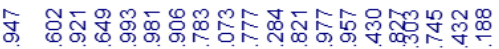

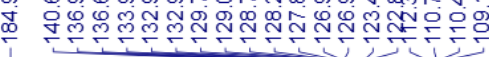

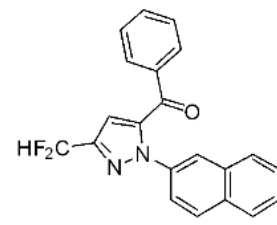

${ }^{13} \mathrm{C}$ NMR $150 \mathrm{MHz}, \mathrm{CDCl}$

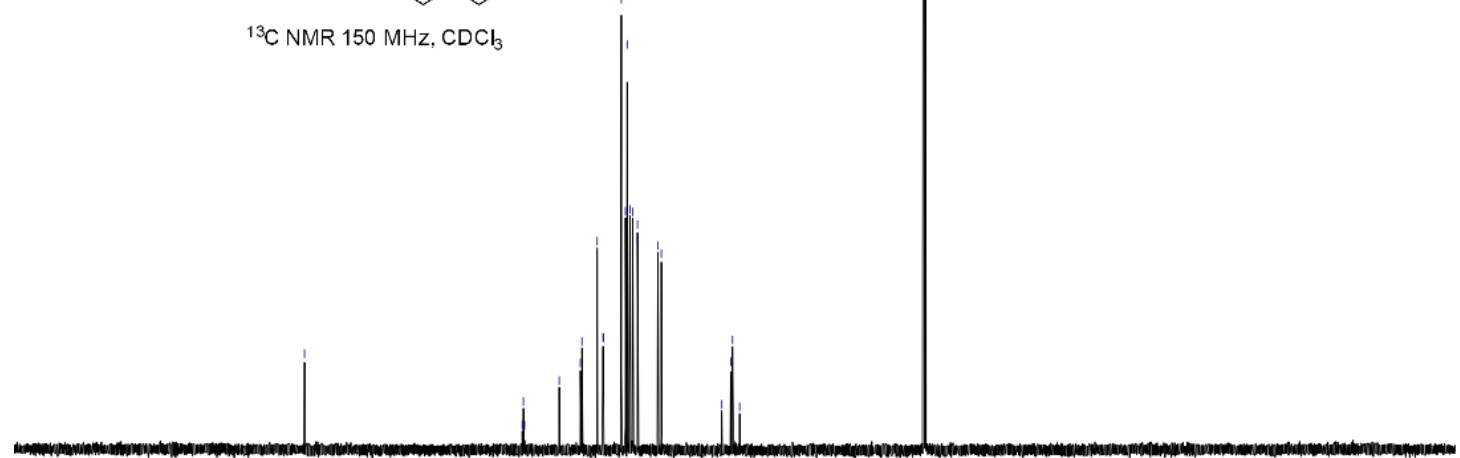

230

$90 \quad 170$

150

130

$\stackrel{110}{110}$

$\begin{array}{lllllllllll}90 & 80 & 70 & 60 & 50 & 40 & 30 & 20 & 10 & 0 & -10\end{array}$ 

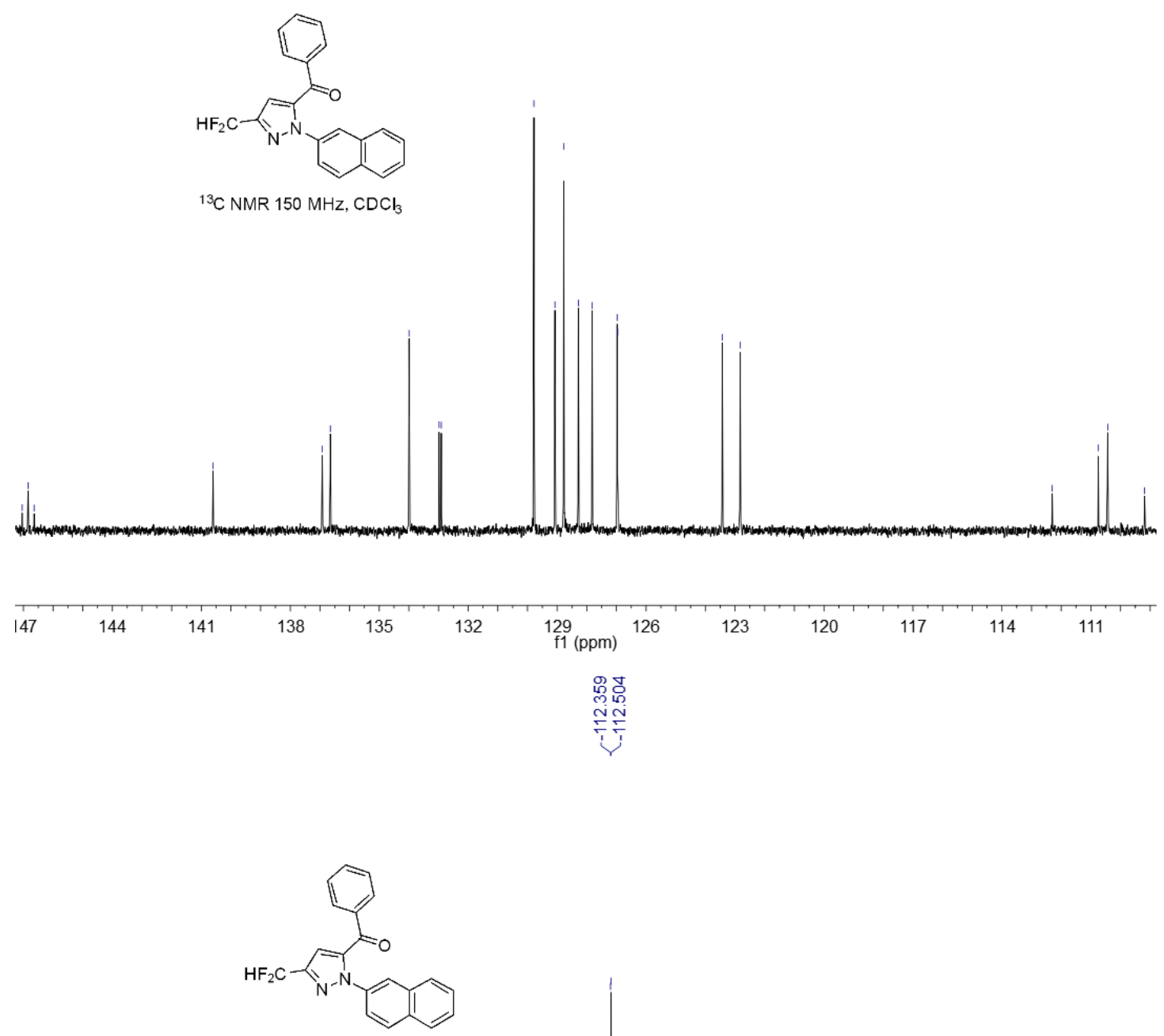

${ }^{19} \mathrm{~F}$ NMR $376 \mathrm{MHz}, \mathrm{CDCl}_{3}$

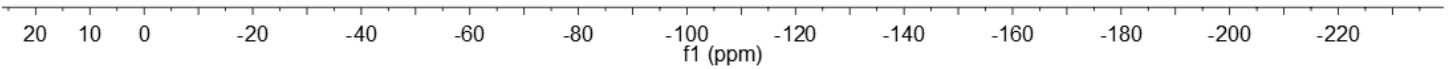




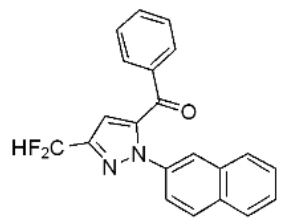

${ }^{19} \mathrm{~F} \mathrm{NMR} 376 \mathrm{MHz}, \mathrm{CDCl}$

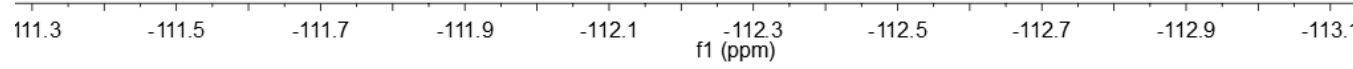

HRMS (ESI) copy of compound 3k:

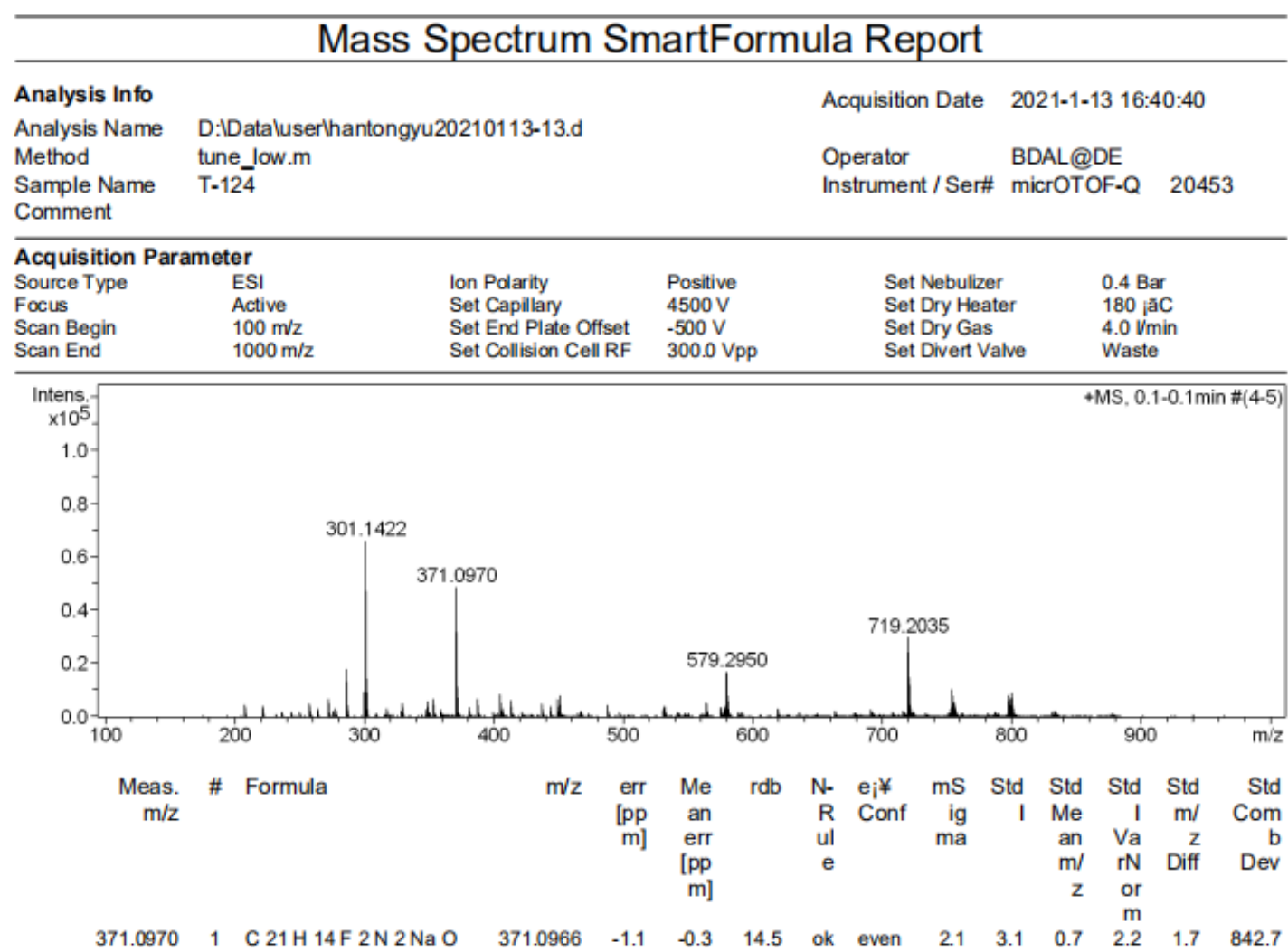


NMR copies of compound 3I:

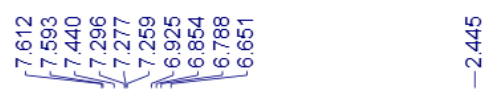

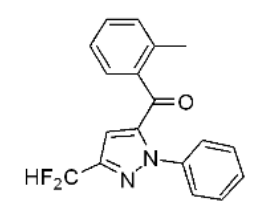

${ }^{1} \mathrm{HNMR} 400 \mathrm{MHz}, \mathrm{CDCl}_{3}$

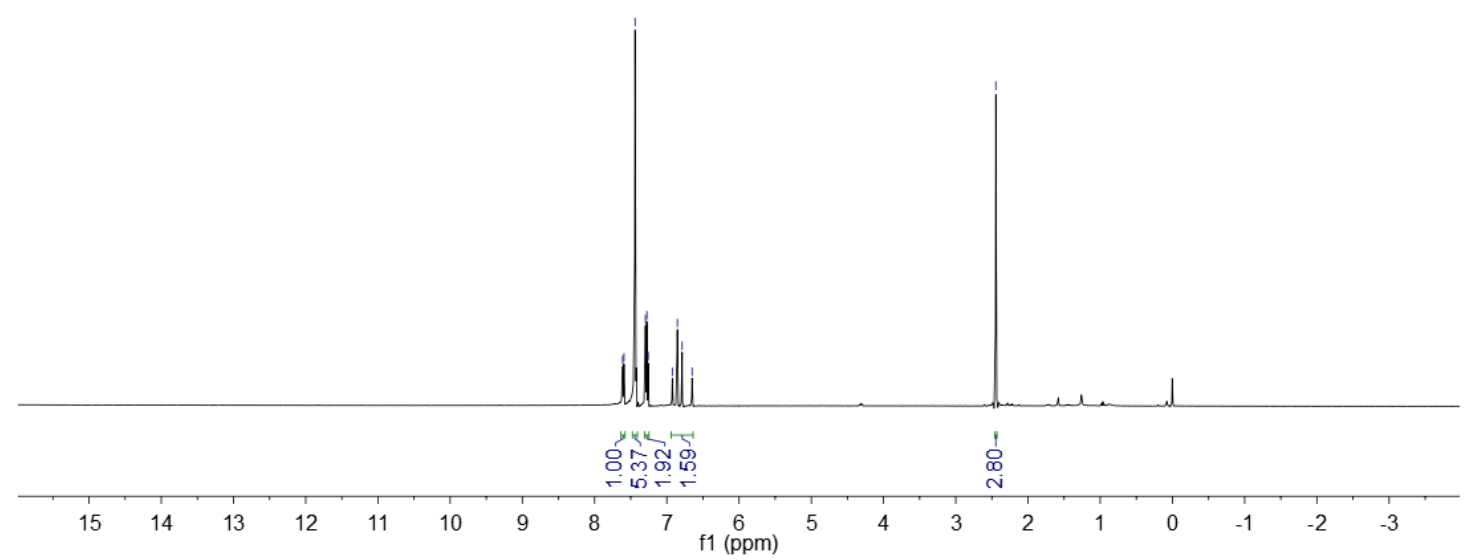

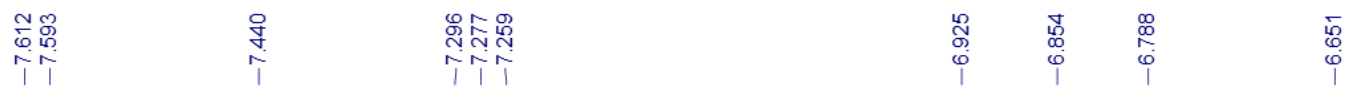

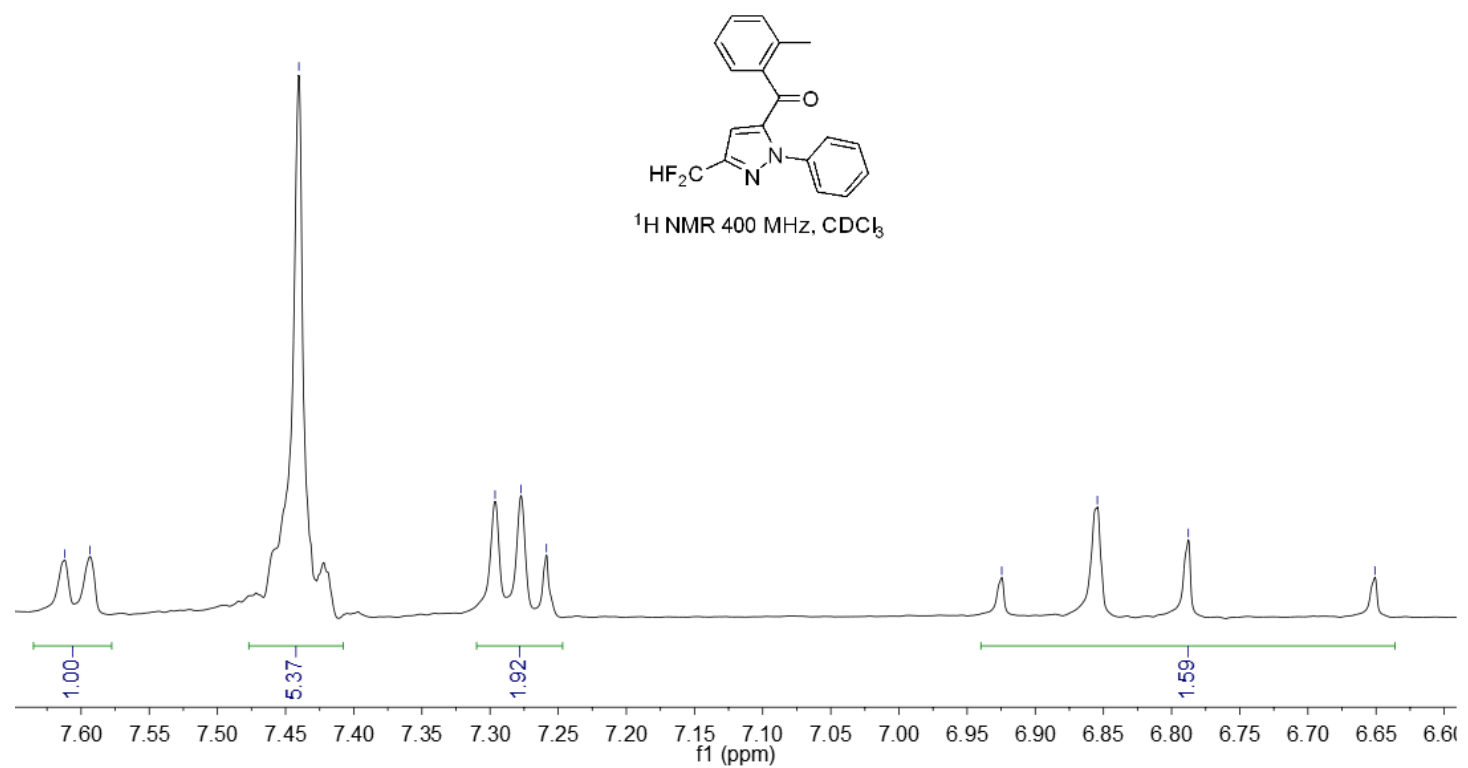




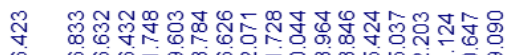

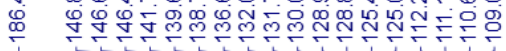
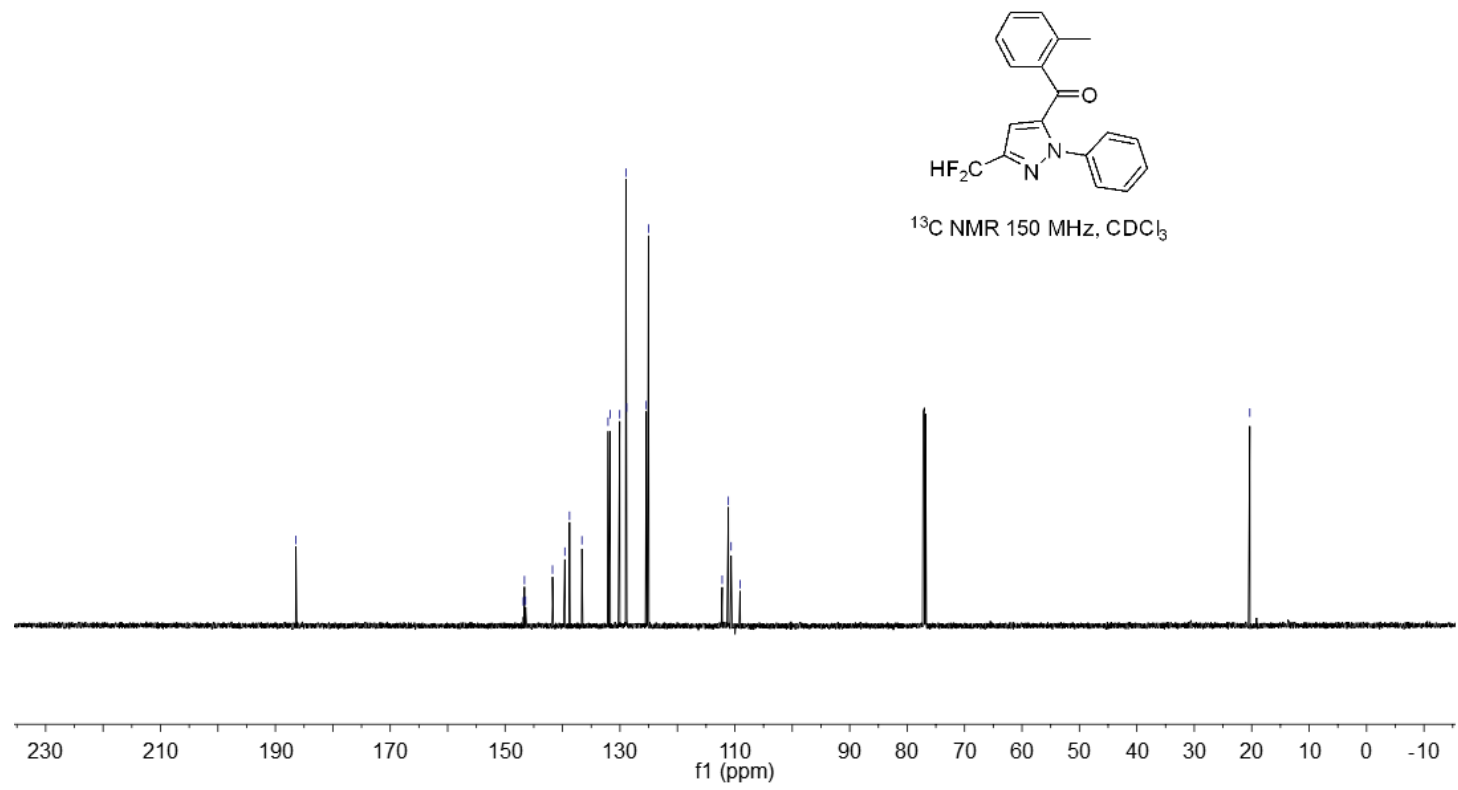

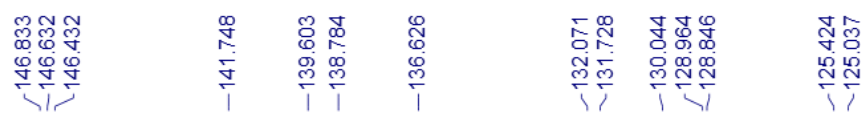

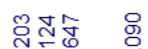

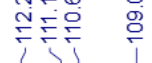

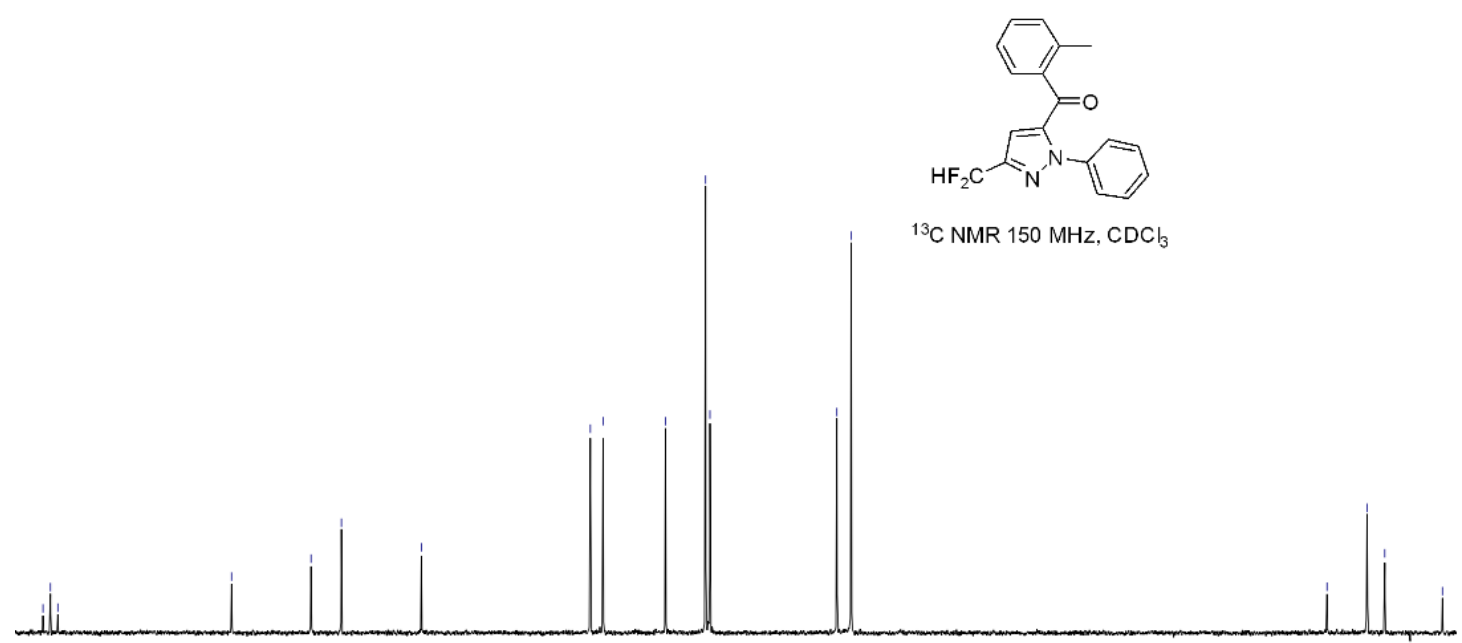

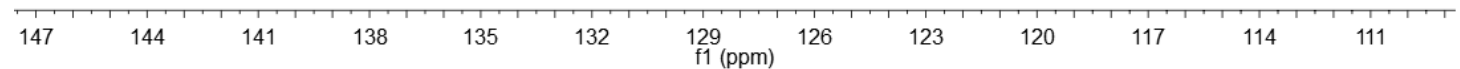



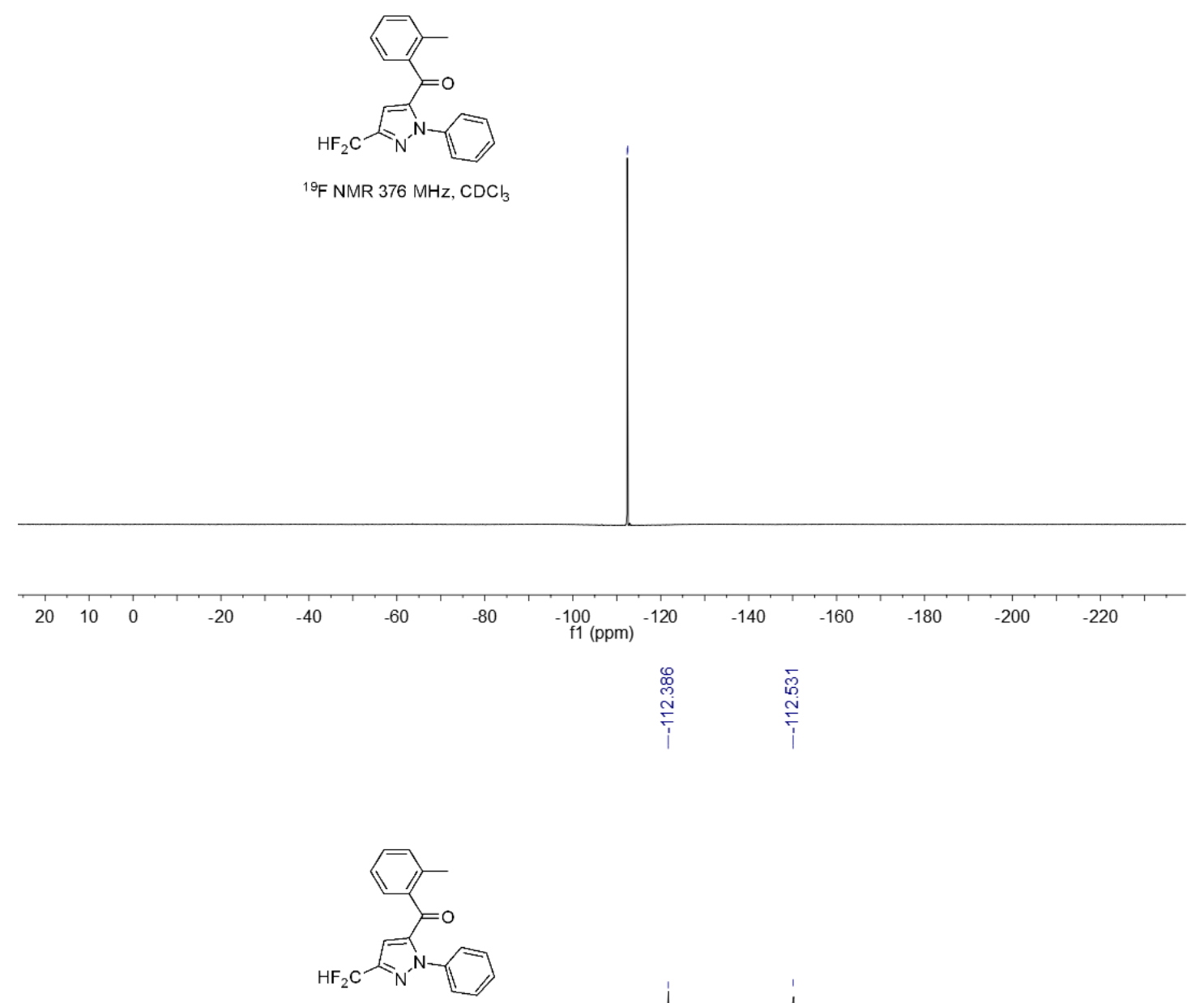

${ }^{19} \mathrm{~F}$ NMR $376 \mathrm{MHz}, \mathrm{CDCl}$

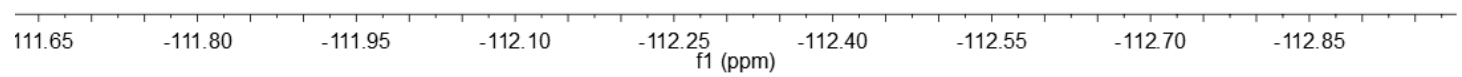


HRMS (ESI) copy of compound 31:

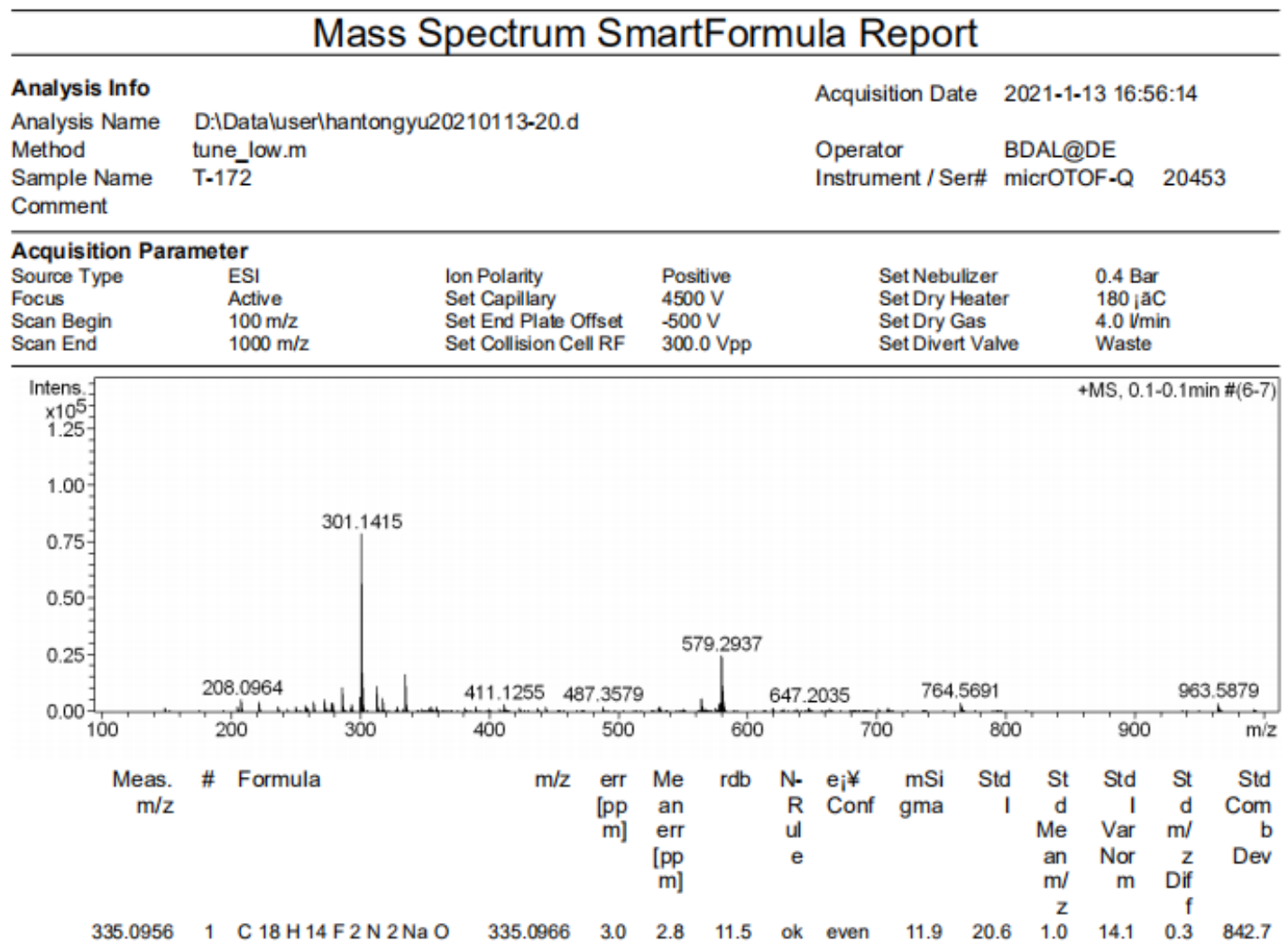

NMR copies of compound $\mathbf{3 m}$ :

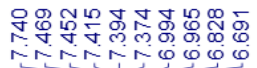

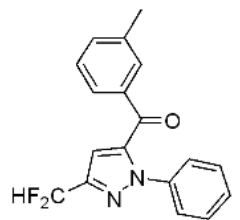

${ }^{1} \mathrm{H} \mathrm{NMR} 400 \mathrm{MHz}, \mathrm{CDCl}_{3}$

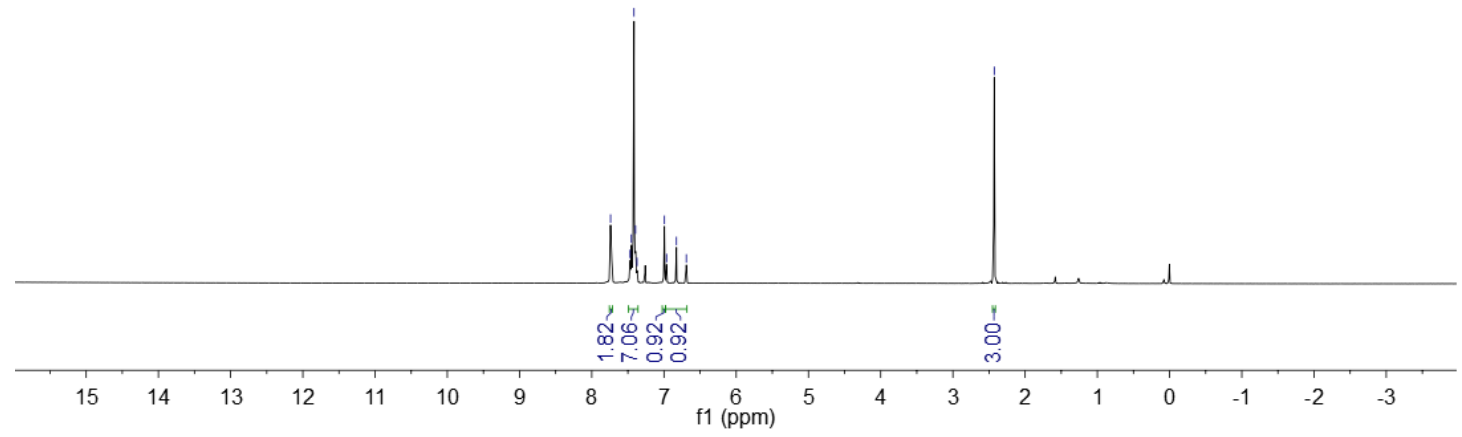



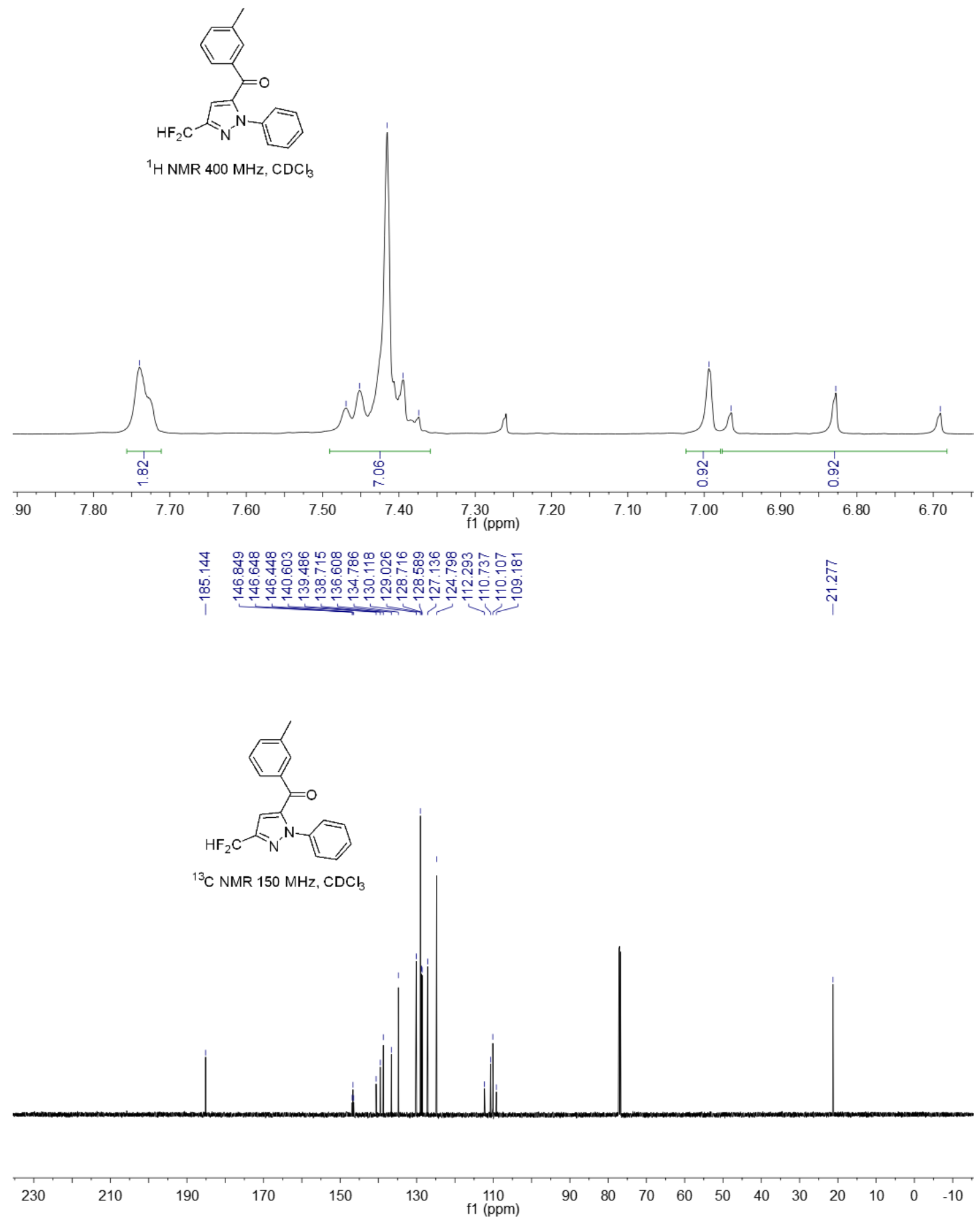

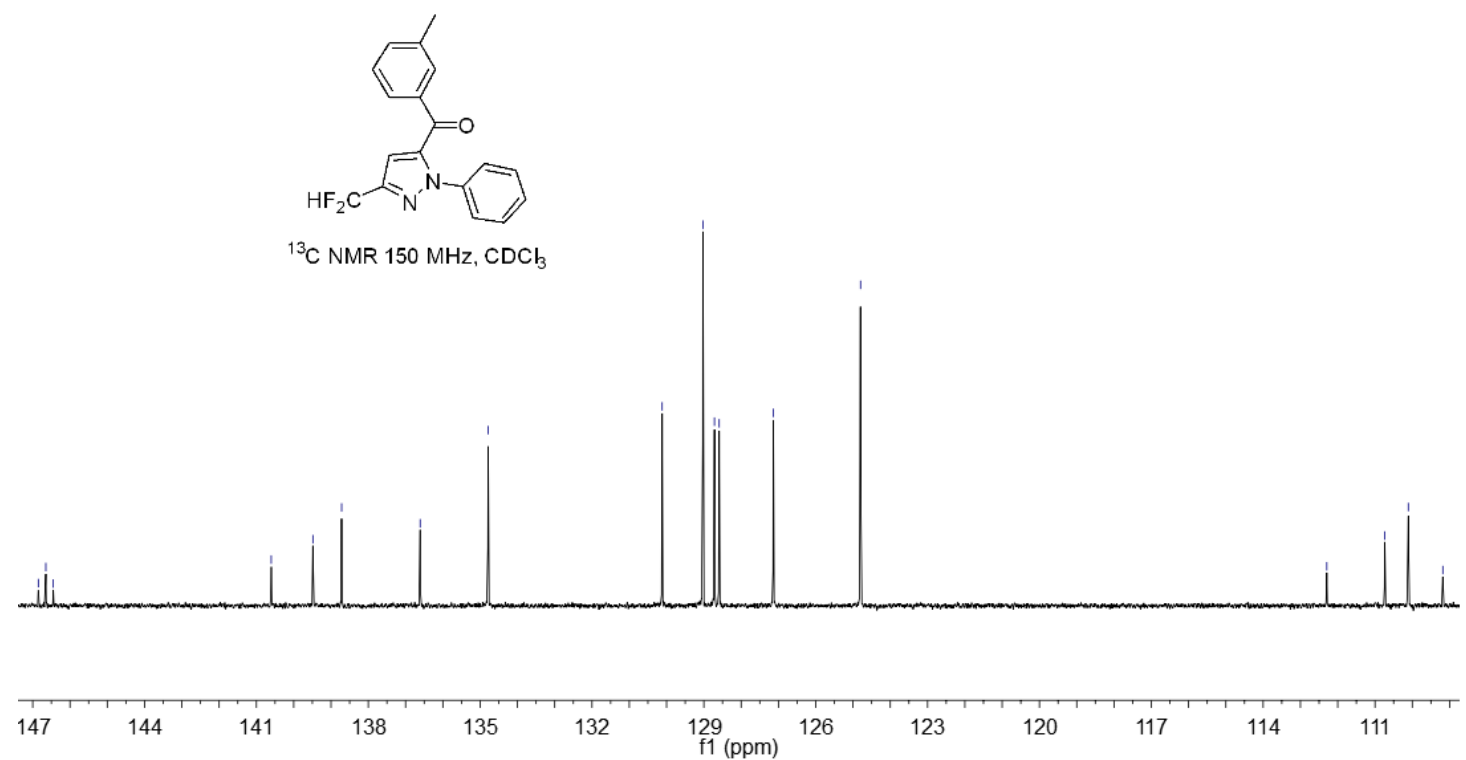

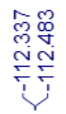

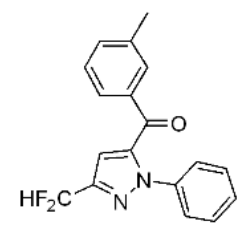

${ }^{19} \mathrm{~F}$ NMR $376 \mathrm{MHz}, \mathrm{CDCl}_{3}$

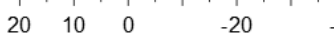

$-100 \quad-120$

$20-140-160$

180
-180 


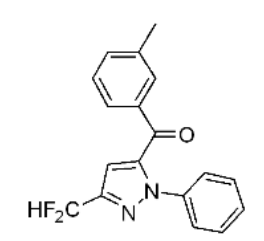

${ }^{19} \mathrm{~F} \mathrm{NMR} 376 \mathrm{MHz}, \mathrm{CDCl}_{3}$

$\begin{array}{lllllllllllllllll}-111.5 & -111.6 & -111.7 & -111.8 & -111.9 & -112.0 & -112.1 & -112.2 & -112.3 & -112.4 & -112.5 & -112.6 & -112.7 & -112.8 & -112.9 & -113.0\end{array}$

HRMS (ESI) copy of compound $\mathbf{3} \mathbf{m}$ :

\section{Mass Spectrum SmartFormula Report}

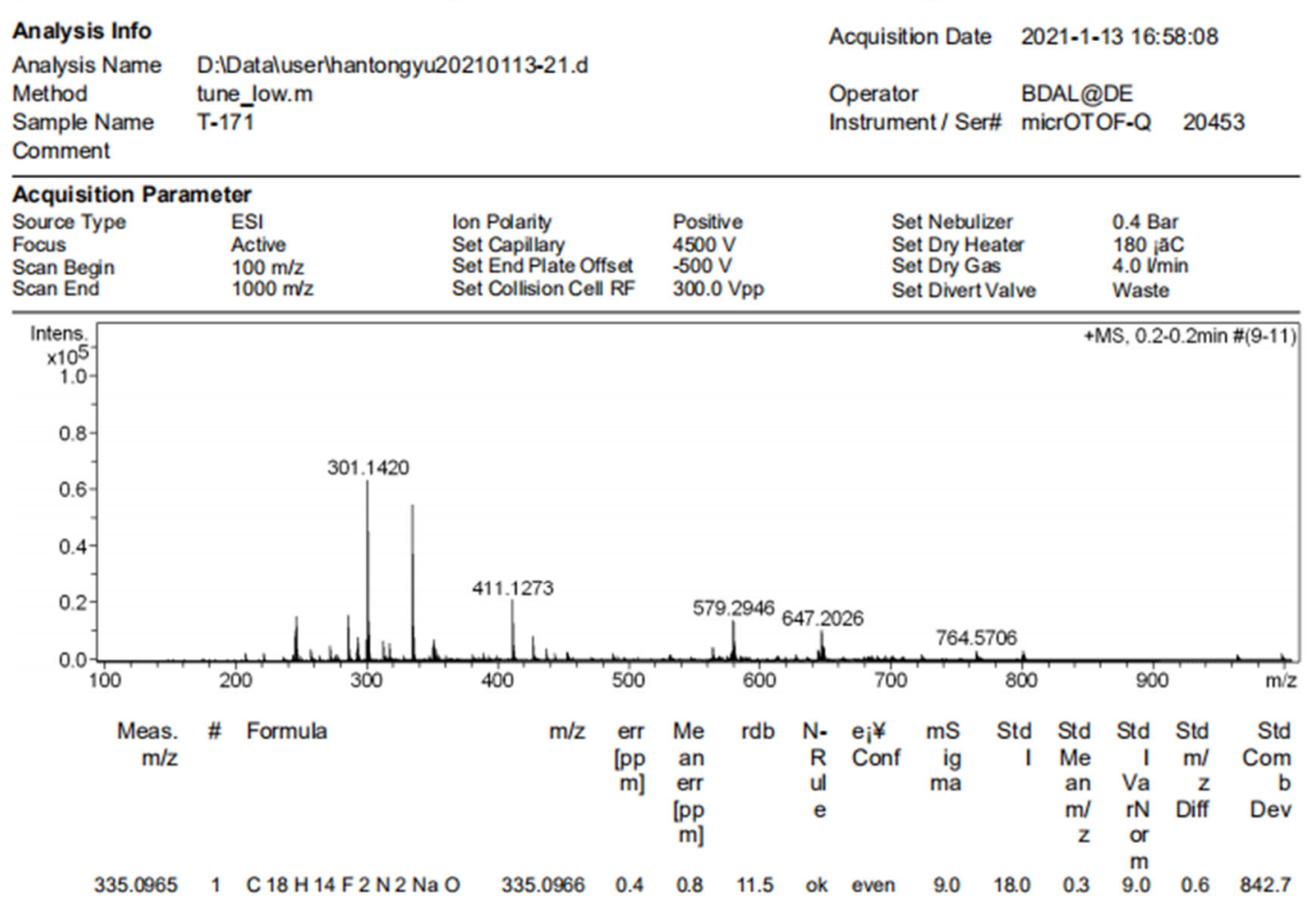


NMR copies of compound 3n:

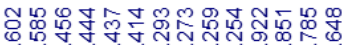

NNNNN

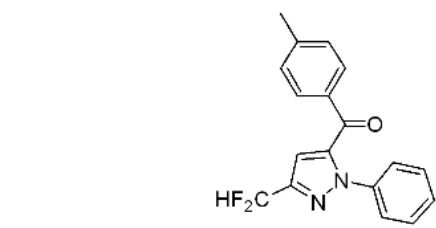

${ }^{1} \mathrm{HNMR} 400 \mathrm{MHz}, \mathrm{CDCl}_{3}$
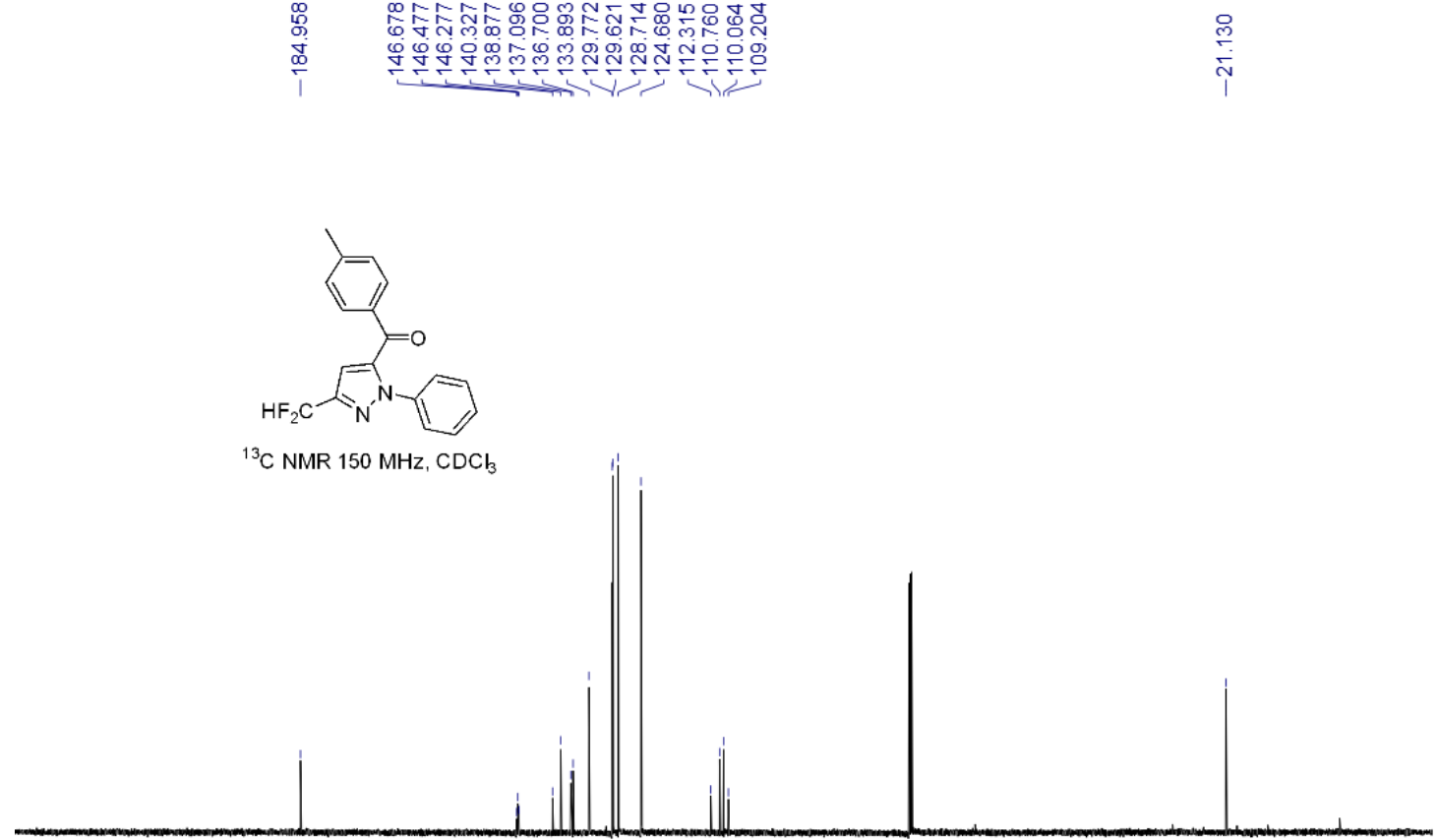

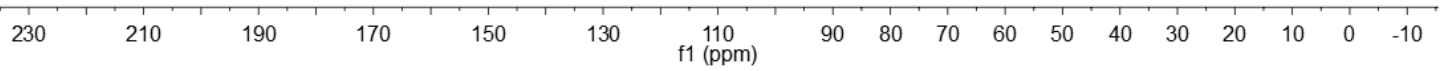




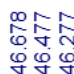

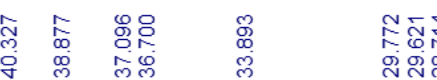

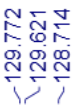

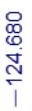

응 웜

ata

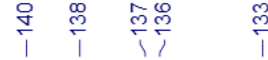

굳 윆융

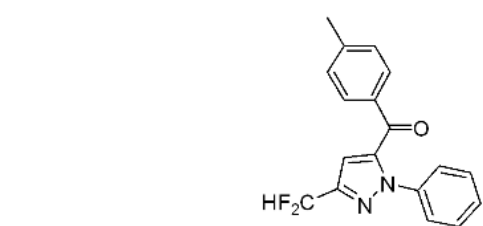

${ }^{13} \mathrm{C}$ NMR $150 \mathrm{MHz}, \mathrm{CDCl}$
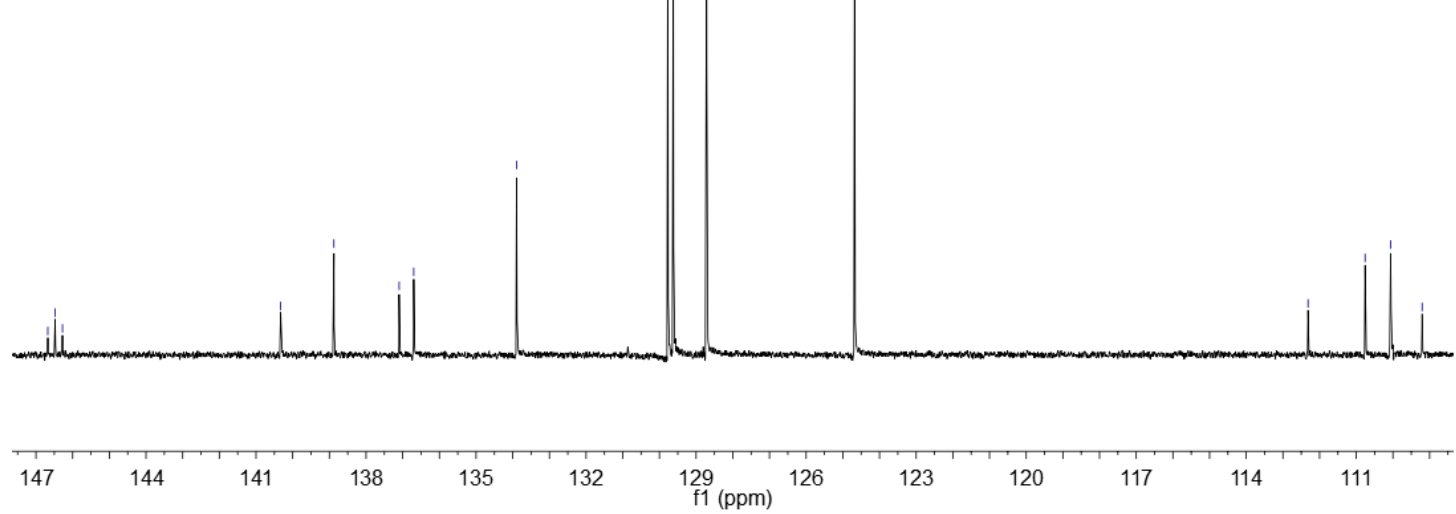

ชึष्ठ

ํํำ

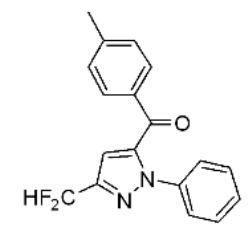

${ }^{19} \mathrm{~F} \mathrm{NMR} 376 \mathrm{MHz}, \mathrm{CDCl}_{3}$

$\begin{array}{lllll}20 & 10 & 0 & -20 & -40\end{array}$

$-100 \quad-120$

$20-140 \quad-160 \quad-18$ 


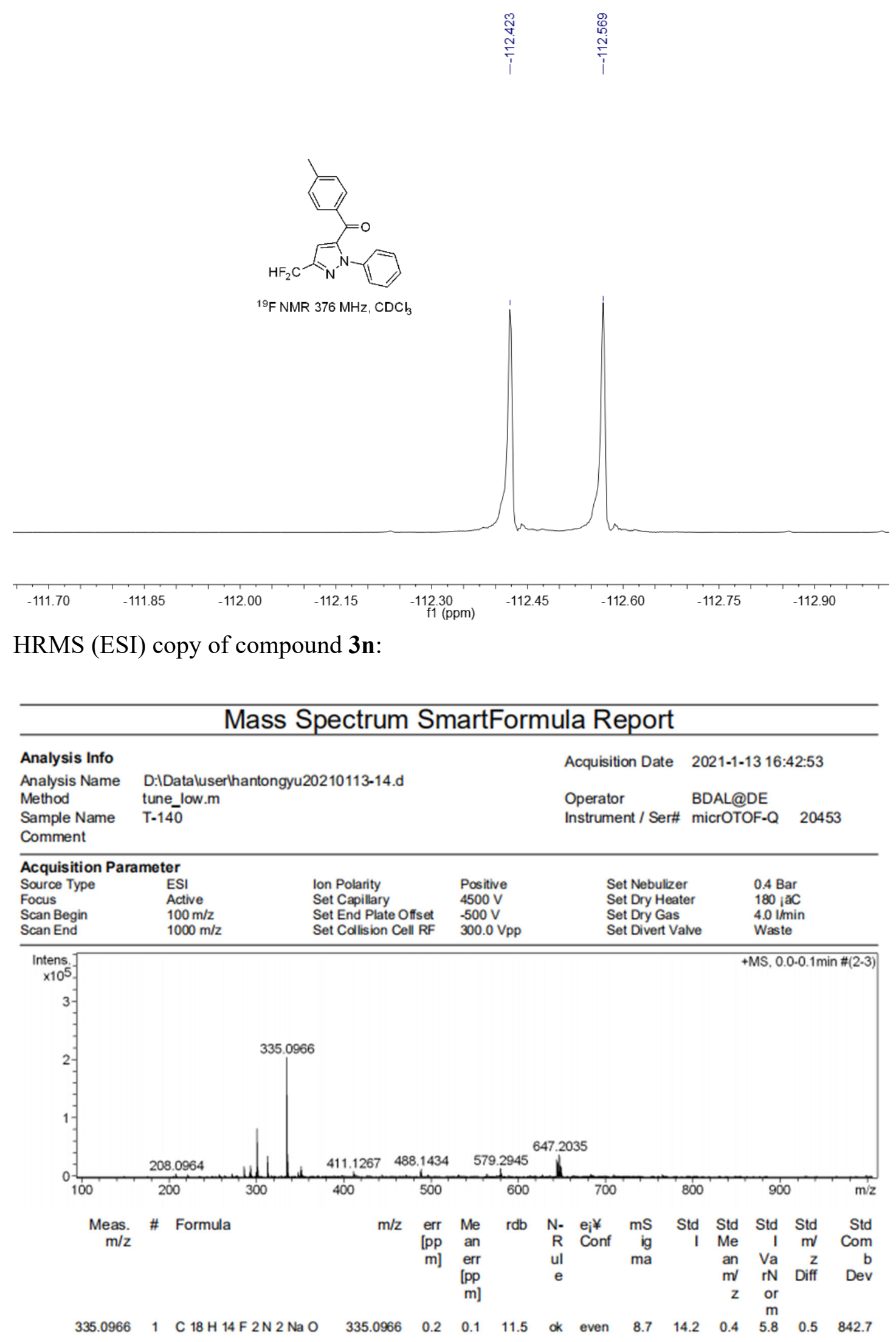


NMR copies of compound 3o:

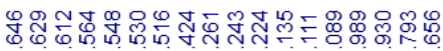

的
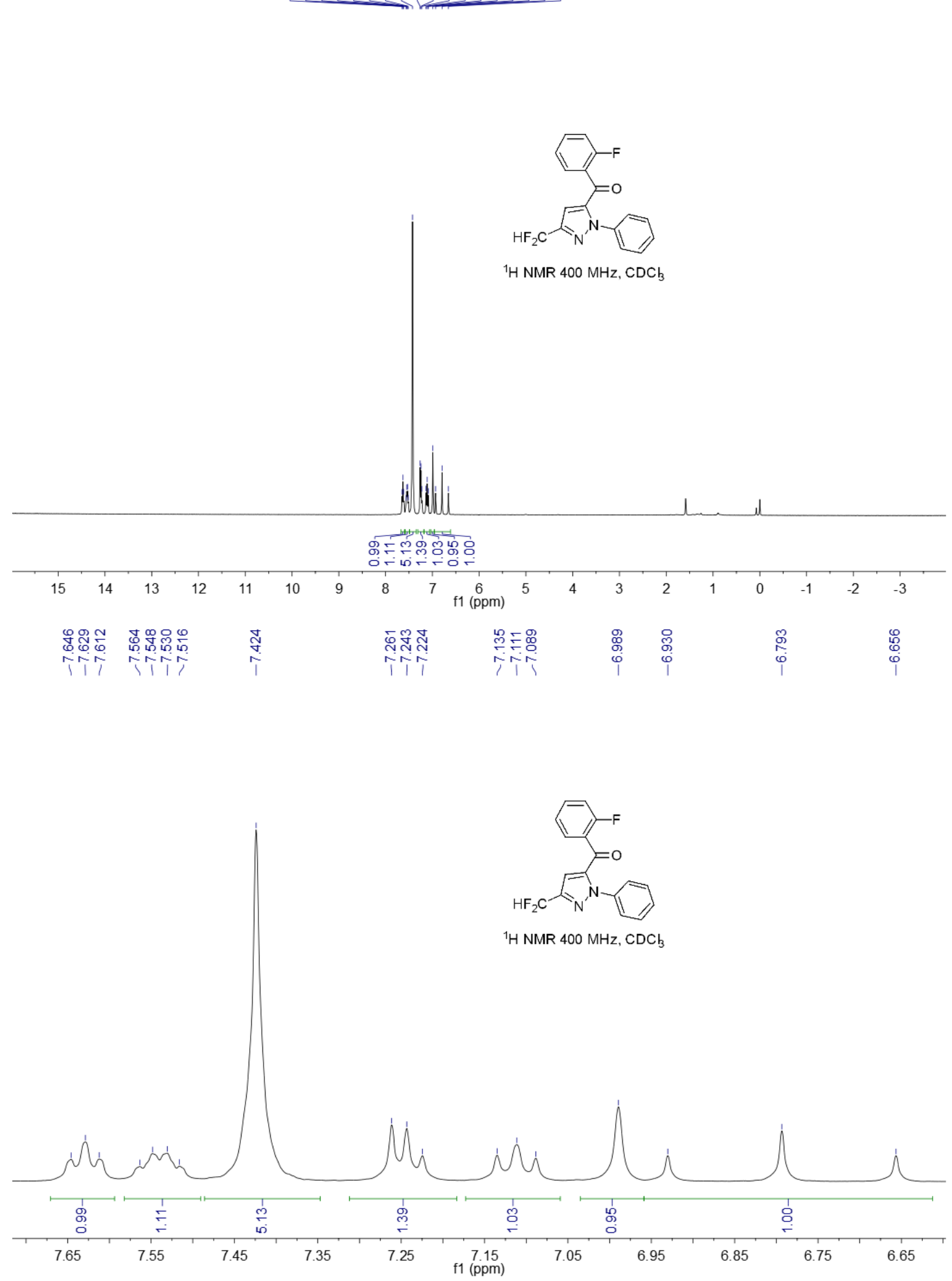


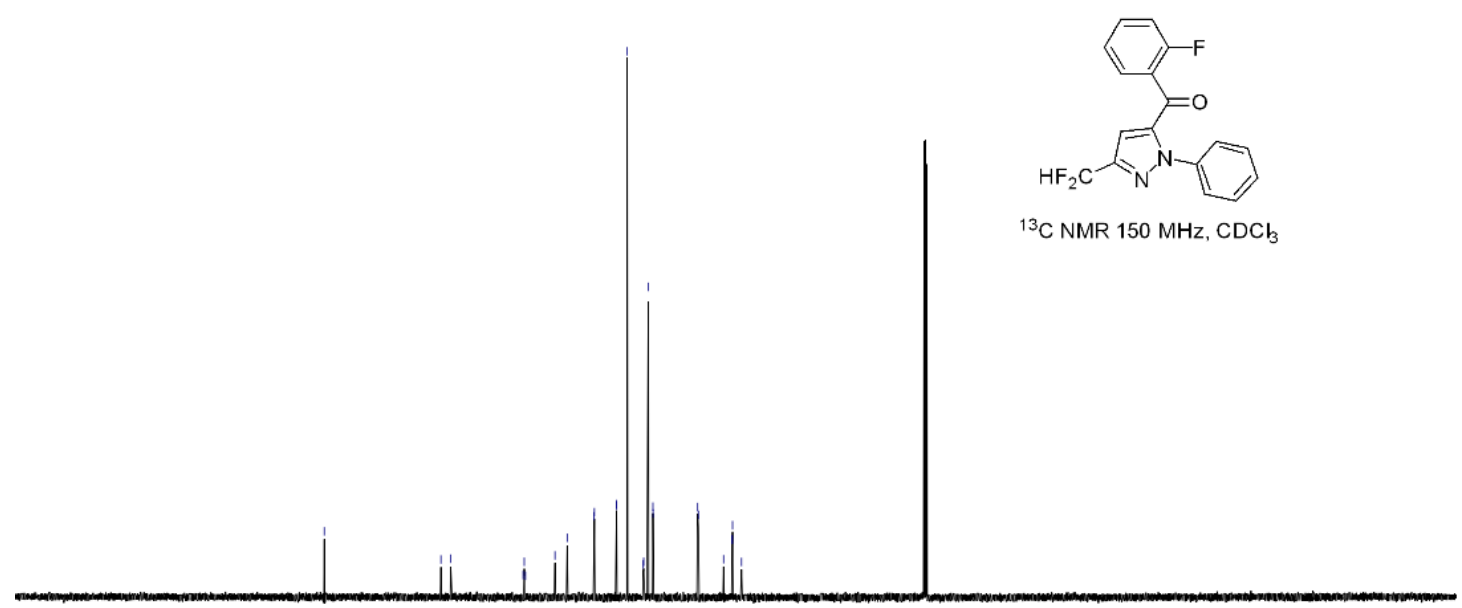

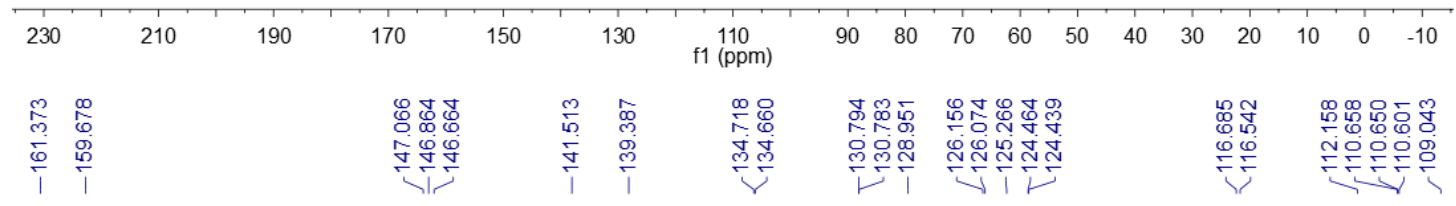

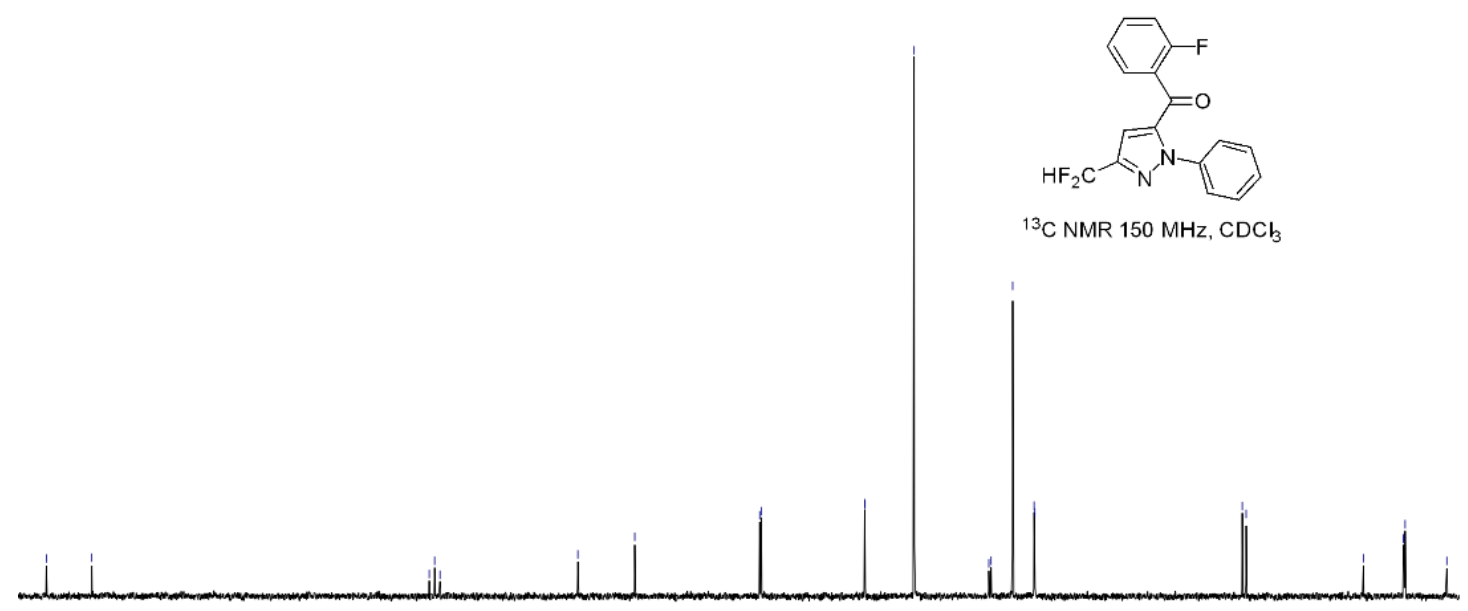

$160 \quad 156 \quad 152 \quad 148 \quad 144 \quad 140 \quad \begin{aligned} & 136 \\ & \mathrm{f}(\mathrm{ppm})\end{aligned}$




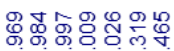

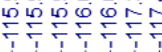

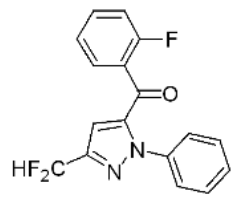

${ }^{19} \mathrm{~F}$ NMR $376 \mathrm{MHz}, \mathrm{CDCl}$

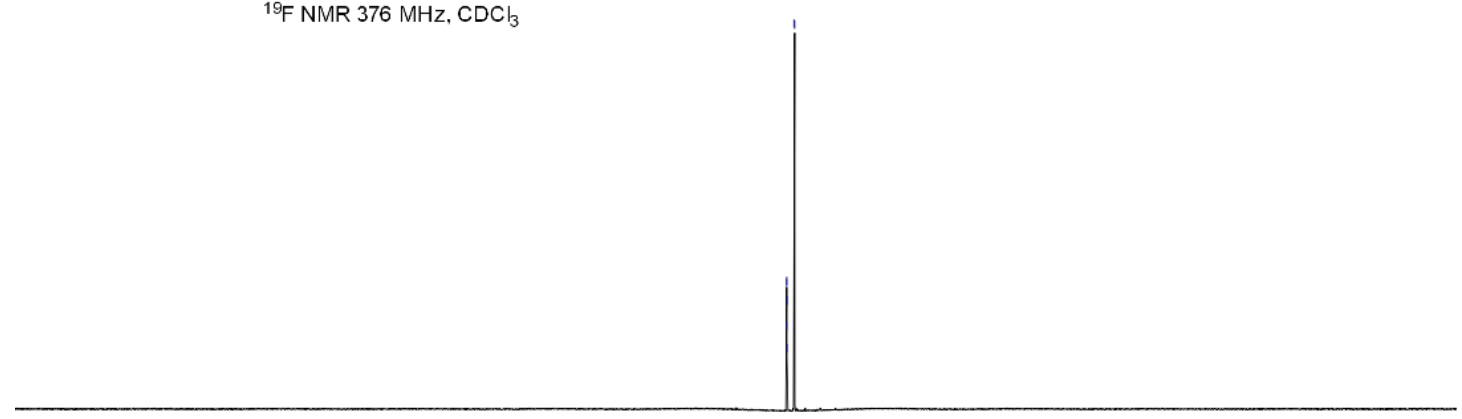

$\begin{array}{rrrrrrrrrrr}1 \\ 20 & 10 & 0 & -20 & -40 & -60 & -80 & -100 \\ f 1(\mathrm{ppm}) & -120 & -140 & -160 & -180 & -200 & -220 & \end{array}$

영효용용

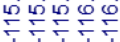

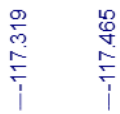

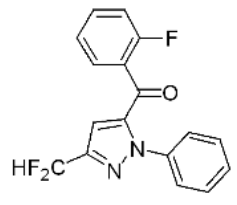

${ }^{19} \mathrm{~F} \mathrm{NMR} 376 \mathrm{MHz}, \mathrm{CDCl}_{3}$

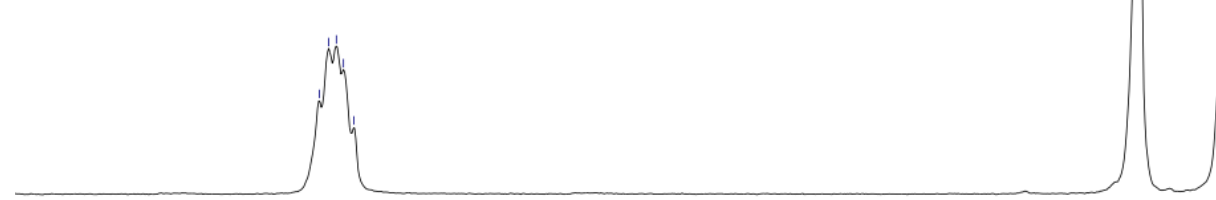

\begin{tabular}{llllllllllll}
\hline 115.5 & -115.7 & -115.9 & -116.1 & -116.3 & -116.5 & -116.7 & -116.9 & -117.1 & -117.3 & -117.5 & -117.7
\end{tabular} 
HRMS (ESI) copy of compound 3o:

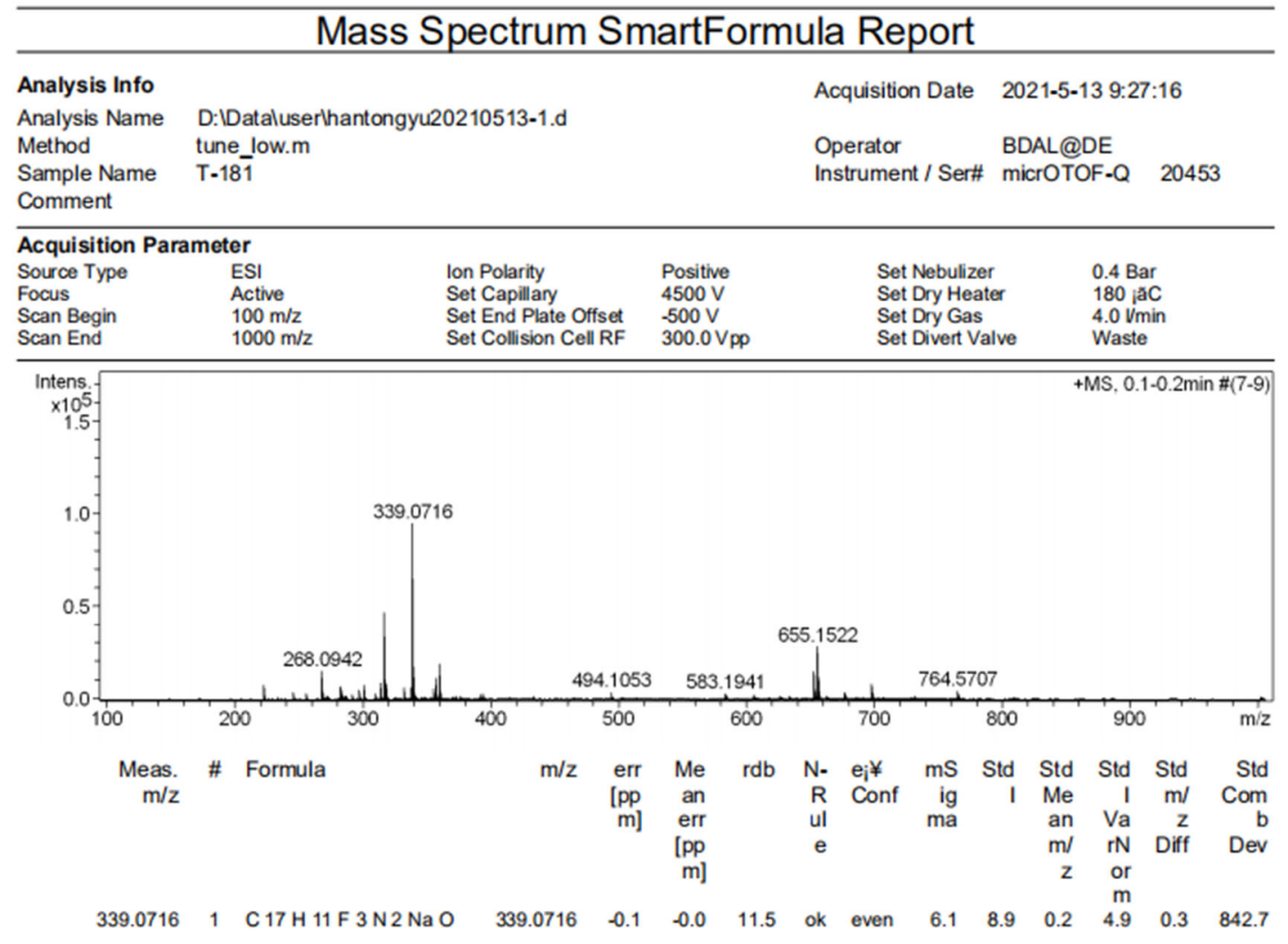

NMR copies of compound $\mathbf{3 p}$ :

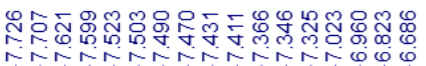

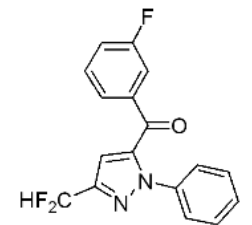

${ }^{1} \mathrm{H}$ NMR $400 \mathrm{MHz}, \mathrm{CDCl}$

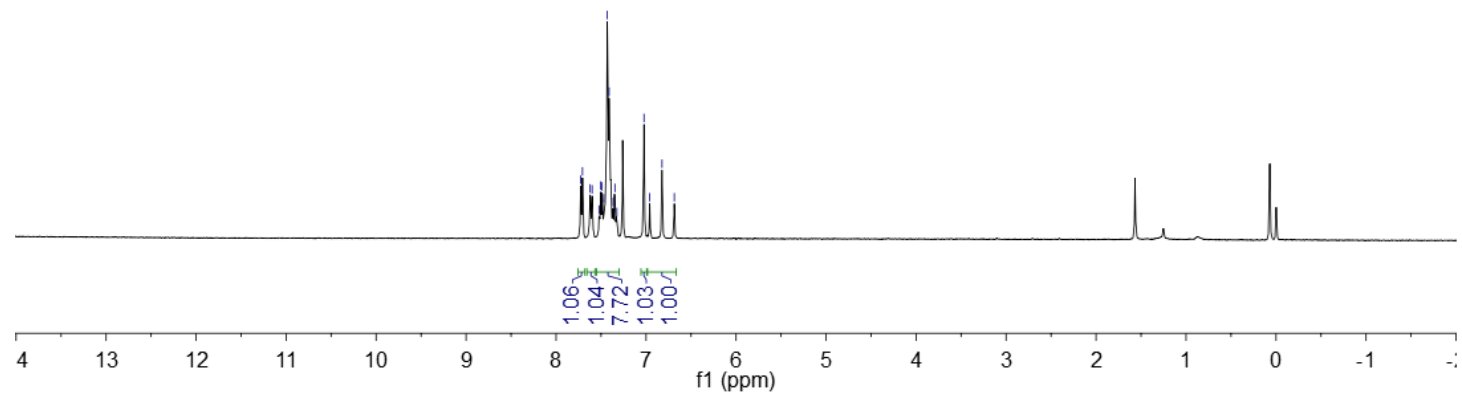




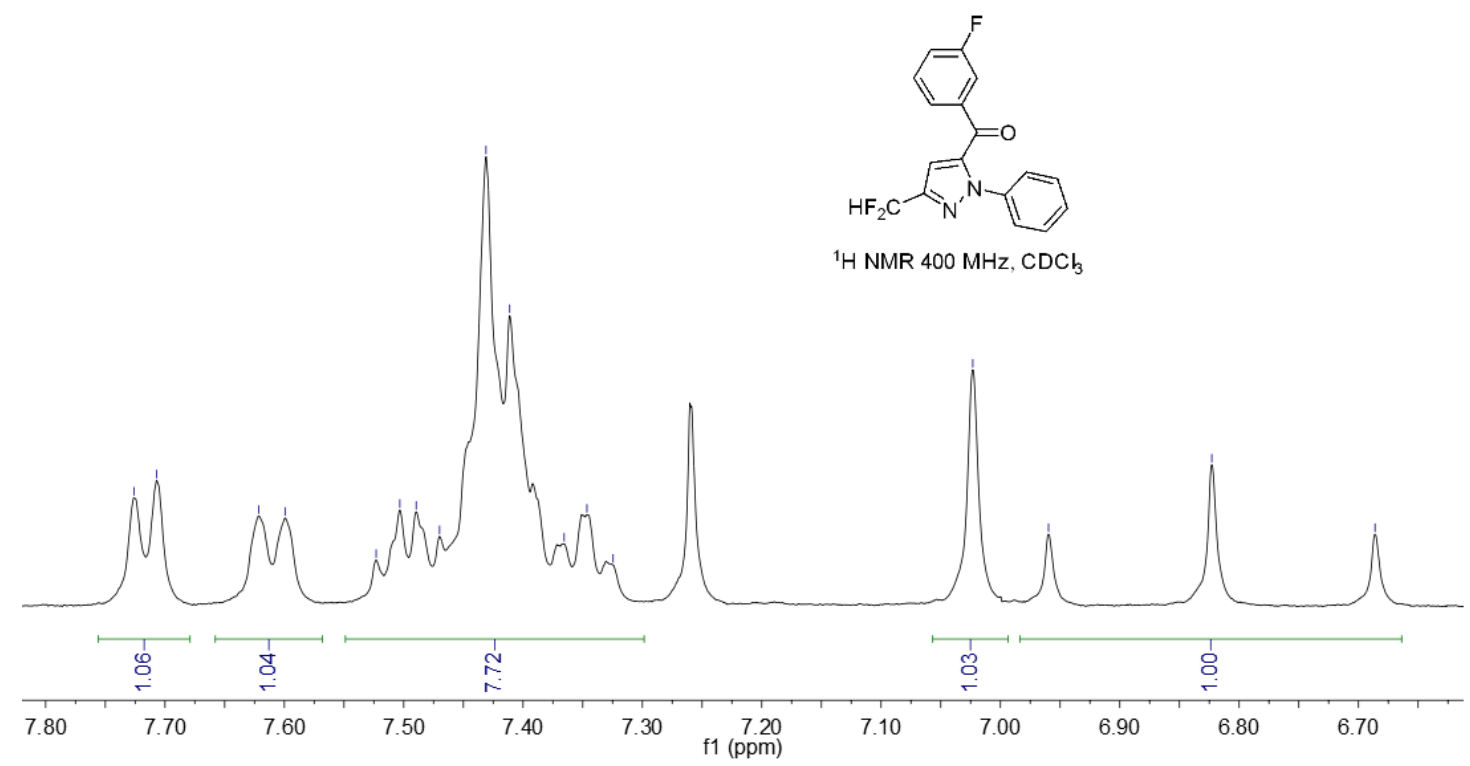

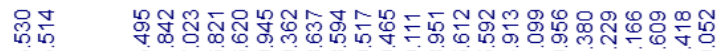

要

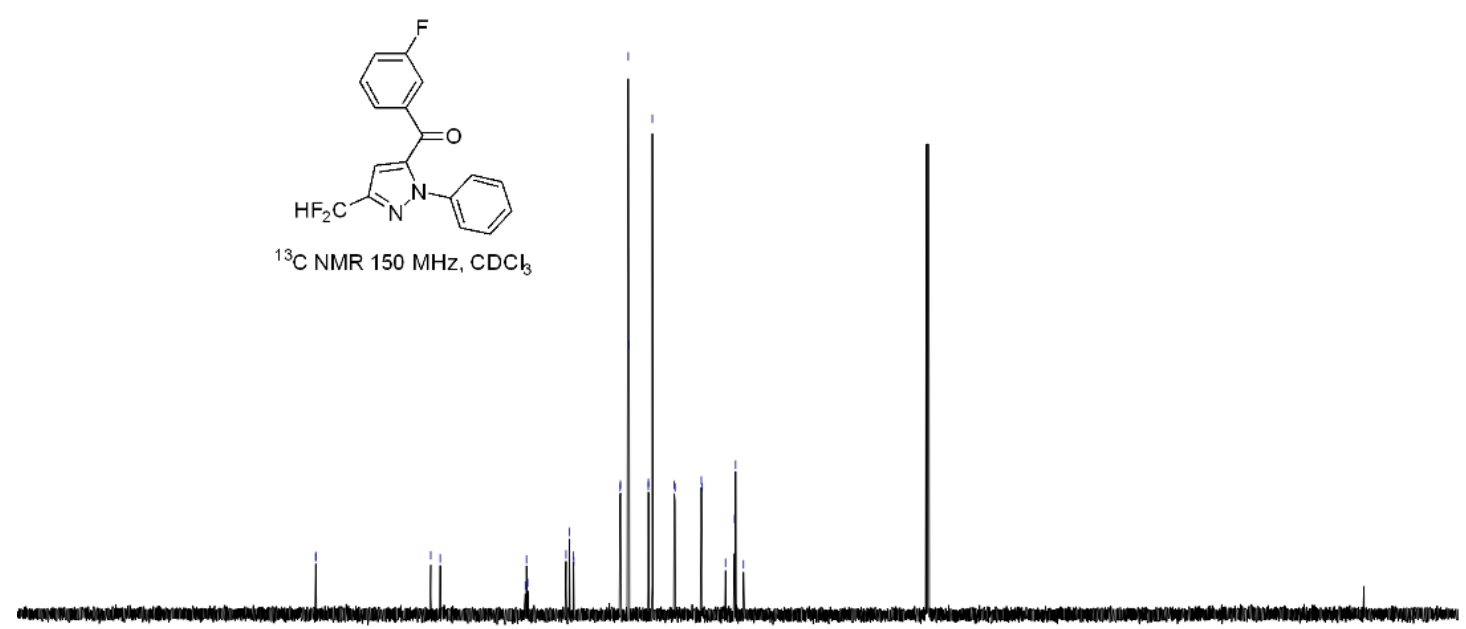

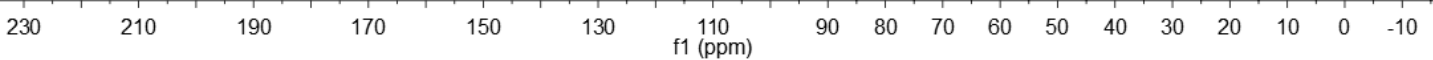




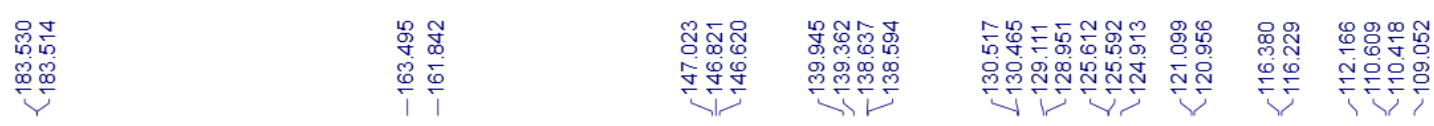

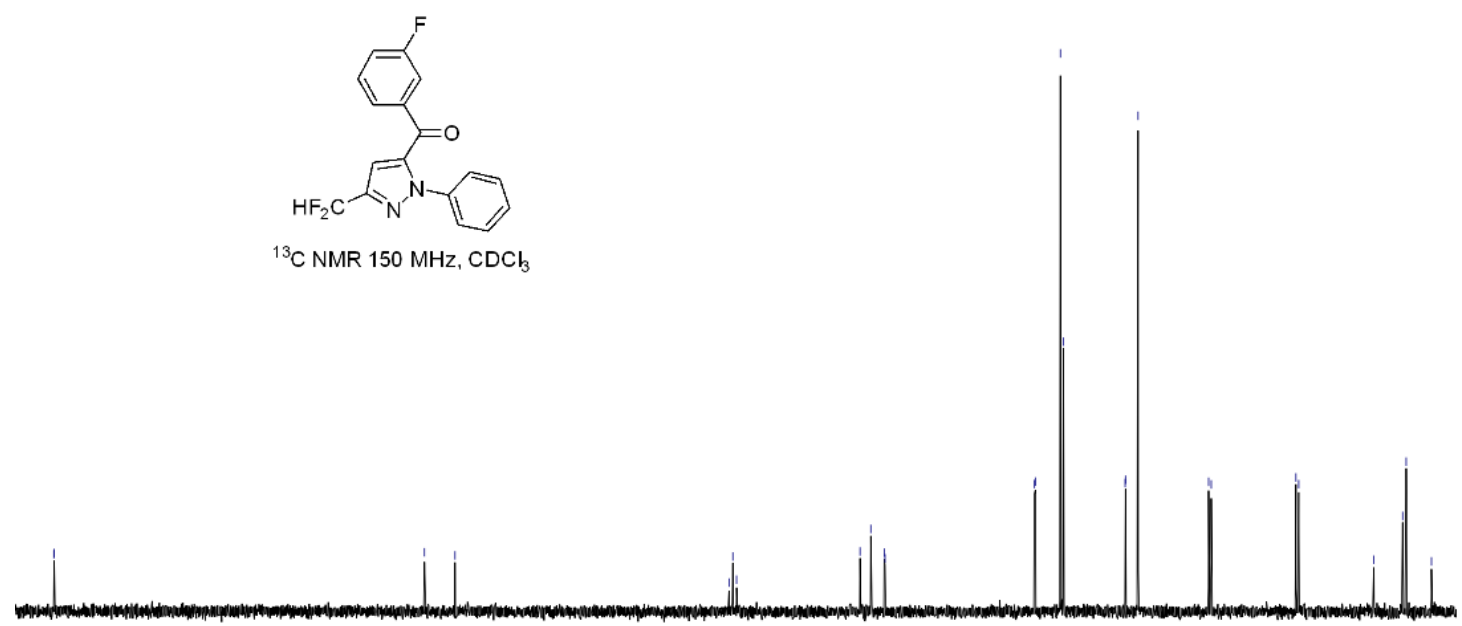

${ }^{13} \mathrm{C} \mathrm{NMR} 150 \mathrm{MHz}, \mathrm{CDCl}$

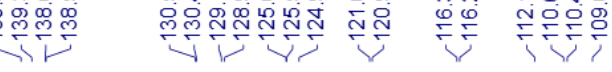
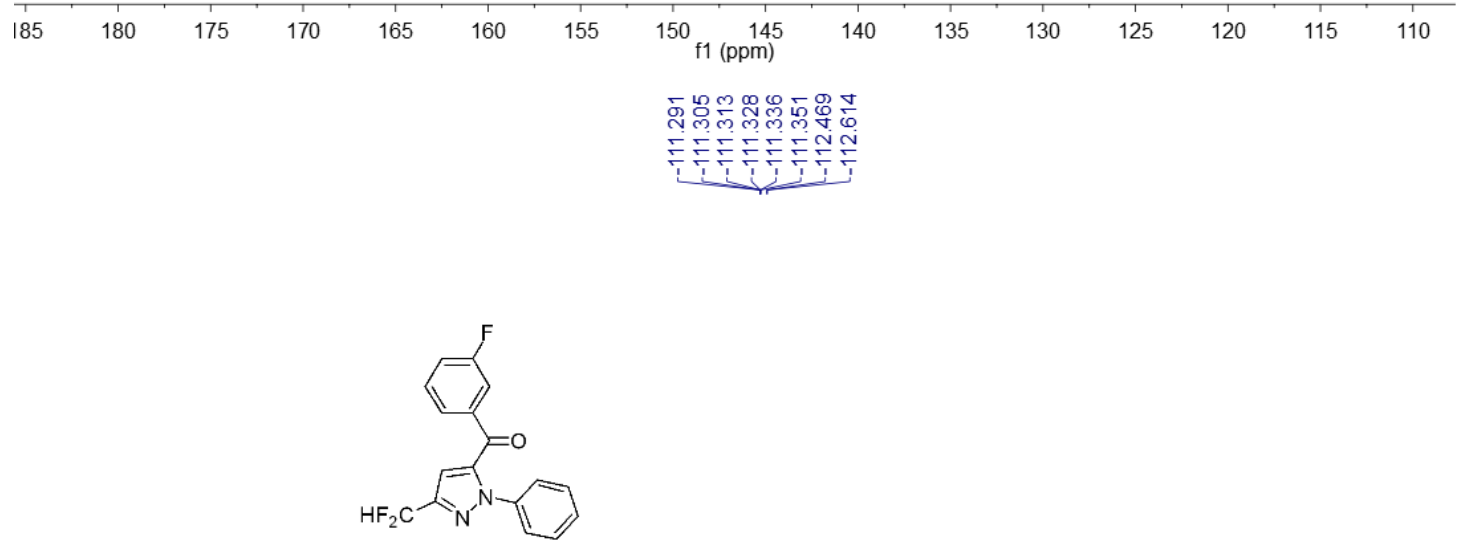

${ }^{19} \mathrm{~F}$ NMR $376 \mathrm{MHz}, \mathrm{CDCl}_{3}$

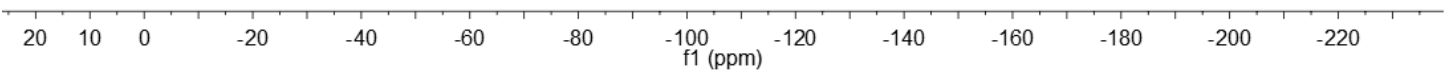




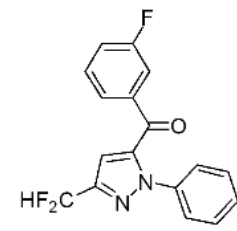

${ }^{19} \mathrm{~F}$ NMR $376 \mathrm{MHz}, \mathrm{CDCl}$

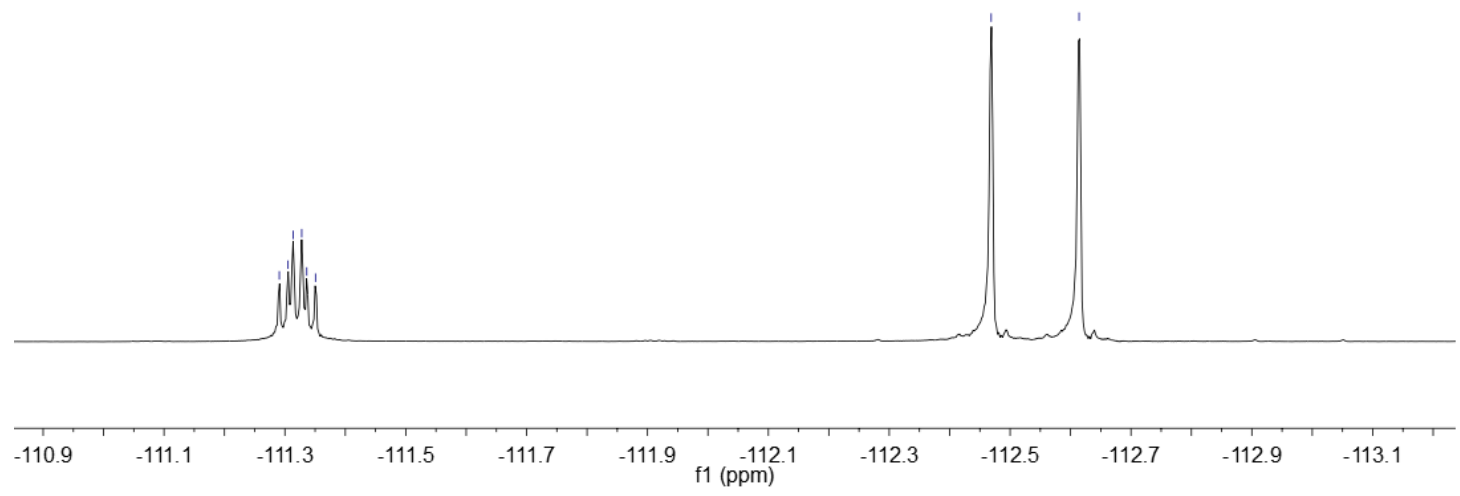

HRMS (ESI) copy of compound 3p:

\section{Mass Spectrum SmartFormula Report}

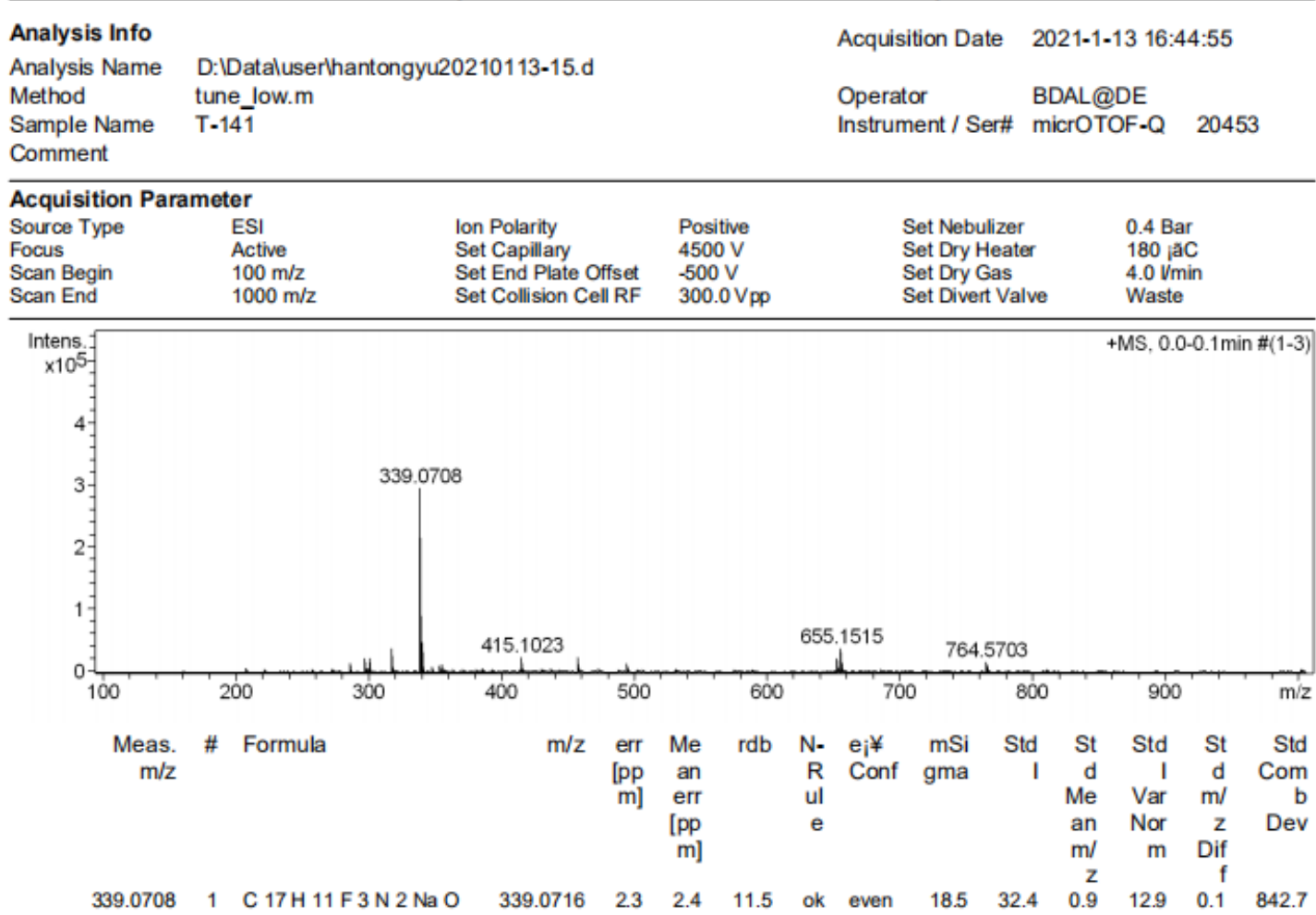


NMR copies of compound 3q:

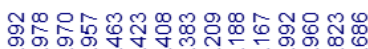

nanananongen

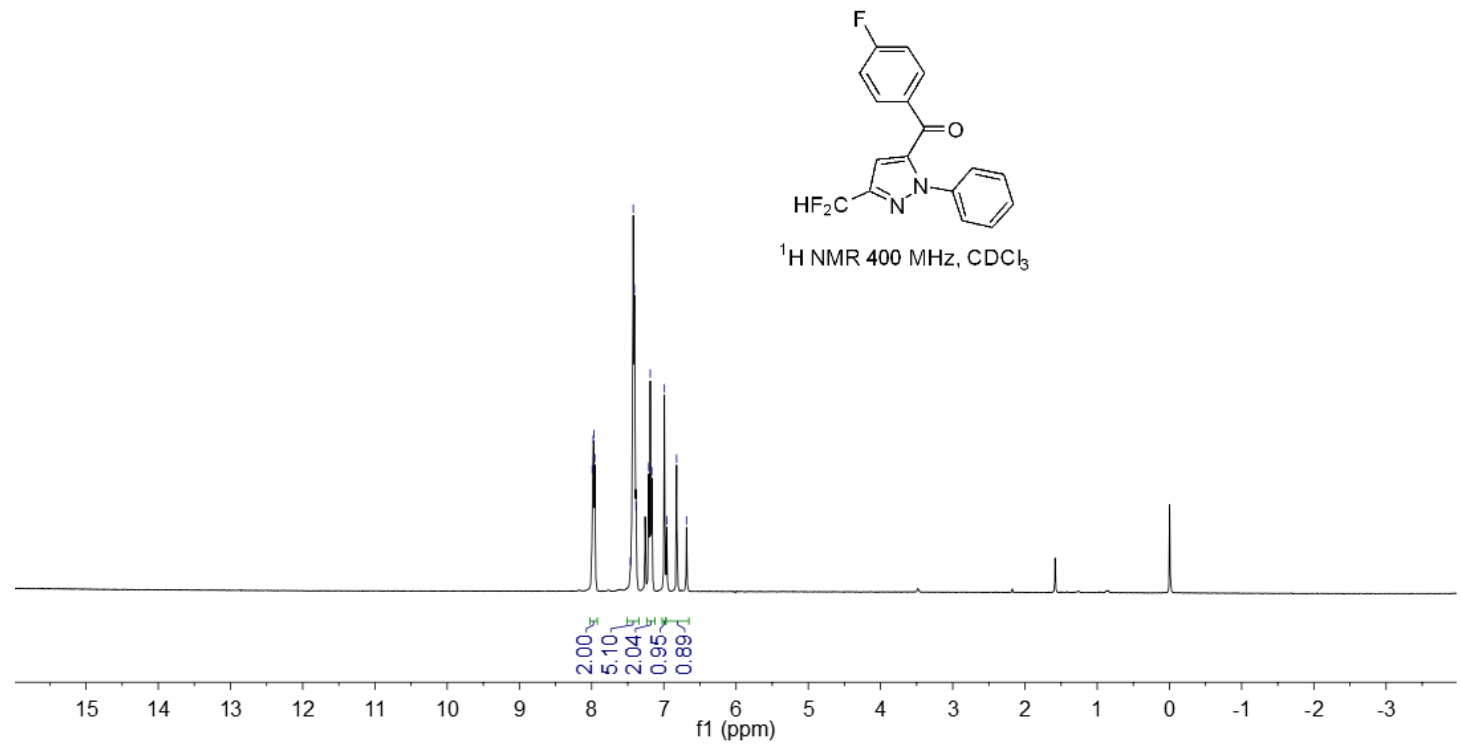

\begin{tabular}{|c|c|}
\hline 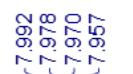 & 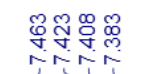 \\
\hline
\end{tabular}

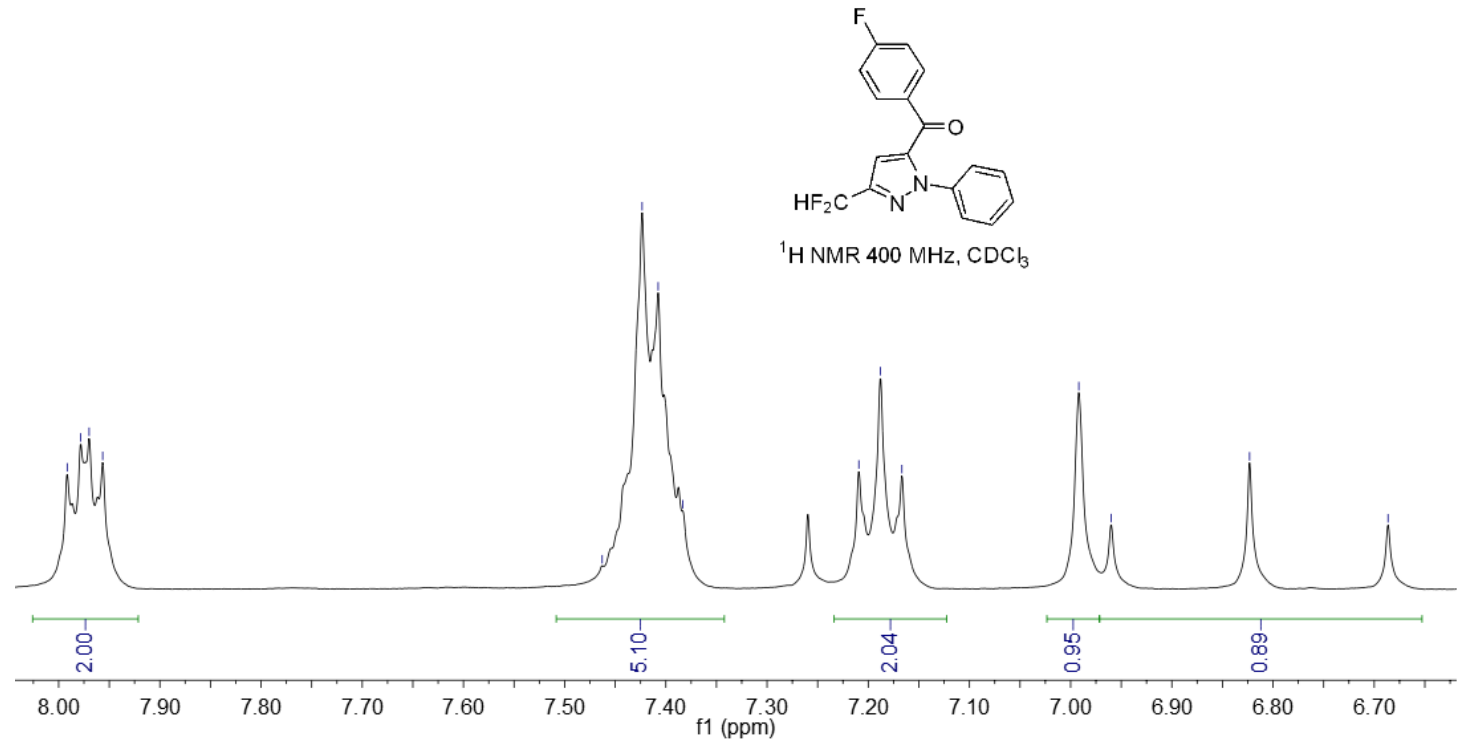




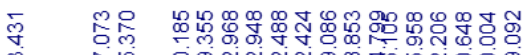

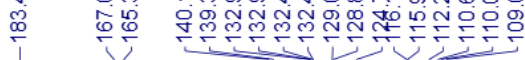

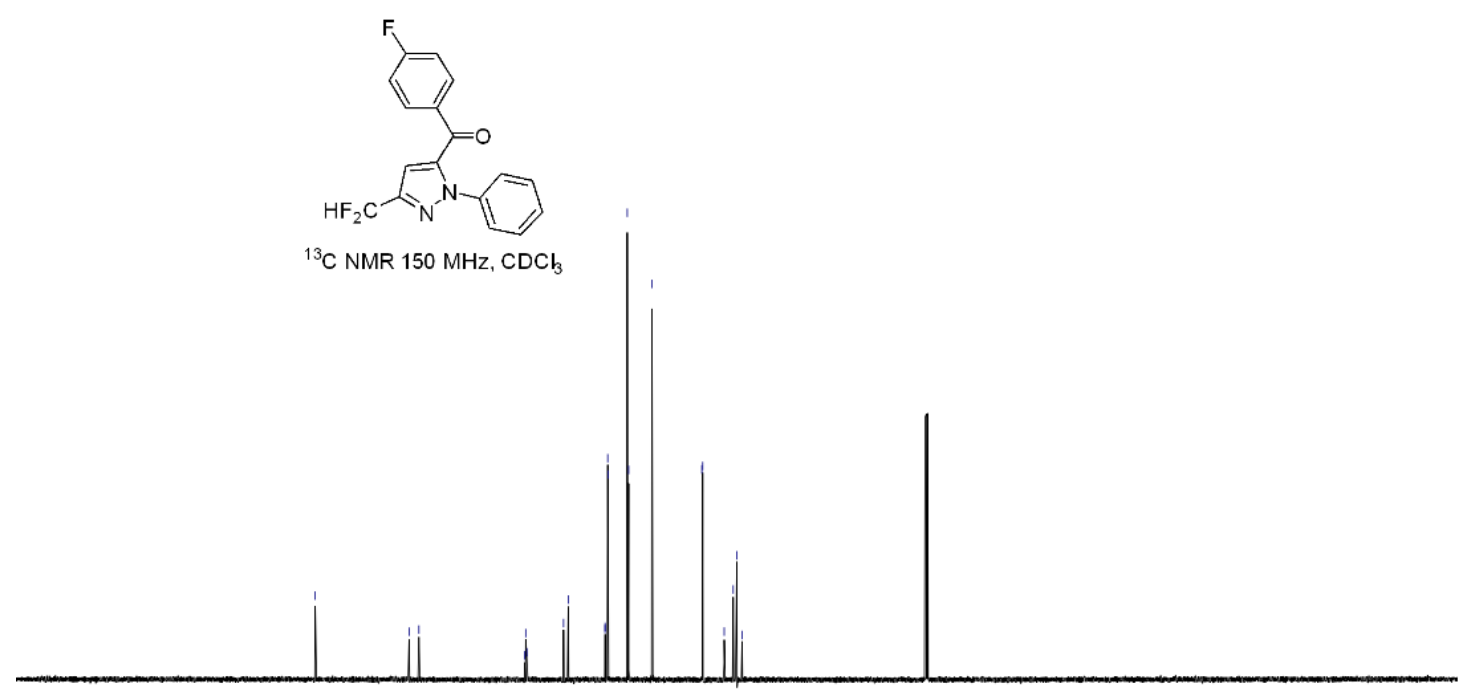

\begin{tabular}{|c|c|c|c|c|c|c|c|c|c|c|c|c|c|c|c|c|c|}
\hline 230 & 210 & 190 & 170 & 150 & 130 & $\begin{array}{c}110 \\
\mathrm{f} 1(\mathrm{ppm})\end{array}$ & 90 & 80 & 70 & 60 & 50 & 40 & 30 & 20 & 10 & 0 & -10 \\
\hline 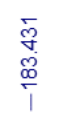 & & & 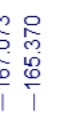 & & & 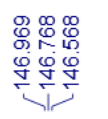 & 点 & & 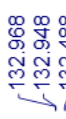 & 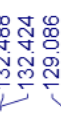 & & 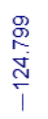 & & & 我 & 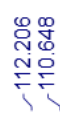 & 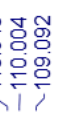 \\
\hline
\end{tabular}

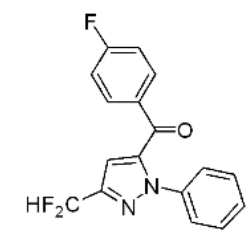

${ }^{13} \mathrm{C} \mathrm{NMR} 150 \mathrm{MHz}, \mathrm{CDCl}_{3}$

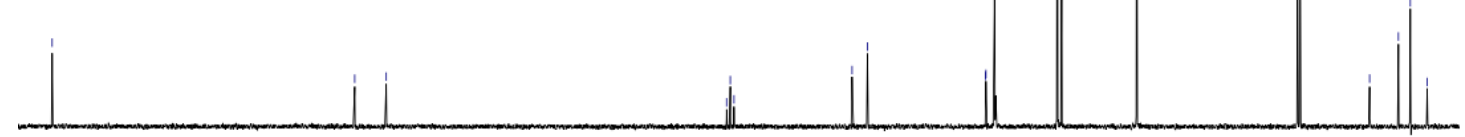

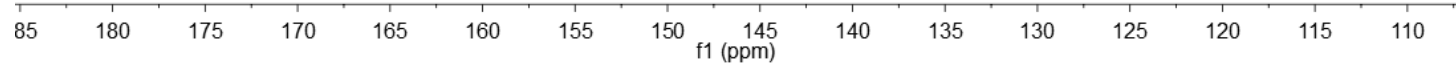




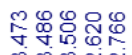

ำำำำ

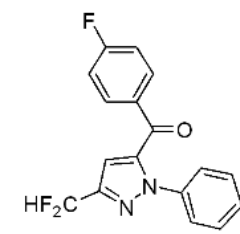

${ }^{19} \mathrm{~F} \mathrm{NMR} 376 \mathrm{MHz}, \mathrm{CDCl}$

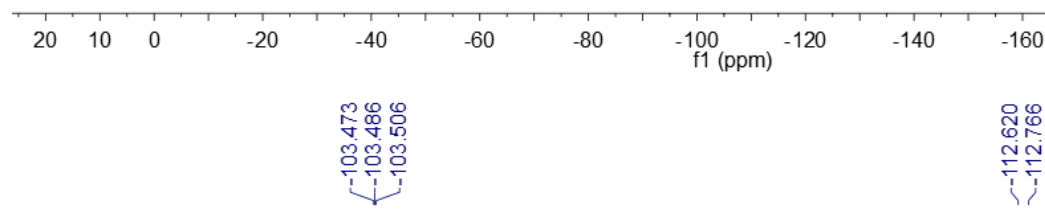

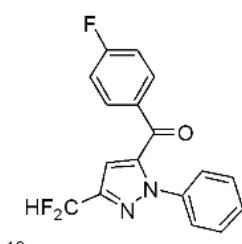

${ }^{19} \mathrm{~F}$ NMR $376 \mathrm{MHz}, \mathrm{CDCl}$

$\begin{array}{llllllllllllllllllllll}-99 & -100 & -101 & -102 & -103 & -104 & -105 & -106 & -107 & -108 & -109 & -110 & -111 & -112 & -113 & -114 & -115 & -116 & -117 & -118\end{array}$ 


\section{HRMS (ESI) copy of compound 3q:}

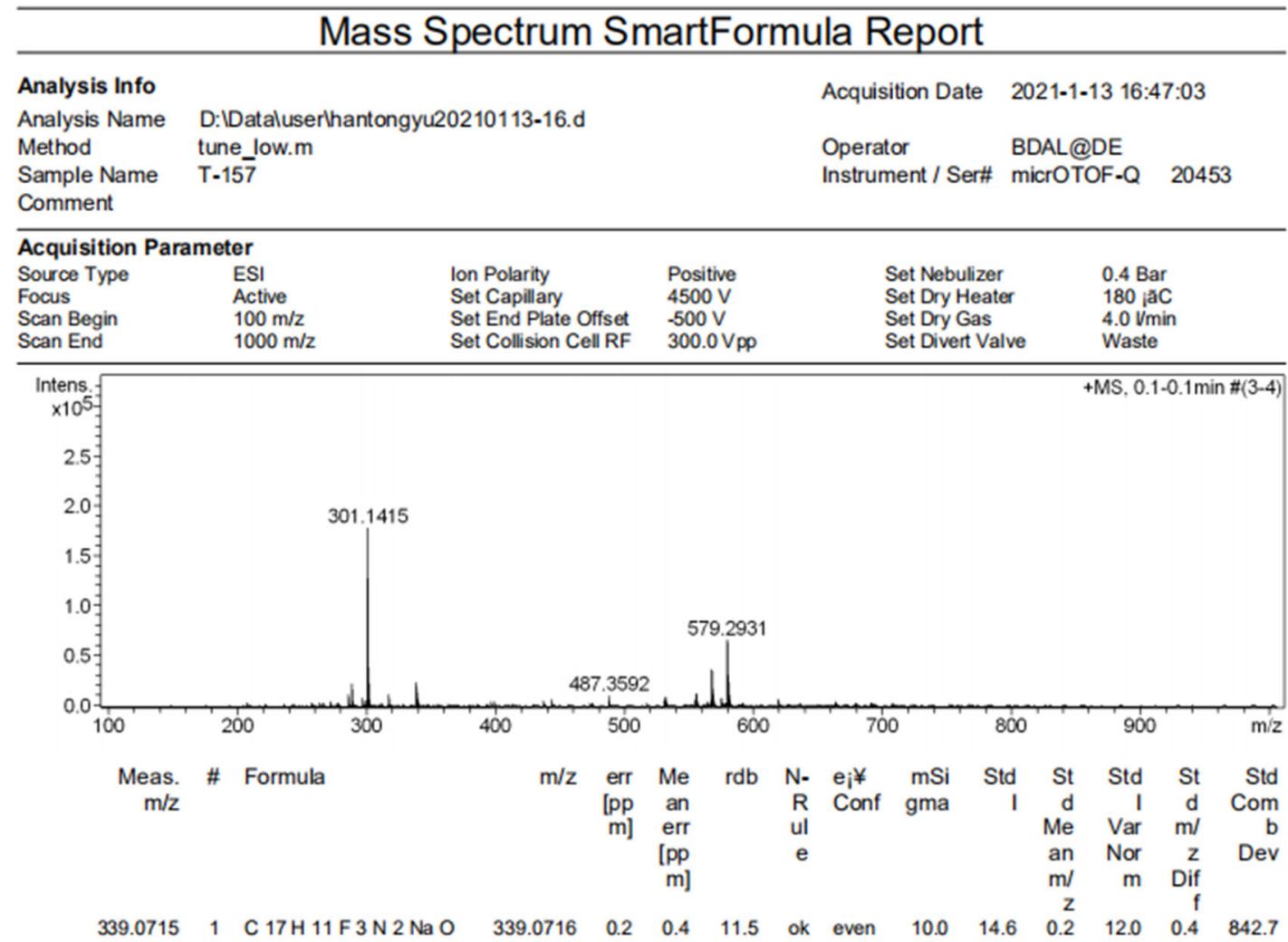

NMR copies of compound 3r:

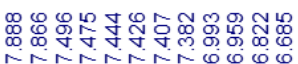

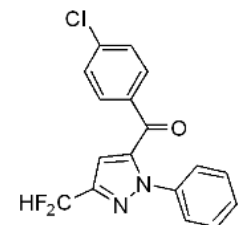

${ }^{1} \mathrm{H}$ NMR $400 \mathrm{MHz}, \mathrm{CDCl}$

舟

$\begin{array}{lllllllllllllllll}14 & 13 & 12 & 11 & 10 & 9 & 8 & 7 & \underset{\mathrm{f} 1}{6}(\mathrm{ppm}) & 5 & 4 & 3 & 2 & 1 & 0 & -1 & -2\end{array}$




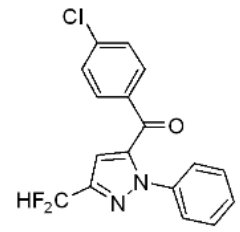

${ }^{1} \mathrm{HNMR} 400 \mathrm{MHz}, \mathrm{CDCl}_{3}$

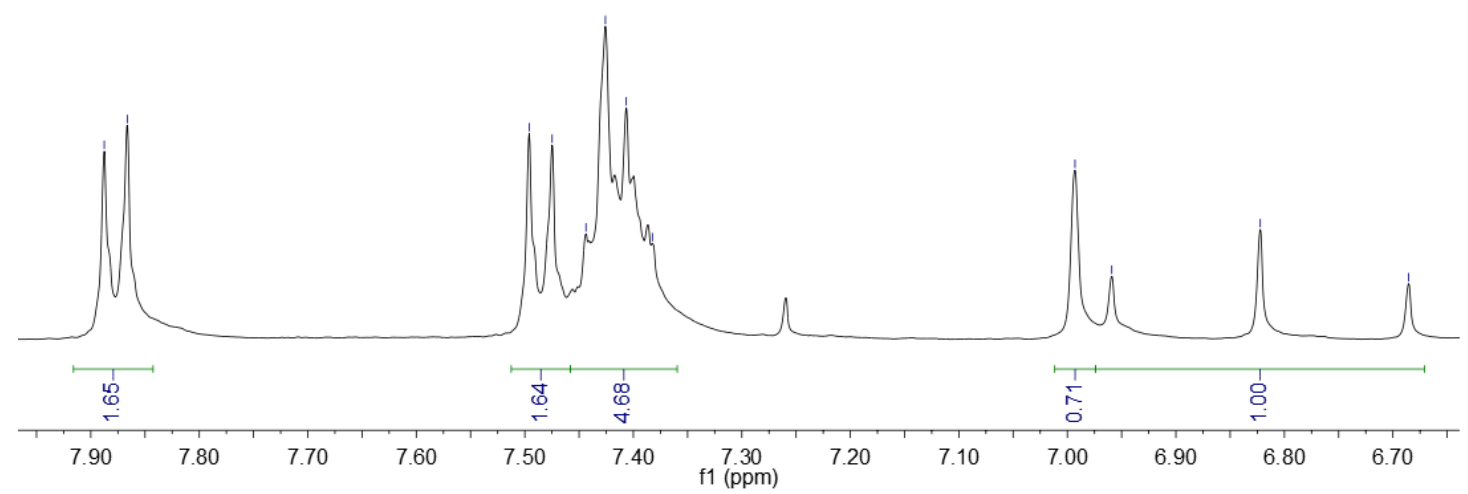

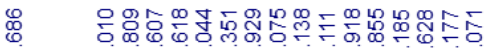

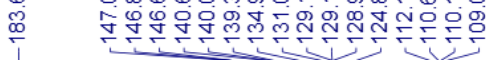

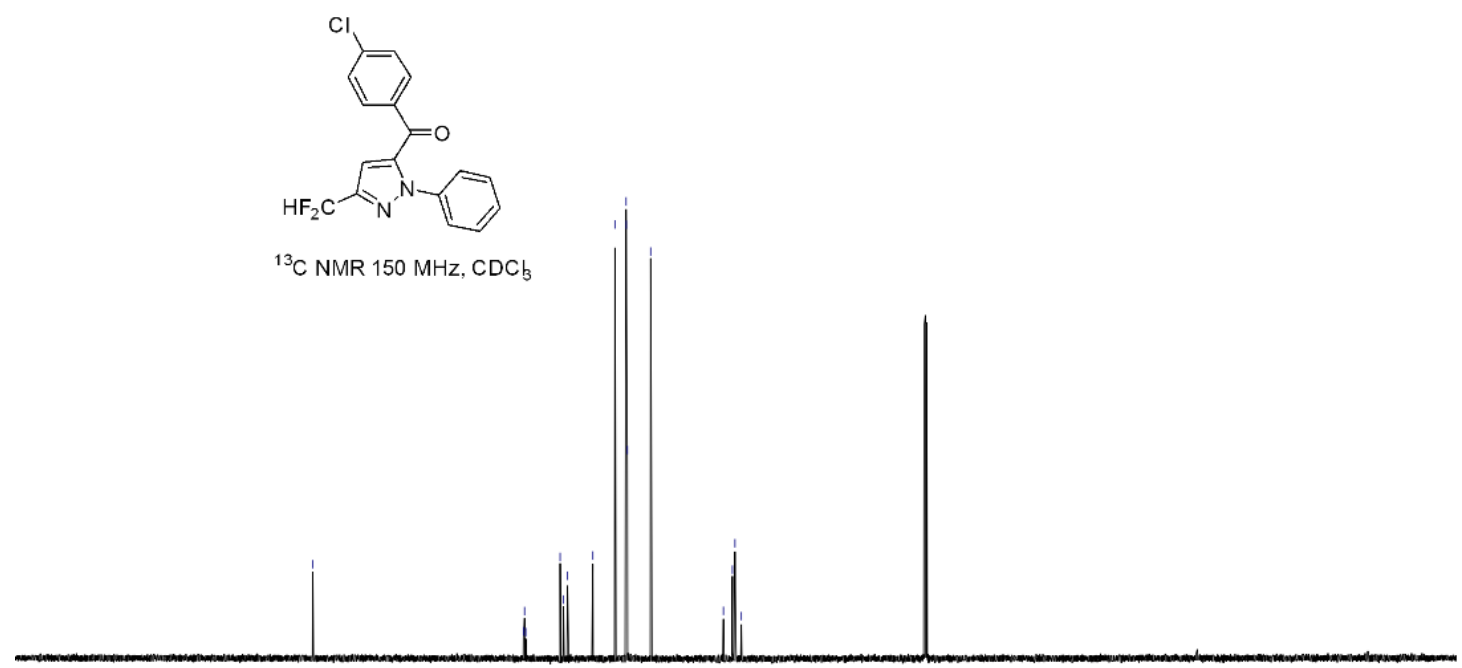

230 210 190 130 110 $\begin{array}{lllllllllll}90 & 80 & 70 & 60 & 50 & 40 & 30 & 20 & 10 & 0 & -10\end{array}$ 


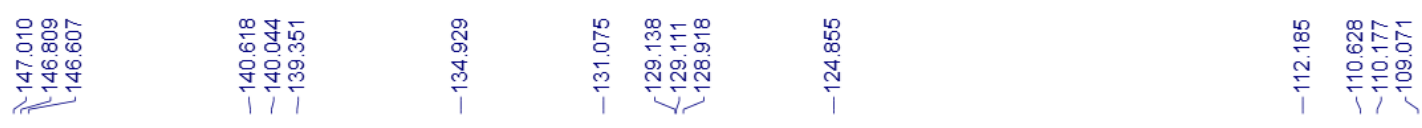
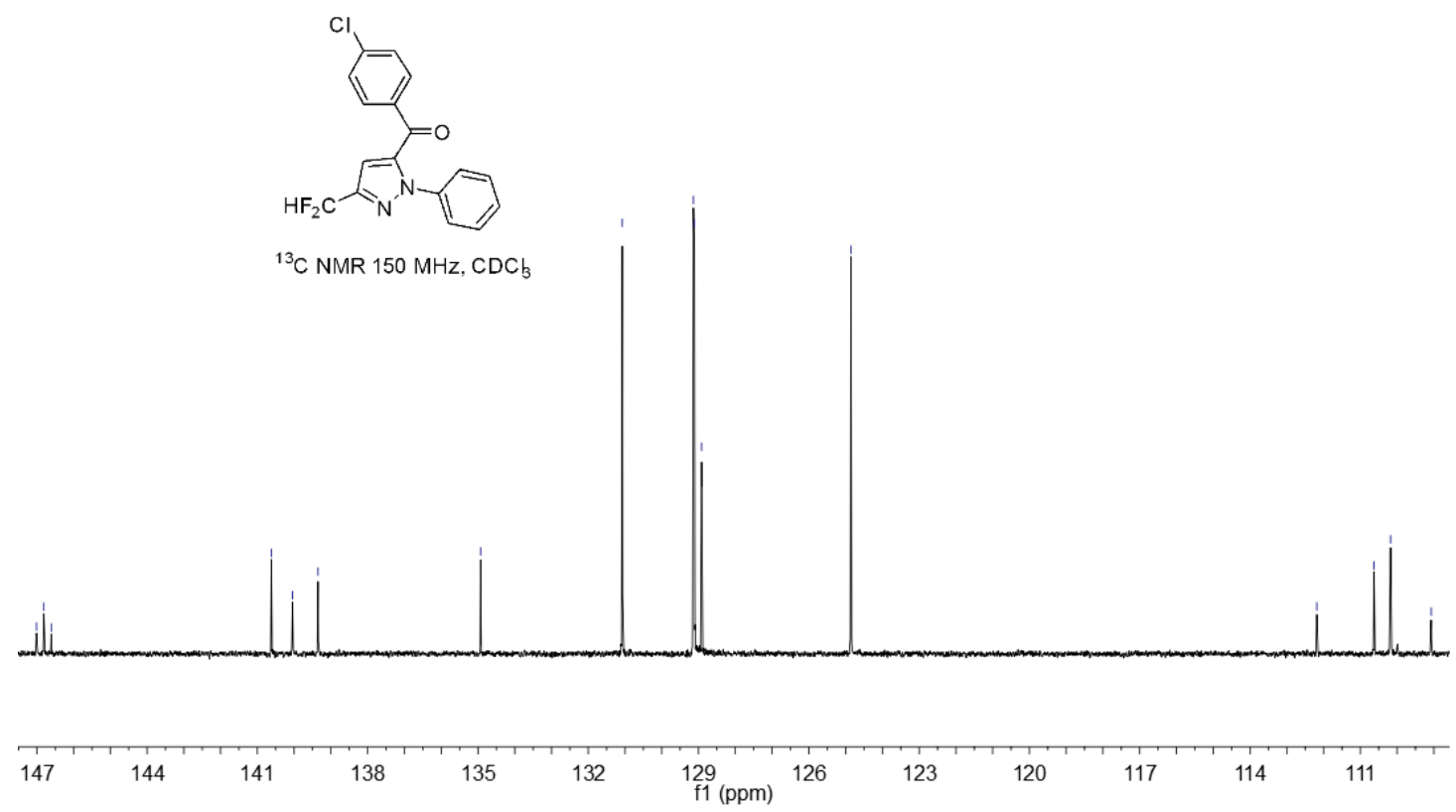

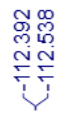

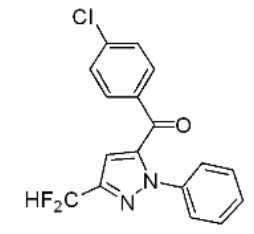

${ }^{19} \mathrm{~F}$ NMR $376 \mathrm{MHz}, \mathrm{CDCl}_{3}$

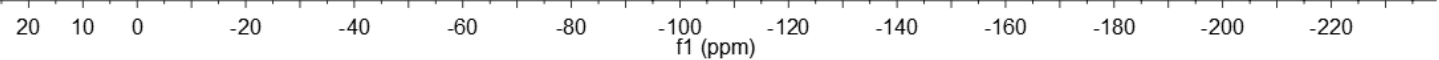




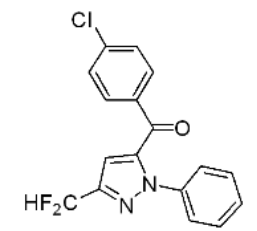

${ }^{19} \mathrm{~F} \mathrm{NMR} 376 \mathrm{MHz}, \mathrm{CDCl}_{3}$

$\begin{array}{lllllllllllllllll}-111.5 & -111.6 & -111.7 & -111.8 & -111.9 & -112.0 & -112.1 & -112.2 & -112.3 & -112.4 & -112.5 & -112.6 & -112.7 & -112.8 & -112.9 & -113.0\end{array}$

HRMS (ESI) copy of compound 3r:

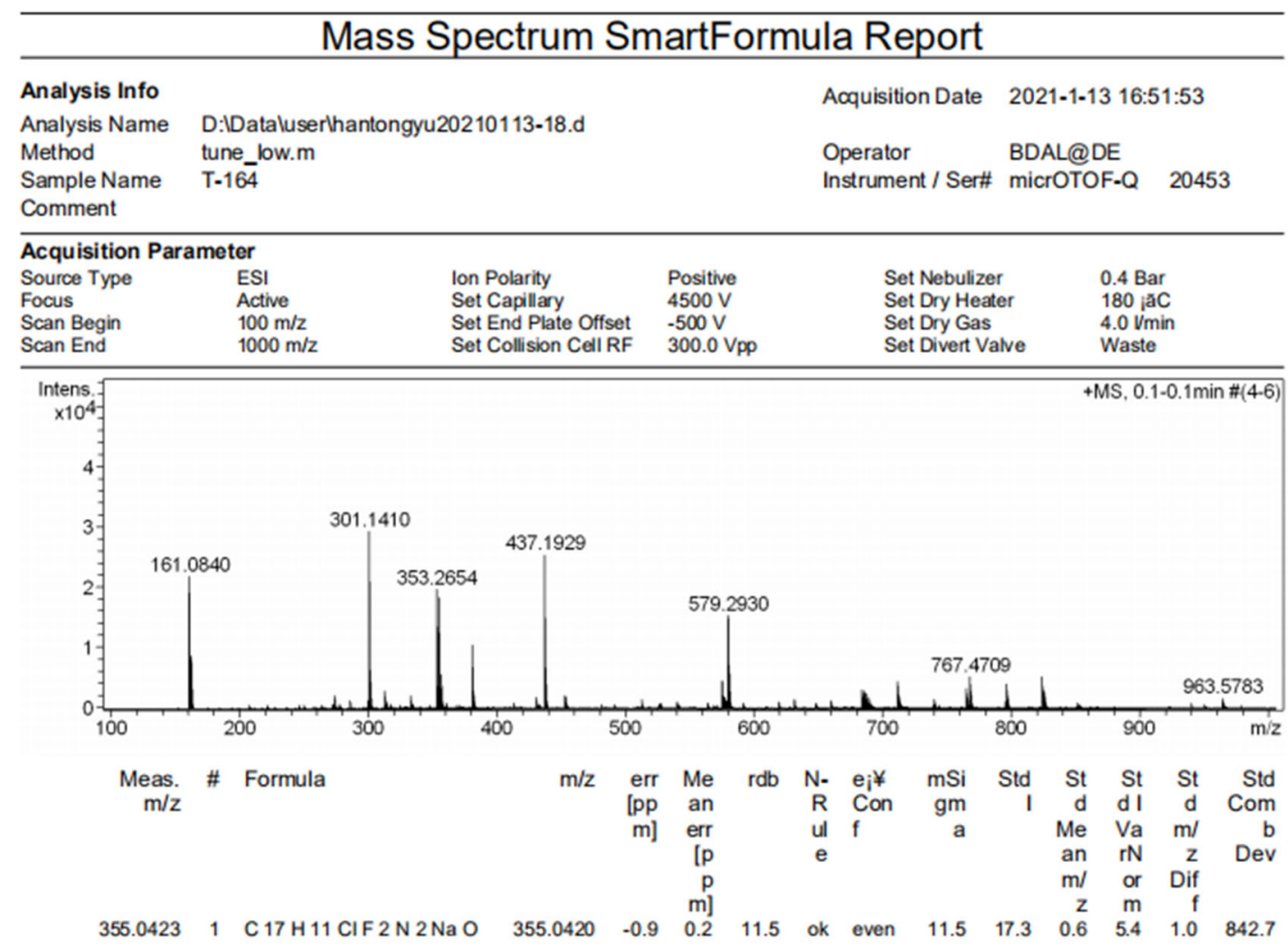


NMR copies of compound 3s:

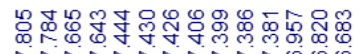

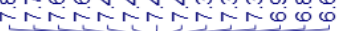

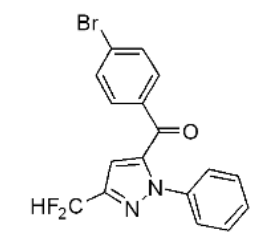

${ }^{1} \mathrm{HNMR} 400 \mathrm{MHz}, \mathrm{CDCl}_{3}$

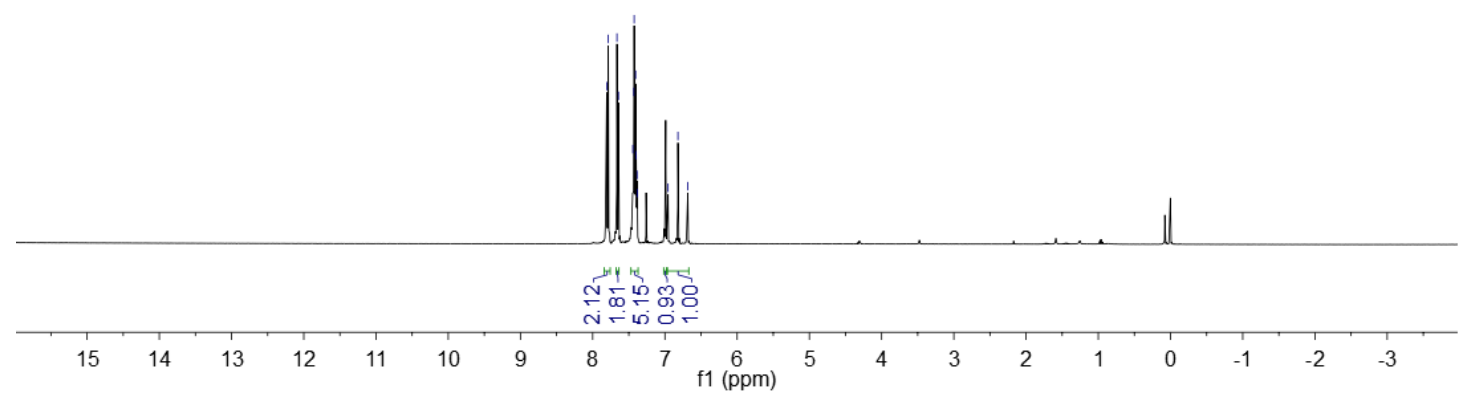

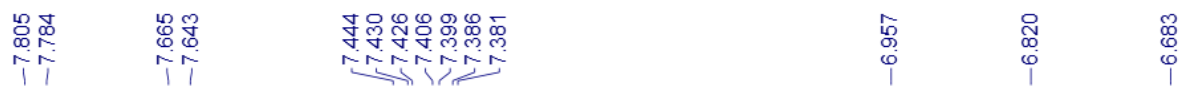

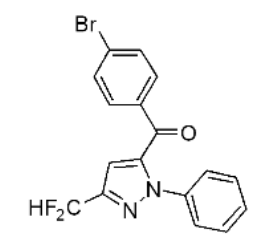

${ }^{1} \mathrm{H}$ NMR $400 \mathrm{MHz}, \mathrm{CDCl}_{3}$

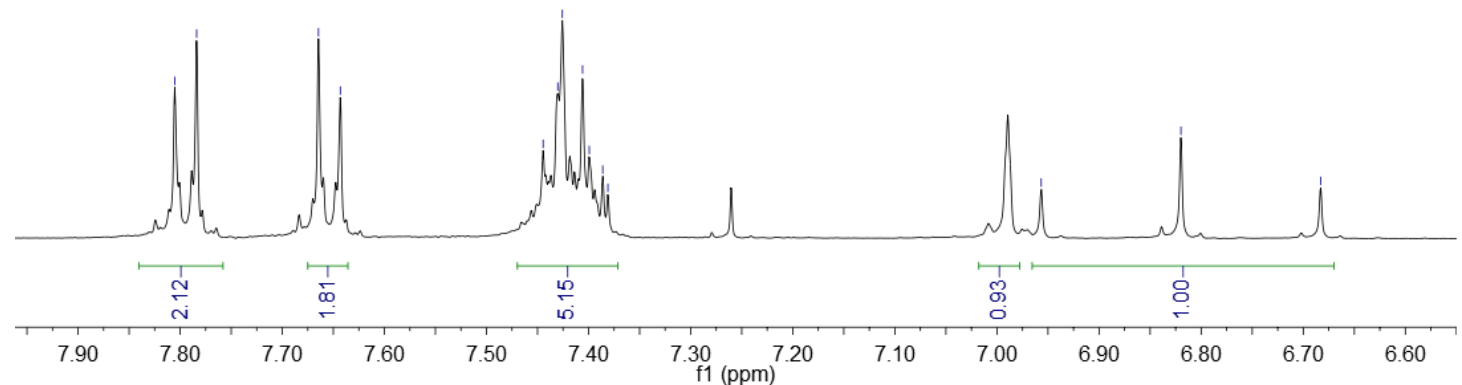




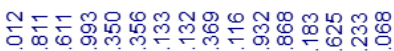

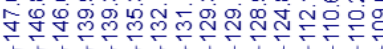
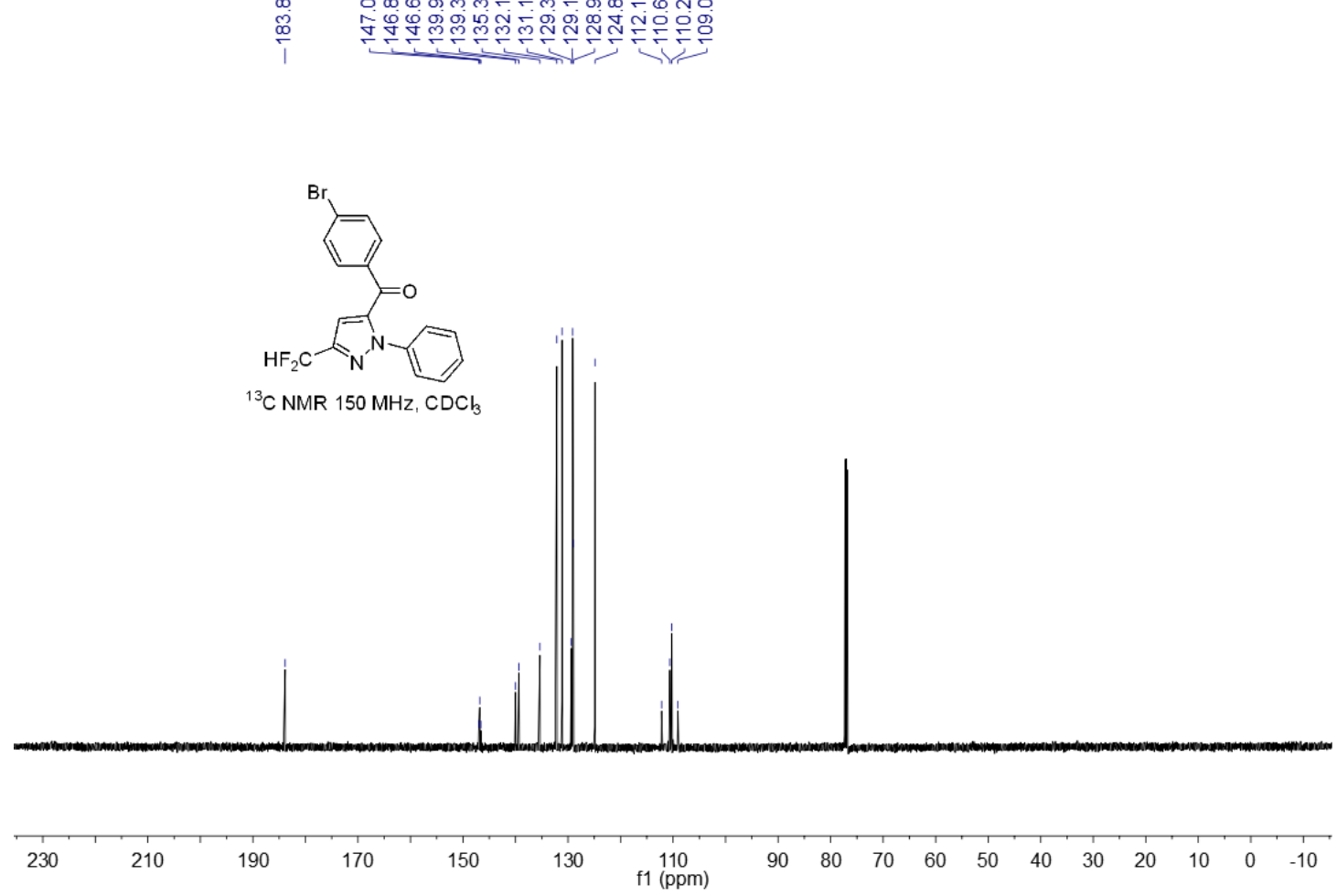

\section{둥두}

守守宁

刃ํ.

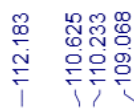

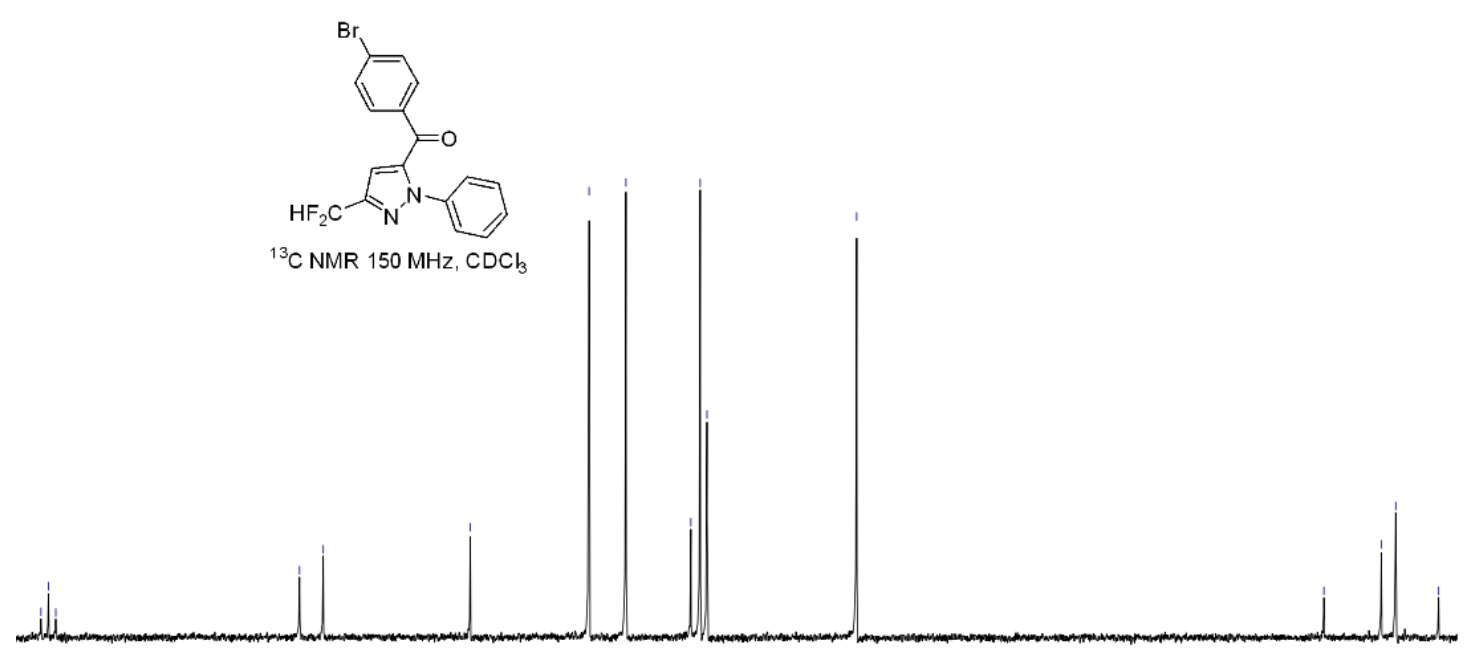

129 f1 (ppm)

120

117

114

111 


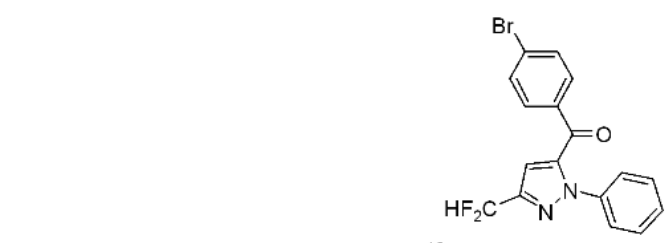

${ }^{19} \mathrm{~F}$ NMR $376 \mathrm{MHz}, \mathrm{CDCl}$
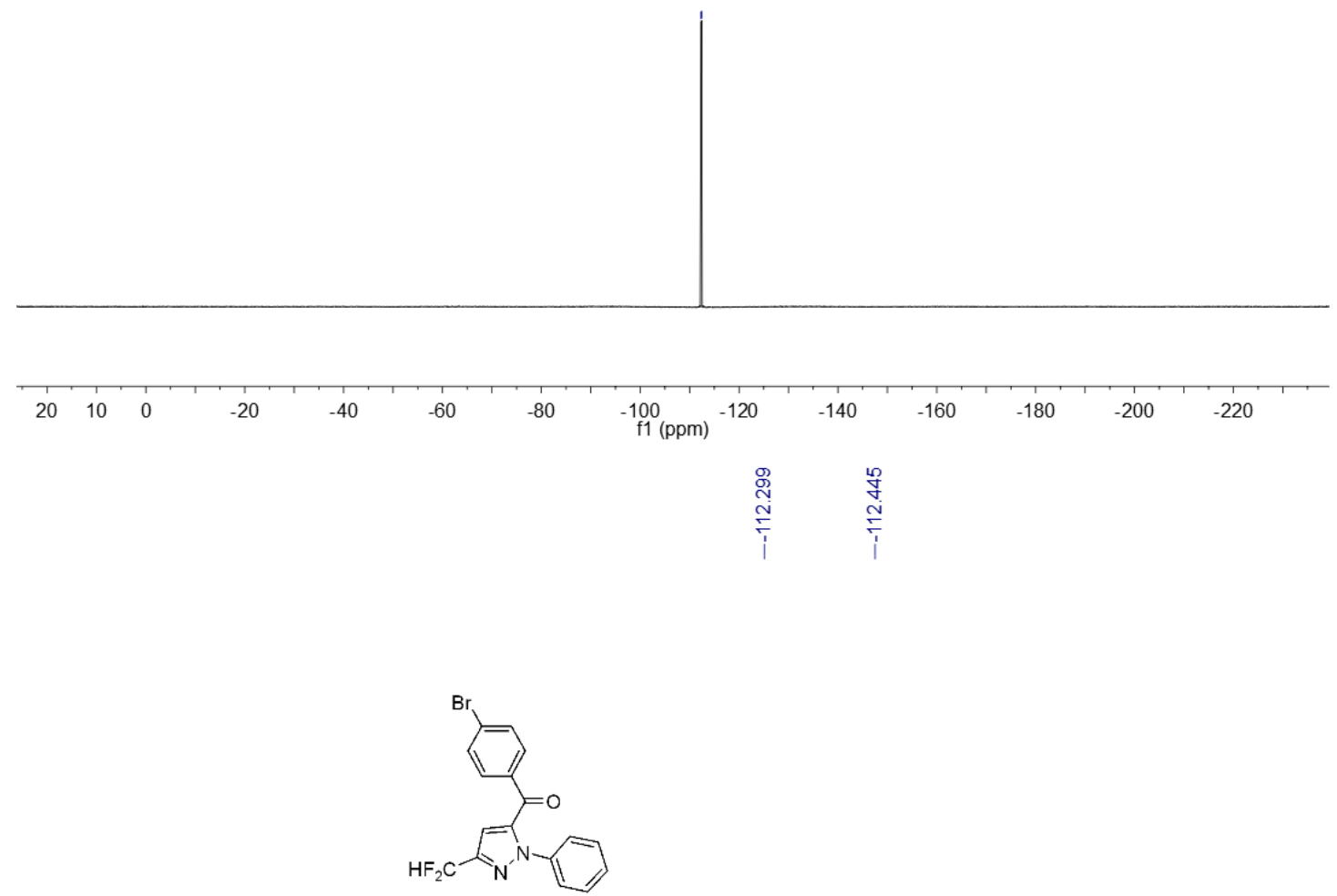

${ }^{19} \mathrm{~F}$ NMR $376 \mathrm{MHz}, \mathrm{CDCl}_{3}$

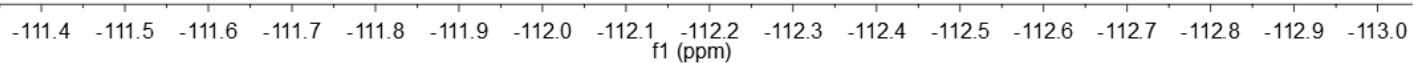

\section{S65}


HRMS (ESI) copy of compound 3s:

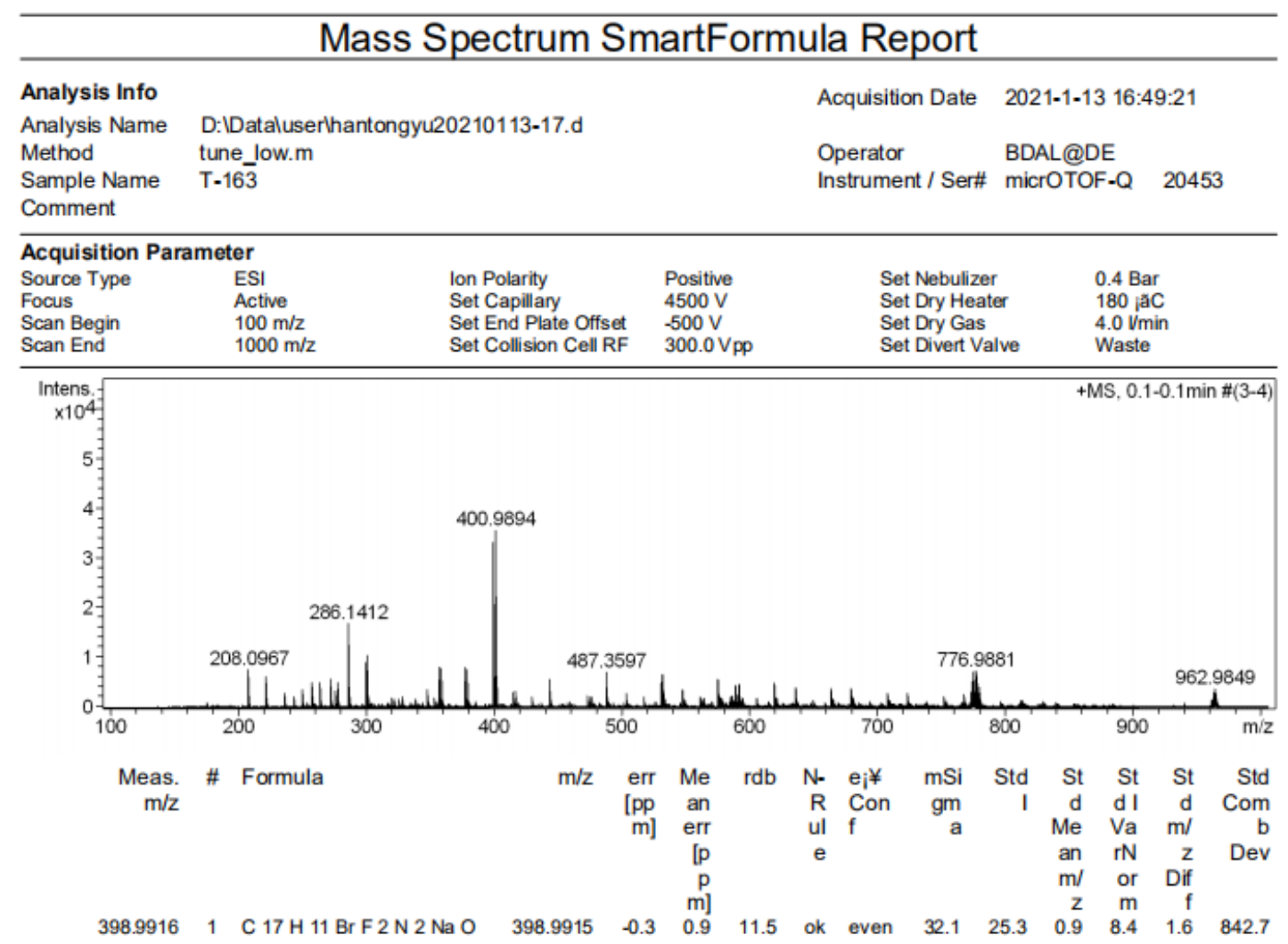

NMR copies of compound $3 t$ :

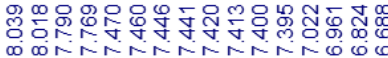

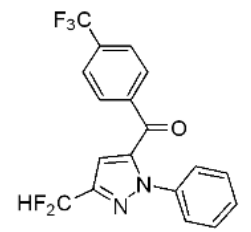

${ }^{1} \mathrm{HNMR} 400 \mathrm{MHz}, \mathrm{CDCl}_{3}$

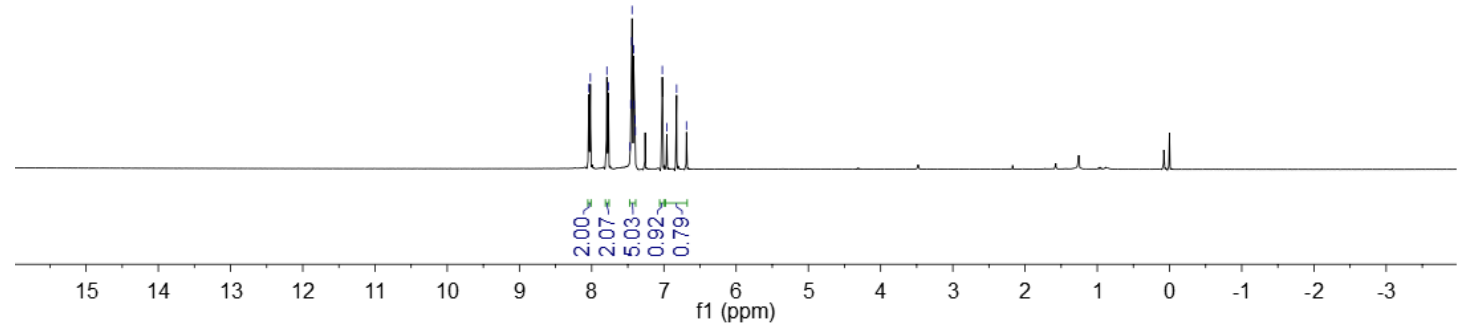



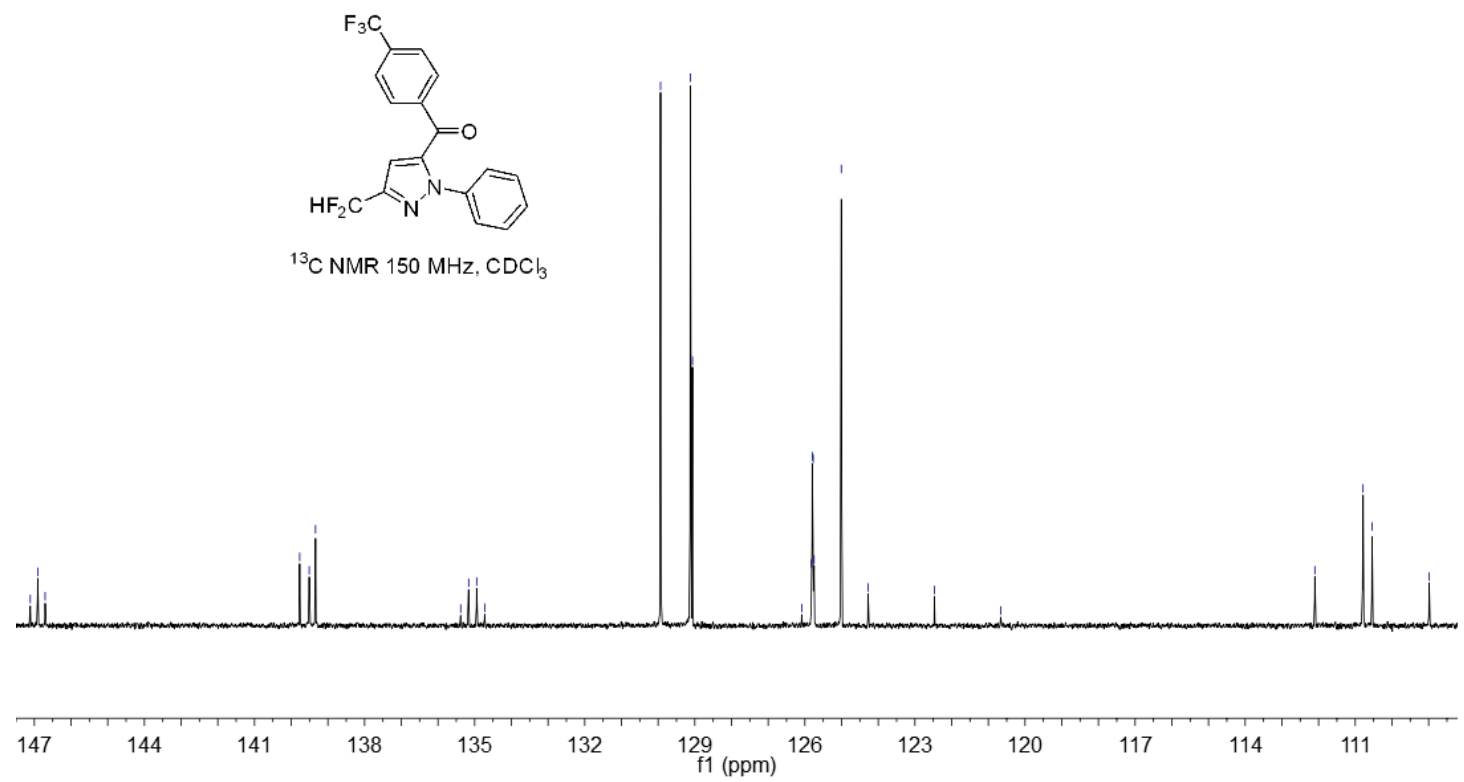

兽

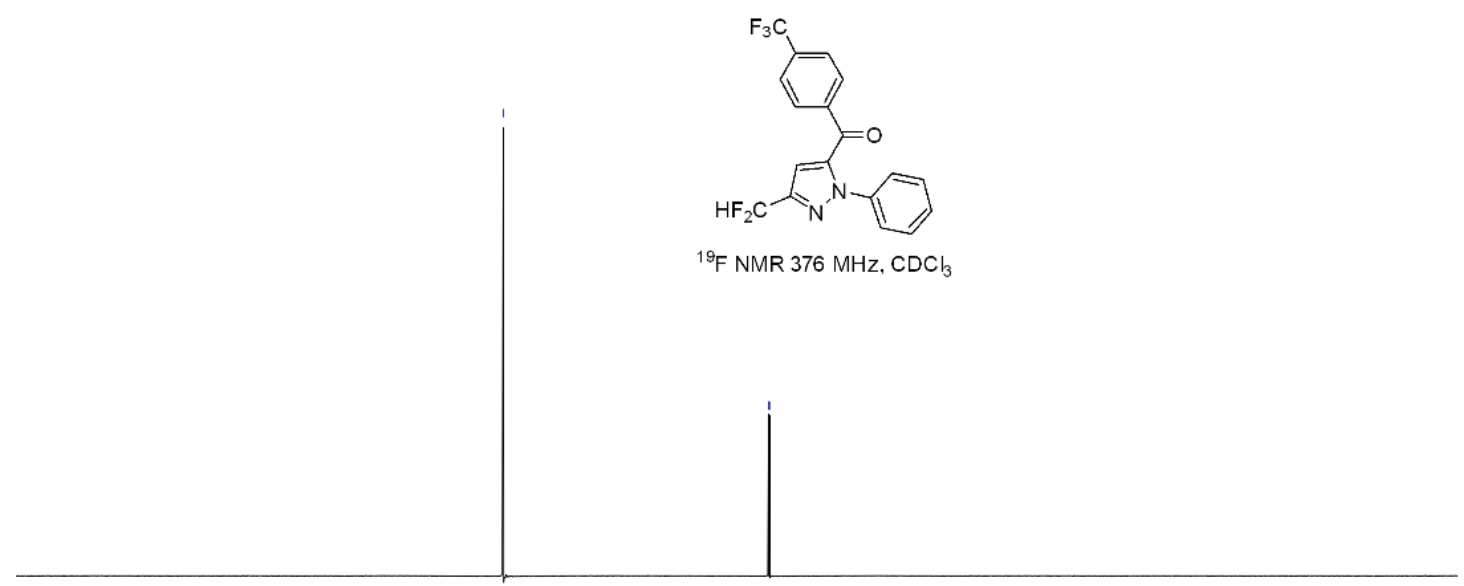

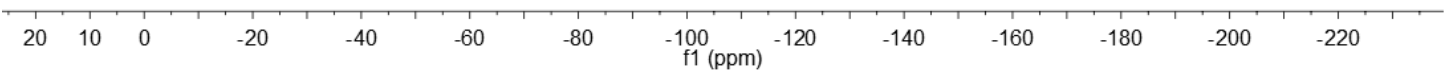




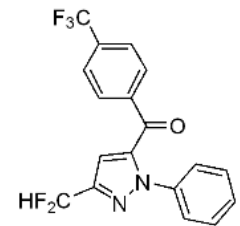

${ }^{19} \mathrm{~F}$ NMR $376 \mathrm{MHz}, \mathrm{CDCl}$

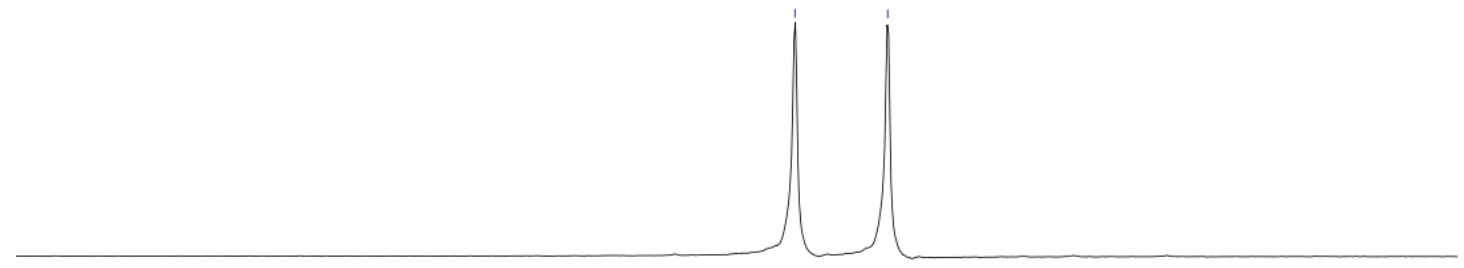

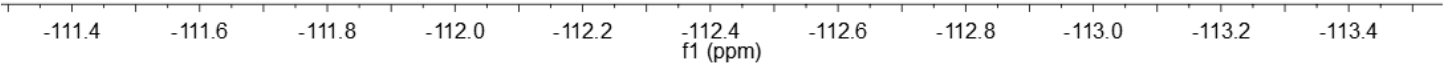

HRMS (ESI) copy of compound 3t:

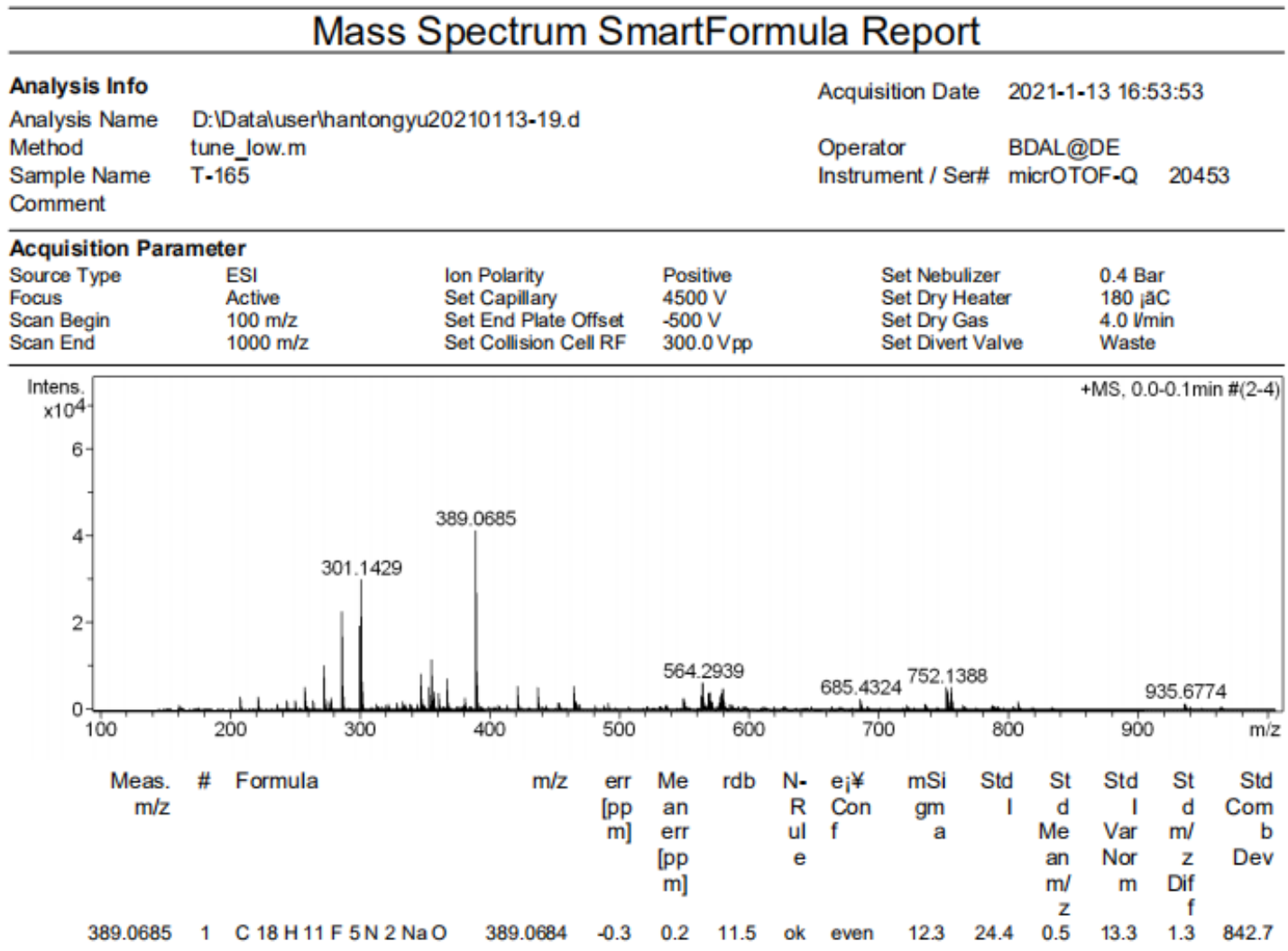


NMR copies of compound $\mathbf{3 u}$ :

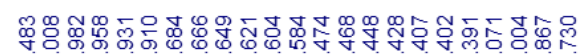

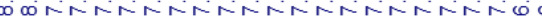

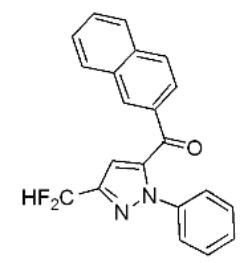

${ }^{1} \mathrm{H} \mathrm{NMR} 400 \mathrm{MHz}, \mathrm{CDCl}_{3}$
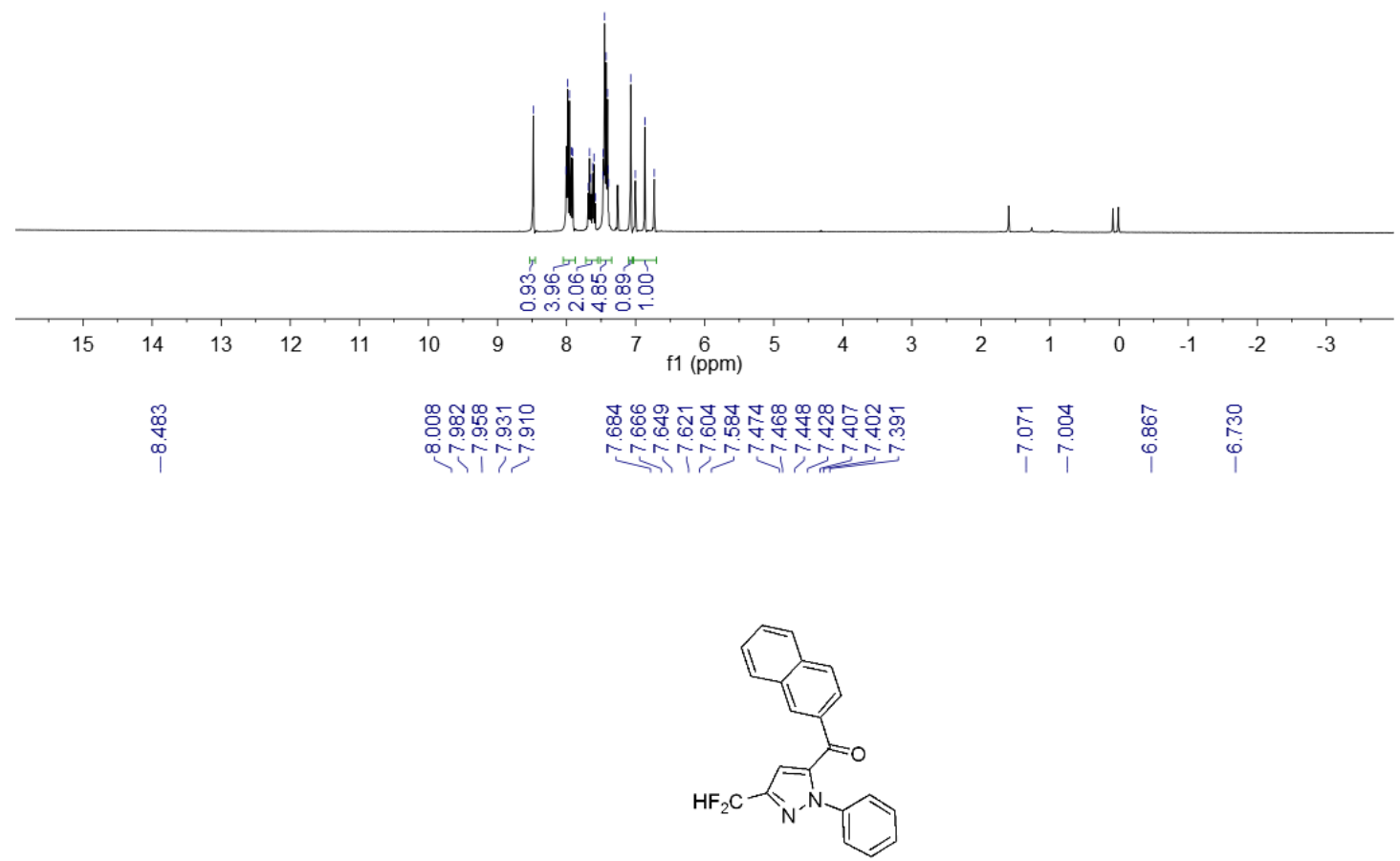

${ }^{1} \mathrm{H} \mathrm{NMR} 400 \mathrm{MHz}, \mathrm{CDCl}_{3}$

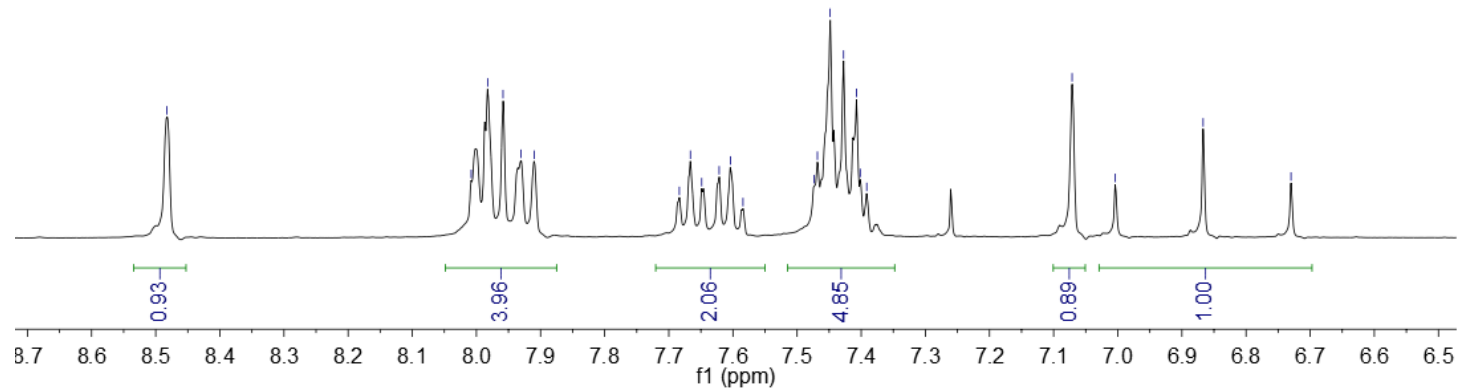




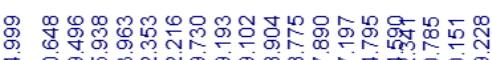

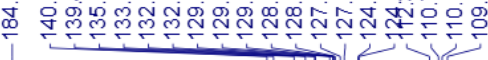

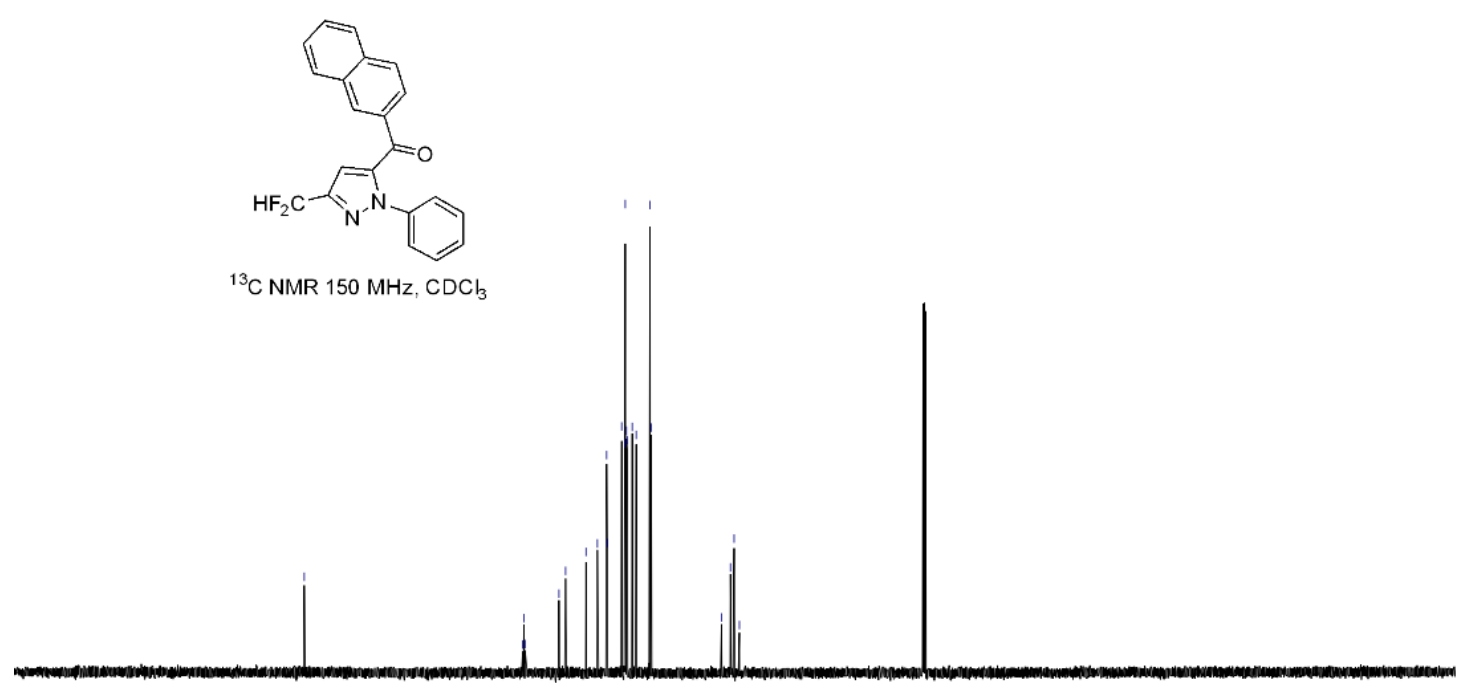

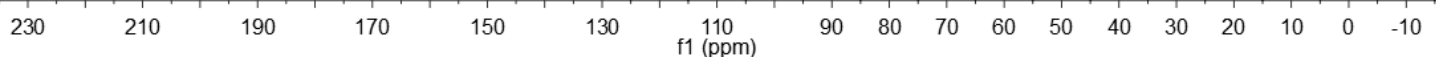

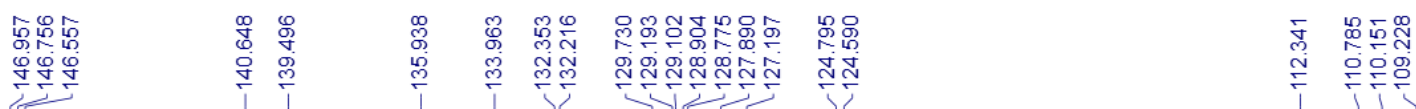

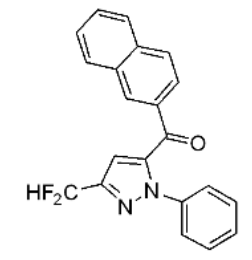

${ }^{13} \mathrm{C} \mathrm{NMR} 150 \mathrm{MHz}, \mathrm{CDCl}$

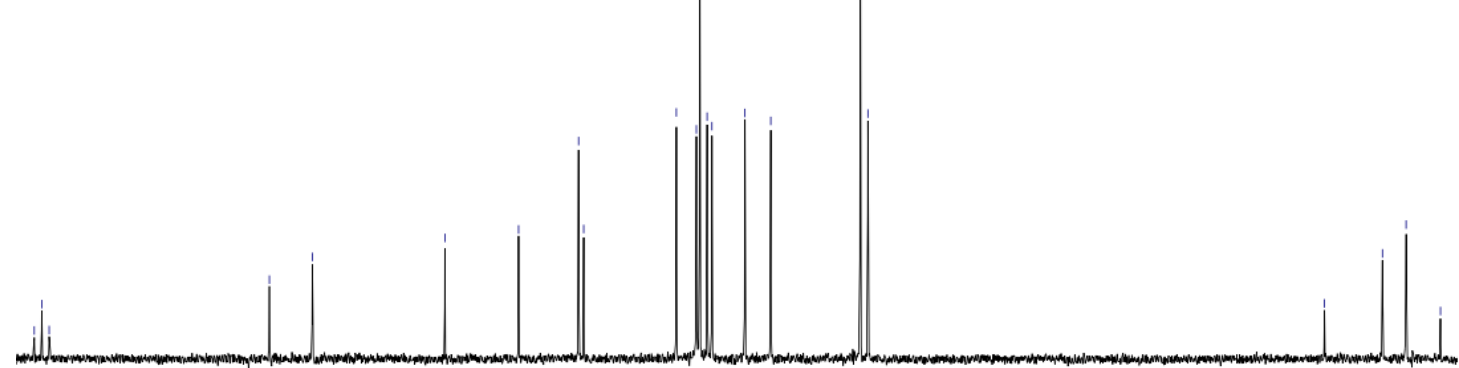

$\begin{array}{lllllllllllll}147 & 144 & 141 & 138 & 135 & 132 & \substack{129 \\ \mathrm{f} 1(\mathrm{ppm})} & 126 & 123 & 120 & 117 & 114 & 111\end{array}$ 


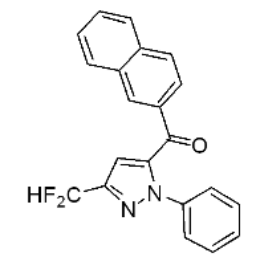

${ }^{19} \mathrm{~F} \mathrm{NMR} 376 \mathrm{MHz}, \mathrm{CDCl}_{3}$
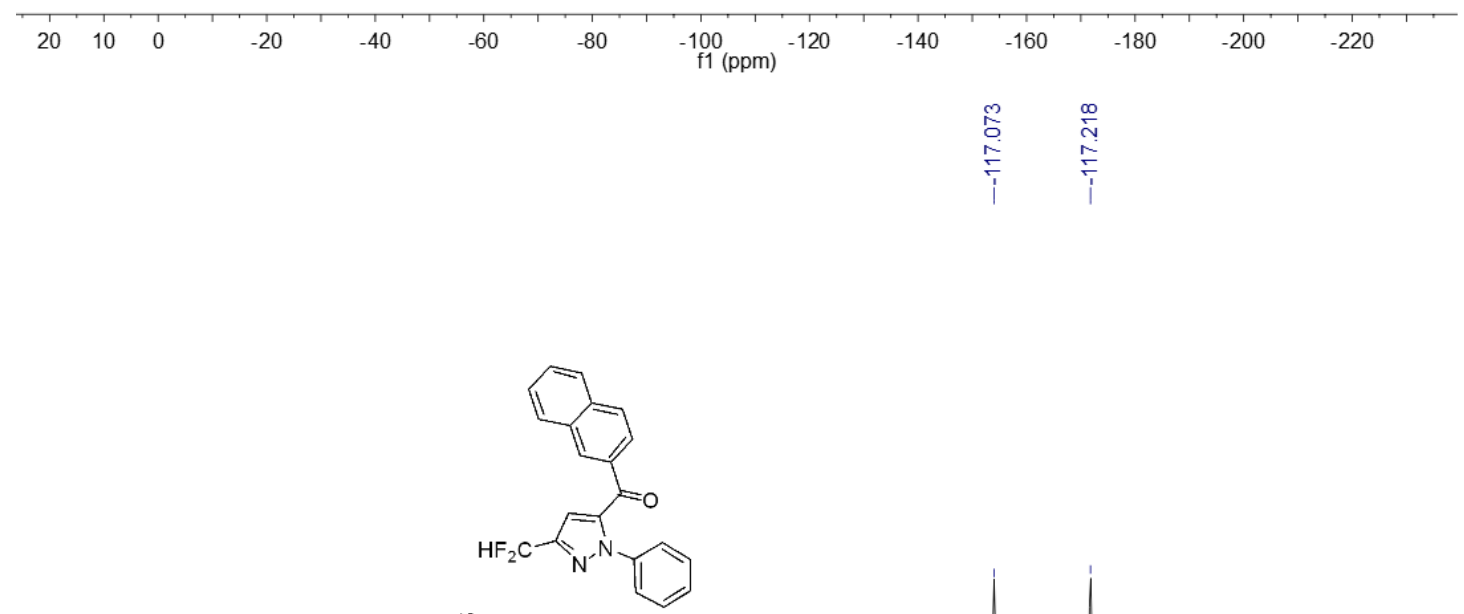

${ }^{19} \mathrm{~F} \mathrm{NMR} 376 \mathrm{MHz}, \mathrm{CDCl}_{3}$

\begin{tabular}{lllllllll}
\hline 5.6 & -115.8 & -116.0 & -116.2 & -116.4 & -116.6 & 1 \\
$\mathrm{f} 1(\mathrm{ppm})$ & -116.8 & -117.0 & -117.2 & -117.4 & -117.6
\end{tabular}


HRMS (ESI) copy of compound $\mathbf{3 u}$ :

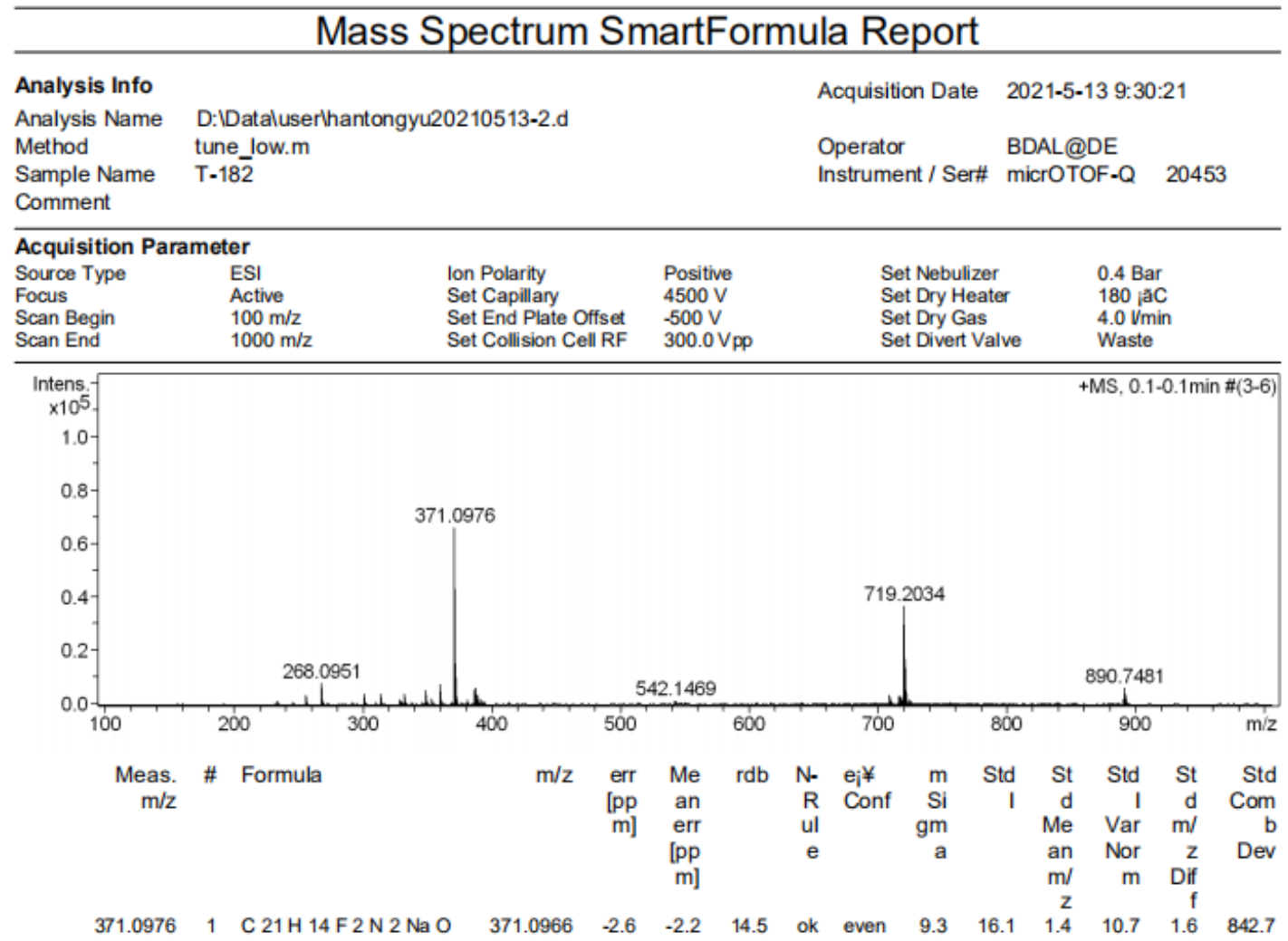

NMR copies of compound $3 \mathbf{v}$ :

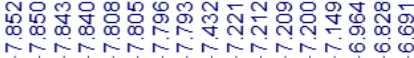

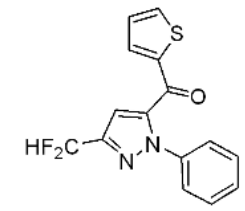

${ }^{1} \mathrm{H} \mathrm{NMR} 400 \mathrm{MHz}, \mathrm{CDCl}_{3}$

\begin{tabular}{|llllllllllllllll}
\hline 4 & 13 & 12 & 11 & 10 & 9 & 8 & 7 & 6 & 5 & 4 & 3 & 2 & 1 & 0 & -1
\end{tabular}




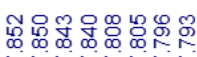
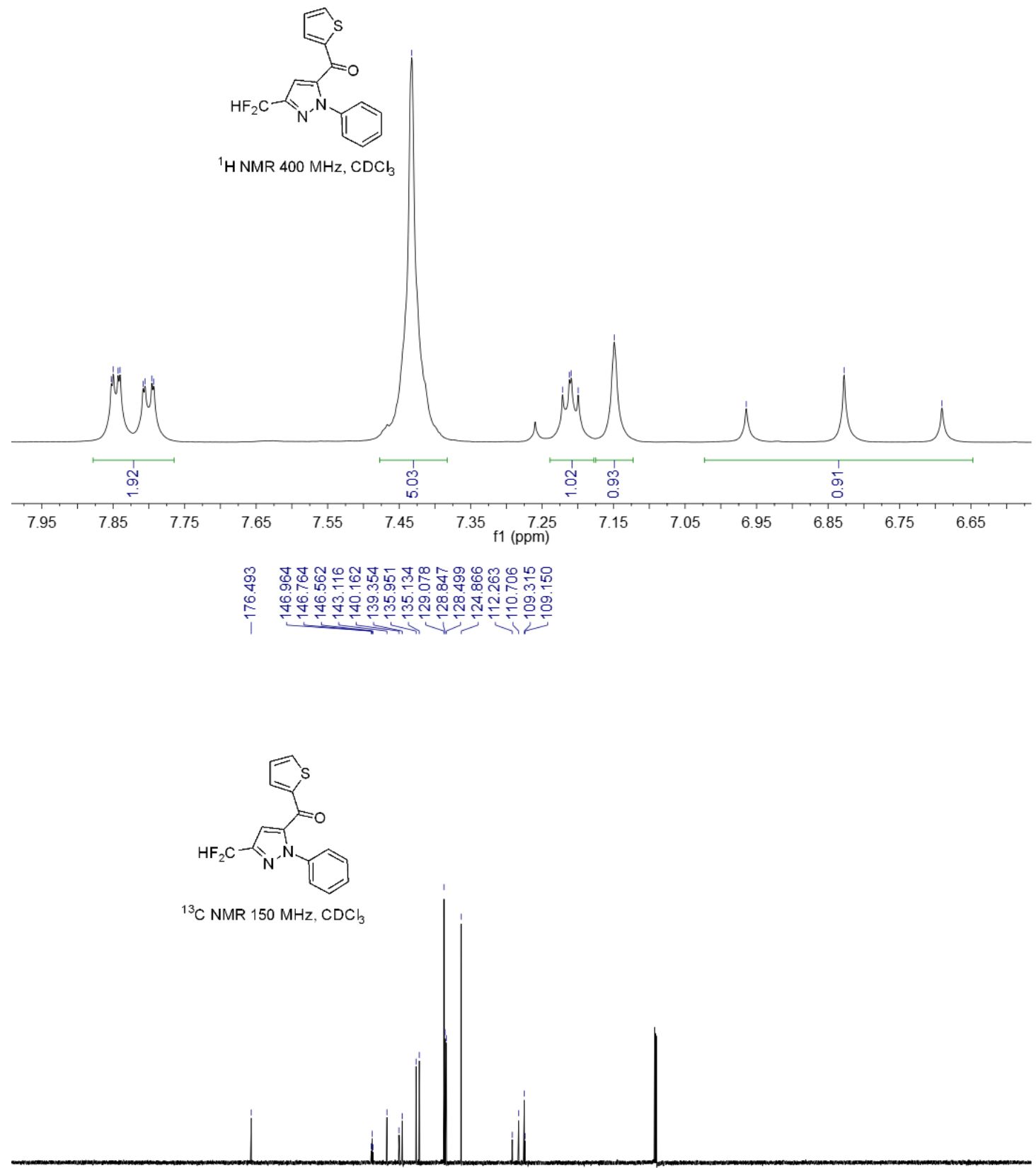

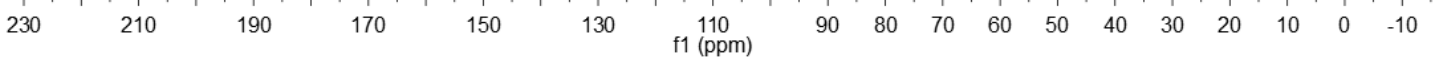




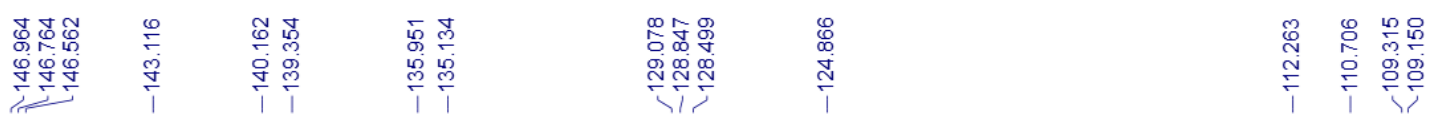
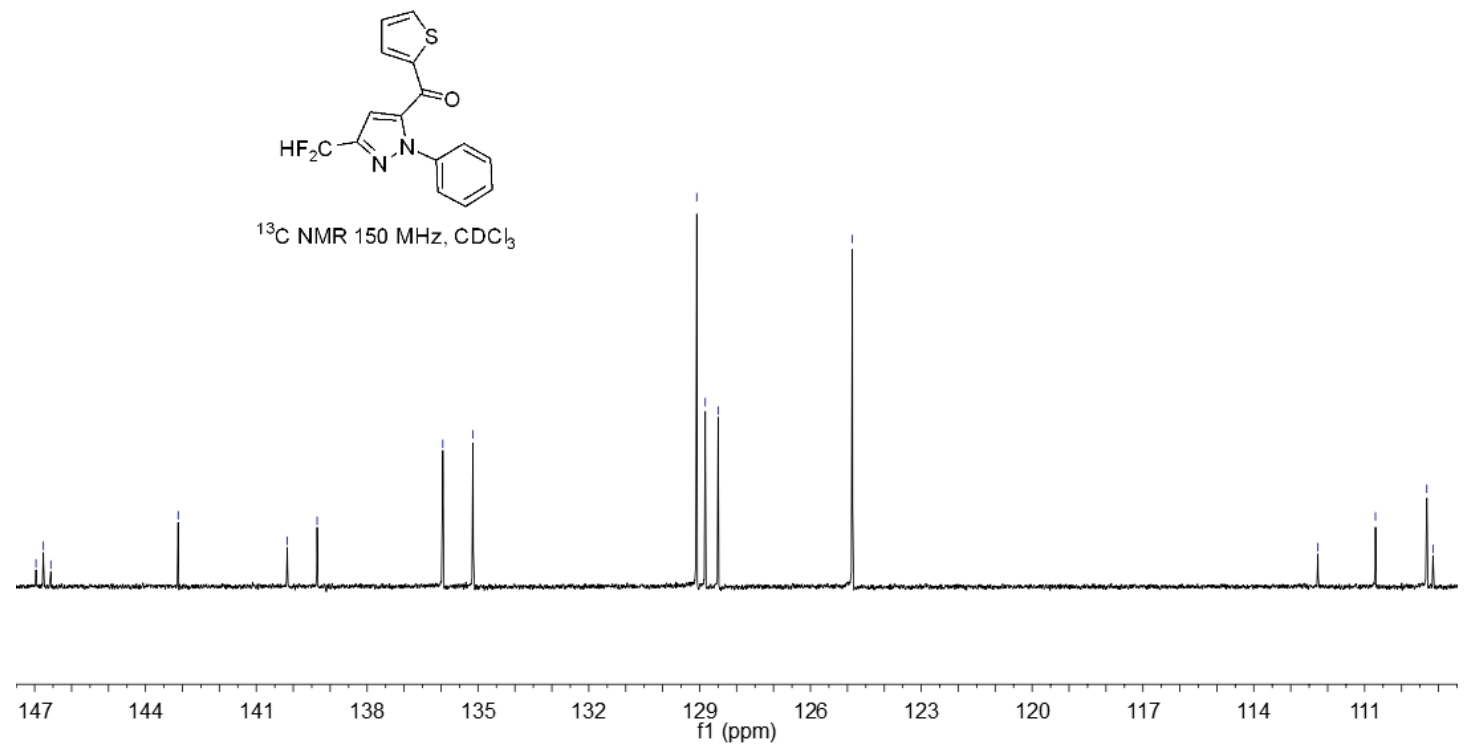

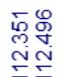

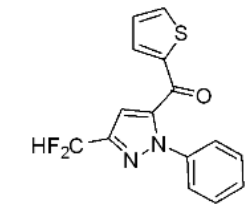

${ }^{19} \mathrm{~F}$ NMR $376 \mathrm{MHz}, \mathrm{CDCl}$

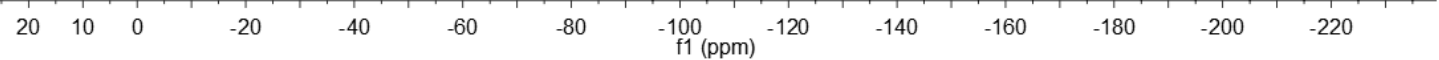




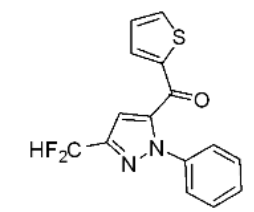

${ }^{19} \mathrm{~F}$ NMR $376 \mathrm{MHz}, \mathrm{CDCl}_{3}$

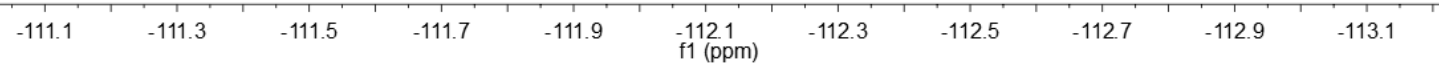

HRMS (ESI) copy of compound $\mathbf{3 v}$ :

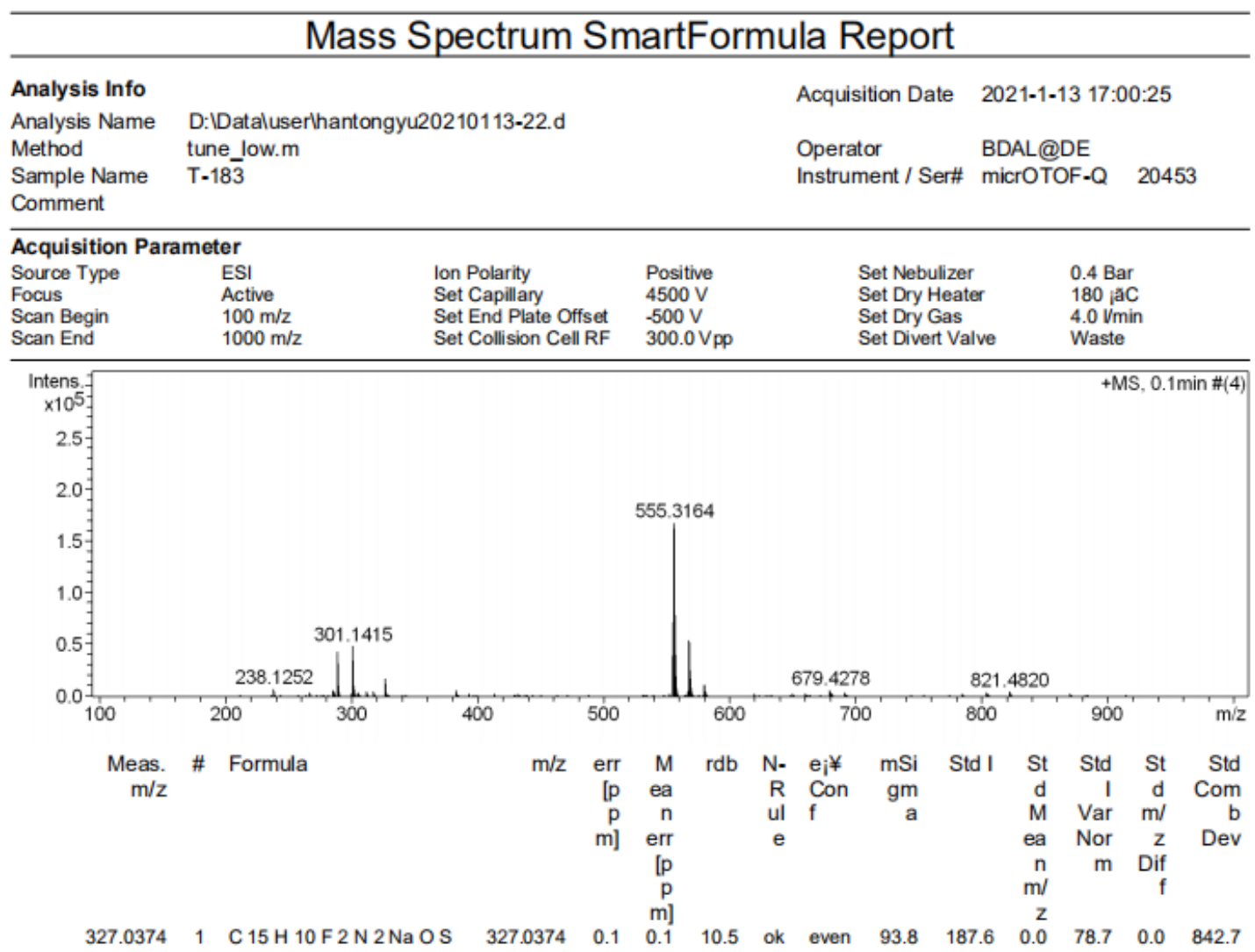


NMR copies of compound $\mathbf{3 w}$ :

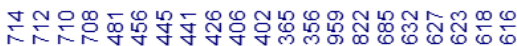

制

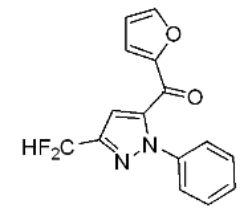

${ }^{1} \mathrm{H} \mathrm{NMR} 400 \mathrm{MHz}, \mathrm{CDCl}_{3}$
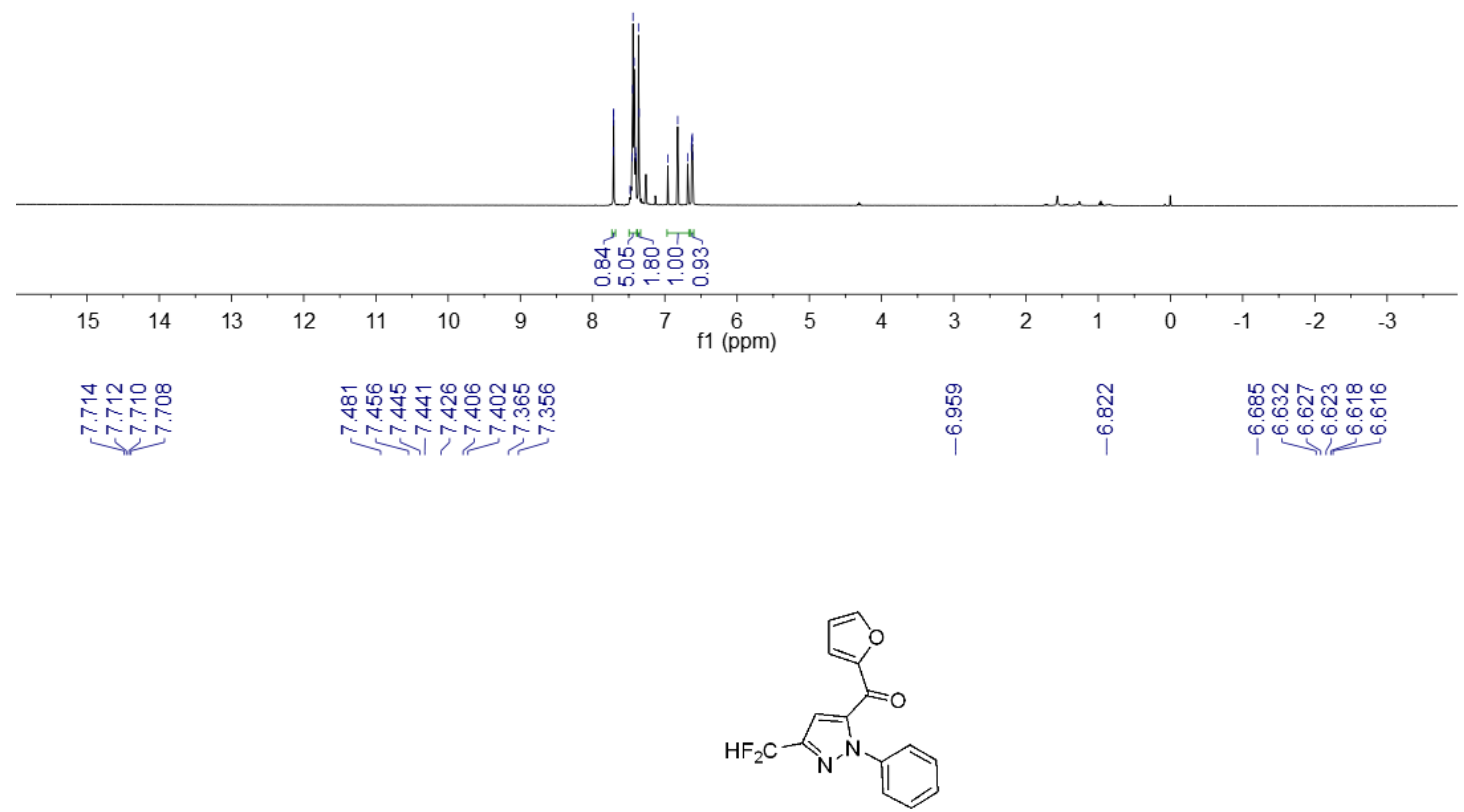

${ }^{1} \mathrm{H}$ NMR $400 \mathrm{MHz}, \mathrm{CDCl}$

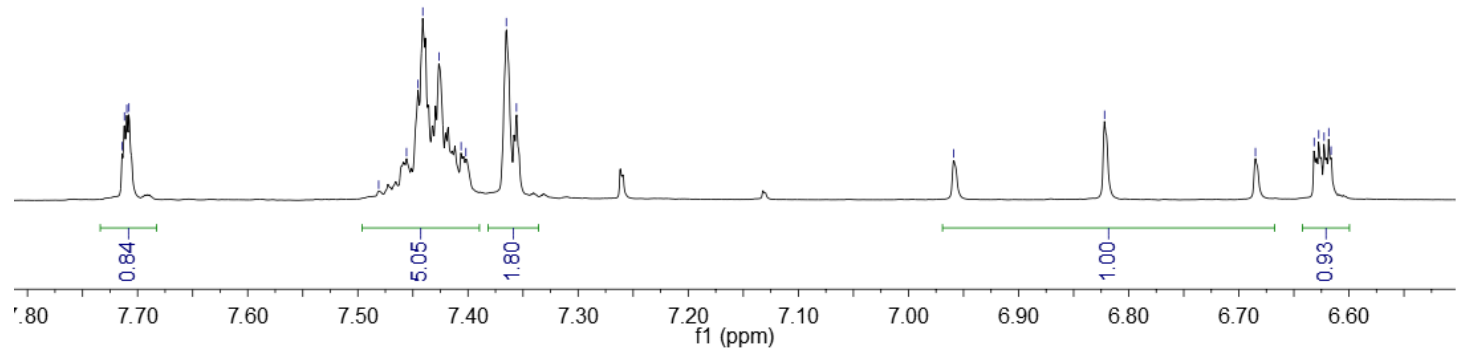




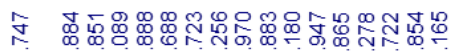

完

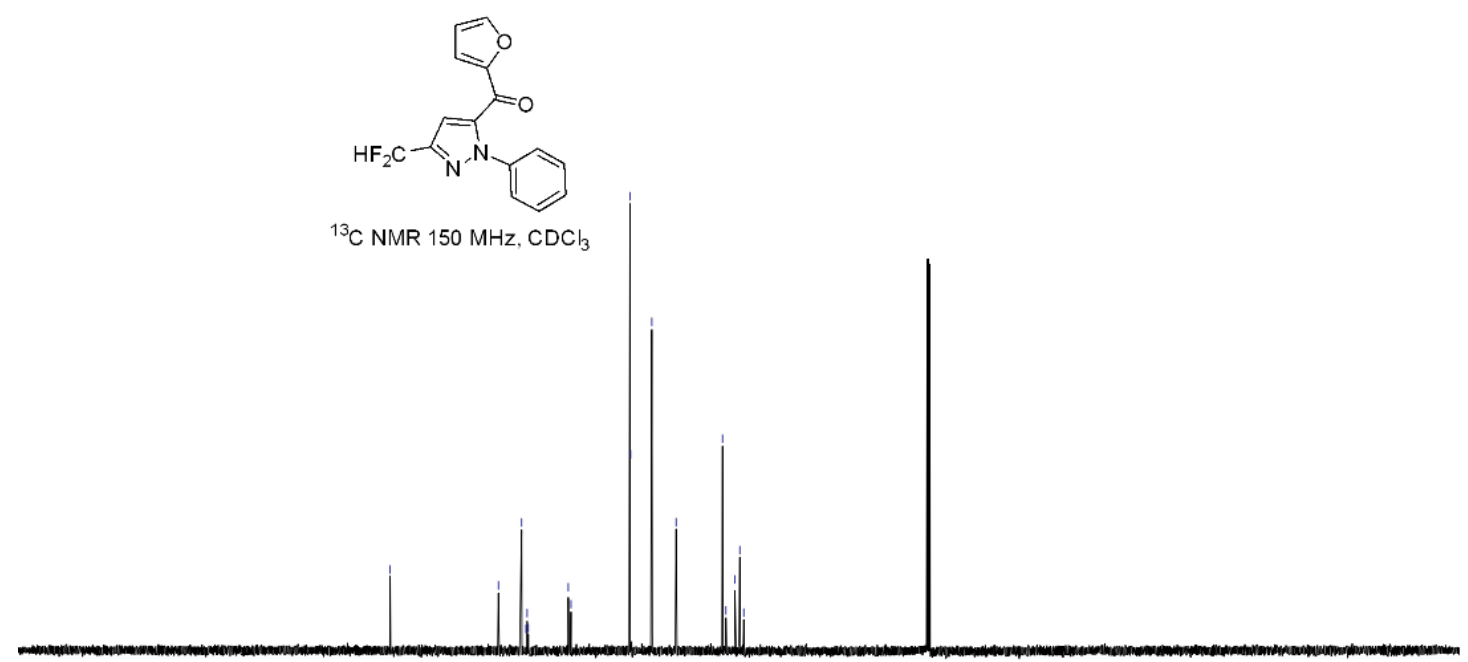

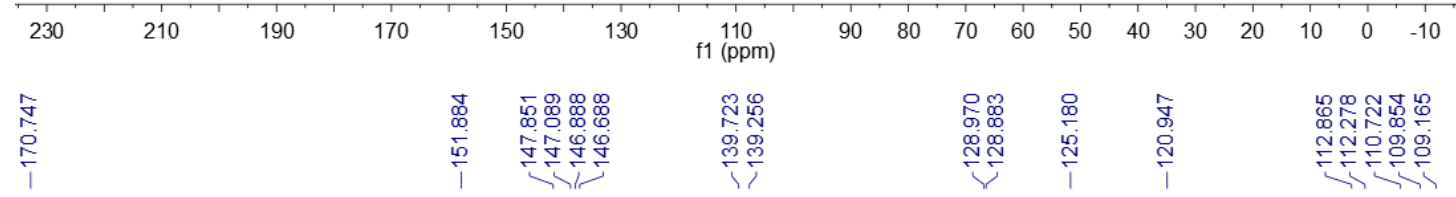

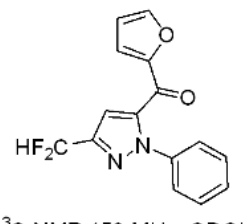

${ }^{13} \mathrm{C} \mathrm{NMR} 150 \mathrm{MHz}, \mathrm{CDCl}_{3}$

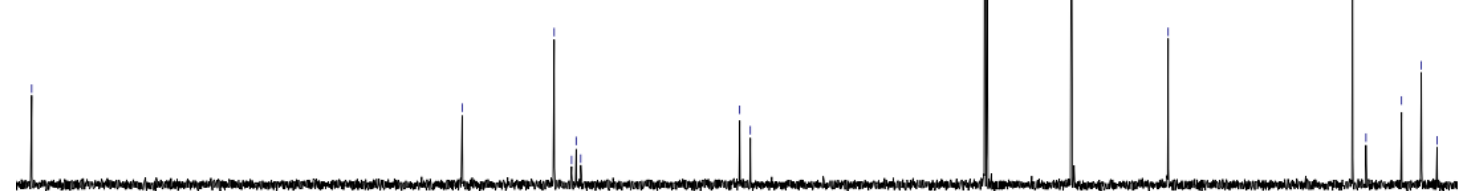

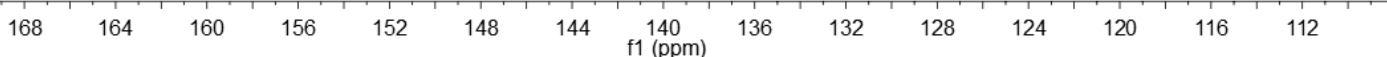




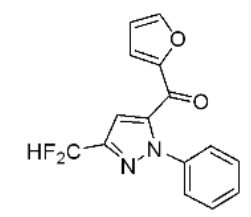

${ }^{19} \mathrm{~F}$ NMR $376 \mathrm{MHz}, \mathrm{CDCl}_{3}$
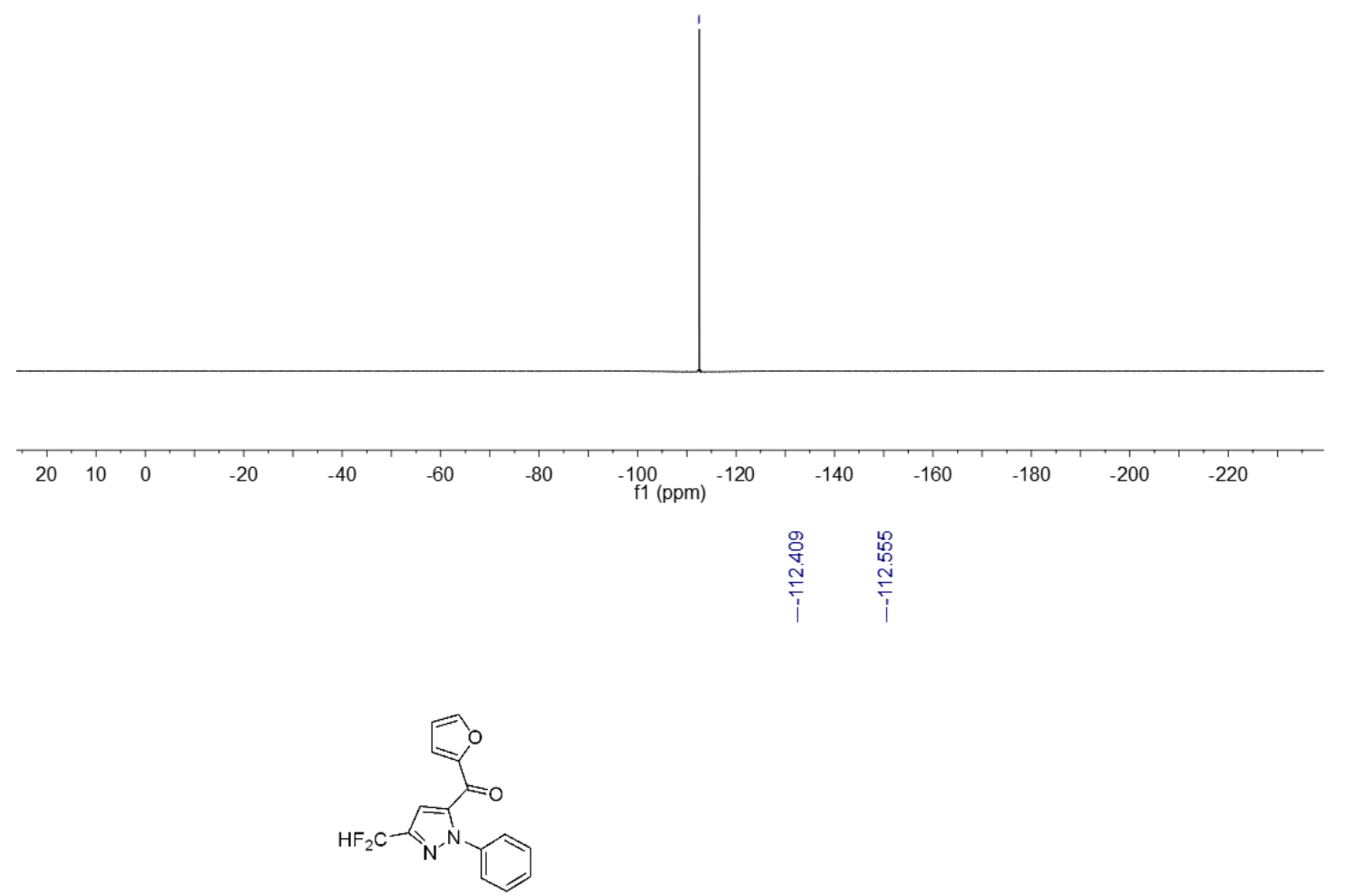

${ }^{19} \mathrm{~F}$ NMR $376 \mathrm{MHz}, \mathrm{CDCl}_{3}$

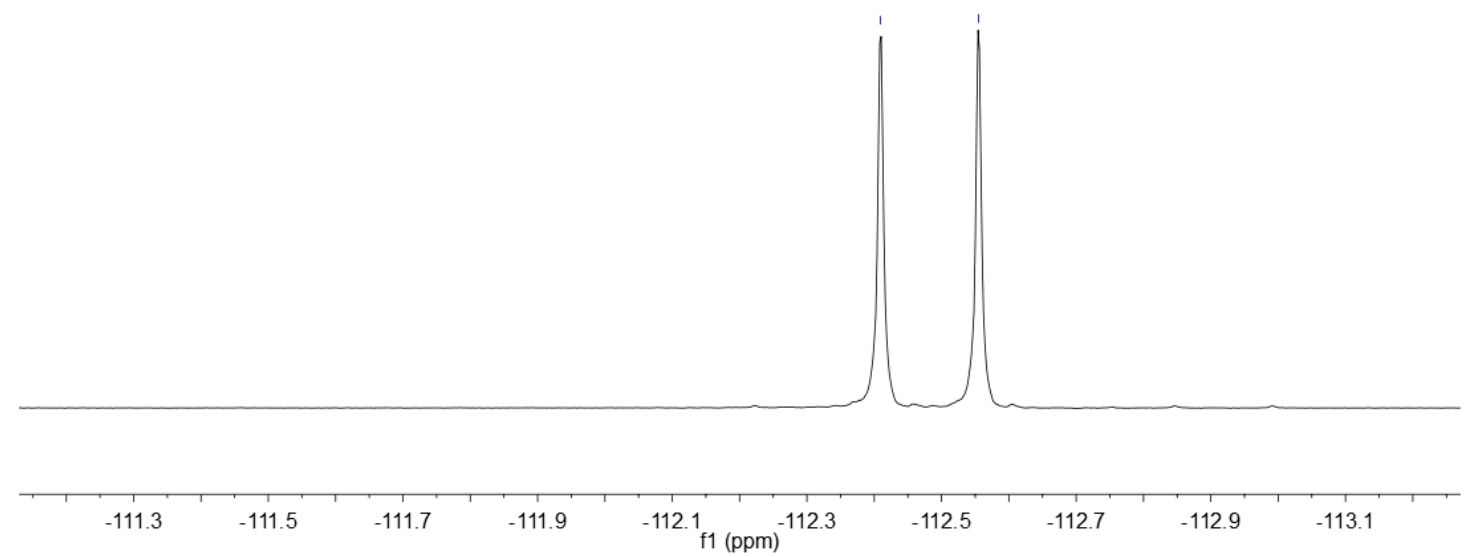


HRMS (ESI) copy of compound $\mathbf{3 w}$ :

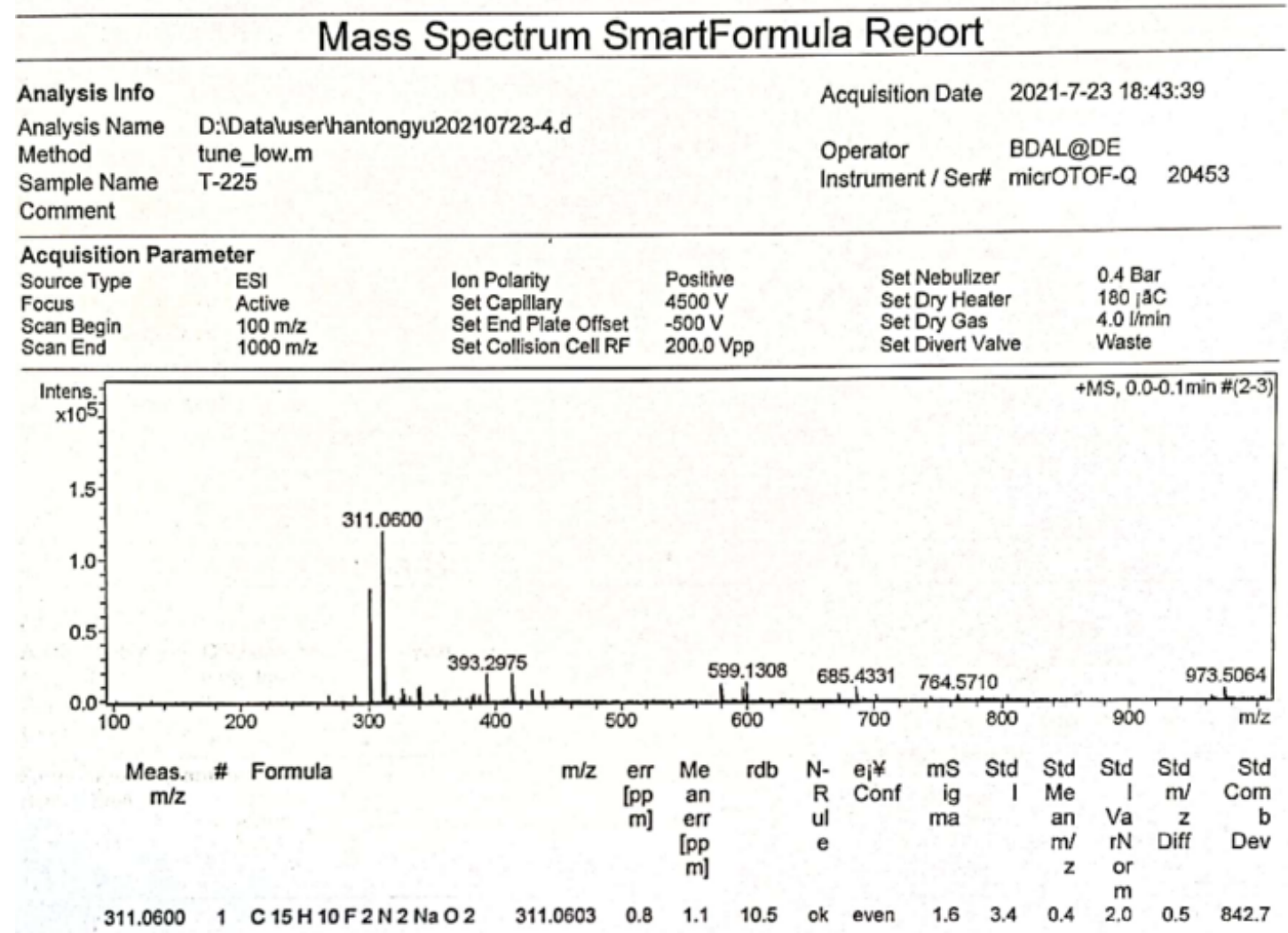

NMR copies of compound $\mathbf{3 x}$ :

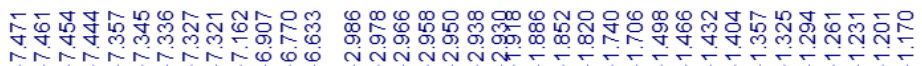

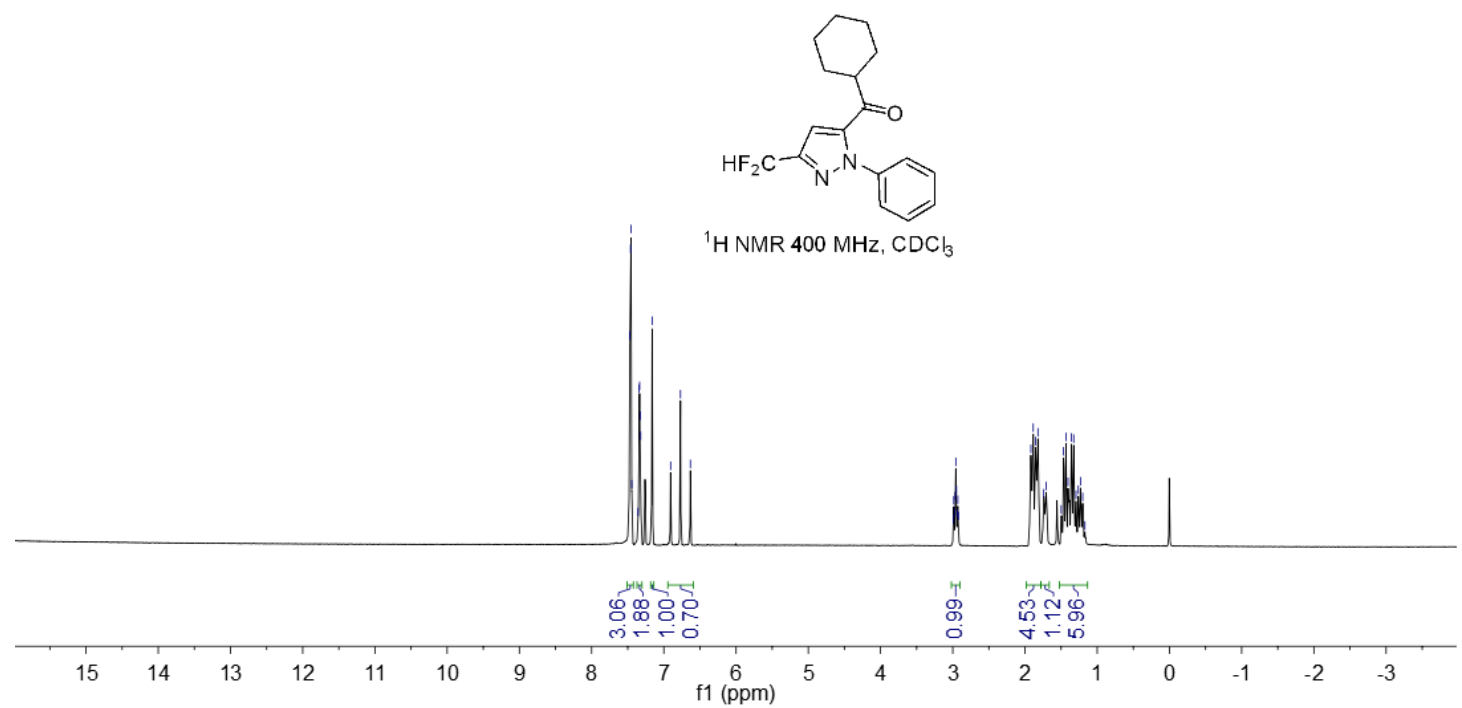



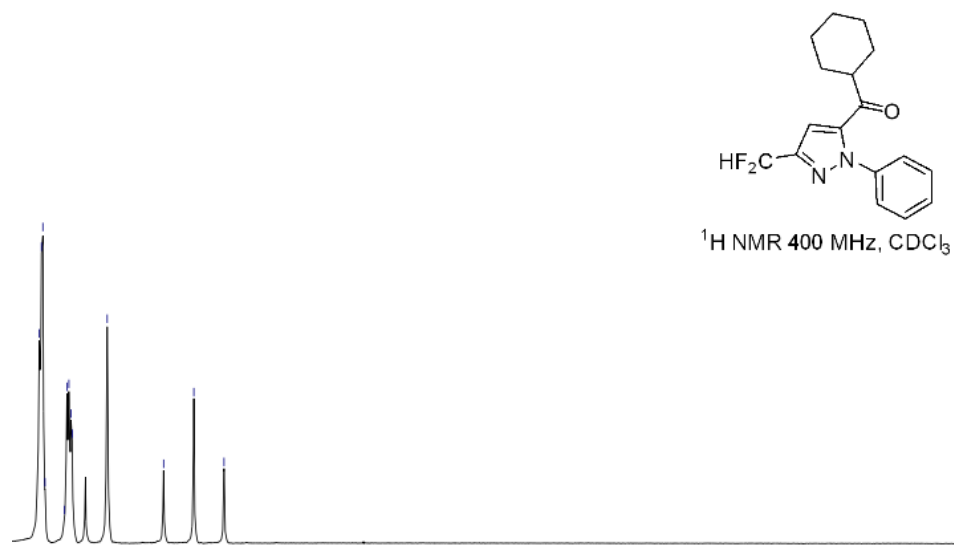

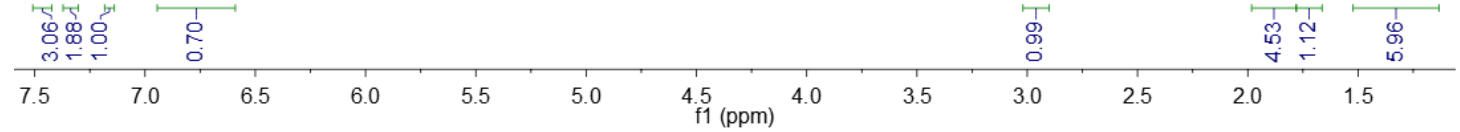

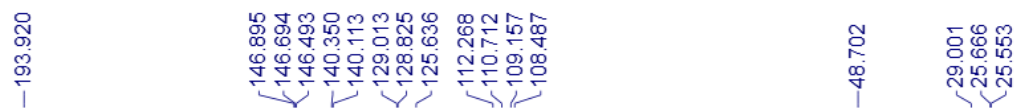
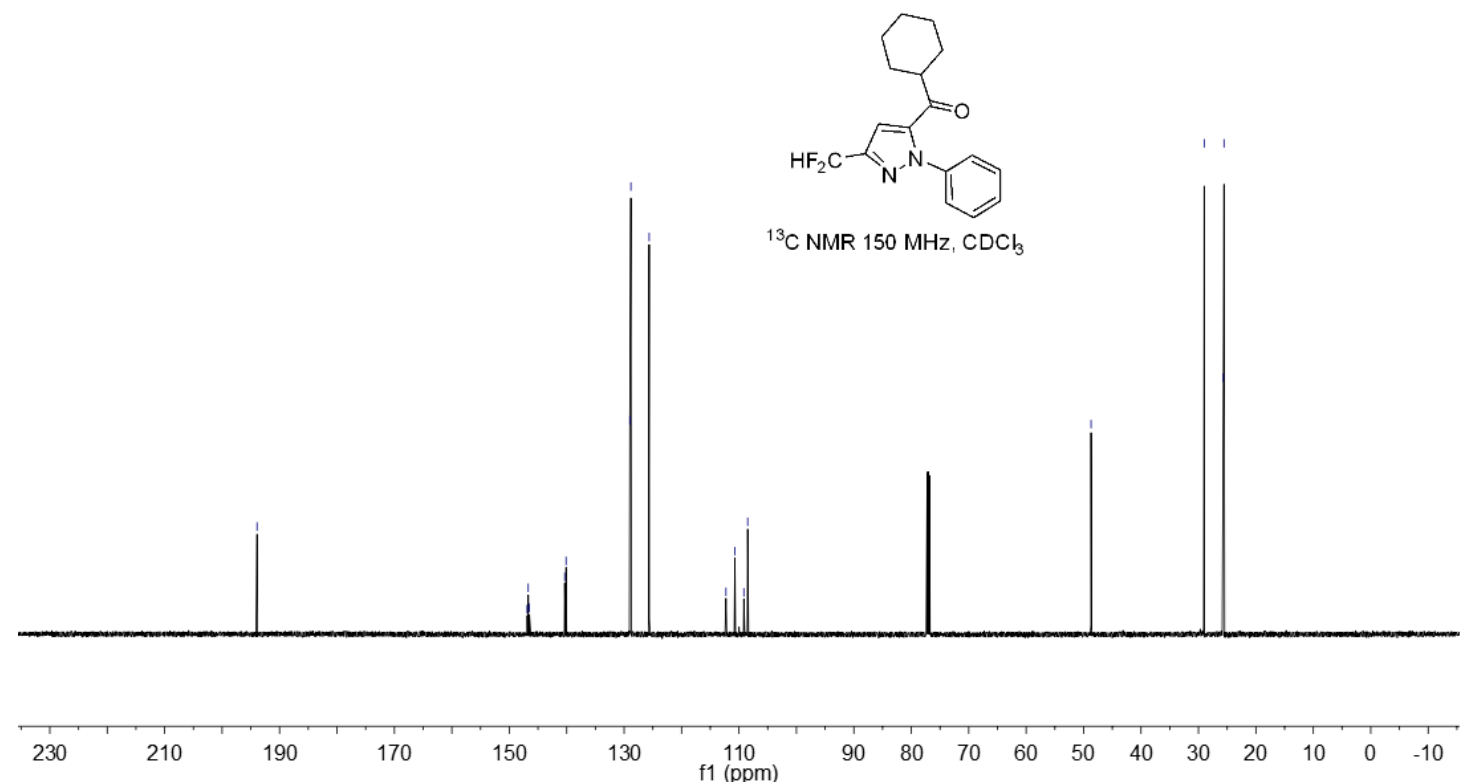


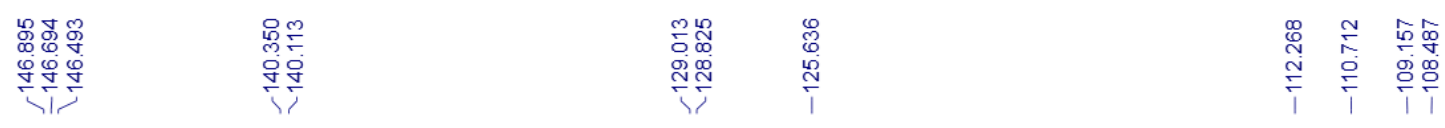
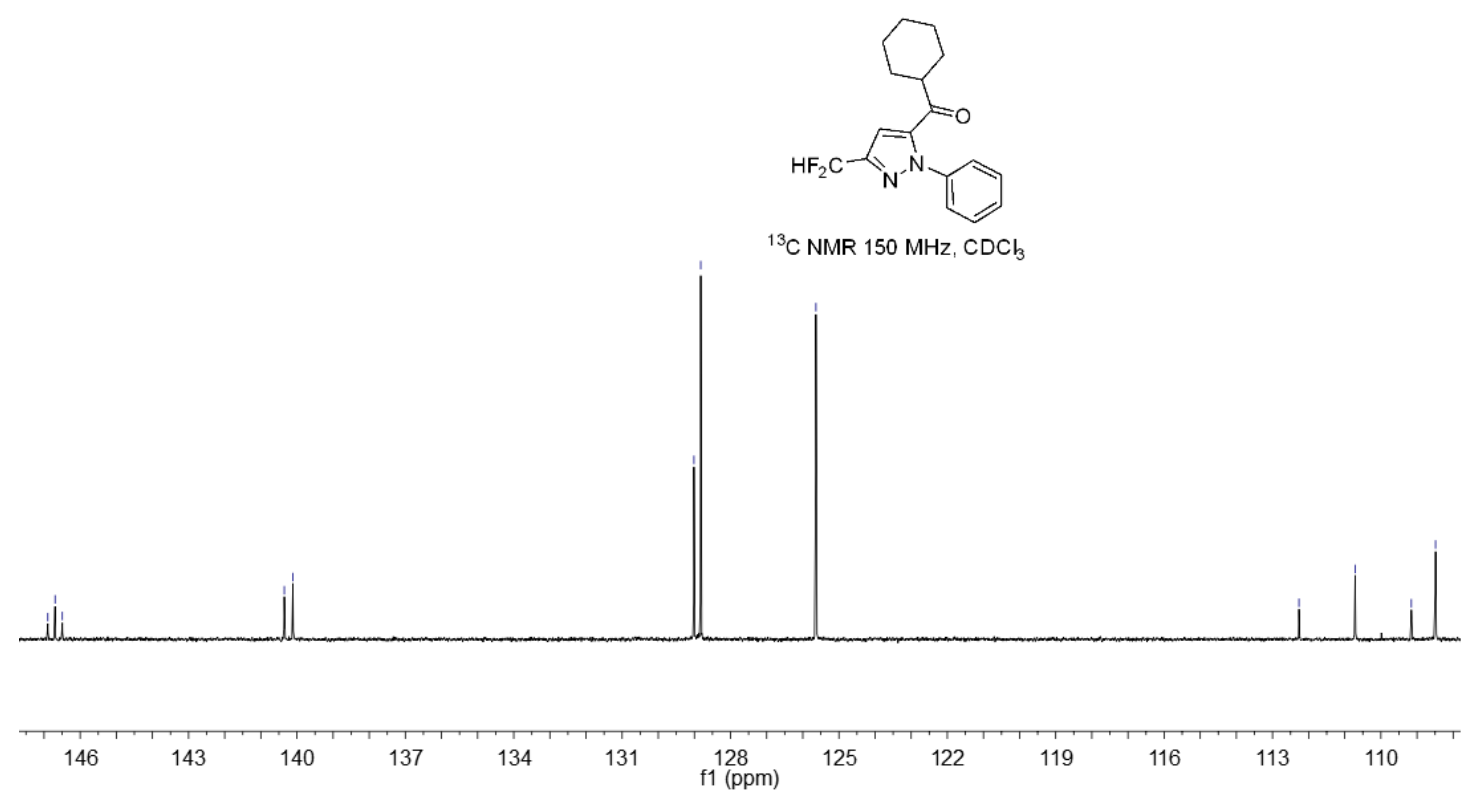

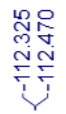

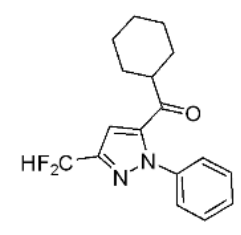

${ }^{19} \mathrm{~F} \mathrm{NMR} 376 \mathrm{MHz}, \mathrm{CDCl}$

\begin{tabular}{|c|c|c|c|c|c|c|c|c|c|c|c|c|c|}
\hline 20 & 10 & 0 & -20 & -40 & -60 & -80 & -100 & -120 & -140 & -160 & -180 & -200 & -220 \\
\hline
\end{tabular}




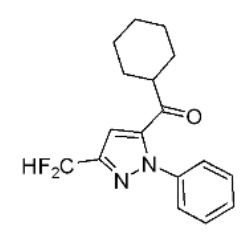

${ }^{19} \mathrm{~F} \mathrm{NMR} 376 \mathrm{MHz}, \mathrm{CDCl}$

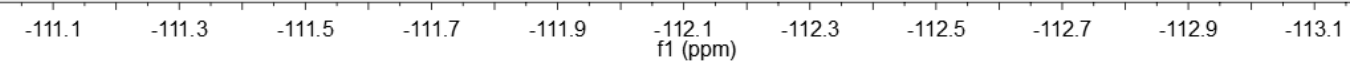

HRMS (ESI) copy of compound $\mathbf{3 x}$ :

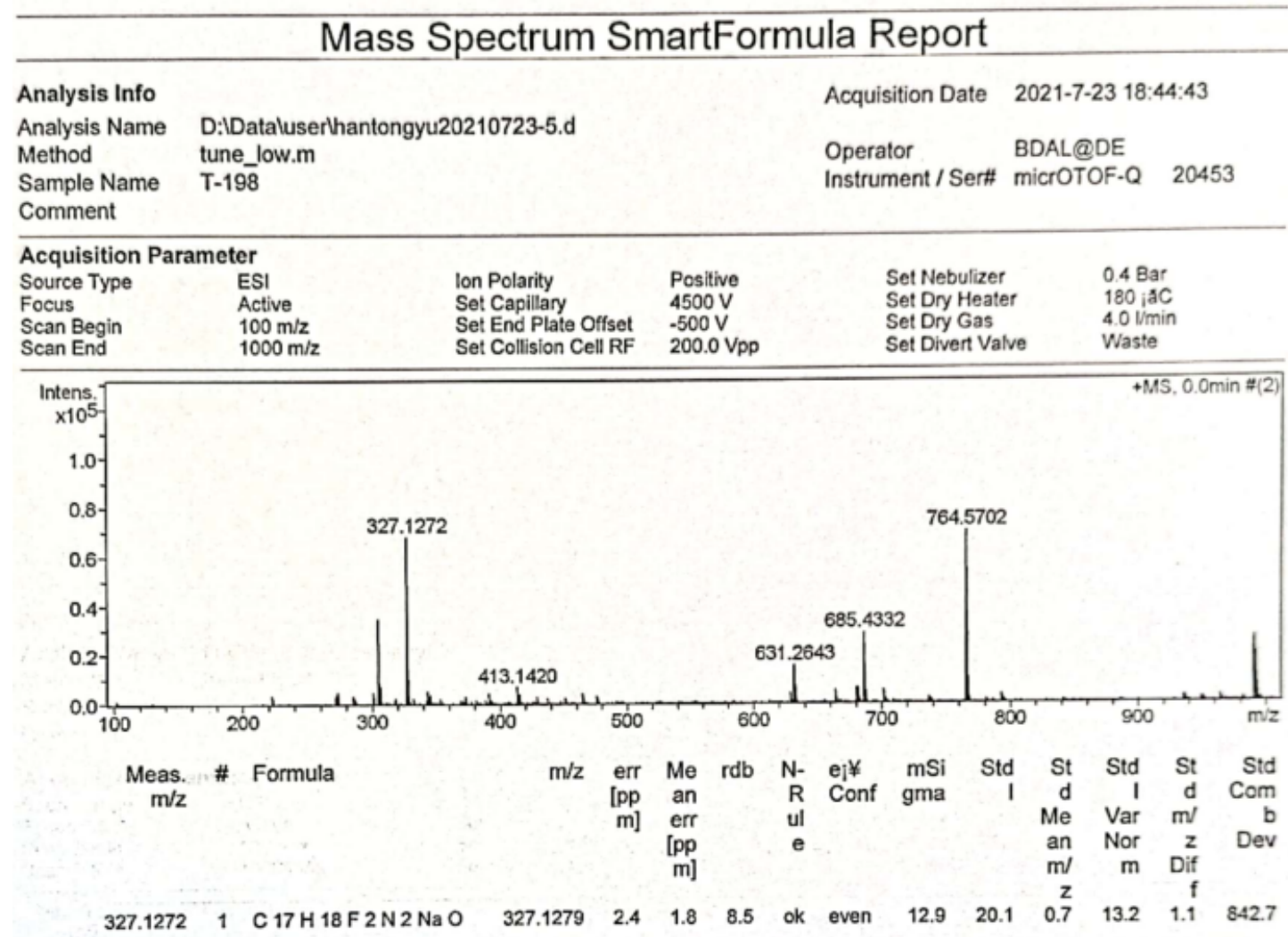


NMR copies of compound $\mathbf{3 y}$ :

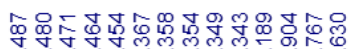

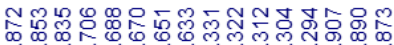

Nin

Nin-tithtriolo

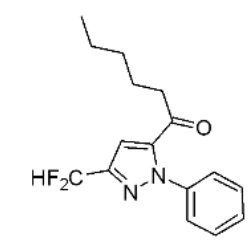

${ }^{1} \mathrm{H}$ NMR $400 \mathrm{MHz}, \mathrm{CDCl}_{3}$

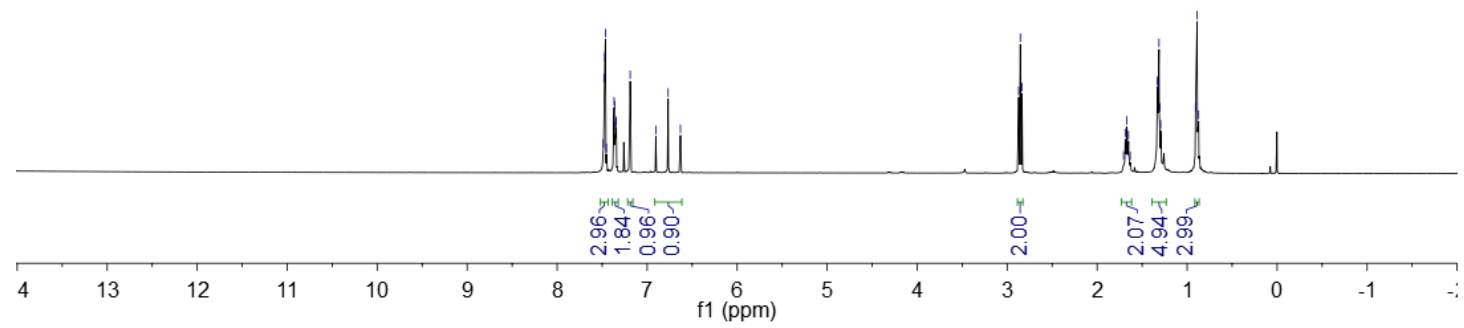

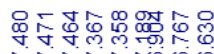

riñino

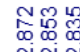

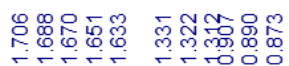

Nen

-ji inio

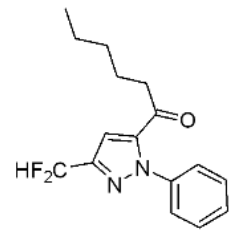

${ }^{1} \mathrm{H}$ NMR $400 \mathrm{MHz}, \mathrm{CDCl}_{3}$

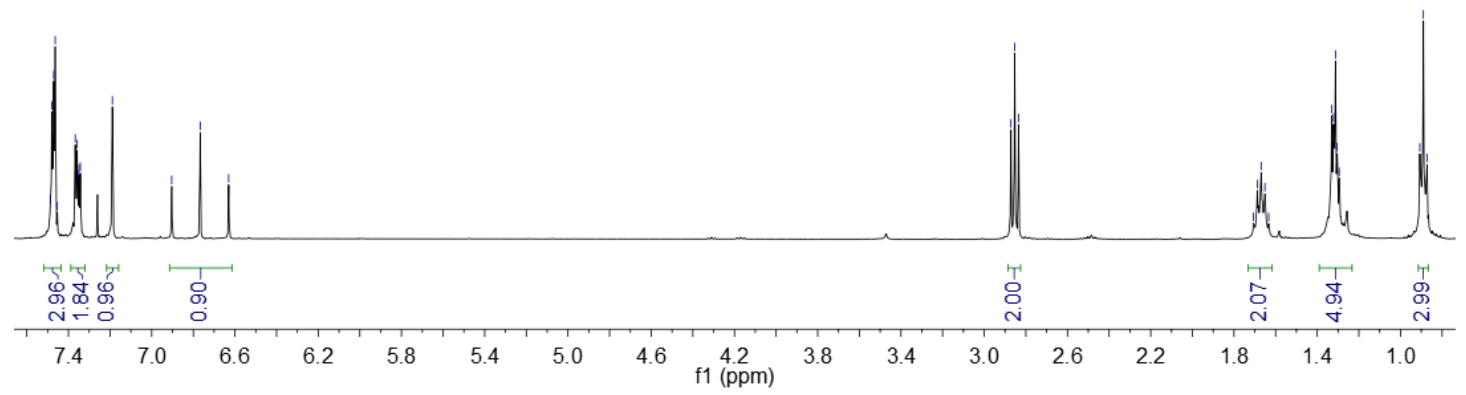




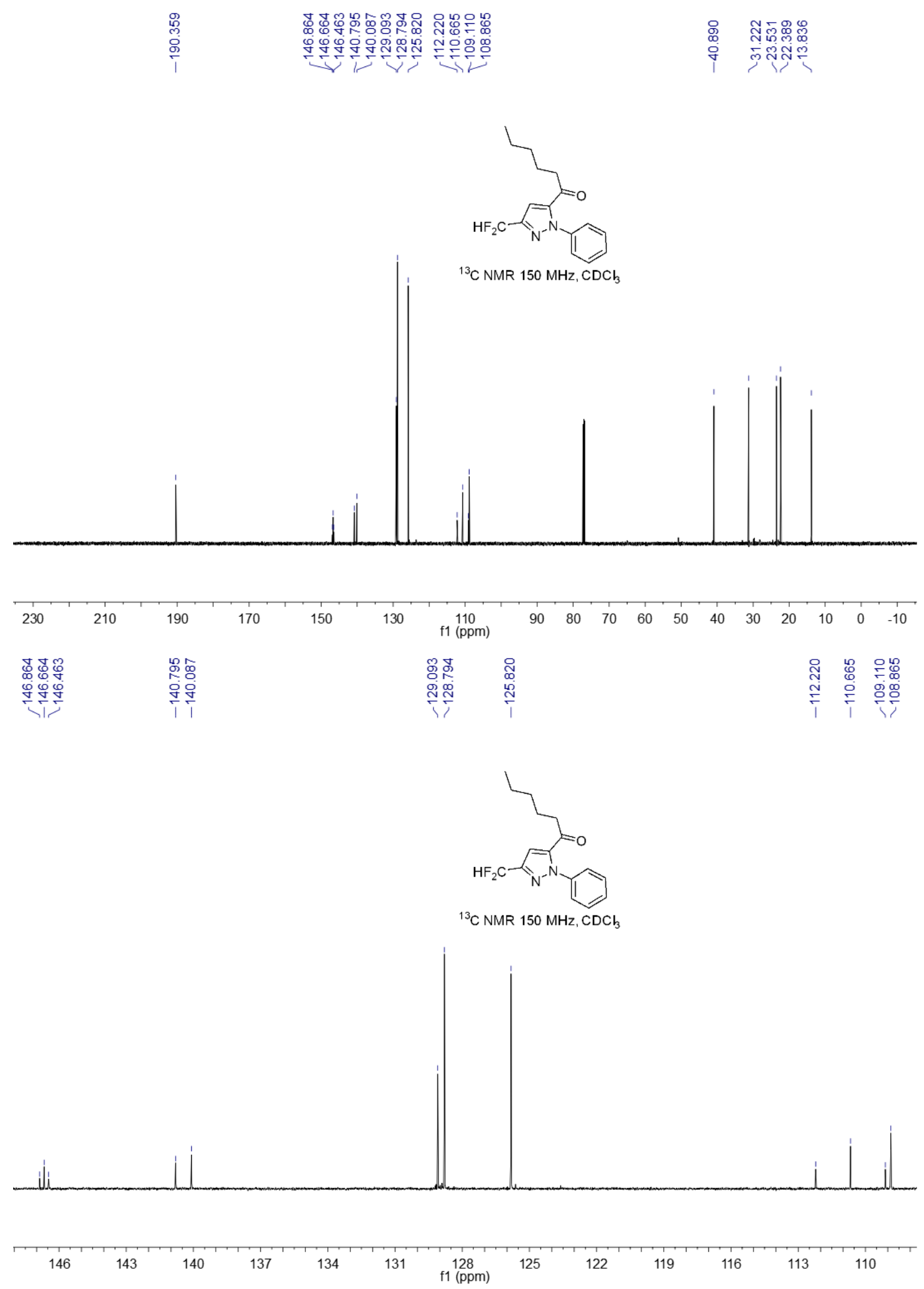




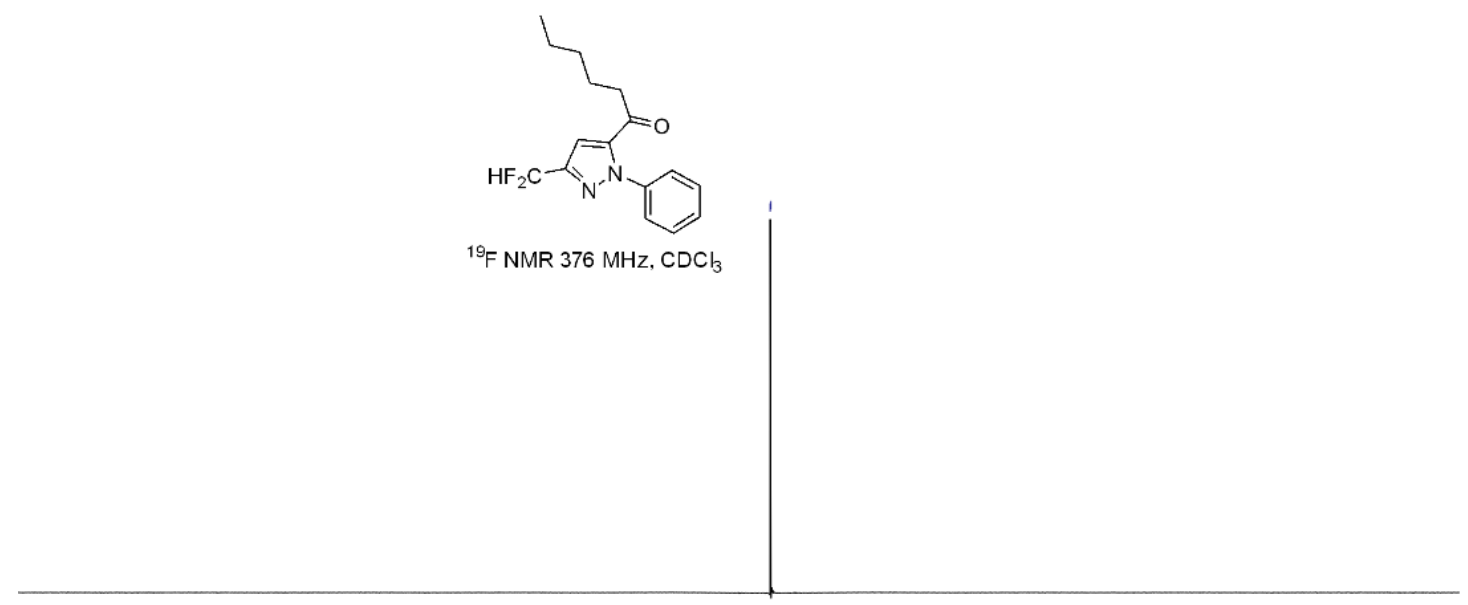

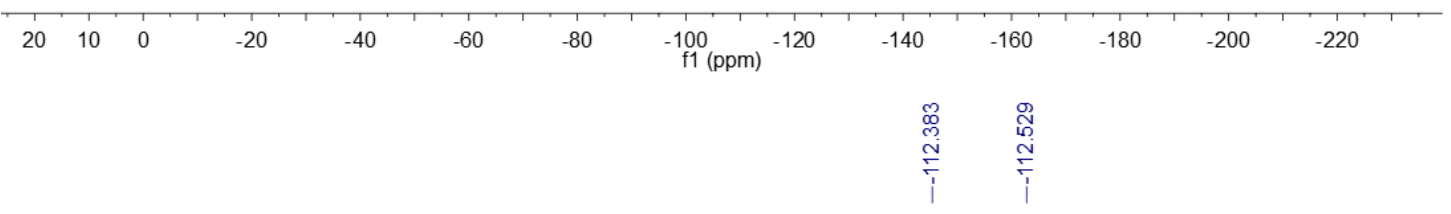

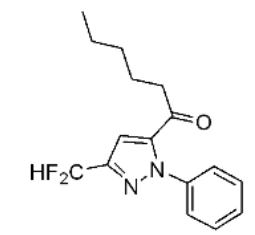

${ }^{19} \mathrm{~F} \mathrm{NMR} 376 \mathrm{MHz}, \mathrm{CDCl}_{3}$

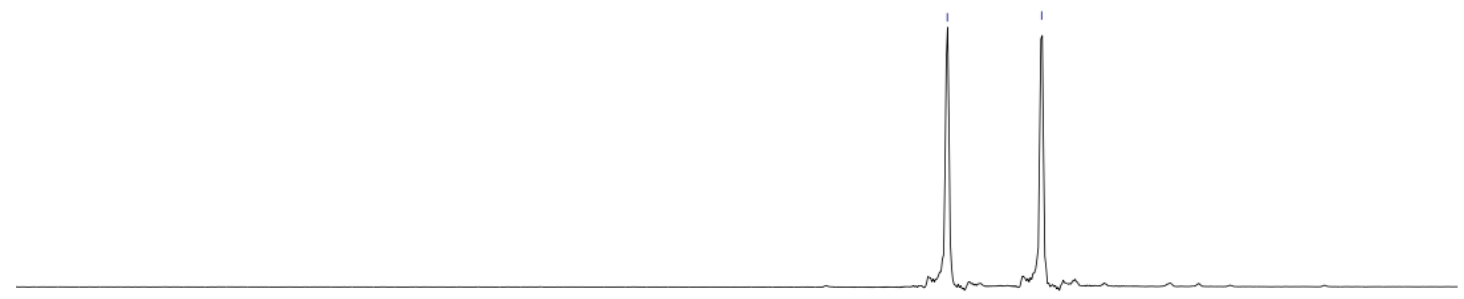

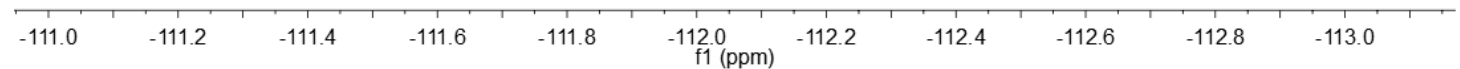


HRMS (ESI) copy of compound $\mathbf{3 y}$ :

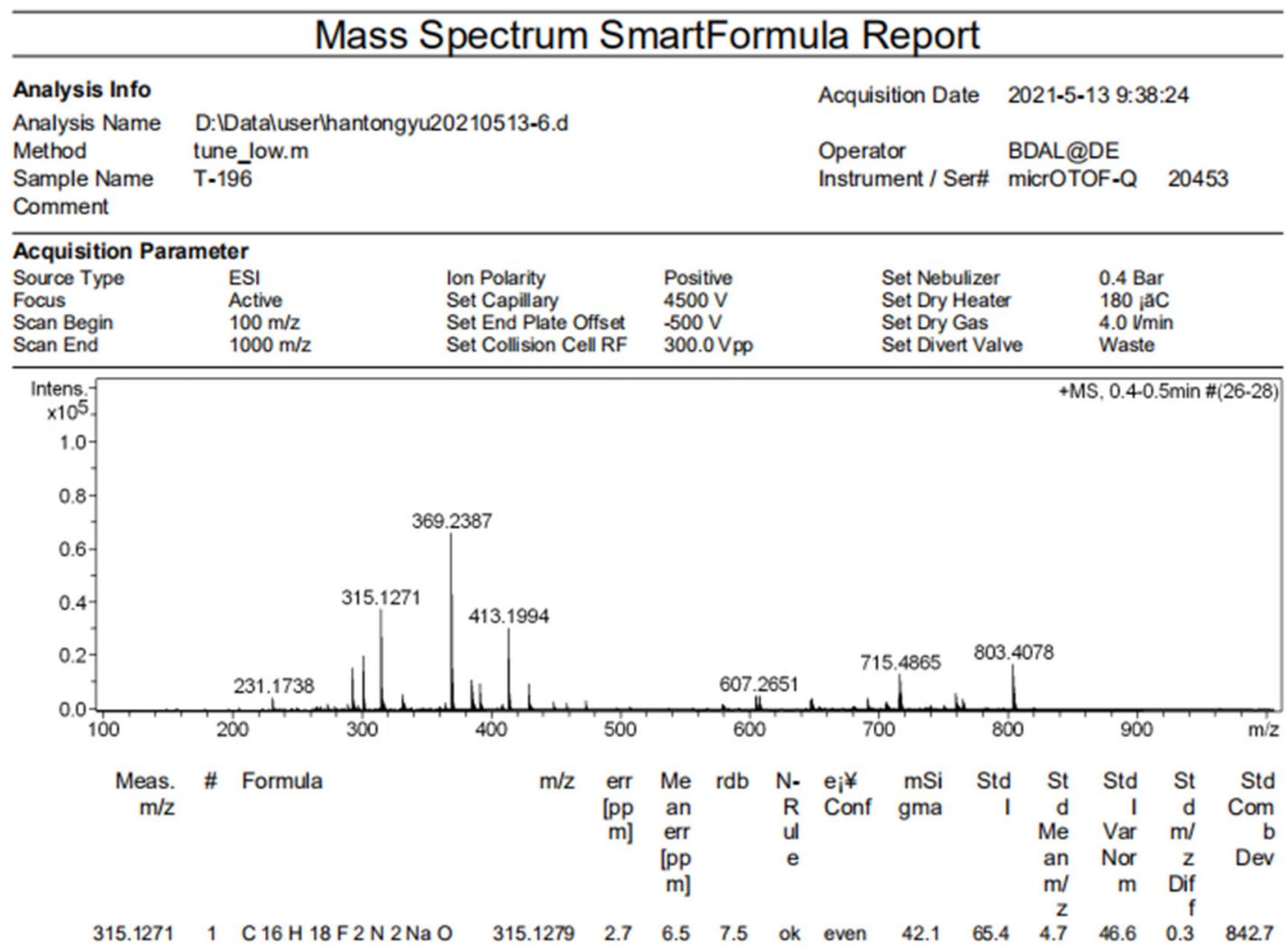

NMR copies of compound $\mathbf{3 z}$ :

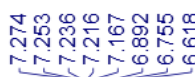

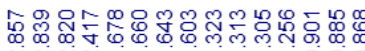

intalos

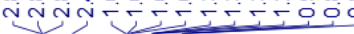

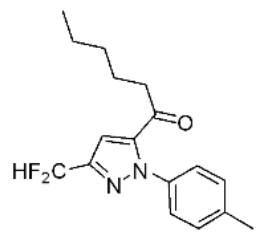

${ }^{1} \mathrm{HNMR} 400 \mathrm{MHz}, \mathrm{CDCl}$

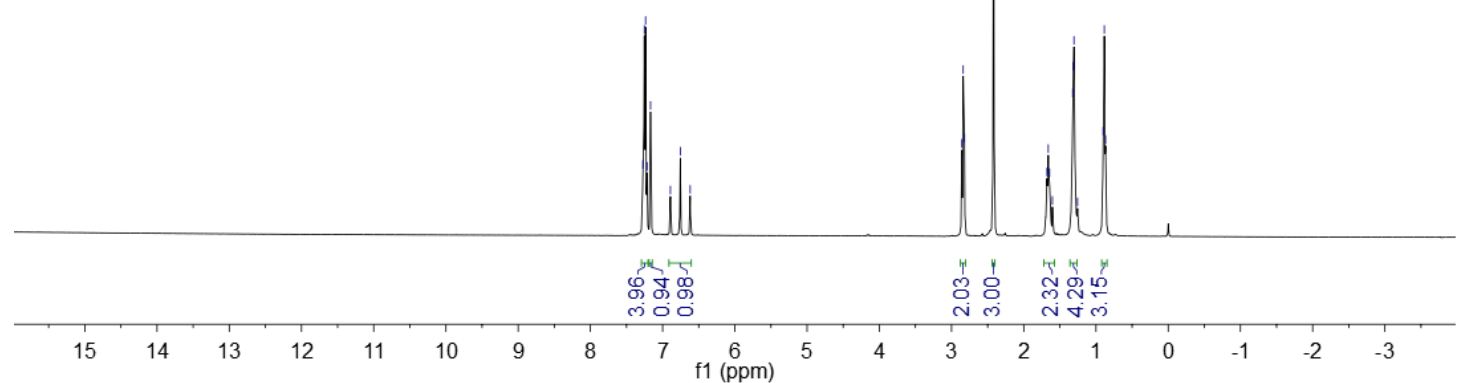



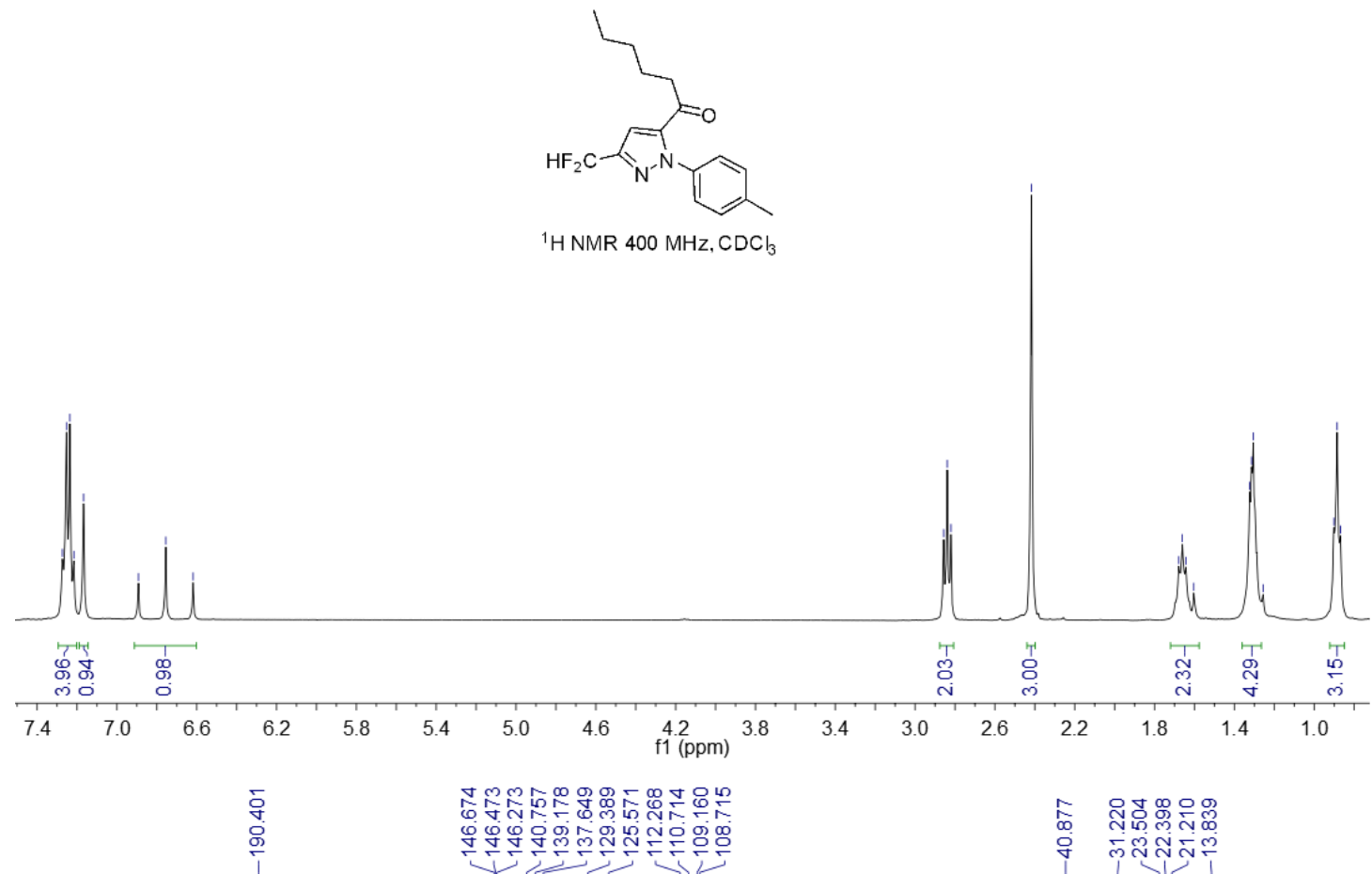

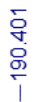
OAn 守守守宁

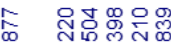

व लㅊำ
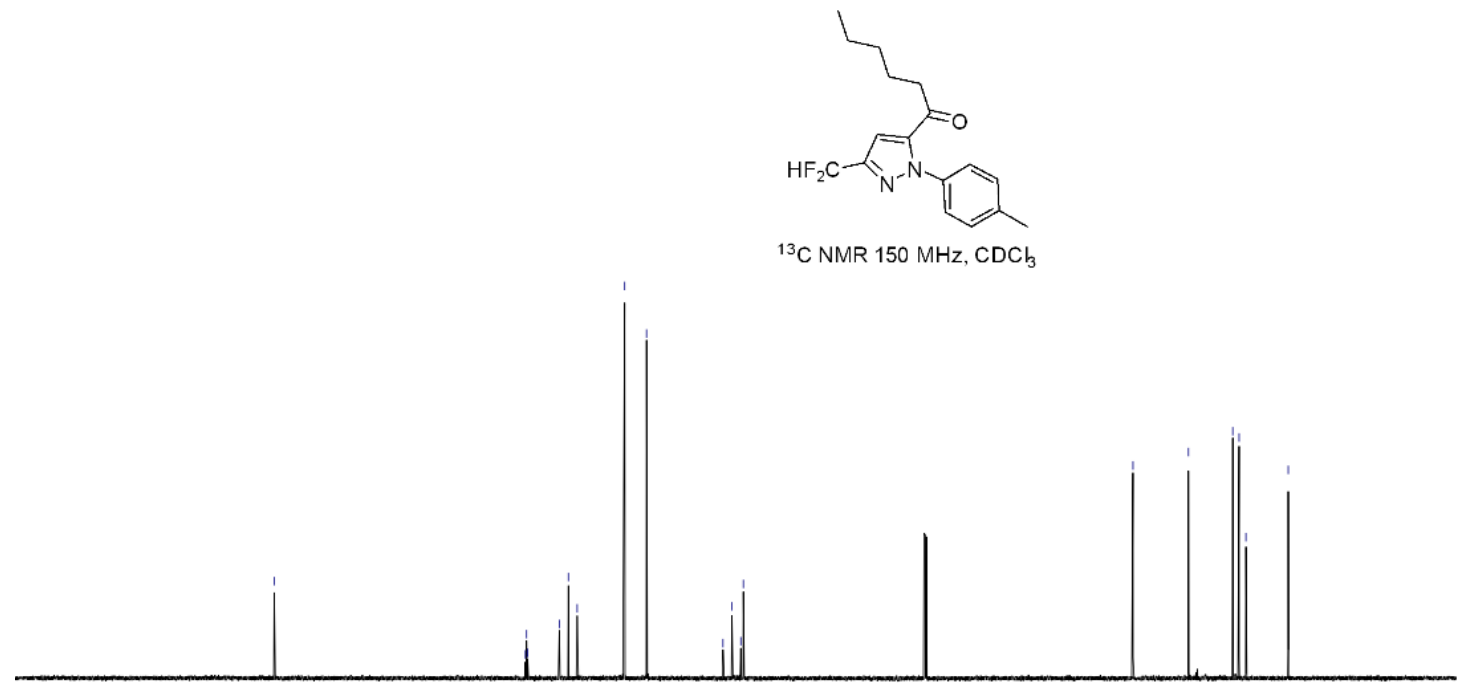
210 190 130 110
$\mathrm{f} 1(\mathrm{ppm})$ $\begin{array}{lllllllllll}90 & 80 & 70 & 60 & 50 & 40 & 30 & 20 & 10 & 0 & -10\end{array}$ 


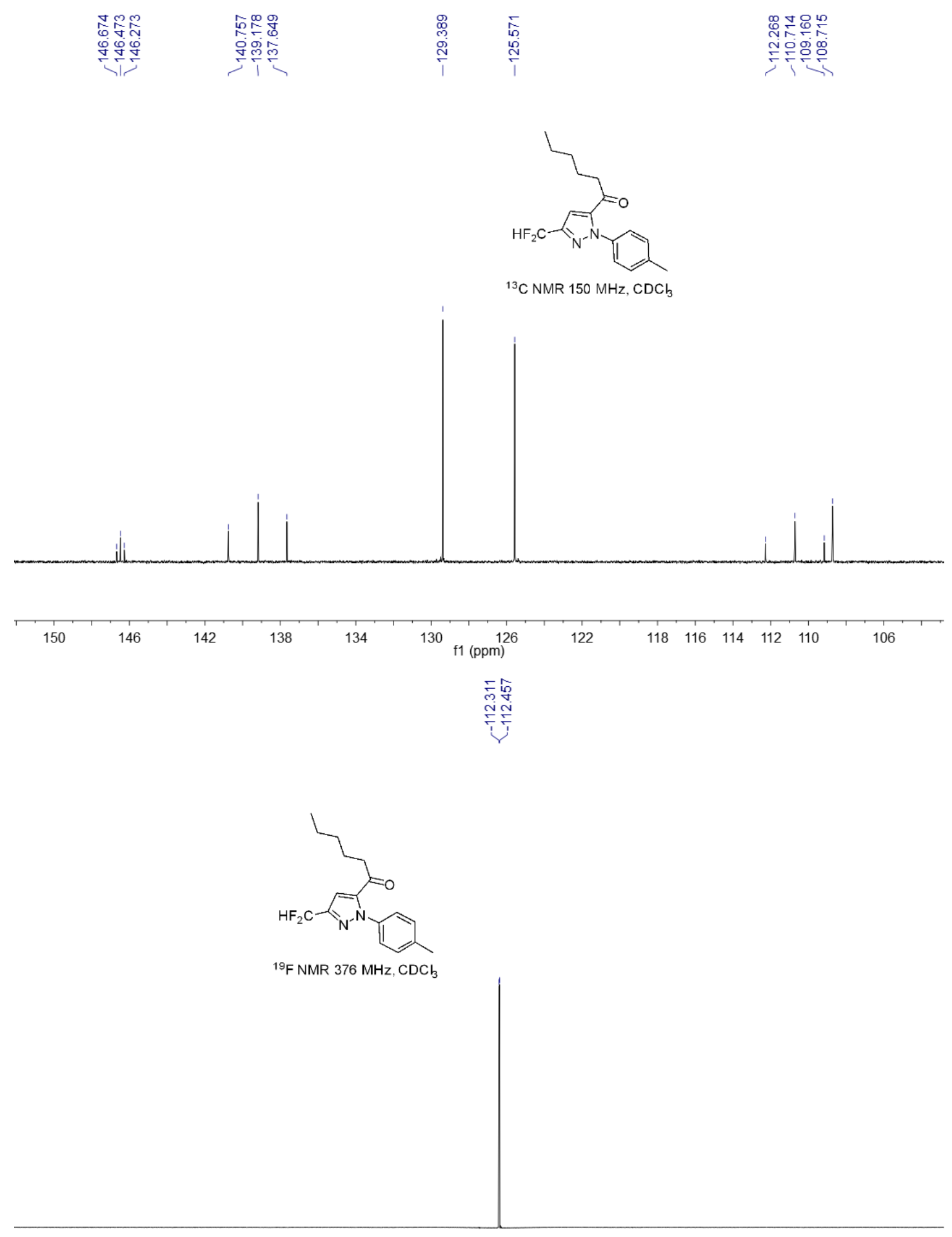

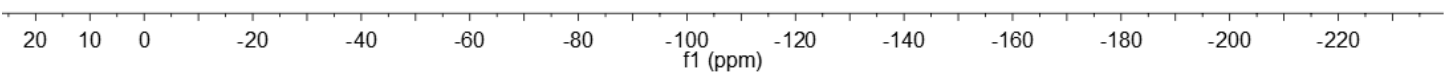




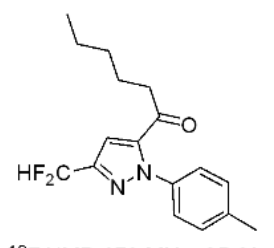

${ }^{19} \mathrm{~F} \mathrm{NMR} 376 \mathrm{MHz}, \mathrm{CDCl}_{3}$

$\begin{array}{lllllll}-111.4 & -111.5 & -111.6 & -111.7 & -111.8 & -111.9 & -112.1 \\ & & & & & \end{array}$

$-112.3$

$-112.5$

$-112.7$

$-112.9$

HRMS (ESI) copy of compound 3z:

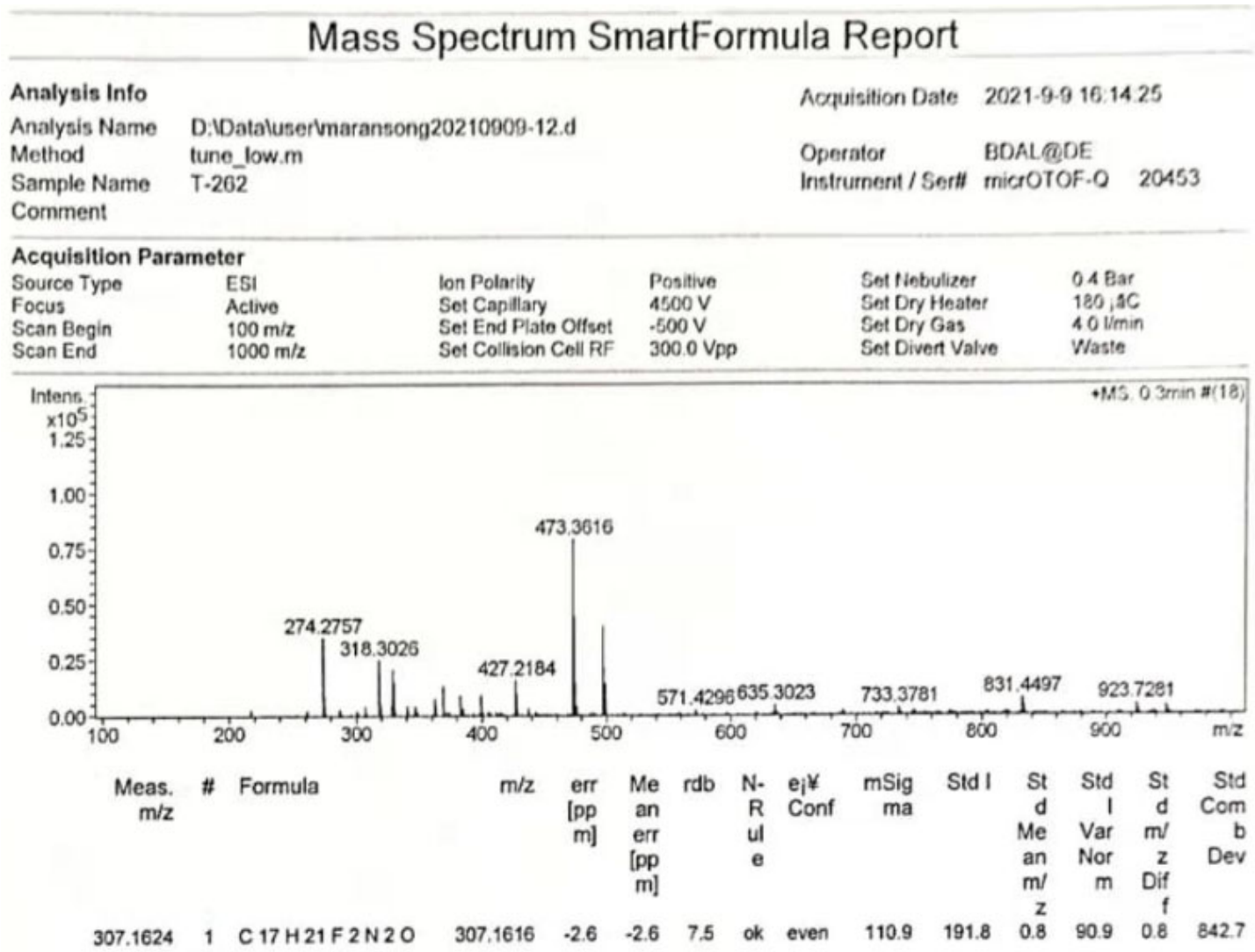


NMR copies of compound 3aa:

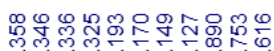

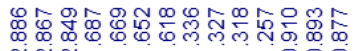

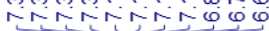

nN4

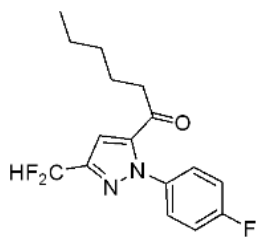

${ }^{1} \mathrm{H}$ NMR $400 \mathrm{MHz}, \mathrm{CDCl}$

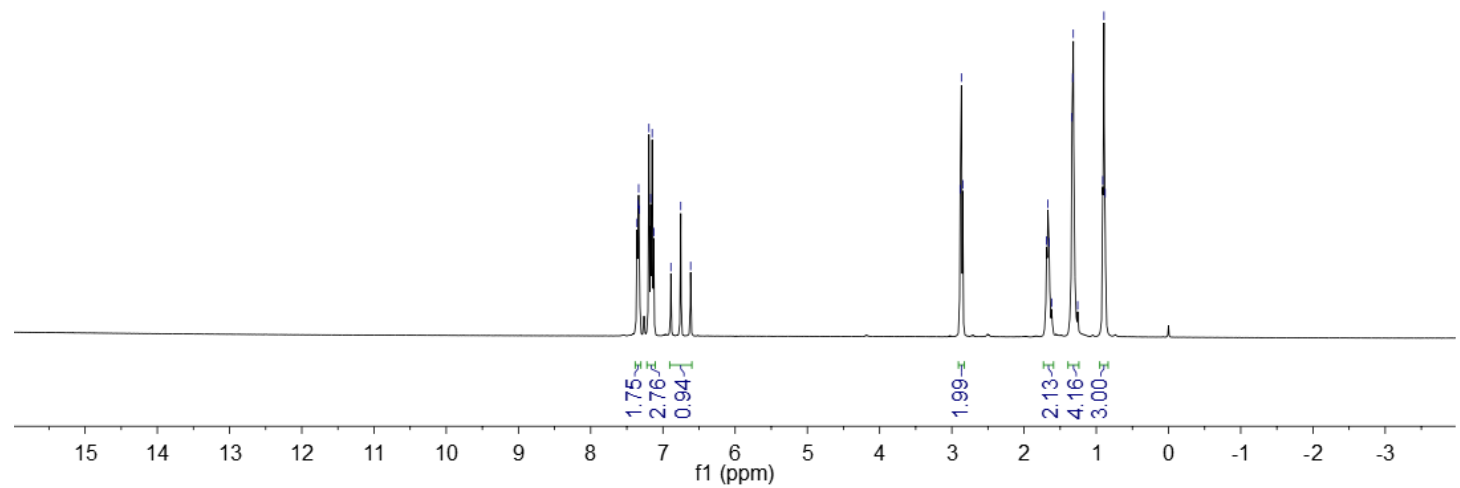

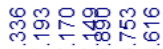

Tino

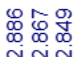

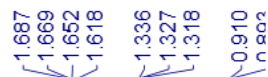

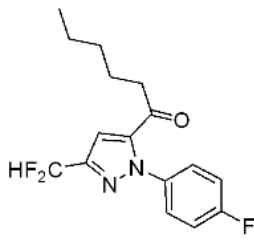

${ }^{1} \mathrm{H}$ NMR $400 \mathrm{MHz}, \mathrm{CDCl}$

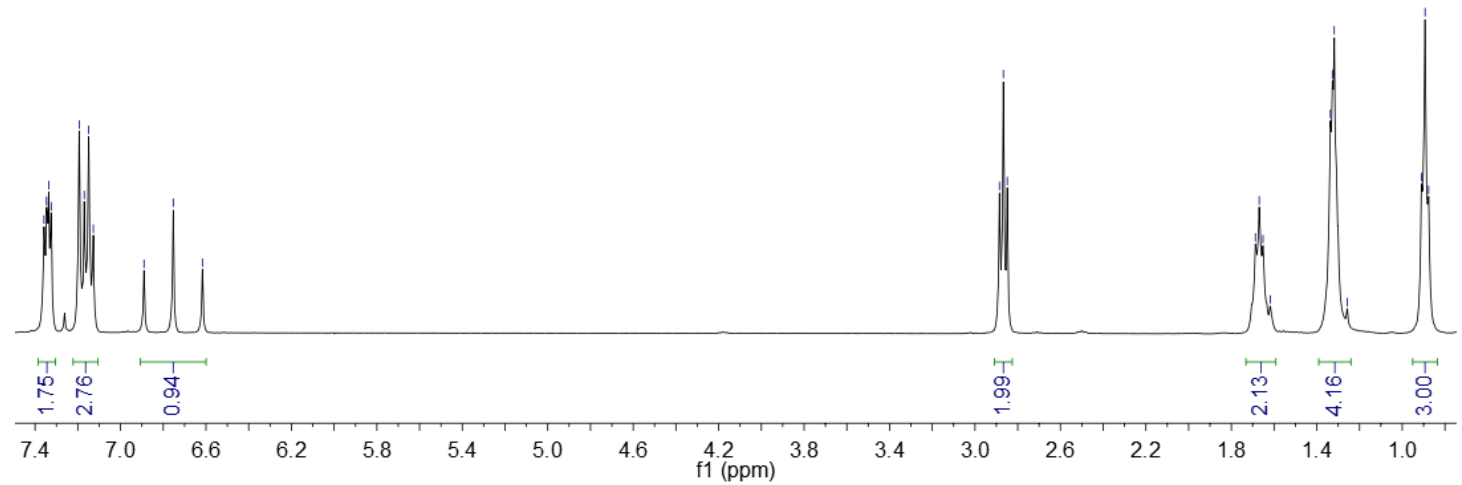




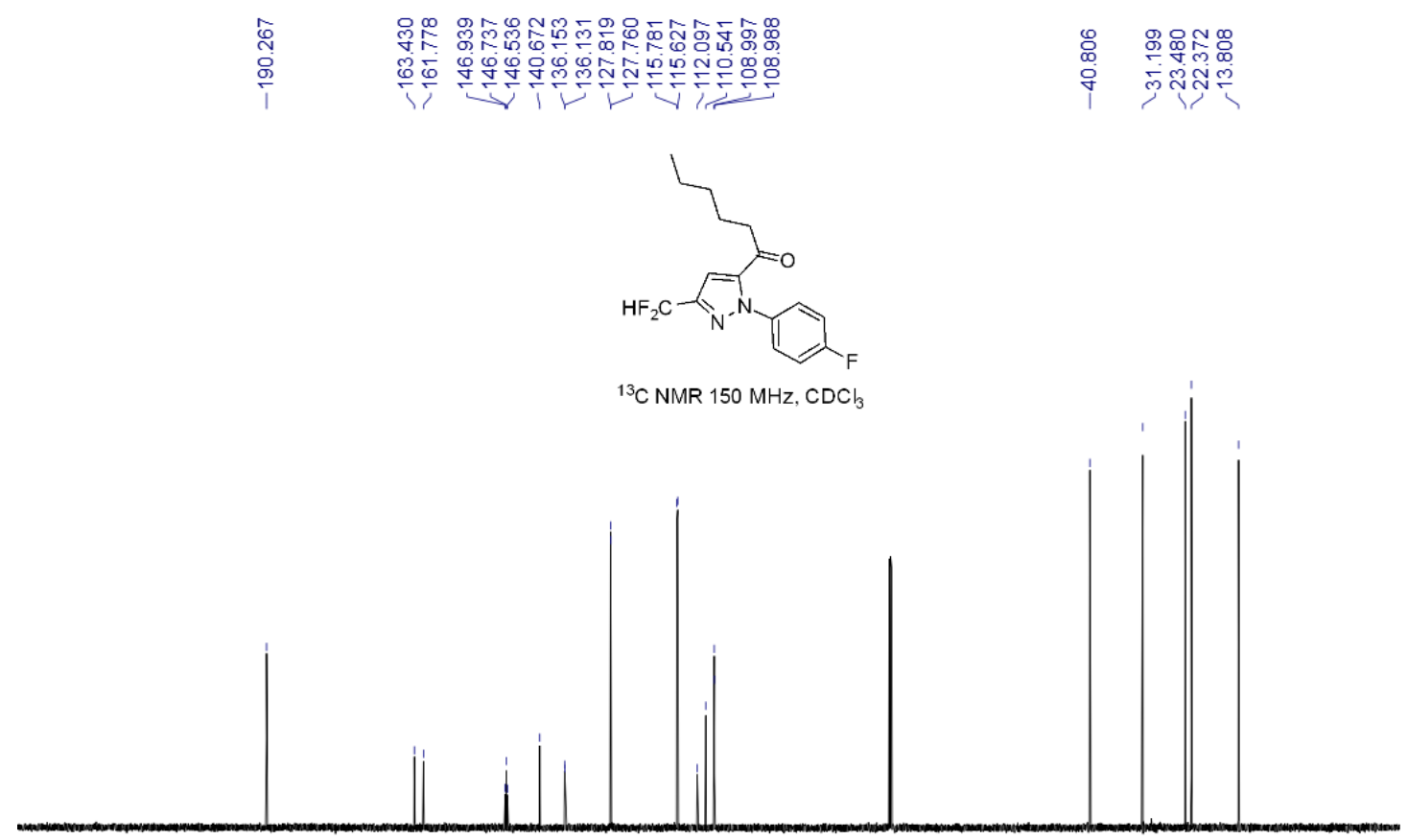

\begin{tabular}{|c|c|c|c|c|c|c|c|c|c|c|c|c|c|c|c|c|}
\hline 230 & 210 & 190 & 170 & 150 & 130 & $\begin{array}{c}110 \\
\mathrm{f} 1(\mathrm{ppm})\end{array}$ & 90 & 80 & 70 & 60 & 50 & 40 & 30 & 20 & 10 & $\begin{array}{ll}0 & -10\end{array}$ \\
\hline 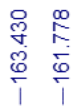 & & & & & $\begin{array}{l}\frac{1}{0} \\
0 \\
0 \\
0 \\
0\end{array}$ & 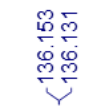 & & & 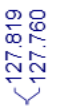 & & & & & 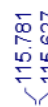 & & 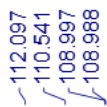 \\
\hline
\end{tabular}

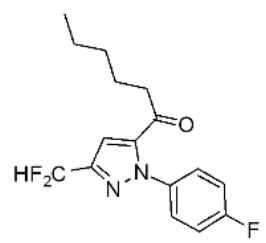

${ }^{13} \mathrm{C}$ NMR $150 \mathrm{MHz}, \mathrm{CDCl}$
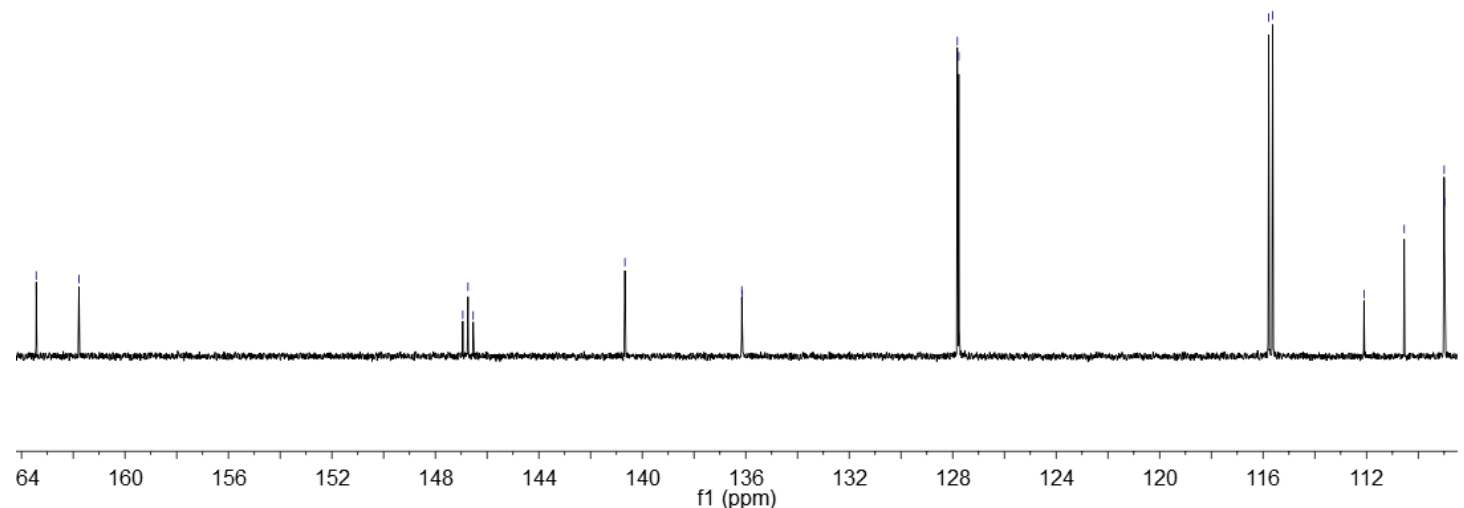


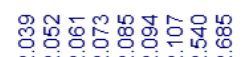

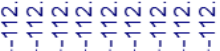

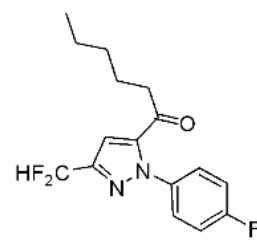

${ }^{19} \mathrm{~F}$ NMR $376 \mathrm{MHz}, \mathrm{CDCl}_{3}$

\begin{tabular}{|c|c|c|c|c|c|c|c|c|c|c|c|c|c|}
\hline 20 & 10 & 0 & -20 & -40 & -60 & -80 & $\begin{array}{l}-100 \\
\quad \mathrm{f} 1(\mathrm{ppm})\end{array}$ & -120 & -140 & -160 & -180 & -200 & -220 \\
\hline & & & & & 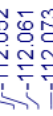 & & & & & $\begin{array}{l}\stackrel{P}{+} \\
\stackrel{5}{N} \\
\stackrel{5}{\leftarrow}\end{array}$ & & 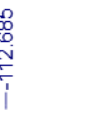 & \\
\hline
\end{tabular}

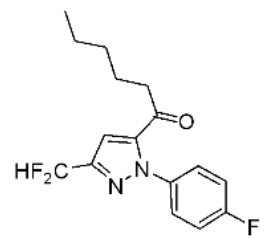

${ }^{19} \mathrm{~F}$ NMR $376 \mathrm{MHz}, \mathrm{CDCl}_{3}$

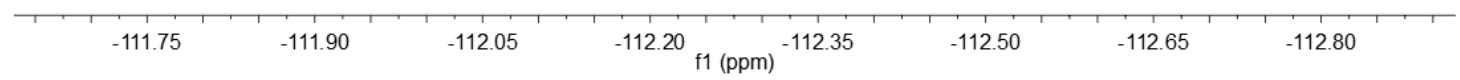


HRMS (ESI) copy of compound 3aa:

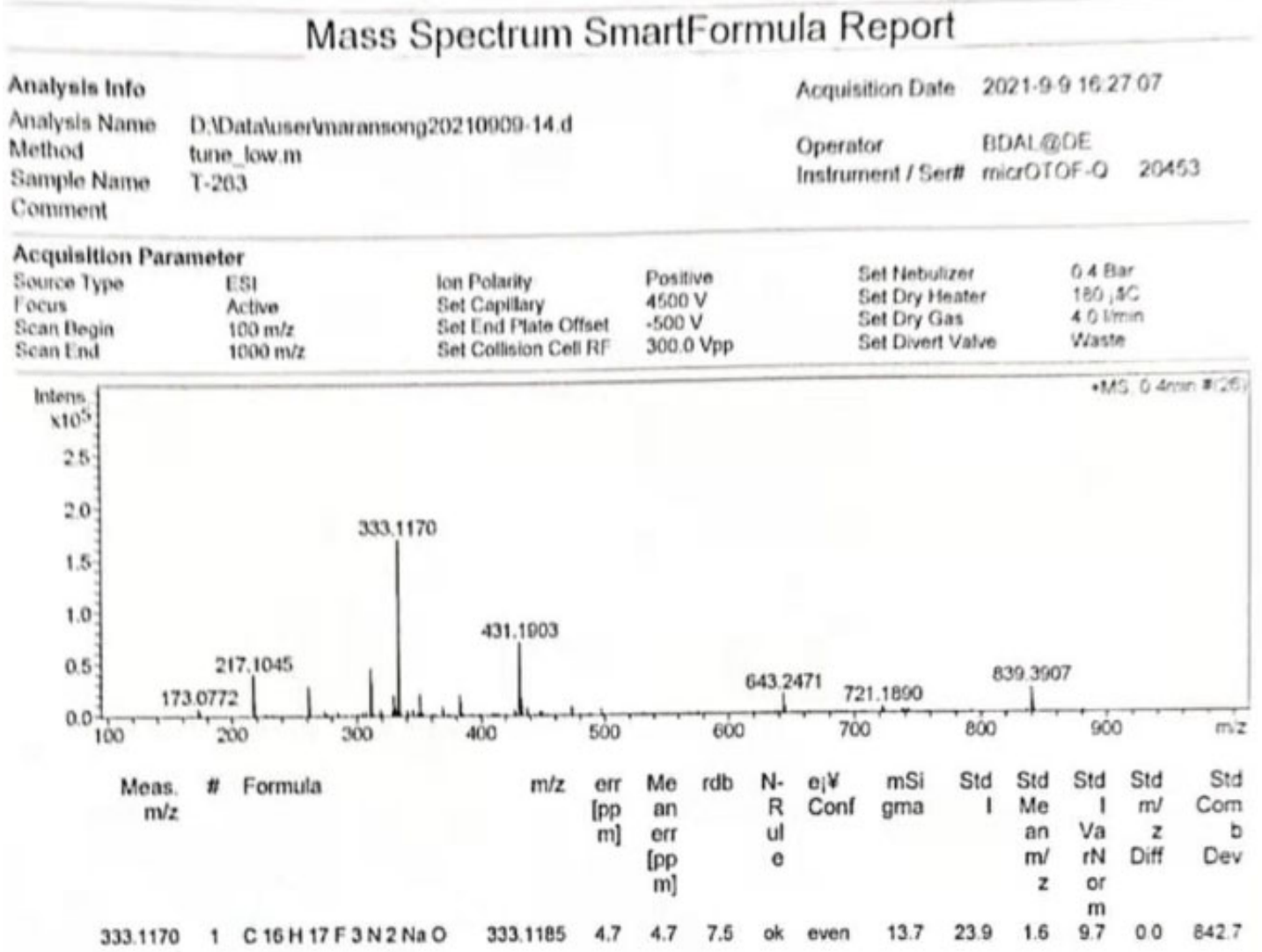

NMR copies of compound $\mathbf{3 a b}$ :

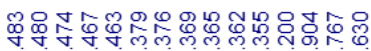

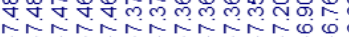

$\stackrel{\infty}{\sim}$

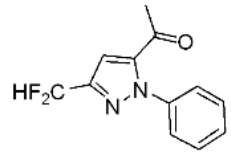

${ }^{1} \mathrm{H}$ NMR $400 \mathrm{MHz}, \mathrm{CDCl}_{3}$

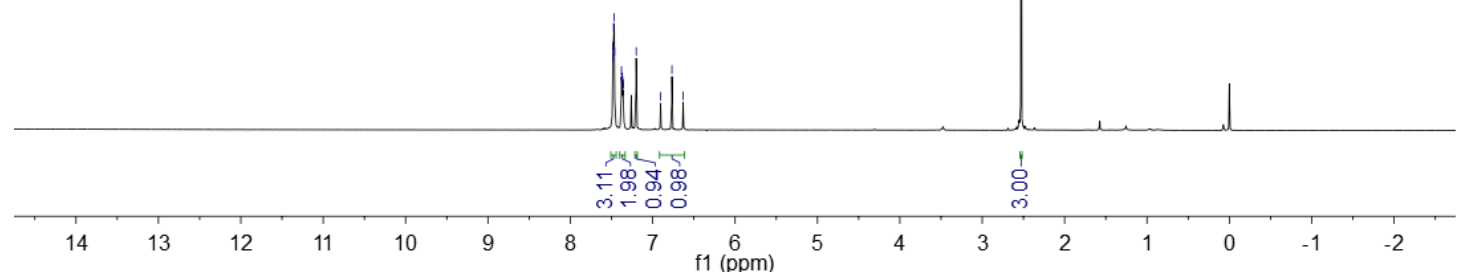




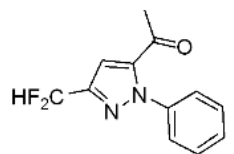

${ }^{1} \mathrm{H}$ NMR $400 \mathrm{MHz}, \mathrm{CDCl}_{3}$

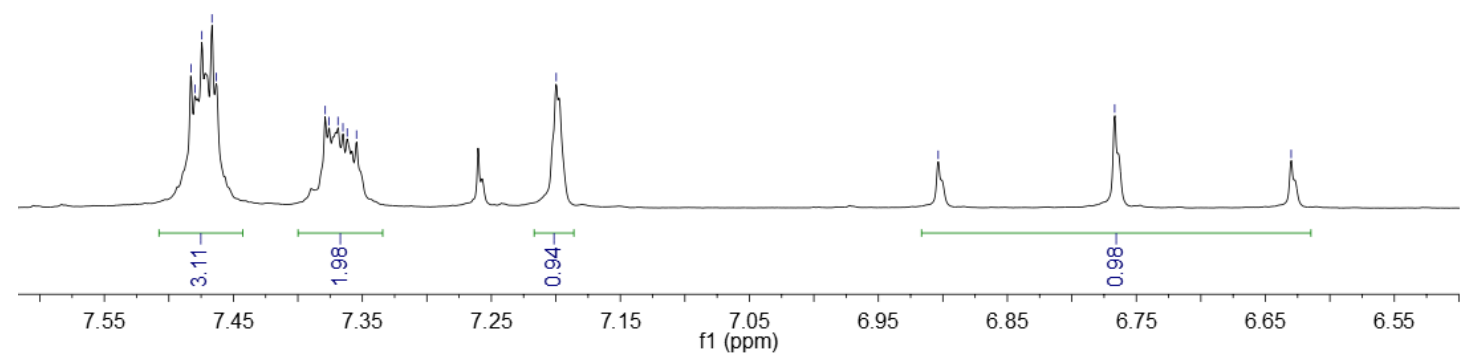

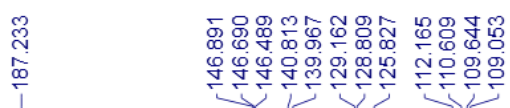

$\underset{\substack{\infty \\ \infty}}{\stackrel{\infty}{\infty}}$

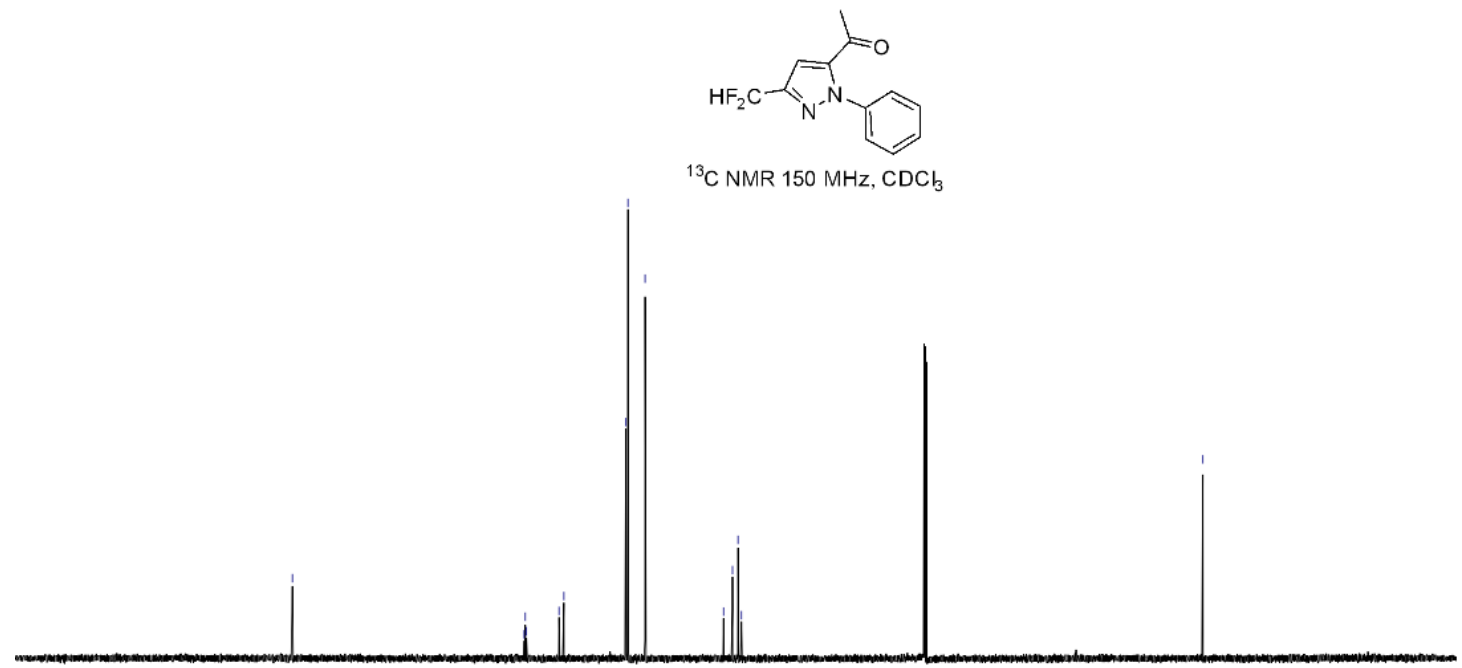

230 210 190 130 f1 $\stackrel{110}{(p p m)}$ $\begin{array}{lllllllllll}90 & 80 & 70 & 60 & 50 & 40 & 30 & 20 & 10 & 0 & -10\end{array}$ 


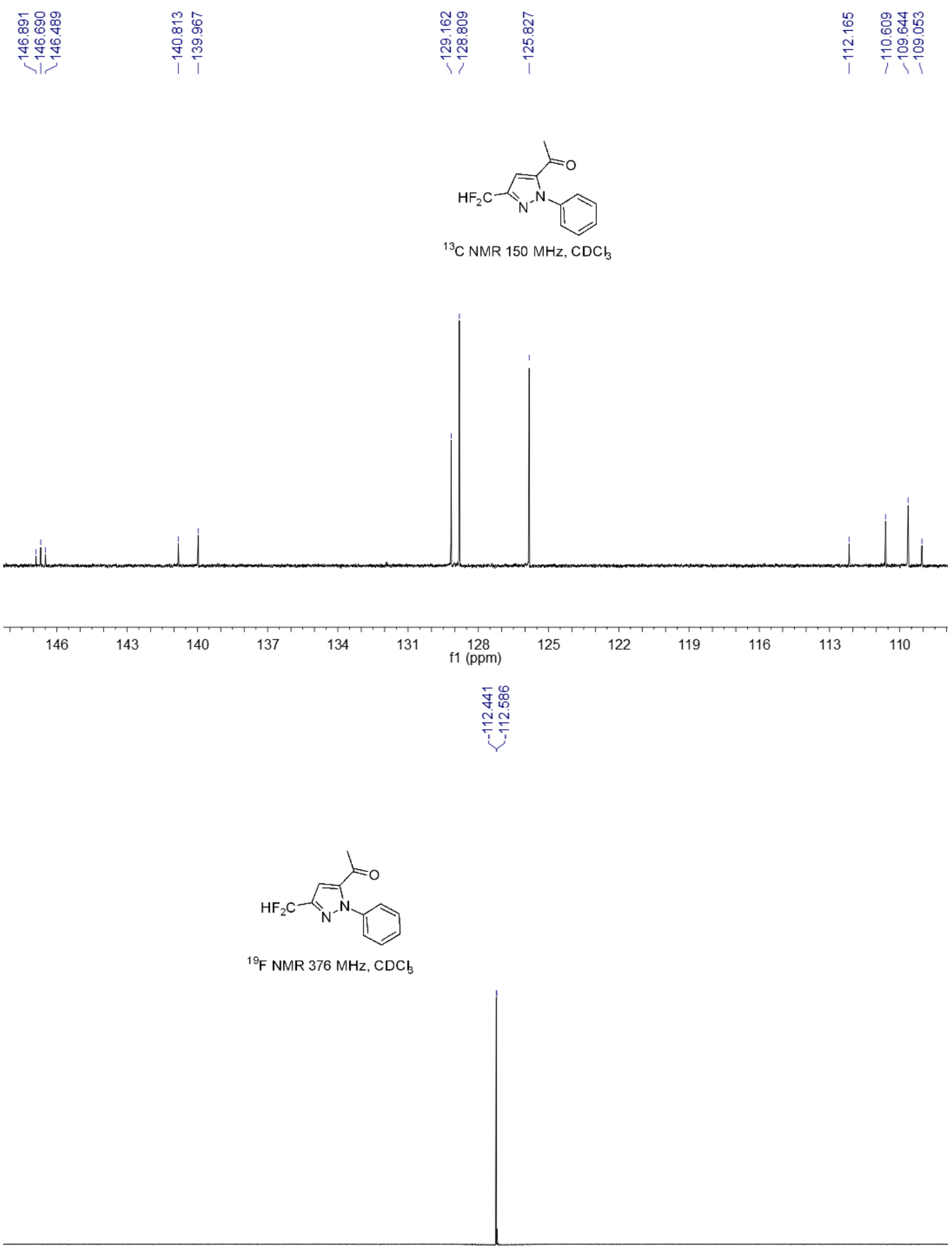

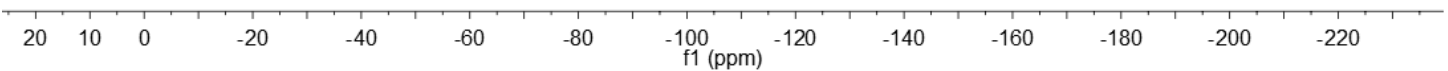




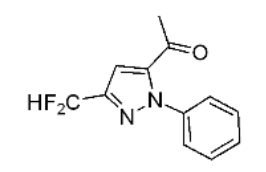

${ }^{19} \mathrm{~F} \mathrm{NMR} 376 \mathrm{MHz}, \mathrm{CDCb}$

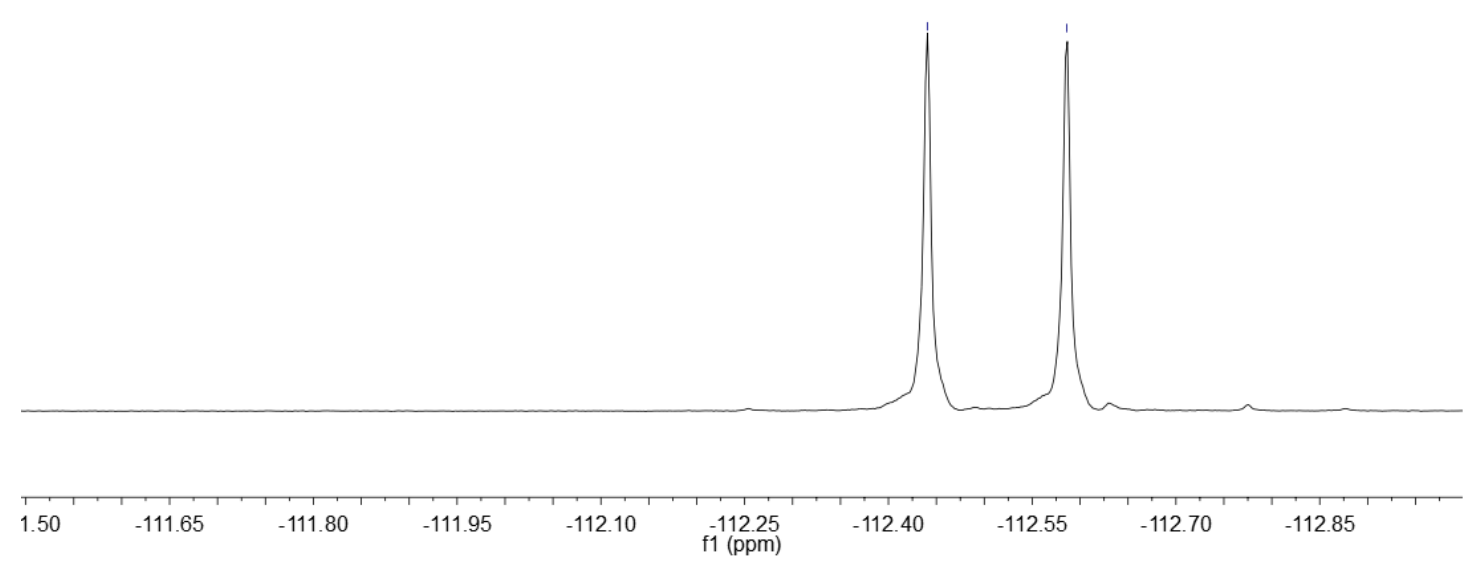

HRMS (ESI) copy of compound 3ab:

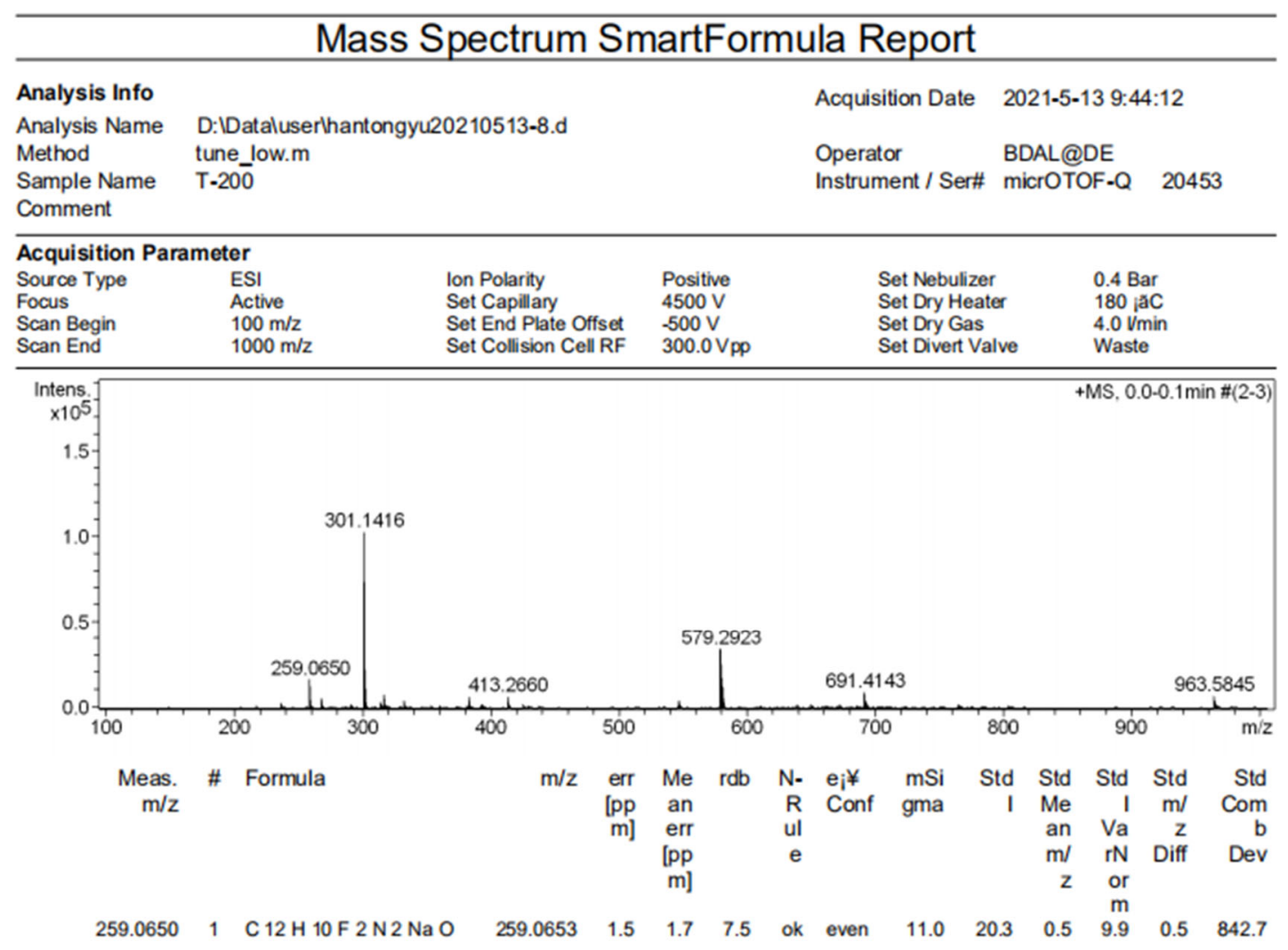


NMR copies of compound 3ac:

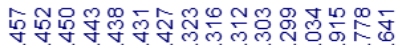

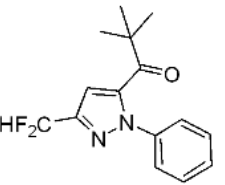

${ }^{1} \mathrm{H} \mathrm{NMR} 400 \mathrm{MHz}, \mathrm{CDCl}$

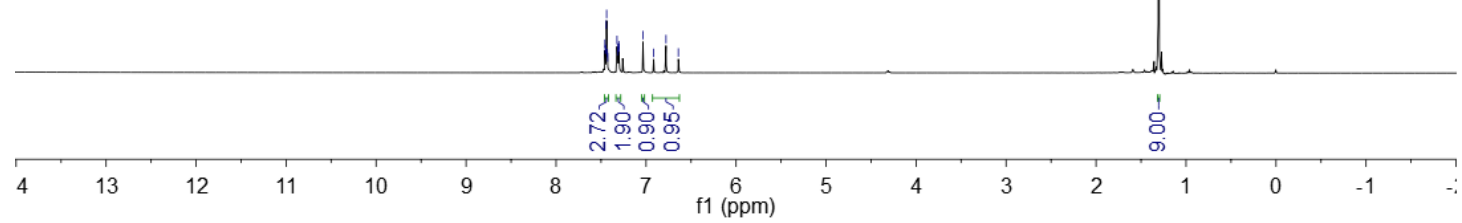

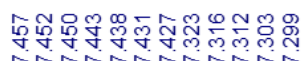

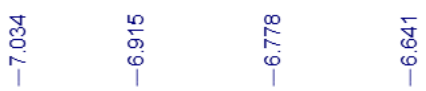

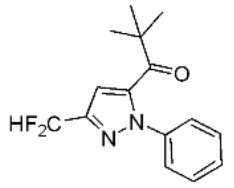

${ }^{1} \mathrm{H}$ NMR $400 \mathrm{MHz}, \mathrm{CDCb}$

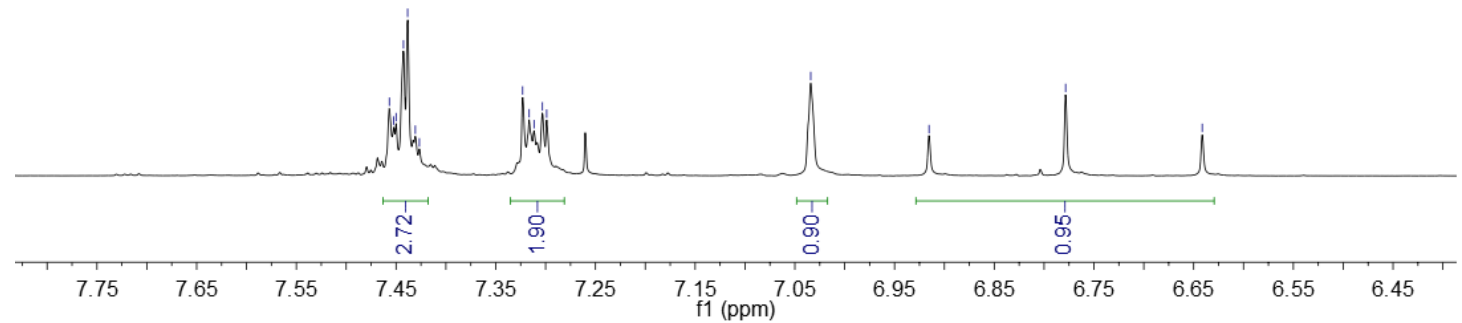




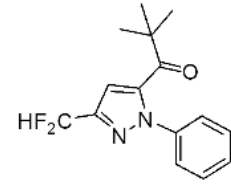

${ }^{13} \mathrm{C}$ NMR $150 \mathrm{MHz}, \mathrm{CDCl}$
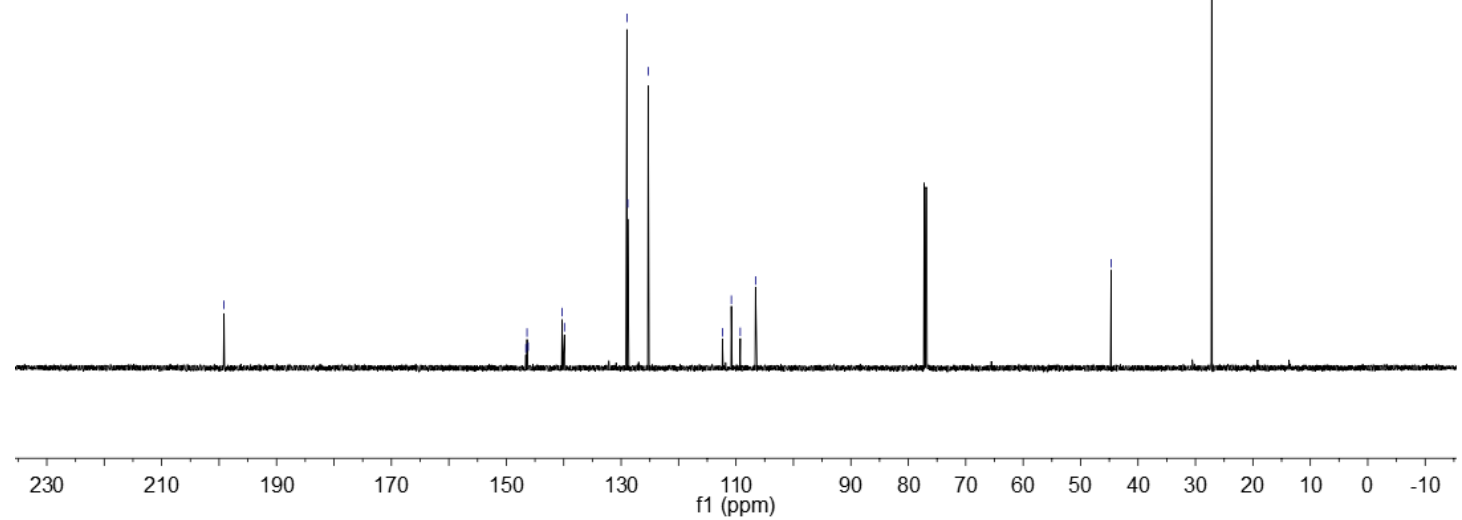

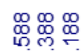

웜

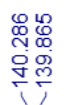

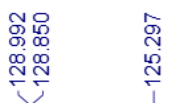

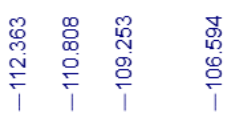

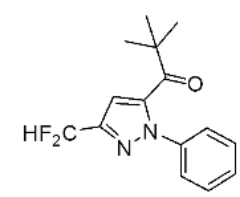

${ }^{13} \mathrm{C}$ NMR $150 \mathrm{MHz}, \mathrm{CDCl}$

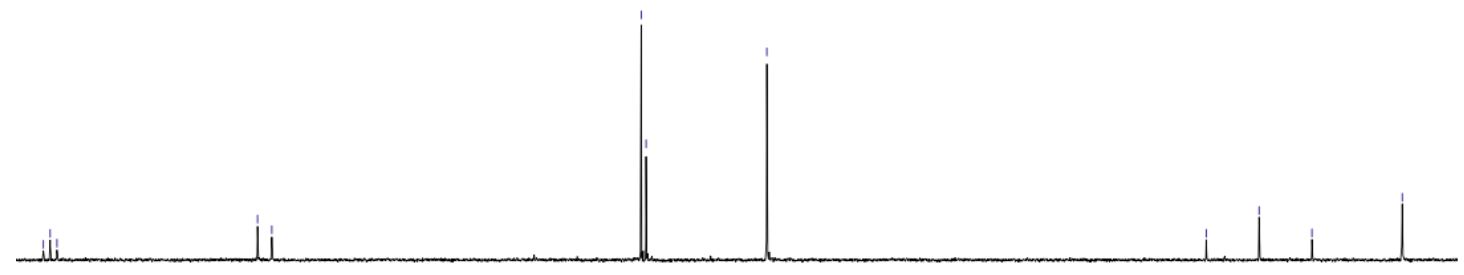

$144 \quad 140$

136

132

$128 \mathrm{f} 1(\mathrm{ppm})$

120

116

112

108 


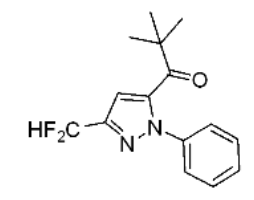

${ }^{19} \mathrm{~F}$ NMR $376 \mathrm{MHz}, \mathrm{CDCl}_{3}$
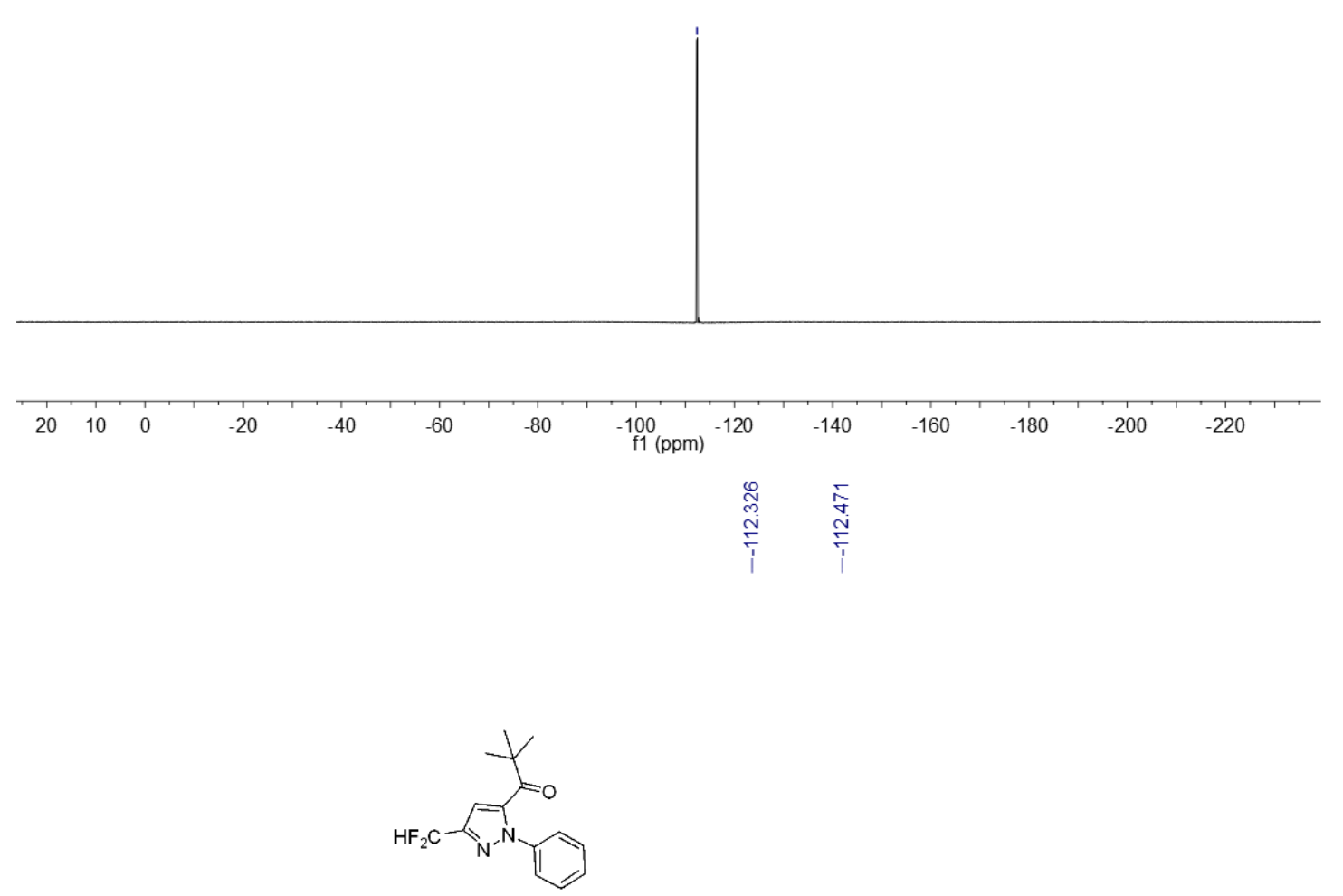

${ }^{19} \mathrm{~F}$ NMR $376 \mathrm{MHz}, \mathrm{CDCl}$

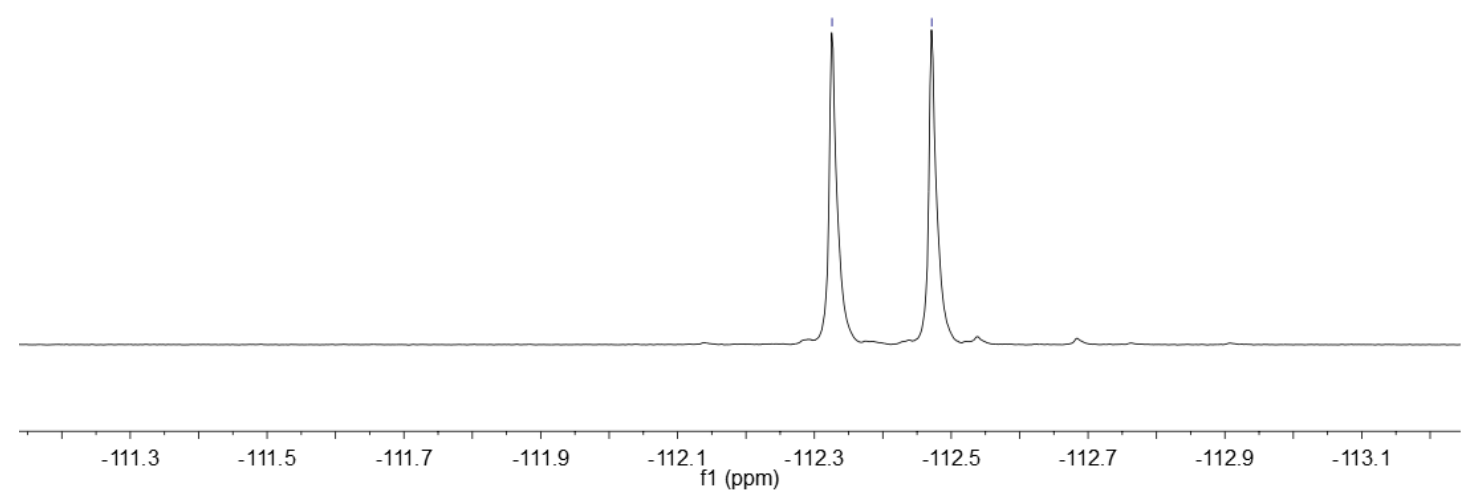


HRMS (ESI) copy of compound 3ac:

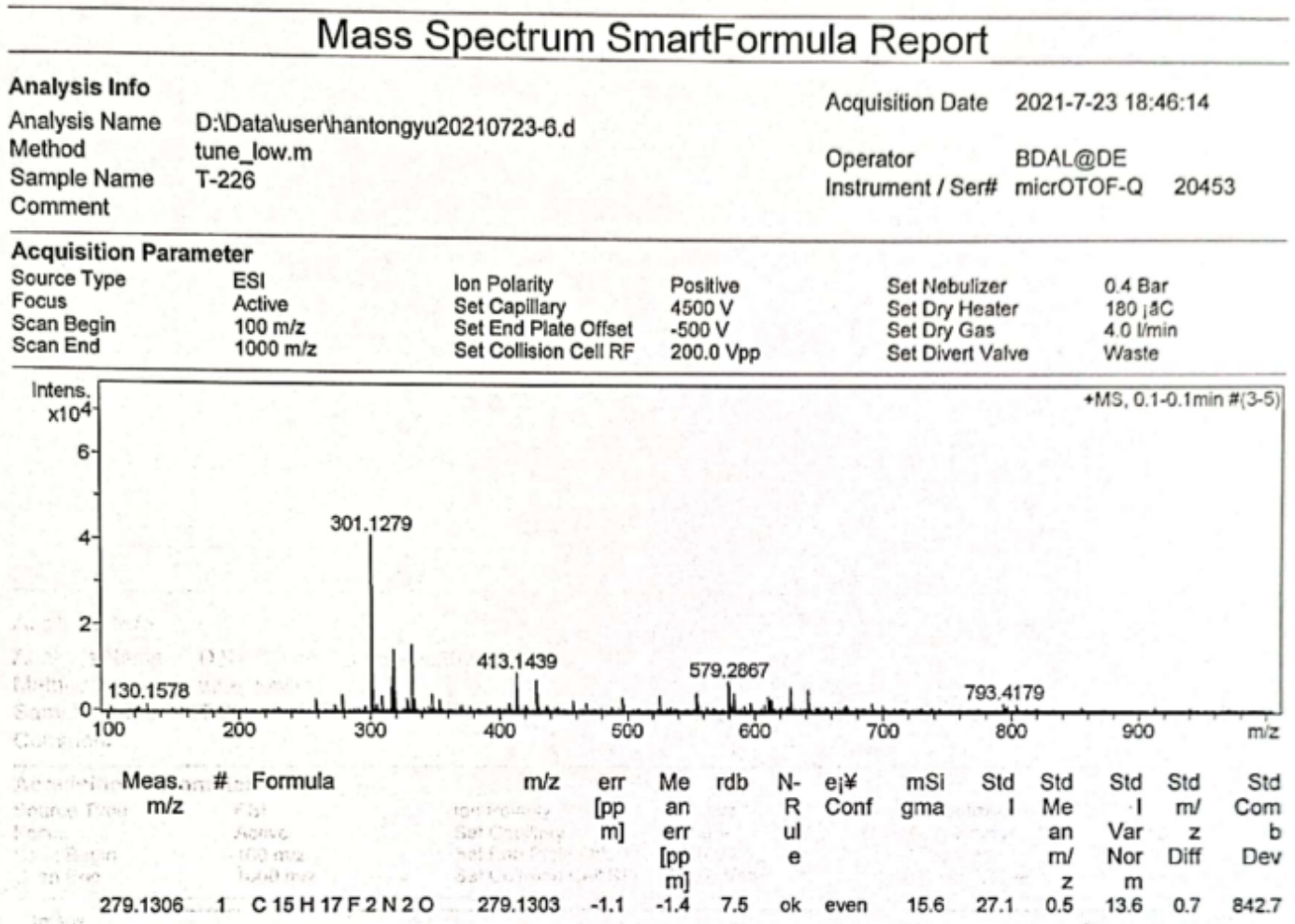

Copies of NMR and HRMS Spectra for Compounds $\mathbf{5 a}-\mathbf{5 k}$

NMR copies of compound $\mathbf{5 a}$ :

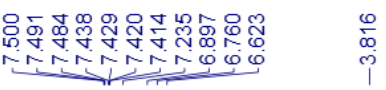

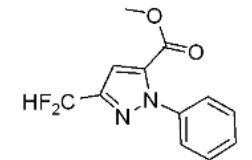

${ }^{1} \mathrm{H}$ NMR $400 \mathrm{MHz}, \mathrm{CDCl}$

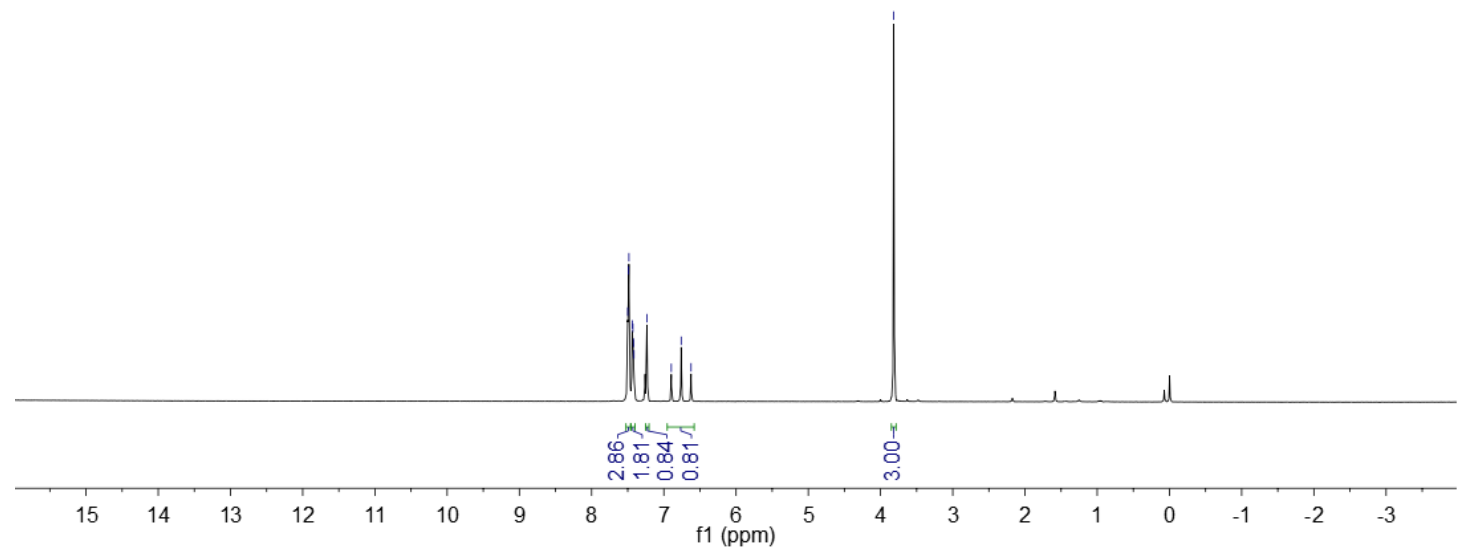




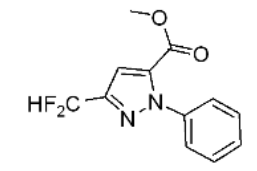

${ }^{1} \mathrm{H}$ NMR $400 \mathrm{MHz}, \mathrm{CDCl}_{3}$
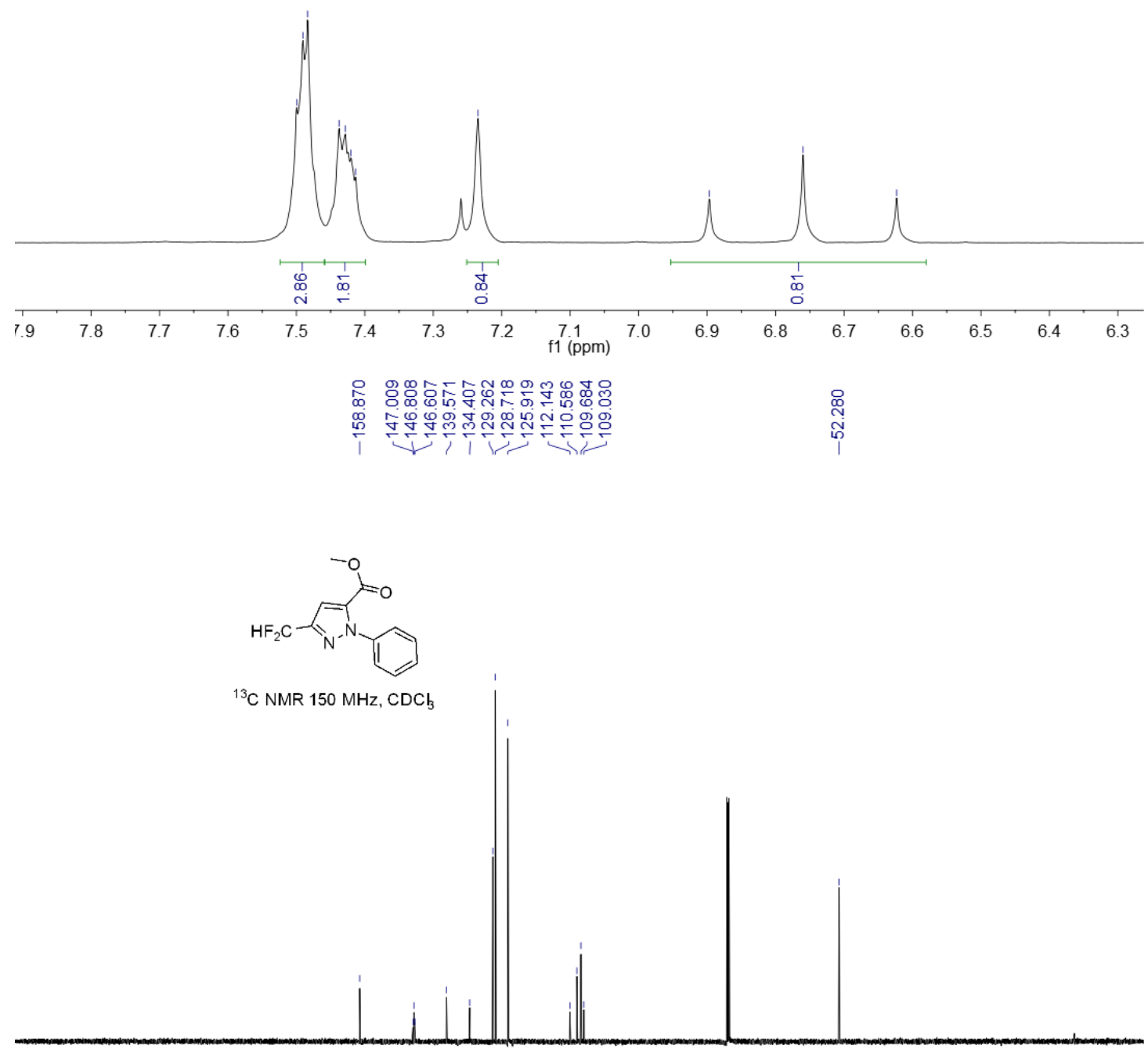

$230 \quad 210 \quad 190$

$190 \quad 170$

150

130

110

$\begin{array}{lllllllllll}90 & 80 & 70 & 60 & 50 & 40 & 30 & 20 & 10 & 0 & -10\end{array}$ 


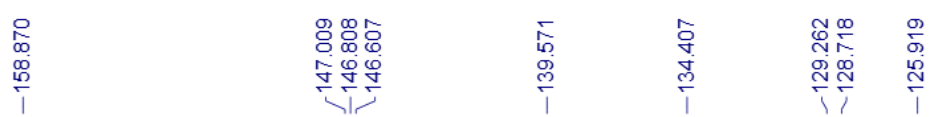

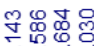

숟유윰유

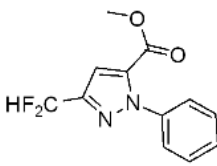

${ }^{13} \mathrm{C}$ NMR $150 \mathrm{MHz}, \mathrm{CDCl}$

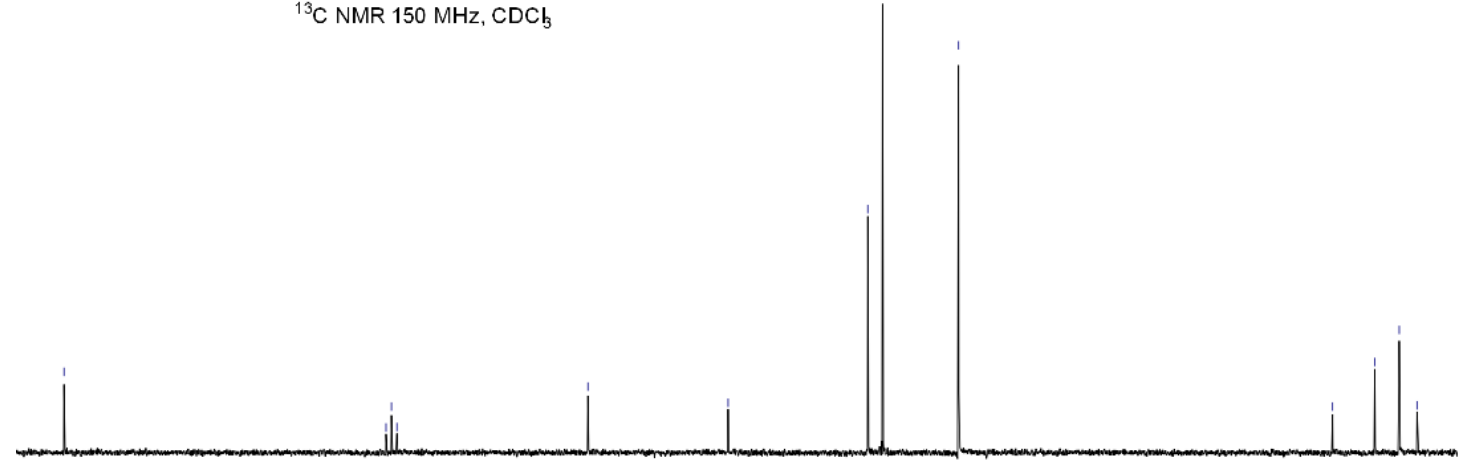

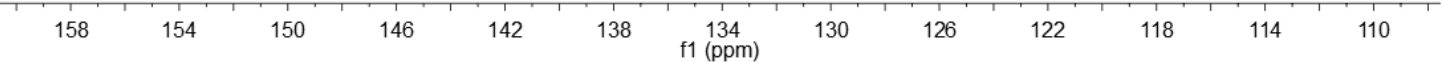

$\underset{\substack{\infty \\ \infty}}{\infty}$

芳芒

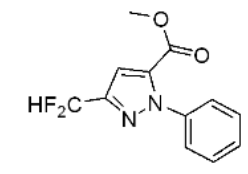

${ }^{19} \mathrm{~F} \mathrm{NMR} 376 \mathrm{MHz}, \mathrm{CDCl}_{3}$

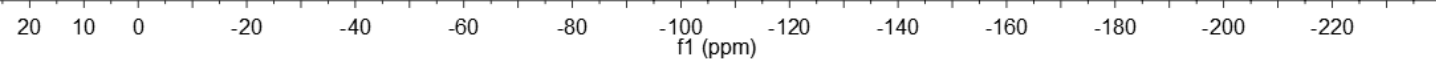




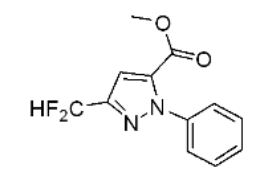

${ }^{19} \mathrm{~F} \mathrm{NMR} 376 \mathrm{MHz}, \mathrm{CDCl}_{3}$

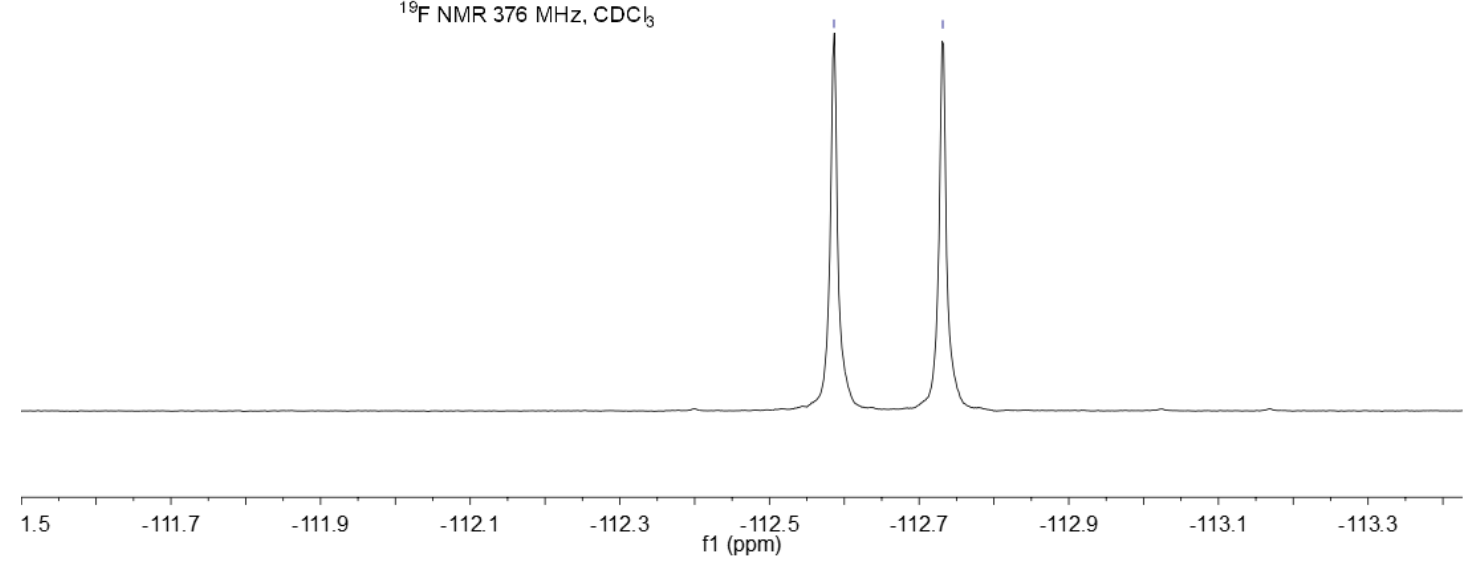

HRMS (ESI) copy of compound $\mathbf{5 a}$ :

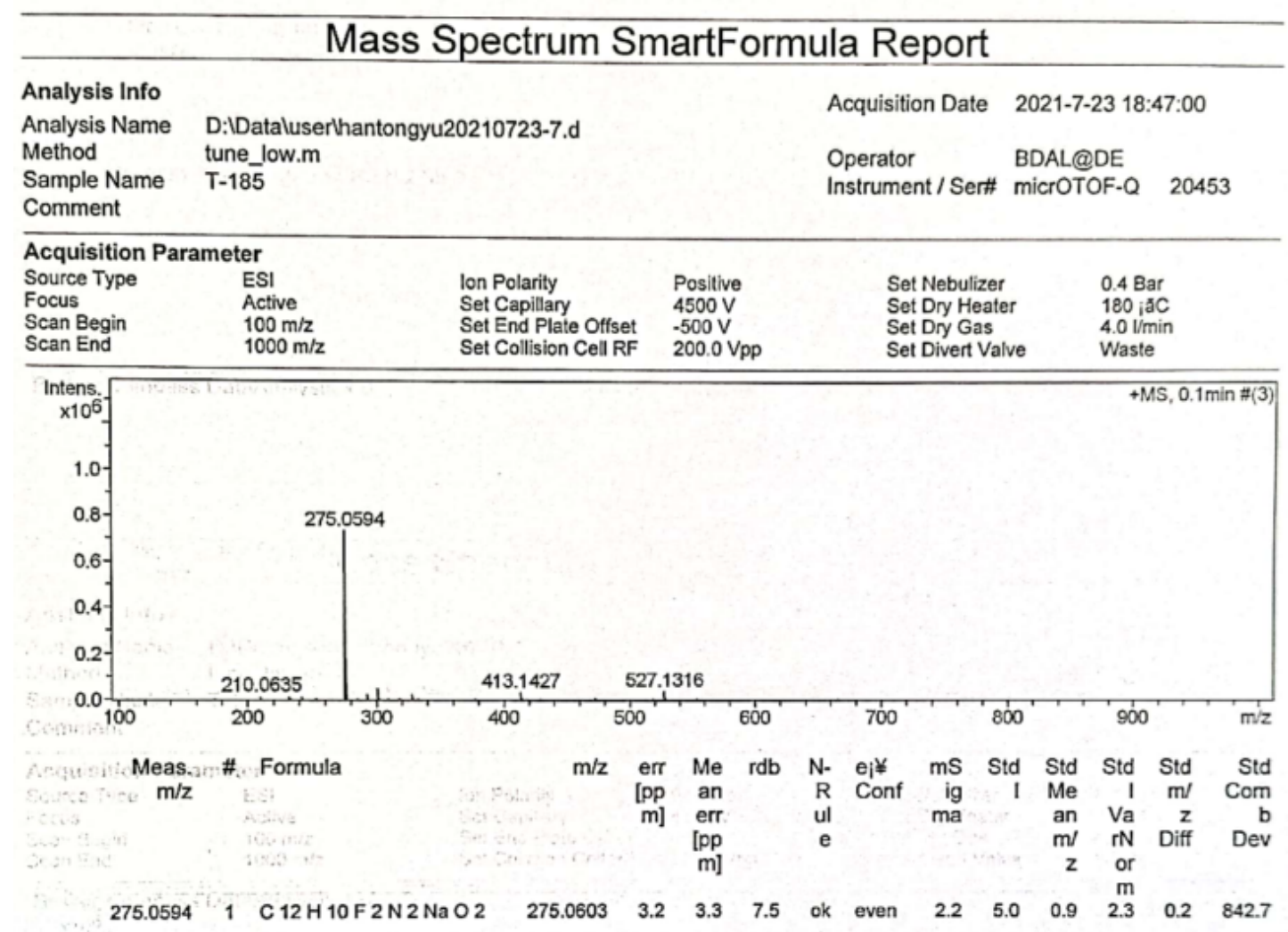


NMR copies of compound $\mathbf{5 b}$ :

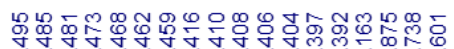

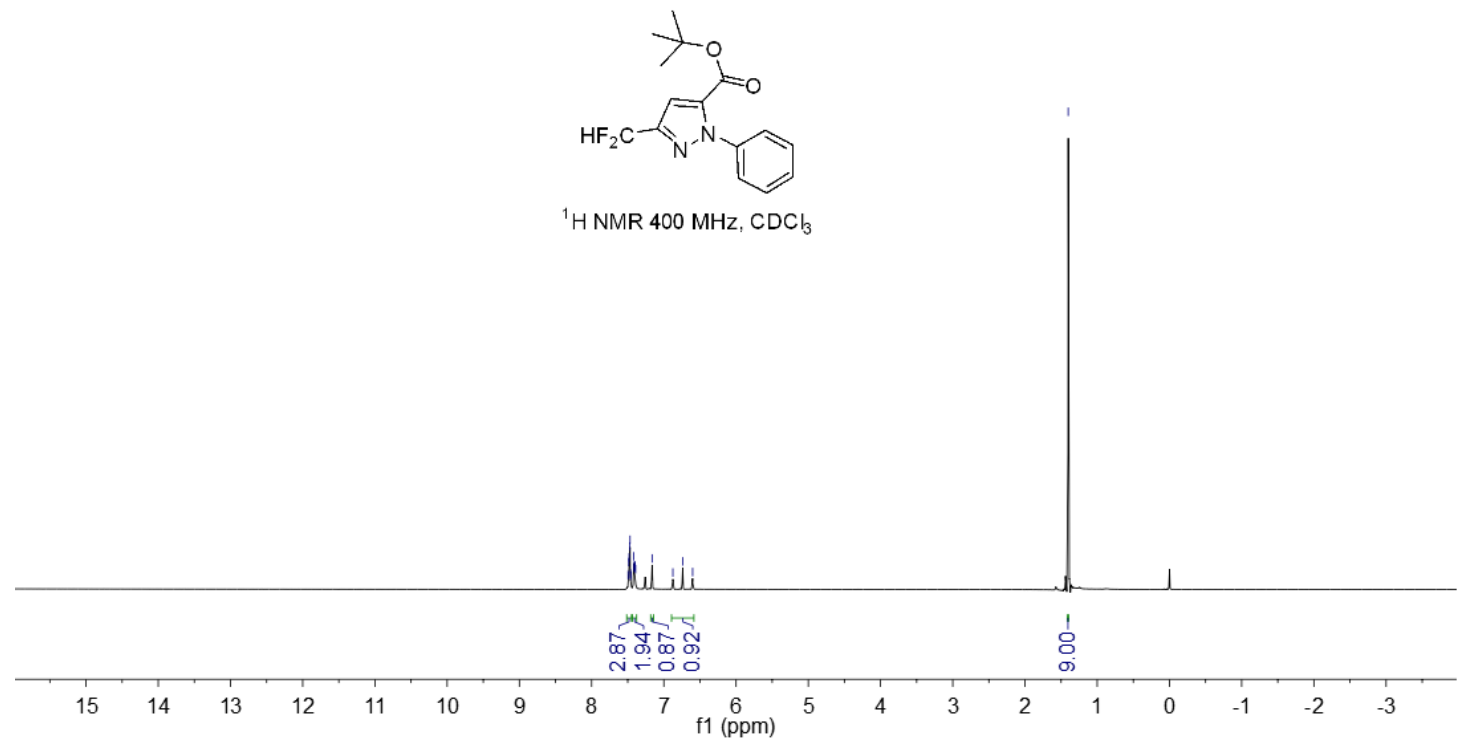

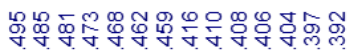

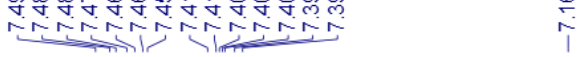

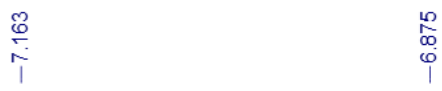

$\underset{\substack{\infty \\ 0}}{\substack{\delta \\ 0}}$

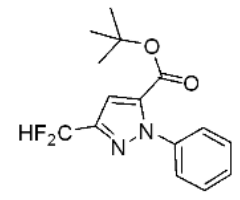

${ }^{1} \mathrm{HNMR} 400 \mathrm{MHz}, \mathrm{CDCl}_{3}$

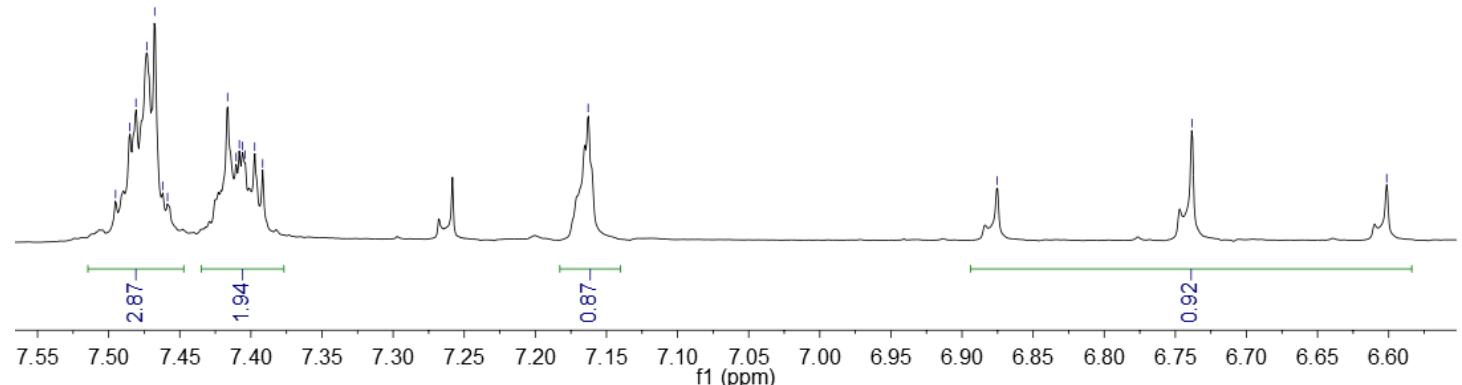




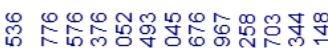

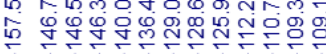

$\underset{\substack{\infty \\ \infty}}{\substack{\infty \\ \text { o. }}}$

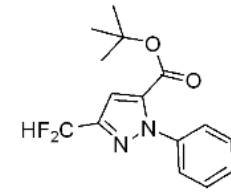

${ }^{13} \mathrm{C}$ NMR $150 \mathrm{MHz}, \mathrm{CDCl}$
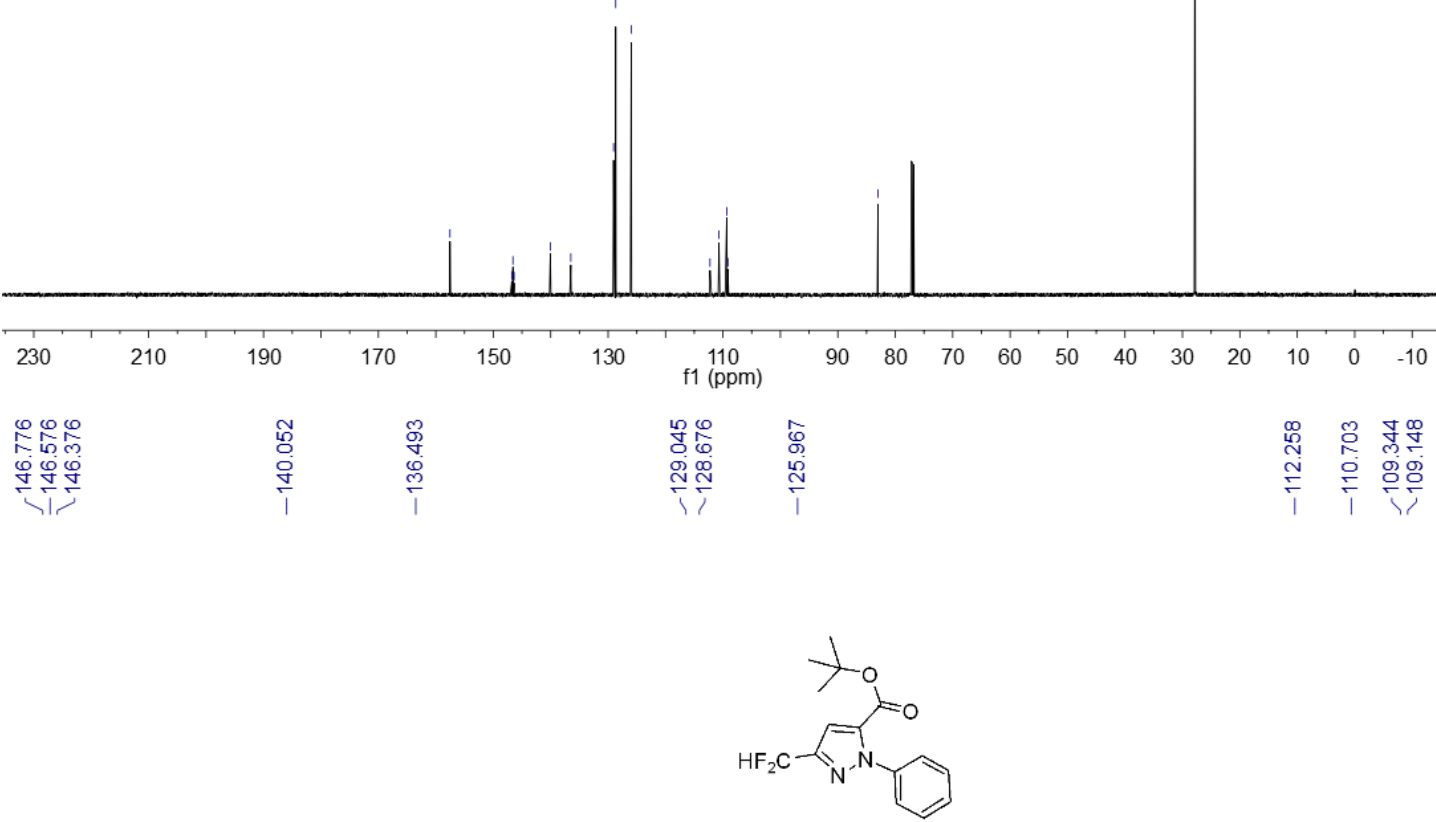

${ }^{13} \mathrm{C}$ NMR $150 \mathrm{MHz}, \mathrm{CDCl}$

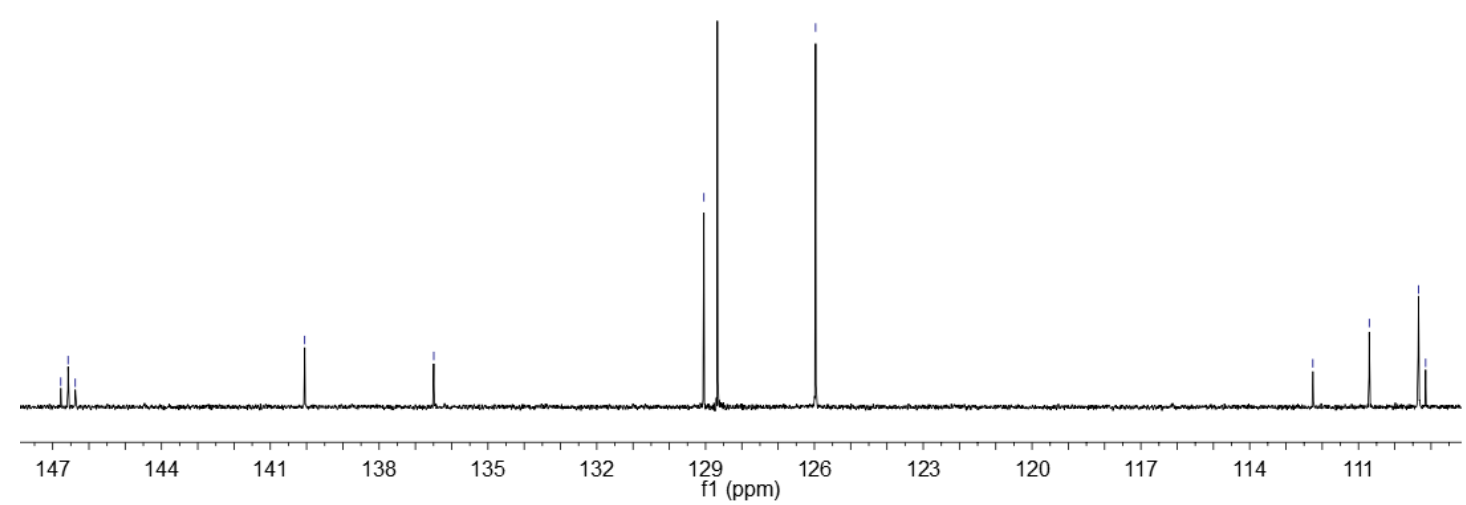



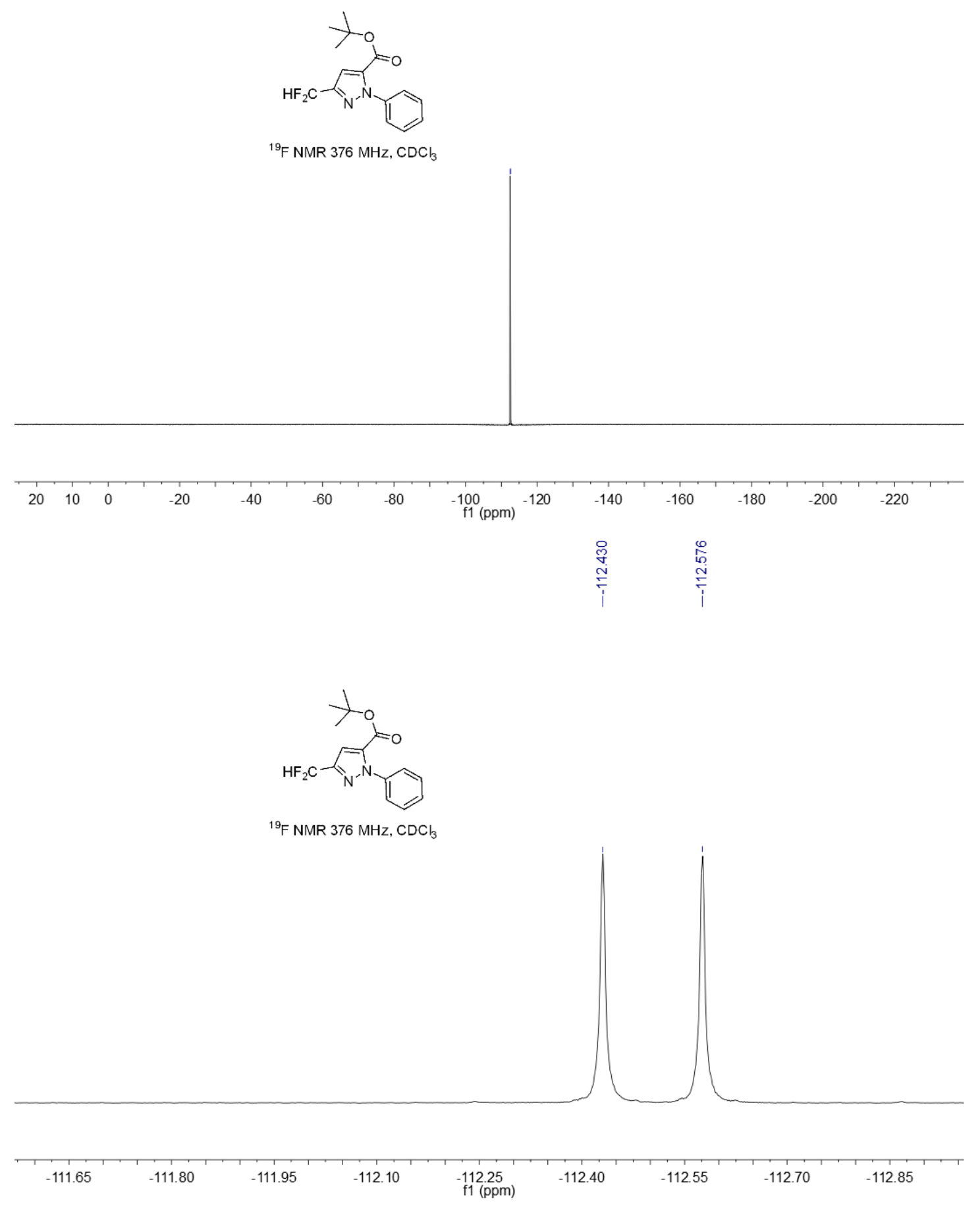
HRMS (ESI) copy of compound $\mathbf{5 b}$ :

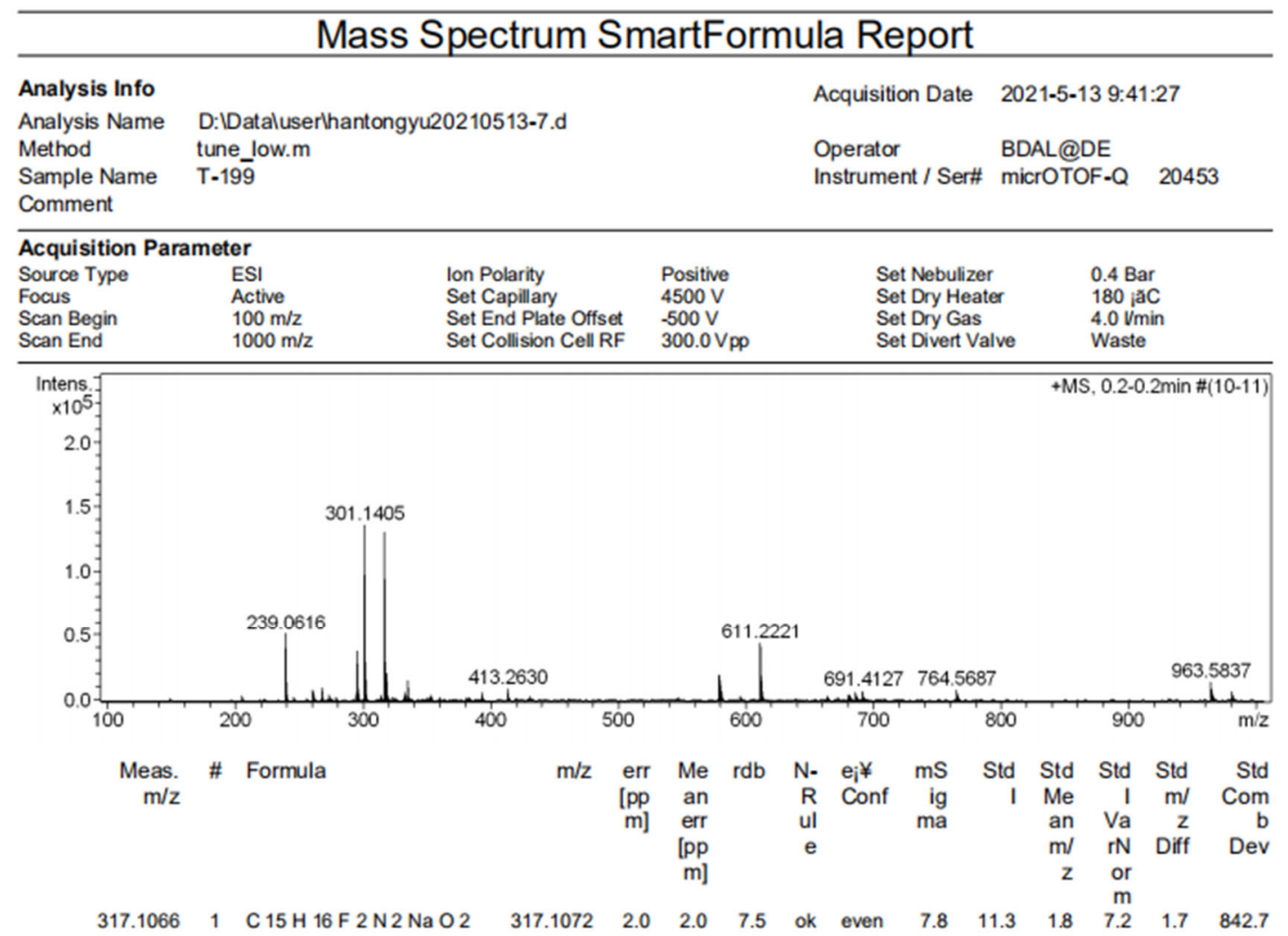

NMR copies of compound $\mathbf{5 c}$ :

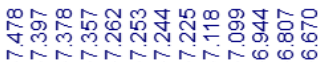

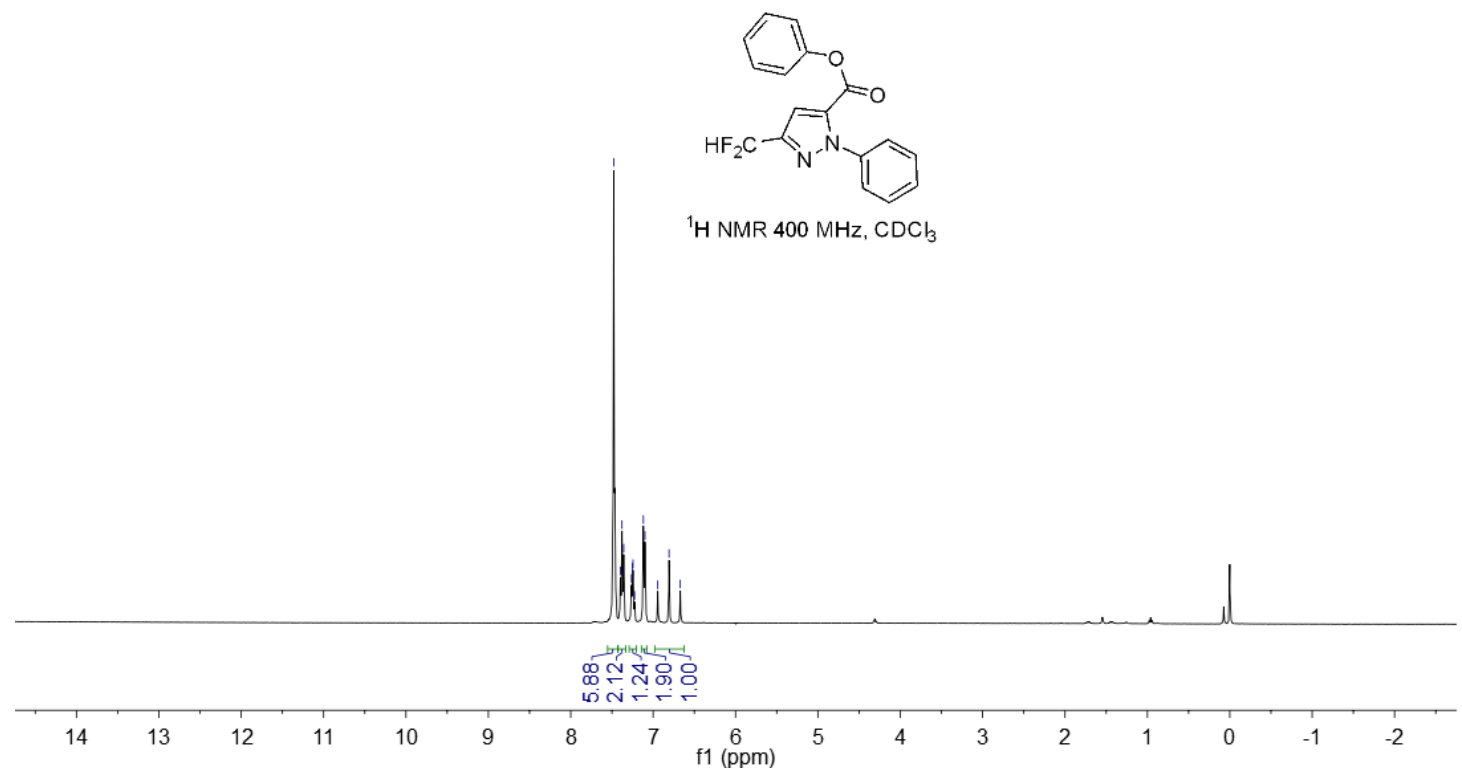




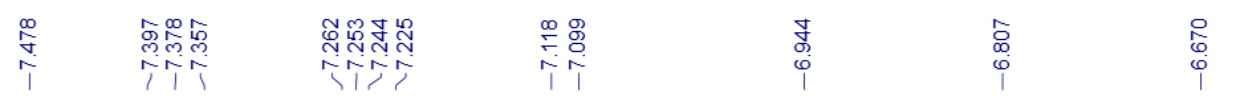

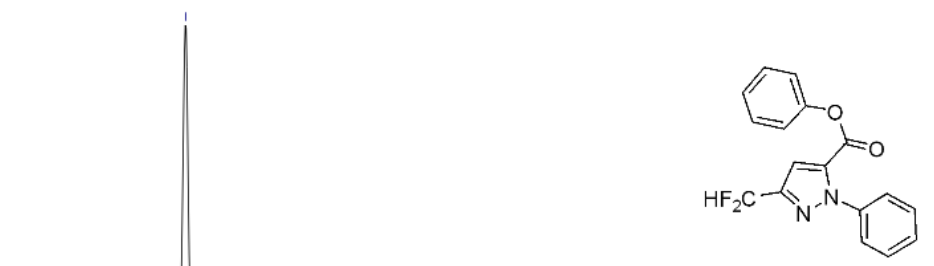

${ }^{1} \mathrm{H}$ NMR $400 \mathrm{MHz}, \mathrm{CDCl}_{3}$

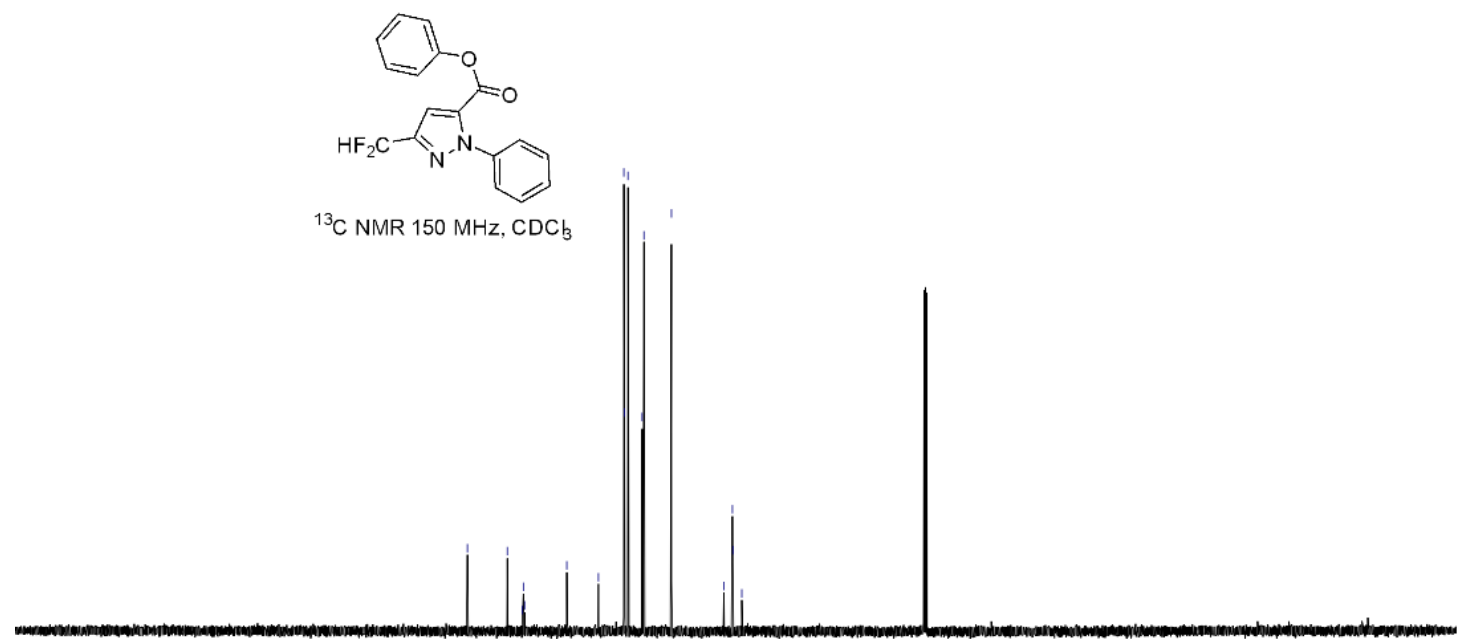

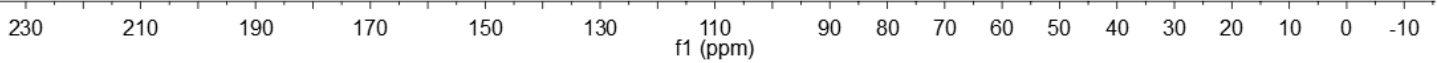




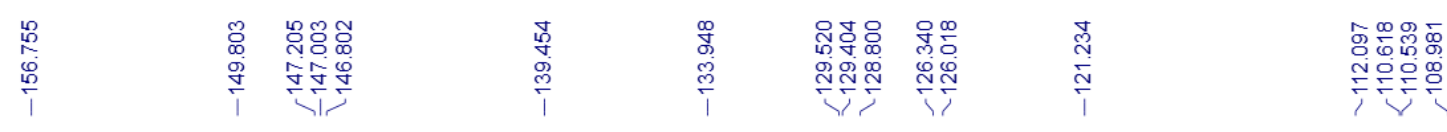
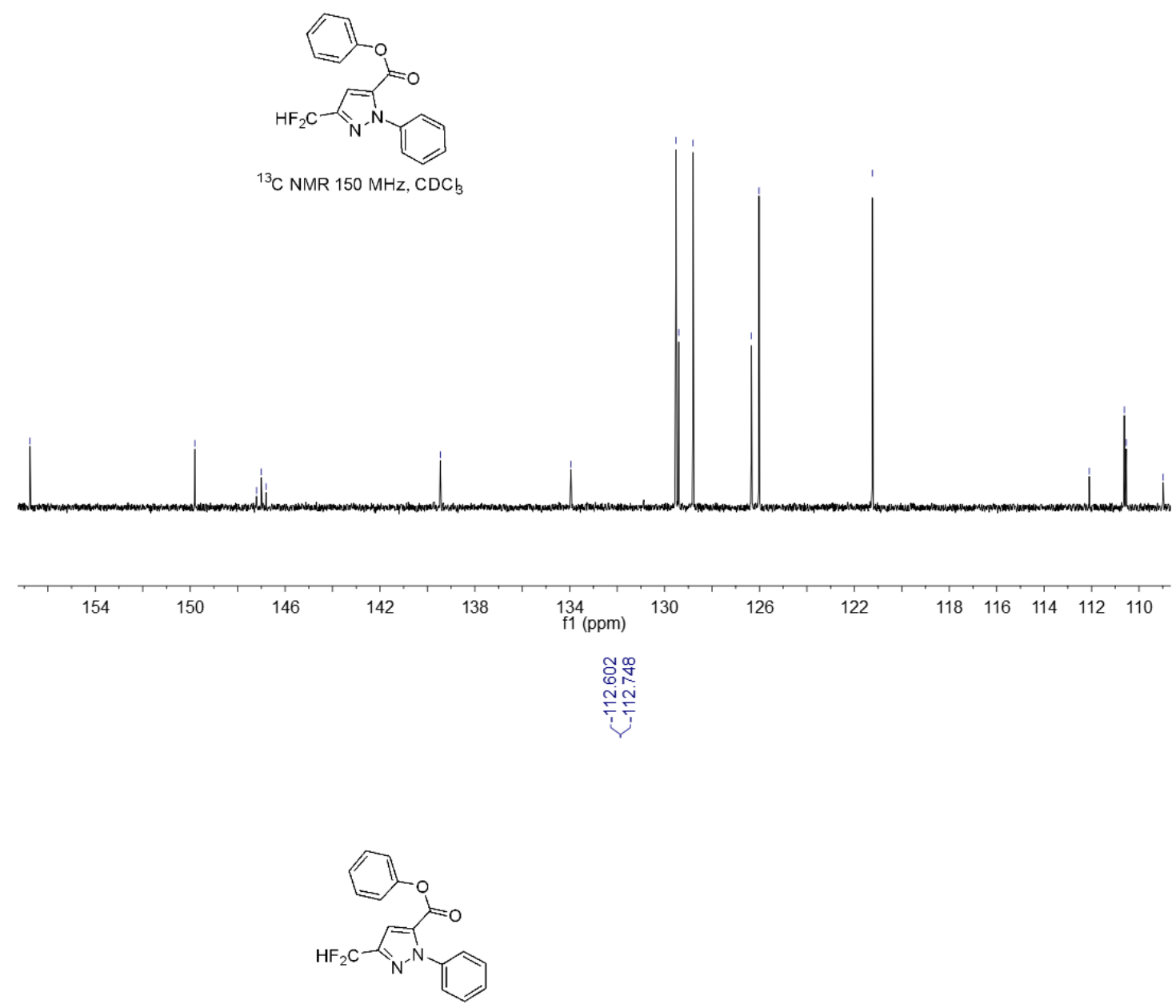

${ }^{19} \mathrm{~F}$ NMR $376 \mathrm{MHz}, \mathrm{CDCl}_{3}$

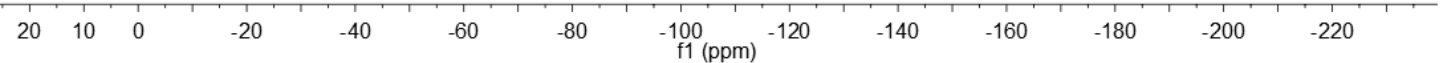




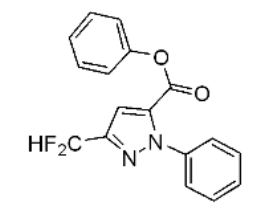

${ }^{19} \mathrm{~F} \mathrm{NMR} 376 \mathrm{MHz}, \mathrm{CDCl}_{3}$

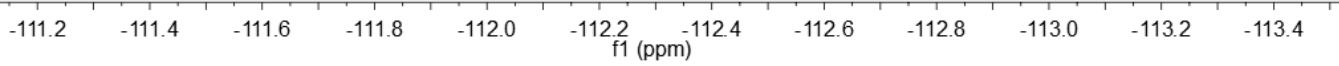

HRMS (ESI) copy of compound 5c:

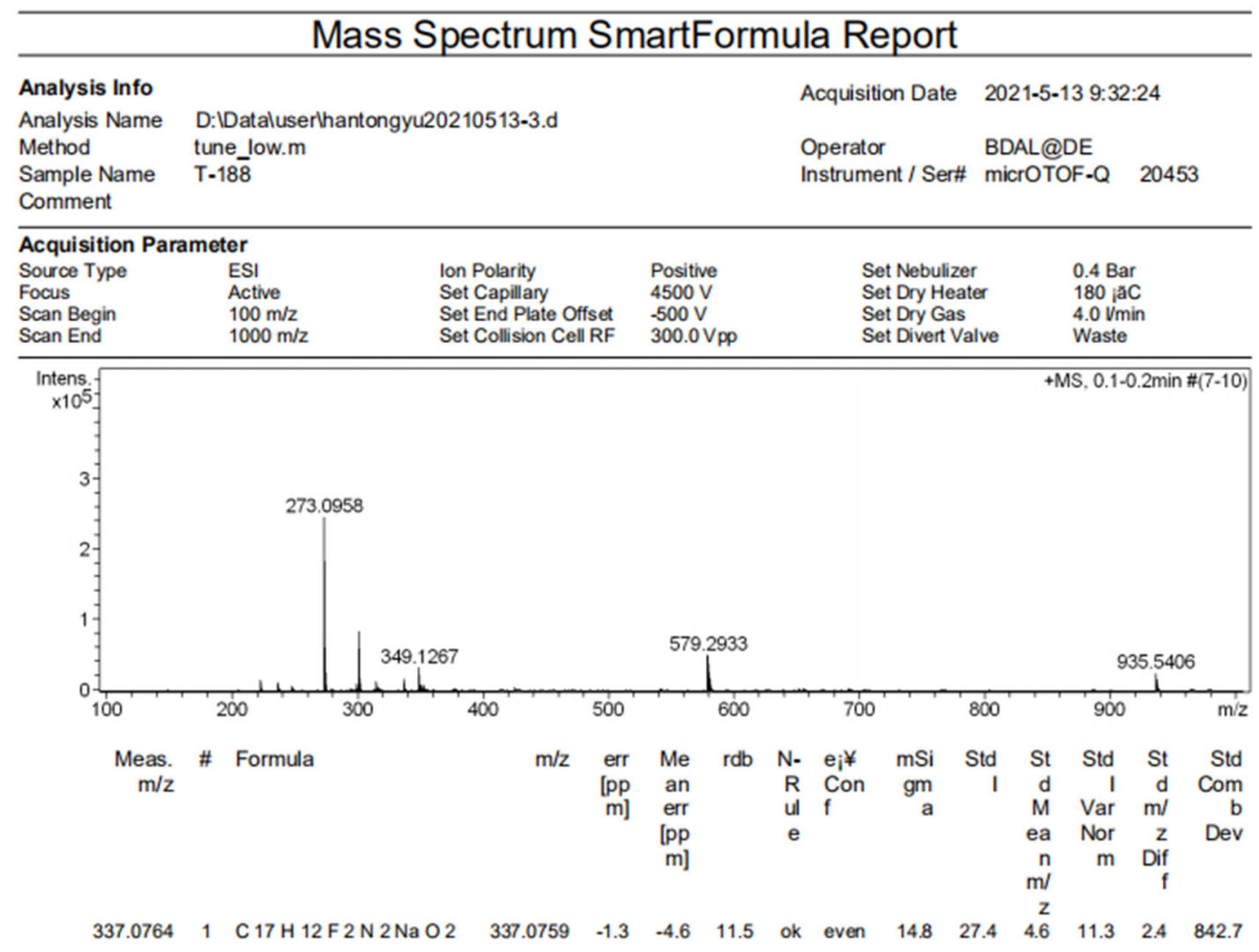


NMR copies of compound $\mathbf{5 d}$ :

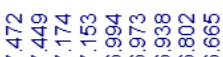

N-Tín

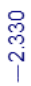

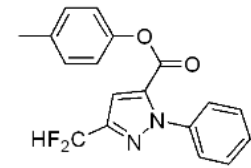

${ }^{1} \mathrm{HNMR} 400 \mathrm{MHz}, \mathrm{CDCl}_{3}$

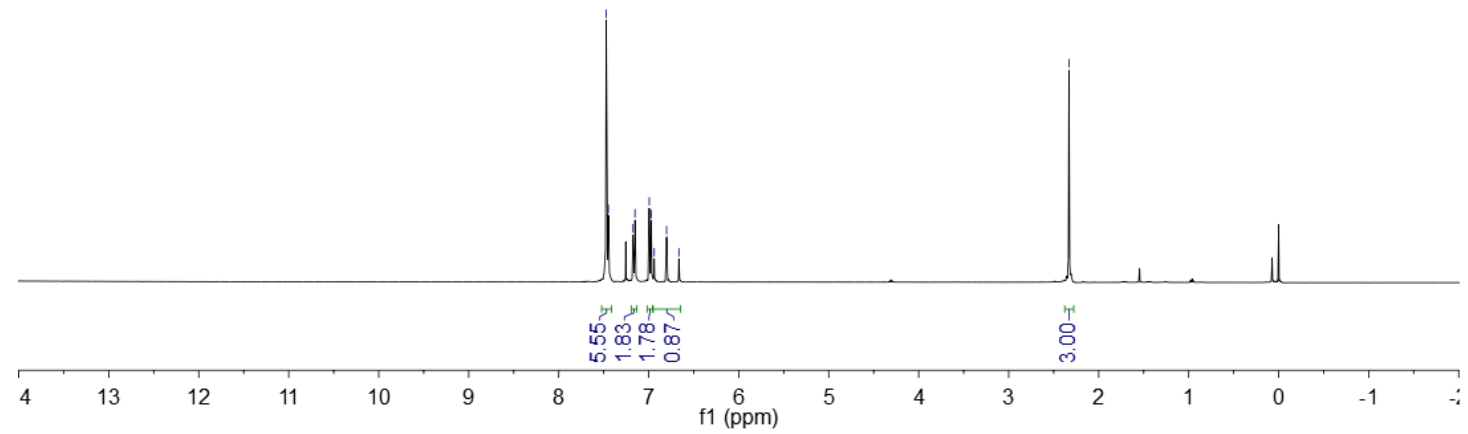

$\frac{1}{\sqrt{2}}$

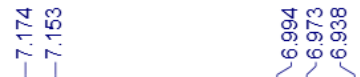

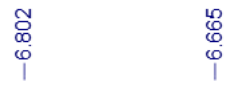

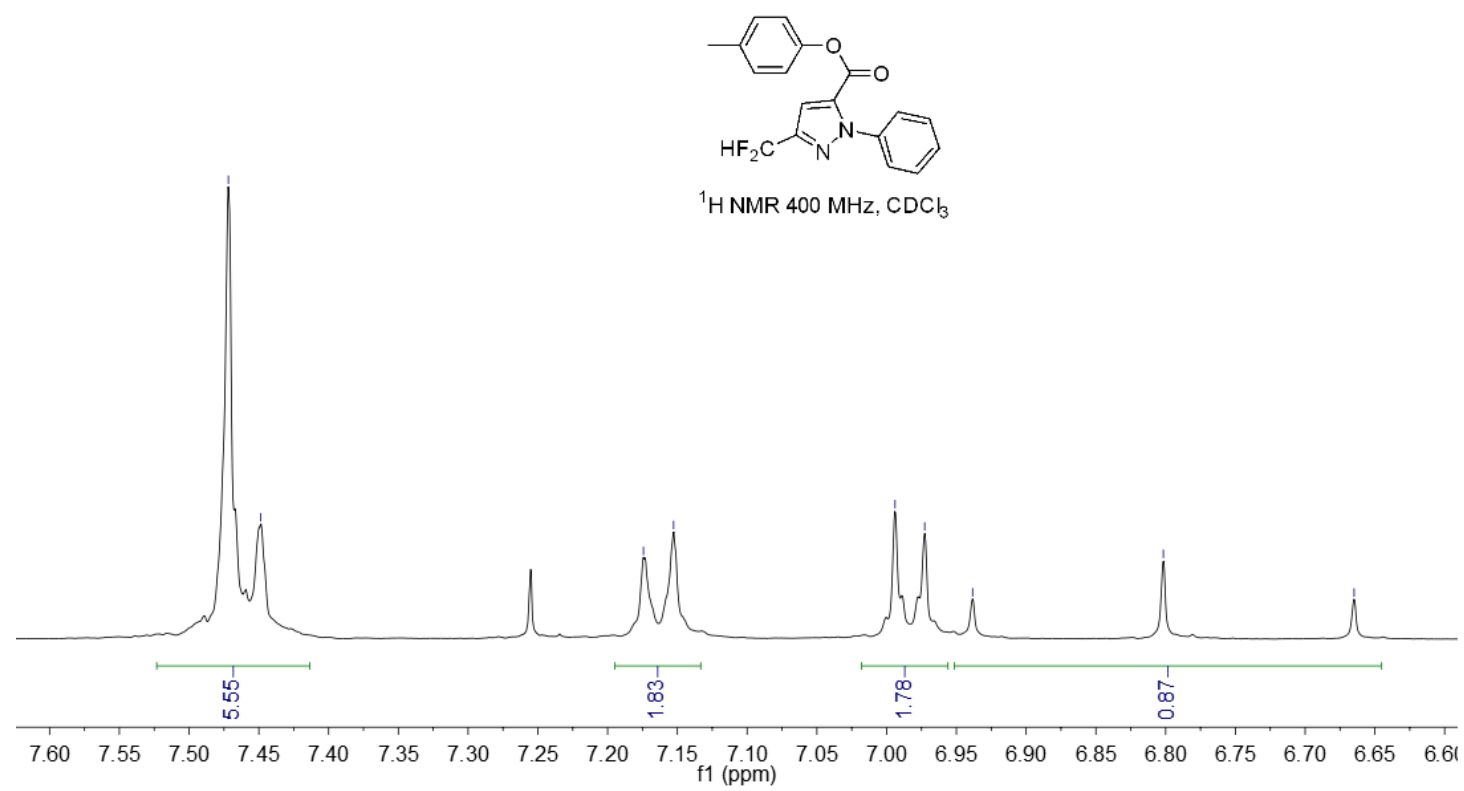




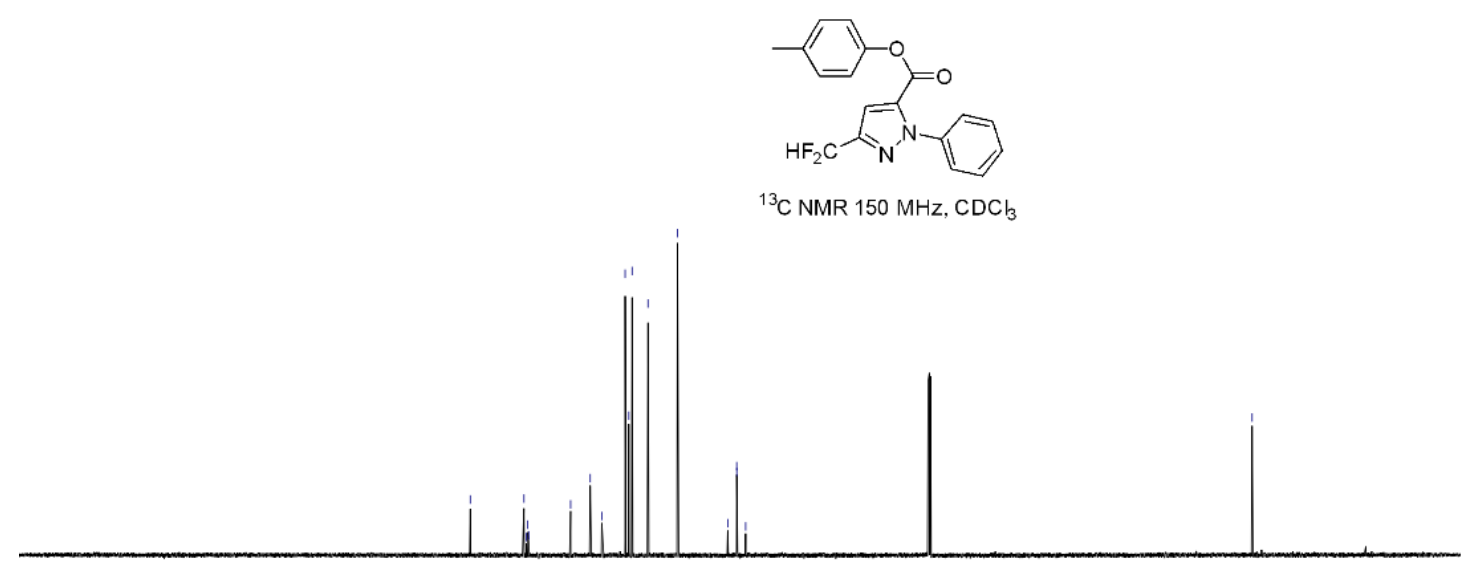

\begin{tabular}{|c|c|c|c|c|c|c|c|c|c|c|c|c|c|c|c|}
\hline 230 & 210 & 190 & 170 & 150 & 130 & $\begin{array}{c}110 \\
\mathrm{f} 1(\mathrm{ppm})\end{array}$ & 90 & 80 & 70 & 60 & 50 & 40 & 30 & 20 & 10 \\
\hline 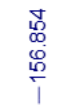 & & & & 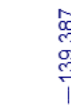 & 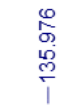 & 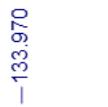 & 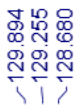 & 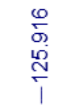 & & & 商 & & & & 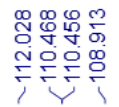 \\
\hline
\end{tabular}

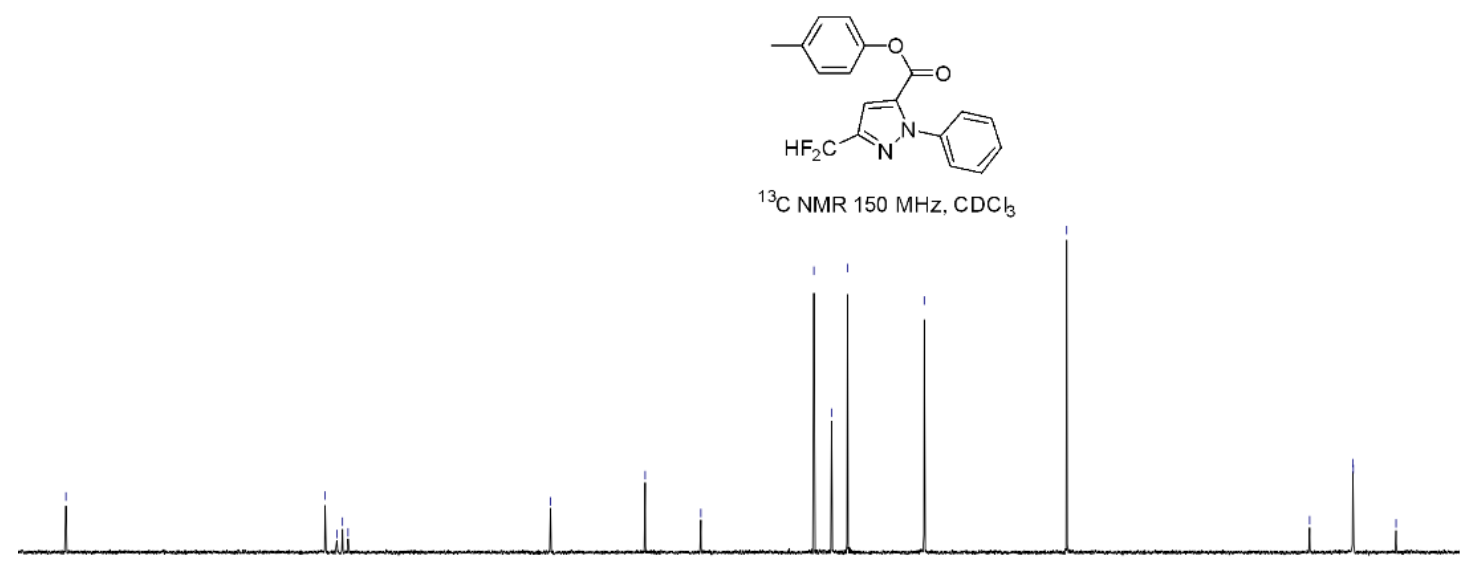

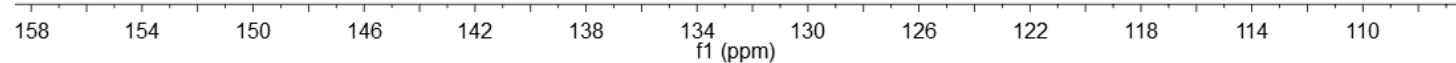




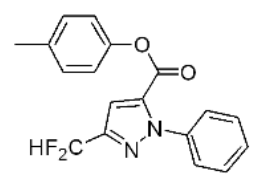

${ }^{19} \mathrm{~F}$ NMR $376 \mathrm{MHz}, \mathrm{CDCb}$
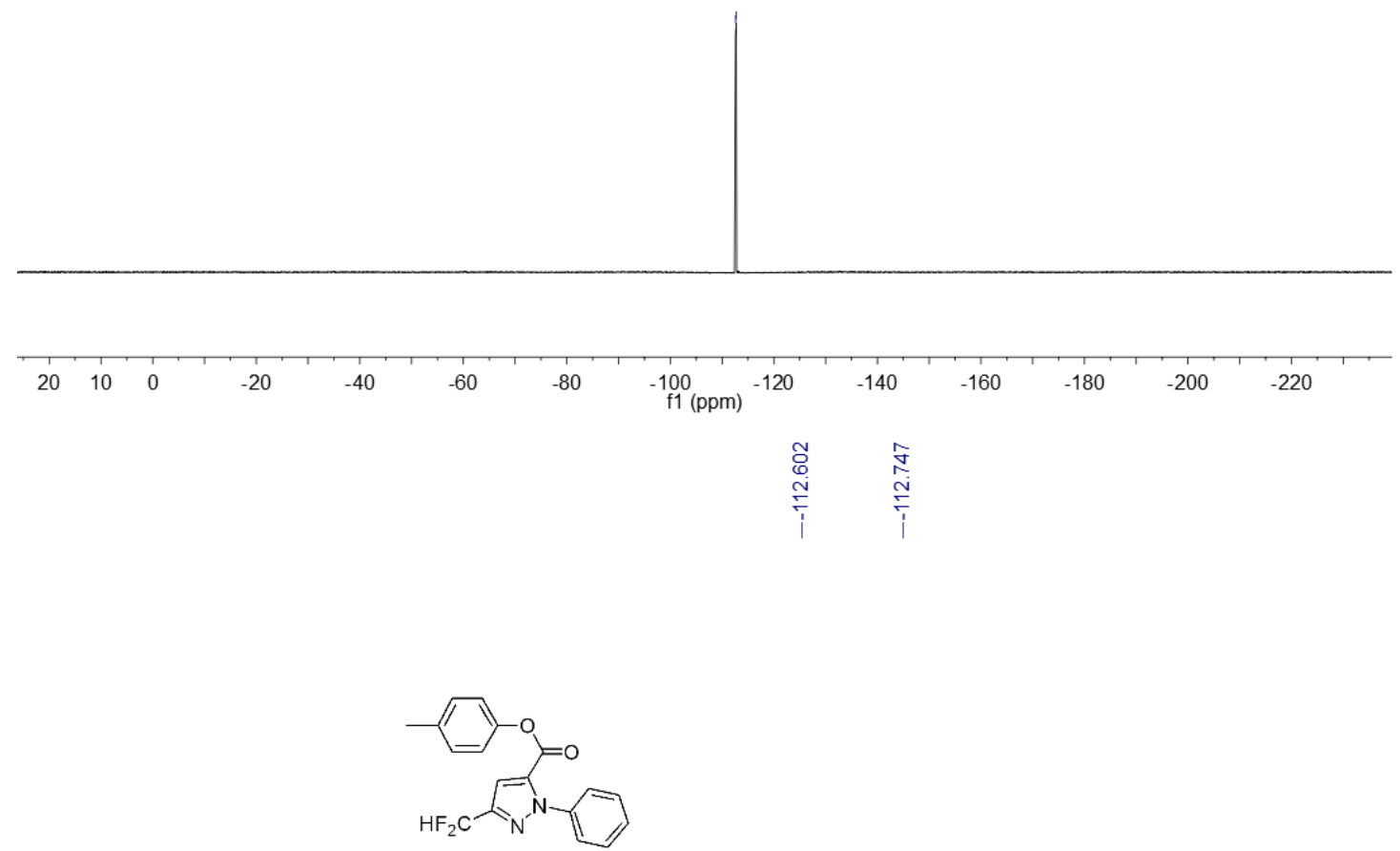

${ }^{19} \mathrm{~F}$ NMR $376 \mathrm{MHz}, \mathrm{CDCl}$

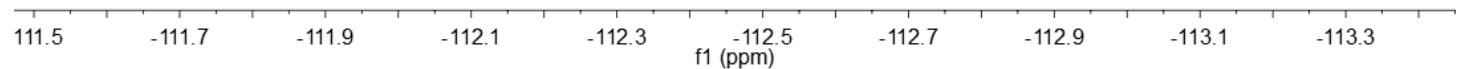


HRMS (ESI) copy of compound 5d:

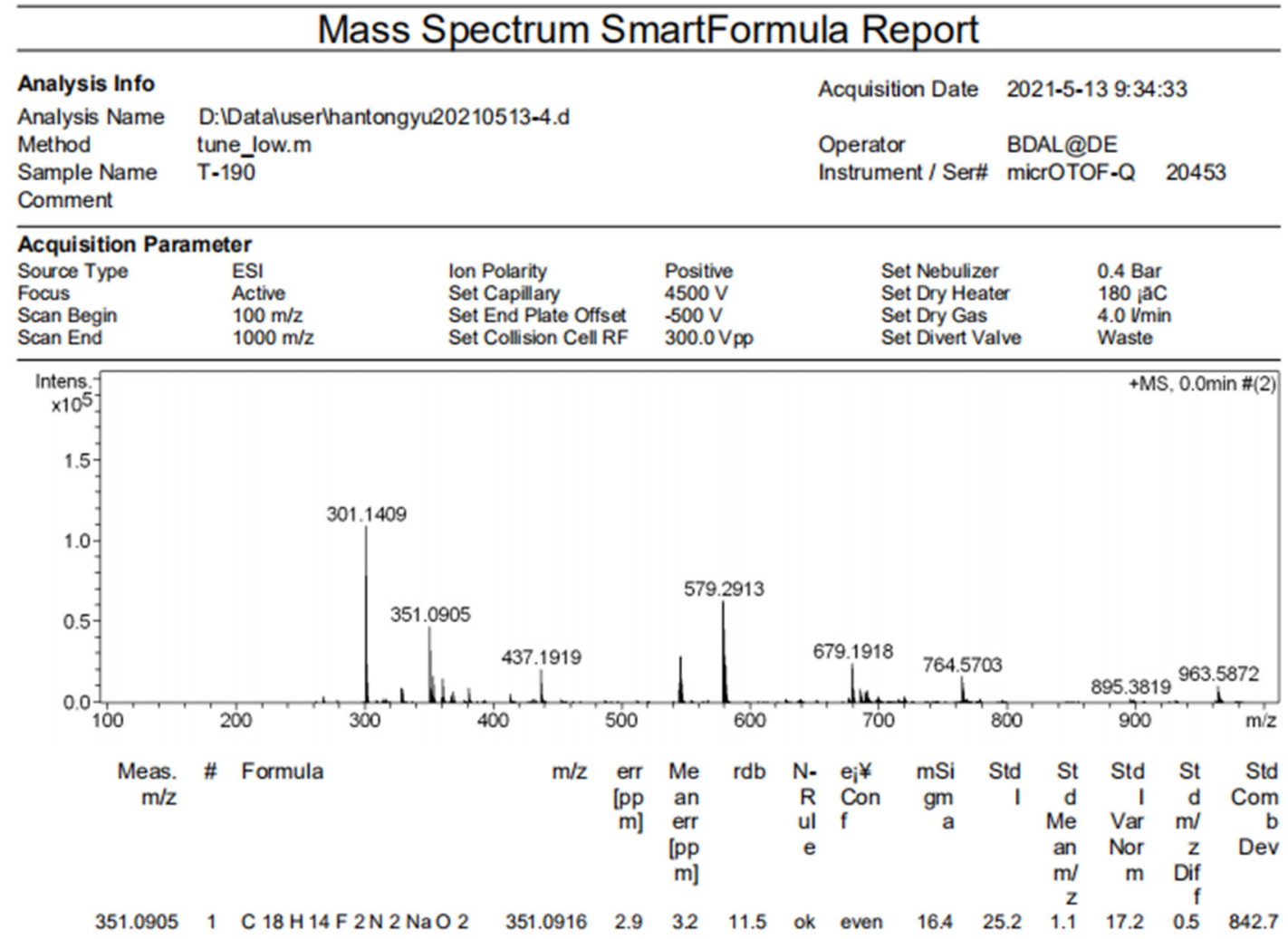

NMR copies of compound $\mathbf{5 e}$ :

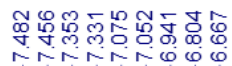

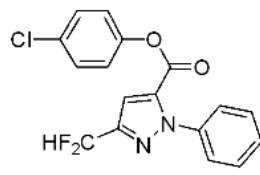

${ }^{1} \mathrm{H} \mathrm{NMR} 400 \mathrm{MHz}, \mathrm{CDCl}_{3}$

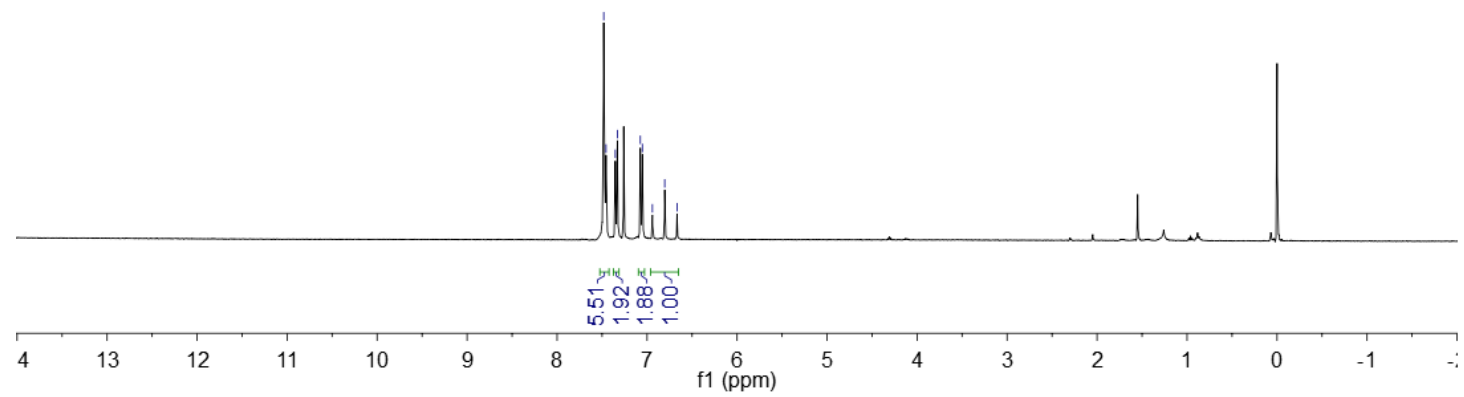




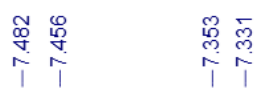

贻苋

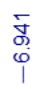

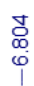

$\stackrel{0}{\emptyset}$
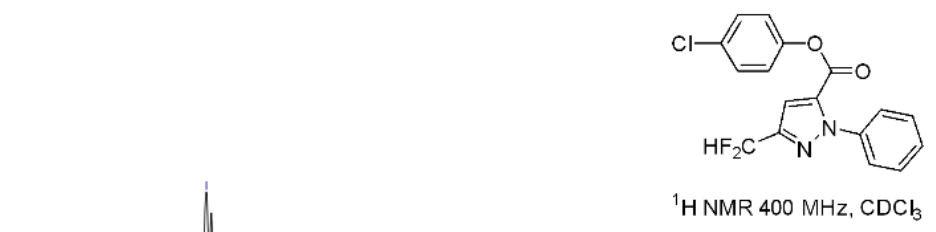

${ }^{1} \mathrm{H}$ NMR $400 \mathrm{MHz}, \mathrm{CDCl}_{3}$

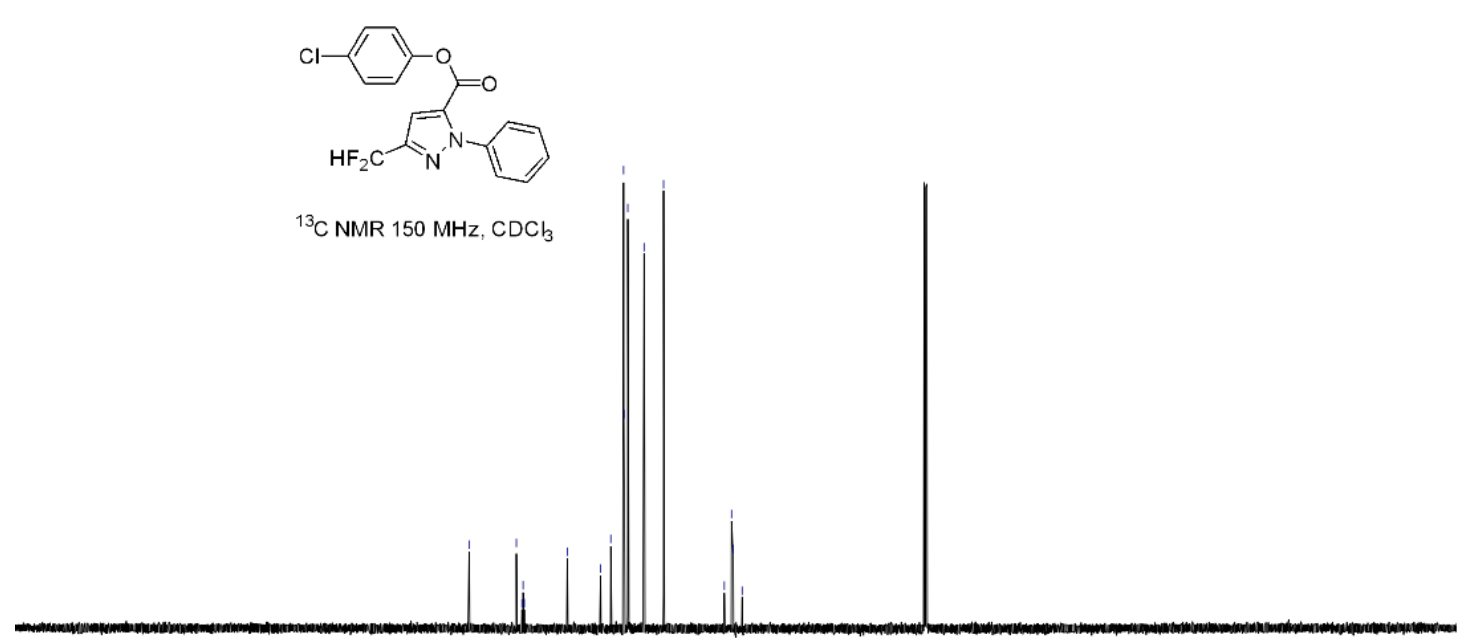

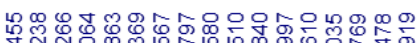

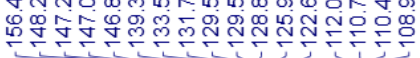
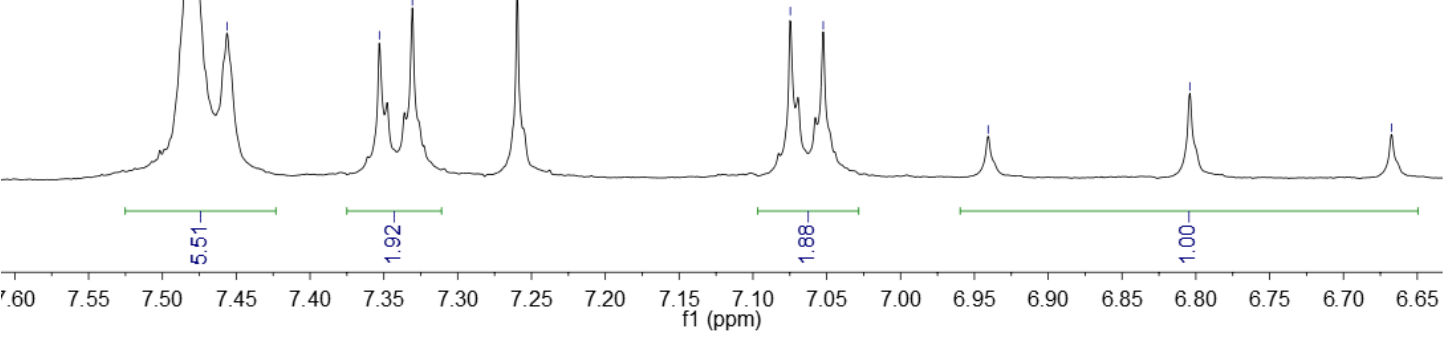

\section{型,}

${ }^{13} \mathrm{C}$ NMR $150 \mathrm{MHz}, \mathrm{CDCl}_{3}$

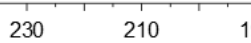

$190 \quad 17$

150

130

$\stackrel{110}{\mathrm{f} 1}(\mathrm{ppm})$

$\begin{array}{lllllllllll}90 & 80 & 70 & 60 & 50 & 40 & 30 & 20 & 10 & 0 & -10\end{array}$ 


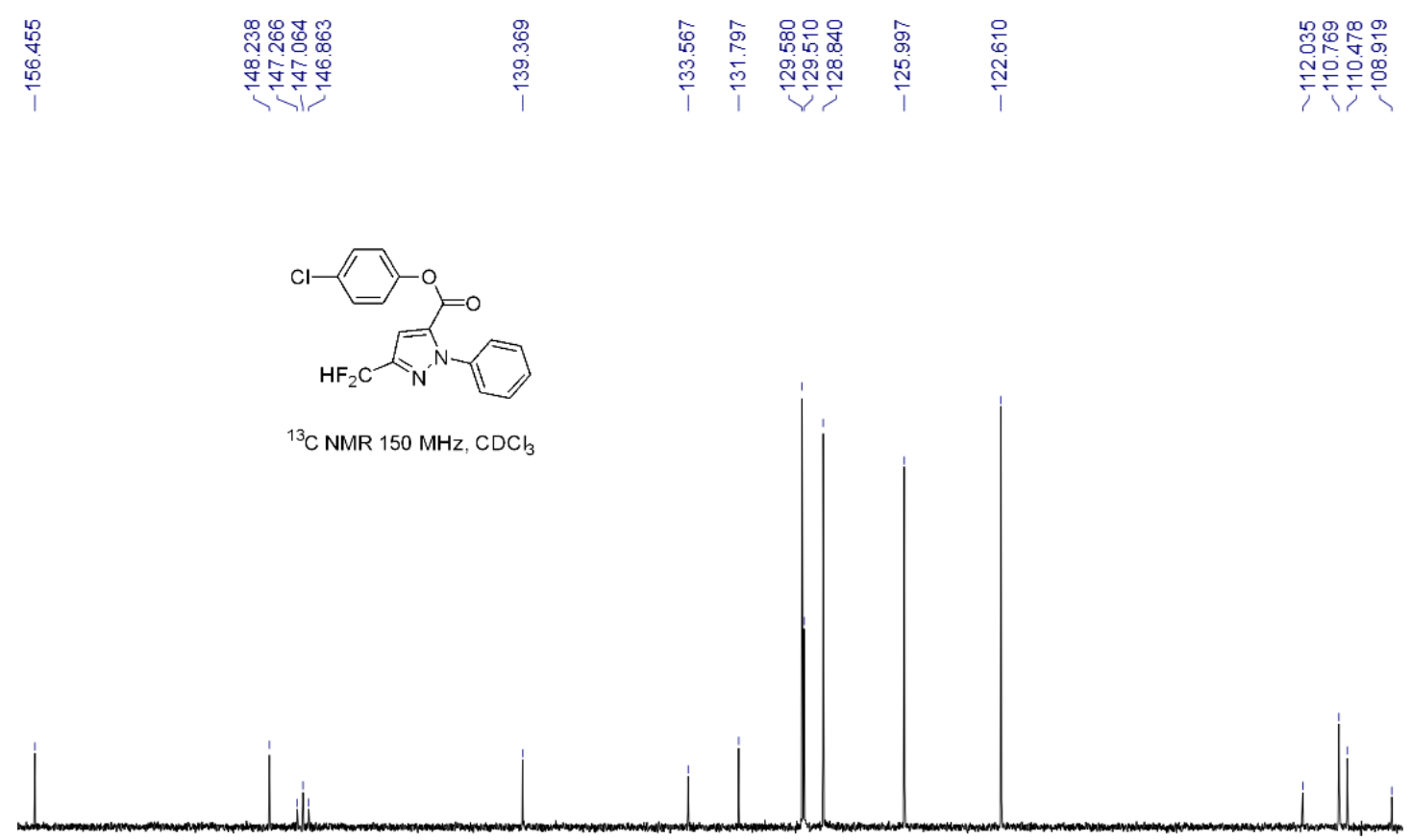

$\begin{array}{lllllllllllllllllllllll}156 & 154 & 152 & 150 & 148 & 146 & 144 & 142 & 140 & 138 & 136 & \begin{array}{r}134 \\ \mathrm{f} 1(\mathrm{ppm})\end{array} & 130 & 128 & 126 & 124 & 122 & 120 & 118 & 116 & 114 & 112 & 110\end{array}$

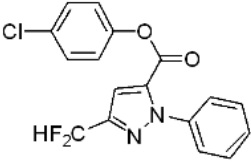

${ }^{19} \mathrm{~F} \mathrm{NMR} 376 \mathrm{MHz}, \mathrm{CDCl}_{3}$

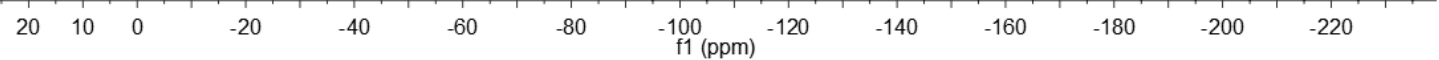




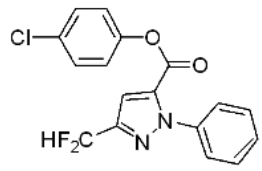

${ }^{19} \mathrm{~F} \mathrm{NMR} 376 \mathrm{MHz}, \mathrm{CDCl}$

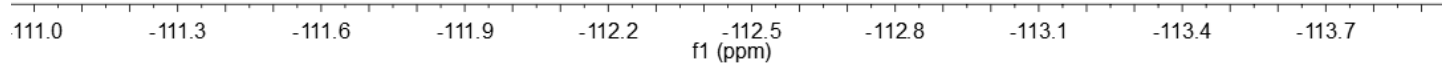

HRMS (ESI) copy of compound 5e:

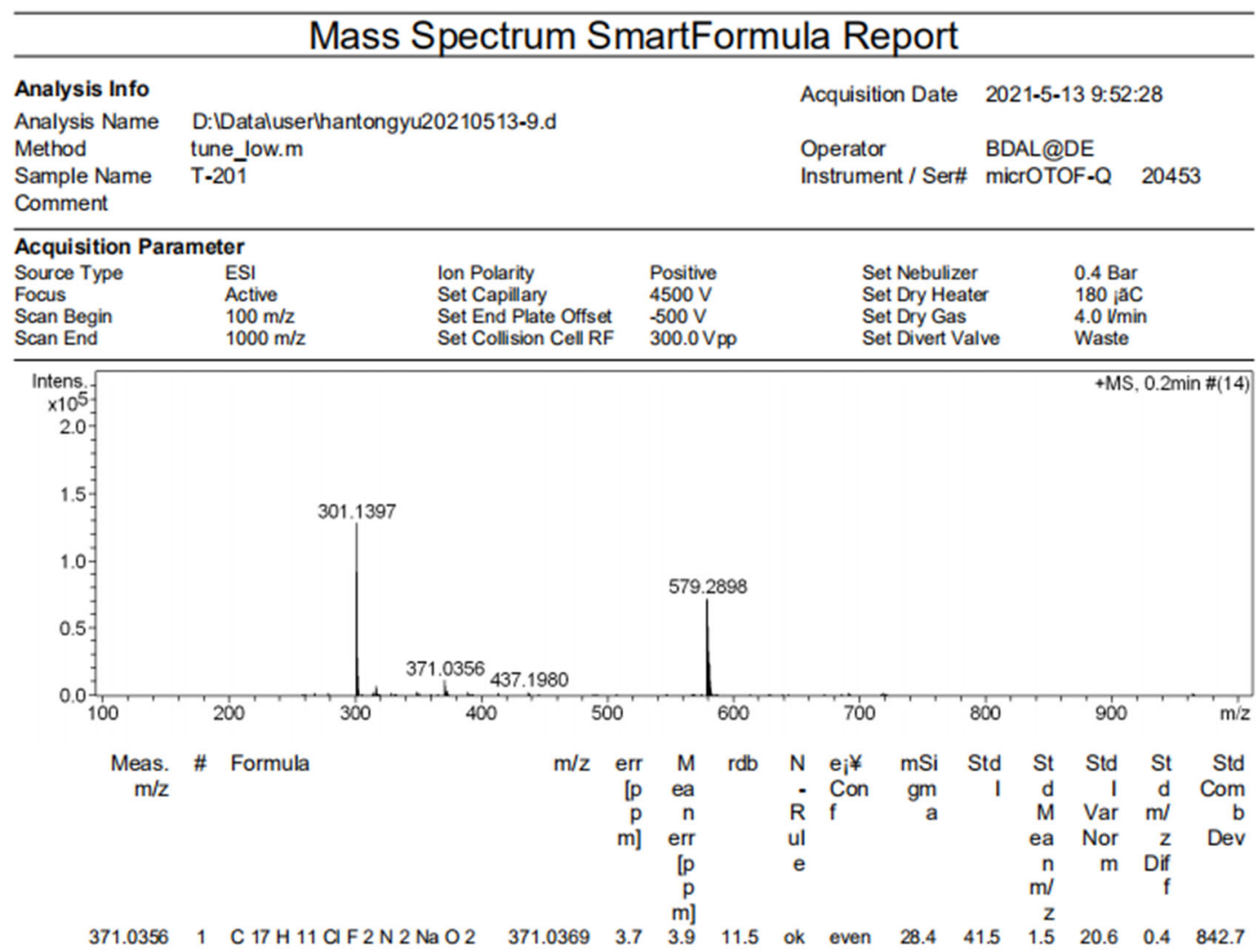


NMR copies of compound $\mathbf{5 f}$ :

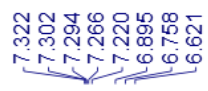

$\underset{\substack{\infty \\ i}}{i}$

$\underset{\substack{\mathscr{g} \\ \text { ָे }}}{ }$
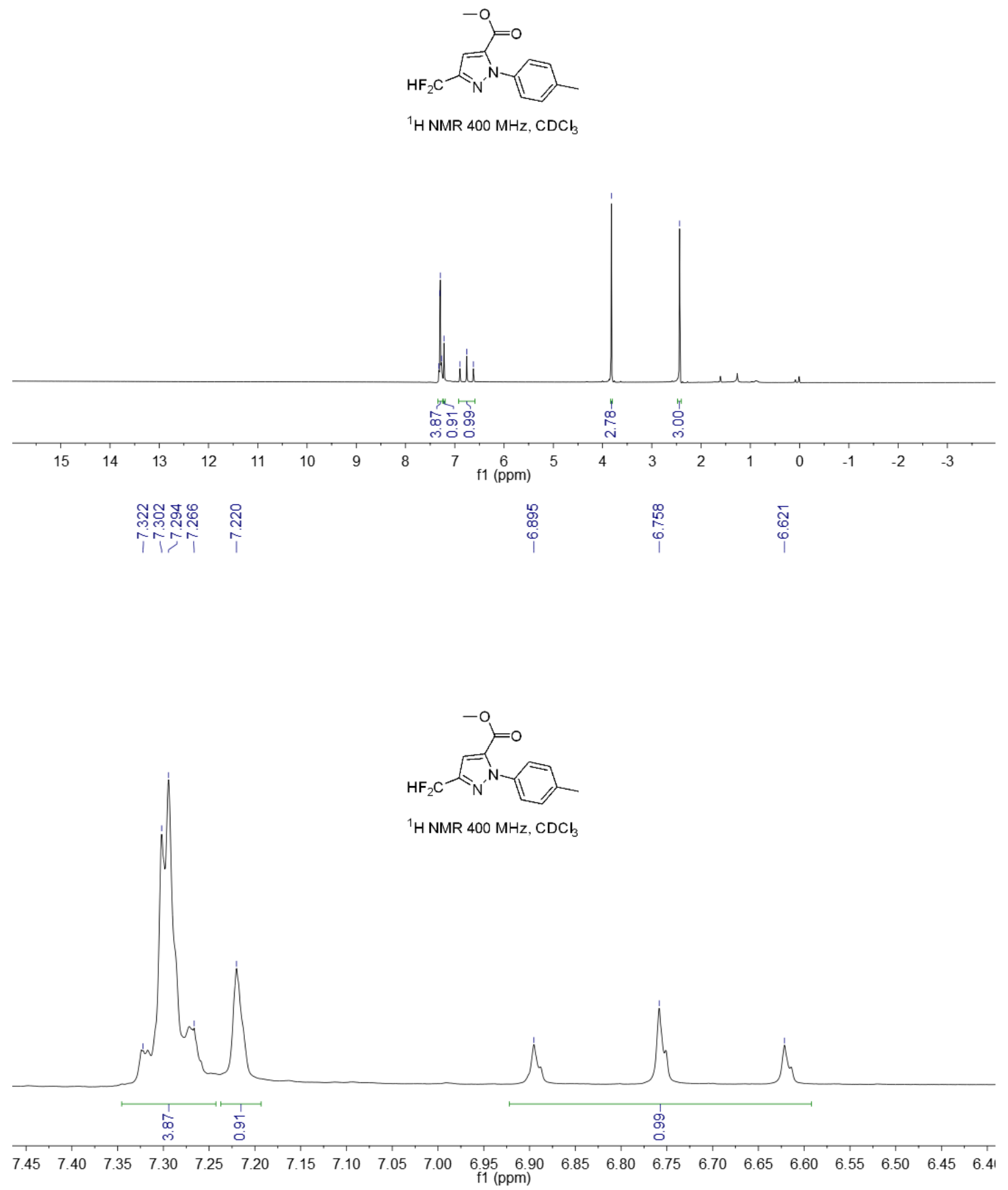

S119 


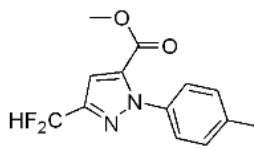

${ }^{13} \mathrm{C}$ NMR $150 \mathrm{MHz}, \mathrm{CDCl}$

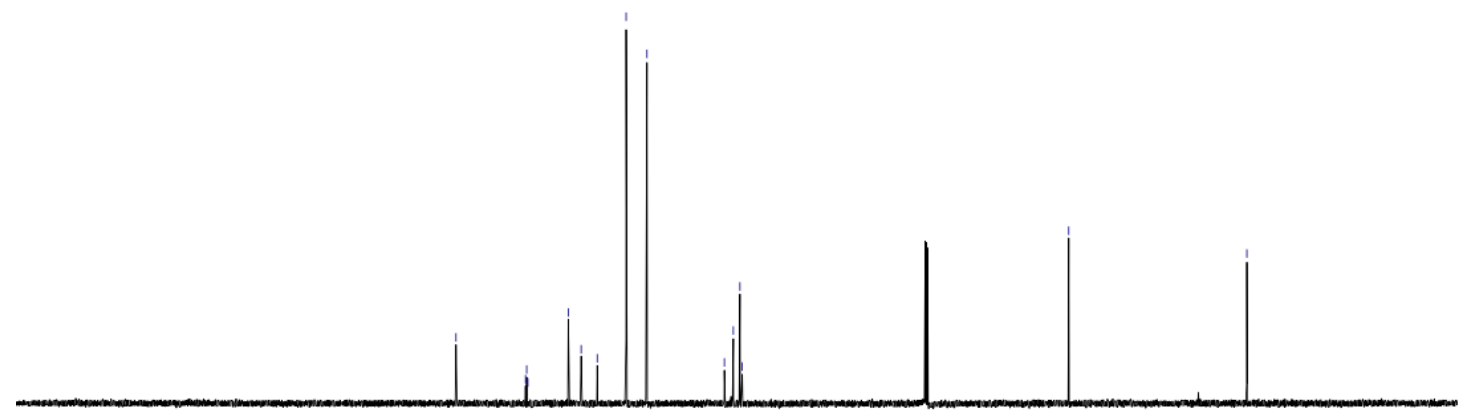

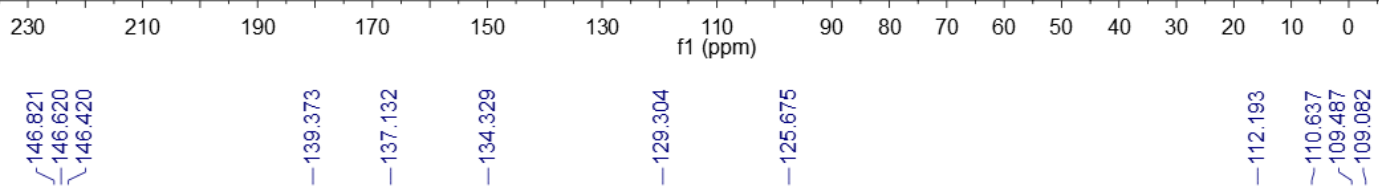

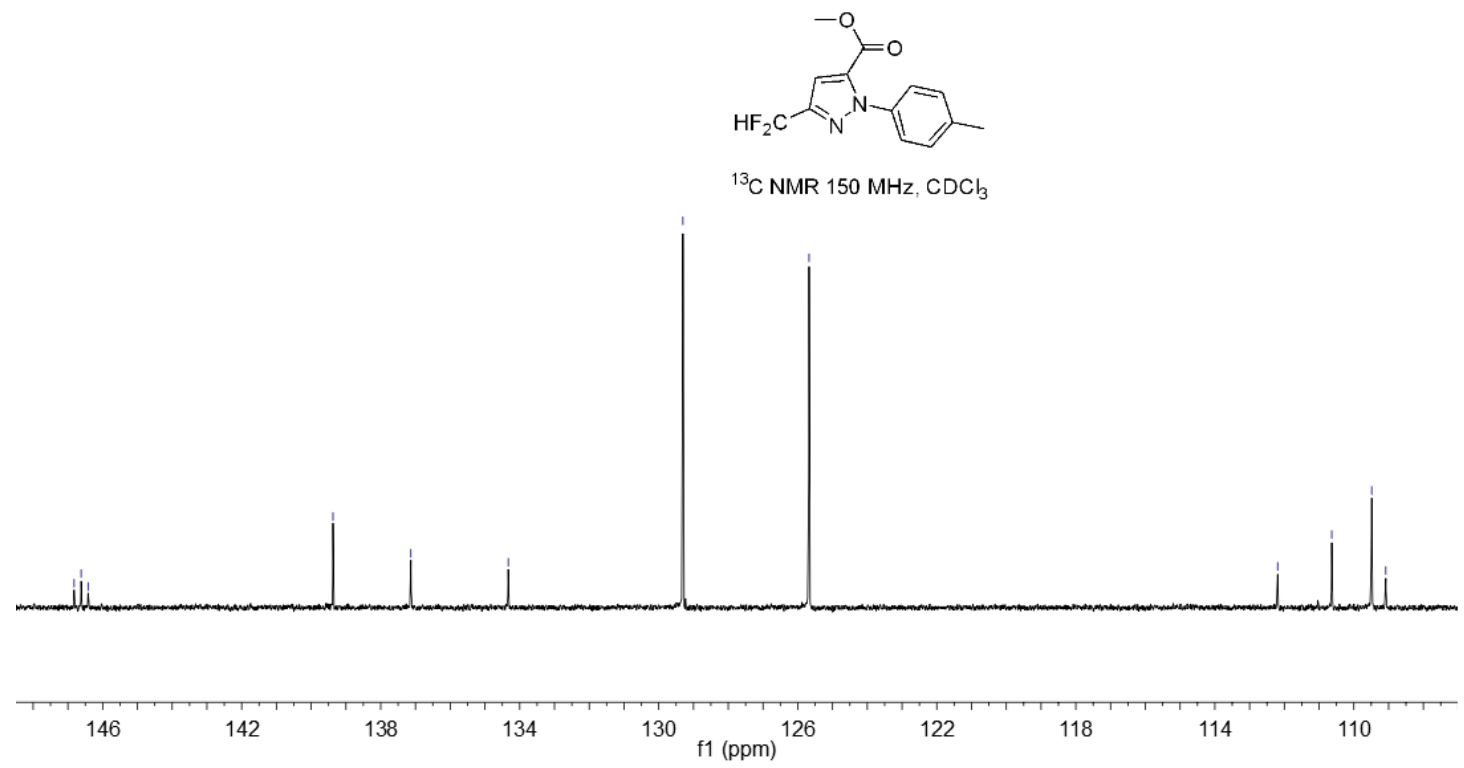




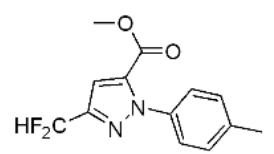

${ }^{19} \mathrm{~F}$ NMR $376 \mathrm{MHz}, \mathrm{CDCl}_{3}$

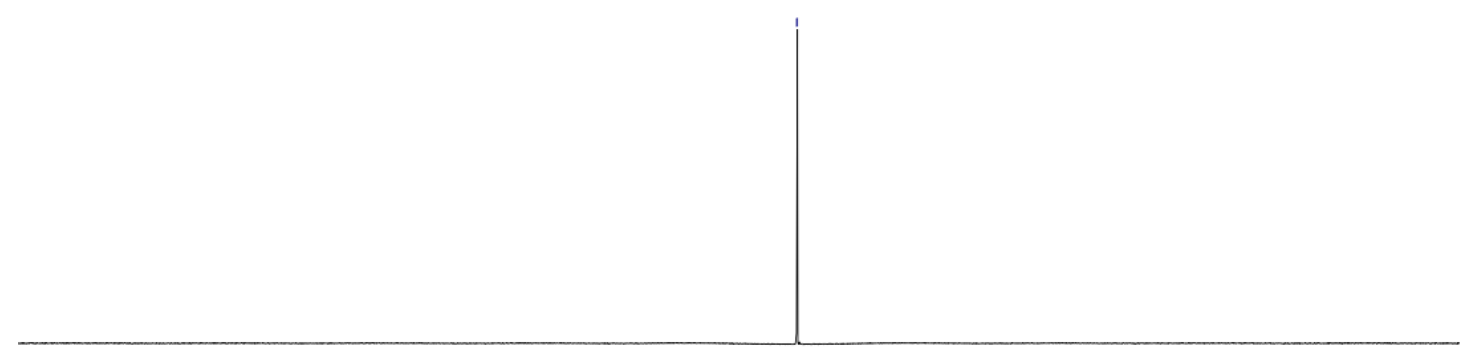

\begin{tabular}{|c|c|c|c|c|c|c|c|c|c|c|c|c|c|}
\hline 20 & 10 & 0 & -20 & -40 & -60 & -80 & $\begin{array}{l}-100 \\
\quad \mathrm{f} 1(\mathrm{ppm})\end{array}$ & -120 & -140 & -160 & -180 & -200 & -220 \\
\hline & & & & & & & & & $\begin{array}{l}\stackrel{\mathbb{N}}{N} \\
\stackrel{i}{F}\end{array}$ & $\frac{\stackrel{0}{v}}{\frac{j}{i}}$ & & & \\
\hline
\end{tabular}

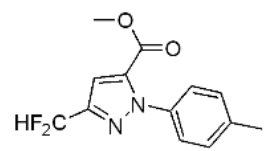

${ }^{19} \mathrm{~F}$ NMR $376 \mathrm{MHz}, \mathrm{CDCl}_{3}$

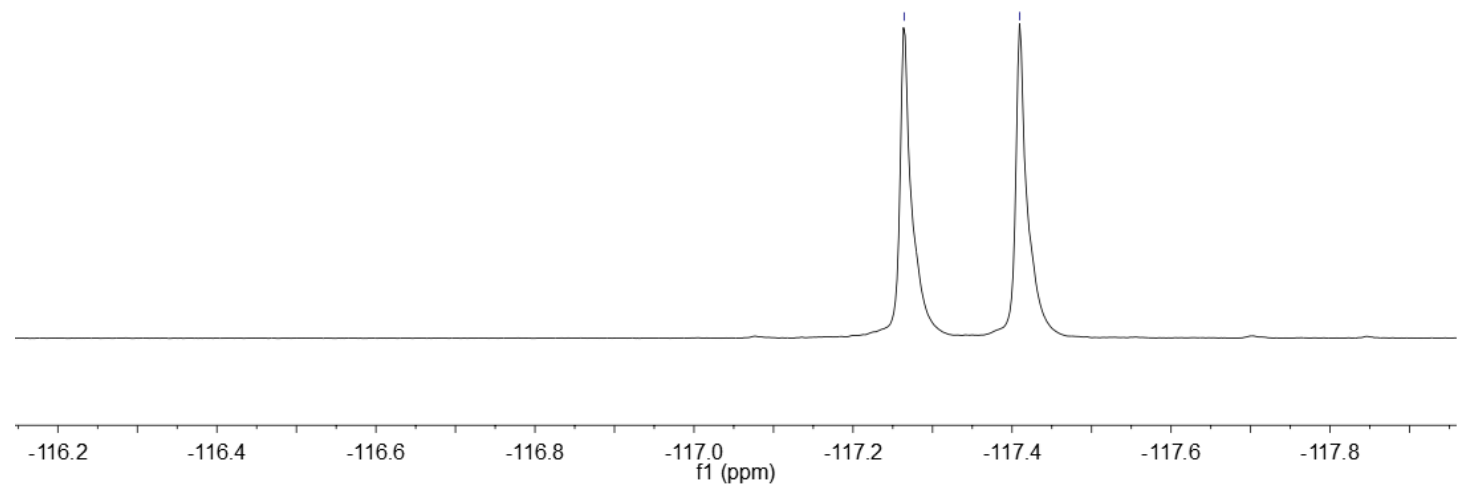


HRMS (ESI) copy of compound $\mathbf{5 f}$ :

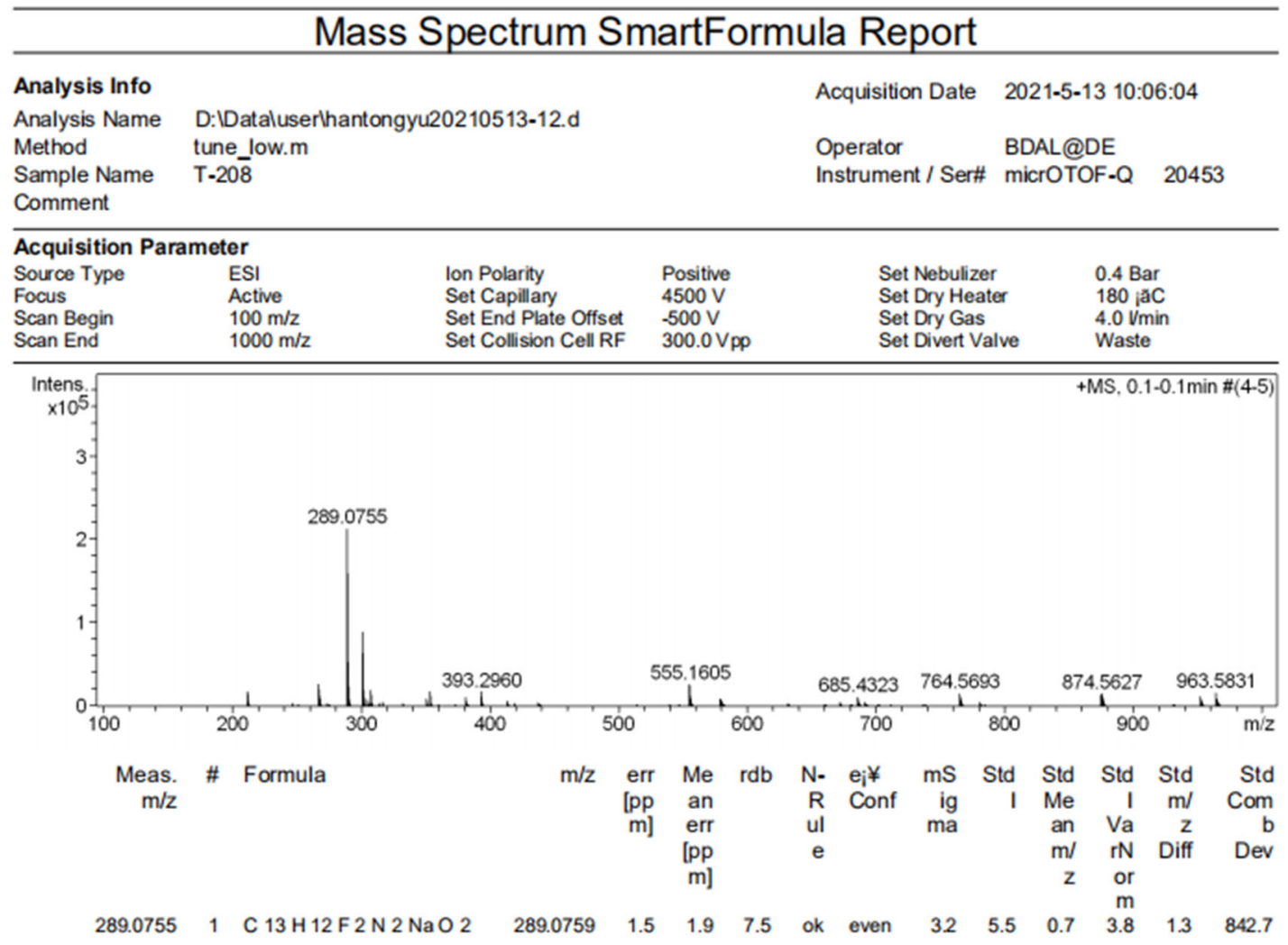

NMR copies of compound 5g:

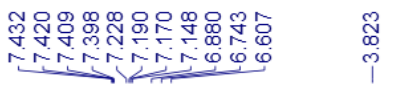

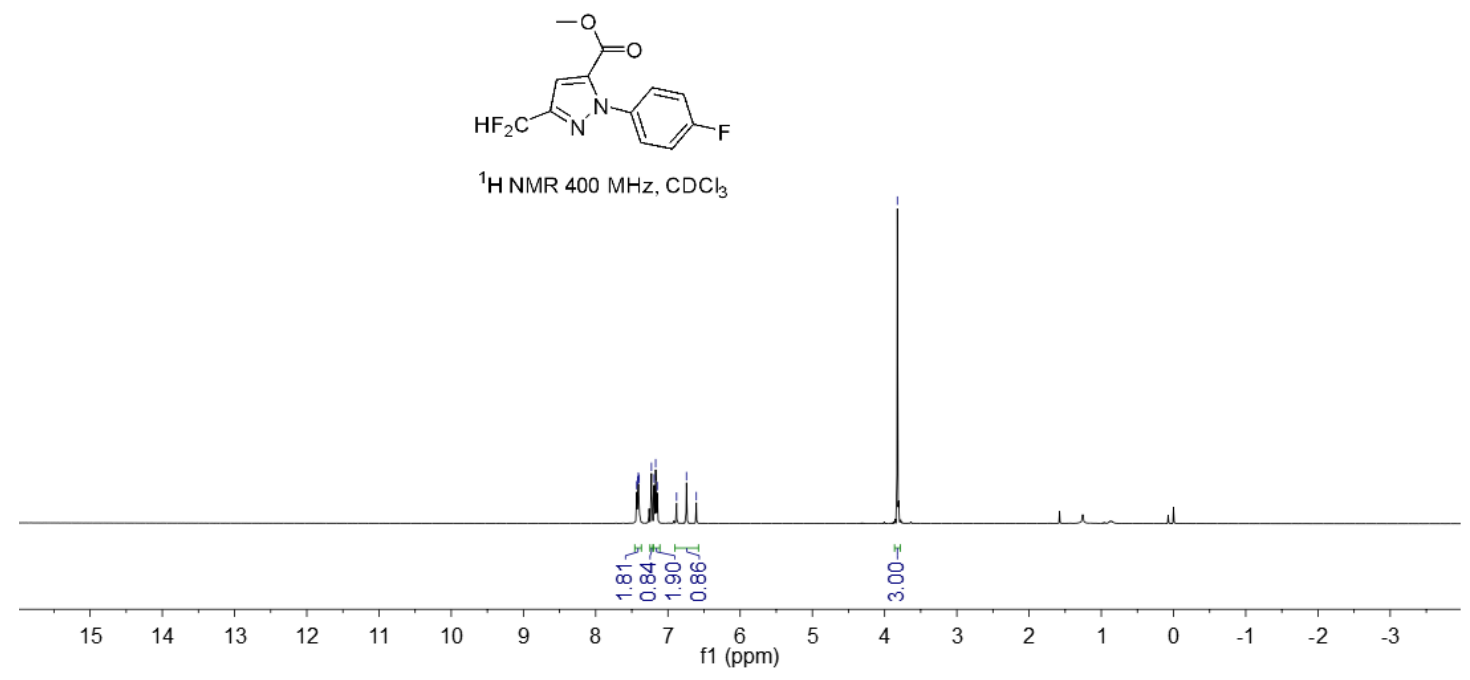




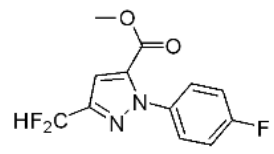

${ }^{1} \mathrm{H} \mathrm{NMR} 400 \mathrm{MHz}, \mathrm{CDCl}_{3}$

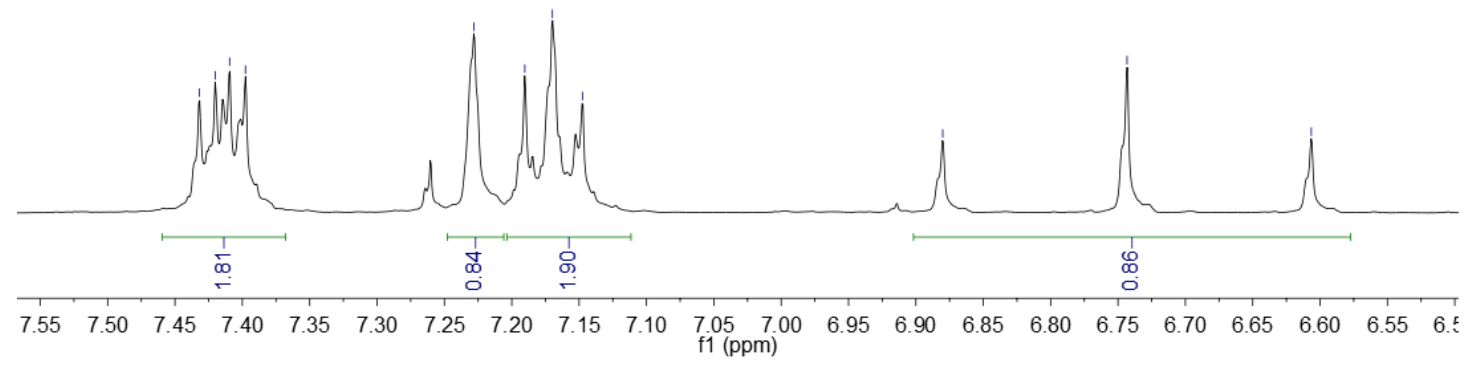

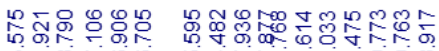

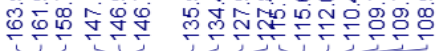

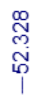

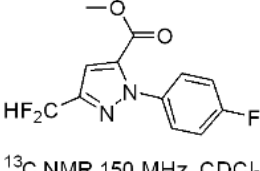

${ }^{13} \mathrm{C} \mathrm{NMR} 150 \mathrm{MHz}, \mathrm{CDCl}_{3}$

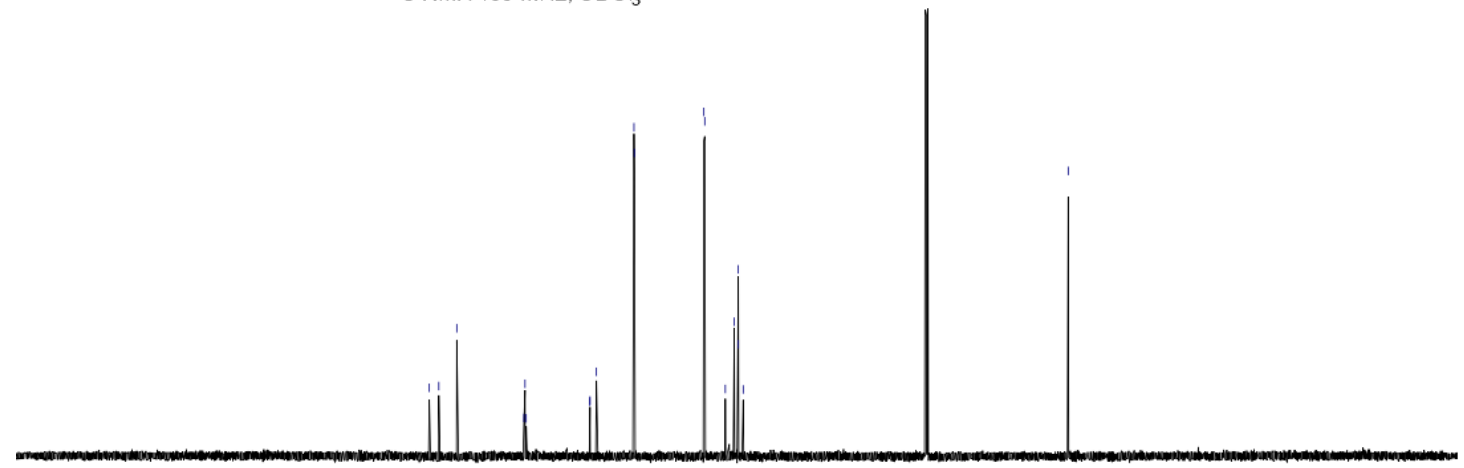

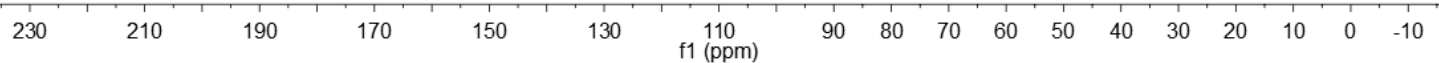




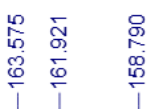

웅요

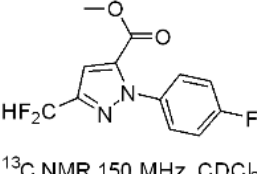

${ }^{13} \mathrm{C} \mathrm{NMR} 150 \mathrm{MHz}, \mathrm{CDCl}_{3}$
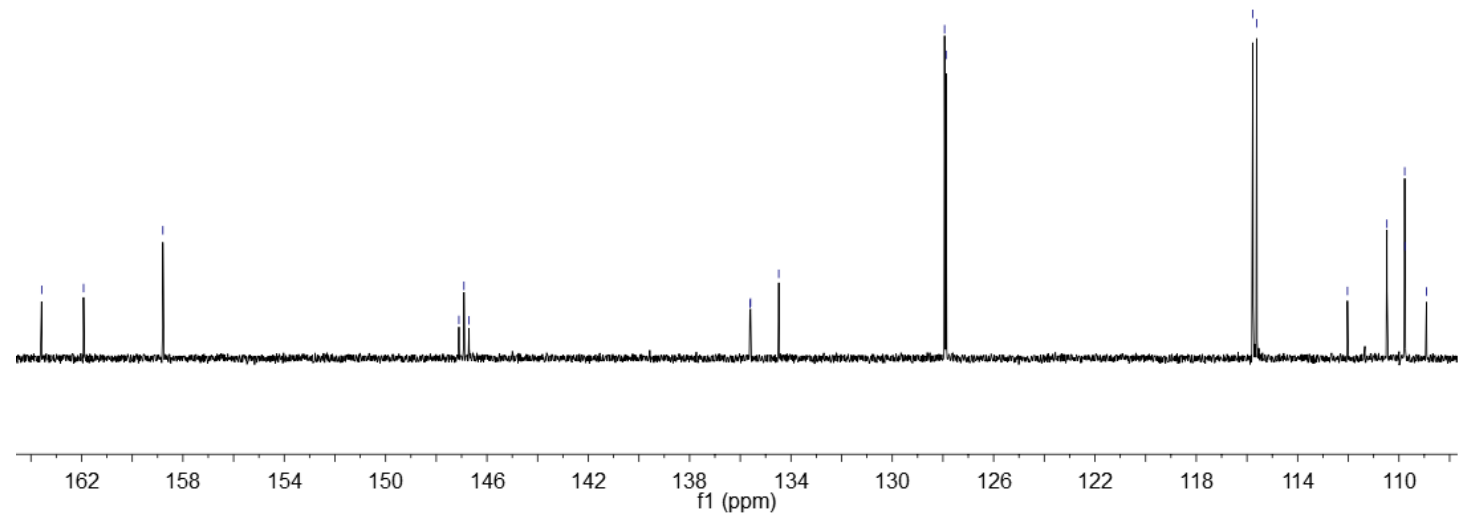

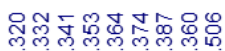

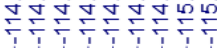

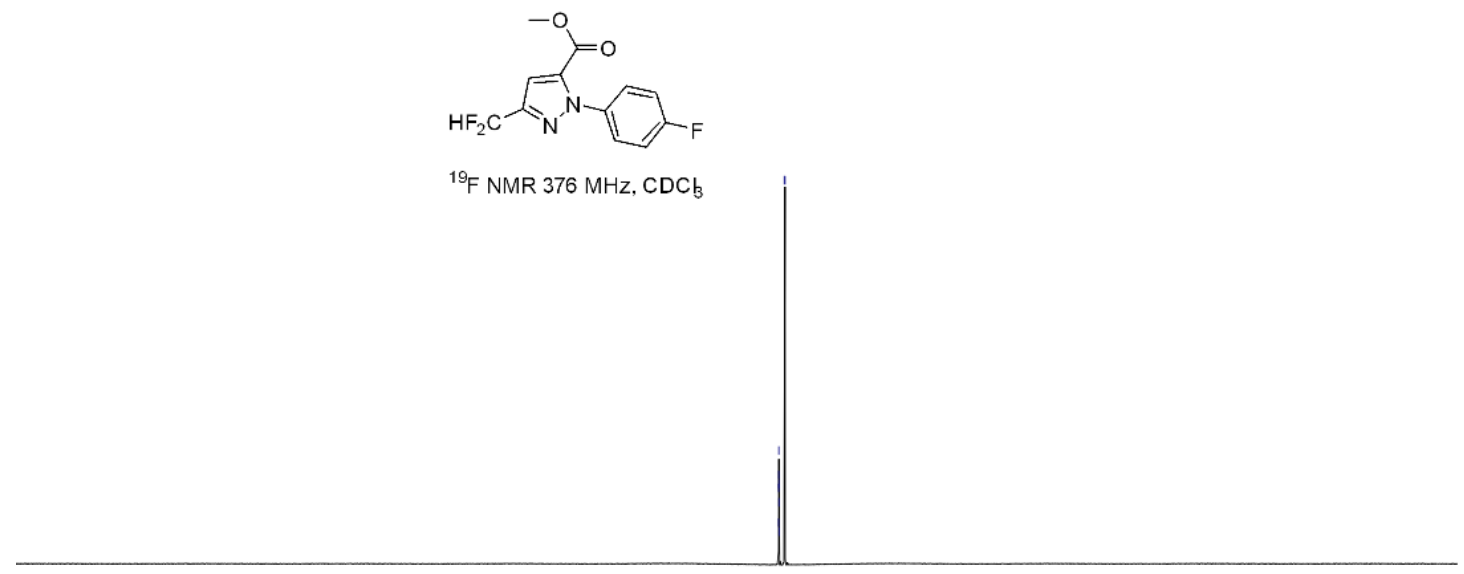

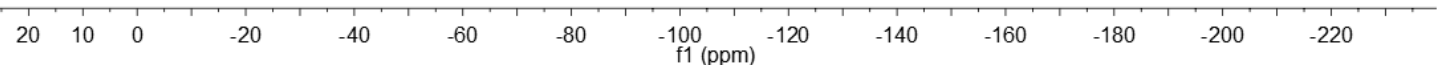




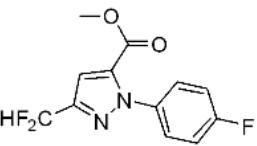

${ }^{19} \mathrm{~F}$ NMR $376 \mathrm{MHz}, \mathrm{CDCl}$

\begin{tabular}{lllllllllllll}
\hline & -113.5 & -113.9 & -114.1 & -114.3 & -114.5 & $\begin{array}{c}-114.7 \\
\mathrm{fpm}\end{array}$ & -114.9 & -115.1 & -115.3 & -115.5 & -115.7
\end{tabular}

HRMS (ESI) copy of compound $\mathbf{5 g}$ :

\section{Mass Spectrum SmartFormula Report}

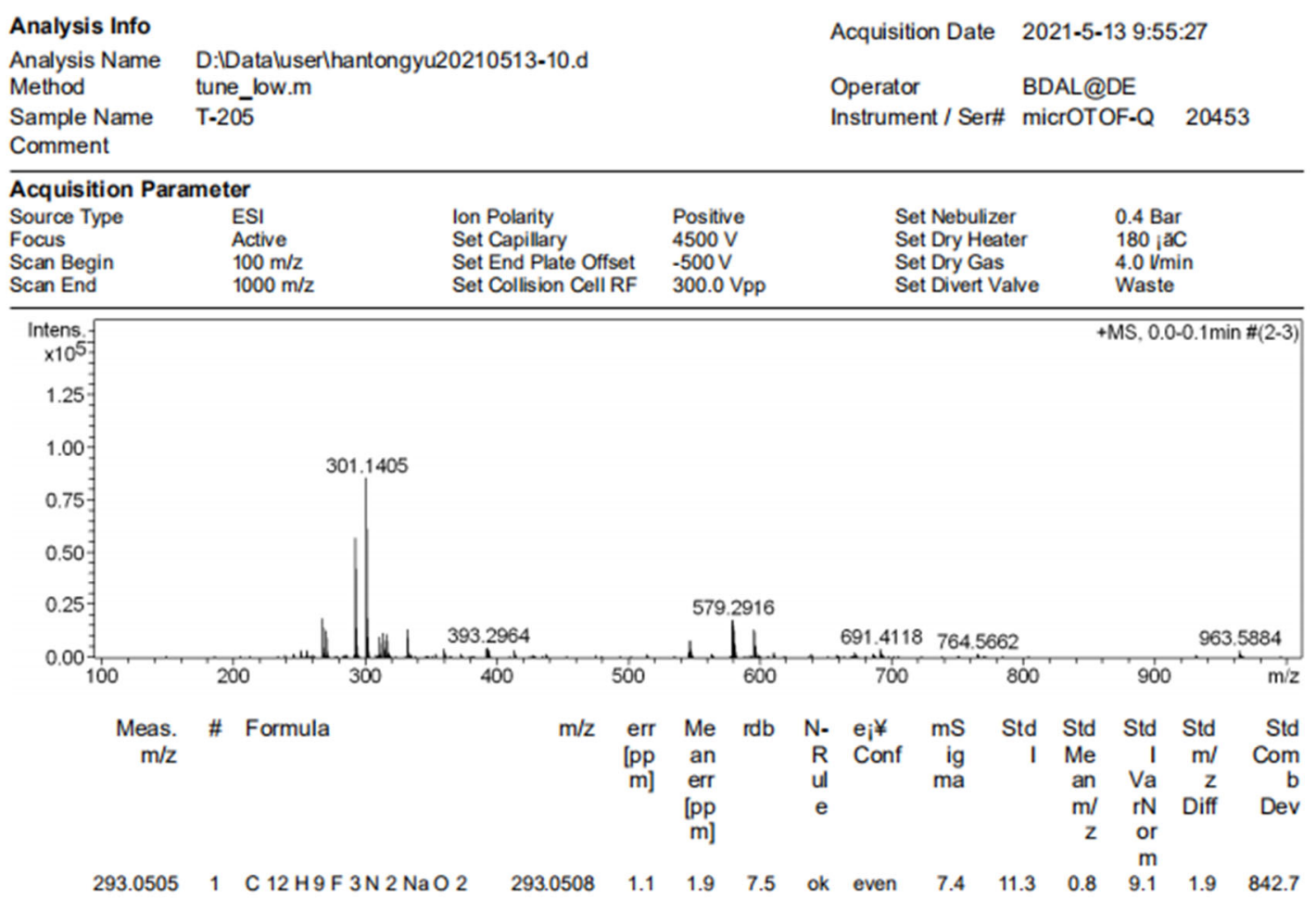


NMR copies of compound $\mathbf{5 h}$ :

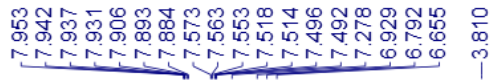
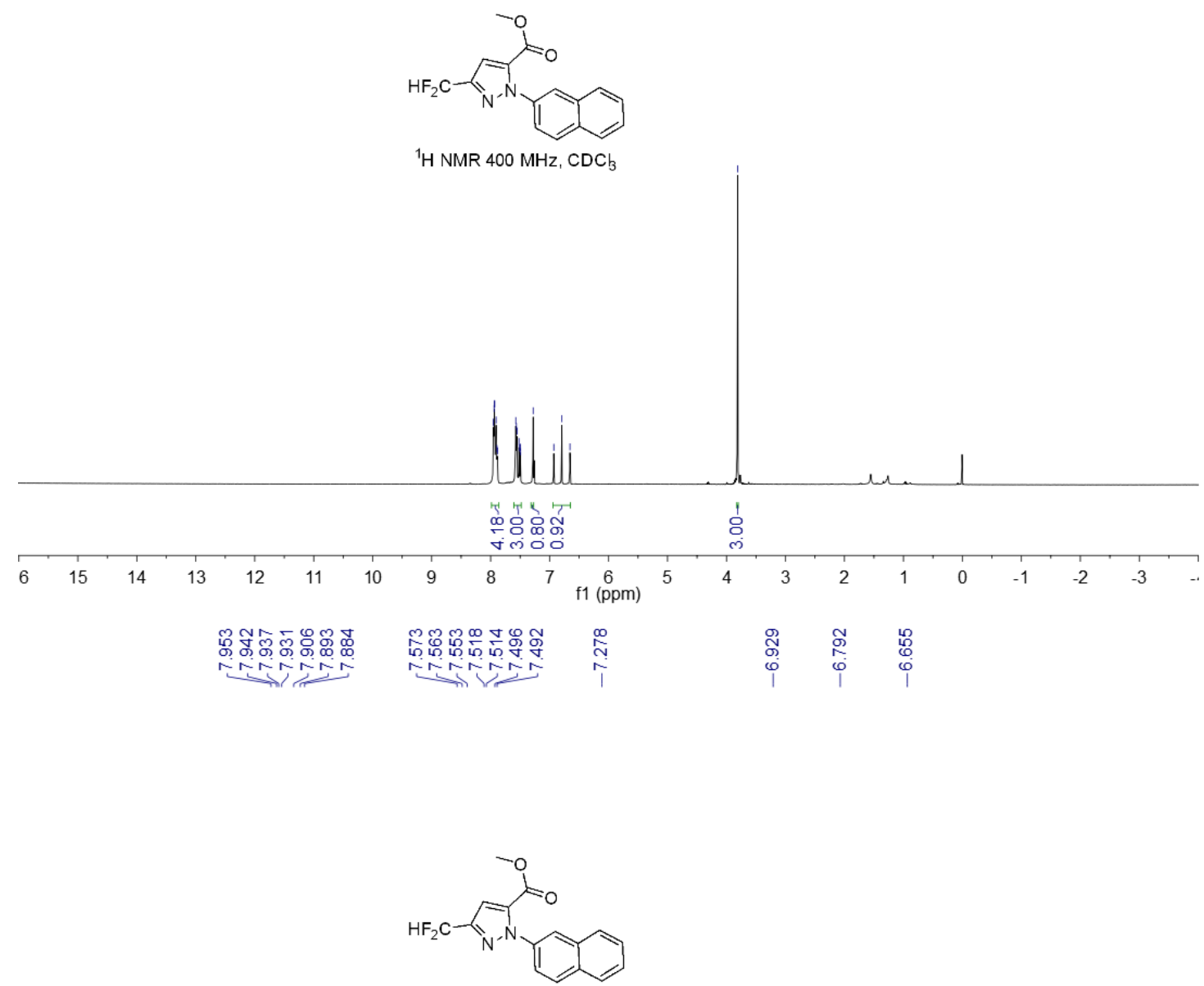

${ }^{1} \mathrm{H}$ NMR $400 \mathrm{MHz}, \mathrm{CDC}_{3}$

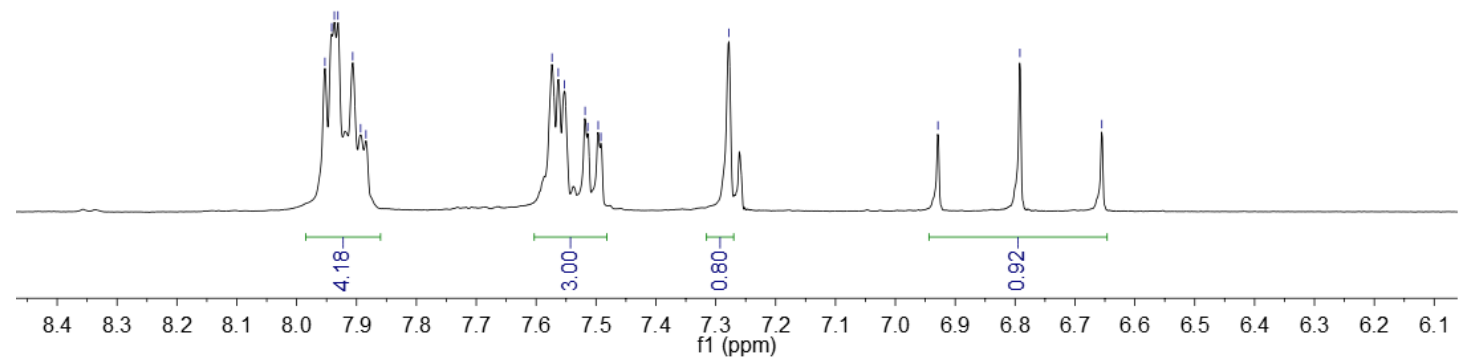




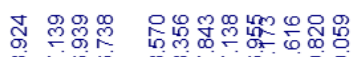

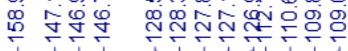

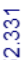

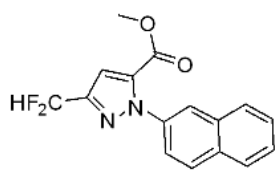

${ }^{13} \mathrm{C}$ NMR $150 \mathrm{MHz}, \mathrm{CDCl}_{3}$

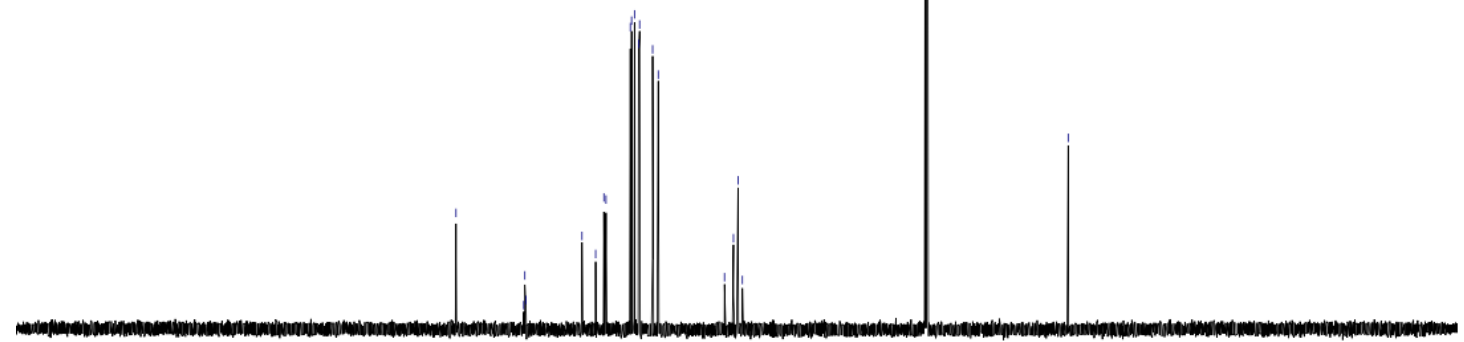

\begin{tabular}{|c|c|c|c|c|c|c|c|c|c|c|c|c|c|c|c|}
\hline 230 & 210 & 190 & 170 & 150 & 130 & $\begin{array}{c}110 \\
\mathrm{f} 1(\mathrm{ppm})\end{array}$ & $\begin{array}{ll}90 & 80\end{array}$ & 70 & 60 & 50 & 40 & 30 & 20 & 10 & $\begin{array}{ll}0 & -10\end{array}$ \\
\hline 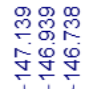 & & & $\begin{array}{l}\text { O } \\
8 \\
0 \\
0 \\
\end{array}$ & 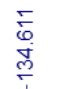 & 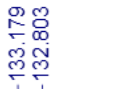 & 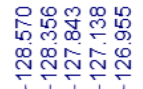 & 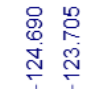 & & & & & & & $\underset{\stackrel{N}{\stackrel{N}{ }}}{\stackrel{\sim}{\leftarrow}}$ & 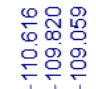 \\
\hline
\end{tabular}

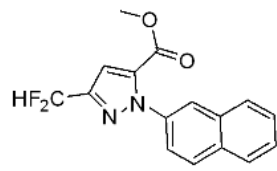

${ }^{13} \mathrm{C} \mathrm{NMR} 150 \mathrm{MHz}, \mathrm{CDCl}_{3}$

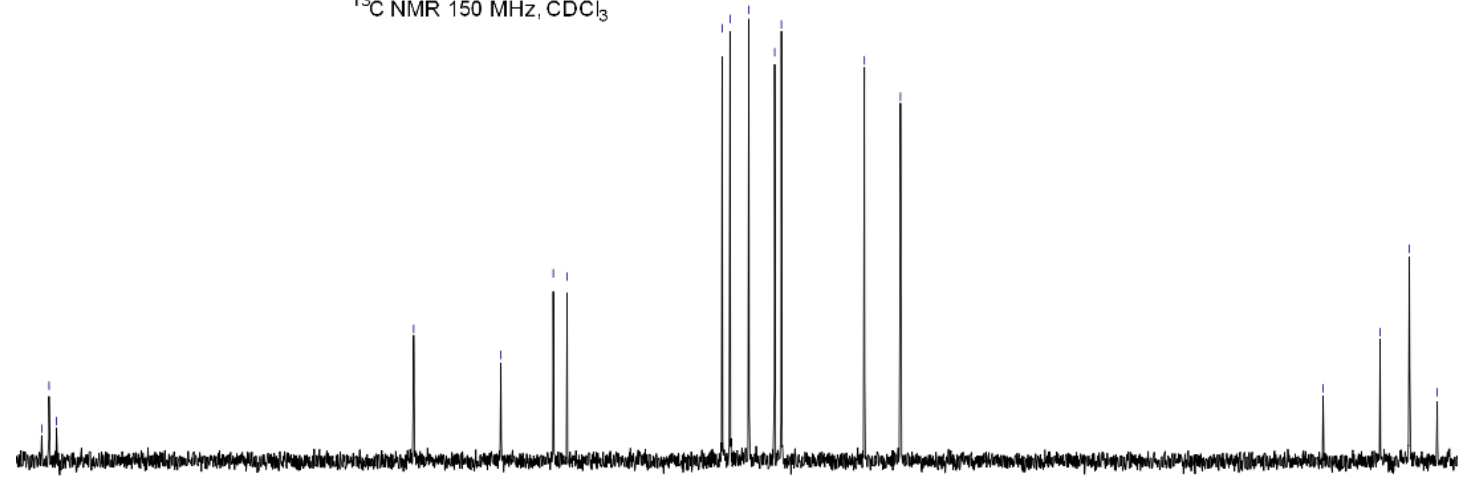

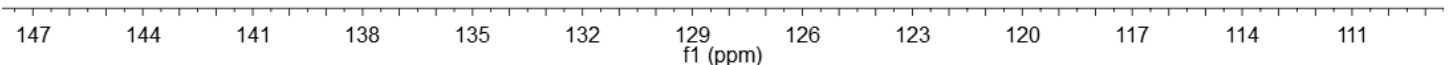



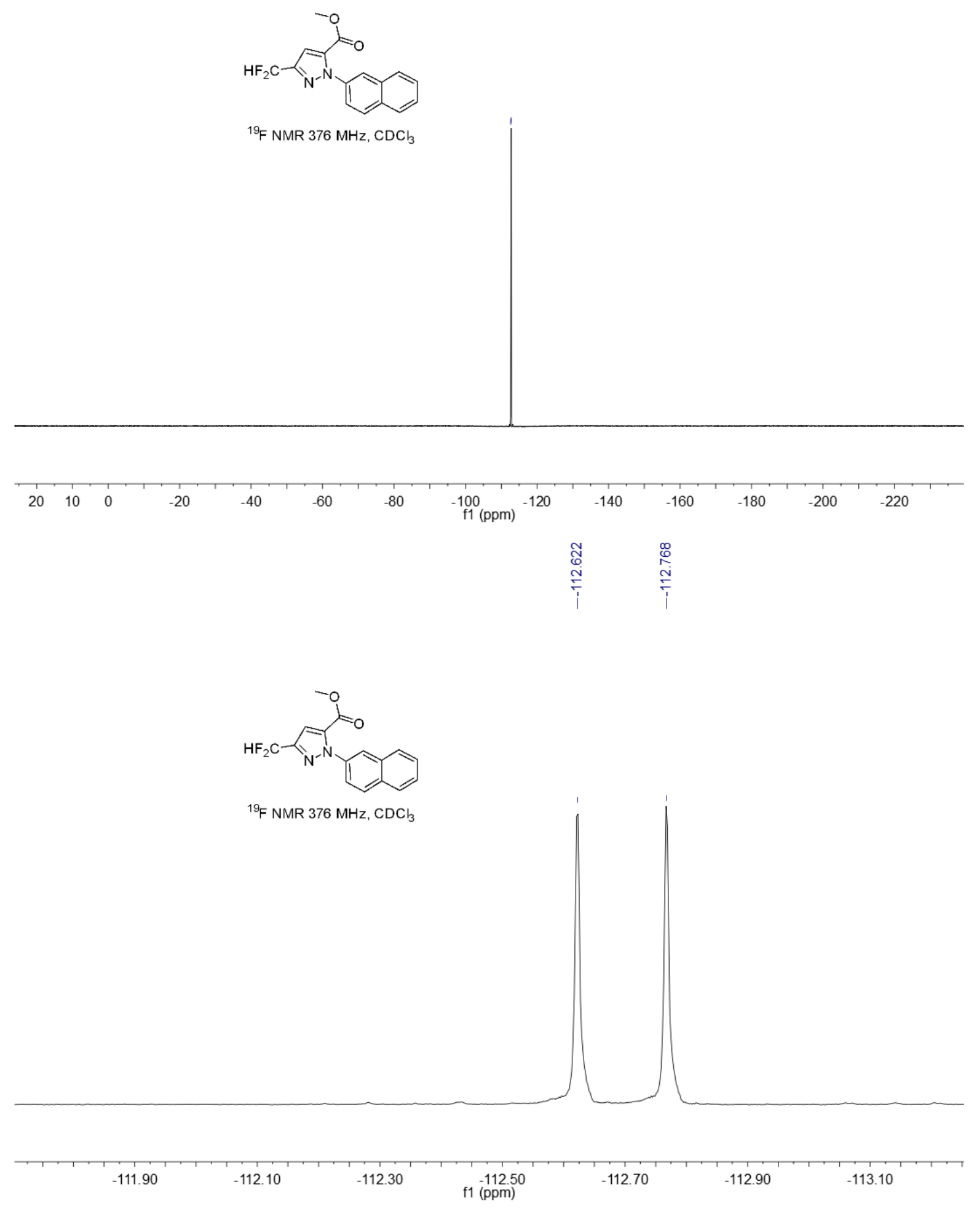
HRMS (ESI) copy of compound $\mathbf{5 h}$ :

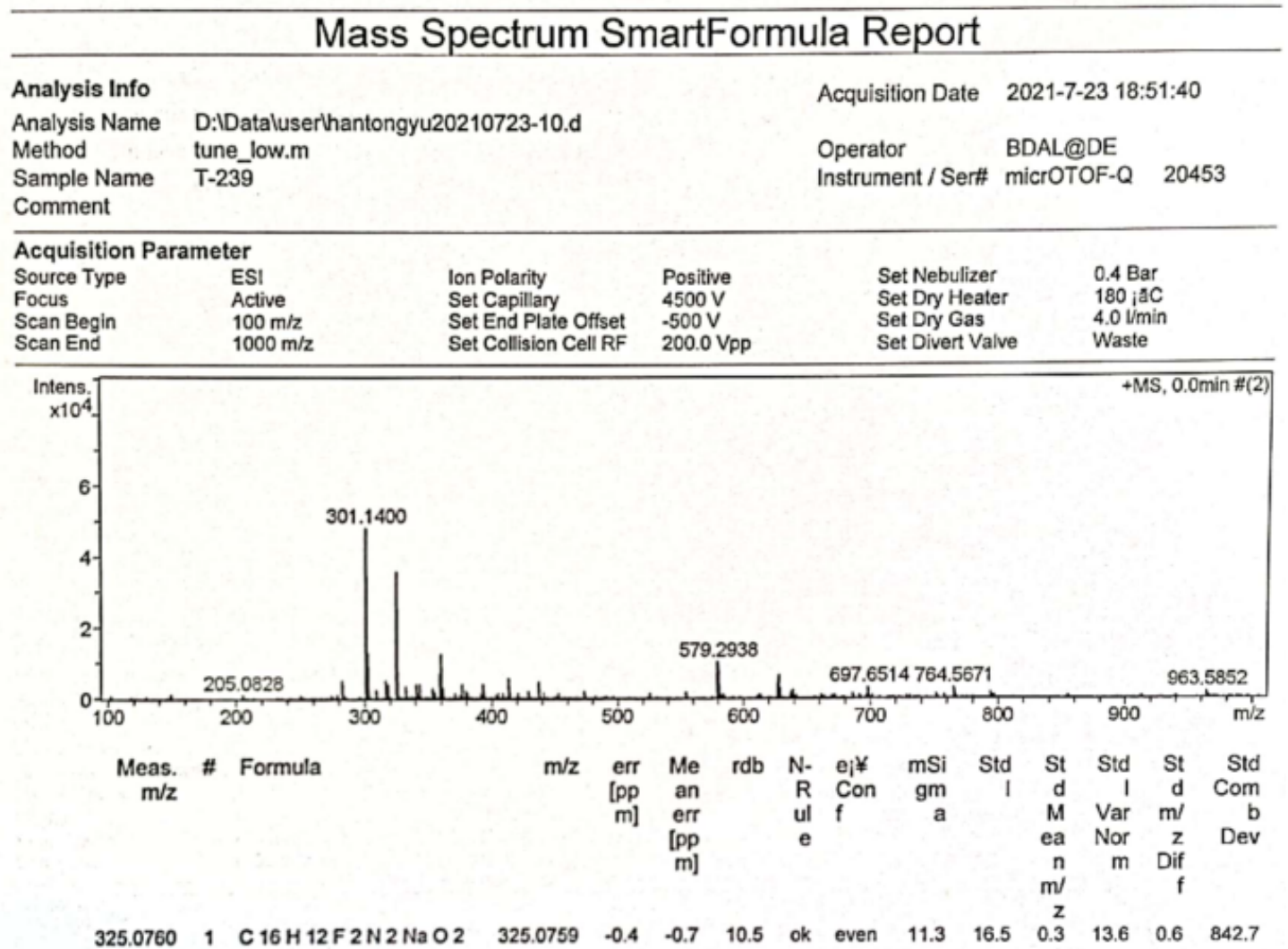

NMR copies of compound $\mathbf{5 i}$ :
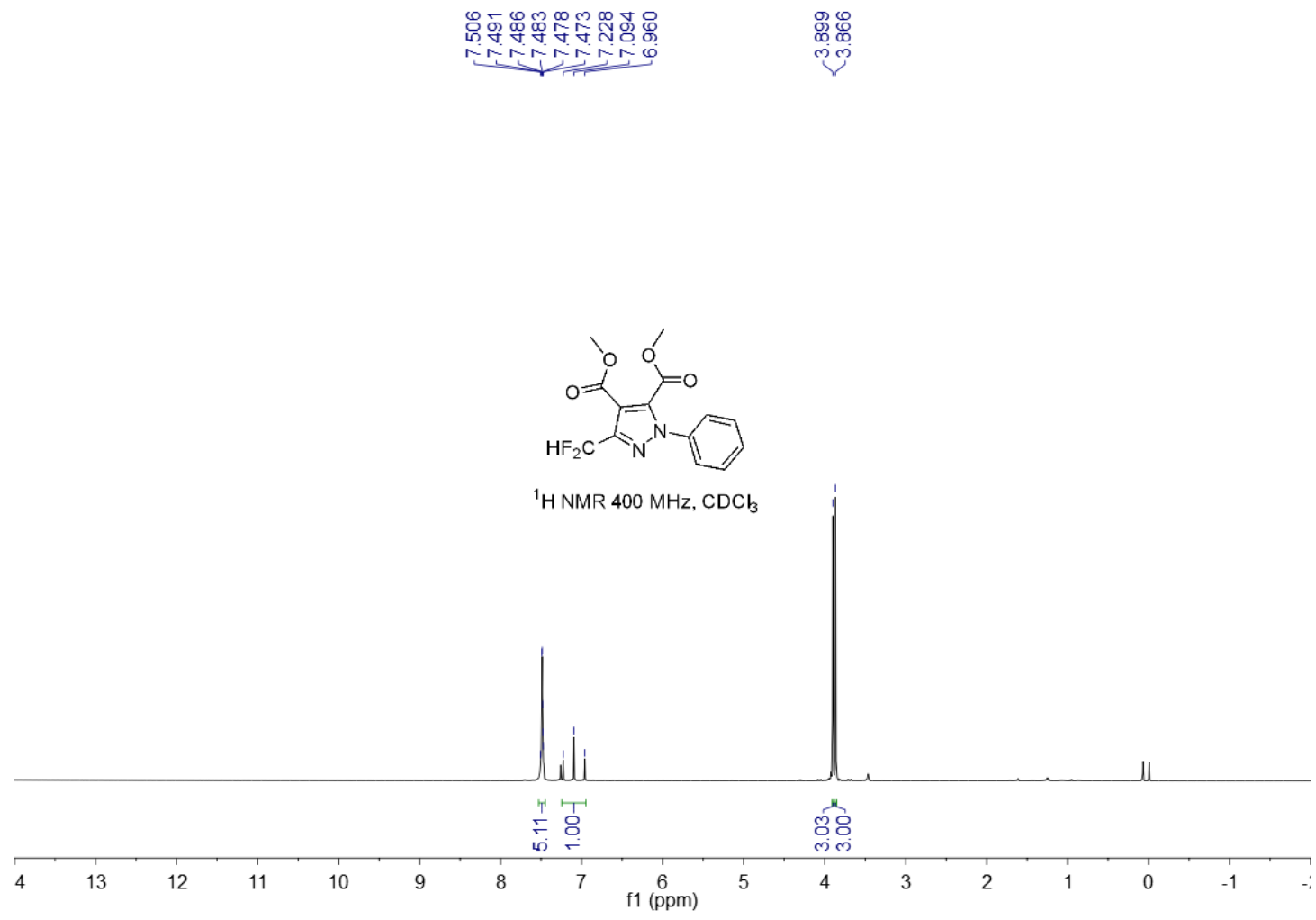


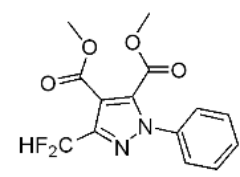

${ }^{1} \mathrm{H} \mathrm{NMR} 400 \mathrm{MHz}, \mathrm{CDCl}_{3}$

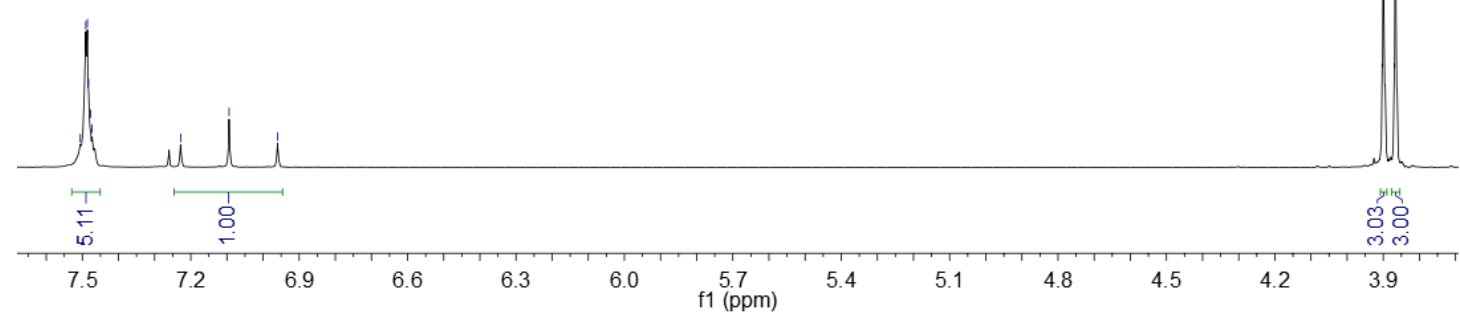

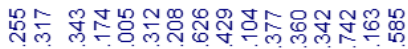

ঢ은

导扁

กุก

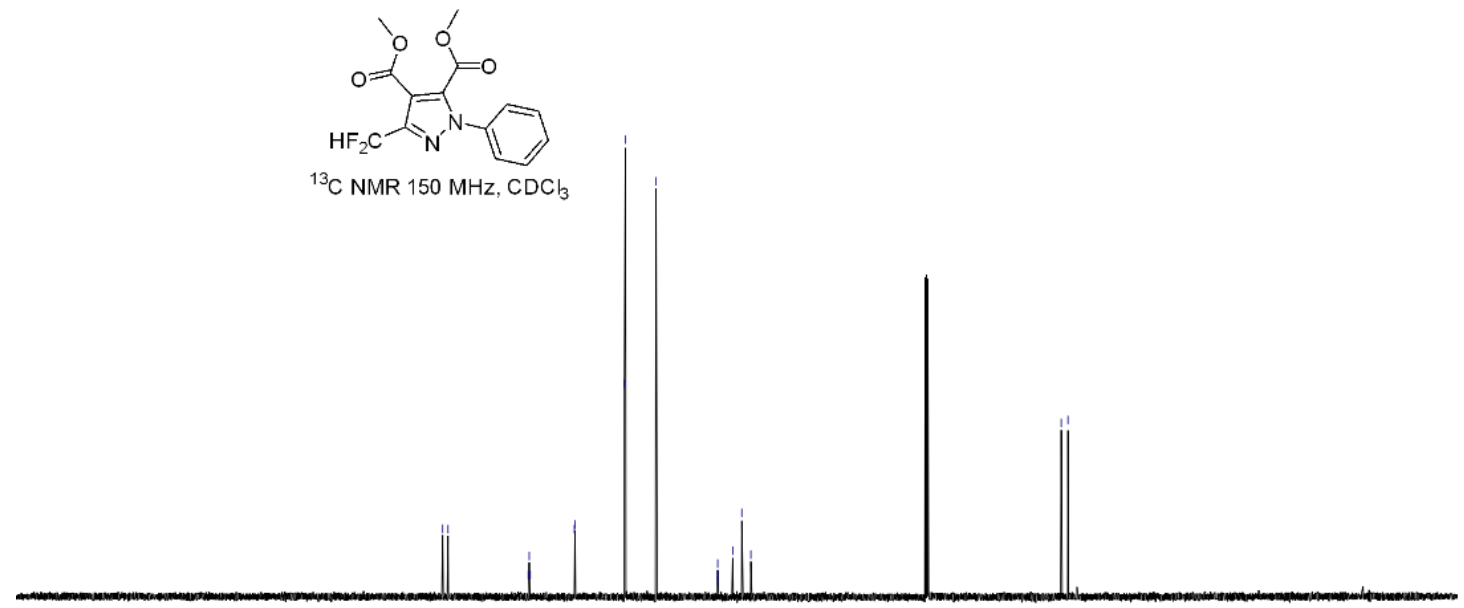

230

210

190

130

$\stackrel{110}{\mathrm{f} 1}(\mathrm{ppm})$

$\begin{array}{lllllllllll}90 & 80 & 70 & 60 & 50 & 40 & 30 & 20 & 10 & 0 & -10\end{array}$ 


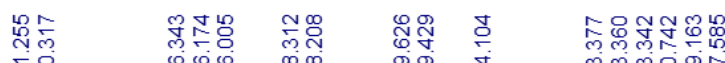

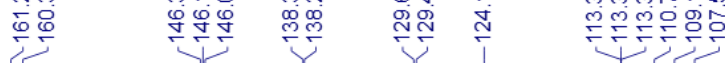

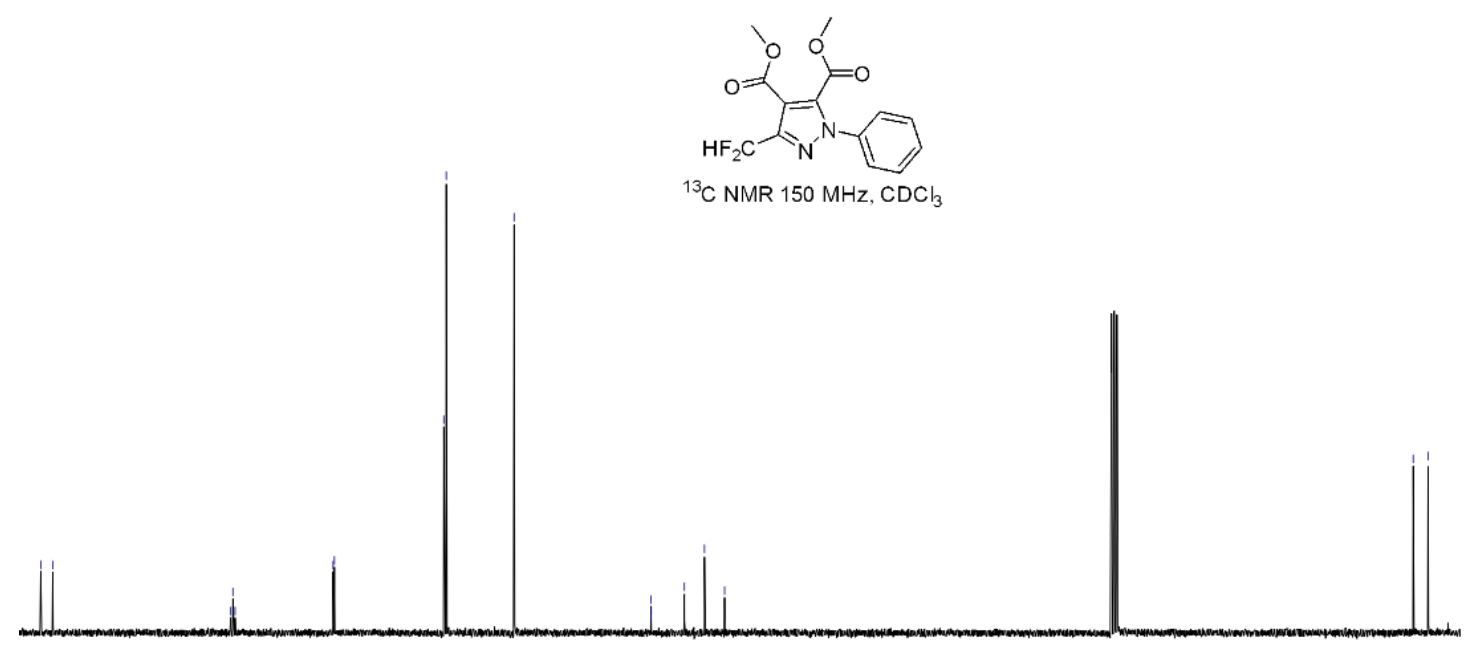

$\begin{array}{lllllllllllllllllllllll}160 & 155 & 150 & 145 & 140 & 135 & 130 & 125 & 120 & 115 & \begin{array}{c}110 \\ \mathrm{f} 1(\mathrm{ppm})\end{array} & 100 & 95 & 90 & 85 & 80 & 75 & 70 & 65 & 60 & 55 & 51\end{array}$

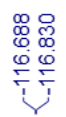

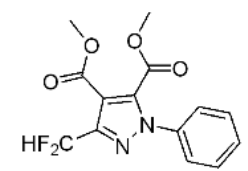

${ }^{19} \mathrm{~F}$ NMR $376 \mathrm{MHz}, \mathrm{CDCl}_{3}$

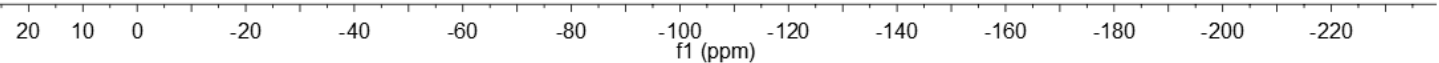




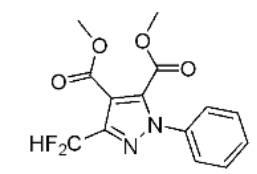

${ }^{19} \mathrm{~F} \mathrm{NMR} 376 \mathrm{MHz}, \mathrm{CDCl}_{3}$

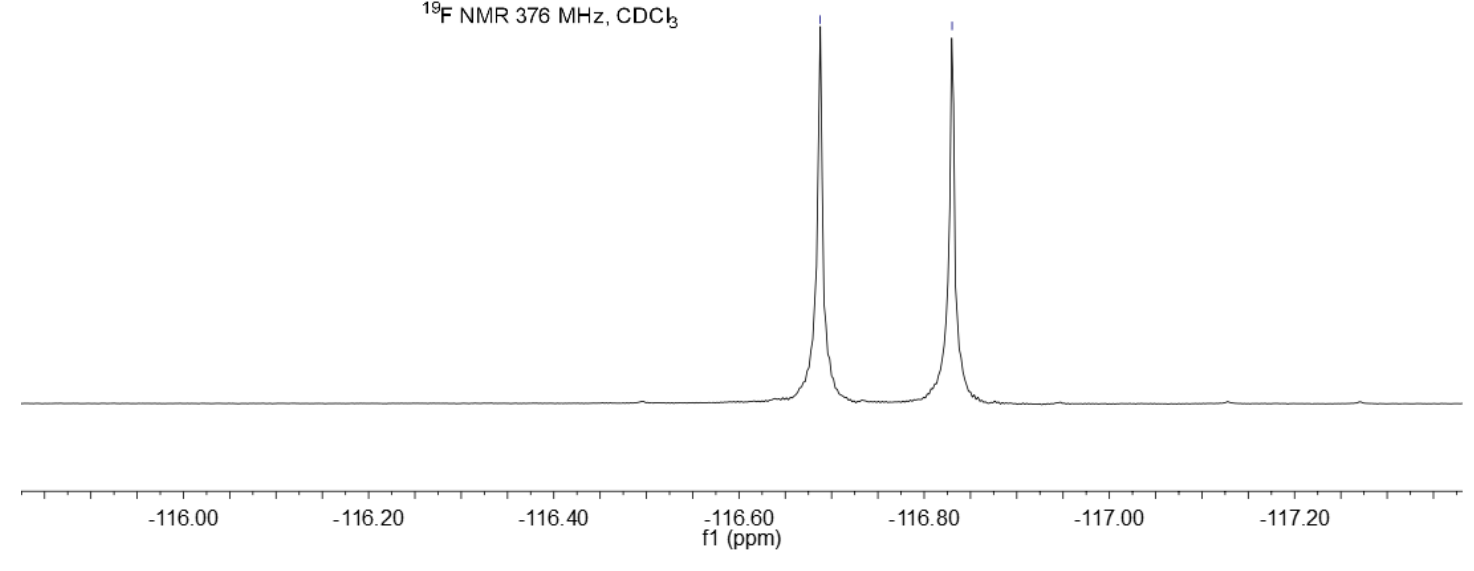

HRMS (ESI) copy of compound $\mathbf{5 i}$ :

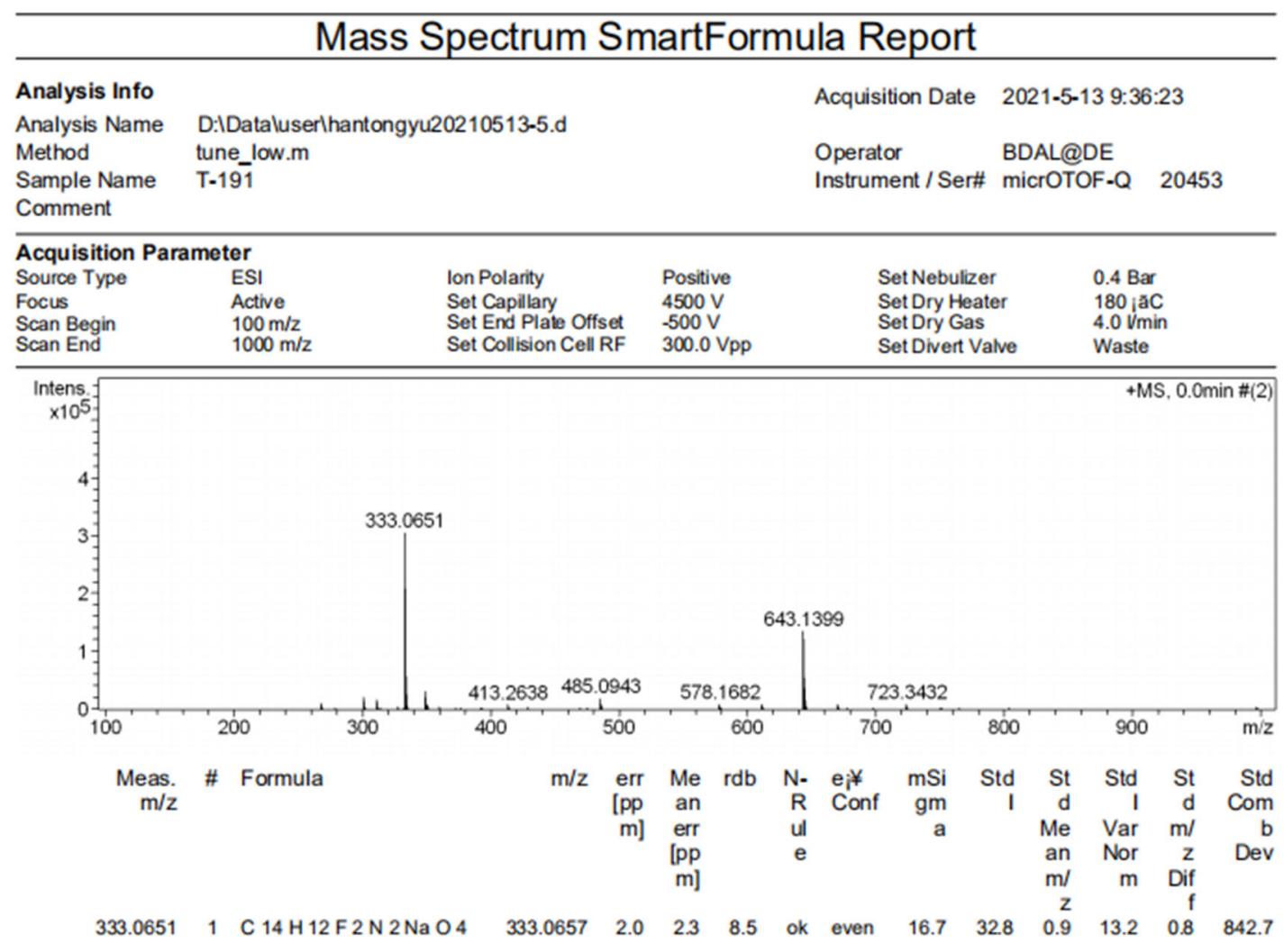


NMR copies of compound $\mathbf{5 j}$ :

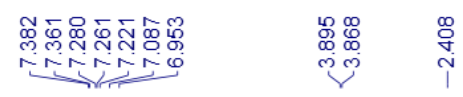

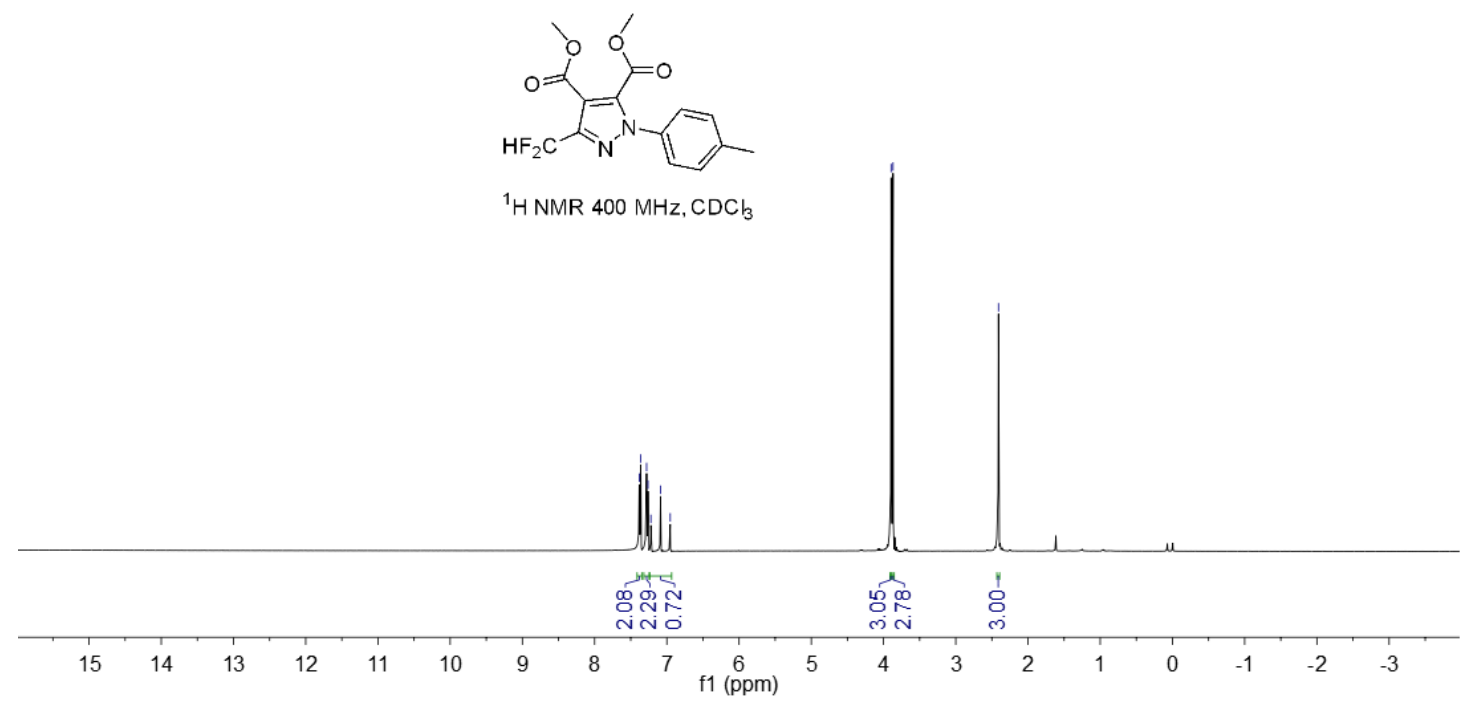

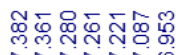

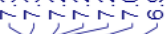

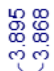

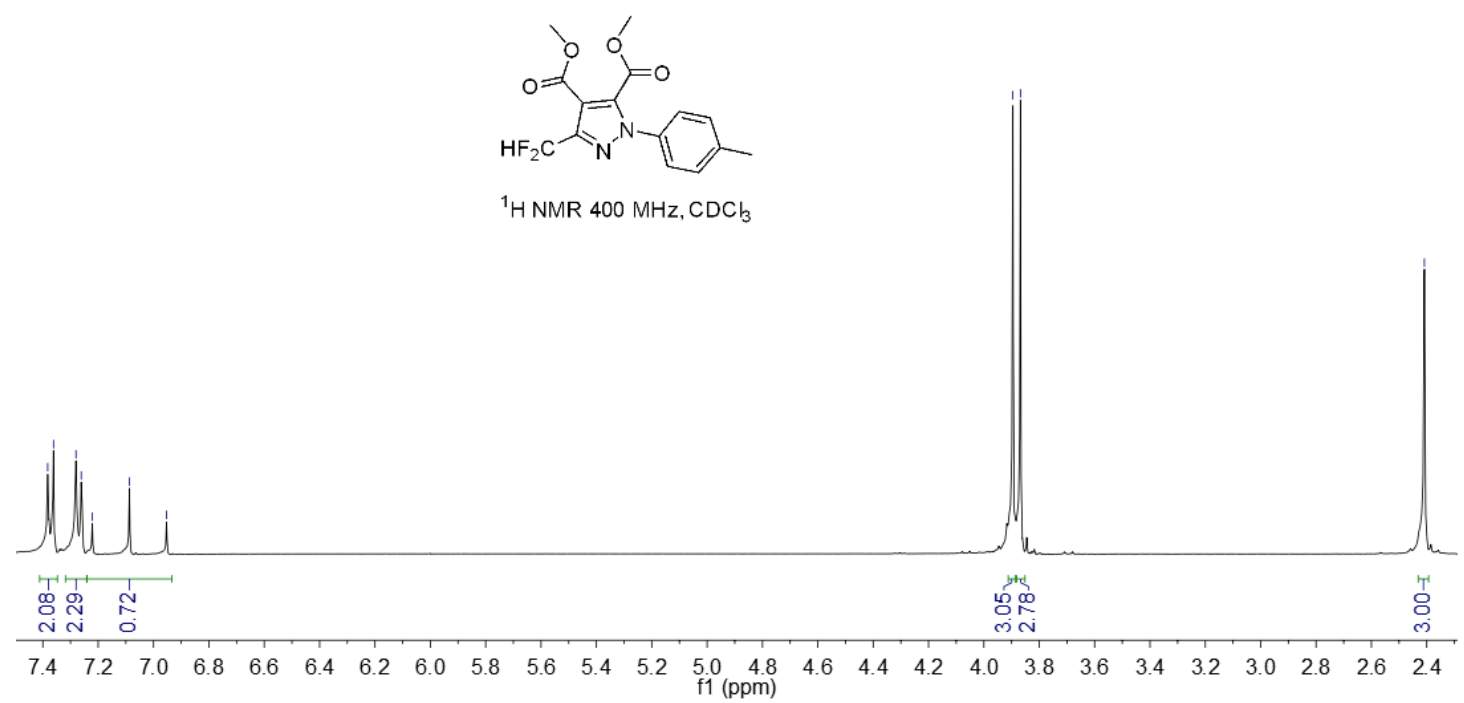




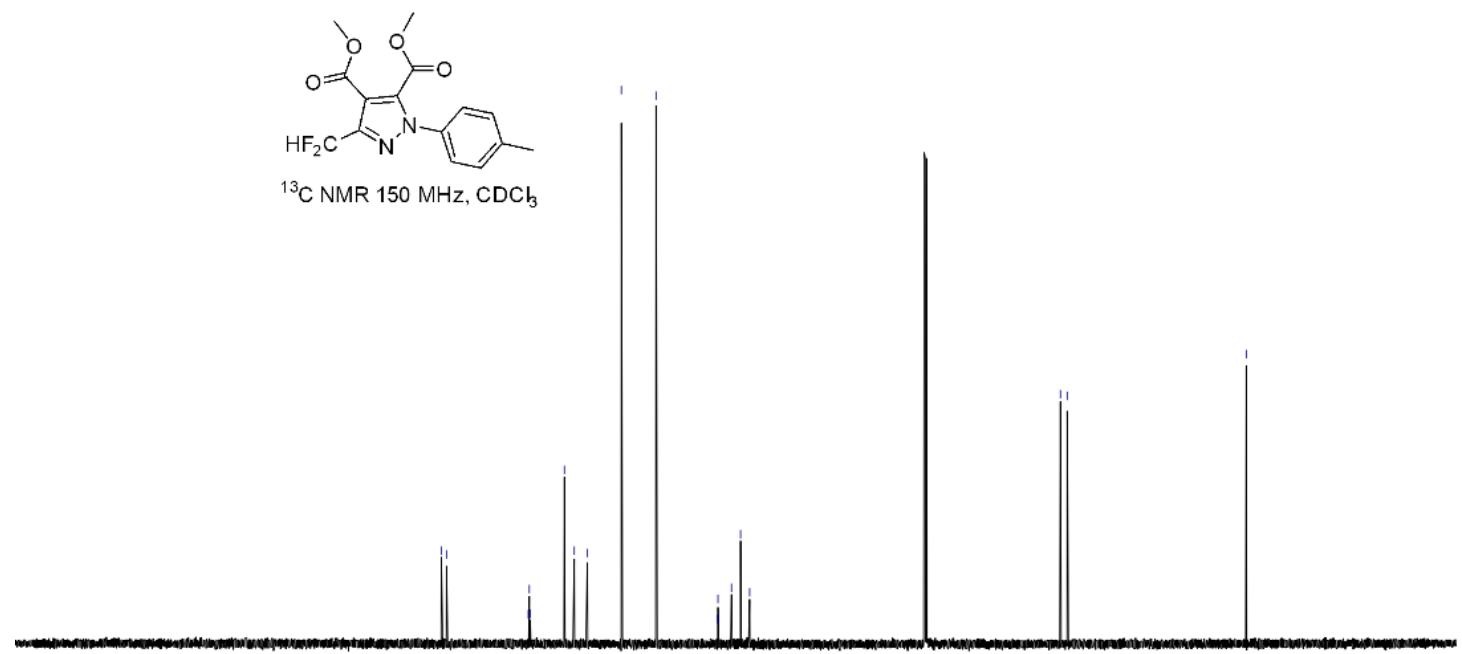

\begin{tabular}{|c|c|c|c|c|c|c|c|c|c|c|c|c|c|c|c|}
\hline 230 & 210 & 190 & 170 & 150 & 130 & $\begin{array}{c}110 \\
\mathrm{f} 1(\mathrm{ppm})\end{array}$ & 90 & 80 & 70 & 60 & 50 & 40 & 30 & $20 \quad 10$ & $\begin{array}{ll}0 & -10\end{array}$ \\
\hline 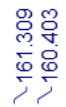 & & & 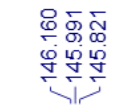 & & 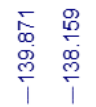 & 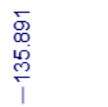 & $\begin{array}{l}\stackrel{\circ}{\circ} \\
\stackrel{\leftrightarrow}{\sigma} \\
\stackrel{\text { j}}{\mid}\end{array}$ & & & 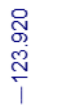 & & & & 点承 & 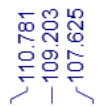 \\
\hline
\end{tabular}

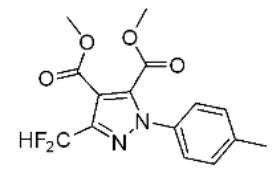

${ }^{13} \mathrm{C} \mathrm{NMR} 150 \mathrm{MHz}, \mathrm{CDCl}_{3}$

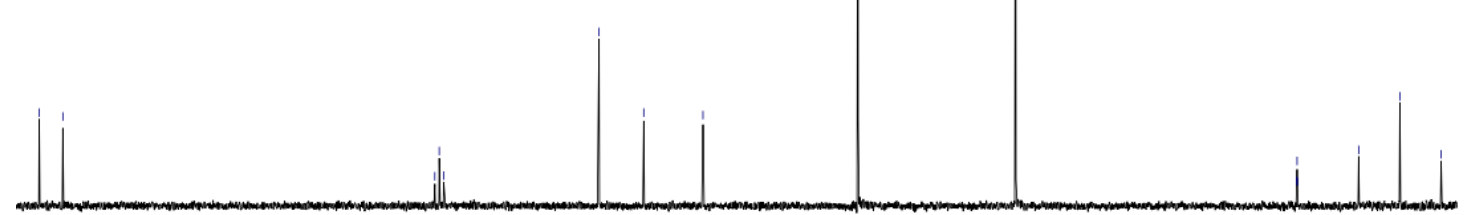

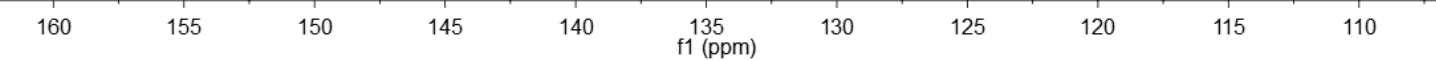




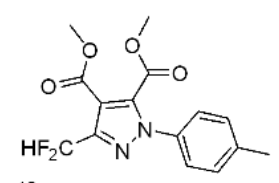

${ }^{19} \mathrm{~F} \mathrm{NMR} 376 \mathrm{MHz}, \mathrm{CDCl}_{3}$

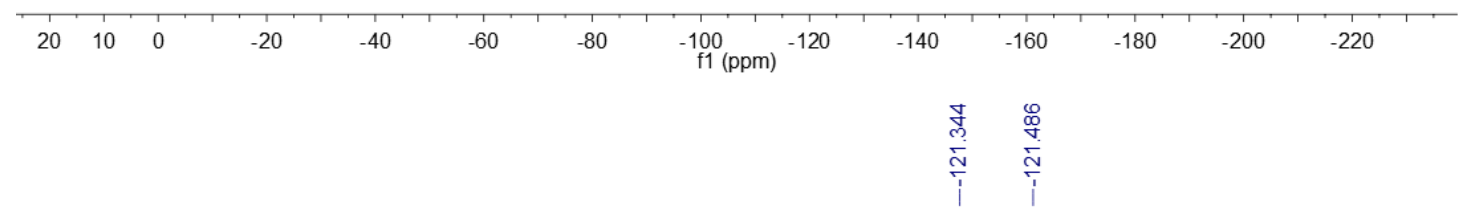

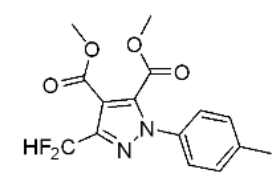

${ }^{19} \mathrm{~F} \mathrm{NMR} 376 \mathrm{MHz}, \mathrm{CDCl}_{3}$

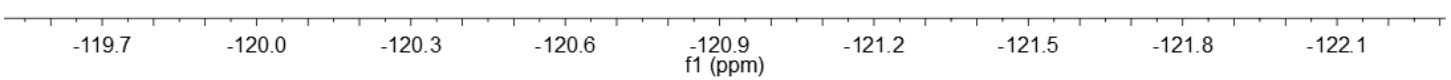


HRMS (ESI) copy of compound $\mathbf{5} \mathbf{j}$ :

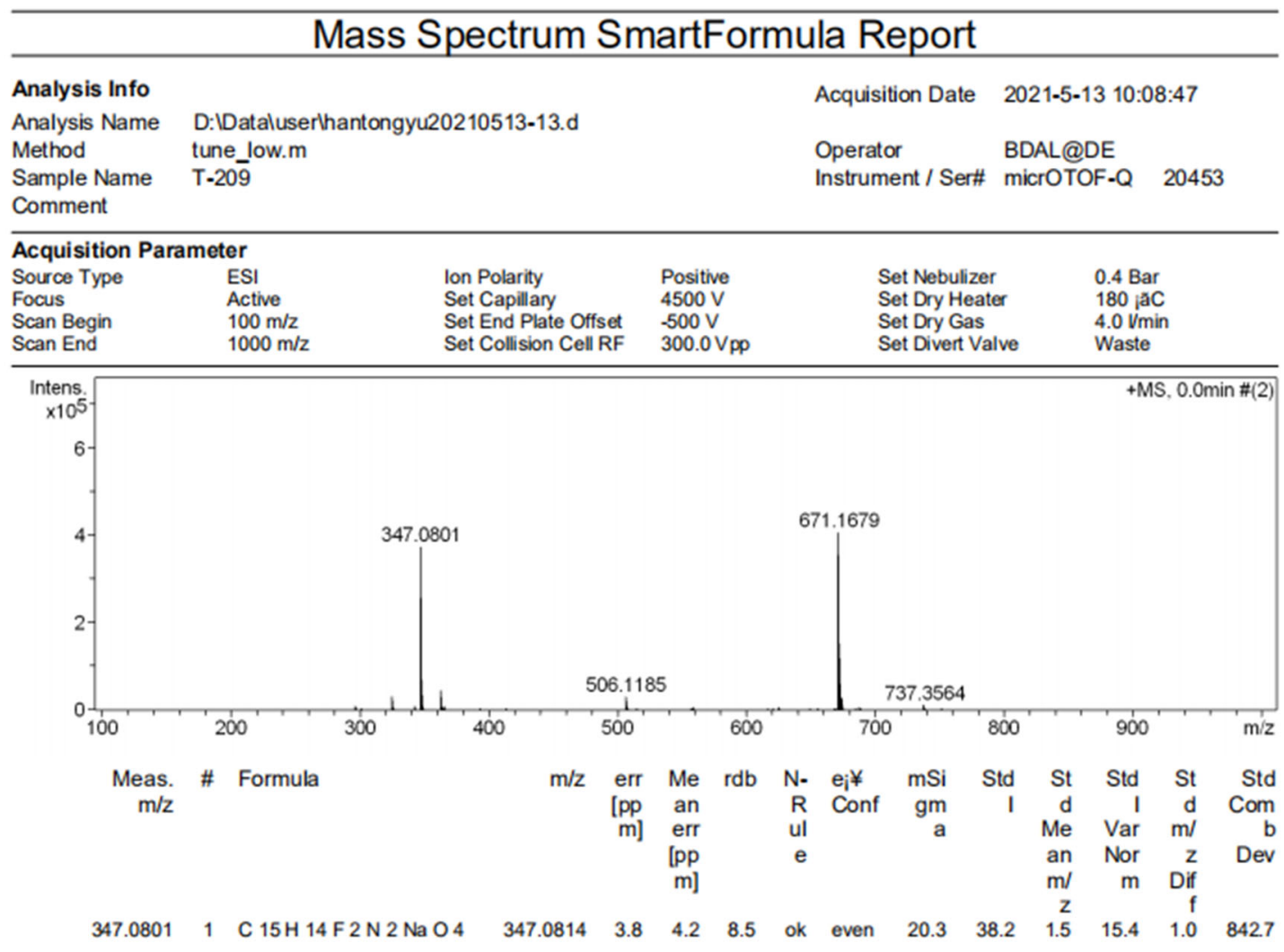

NMR copies of compound $\mathbf{5 k}$ :

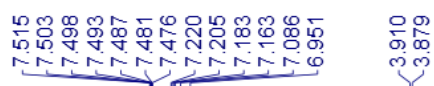

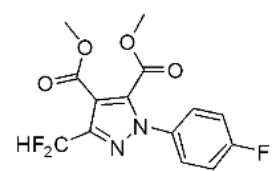

${ }^{1} \mathrm{H}$ NMR $400 \mathrm{MHz}, \mathrm{CDCl}_{3}$

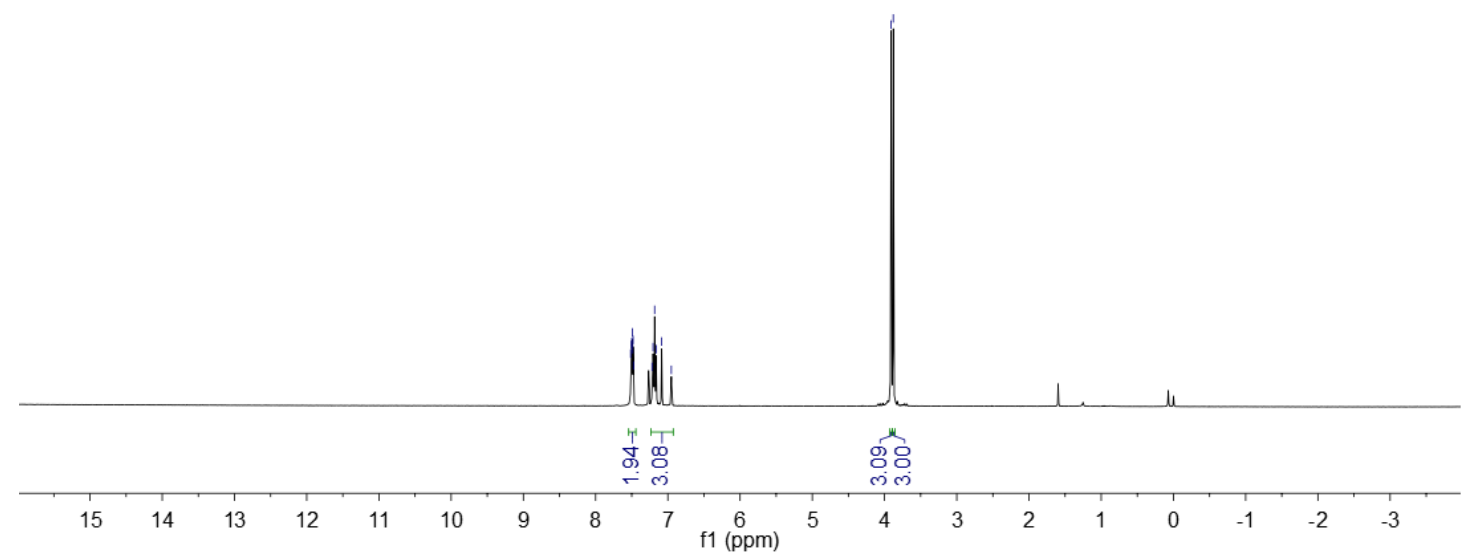




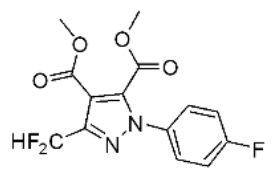

${ }^{1} \mathrm{H} \mathrm{NMR} 400 \mathrm{MHz}, \mathrm{CDCl}_{3}$

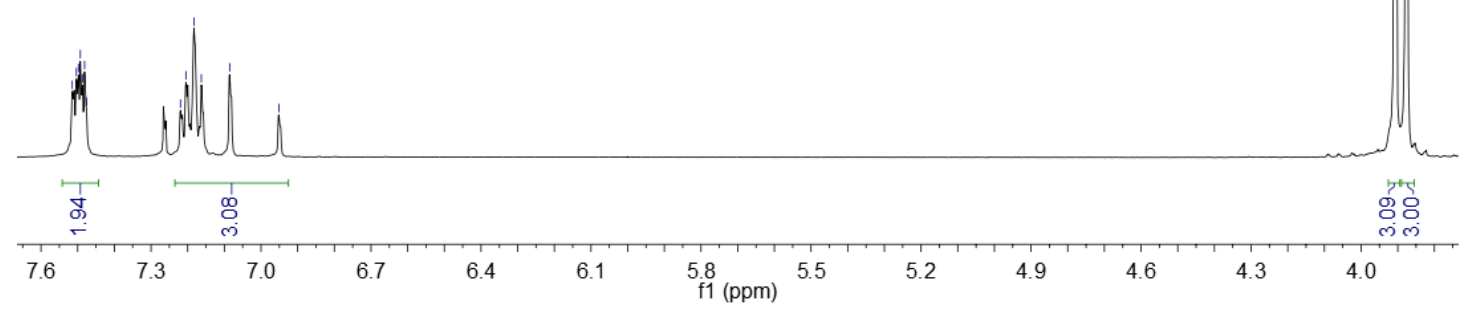

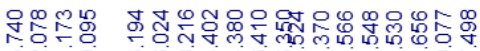

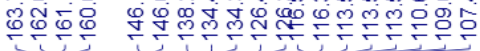

ภ̊ำ

ถูก

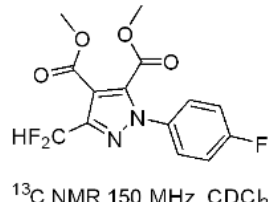

${ }^{13} \mathrm{C} \mathrm{NMR} 150 \mathrm{MHz}, \mathrm{CDCl}$

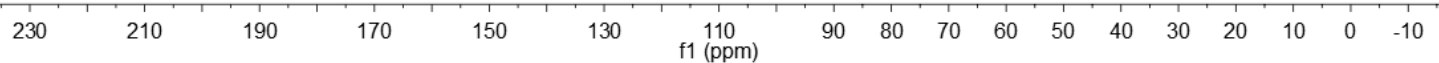




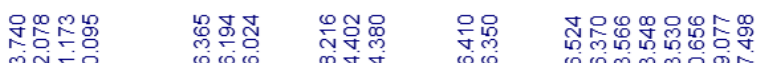

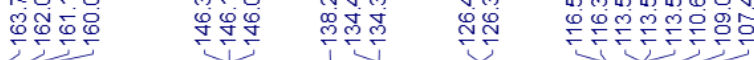

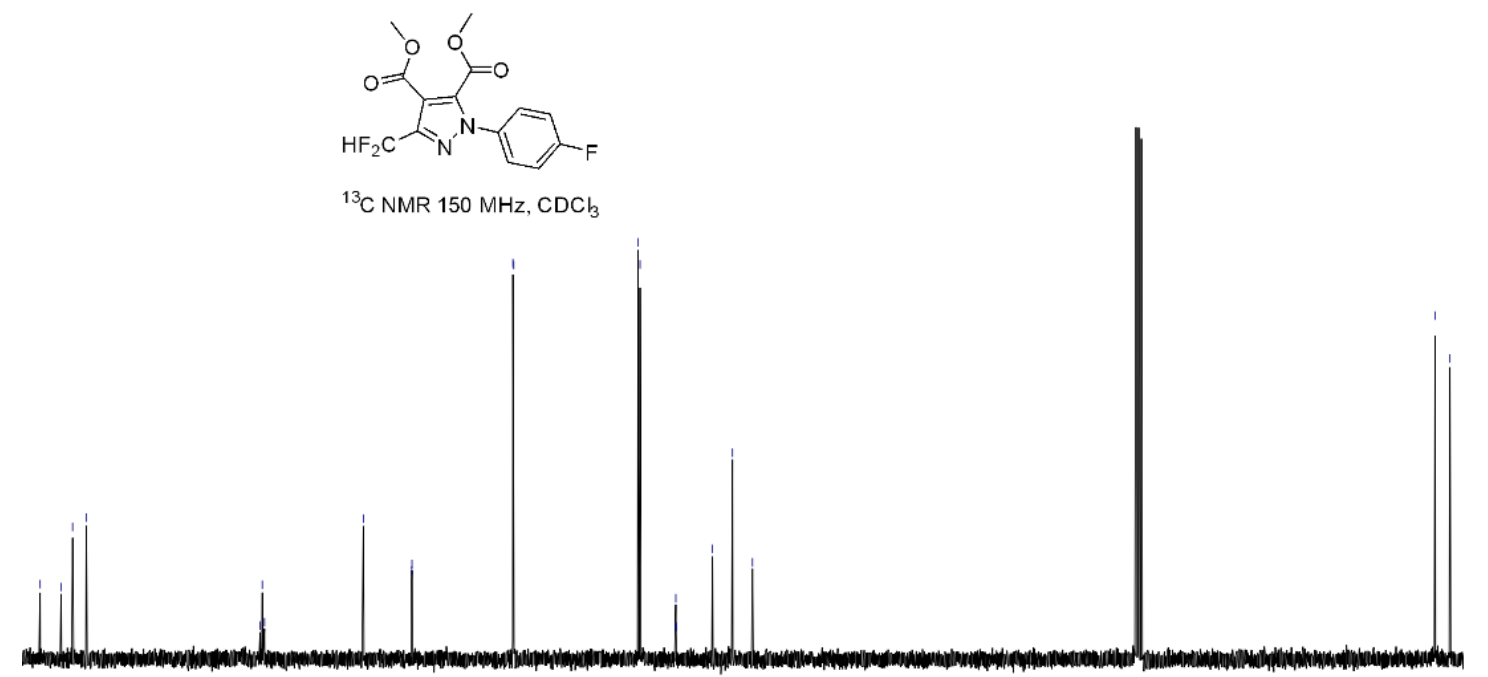

\begin{tabular}{llllllllllllllllllllllllllll}
\hline 5 & 160 & 155 & 150 & 145 & 140 & 135 & 130 & 125 & 120 & 115 & $\begin{array}{l}110 \\
\mathrm{f} 1(\mathrm{ppm})\end{array}$ & 105 & 100 & 95 & 90 & 85 & 80 & 75 & 70 & 65 & 60 & 55
\end{tabular}

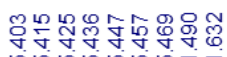

눈

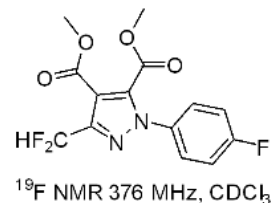

$20 \quad 10 \quad 0 \quad-20$

-100
$\mathrm{f} 1(\mathrm{ppm})$

$40-160$

$-180$

$-200$ 


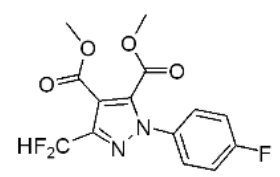

${ }^{19} \mathrm{~F}$ NMR $376 \mathrm{MHz}, \mathrm{CDCl}$

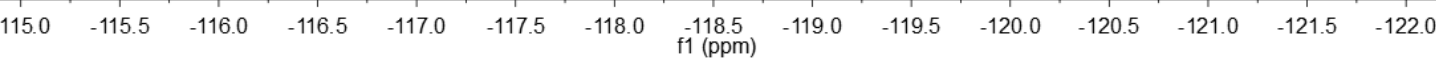

HRMS (ESI) copy of compound $\mathbf{5 k}$ :

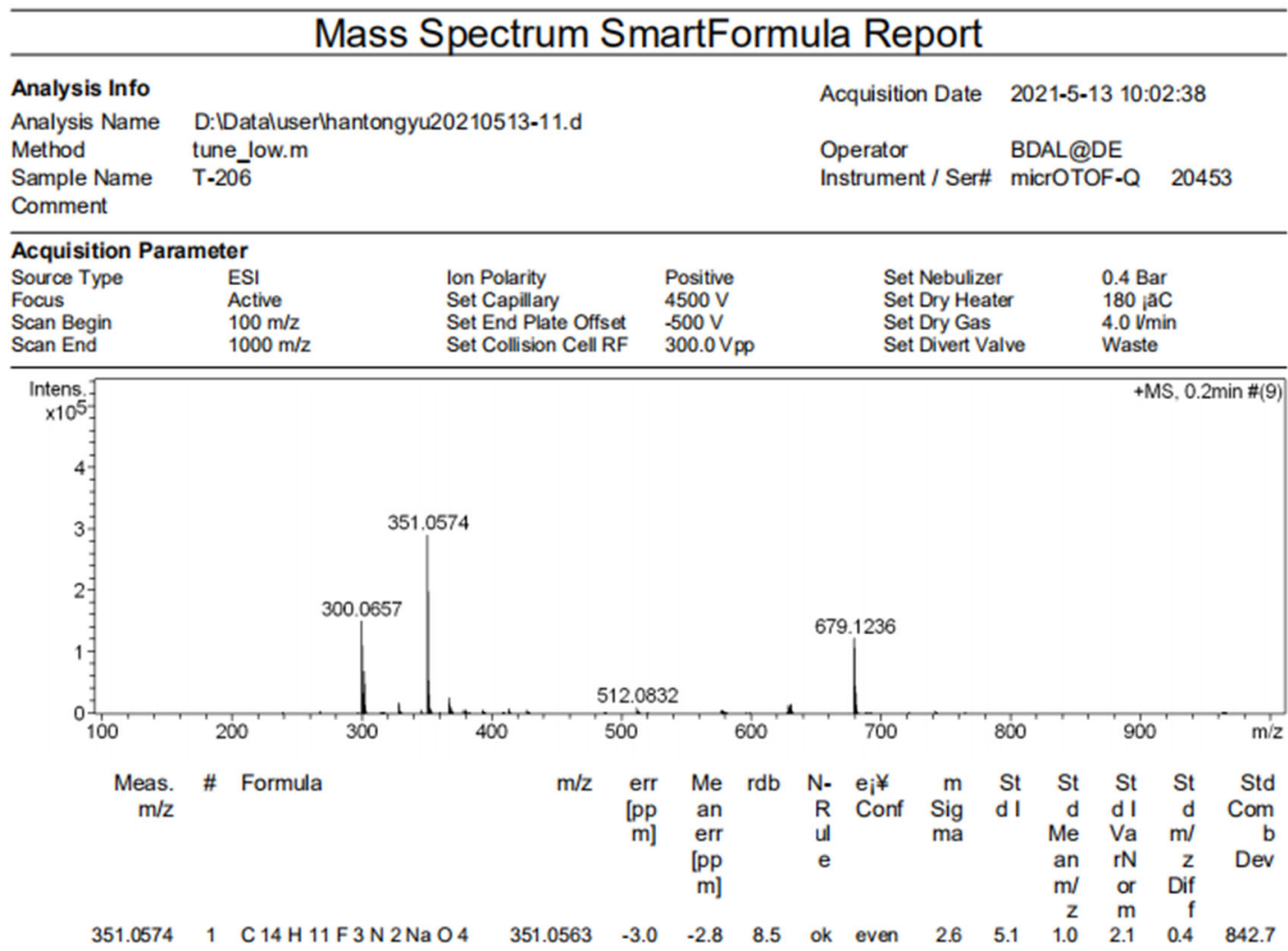


Copies of NMR and HRMS Spectra for Compounds $\mathbf{7 a}-7 \mathbf{k}$

NMR copies of compound 7a:

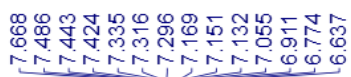
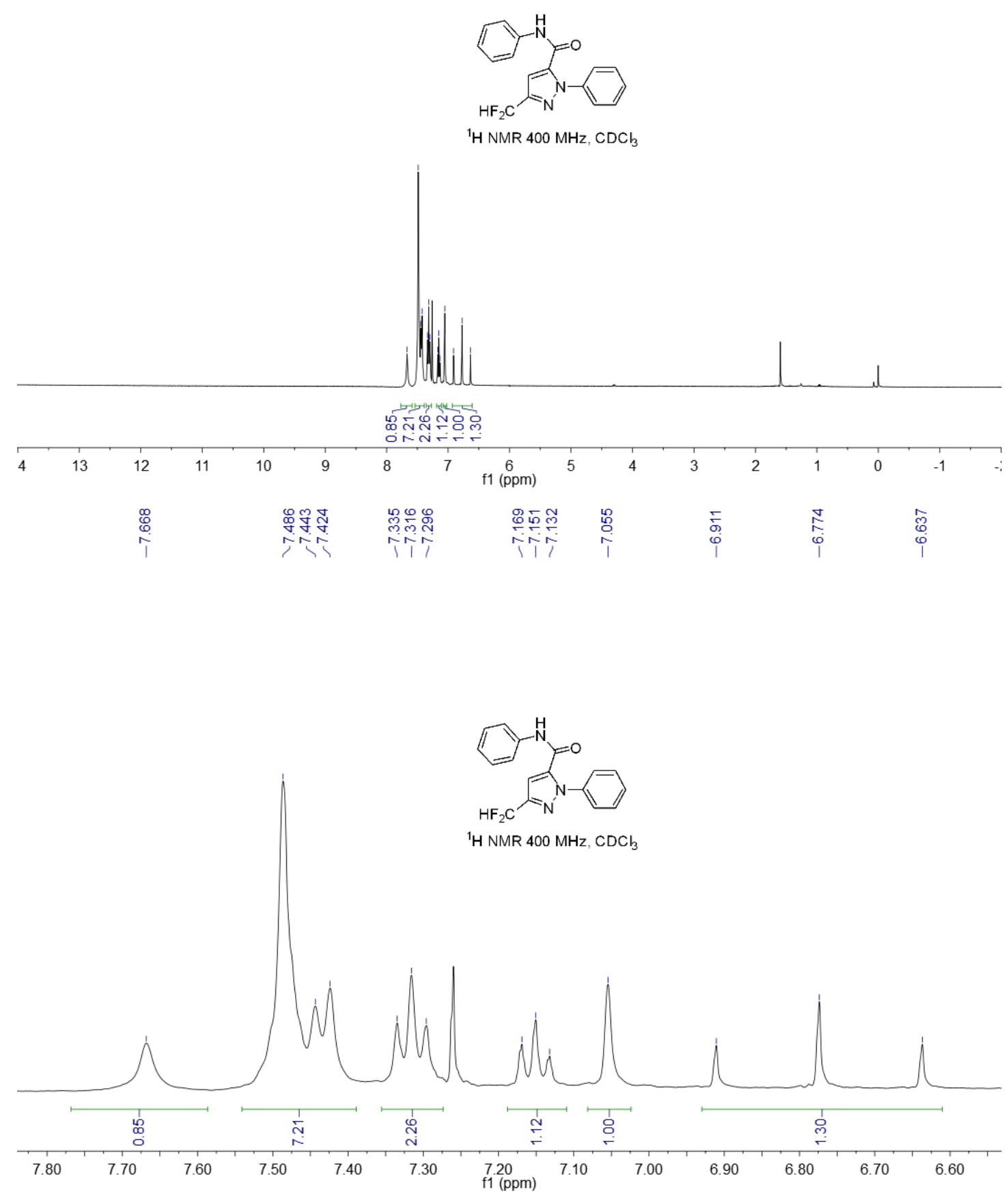


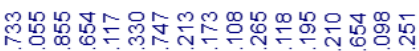

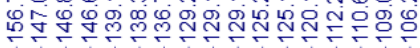

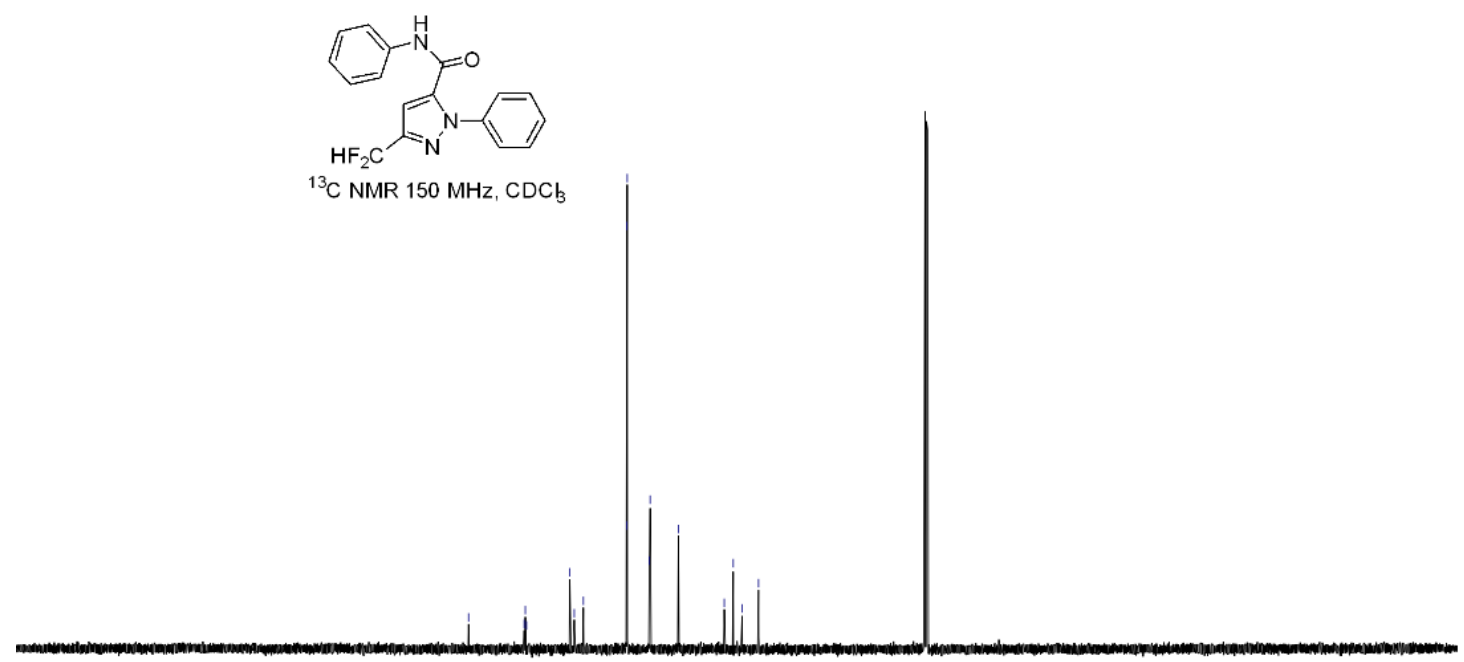

${ }^{13} \mathrm{C}$ NMR $150 \mathrm{MHz}, \mathrm{CDCb}$

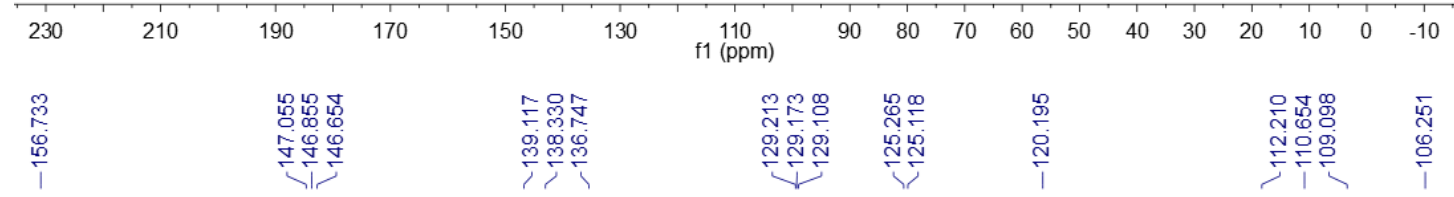

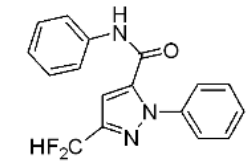

${ }^{13} \mathrm{C}$ NMR $150 \mathrm{MHz}, \mathrm{CDCb}$

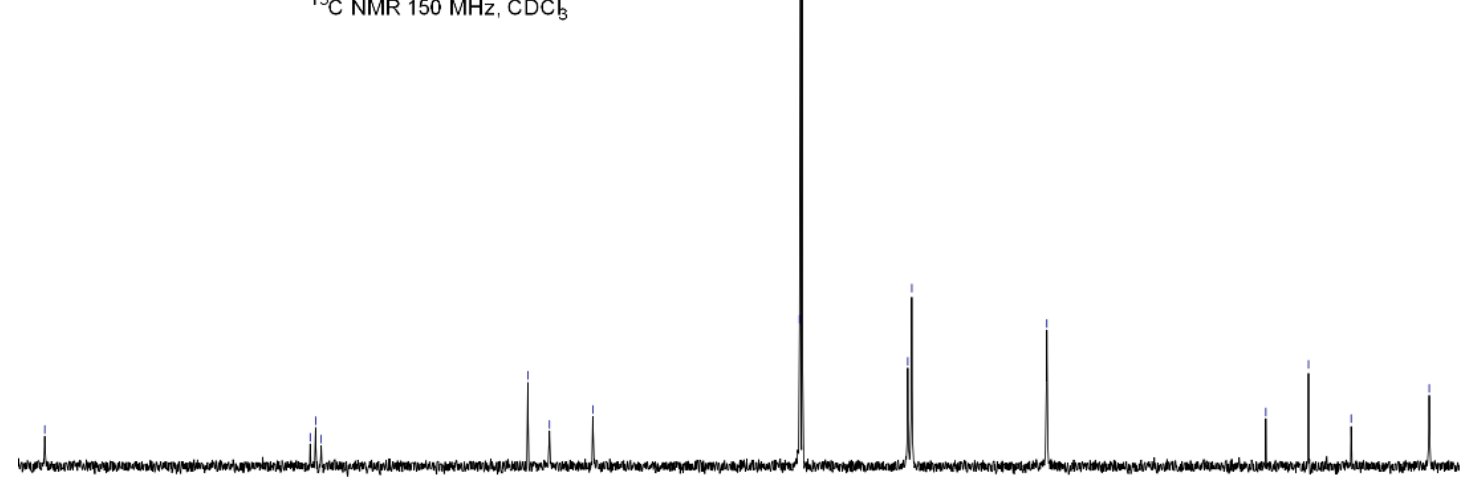

\begin{tabular}{|c|c|c|c|c|c|c|c|c|c|c|c|}
\hline 156 & 152 & 148 & 144 & 140 & 136 & $\begin{array}{c}132 \\
\text { f1 (ppm) }\end{array}$ & 128 & 124 & 120 & 116 & 112 \\
\hline
\end{tabular}



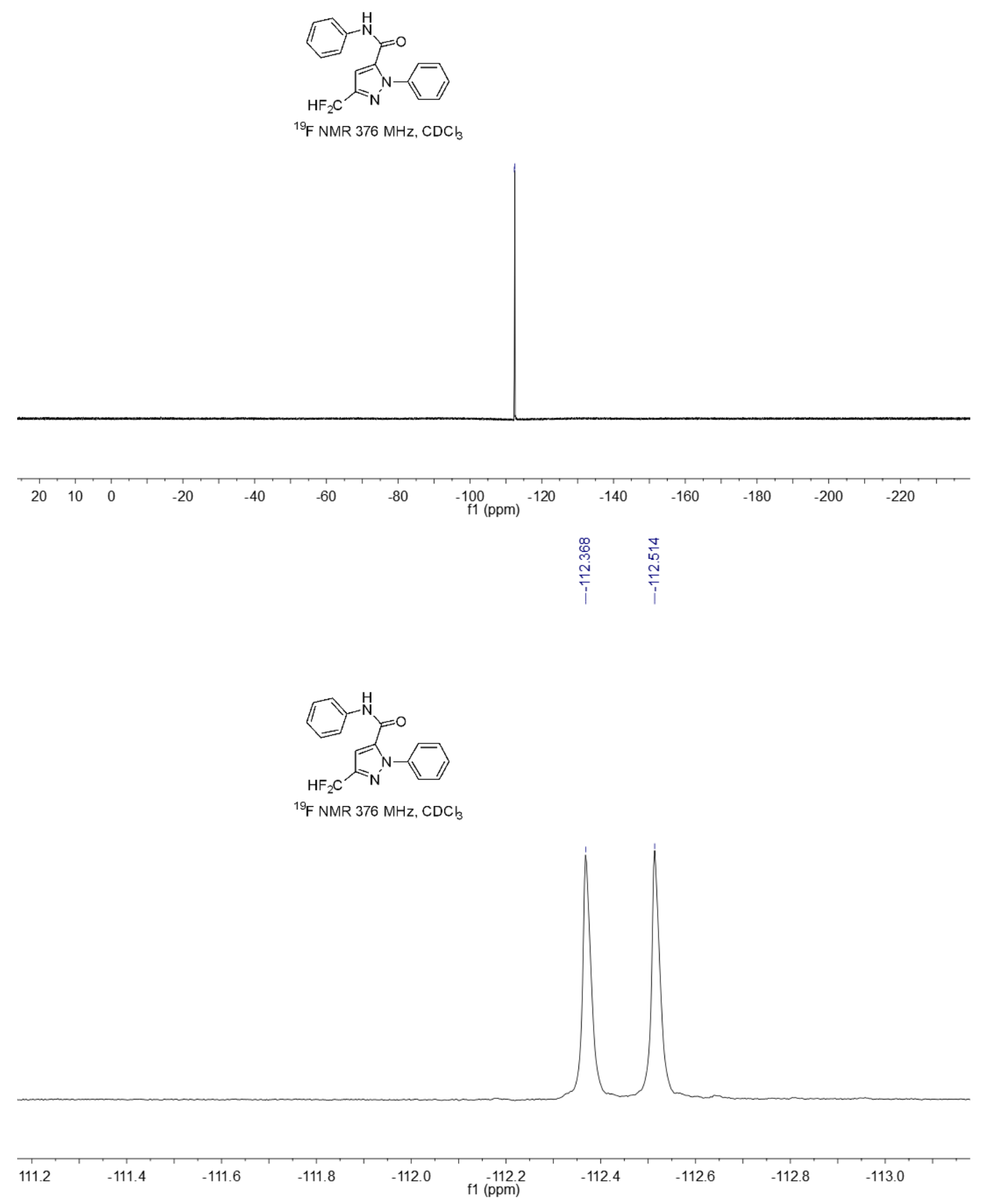
HRMS (ESI) copy of compound 7a:

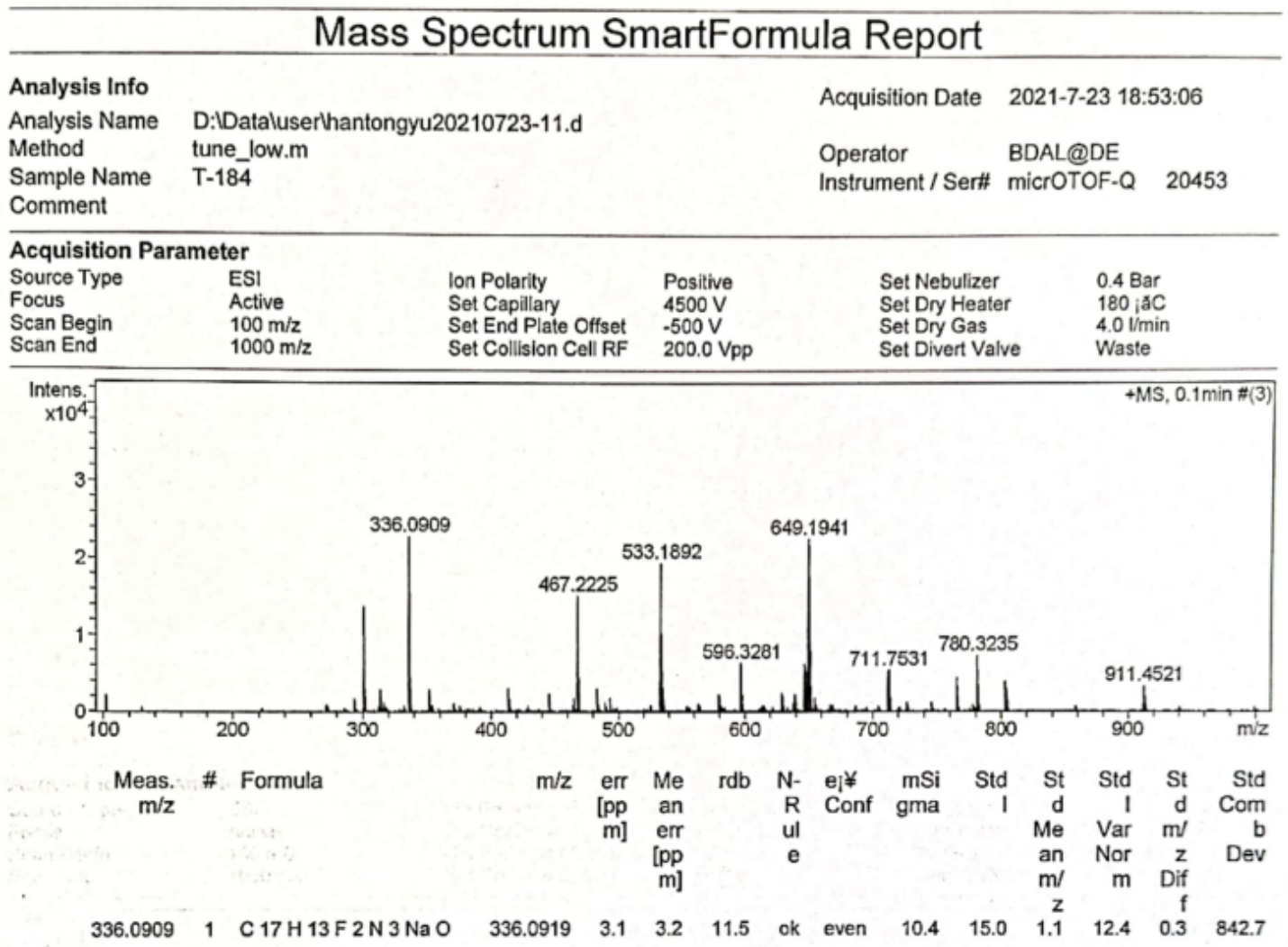

NMR copies of compound $\mathbf{7 b}$ :

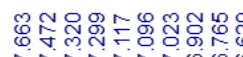

N-Nine

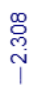

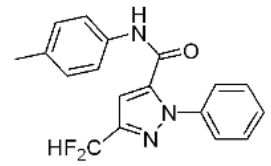

${ }^{1} \mathrm{H}$ NMR $400 \mathrm{MHz}, \mathrm{CDCl}_{3}$

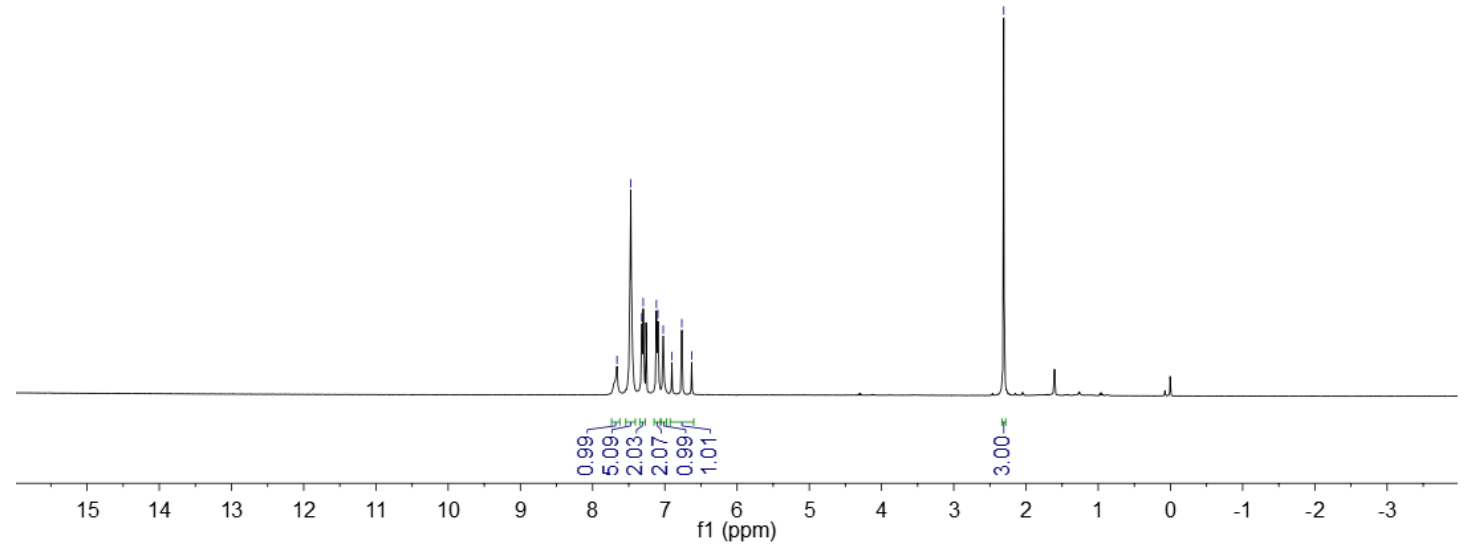




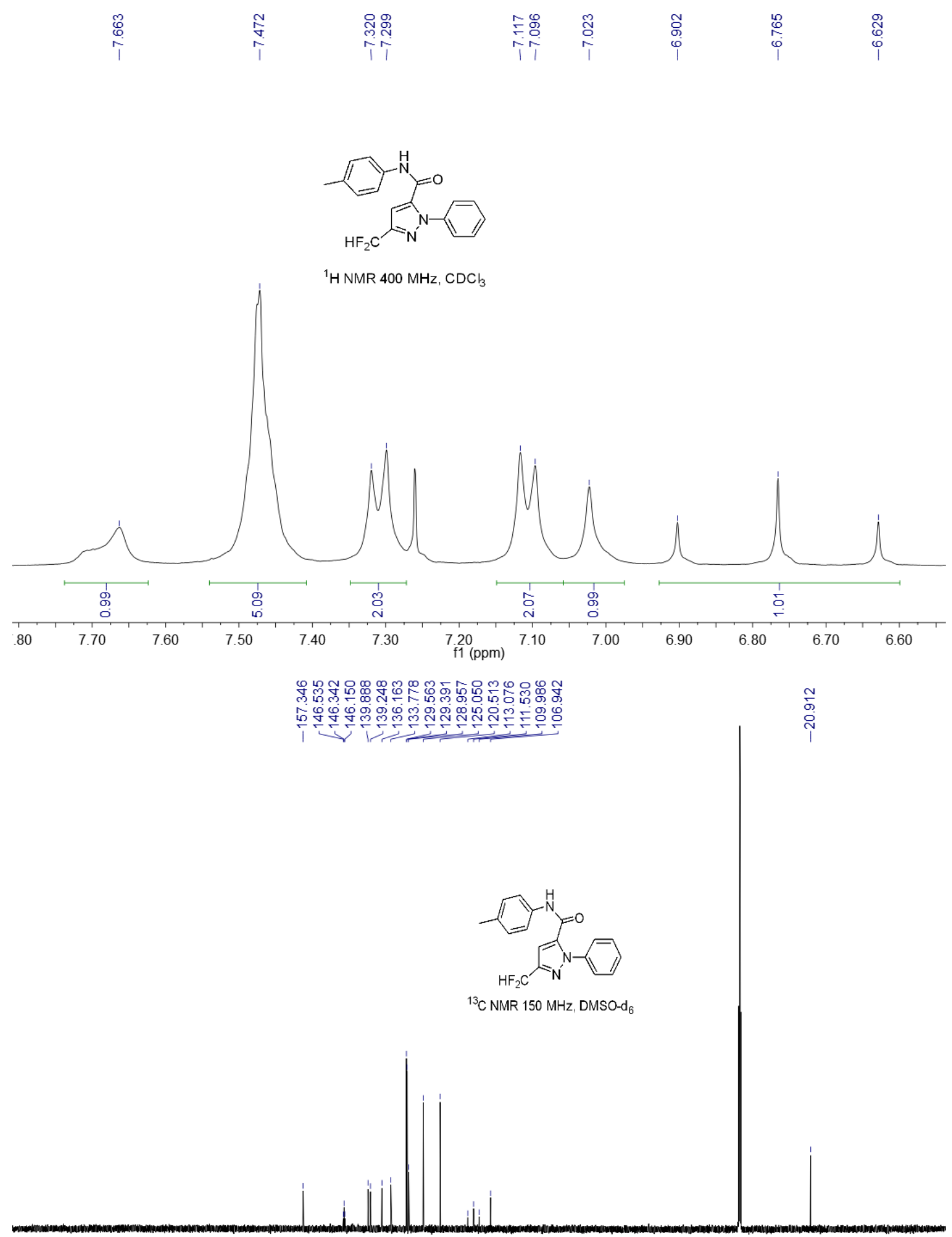

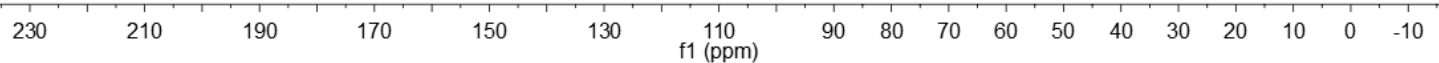




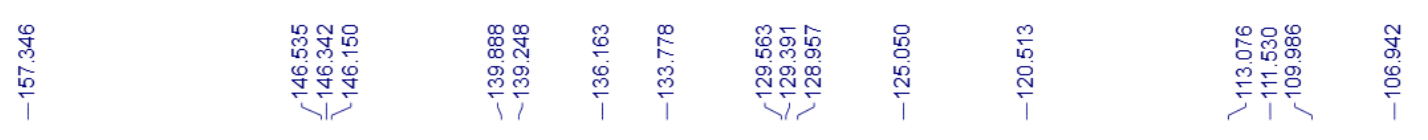
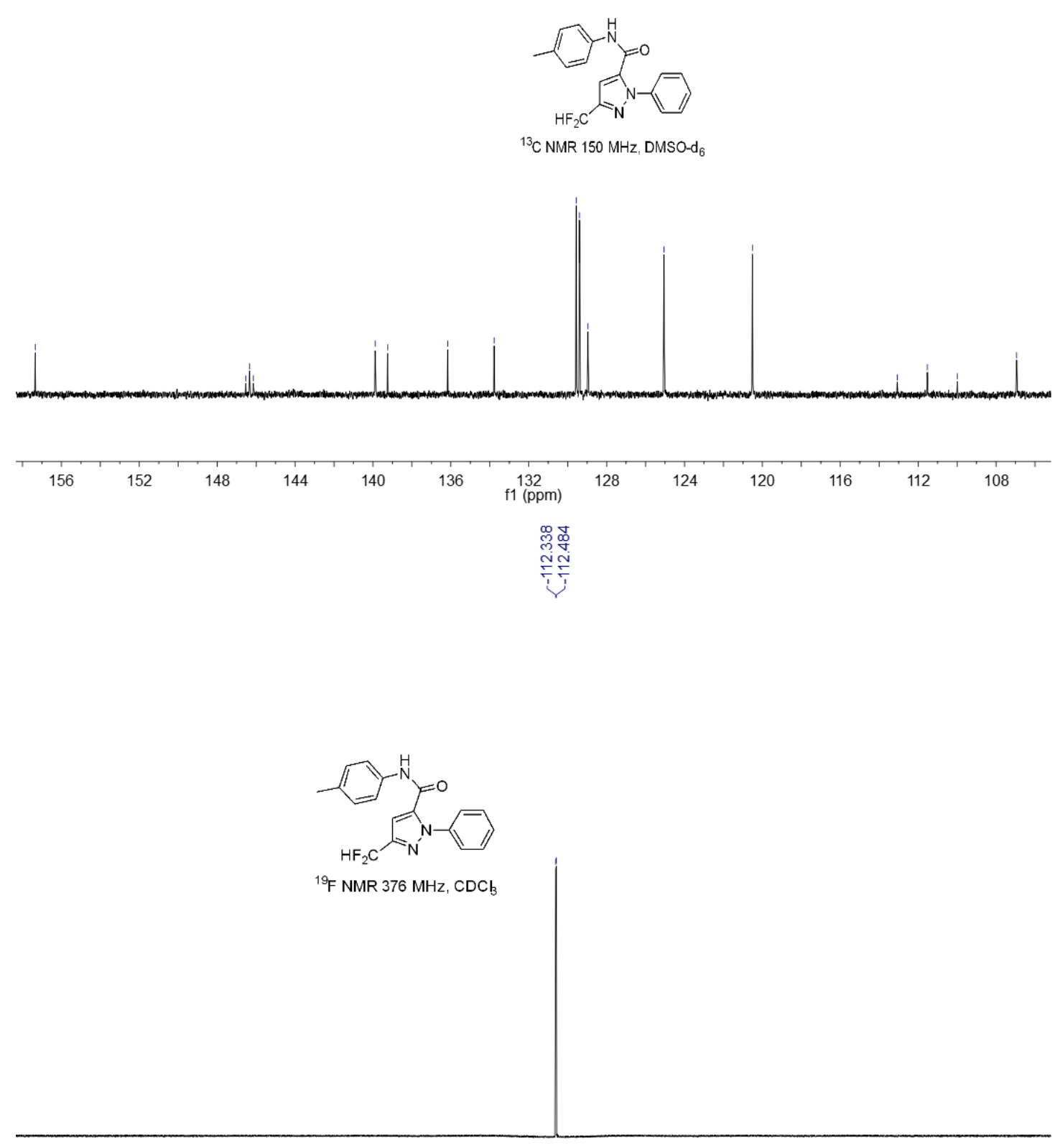

\begin{tabular}{|c|c|c|c|c|c|c|c|c|c|c|c|c|c|}
\hline 20 & 10 & 0 & -20 & -40 & -60 & -80 & -100 & -120 & -140 & -160 & -180 & -200 & -220 \\
\hline
\end{tabular}




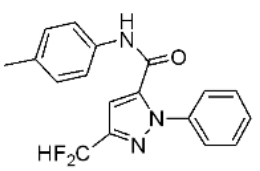

${ }^{19} \mathrm{~F}$ NMR $376 \mathrm{MHz}, \mathrm{CDCl}$

\begin{tabular}{lllllllll}
\hline-111.55 & -111.70 & -111.85 & -112.00 & $\mathrm{f} 1112.15$ & -112.30 & -112.45 & -112.60 & -112.75 \\
\hline
\end{tabular}

HRMS (ESI) copy of compound $7 \mathbf{b}$ :

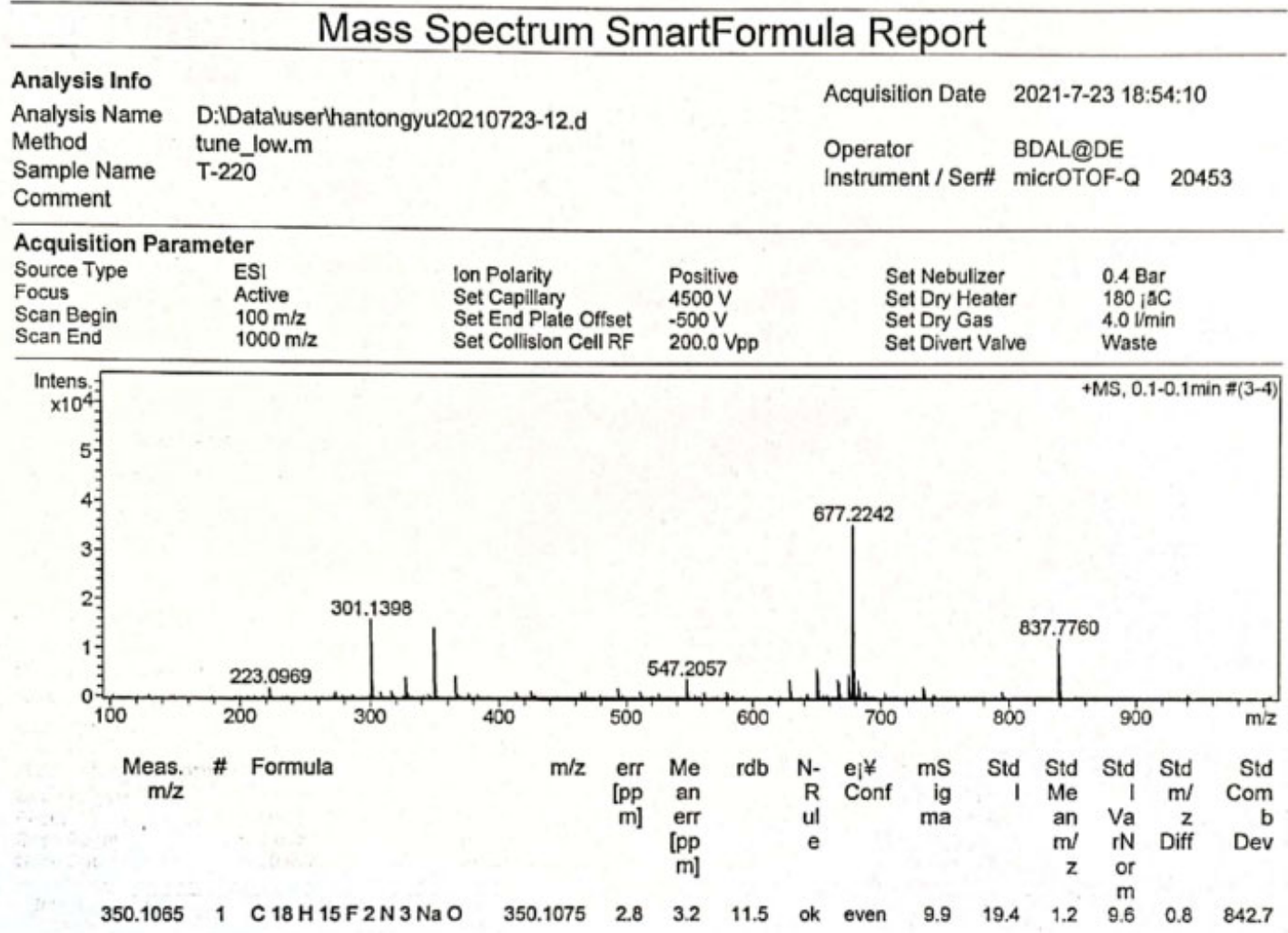


NMR copies of compound $7 \mathbf{c}$ :

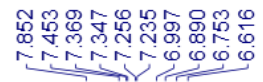
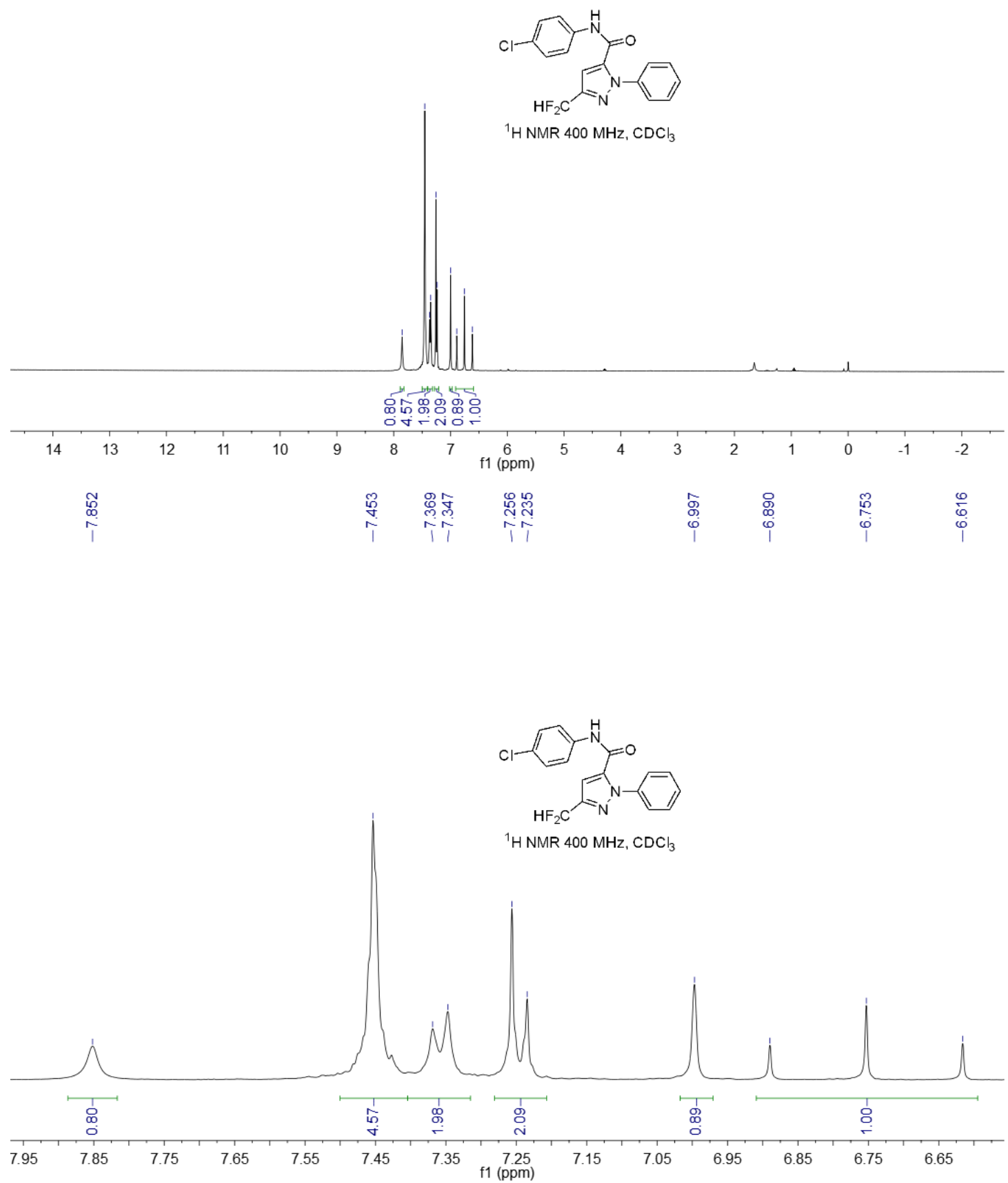


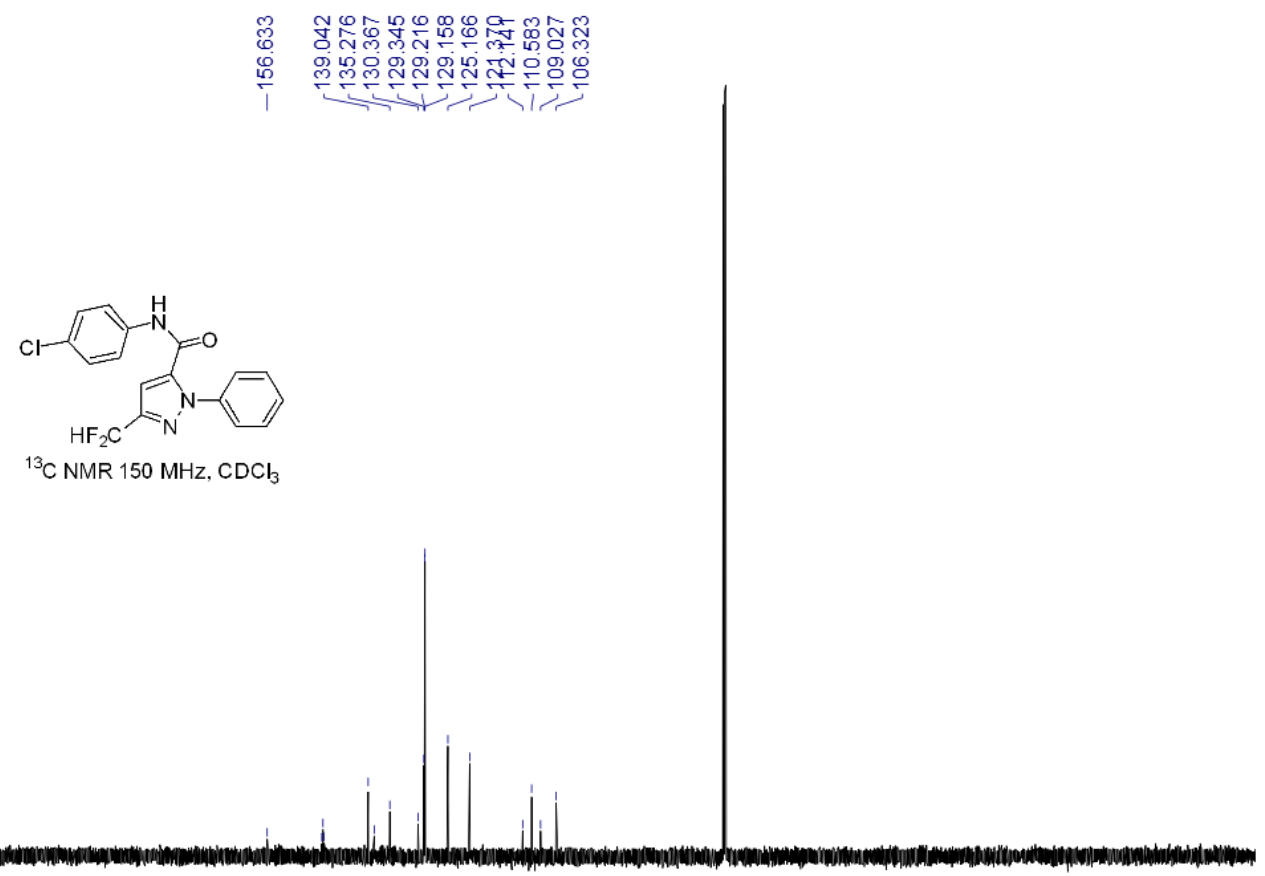

\begin{tabular}{|c|c|c|c|c|c|c|c|c|c|c|c|c|c|c|c|c|c|}
\hline 230 & 210 & 190 & 170 & 150 & 130 & $\begin{array}{l}110 \\
\mathrm{f} 1(\mathrm{ppm})\end{array}$ & 90 & 80 & 70 & 60 & 50 & 40 & 30 & 20 & 10 & 0 & -10 \\
\hline 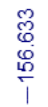 & & & & 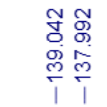 & $\begin{array}{l}\stackrel{0}{\sim} \\
\underset{\sim}{\infty} \\
\stackrel{\rho}{1}\end{array}$ & 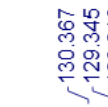 & & 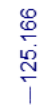 & & $\underset{\substack{\stackrel{p}{i} \\
\stackrel{m}{i}}}{ }$ & & & & & 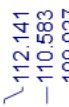 & & $\mathcal{N}$ \\
\hline
\end{tabular}
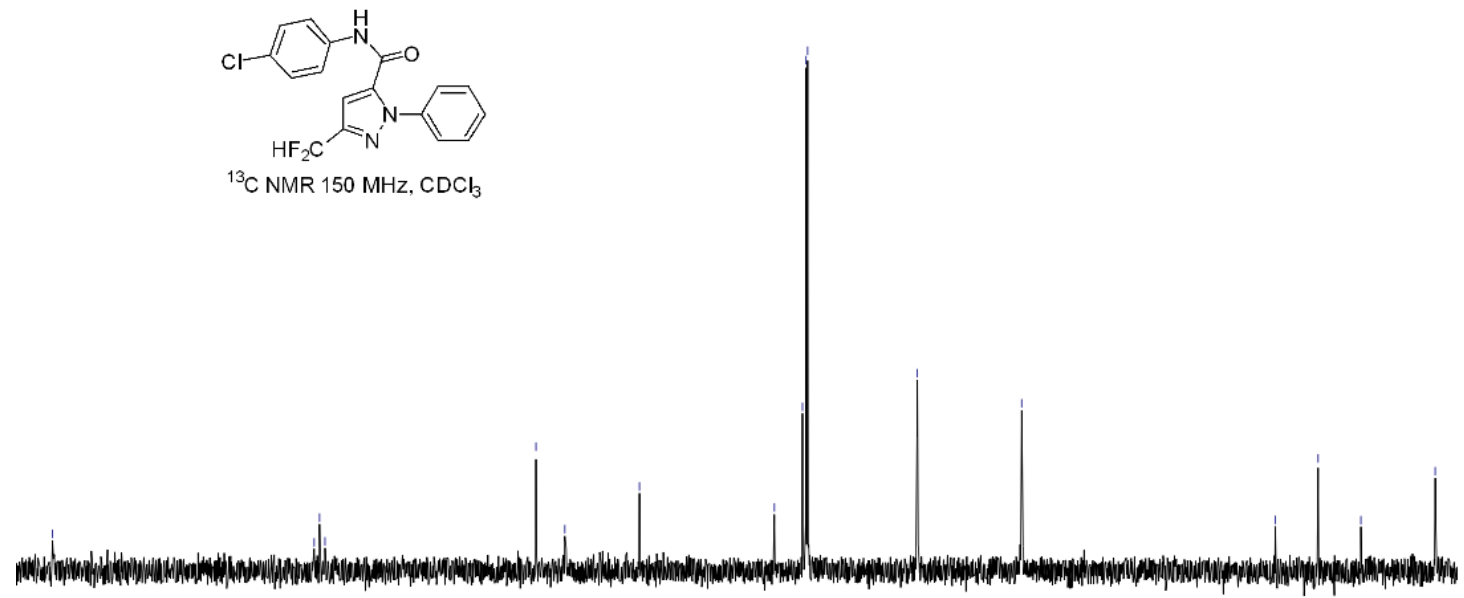

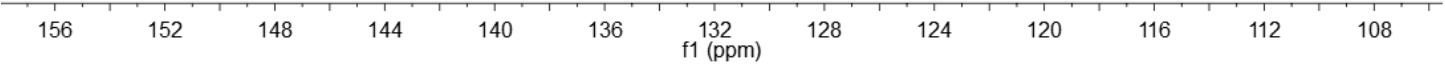




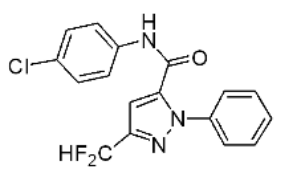

${ }^{19} \mathrm{~F}$ NMR $376 \mathrm{MHz}, \mathrm{CDCl}_{3}$
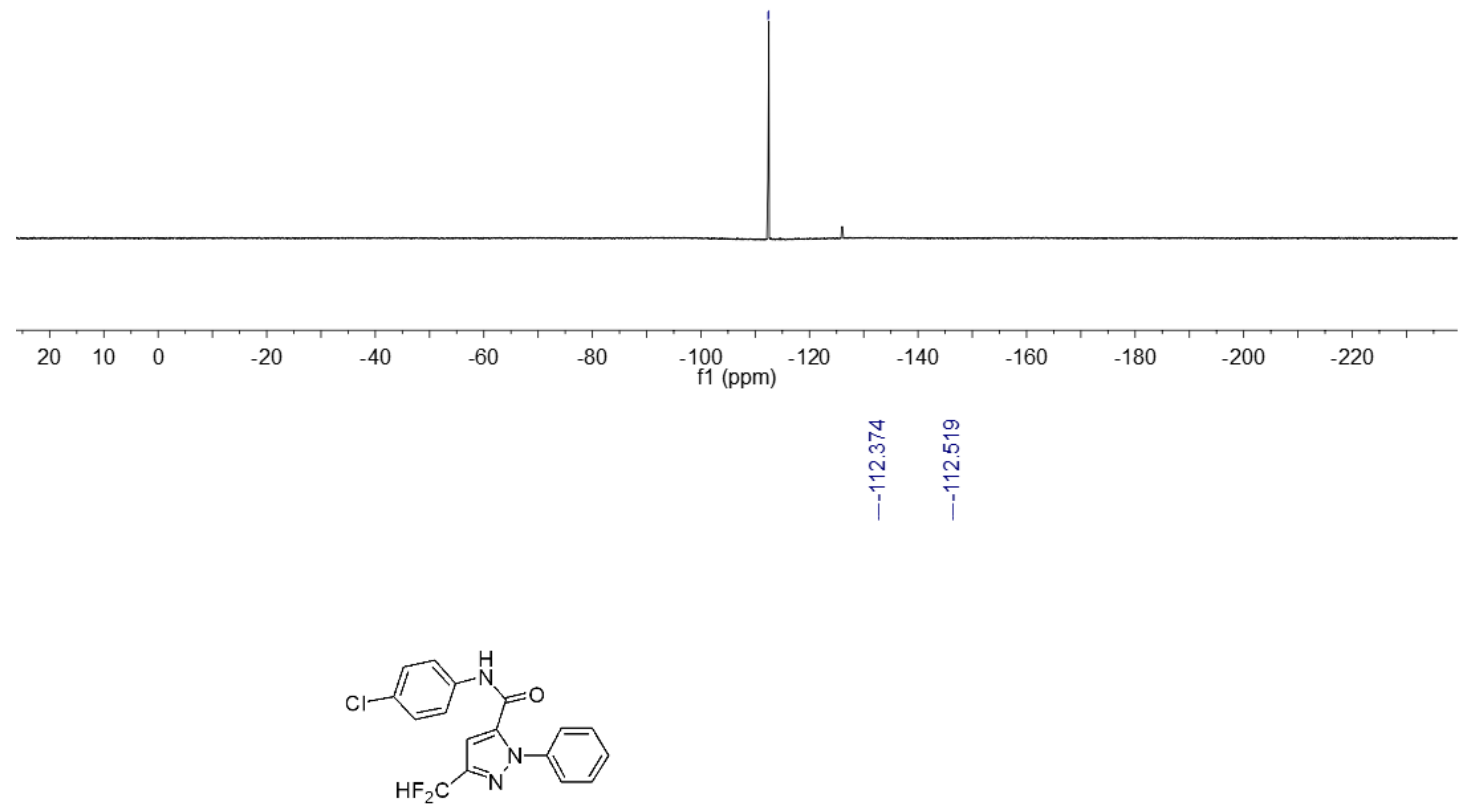

${ }^{19} \mathrm{~F}$ NMR $376 \mathrm{MHz}, \mathrm{CDCl}_{3}$

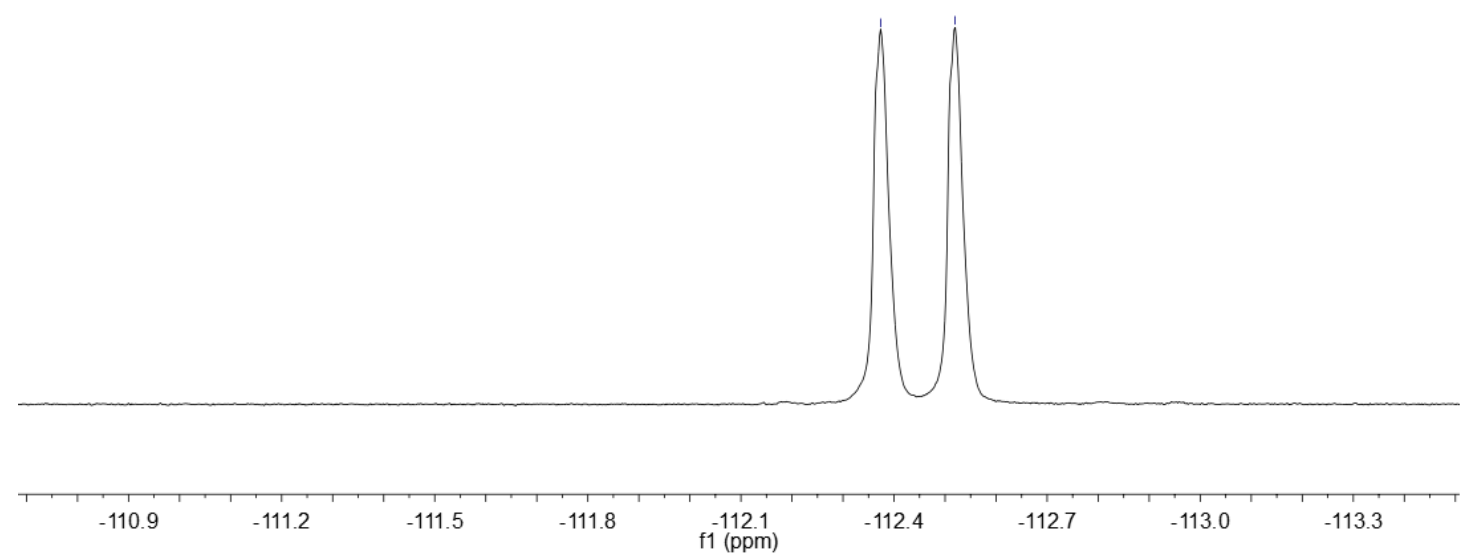


HRMS (ESI) copy of compound 7c:

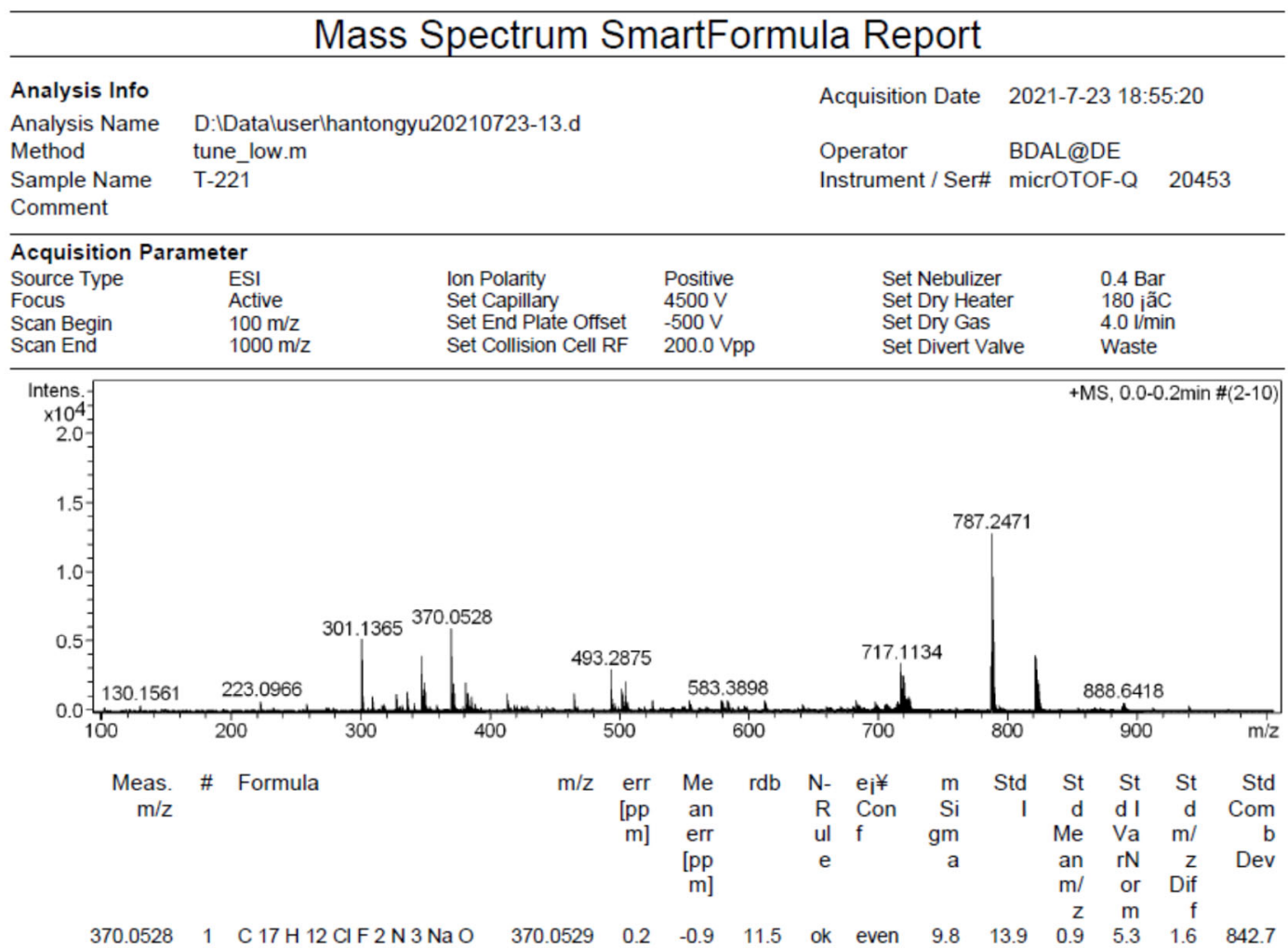

NMR copies of compound $\mathbf{7 d}$ :

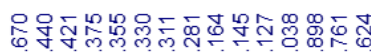

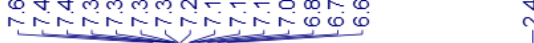

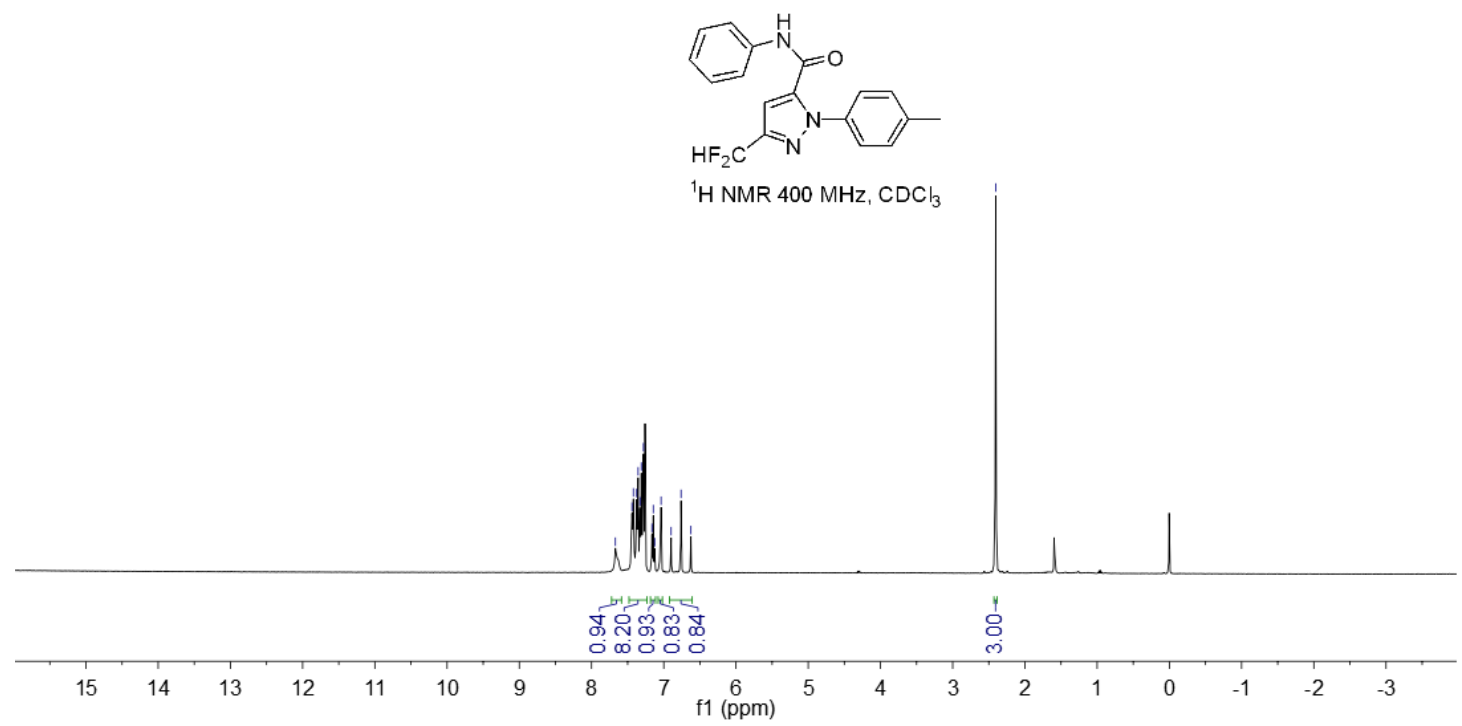




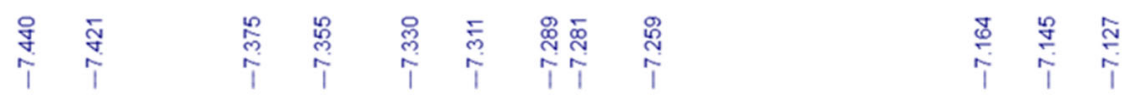

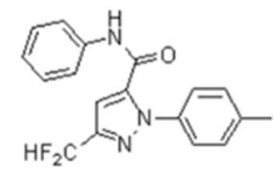

${ }^{1} \mathrm{H}$ NMR $400 \mathrm{MHz}, \mathrm{CDCl}_{3}$

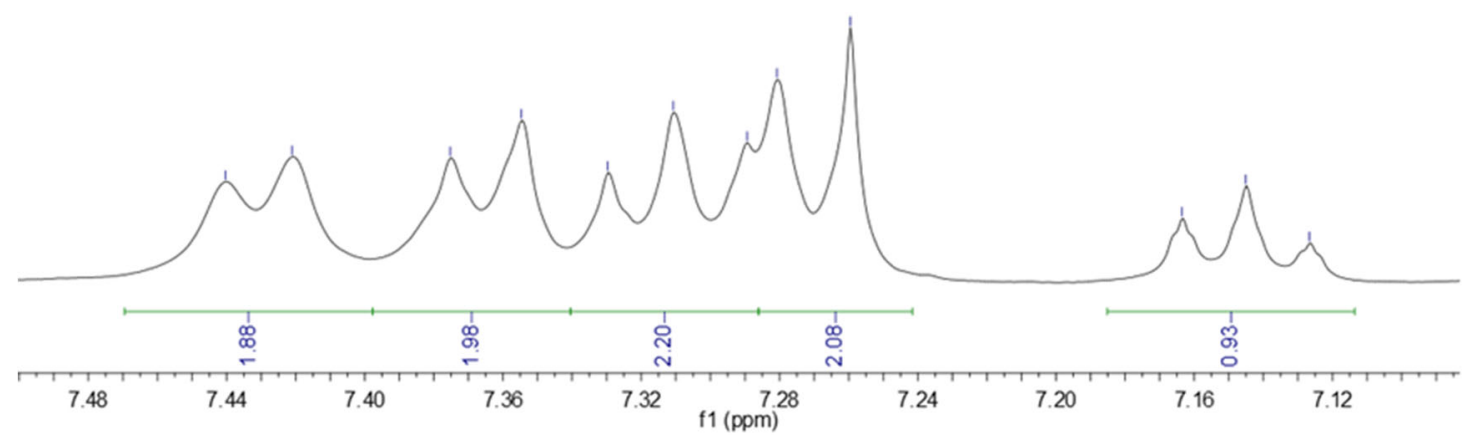

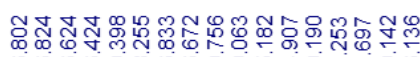

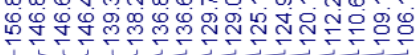

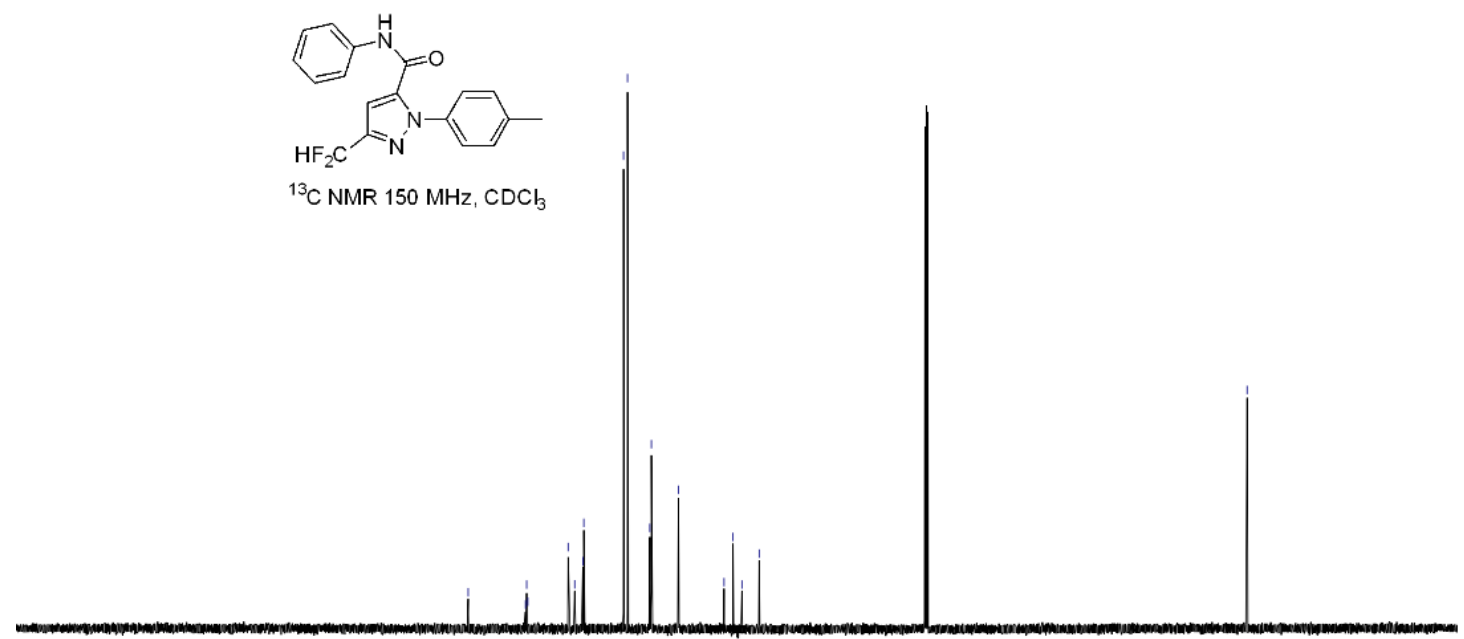

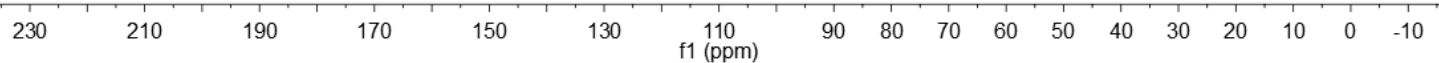




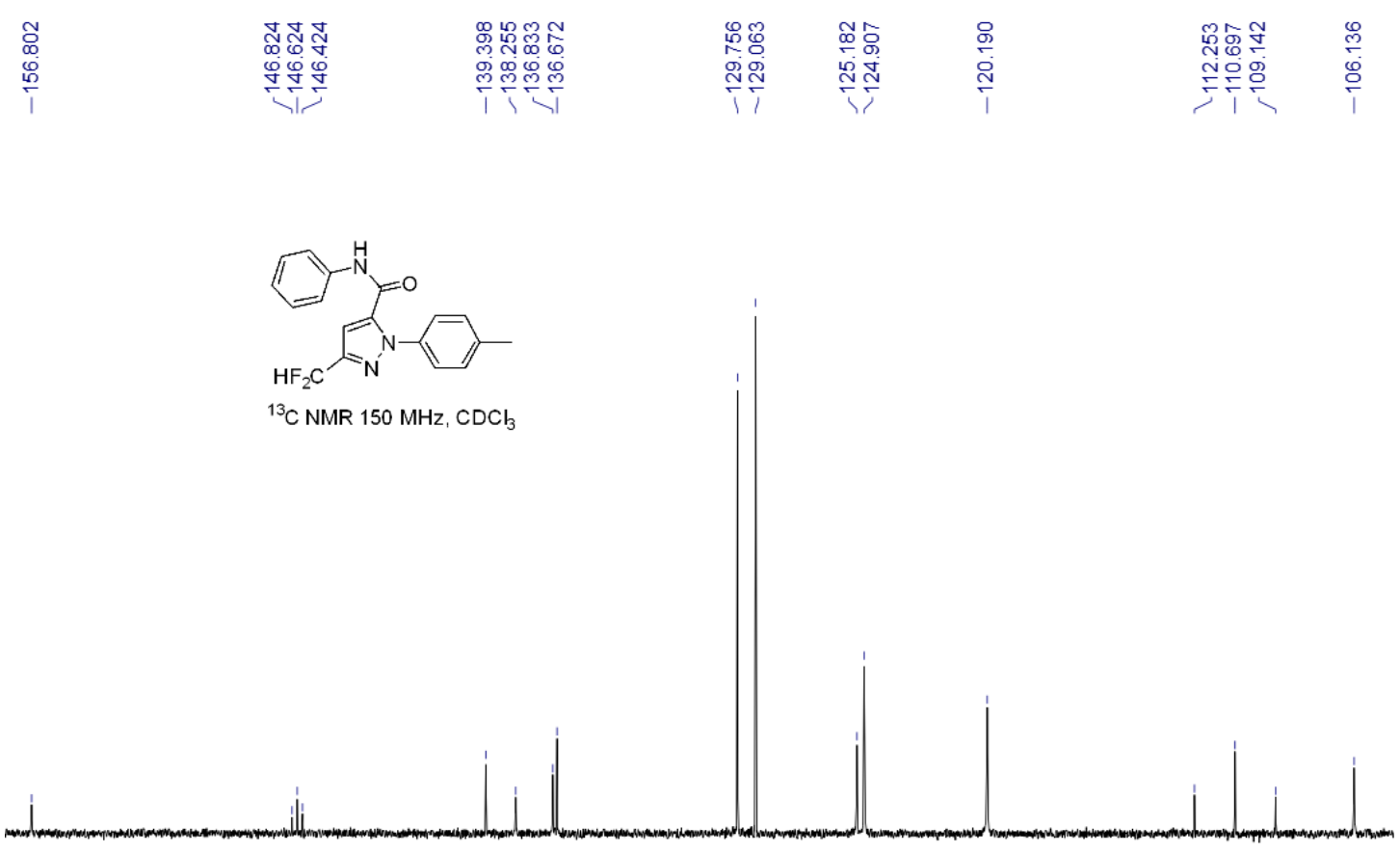

\begin{tabular}{|c|c|c|c|c|c|c|c|c|c|c|c|c|}
\hline 156 & 152 & 148 & 144 & 140 & 136 & $\begin{array}{l}132 \\
\mathrm{f} 1 \text { (ppm) }\end{array}$ & 128 & 124 & 120 & 116 & 112 & 108 \\
\hline
\end{tabular}

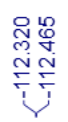

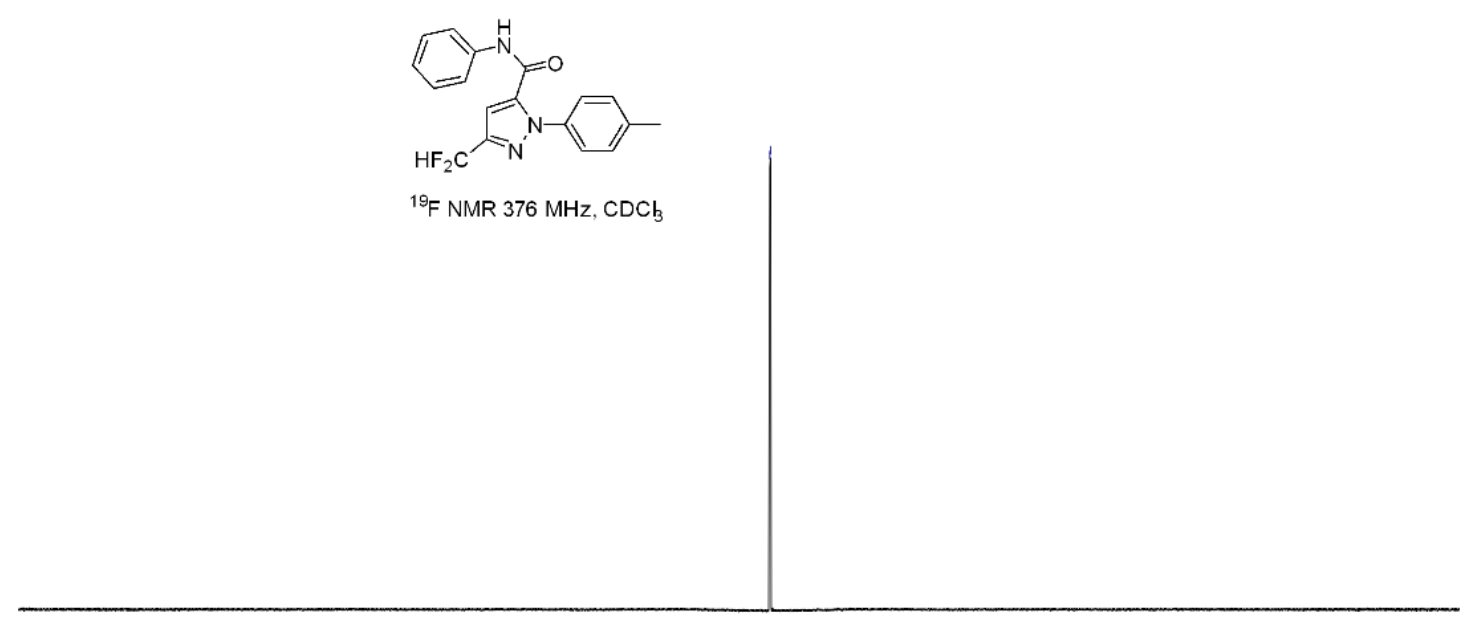

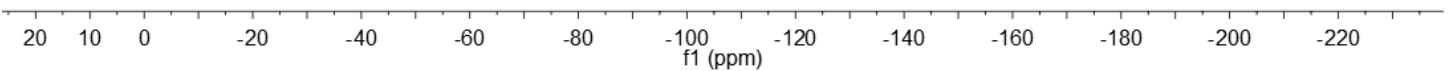




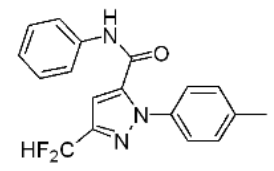

${ }^{19} \mathrm{~F}$ NMR $376 \mathrm{MHz}, \mathrm{CDCl}$

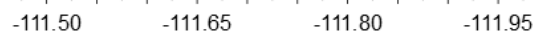

-112.10
$\mathrm{f} 1(\mathrm{ppm})$

$-112.25$

$-112.40$

$-112.55$

$-112.70$

HRMS (ESI) copy of compound 7d:

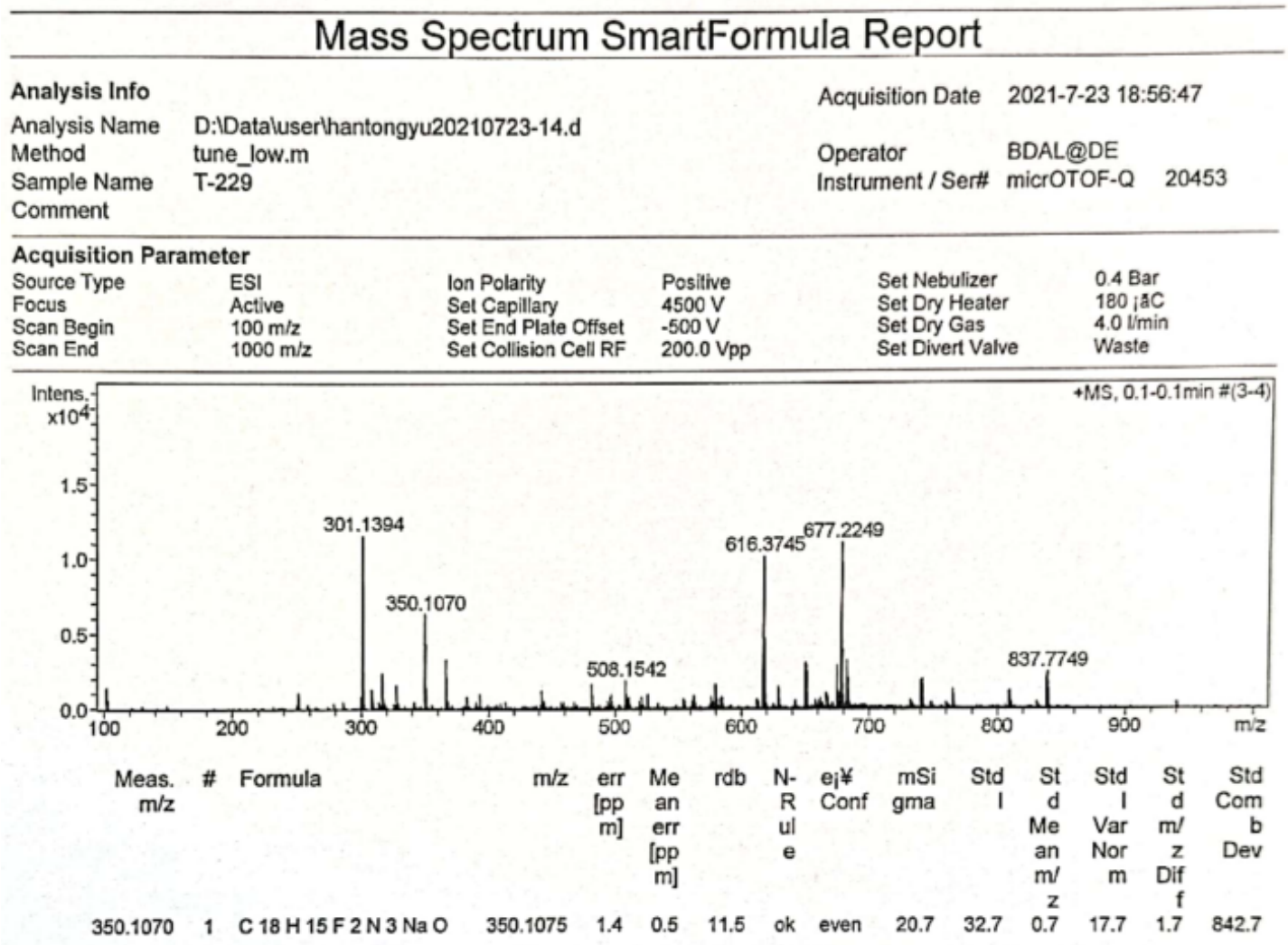


NMR copies of compound 7e:

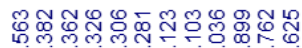

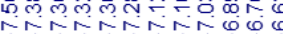

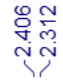

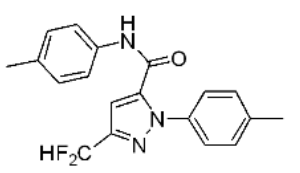

${ }^{1} \mathrm{HNMR} 400 \mathrm{MHz}, \mathrm{CDCl}_{3}$

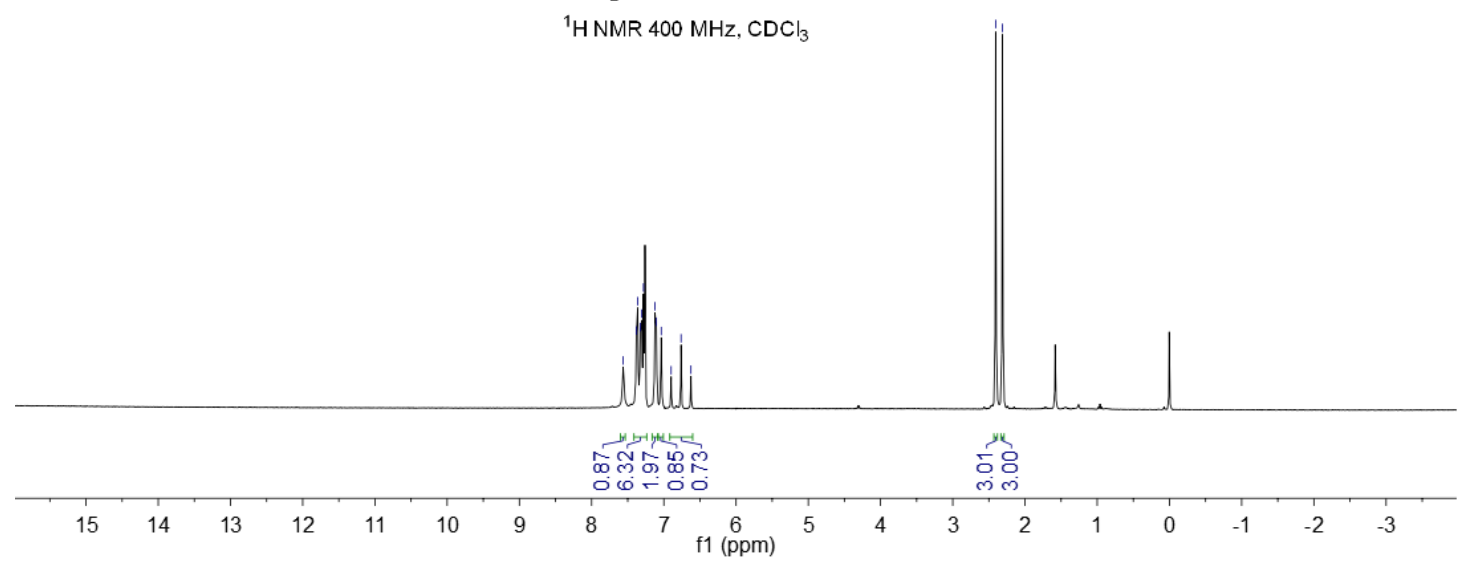

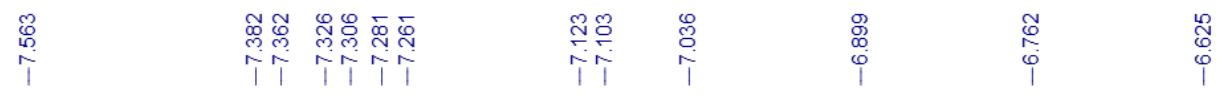

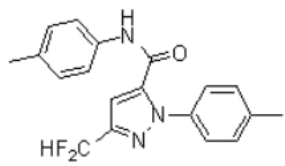

${ }^{1} \mathrm{HNMR} 400 \mathrm{MHz}, \mathrm{CDCl}$

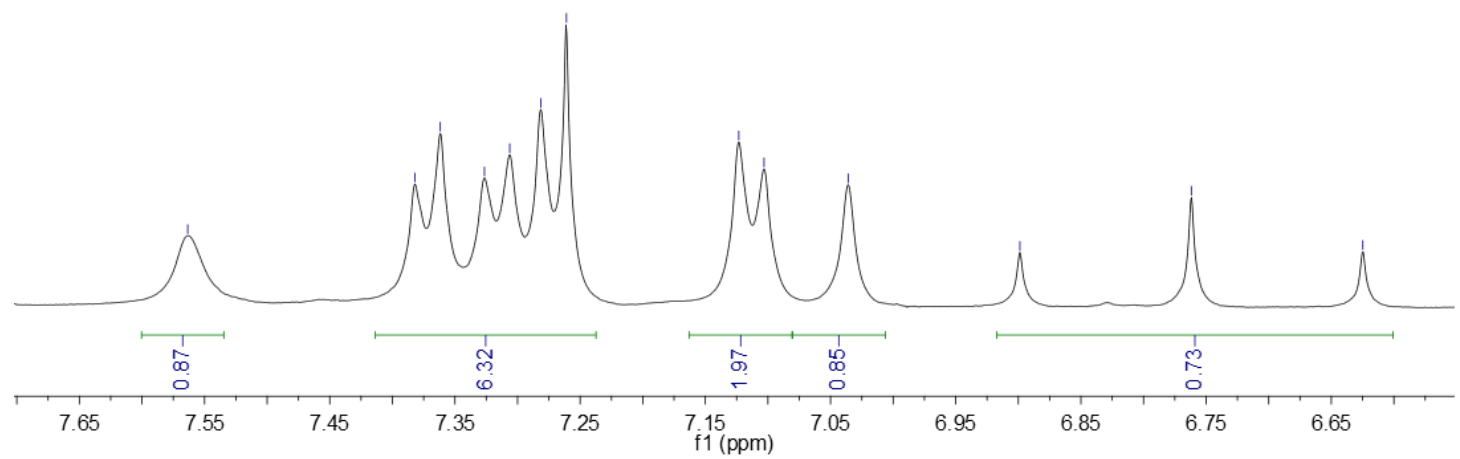




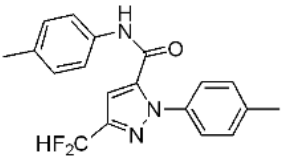

${ }^{13} \mathrm{C} \mathrm{NMR} 150 \mathrm{MHz}, \mathrm{CDCl}_{3}$

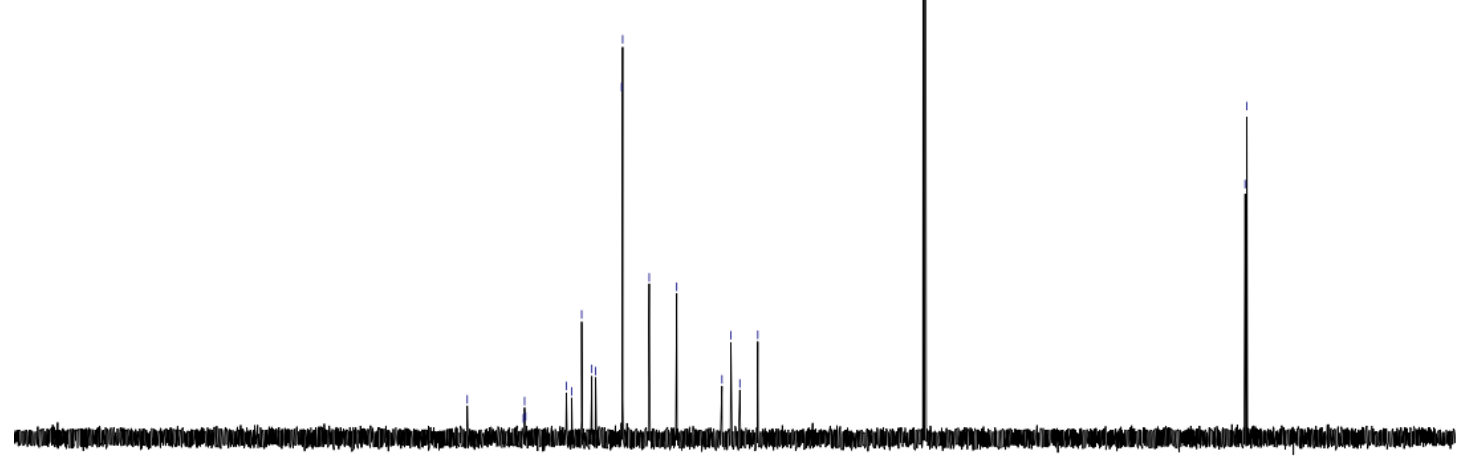

\begin{tabular}{|c|c|c|c|c|c|c|c|c|c|c|c|c|c|c|c|c|c|}
\hline 230 & 210 & 190 & 170 & 150 & 130 & $\begin{array}{c}110 \\
\mathrm{f} 1(\mathrm{ppm})\end{array}$ & 90 & 80 & 70 & 60 & 50 & 40 & 30 & 20 & 10 & 0 & -10 \\
\hline $\begin{array}{l}\sigma \\
\frac{\sigma}{6} \\
6 \\
0 \\
5 \\
\end{array}$ & & & & & 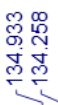 & 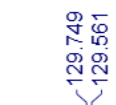 & & 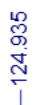 & & 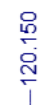 & & & & \begin{tabular}{l}
\multirow{0}{*}{} \\
\multirow{\Sigma}{*}{}
\end{tabular} & $\frac{5}{8}$ & & 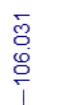 \\
\hline
\end{tabular}

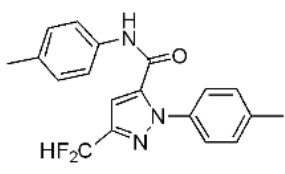

${ }^{13} \mathrm{C} \mathrm{NMR} 150 \mathrm{MHz}, \mathrm{CDCl}_{3}$

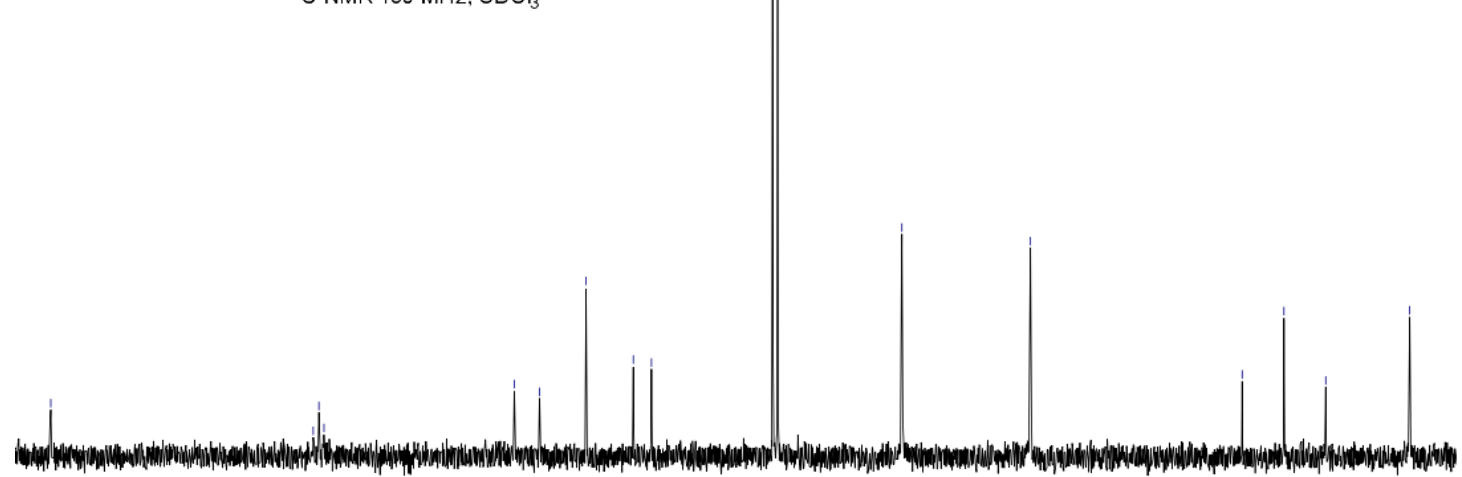

\begin{tabular}{llllllllllllll}
\hline 156 & 152 & 148 & 144 & 140 & 136 & $\begin{array}{l}132 \\
\mathrm{f} 1(\mathrm{ppm})\end{array}$ & 128 & 124 & 120 & 116 & 112 & 108
\end{tabular}



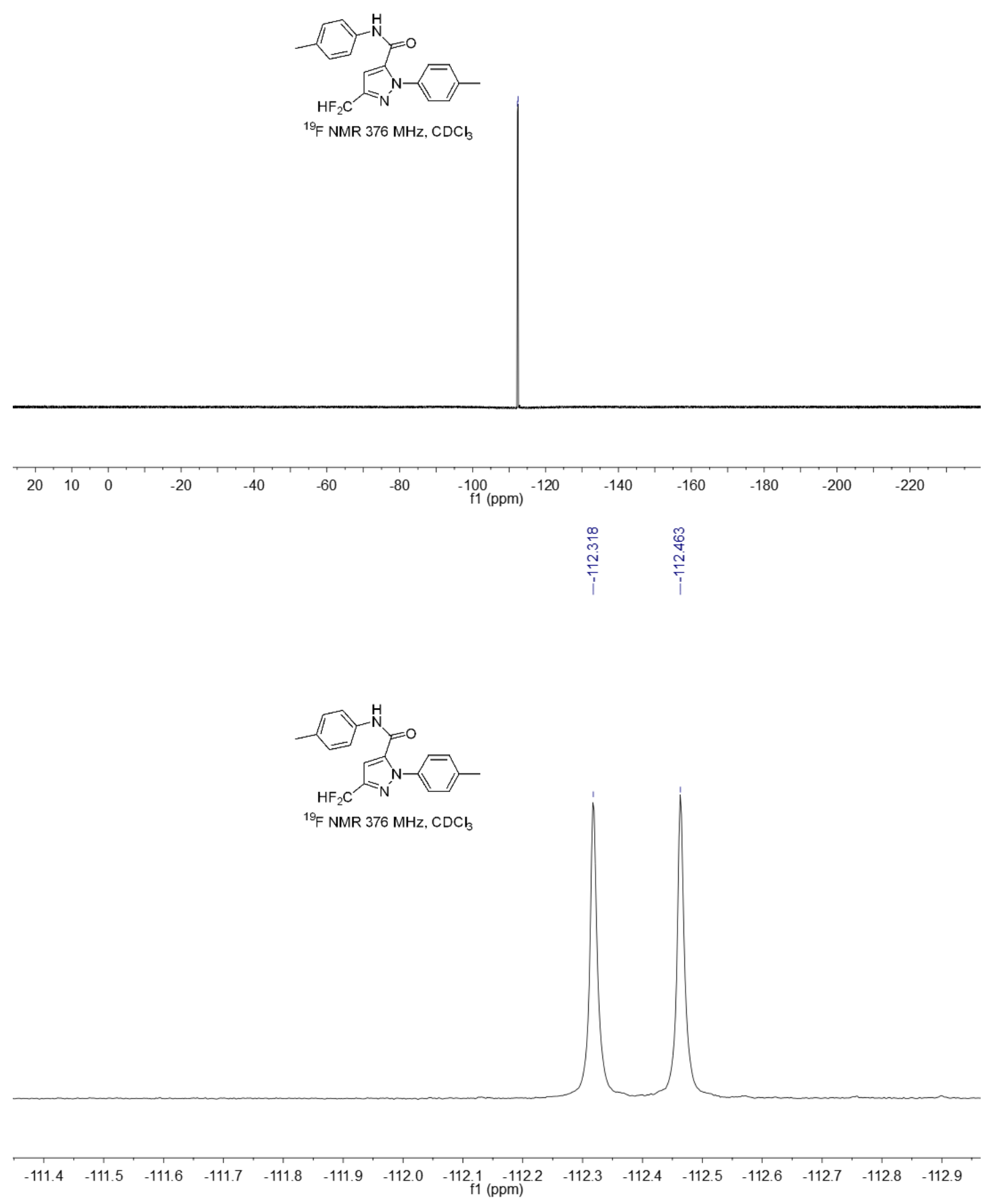
HRMS (ESI) copy of compound 7e:

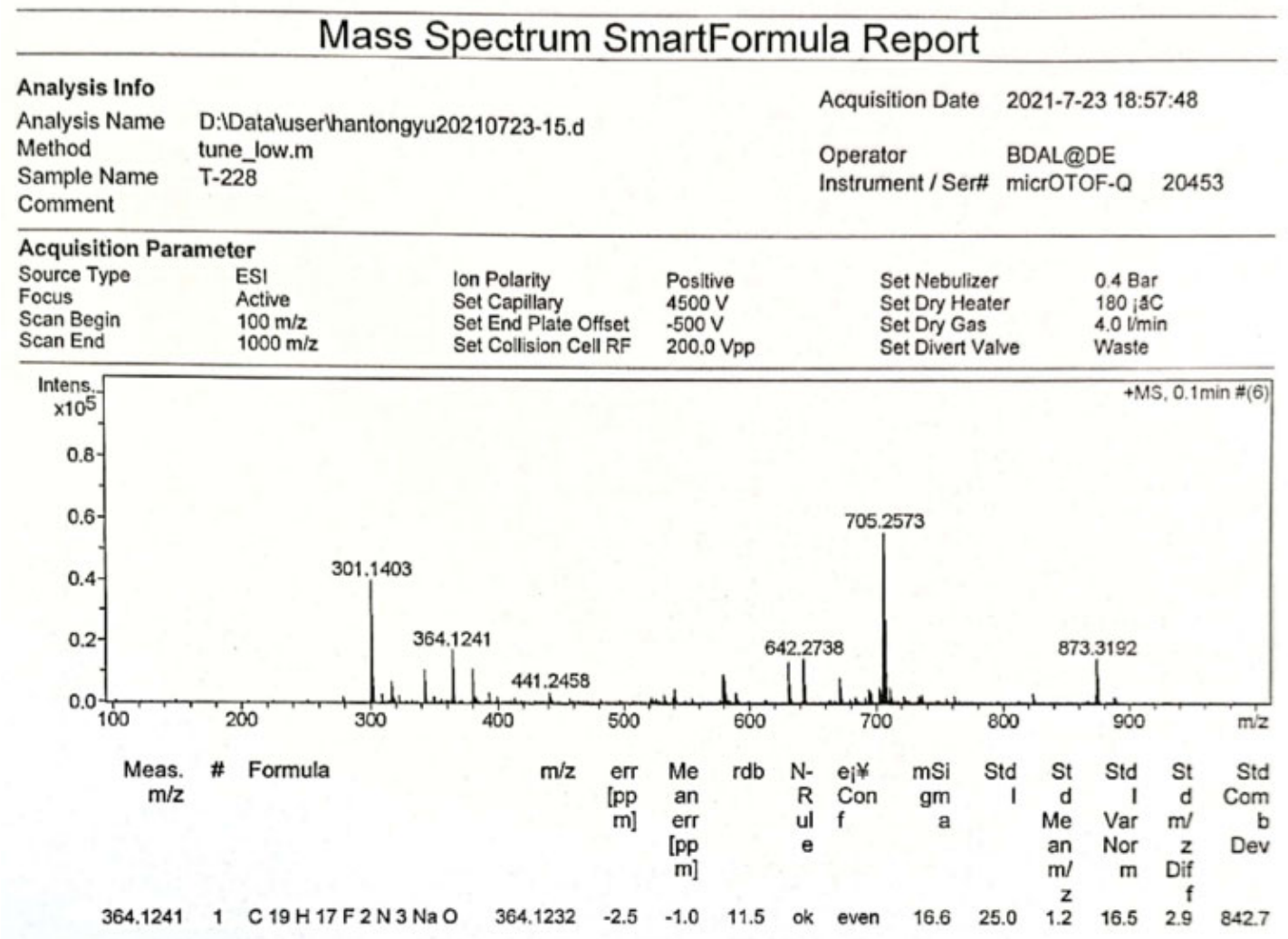

NMR copies of compound 7f:

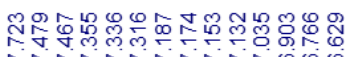

NNMNiñ

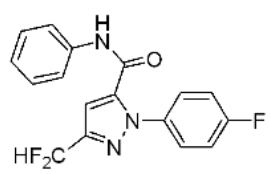

${ }^{1} \mathrm{H}$ NMR $400 \mathrm{MHz}, \mathrm{CDCl}_{3}$

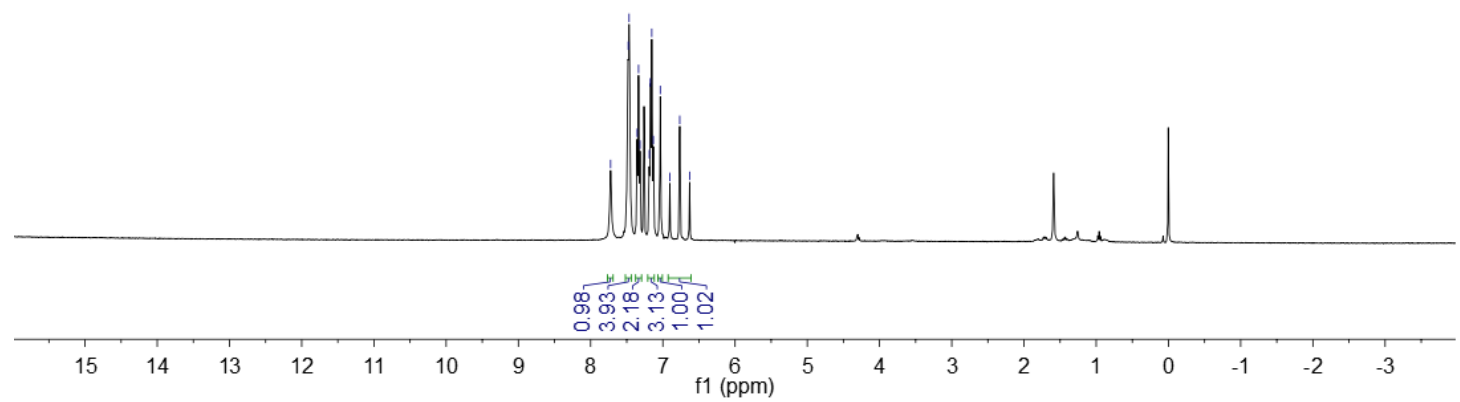




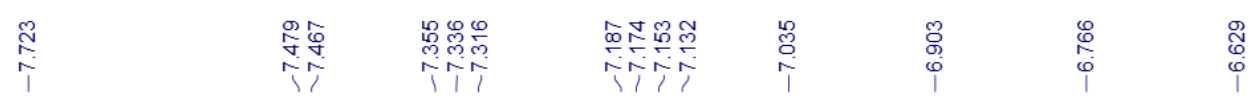

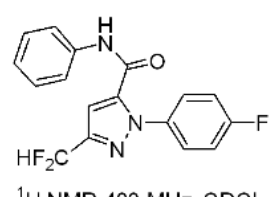

${ }^{1} \mathrm{H} \mathrm{NMR} 400 \mathrm{MHz}, \mathrm{CDCl}_{3}$

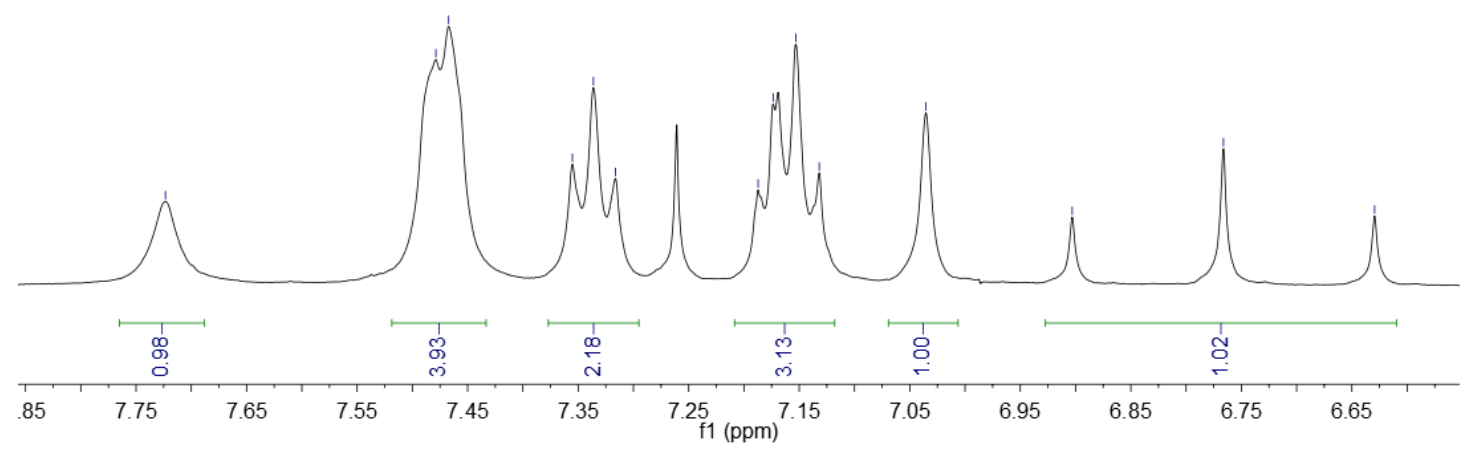

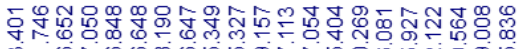

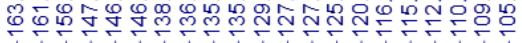

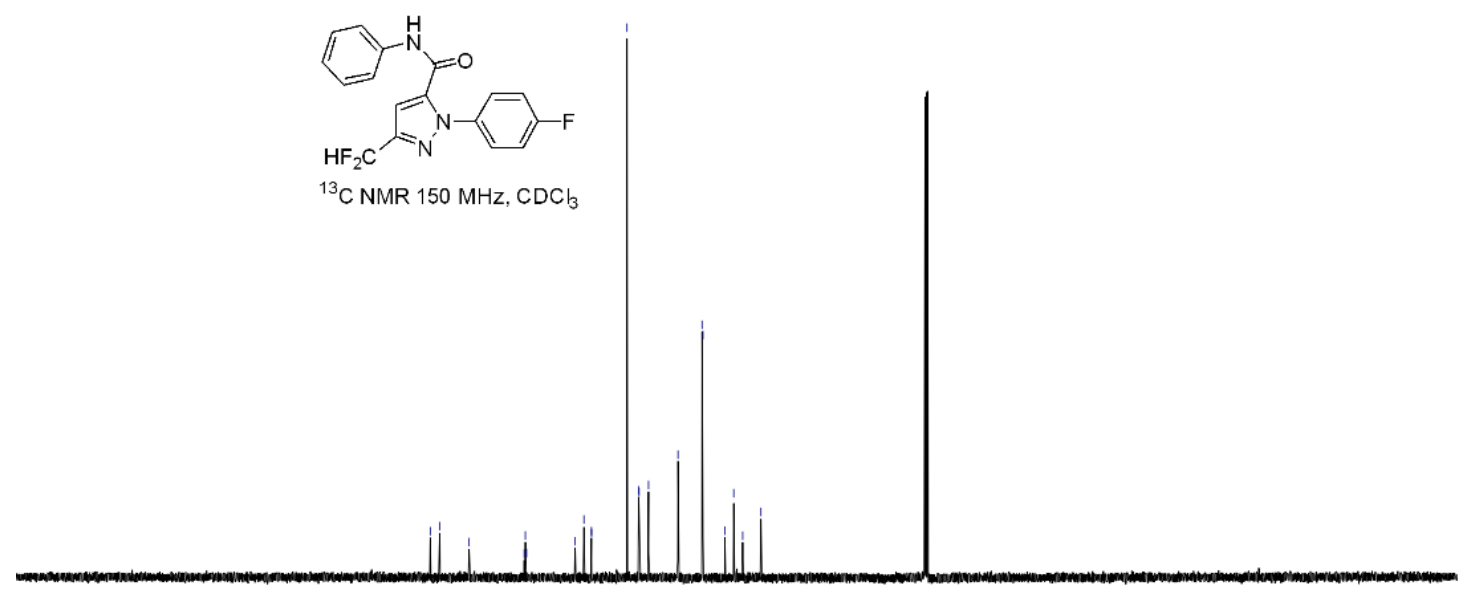

230

190

170

50

130

11 (ppm)

$\begin{array}{lllllllllll}90 & 80 & 70 & 60 & 50 & 40 & 30 & 20 & 10 & 0 & -10\end{array}$ 


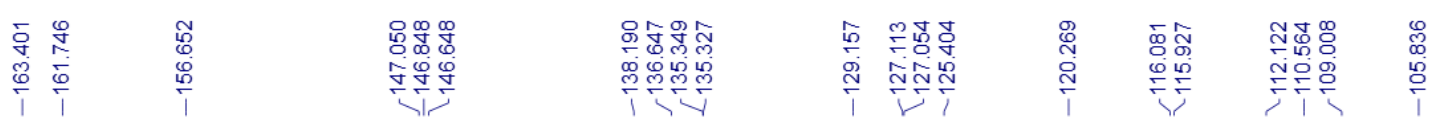
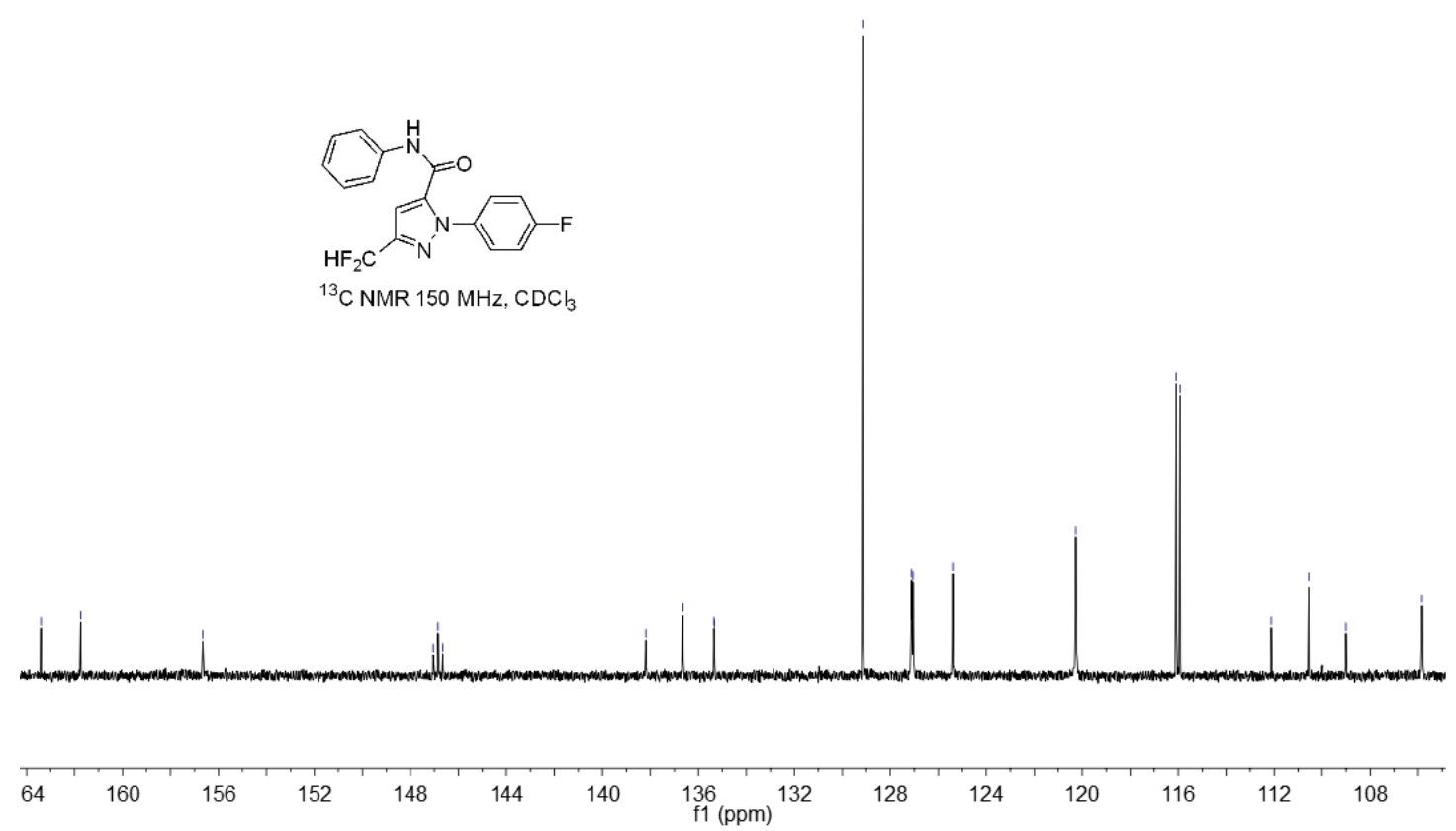

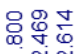

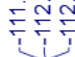
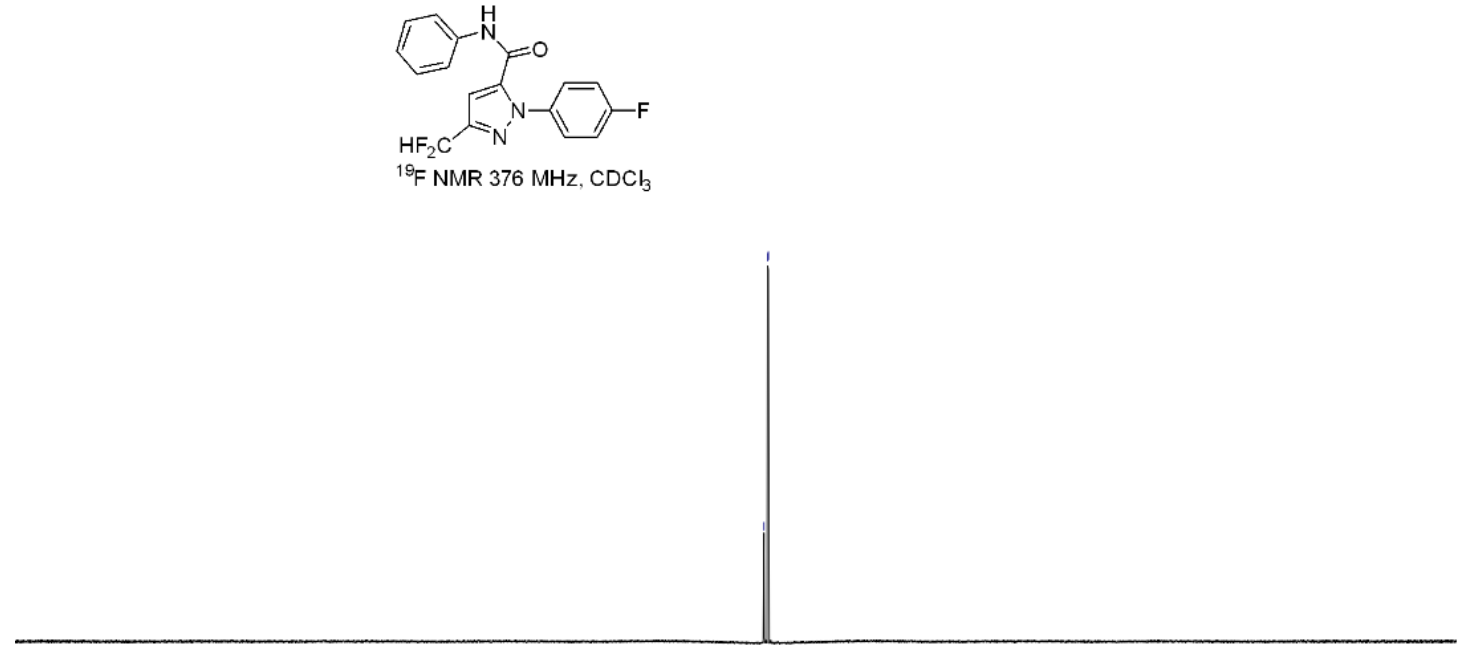

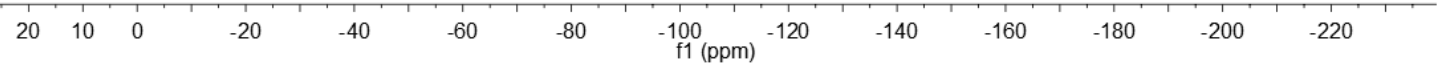




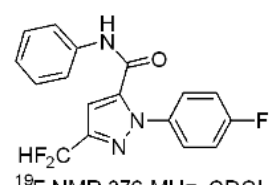

${ }^{19} \mathrm{~F}$ NMR $376 \mathrm{MHz}, \mathrm{CDCl}_{3}$

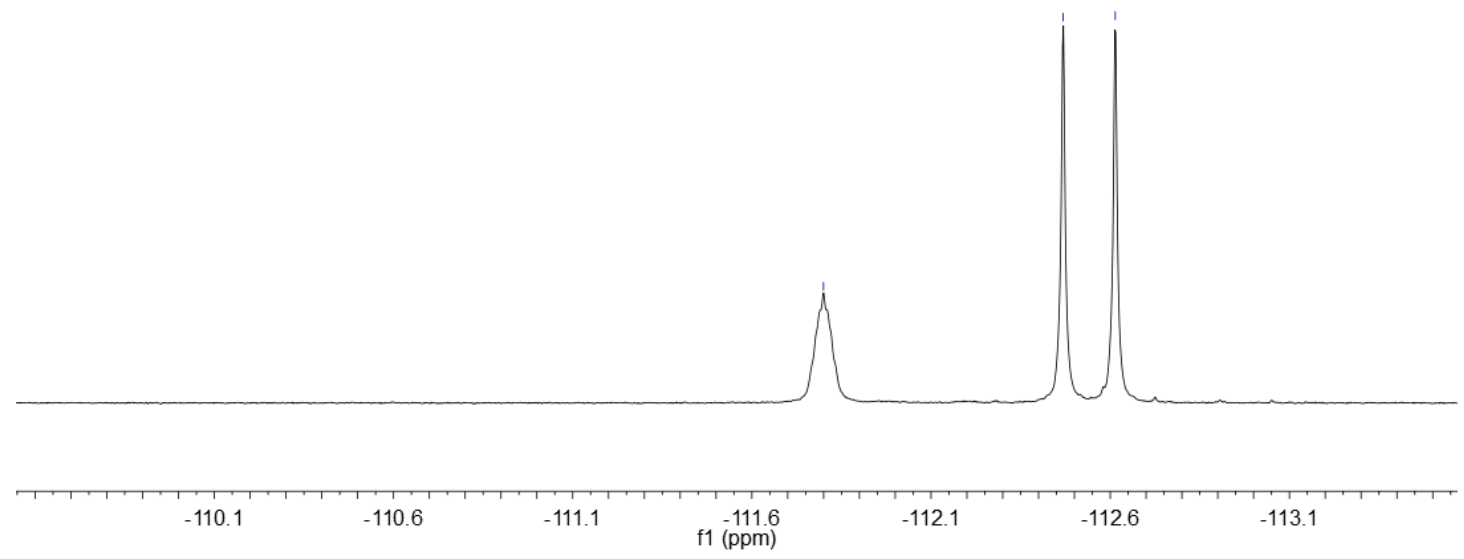

HRMS (ESI) copy of compound 7f:

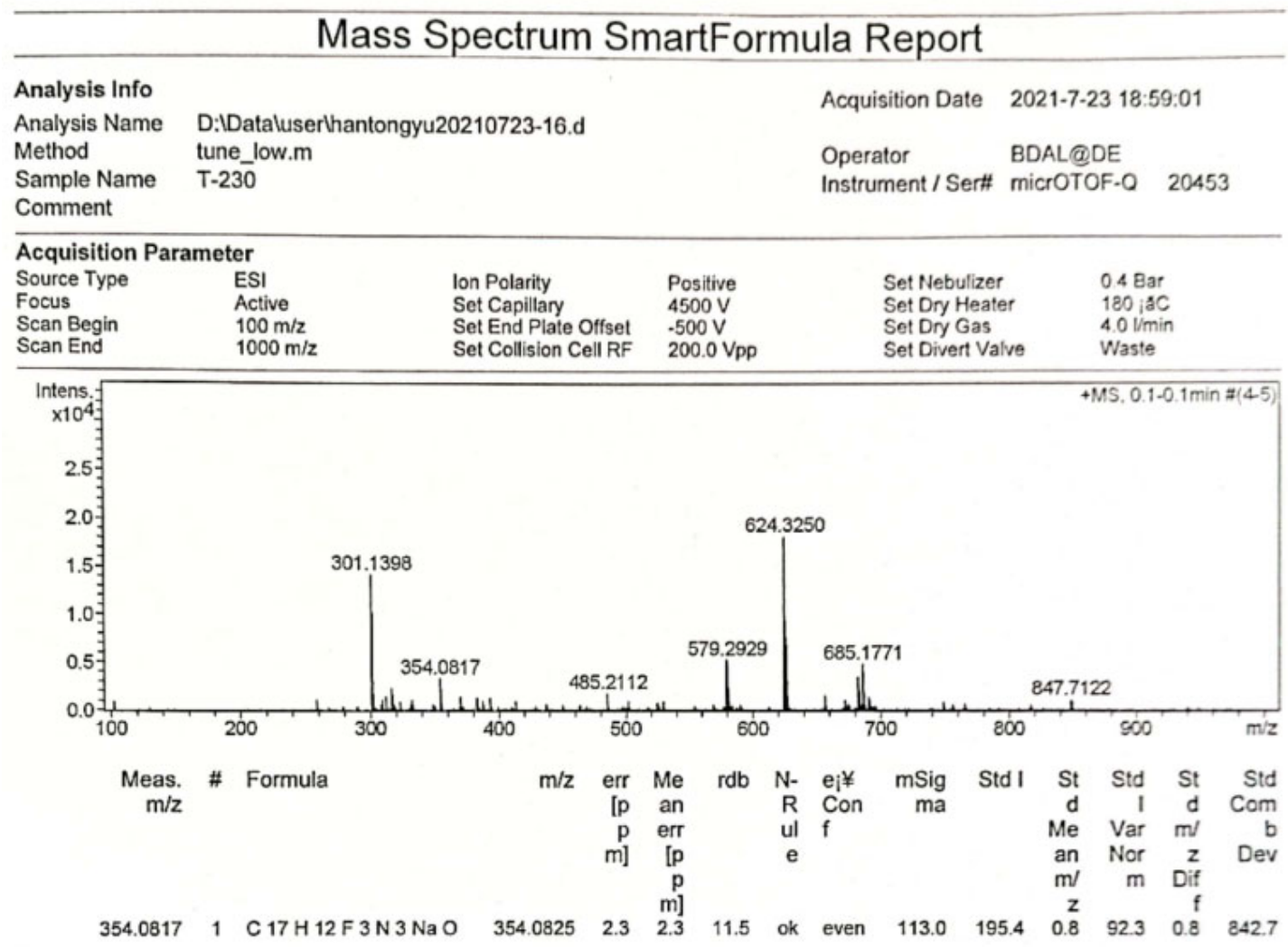




\section{NMR copies of compound $7 \mathrm{~g}$ :}

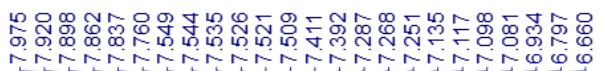

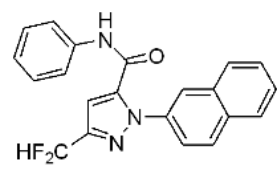

${ }^{1} \mathrm{H}$ NMR $400 \mathrm{MHz}, \mathrm{CDCl}_{3}$
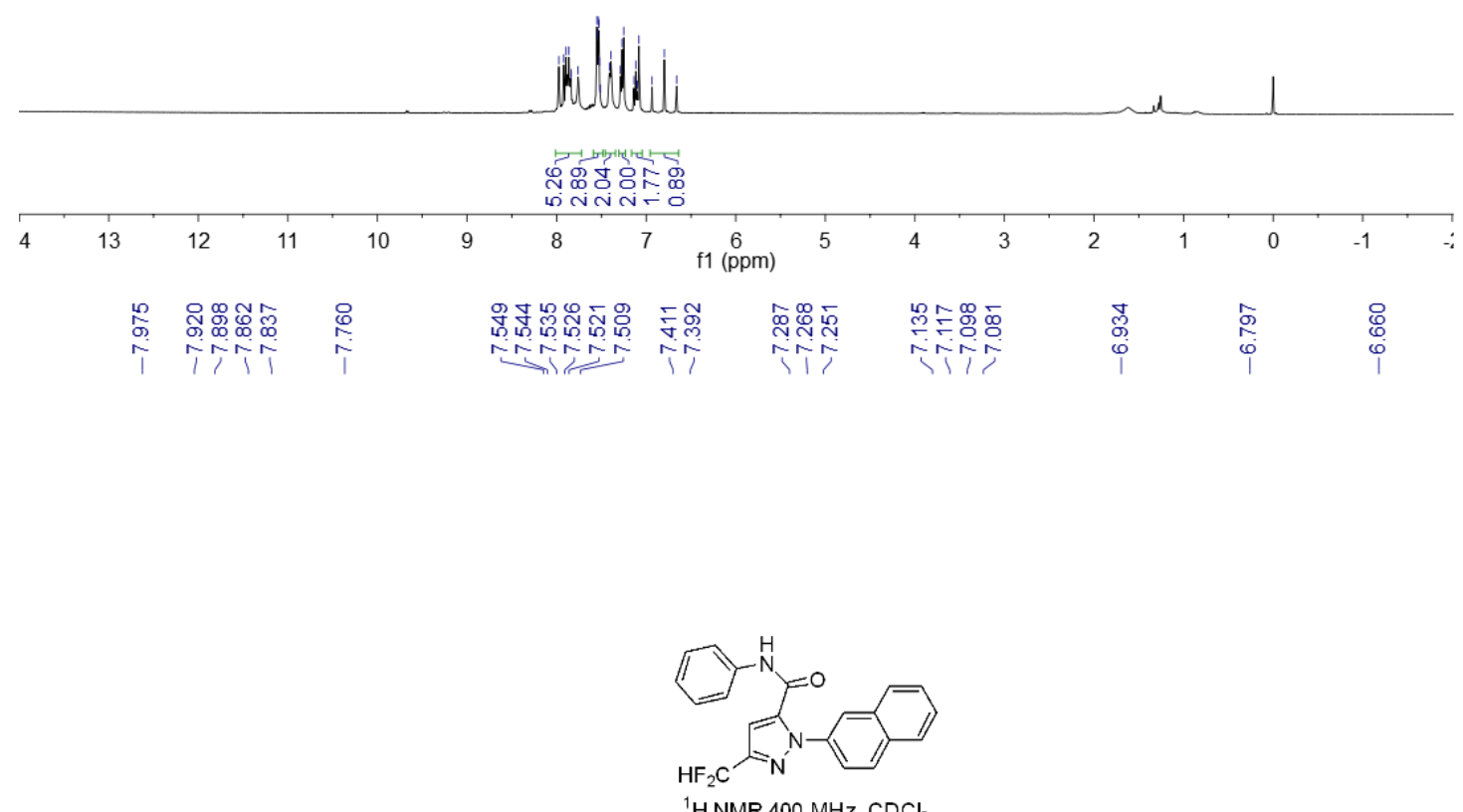

${ }^{1} \mathrm{HNMR} 400 \mathrm{MHz}, \mathrm{CDCl}_{3}$

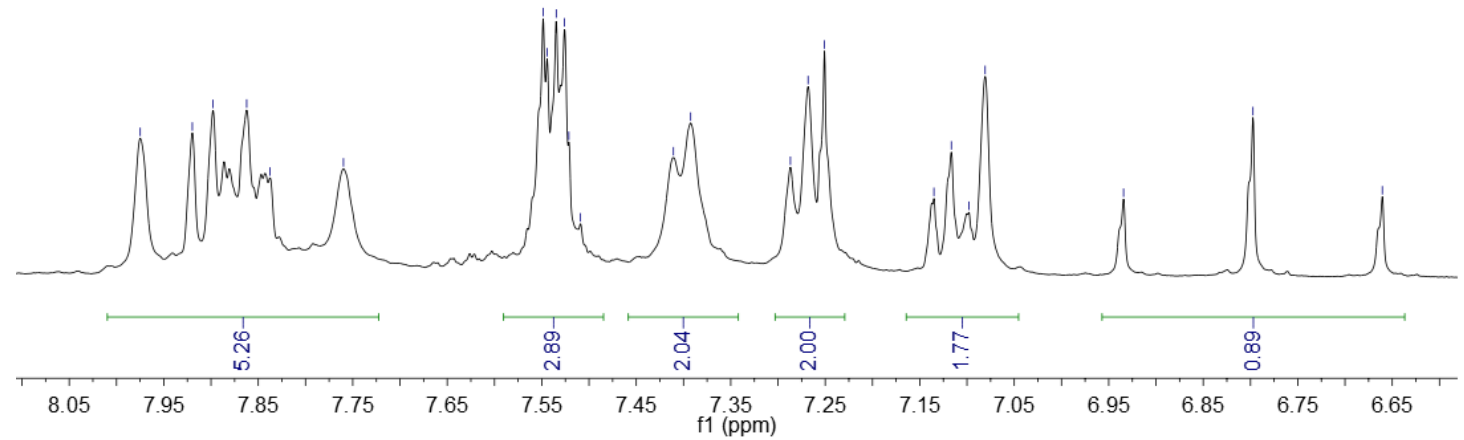




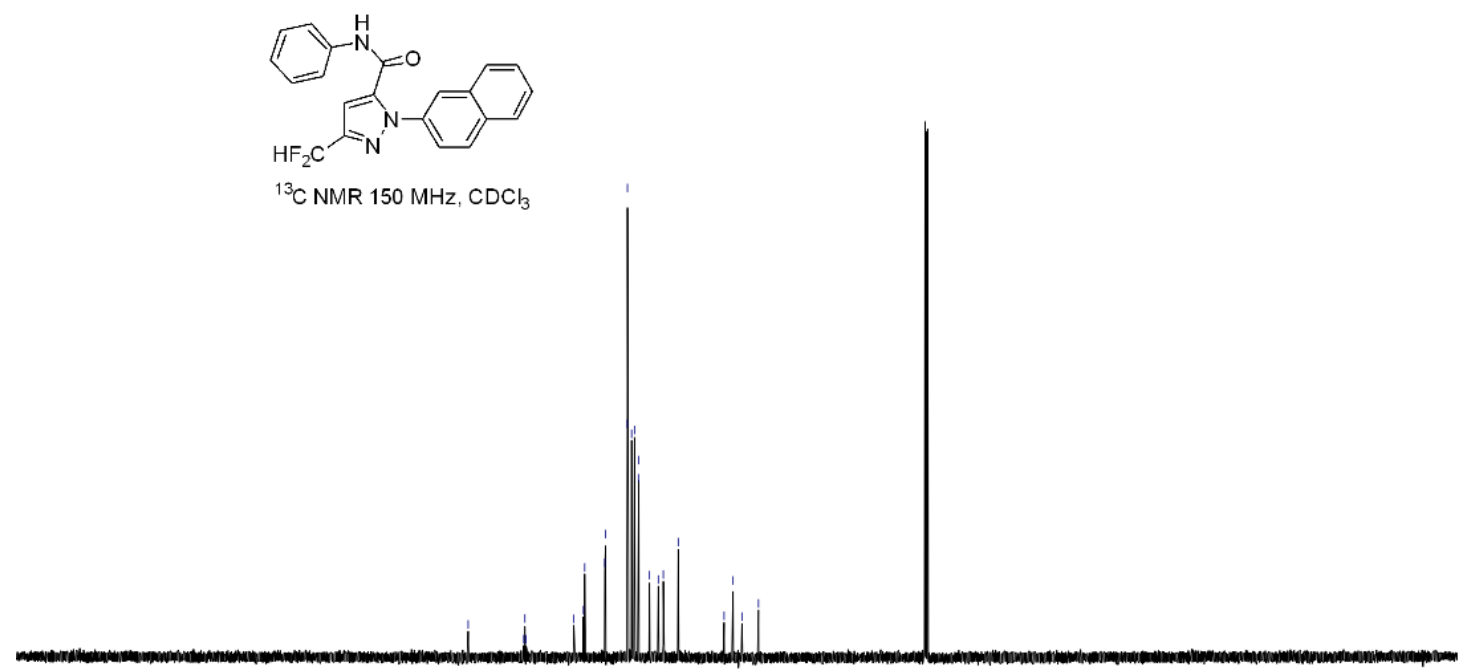

\begin{tabular}{|c|c|c|c|c|c|c|c|c|c|c|c|c|c|c|c|c|c|}
\hline 230 & 210 & 190 & 170 & 150 & 130 & $\begin{array}{c}110 \\
\mathrm{f} 1(\mathrm{ppm})\end{array}$ & 90 & 80 & 70 & 60 & 50 & 40 & 30 & 20 & 10 & 0 & -10 \\
\hline $\begin{array}{l}\stackrel{2}{0} \\
0 \\
0 \\
0 \\
0 \\
\end{array}$ & & & & & & 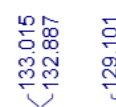 & 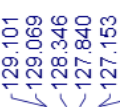 & 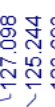 & 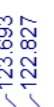 & 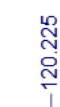 & & & & & 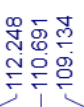 & & $\begin{array}{l}\overline{\mathscr{g}} \\
\stackrel{0}{0}\end{array}$ \\
\hline
\end{tabular}

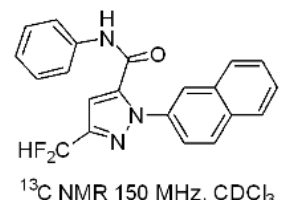

${ }^{13} \mathrm{C} \mathrm{NMR} 150 \mathrm{MHz}, \mathrm{CDCl}_{3}$

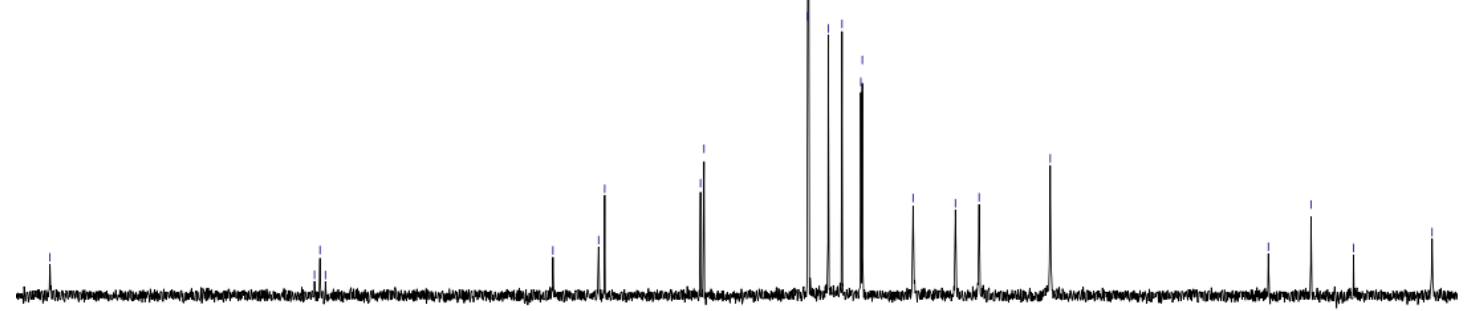

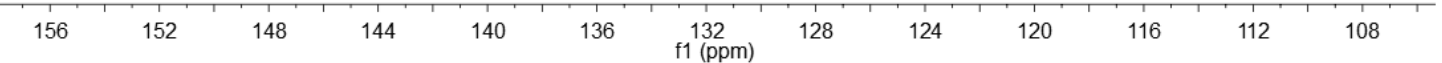



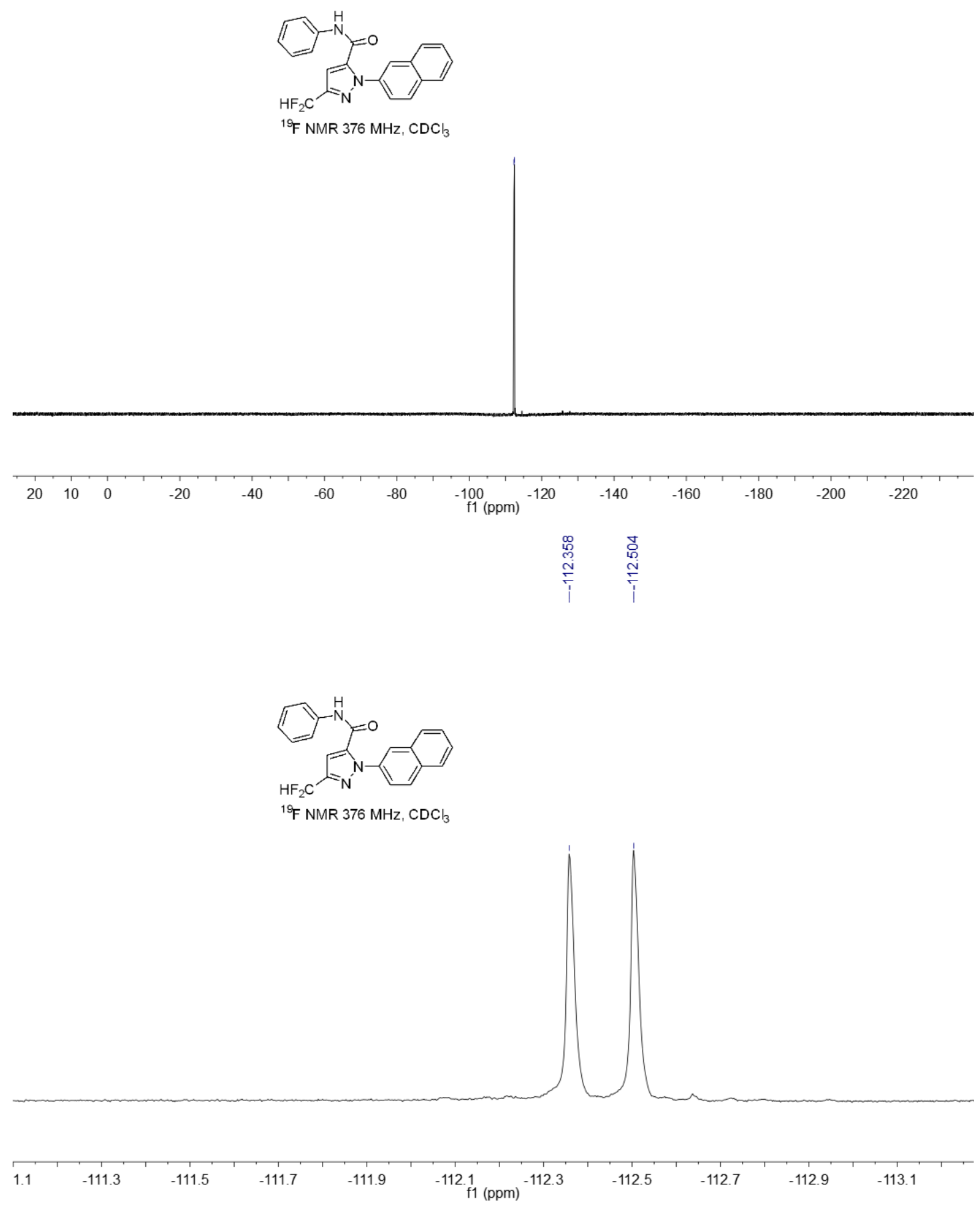
HRMS (ESI) copy of compound 7g:

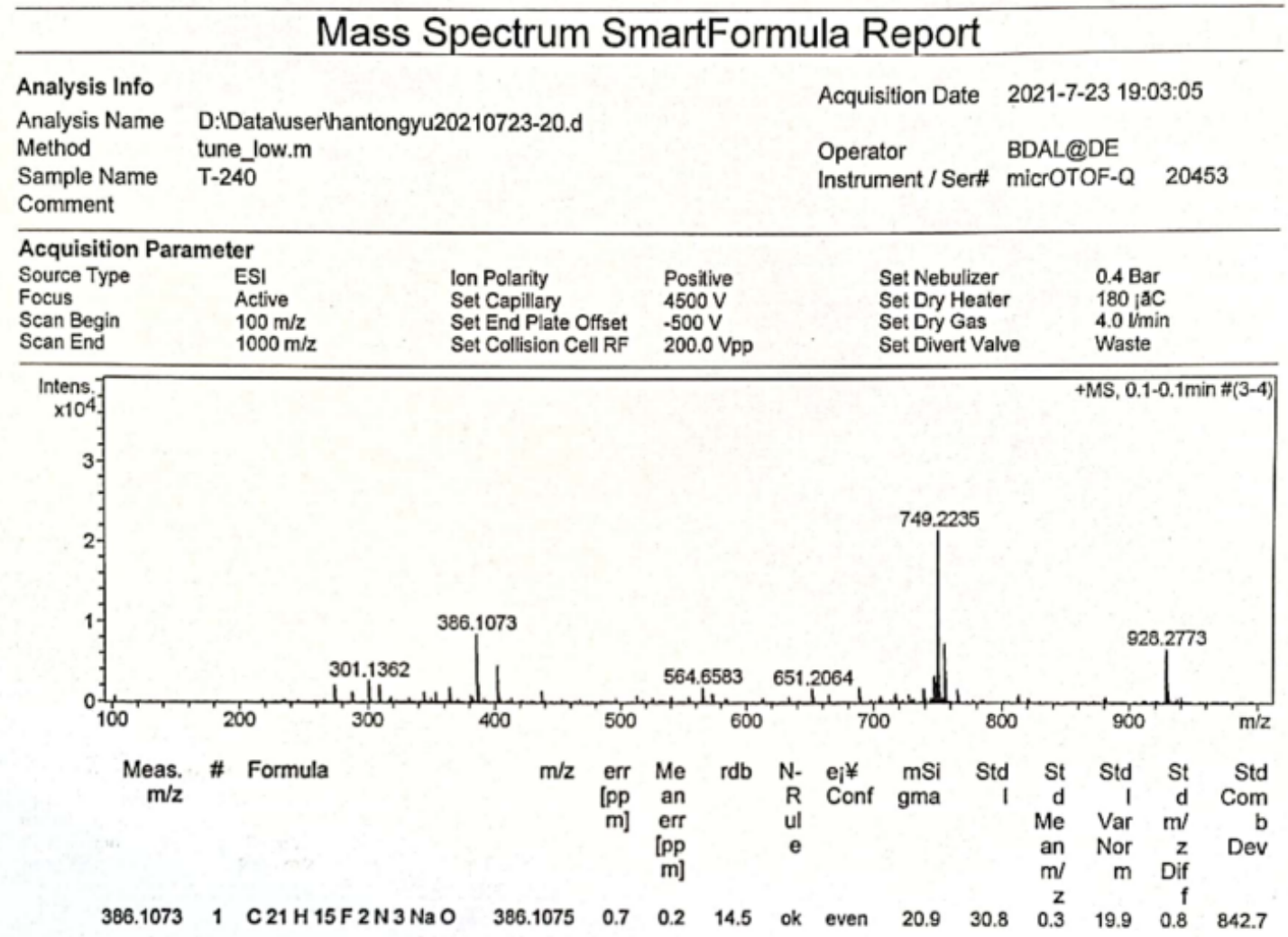

NMR copies of compound $\mathbf{7 h}$ :

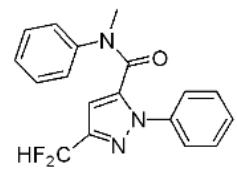

${ }^{1} \mathrm{H} \mathrm{NMR} 400 \mathrm{MHz}, \mathrm{CDCl}_{3}$

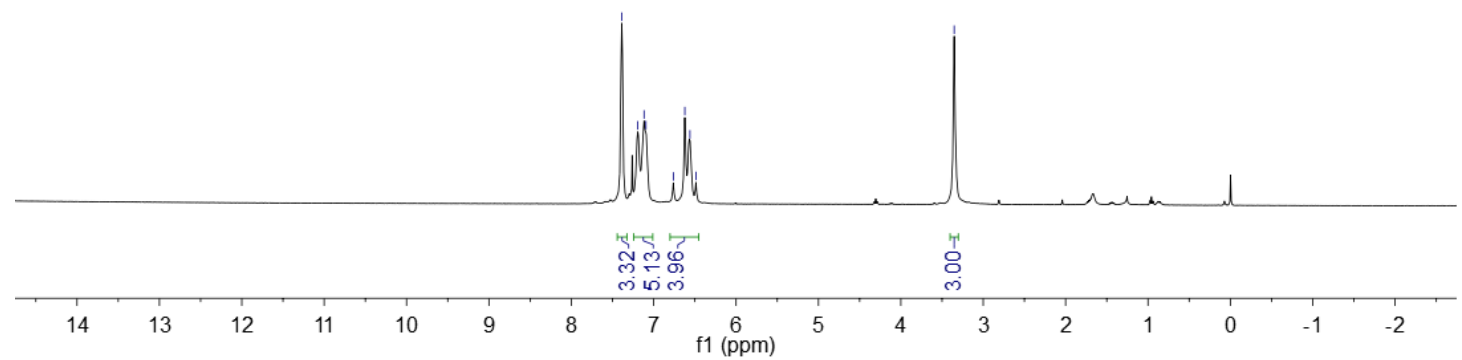




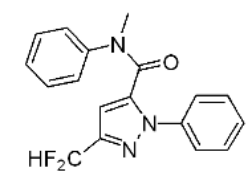

${ }^{1} \mathrm{H} \mathrm{NMR} 400 \mathrm{MHz}, \mathrm{CDCl}_{3}$
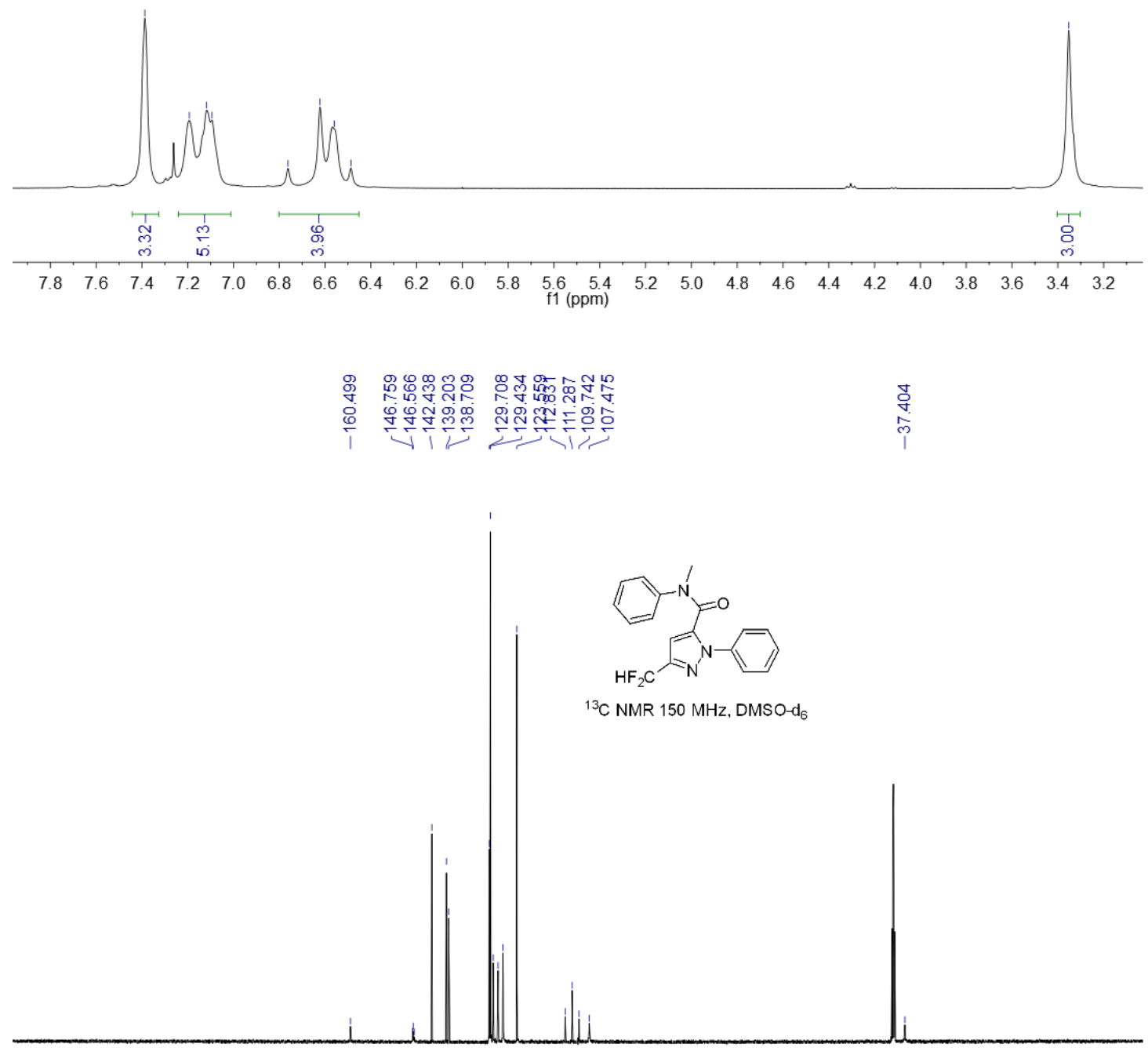

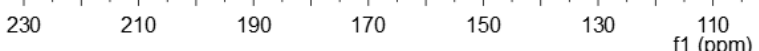

$\begin{array}{lllllllllll}90 & 80 & 70 & 60 & 50 & 40 & 30 & 20 & 10 & 0 & -10\end{array}$ 
g.

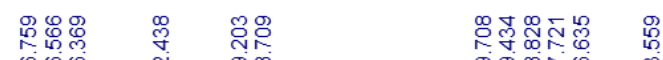

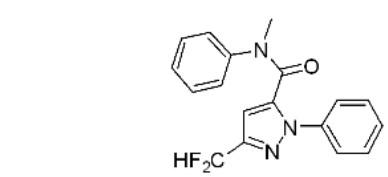

${ }^{13} \mathrm{C}$ NMR $150 \mathrm{MHz}$, DMSO-d
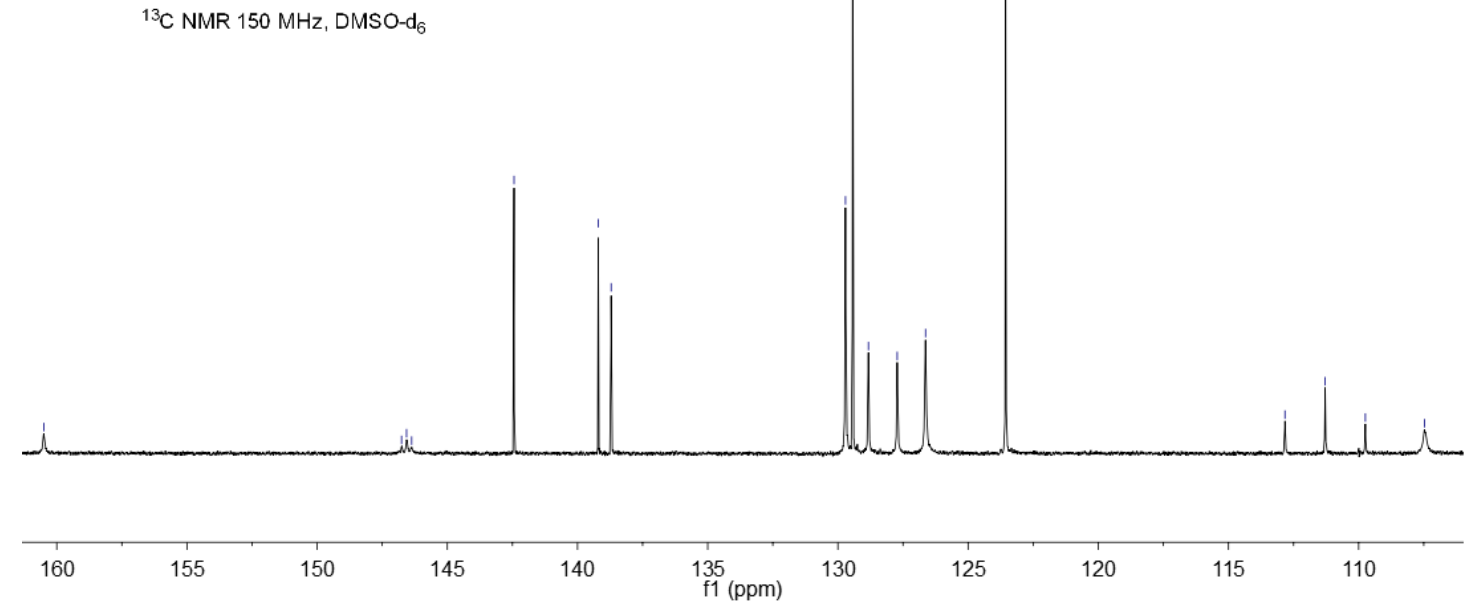

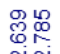

芒

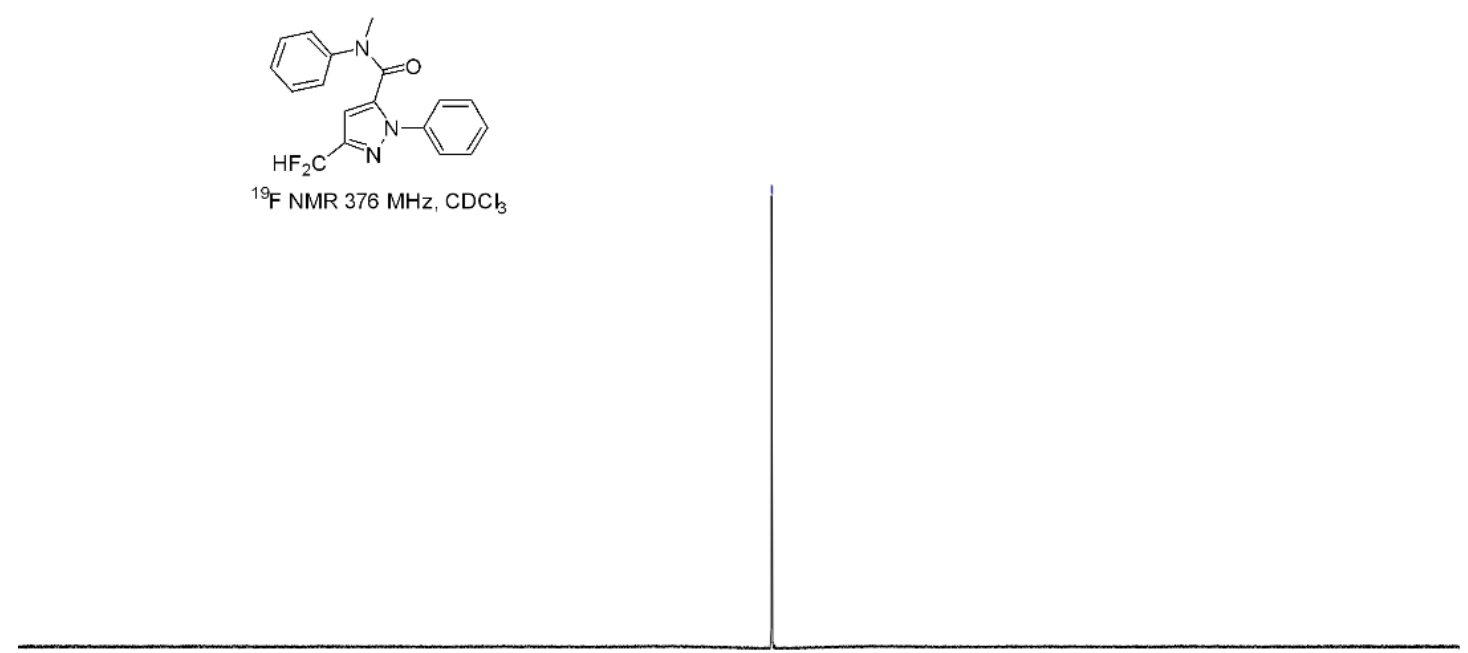

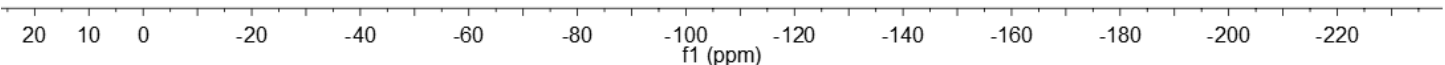




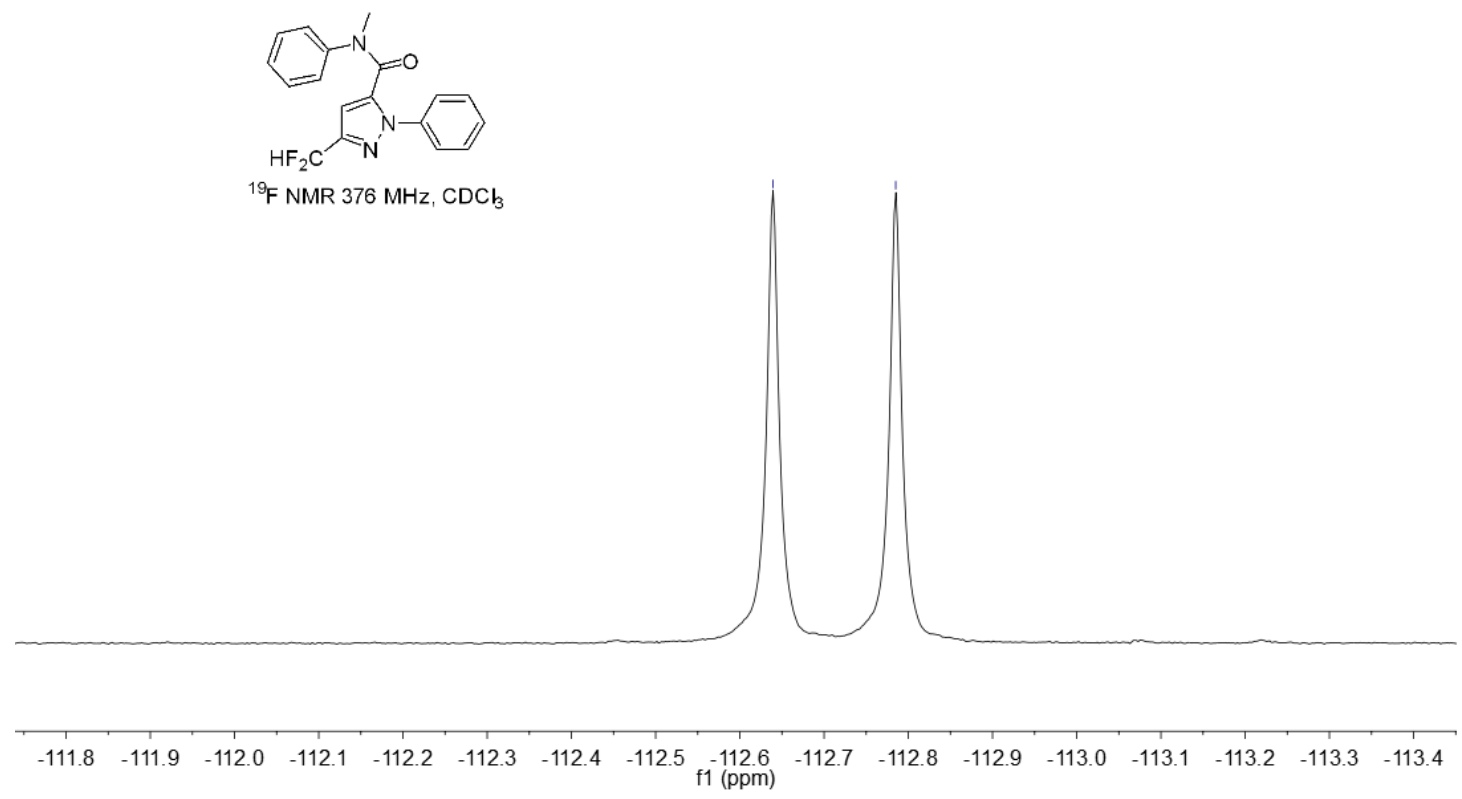

HRMS (ESI) copy of compound $\mathbf{7 h}$ :

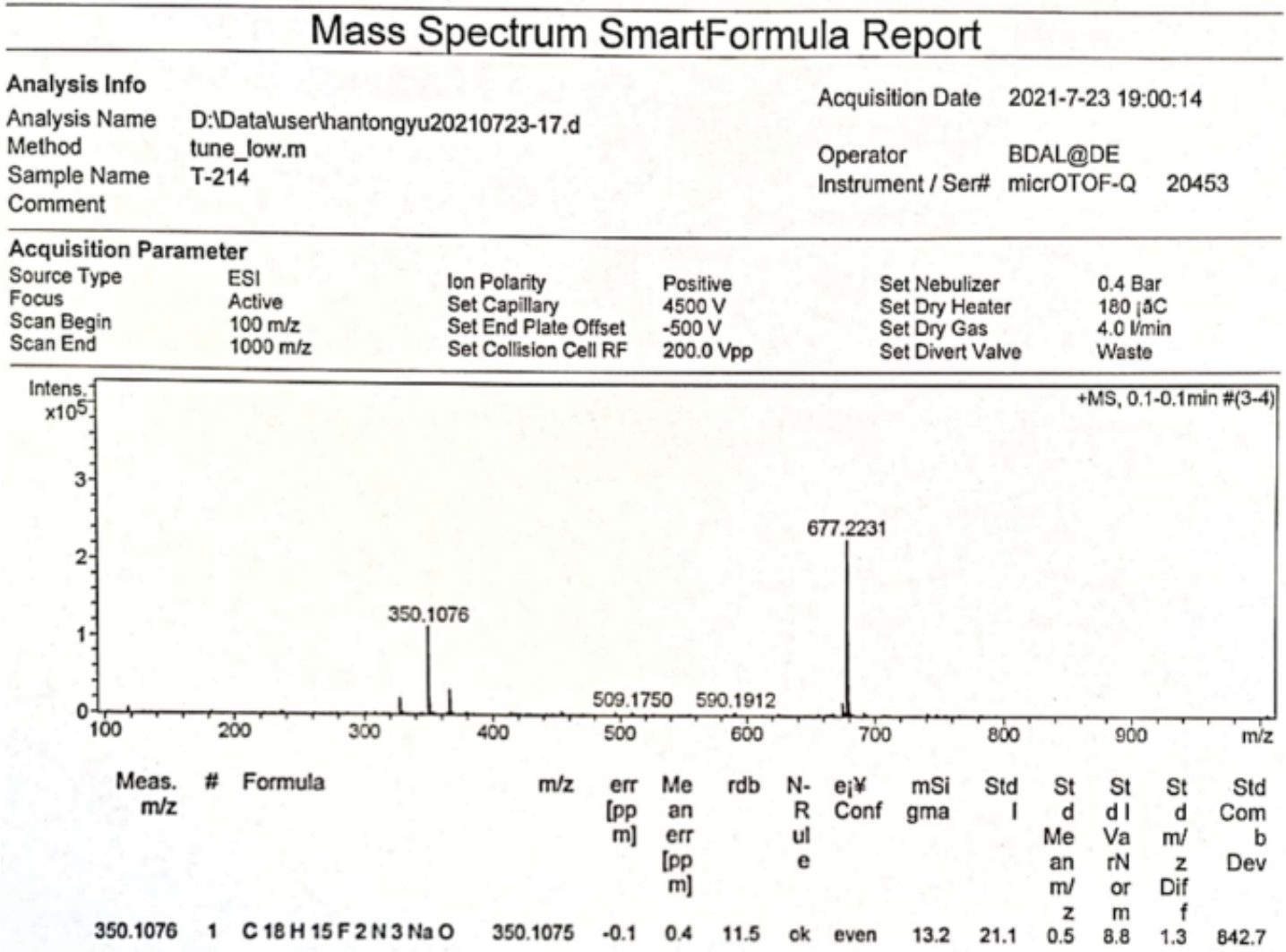


NMR copies of compound 7i:

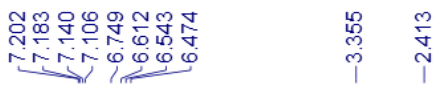

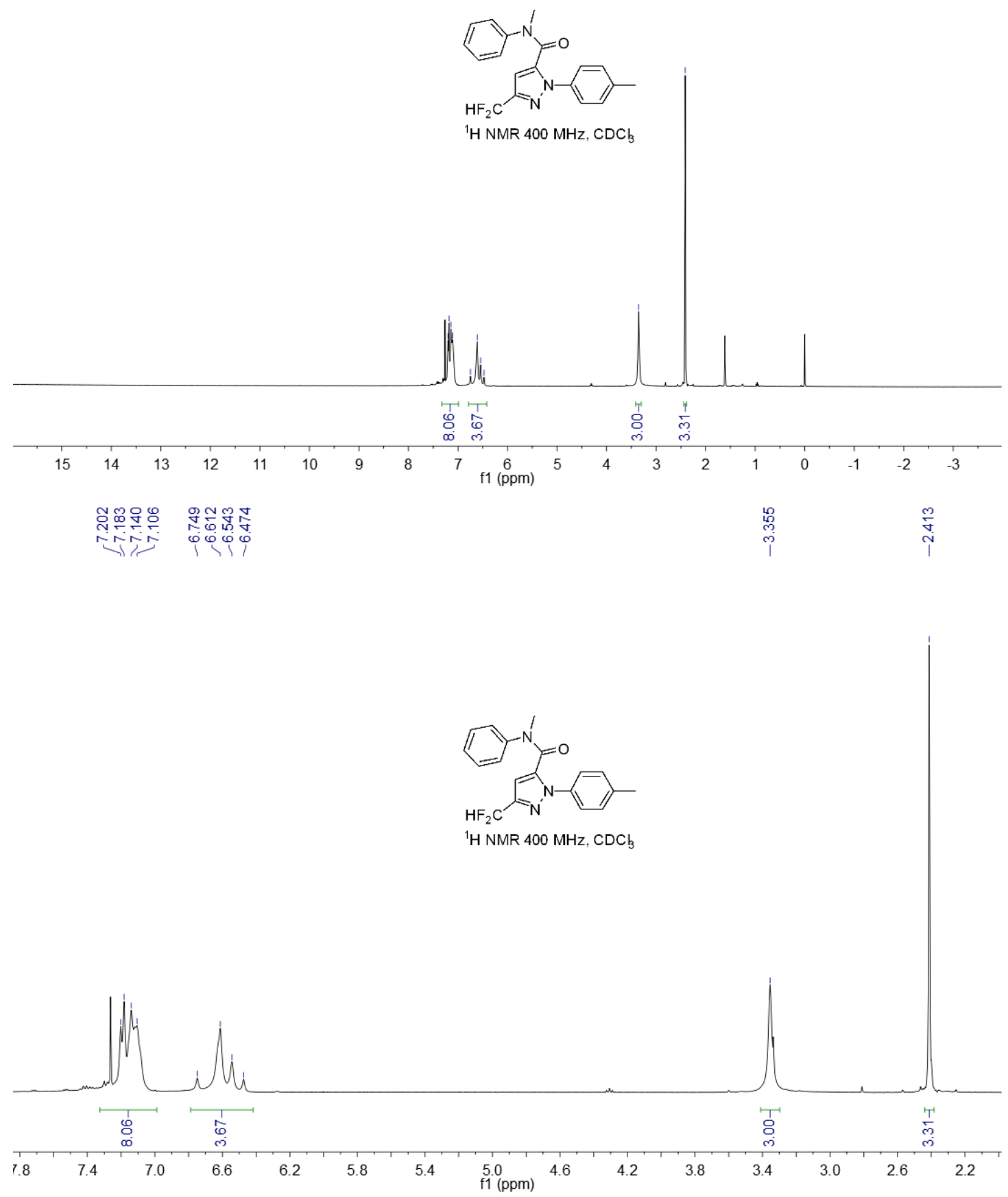




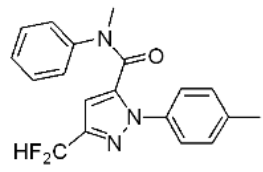

${ }^{13} \mathrm{C} N M R 150 \mathrm{MHz}, \mathrm{CDCl}$

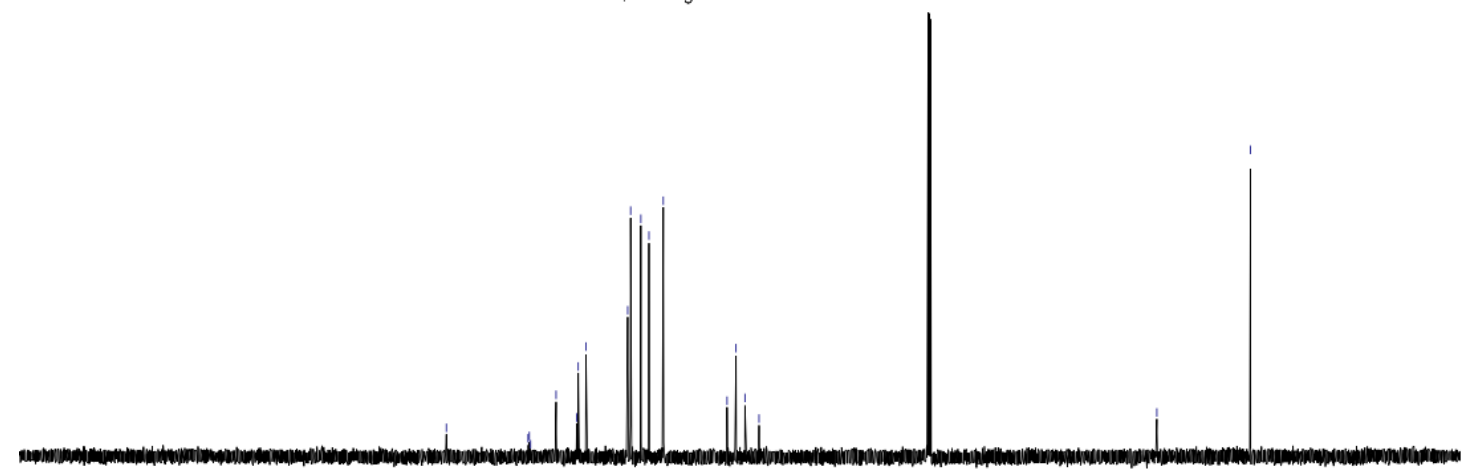

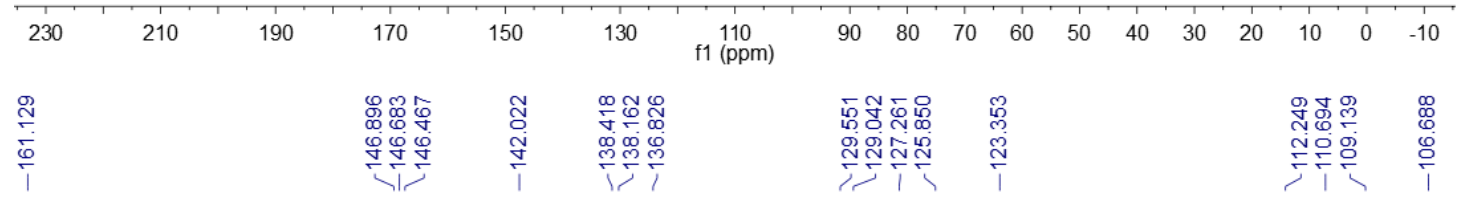

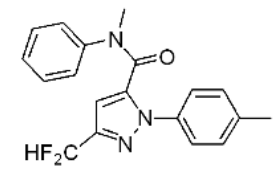

${ }^{13} \mathrm{C}$ NMR $150 \mathrm{MHz}, \mathrm{CDCl}$

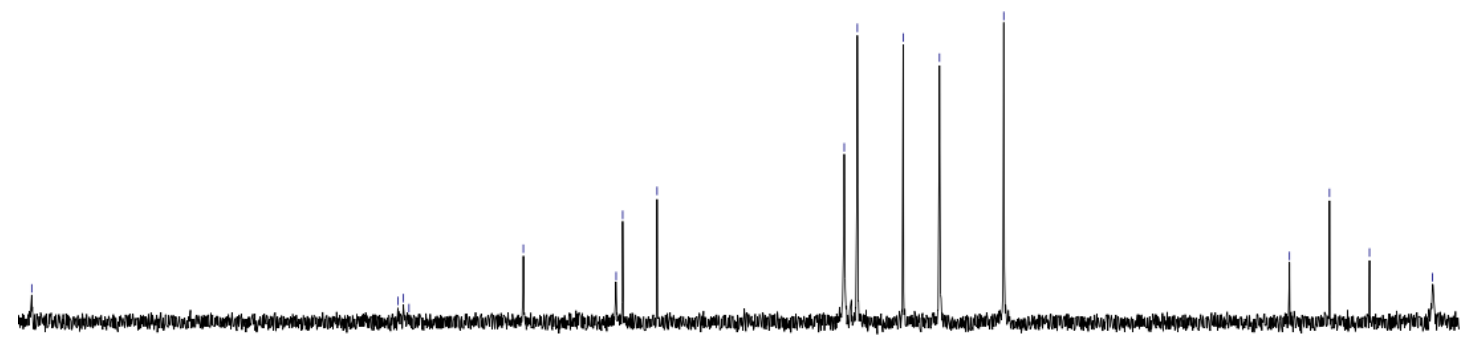

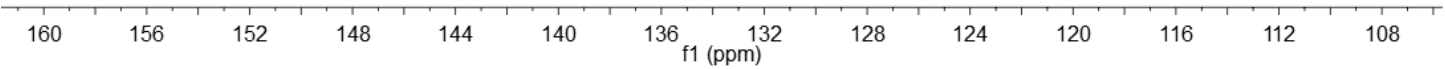




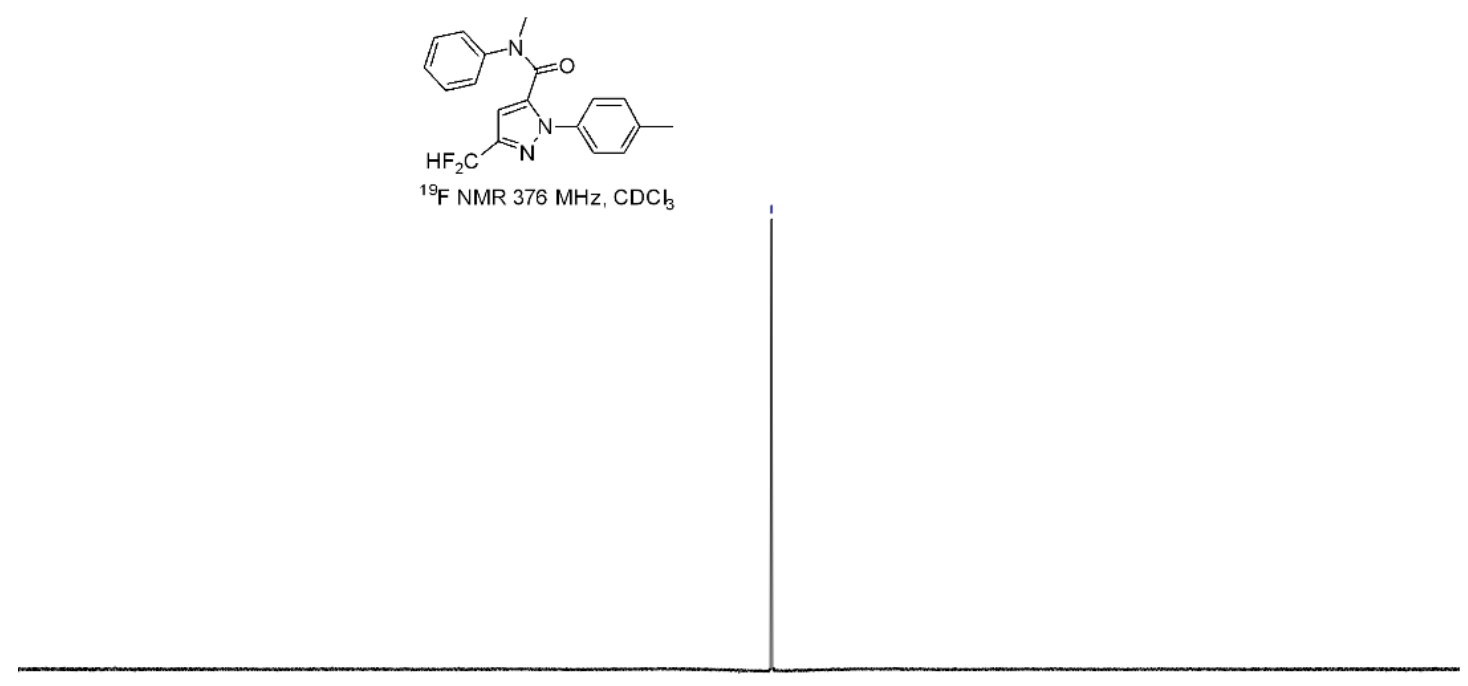

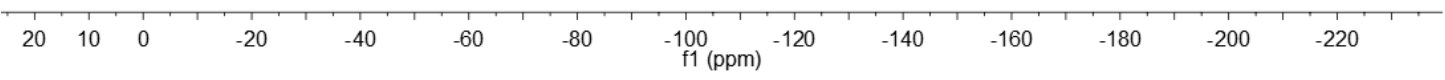

귷

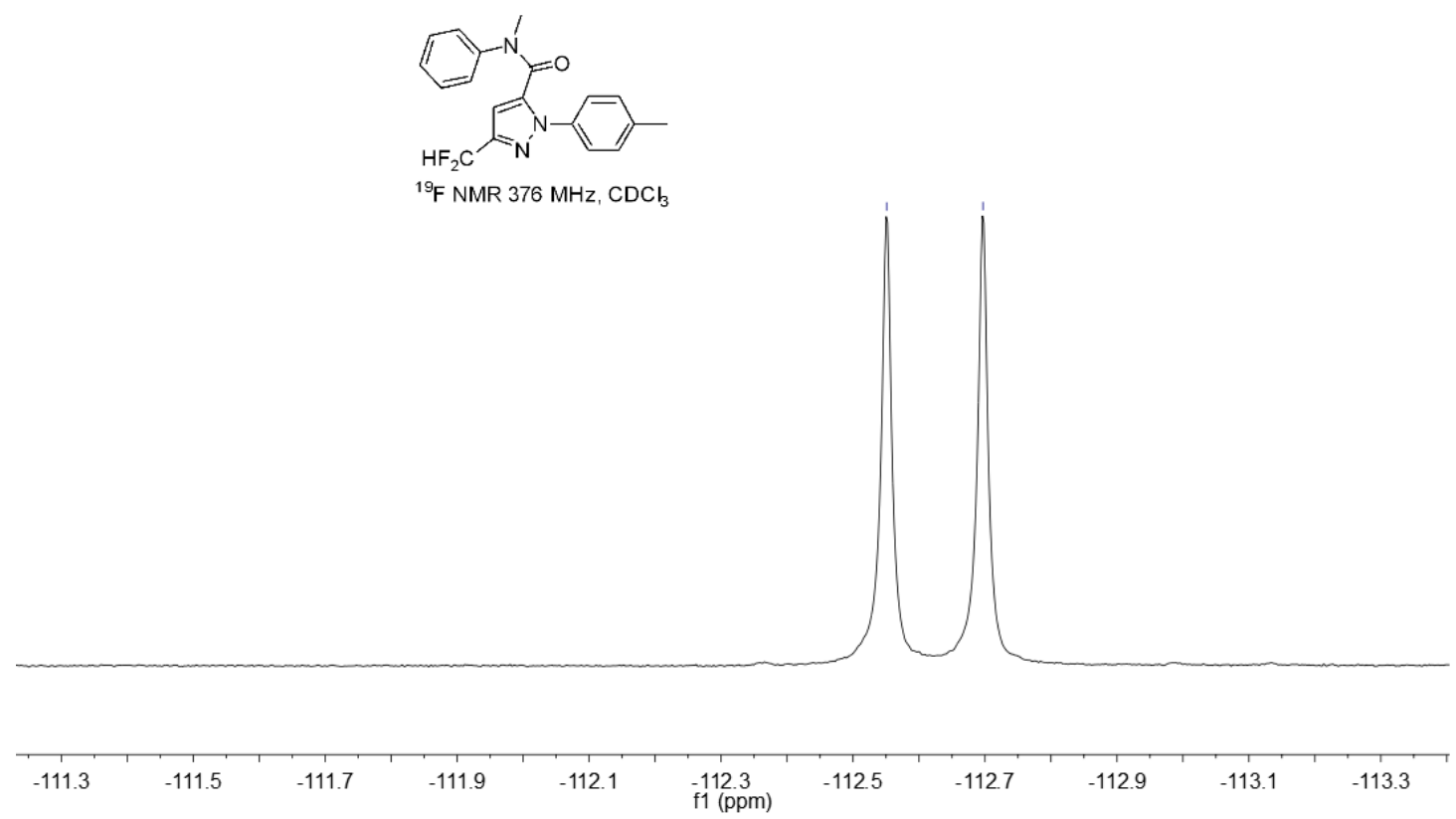


HRMS (ESI) copy of compound 7i:

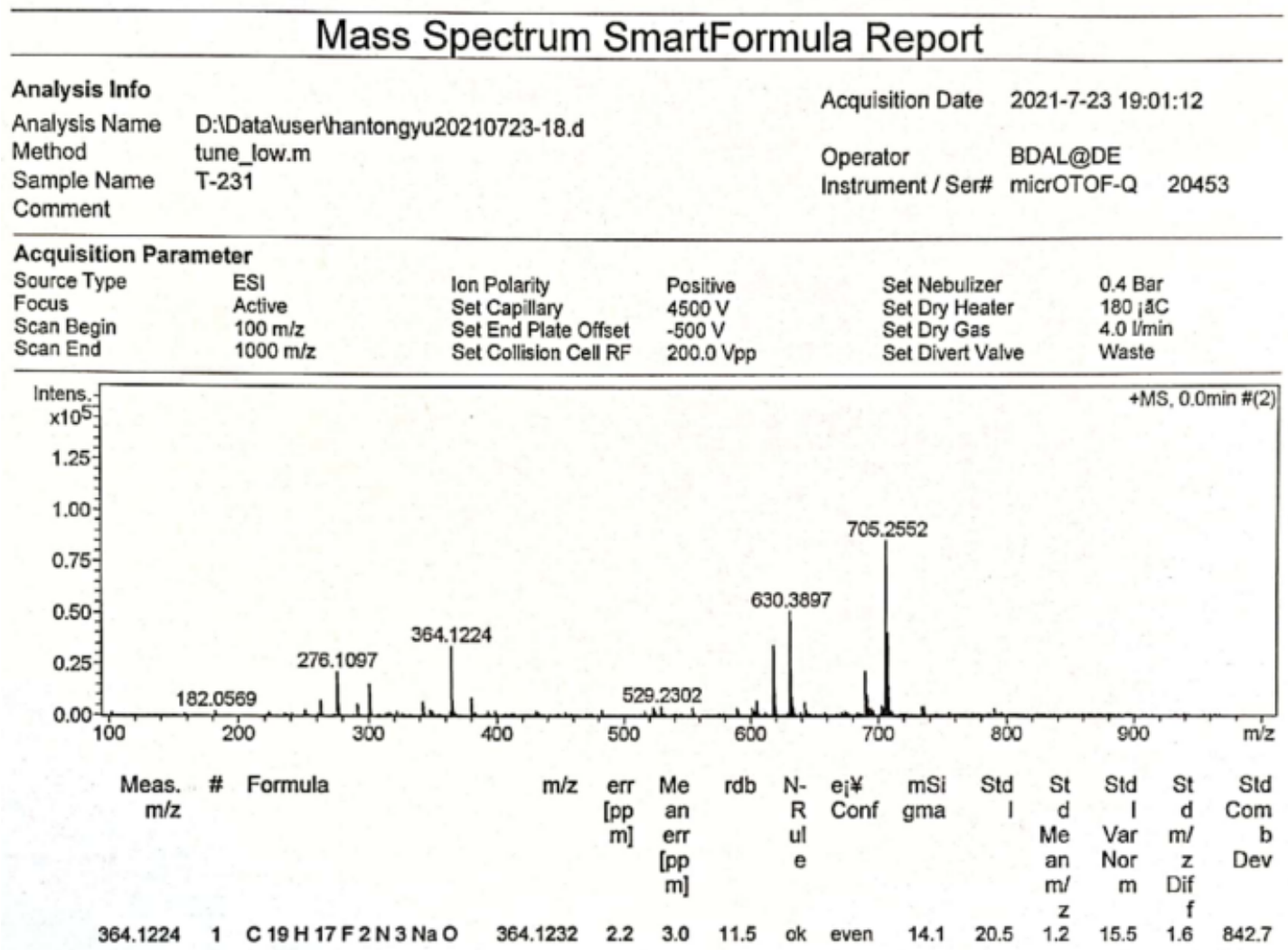

NMR copies of compound $\mathbf{7 j}$ :

\begin{tabular}{|c|c|}
\hline 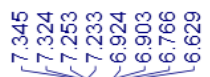 & $\begin{array}{ll}\infty & \infty \\
\infty & \stackrel{N}{\infty} \\
\infty & m\end{array}$ \\
\hline
\end{tabular}

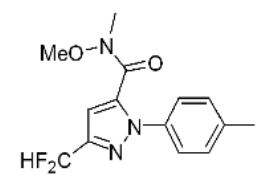

${ }^{1} \mathrm{HNMR} 400 \mathrm{MHz}, \mathrm{CDCl}_{3}$

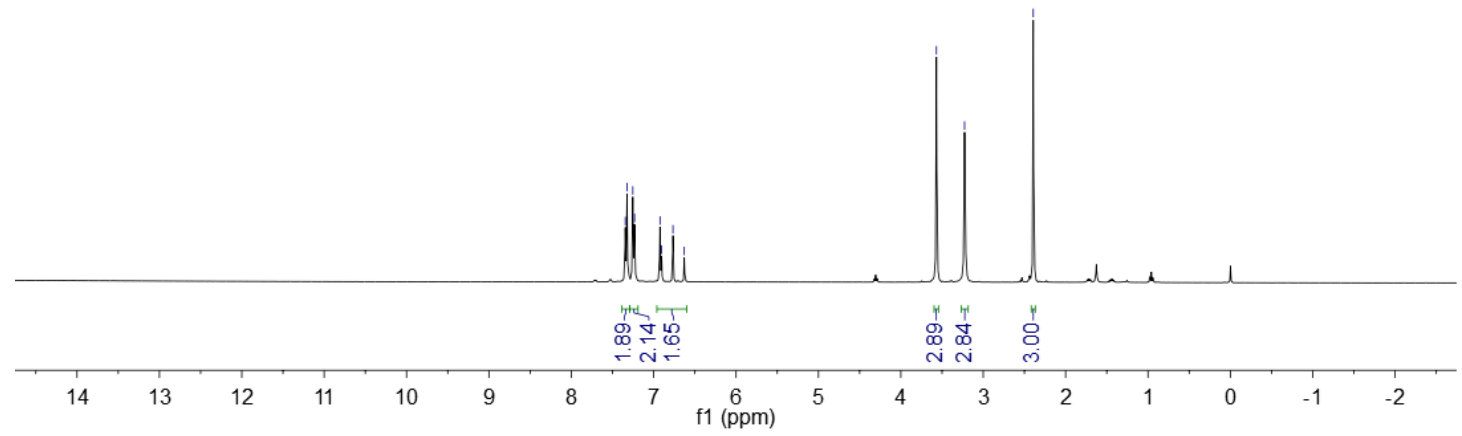




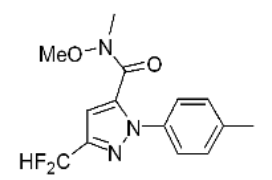

${ }^{1} \mathrm{HNMR} 400 \mathrm{MHz}, \mathrm{CDCl}_{3}$
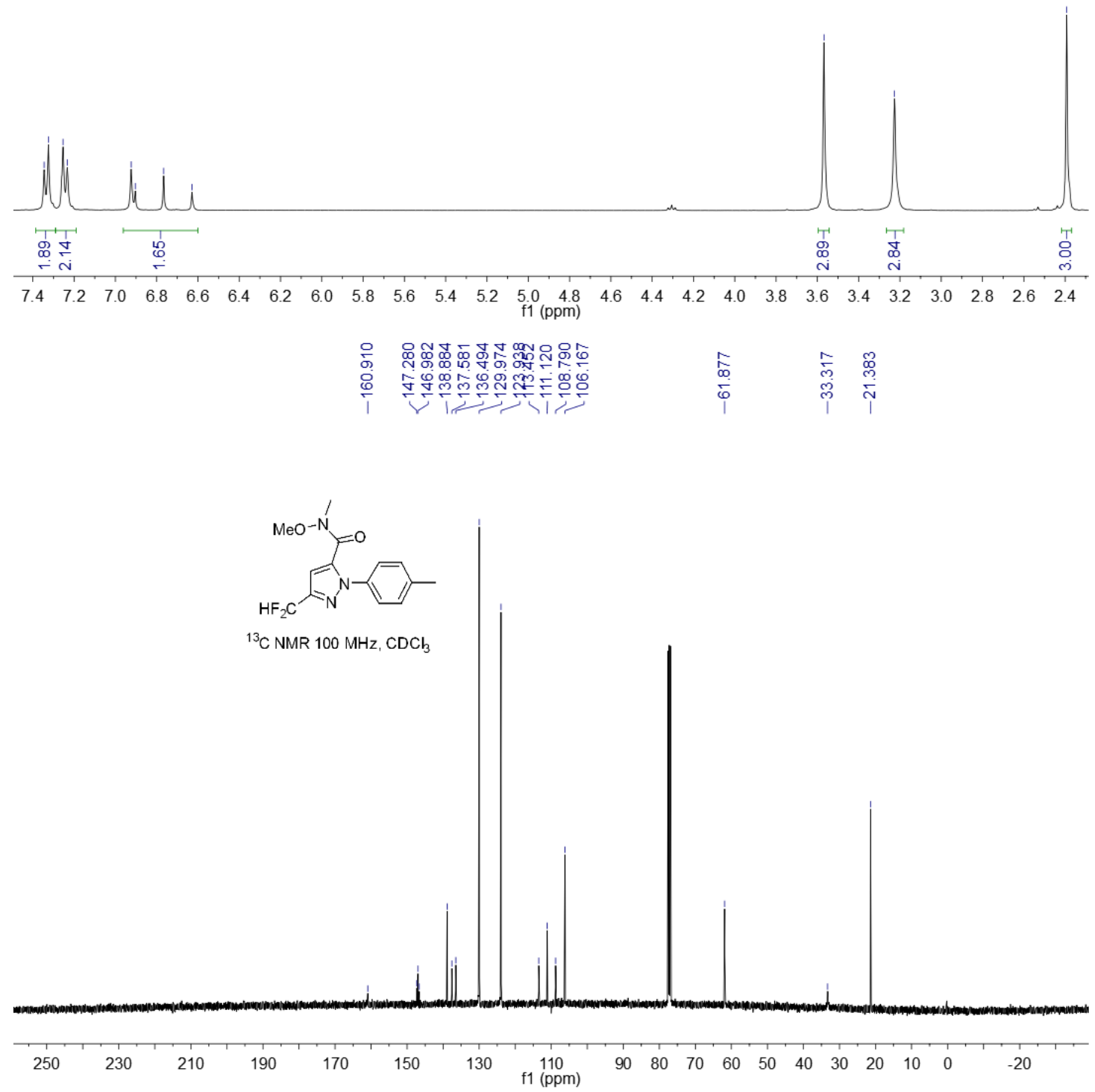


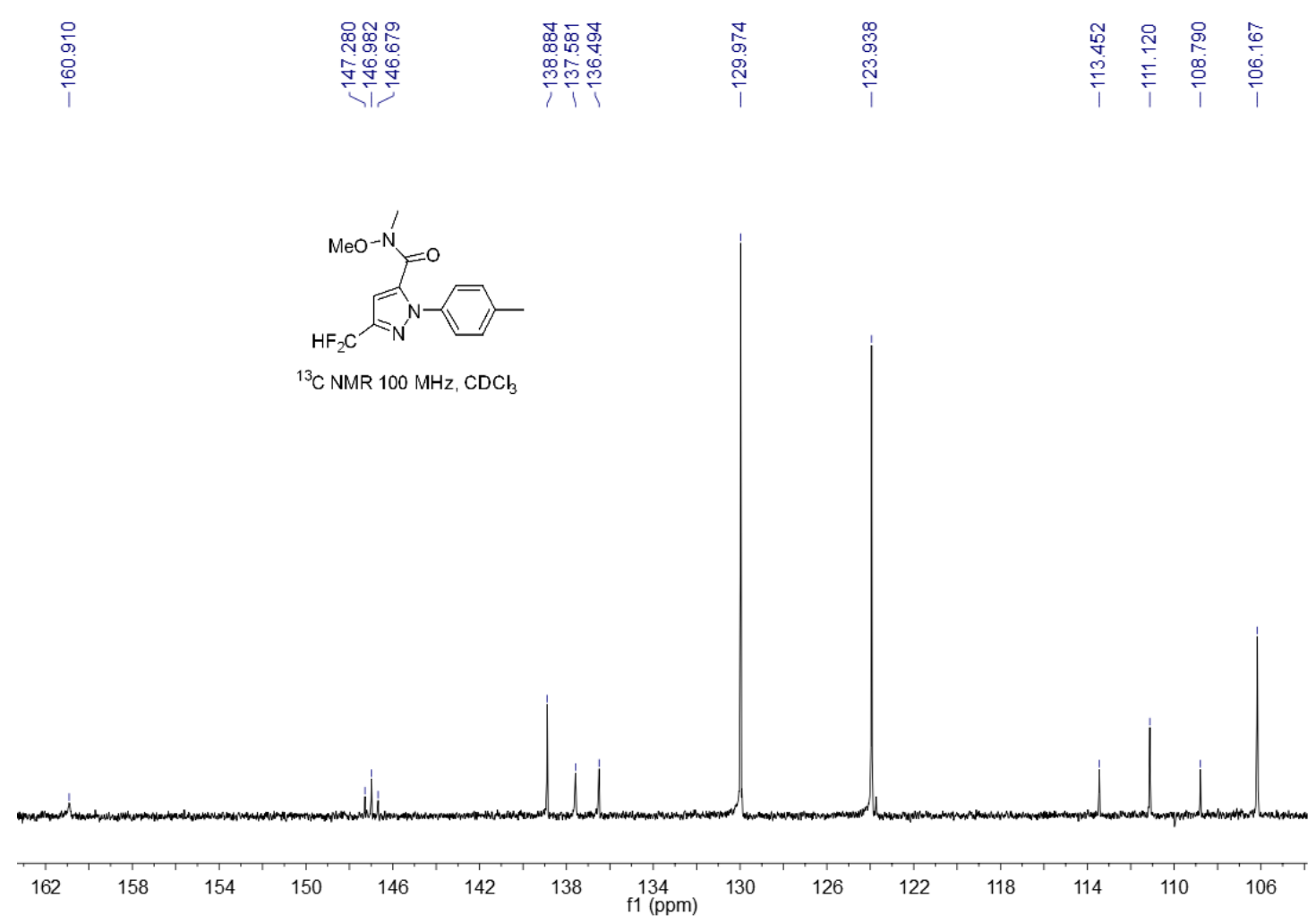

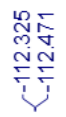

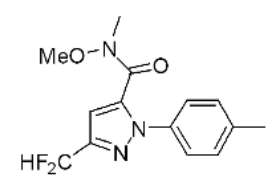

${ }^{19} \mathrm{~F}$ NMR $376 \mathrm{MHz}, \mathrm{CDCl}_{3}$

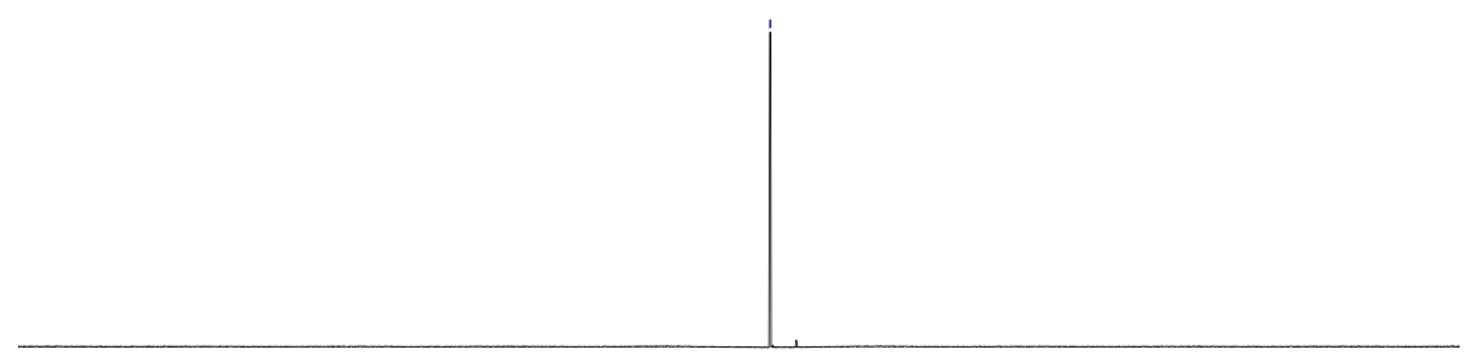

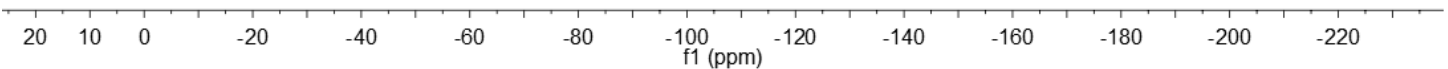




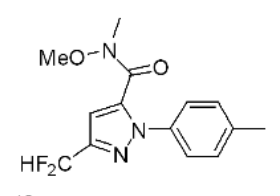

${ }^{19}{ }^{\mathrm{F}} \mathrm{NMR} 376 \mathrm{MHz}, \mathrm{CDCl}_{3}$

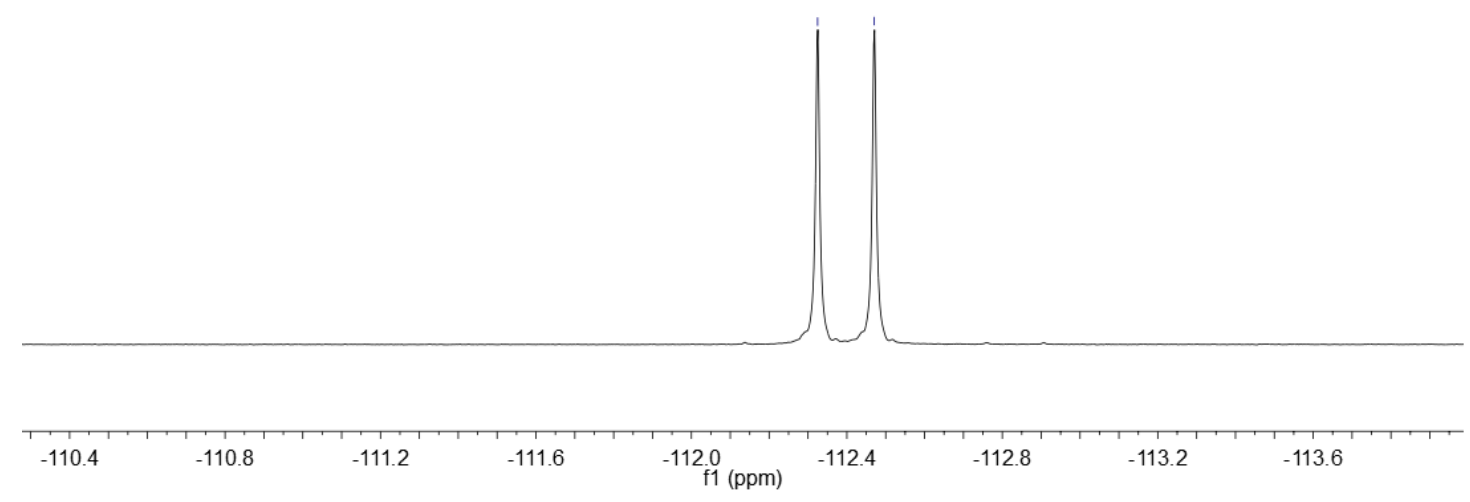

HRMS (ESI) copy of compound $\mathbf{7 j}$ :

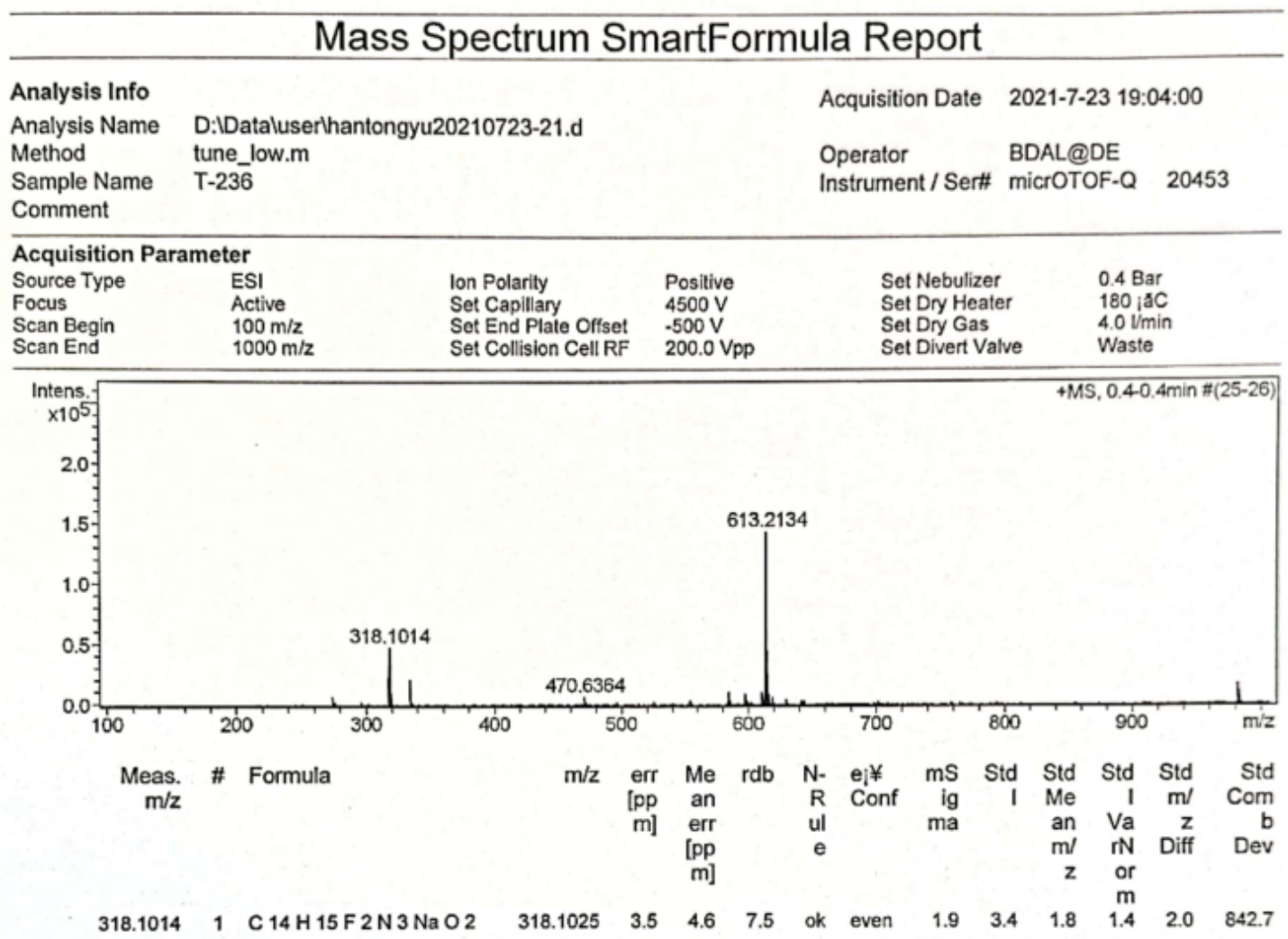


NMR copies of compound 7k:

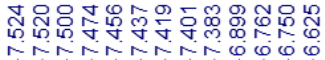

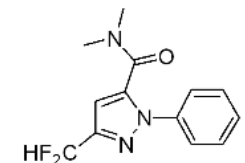

${ }^{1} \mathrm{H}$ NMR $400 \mathrm{MHz}, \mathrm{CDCl}_{3}$

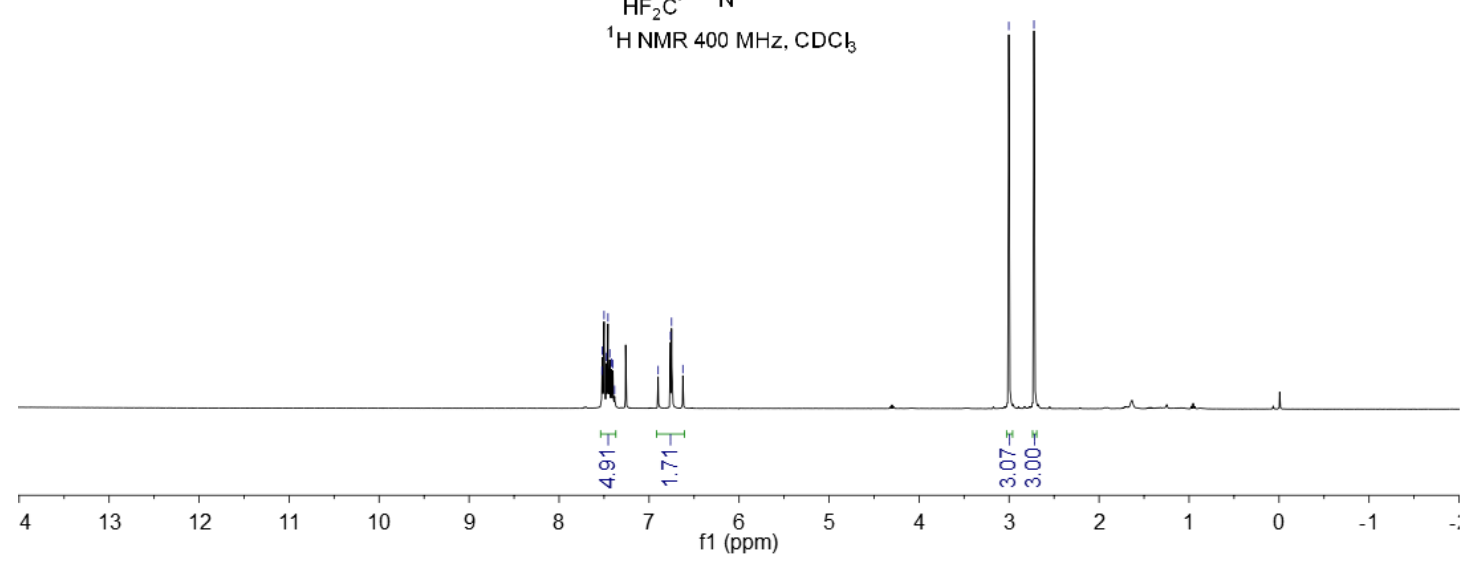

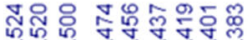

NTTイTイT

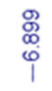

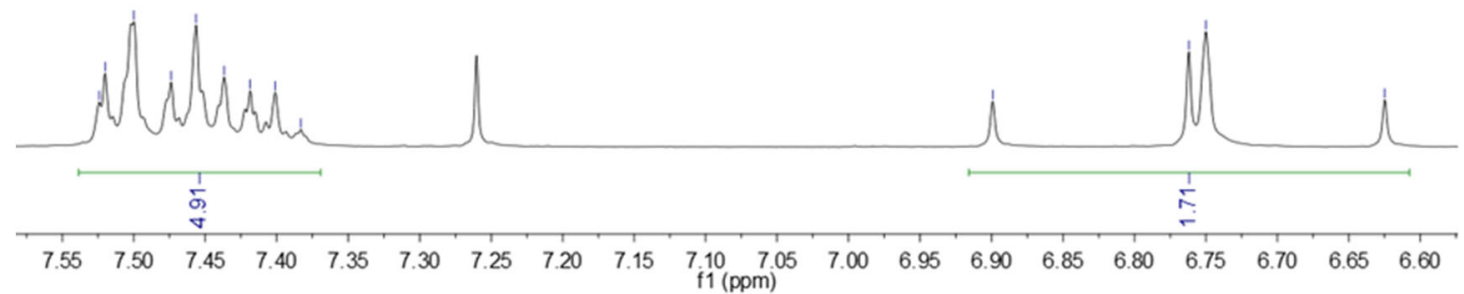




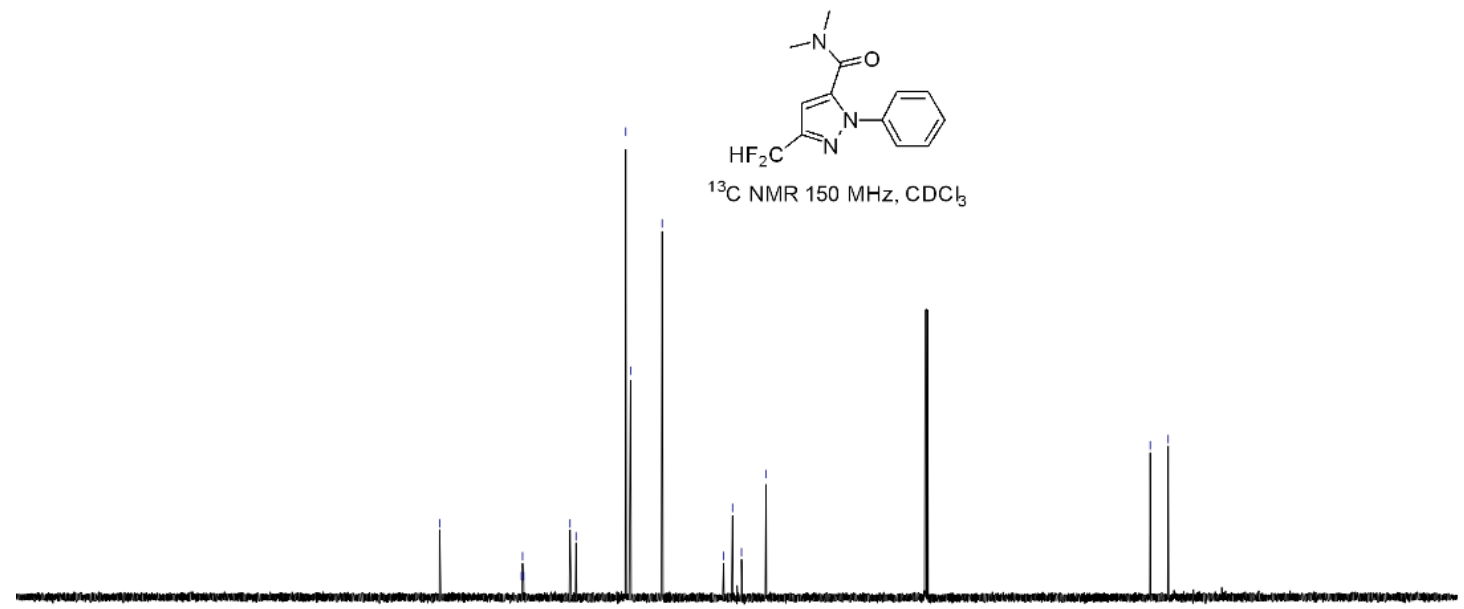

\begin{tabular}{|c|c|c|c|c|c|c|c|c|c|c|c|c|c|c|c|c|}
\hline 230 & 210 & 190 & 170 & 150 & 130 & $\begin{array}{c}110 \\
\mathrm{f} 1(\mathrm{ppm})\end{array}$ & 90 & 80 & 70 & 60 & 50 & 40 & 30 & 20 & 10 & 0 \\
\hline & & & 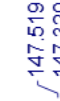 & & 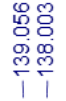 & & 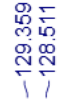 & & $\begin{array}{l}\stackrel{\leftrightarrow}{0} \\
\stackrel{\sim}{N} \\
i\end{array}$ & & & & & 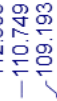 & & 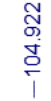 \\
\hline
\end{tabular}

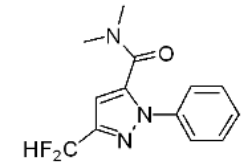

${ }^{13} \mathrm{C}$ NMR $150 \mathrm{MHz}, \mathrm{CDCl}_{3}$

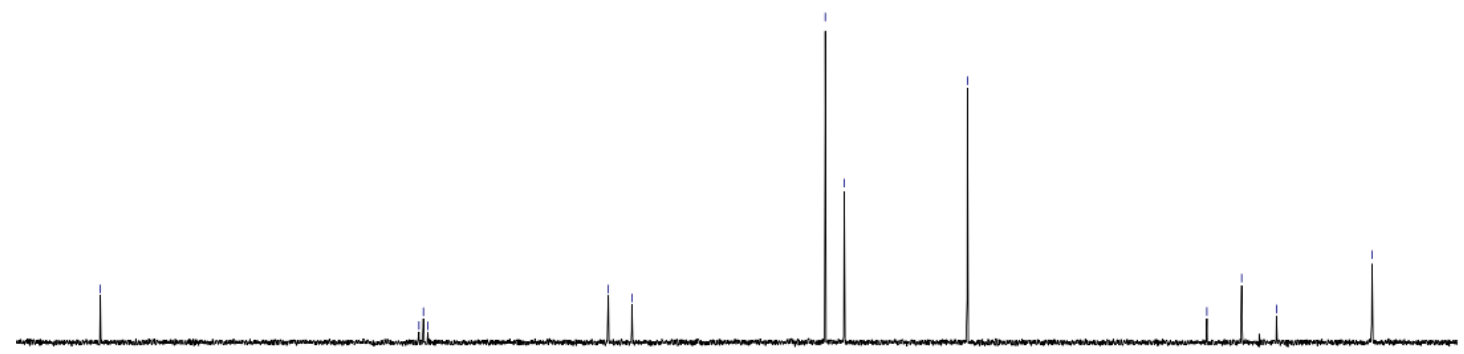

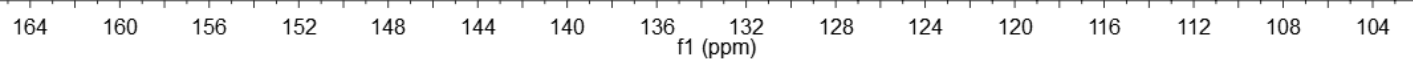




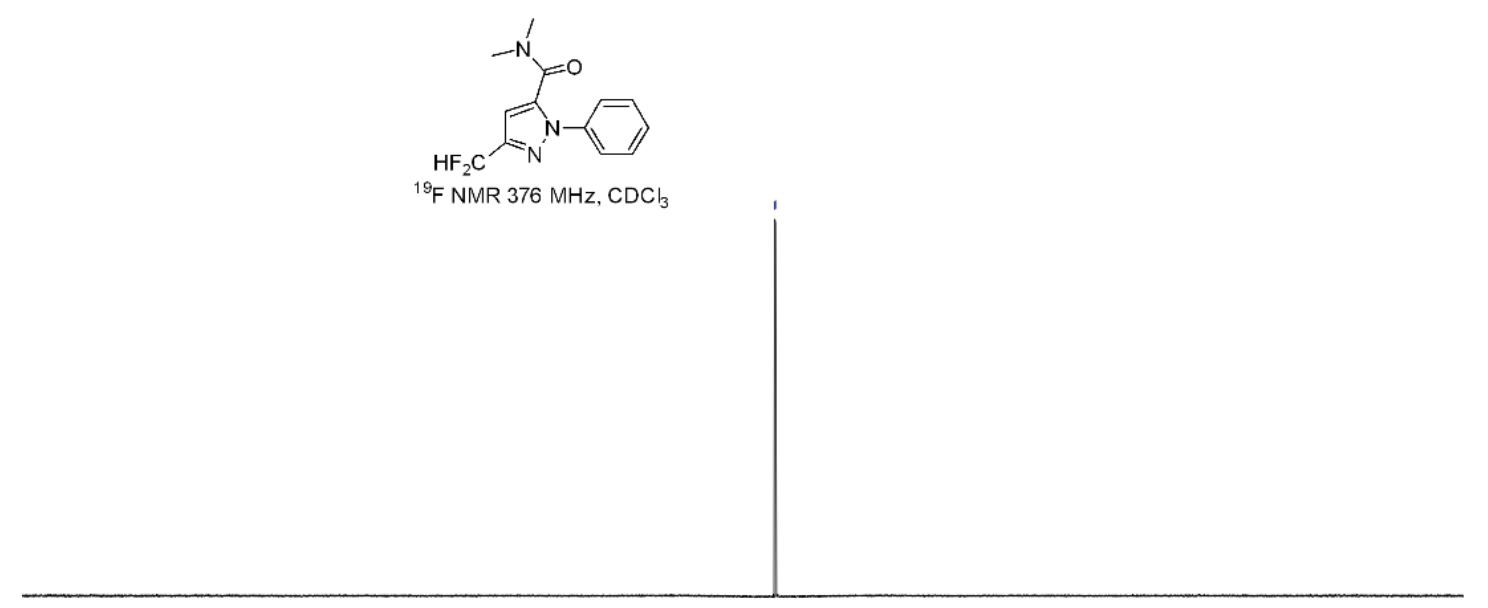

\begin{tabular}{|c|c|c|c|c|c|c|c|c|c|c|c|c|c|}
\hline \multirow[t]{2}{*}{20} & 10 & 0 & -20 & -40 & -60 & -80 & $\begin{array}{l}-100 \\
\mathrm{f} 1 \text { (ppm) }\end{array}$ & -120 & -140 & -160 & -180 & -200 & -220 \\
\hline & & & & & & & & & & & & & \\
\hline
\end{tabular}

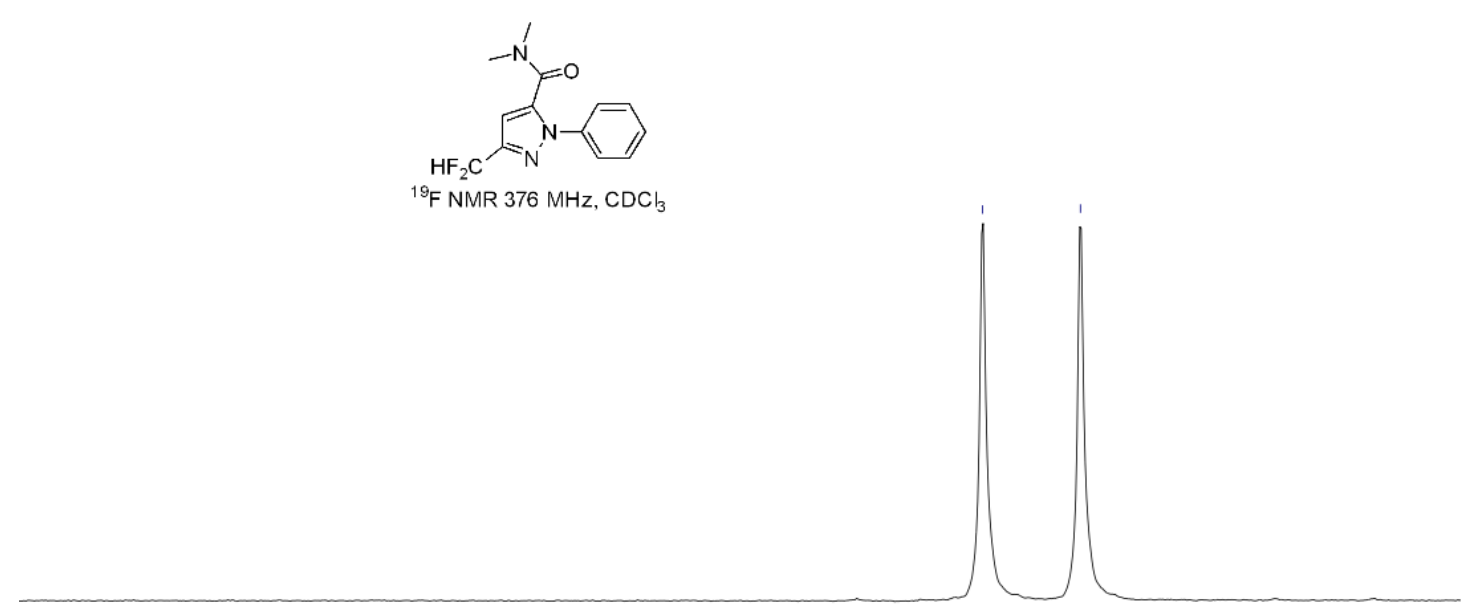

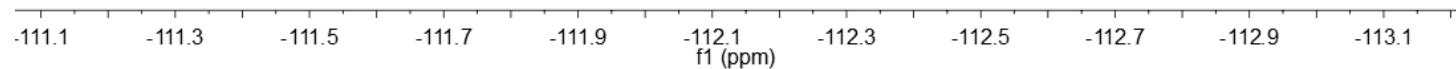


HRMS (ESI) copy of compound 7k:

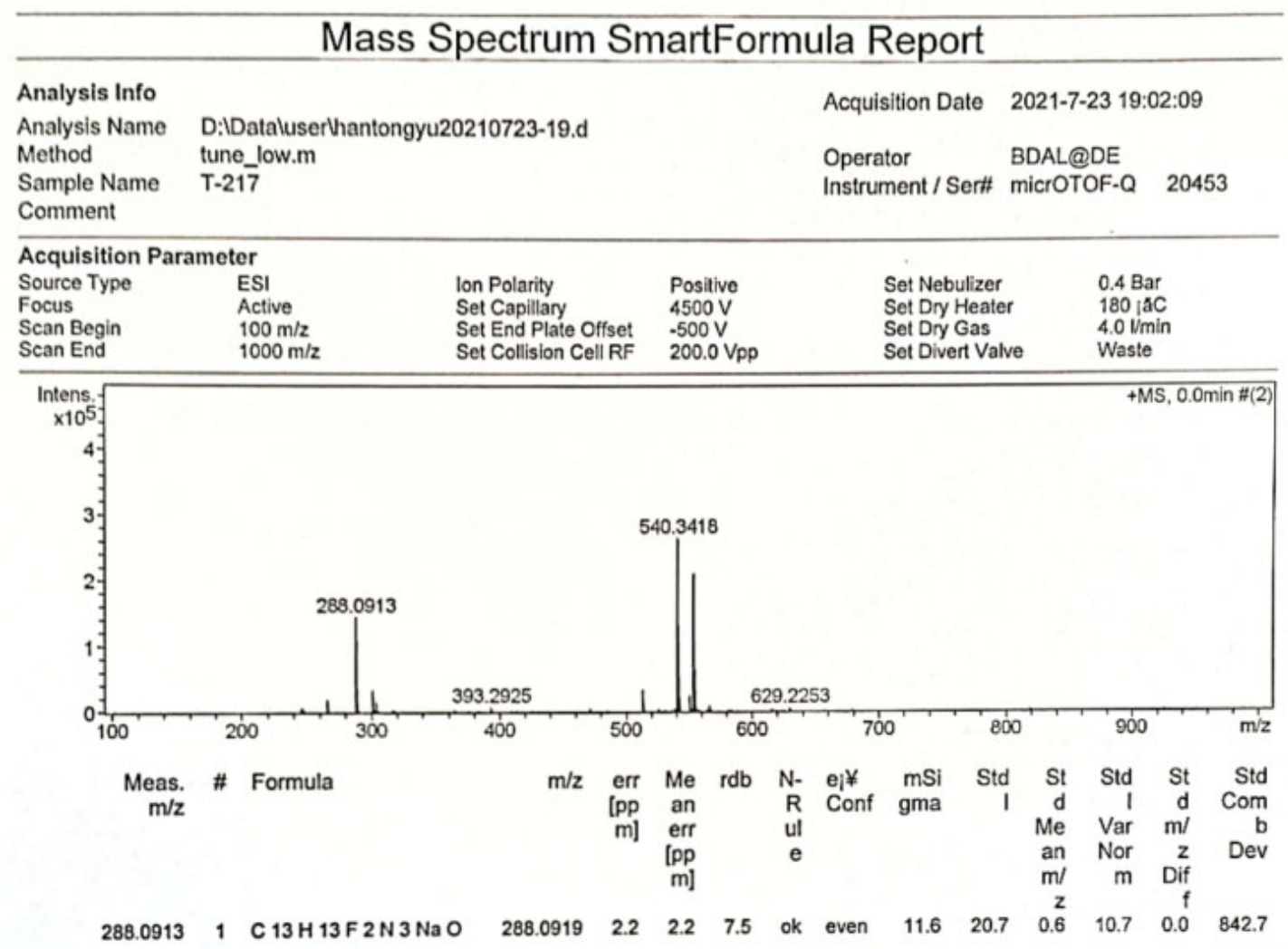




\section{X-ray Crystallographic Data of Compound 3g}

Thermal ellipsoids are set at a 50\% probability level. Crystal data have been deposited to CCDC, number 2071160.

Crystallization Details

The obtained compound $\mathbf{3 g}(48 \mathrm{mg})$ was dissolved in THF $(0.3 \mathrm{~mL})$ in a NMR tube at room temperature. Then petroleum ether $(2 \mathrm{~mL})$ was added to the solution slowly along the tube wall, resulting in a two-phase mixture. The colorless crystal of 3g was formed after the two-phase mixture has diffused.

\section{Experimental}

A suitable crystal was selected and placed on a ROD, Synergy Custom system, HyPix diffractometer. The crystal was kept at 293(2) K during data collection. Using Olex2 ${ }^{1}$, the structure was solved with the SHELXS ${ }^{2}$ structure solution program using Direct Methods and refined with the SHELXL ${ }^{3}$ refinement package using Least Squares minimisation.

\section{Crystal structure determination}

Crystal Data for $\mathrm{C}_{17} \mathrm{H}_{11} \mathrm{BrF}_{2} \mathrm{~N}_{2} \mathrm{O}(M=377.19 \mathrm{~g} / \mathrm{mol})$ : monoclinic, space group $\mathrm{P} 21 / \mathrm{n}$ (no. 14), $a=9.9118(2) \AA, b=7.8540(2) \AA, c=20.2220(5) \AA, \beta=92.012(2)^{\circ}, V=$ 1573.27(7) $\AA^{3}, Z=4, T=293(2) \mathrm{K}, \mu(\mathrm{Cu} \mathrm{K} \alpha)=3.809 \mathrm{~mm}^{-1}$, Dcalc $=1.592 \mathrm{~g} / \mathrm{cm}^{3}$, 11444 reflections measured $\left(8.75^{\circ} \leqslant 2 \Theta \leqslant 170.308^{\circ}\right), 3078$ unique $\left(\mathrm{R}_{\text {int }}=0.0444\right.$, $\left.R_{\text {sigma }}=0.0310\right)$ which were used in all calculations. The final $R_{1}$ was $0.0626(I>2 \sigma$ (I)) and $w R_{2}$ was 0.1771 (all data).

\section{Refinement model description}


Number of restraints - 0, number of constraints - unknown.

Details:

1. Fixed Uiso

At 1.2 times of:

All $\mathrm{C}(\mathrm{H})$ groups

2.a Ternary $\mathrm{CH}$ refined with riding coordinates:

C10(H10)

3.b Aromatic/amide $\mathrm{H}$ refined with riding coordinates:

C2(H2), C5(H5), C6(H6), C8(H8), C9(H9), C13(H13), C14(H14), C15(H15),

C16(H16), C17(H17)

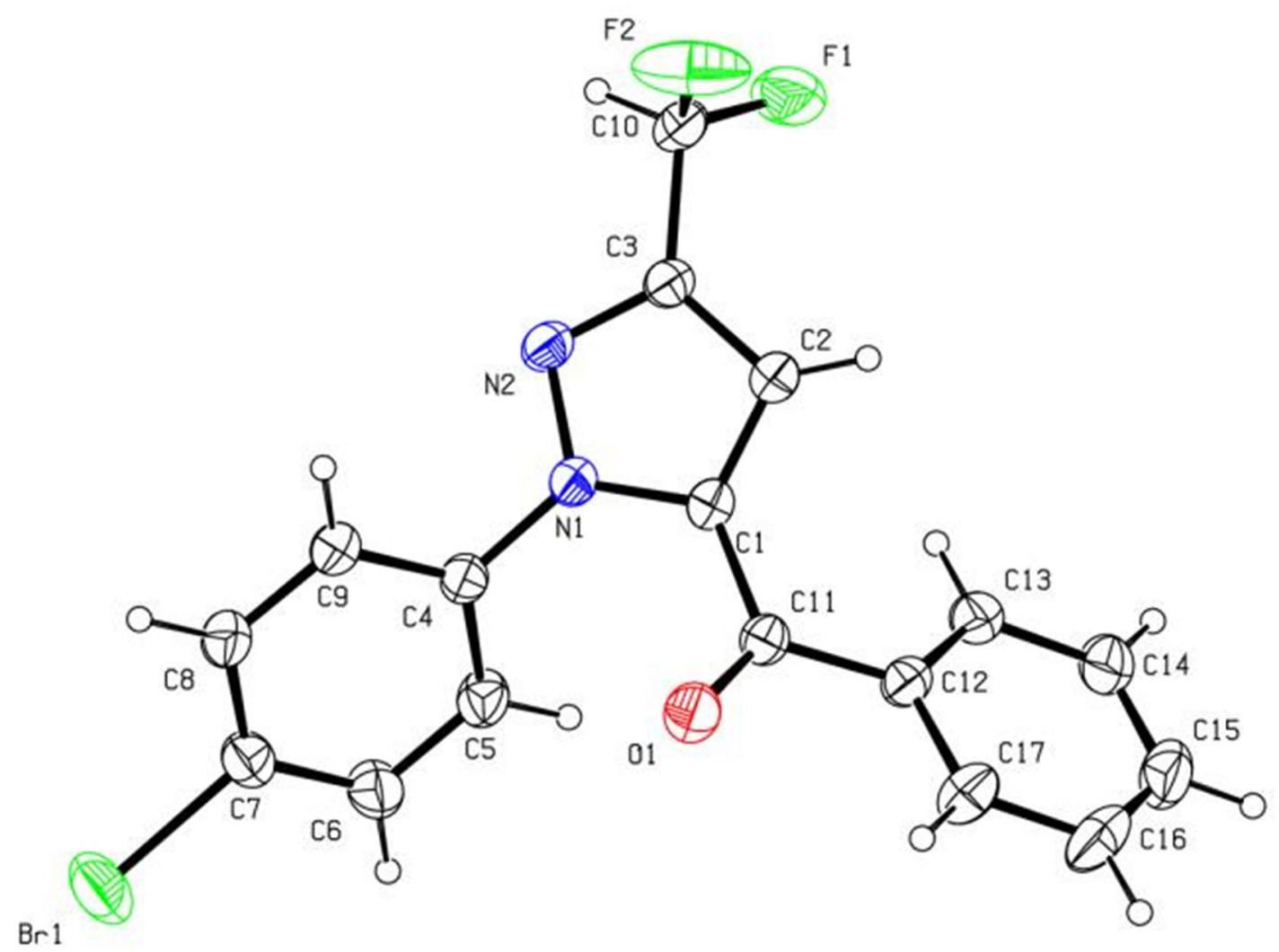


Table S1 Crystallographic Data of Compound 3g

\begin{tabular}{|c|c|}
\hline Empirical formula & $\mathrm{C}_{17} \mathrm{H}_{11} \mathrm{BrF}_{2} \mathrm{~N}_{2} \mathrm{O}$ \\
\hline Formula weight & 377.19 \\
\hline Temperature/K & 293(2) \\
\hline Crystal system & monoclinic \\
\hline Space group & $\mathrm{P} 21 / \mathrm{n}$ \\
\hline $\mathrm{a} / \AA$ & $9.9118(2)$ \\
\hline $\mathrm{b} / \AA$ & $7.8540(2)$ \\
\hline $\mathrm{c} / \AA ̊$ & $20.2220(5)$ \\
\hline$\alpha /{ }^{\circ}$ & 90 \\
\hline$\beta /{ }^{\circ}$ & $92.012(2)$ \\
\hline$\gamma /{ }^{\circ}$ & 90 \\
\hline Volume $/ \AA^{3}$ & $1573.27(7)$ \\
\hline $\mathrm{Z}$ & 4 \\
\hline$\rho_{\text {calc }} / \mathrm{cm}^{3}$ & 1.592 \\
\hline$\mu / \mathrm{mm}^{-1}$ & 3.809 \\
\hline $\mathrm{F}(000)$ & 752.0 \\
\hline Crystal size $/ \mathrm{mm}^{3}$ & $0.12 \times 0.05 \times 0.03$ \\
\hline Radiation & $\mathrm{Cu} \mathrm{K \alpha}(\lambda=1.54184)$ \\
\hline $2 \Theta$ range for data collection $/{ }^{\circ}$ & 8.75 to 170.308 \\
\hline Index ranges & $-12 \leq \mathrm{h} \leq 11,-9 \leq \mathrm{k} \leq 9,-25 \leq 1 \leq 24$ \\
\hline Reflections collected & 11444 \\
\hline Independent reflections & $3078\left[\mathrm{R}_{\text {int }}=0.0444, \mathrm{R}_{\text {sigma }}=0.0310\right]$ \\
\hline Data/restraints/parameters & $3078 / 0 / 208$ \\
\hline Goodness-of-fit on $\mathrm{F}^{2}$ & 1.045 \\
\hline Final $R$ indexes $[\mathrm{I}>=2 \sigma(\mathrm{I})]$ & $\mathrm{R}_{1}=0.0626, \mathrm{wR}_{2}=0.1700$ \\
\hline Final $\mathrm{R}$ indexes [all data] & $\mathrm{R}_{1}=0.0695, \mathrm{wR}_{2}=0.1771$ \\
\hline Largest diff. peak/hole / e $\AA^{-3}$ & $0.76 /-1.40$ \\
\hline
\end{tabular}


Table S2 Fractional Atomic Coordinates $\left(\times 10^{4}\right)$ and Equivalent Isotropic Displacement Parameters $\left(\AA^{2} \times 1^{3}\right)$ for Compound $3 g$. $U_{\text {eq }}$ is defined as $1 / 3$ of the trace of the orthogonalised $U_{I J}$ tensor.

\begin{tabular}{|l|l|l|l|l|}
\hline Atom & $\mathrm{x}$ & $\mathrm{y}$ & $\mathrm{z}$ & $\mathrm{U}(\mathrm{eq})$ \\
\hline $\mathrm{Br}^{(1)}$ & $7091.2(6)$ & $7870.3(7)$ & $1356.3(3)$ & $92.1(3)$ \\
\hline $\mathrm{F}^{(1)}$ & $12186(4)$ & $-2819(3)$ & $158.5(15)$ & $88.9(9)$ \\
\hline $\mathrm{F}^{(2)}$ & $13695(3)$ & $-904(6)$ & $119.7(18)$ & $117.2(14)$ \\
\hline $\mathrm{O}^{(1)}$ & $11099(3)$ & $2364(3)$ & $2589.3(12)$ & $56.1(6)$ \\
\hline $\mathrm{N}^{(1)}$ & $10689(3)$ & $1660(4)$ & $1189.4(12)$ & $40.3(6)$ \\
\hline $\mathrm{N}^{(2)}$ & $11063(3)$ & $1157(4)$ & $582.4(12)$ & $45.2(6)$ \\
\hline $\mathrm{C}^{(1)}$ & $11139(3)$ & $556(4)$ & $1673.0(14)$ & $39.5(6)$ \\
\hline $\mathrm{C}^{(2)}$ & $11839(3)$ & $-700(5)$ & $1361.3(15)$ & $44.7(7)$ \\
\hline $\mathrm{C}^{(3)}$ & $11772(3)$ & $-262(4)$ & $688.4(15)$ & $42.4(7)$ \\
\hline $\mathrm{C}^{(4)}$ & $9841(3)$ & $3111(4)$ & $1238.7(15)$ & $40.1(7)$ \\
\hline $\mathrm{C}^{(5)}$ & $8697(4)$ & $3057(5)$ & $1606.8(19)$ & $50.5(8)$ \\
\hline $\mathrm{C}^{(6)}$ & $7880(4)$ & $4463(5)$ & $1638(2)$ & $56.7(9)$ \\
\hline $\mathrm{C}^{(7)}$ & $8202(4)$ & $5908(5)$ & $1292.7(19)$ & $52.5(8)$ \\
\hline $\mathrm{C}^{(8)}$ & $9326(4)$ & $5980(5)$ & $910.5(17)$ & $51.4(8)$ \\
\hline $\mathrm{C}^{(9)}$ & $10160(3)$ & $4575(4)$ & $889.1(16)$ & $46.5(7)$ \\
\hline $\mathrm{C}^{(10)}$ & $12397(4)$ & $-1201(5)$ & $108.0(15)$ & $47.4(7)$ \\
\hline $\mathrm{C}^{(11)}$ & $11012(3)$ & $902(4)$ & $2388.6(15)$ & $40.5(7)$ \\
\hline $\mathrm{C}^{(12)}$ & $10790(3)$ & $-556(4)$ & $2840.7(15)$ & $42.4(7)$ \\
\hline $\mathrm{C}^{(13)}$ & $10369(4)$ & $-2143(4)$ & $2613.0(19)$ & $51.0(8)$ \\
\hline $\mathrm{C}^{(14)}$ & $10118(5)$ & $-3444(5)$ & $3055(2)$ & $63.4(10)$ \\
\hline $\mathrm{C}^{(15)}$ & $10277(5)$ & $-3170(6)$ & $3720(2)$ & $71.8(12)$ \\
\hline $\mathrm{C}^{(16)}$ & $10697(5)$ & $-1605(8)$ & $3955(2)$ & $74.4(13)$ \\
\hline $\mathrm{C}^{(17)}$ & $10946(4)$ & $-290(6)$ & $3520.5(17)$ & $59.3(9)$ \\
\hline & & & & \\
\hline
\end{tabular}


Table S3 Anisotropic Displacement Parameters $\left(\AA^{2} \times 10^{3}\right)$ for Compound 3g. The Anisotropic displacement factor exponent takes the form: $-2 \pi$ ${ }^{2}\left[h^{2} a^{* 2} U_{11}+2 h k a * b * U_{12}+\cdots\right]$.

\begin{tabular}{|l|l|l|l|l|l|l|}
\hline Atom & $\mathrm{U}_{11}$ & $\mathrm{U}_{22}$ & $\mathrm{U}_{33}$ & $\mathrm{U}_{23}$ & $\mathrm{U}_{13}$ & $\mathrm{U}_{12}$ \\
\hline $\mathrm{Br}^{(1)}$ & $87.3(4)$ & $65.0(4)$ & $126.0(6)$ & $16.2(3)$ & $30.3(3)$ & $32.8(3)$ \\
\hline $\mathrm{F}^{(1)}$ & $127(3)$ & $57.5(16)$ & $84.8(19)$ & $-14.8(13)$ & $38.9(17)$ & $-1.6(15)$ \\
\hline $\mathrm{F}^{(2)}$ & $73.5(17)$ & $160(4)$ & $121(2)$ & $-76(3)$ & $45.3(17)$ & $-31(2)$ \\
\hline $\mathrm{O}^{(1)}$ & $76.5(17)$ & $45.2(14)$ & $46.1(13)$ & $-4.3(10)$ & $-4.2(12)$ & $-6.5(12)$ \\
\hline $\mathrm{N}^{(1)}$ & $46.0(13)$ & $39.7(13)$ & $35.2(12)$ & $1.9(10)$ & $1.8(10)$ & $0.9(11)$ \\
\hline $\mathrm{N}^{(2)}$ & $54.7(15)$ & $47.3(15)$ & $33.8(12)$ & $1.4(11)$ & $4.6(11)$ & $-1.2(12)$ \\
\hline $\mathrm{C}^{(1)}$ & $41.7(14)$ & $39.3(16)$ & $37.5(15)$ & $4.2(12)$ & $1.4(11)$ & $-2.0(12)$ \\
\hline $\mathrm{C}^{(2)}$ & $49.1(16)$ & $46.7(18)$ & $38.3(15)$ & $4.6(13)$ & $3.9(12)$ & $3.6(14)$ \\
\hline $\mathrm{C}^{(3)}$ & $48.1(16)$ & $42.9(17)$ & $36.5(15)$ & $0.6(12)$ & $4.6(12)$ & $-0.5(13)$ \\
\hline $\mathrm{C}^{(4)}$ & $43.9(16)$ & $38.9(16)$ & $37.5(15)$ & $2.1(12)$ & $0.9(12)$ & $-0.4(13)$ \\
\hline $\mathrm{C}^{(5)}$ & $46.6(17)$ & $49.1(19)$ & $56.2(19)$ & $12.1(15)$ & $8.5(14)$ & $-2.6(15)$ \\
\hline $\mathrm{C}^{(6)}$ & $46.7(18)$ & $54(2)$ & $70(2)$ & $10.2(17)$ & $15.2(16)$ & $5.5(16)$ \\
\hline $\mathrm{C}^{(7)}$ & $51.3(18)$ & $47.9(19)$ & $59(2)$ & $2.6(15)$ & $4.6(15)$ & $7.8(15)$ \\
\hline $\mathrm{C}^{(8)}$ & $62(2)$ & $42.1(18)$ & $49.9(18)$ & $6.8(14)$ & $5.9(15)$ & $-0.7(15)$ \\
\hline $\mathrm{C}^{(9)}$ & $51.0(17)$ & $44.6(18)$ & $44.3(16)$ & $5.9(13)$ & $7.3(13)$ & $-0.9(14)$ \\
\hline $\mathrm{C}^{(10)}$ & $57.1(18)$ & $49.9(19)$ & $35.5(15)$ & $2.1(13)$ & $6.6(13)$ & $-7.0(15)$ \\
\hline $\mathrm{C}^{(11)}$ & $40.7(14)$ & $42.0(17)$ & $38.7(15)$ & $1.1(12)$ & $-1.0(11)$ & $-1.2(12)$ \\
\hline $\mathrm{C}^{(12)}$ & $38.9(14)$ & $51.0(18)$ & $37.3(15)$ & $4.6(13)$ & $2.9(11)$ & $1.1(13)$ \\
\hline $\mathrm{C}^{(13)}$ & $57.2(19)$ & $46(2)$ & $50.4(18)$ & $1.7(14)$ & $11.9(15)$ & $1.5(15)$ \\
\hline $\mathrm{C}^{(14)}$ & $74(2)$ & $46(2)$ & $72(3)$ & $11.7(18)$ & $22.7(19)$ & $1.7(19)$ \\
\hline $\mathrm{C}^{(15)}$ & $69(2)$ & $72(3)$ & $76(3)$ & $34(2)$ & $17(2)$ & $7(2)$ \\
\hline $\mathrm{C}^{(16)}$ & $78(3)$ & $105(4)$ & $40.8(19)$ & $21(2)$ & $3.4(18)$ & $-4(3)$ \\
\hline $\mathrm{C}^{(17)}$ & $66(2)$ & $70(3)$ & $41.3(17)$ & $4.5(16)$ & $-0.3(15)$ & $-10.4(19)$ \\
\hline
\end{tabular}


Table S4 Bond Lengths for Compound 3g.

\begin{tabular}{|l|l|l|}
\hline Atom & Atom & Length/ \\
\hline $\mathrm{Br}^{(1)}$ & $\mathrm{C}^{(7)}$ & $1.901(4)$ \\
\hline $\mathrm{F}^{(1)}$ & $\mathrm{C}^{(10)}$ & $1.292(5)$ \\
\hline $\mathrm{F}^{(2)}$ & $\mathrm{C}^{(10)}$ & $1.307(4)$ \\
\hline $\mathrm{O}^{(1)}$ & $\mathrm{C}^{(11)}$ & $1.220(4)$ \\
\hline $\mathrm{N}^{(1)}$ & $\mathrm{N}^{(2)}$ & $1.353(4)$ \\
\hline $\mathrm{N}^{(1)}$ & $\mathrm{C}^{(1)}$ & $1.370(4)$ \\
\hline $\mathrm{N}^{(1)}$ & $\mathrm{C}^{(4)}$ & $1.422(4)$ \\
\hline $\mathrm{N}^{(2)}$ & $\mathrm{C}^{(3)}$ & $1.331(4)$ \\
\hline $\mathrm{C}^{(1)}$ & $\mathrm{C}^{(2)}$ & $1.373(5)$ \\
\hline $\mathrm{C}^{(1)}$ & $\mathrm{C}^{(11)}$ & $1.482(4)$ \\
\hline $\mathrm{C}^{(2)}$ & $\mathrm{C}^{(3)}$ & $1.403(4)$ \\
\hline $\mathrm{C}^{(3)}$ & $\mathrm{C}^{(10)}$ & $1.535(4)$ \\
\hline $\mathrm{C}^{(4)}$ & $\mathrm{C}^{(5)}$ & $1.379(5)$ \\
\hline
\end{tabular}

\begin{tabular}{|l|l|l|}
\hline Atom & Atom & Length/ \\
\hline $\mathrm{C}^{(4)}$ & $\mathrm{C}^{(9)}$ & $1.392(5)$ \\
\hline $\mathrm{C}^{(5)}$ & $\mathrm{C}^{(6)}$ & $1.372(5)$ \\
\hline $\mathrm{C}^{(6)}$ & $\mathrm{C}^{(7)}$ & $1.376(5)$ \\
\hline $\mathrm{C}^{(7)}$ & $\mathrm{C}^{(8)}$ & $1.380(5)$ \\
\hline $\mathrm{C}^{(8)}$ & $\mathrm{C}^{(9)}$ & $1.380(5)$ \\
\hline $\mathrm{C}^{(11)}$ & $\mathrm{C}^{(12)}$ & $1.486(4)$ \\
\hline $\mathrm{C}^{(12)}$ & $\mathrm{C}^{(13)}$ & $1.388(5)$ \\
\hline $\mathrm{C}^{(12)}$ & $\mathrm{C}^{(17)}$ & $1.394(5)$ \\
\hline $\mathrm{C}^{(13)}$ & $\mathrm{C}^{(14)}$ & $1.386(5)$ \\
\hline $\mathrm{C}^{(14)}$ & $\mathrm{C}^{(15)}$ & $1.366(7)$ \\
\hline $\mathrm{C}^{(15)}$ & $\mathrm{C}^{(16)}$ & $1.377(8)$ \\
\hline $\mathrm{C}^{(16)}$ & $\mathrm{C}^{(17)}$ & $1.384(6)$ \\
\hline & & \\
\hline
\end{tabular}

Table S5 Bond Angles for Compound 3g.

\begin{tabular}{|l|l|l|l|}
\hline Atom & Atom & Atom & Angle/ $^{\circ}$ \\
\hline $\mathrm{N}^{(2)}$ & $\mathrm{N}^{(1)}$ & $\mathrm{C}^{(1)}$ & $111.7(3)$ \\
\hline $\mathrm{N}^{(2)}$ & $\mathrm{N}^{(1)}$ & $\mathrm{C}^{(4)}$ & $118.6(2)$ \\
\hline $\mathrm{C}^{(1)}$ & $\mathrm{N}^{(1)}$ & $\mathrm{C}^{(4)}$ & $129.5(3)$ \\
\hline $\mathrm{C}^{(3)}$ & $\mathrm{N}^{(2)}$ & $\mathrm{N}^{(1)}$ & $105.0(2)$ \\
\hline $\mathrm{N}^{(1)}$ & $\mathrm{C}^{(1)}$ & $\mathrm{C}^{(2)}$ & $106.5(3)$ \\
\hline $\mathrm{N}^{(1)}$ & $\mathrm{C}^{(1)}$ & $\mathrm{C}^{(11)}$ & $123.0(3)$ \\
\hline $\mathrm{C}^{(2)}$ & $\mathrm{C}^{(1)}$ & $\mathrm{C}^{(11)}$ & $129.9(3)$ \\
\hline $\mathrm{C}^{(1)}$ & $\mathrm{C}^{(2)}$ & $\mathrm{C}^{(3)}$ & $105.1(3)$ \\
\hline $\mathrm{N}^{(2)}$ & $\mathrm{C}^{(3)}$ & $\mathrm{C}^{(2)}$ & $111.6(3)$ \\
\hline $\mathrm{N}^{(2)}$ & $\mathrm{C}^{(3)}$ & $\mathrm{C}^{(10)}$ & $120.3(3)$ \\
\hline $\mathrm{C}^{(2)}$ & $\mathrm{C}^{(3)}$ & $\mathrm{C}^{(10)}$ & $128.1(3)$ \\
\hline $\mathrm{C}^{(5)}$ & $\mathrm{C}^{(4)}$ & $\mathrm{N}^{(1)}$ & $120.8(3)$ \\
\hline $\mathrm{C}^{(5)}$ & $\mathrm{C}^{(4)}$ & $\mathrm{C}^{(9)}$ & $120.4(3)$ \\
\hline $\mathrm{C}^{(9)}$ & $\mathrm{C}^{(4)}$ & $\mathrm{N}^{(1)}$ & $118.7(3)$ \\
\hline $\mathrm{C}^{(6)}$ & $\mathrm{C}^{(5)}$ & $\mathrm{C}^{(4)}$ & $119.9(3)$ \\
\hline $\mathrm{C}^{(5)}$ & $\mathrm{C}^{(6)}$ & $\mathrm{C}^{(7)}$ & $119.5(3)$ \\
\hline $\mathrm{C}^{(6)}$ & $\mathrm{C}^{(7)}$ & $\mathrm{Br}^{(1)}$ & $119.3(3)$ \\
\hline $\mathrm{C}^{(6)}$ & $\mathrm{C}^{(7)}$ & $\mathrm{C}^{(8)}$ & $121.6(3)$ \\
\hline
\end{tabular}

\begin{tabular}{|l|l|l|l|}
\hline Atom & Atom & Atom & Angle/ $^{\circ}$ \\
\hline $\mathrm{C}^{(8)}$ & $\mathrm{C}^{(7)}$ & $\mathrm{Br}^{(1)}$ & $119.1(3)$ \\
\hline $\mathrm{C}^{(7)}$ & $\mathrm{C}^{(8)}$ & $\mathrm{C}^{(9)}$ & $118.8(3)$ \\
\hline $\mathrm{C}^{(8)}$ & $\mathrm{C}^{(9)}$ & $\mathrm{C}^{(4)}$ & $119.8(3)$ \\
\hline $\mathrm{F}^{(1)}$ & $\mathrm{C}^{(10)}$ & $\mathrm{F}^{(2)}$ & $109.6(4)$ \\
\hline $\mathrm{F}^{(1)}$ & $\mathrm{C}^{(10)}$ & $\mathrm{C}^{(3)}$ & $110.0(3)$ \\
\hline $\mathrm{F}^{(2)}$ & $\mathrm{C}^{(10)}$ & $\mathrm{C}^{(3)}$ & $108.9(3)$ \\
\hline $\mathrm{O}^{(1)}$ & $\mathrm{C}^{(11)}$ & $\mathrm{C}^{(1)}$ & $119.3(3)$ \\
\hline $\mathrm{O}^{(1)}$ & $\mathrm{C}^{(11)}$ & $\mathrm{C}^{(12)}$ & $122.1(3)$ \\
\hline $\mathrm{C}^{(1)}$ & $\mathrm{C}^{(11)}$ & $\mathrm{C}^{(12)}$ & $118.6(3)$ \\
\hline $\mathrm{C}^{(13)}$ & $\mathrm{C}^{(12)}$ & $\mathrm{C}^{(11)}$ & $122.5(3)$ \\
\hline $\mathrm{C}^{(13)}$ & $\mathrm{C}^{(12)}$ & $\mathrm{C}^{(17)}$ & $118.9(3)$ \\
\hline $\mathrm{C}^{(17)}$ & $\mathrm{C}^{(12)}$ & $\mathrm{C}^{(11)}$ & $118.5(3)$ \\
\hline $\mathrm{C}^{(14)}$ & $\mathrm{C}^{(13)}$ & $\mathrm{C}^{(12)}$ & $120.5(4)$ \\
\hline $\mathrm{C}^{(15)}$ & $\mathrm{C}^{(14)}$ & $\mathrm{C}^{(13)}$ & $120.1(4)$ \\
\hline $\mathrm{C}^{(14)}$ & $\mathrm{C}^{(15)}$ & $\mathrm{C}^{(16)}$ & $120.2(4)$ \\
\hline $\mathrm{C}^{(15)}$ & $\mathrm{C}^{(16)}$ & $\mathrm{C}^{(17)}$ & $120.3(4)$ \\
\hline $\mathrm{C}^{(16)}$ & $\mathrm{C}^{(17)}$ & $\mathrm{C}^{(12)}$ & $119.9(4)$ \\
\hline & & & \\
\hline
\end{tabular}


Table S6 Torsion Angles for Compound 3g.

\begin{tabular}{|c|c|c|c|c|c|c|c|c|c|}
\hline A & $\mathrm{B}$ & $\mathrm{C}$ & $\mathrm{D}$ & Angle $/^{\circ}$ & $\mathrm{A}$ & $\mathrm{B}$ & $\mathrm{C}$ & $\mathrm{D}$ & Angle $/^{\circ}$ \\
\hline $\mathrm{Br}^{(1)}$ & $C^{(7)}$ & $\mathrm{C}^{(8)}$ & $\mathrm{C}^{(9)}$ & $177.3(3)$ & $\mathrm{C}^{(2)}$ & $\mathrm{C}^{(1)}$ & $\mathrm{C}^{(11)}$ & $\mathrm{O}^{(1)}$ & $136.4(4)$ \\
\hline $\mathrm{O}^{(1)}$ & $\mathrm{C}^{(11)}$ & $\mathrm{C}^{(12)}$ & $\mathrm{C}^{(13)}$ & $164.1(3)$ & $\mathrm{C}^{(2)}$ & $\mathrm{C}^{(1)}$ & $\mathrm{C}^{(11)}$ & $\mathrm{C}^{(12)}$ & $-43.2(5)$ \\
\hline $\mathrm{O}^{(1)}$ & $\mathrm{C}^{(11)}$ & $\mathrm{C}^{(12)}$ & $C^{(17)}$ & $-12.6(5)$ & $\mathrm{C}^{(2)}$ & $\mathrm{C}^{(3)}$ & $\mathrm{C}^{(10)}$ & $\mathrm{F}^{(1)}$ & $44.6(5)$ \\
\hline $\mathrm{N}^{(1)}$ & $\mathrm{N}^{(2)}$ & $\mathrm{C}^{(3)}$ & $\mathrm{C}^{(2)}$ & $1.1(4)$ & $\mathrm{C}^{(2)}$ & $\mathrm{C}^{(3)}$ & $\mathrm{C}^{(10)}$ & $\mathrm{F}^{(2)}$ & $-75.5(5)$ \\
\hline $\mathrm{N}^{(1)}$ & $\mathrm{N}^{(2)}$ & $\mathrm{C}^{(3)}$ & $\mathrm{C}^{(10)}$ & $-178.8(3)$ & $\mathrm{C}^{(4)}$ & $\mathrm{N}^{(1)}$ & $\mathrm{N}^{(2)}$ & $\mathrm{C}^{(3)}$ & $-176.5(3)$ \\
\hline $\mathrm{N}^{(1)}$ & $C^{(1)}$ & $C^{(2)}$ & $\mathrm{C}^{(3)}$ & $0.2(4)$ & $\mathrm{C}^{(4)}$ & $\mathrm{N}^{(1)}$ & $\mathrm{C}^{(1)}$ & $\mathrm{C}^{(2)}$ & $175.3(3)$ \\
\hline $\mathrm{N}^{(1)}$ & $\mathrm{C}^{(1)}$ & $C^{(11)}$ & $\mathrm{O}^{(1)}$ & $-33.8(5)$ & $\mathrm{C}^{(4)}$ & $\mathrm{N}^{(1)}$ & $\mathrm{C}^{(1)}$ & $\mathrm{C}^{(11)}$ & $-12.5(5)$ \\
\hline $\mathrm{N}^{(1)}$ & $\mathrm{C}^{(1)}$ & $\mathrm{C}^{(11)}$ & $\mathrm{C}^{(12)}$ & & $\mathrm{C}^{(4)}$ & $\mathrm{C}^{(5)}$ & $\mathrm{C}^{(6)}$ & $C^{(7)}$ & $1.0(6)$ \\
\hline $\mathrm{N}^{(1)}$ & $\mathrm{C}^{(4)}$ & $\mathrm{C}^{(5)}$ & $\mathrm{C}^{(6)}$ & $-178.9(3)$ & $C^{(5)}$ & $\mathrm{C}^{(4)}$ & $C^{(9)}$ & $\mathrm{C}^{(8)}$ & $0.0(5)$ \\
\hline $\mathrm{N}^{(1)}$ & $\mathrm{C}^{(4)}$ & $\mathrm{C}^{(9)}$ & $\mathrm{C}^{(8)}$ & $177.7(3)$ & $C^{(5)}$ & $\mathrm{C}^{(6)}$ & $C^{(7)}$ & $\mathrm{Br}^{(1)}$ & $-178.4(3)$ \\
\hline $\mathrm{N}^{(2)}$ & $\mathrm{N}^{(1)}$ & $\mathrm{C}^{(1)}$ & $\mathrm{C}^{(2)}$ & $0.5(4)$ & $\mathrm{C}^{(5)}$ & $\mathrm{C}^{(6)}$ & $C^{(7)}$ & $\mathrm{C}^{(8)}$ & $0.3(6)$ \\
\hline $\mathrm{N}^{(2)}$ & $\mathrm{N}^{(1)}$ & $\mathrm{C}^{(1)}$ & $\mathrm{C}^{(11)}$ & $172.6(3)$ & $\mathrm{C}^{(6)}$ & $\mathrm{C}^{(7)}$ & $\mathrm{C}^{(8)}$ & $\mathrm{C}^{(9)}$ & $-1.5(6)$ \\
\hline $\mathrm{N}^{(2)}$ & $\mathrm{N}^{(1)}$ & $\mathrm{C}^{(4)}$ & $\mathrm{C}^{(5)}$ & $130.7(3)$ & $C^{(7)}$ & $\mathrm{C}^{(8)}$ & $\mathrm{C}^{(9)}$ & $\mathrm{C}^{(4)}$ & $1.3(5)$ \\
\hline $\mathrm{N}^{(2)}$ & $\mathrm{N}^{(1)}$ & $\mathrm{C}^{(4)}$ & $\mathrm{C}^{(9)}$ & $-47.1(4)$ & $\mathrm{C}^{(9)}$ & $\mathrm{C}^{(4)}$ & $\mathrm{C}^{(5)}$ & $\mathrm{C}^{(6)}$ & $-1.1(5)$ \\
\hline $\mathrm{N}^{(2)}$ & $\mathrm{C}^{(3)}$ & $\mathrm{C}^{(10)}$ & $\mathrm{F}^{(1)}$ & $-135.4(3)$ & $\mathrm{C}^{(11)}$ & $\mathrm{C}^{(1)}$ & $\mathrm{C}^{(2)}$ & $\mathrm{C}^{(3)}$ & $-171.2(3)$ \\
\hline $\mathrm{N}^{(2)}$ & $\mathrm{C}^{(3)}$ & $\mathrm{C}^{(10)}$ & $F^{(2)}$ & $104.5(4)$ & $\mathrm{C}^{(11)}$ & $\mathrm{C}^{(12)}$ & $\mathrm{C}^{(13)}$ & $C^{(14)}$ & $-177.1(3)$ \\
\hline $\mathrm{C}^{(1)}$ & $\mathrm{N}^{(1)}$ & $\mathrm{N}^{(2)}$ & $\mathrm{C}^{(3)}$ & $-1.0(3)$ & $\mathrm{C}^{(11)}$ & $\mathrm{C}^{(12)}$ & $\mathrm{C}^{(17)}$ & $C^{(16)}$ & $177.6(4)$ \\
\hline $\mathrm{C}^{(1)}$ & $\mathrm{N}^{(1)}$ & $\mathrm{C}^{(4)}$ & $\mathrm{C}^{(5)}$ & $-43.9(5)$ & $\mathrm{C}^{(12)}$ & $\mathrm{C}^{(13)}$ & $\mathrm{C}^{(14)}$ & $C^{(15)}$ & $0.3(6)$ \\
\hline $\mathrm{C}^{(1)}$ & $\mathrm{N}^{(1)}$ & $\mathrm{C}^{(4)}$ & $\mathrm{C}^{(9)}$ & $138.4(3)$ & $\mathrm{C}^{(13)}$ & $\mathrm{C}^{(12)}$ & $\mathrm{C}^{(17)}$ & $\mathrm{C}^{(16)}$ & $0.8(6)$ \\
\hline $\mathrm{C}^{(1)}$ & $\mathrm{C}^{(2)}$ & $\mathrm{C}^{(3)}$ & $\mathrm{N}^{(2)}$ & $-0.9(4)$ & $\mathrm{C}^{(13)}$ & $\mathrm{C}^{(14)}$ & $\mathrm{C}^{(15)}$ & $\mathrm{C}^{(16)}$ & $-0.5(7)$ \\
\hline $\mathrm{C}^{(1)}$ & $\mathrm{C}^{(2)}$ & $\mathrm{C}^{(3)}$ & $\mathrm{C}^{(10)}$ & $179.1(3)$ & $\mathrm{C}^{(14)}$ & $\mathrm{C}^{(15)}$ & $\mathrm{C}^{(16)}$ & $\mathrm{C}^{(17)}$ & $0.8(7)$ \\
\hline $\mathrm{C}^{(1)}$ & $\mathrm{C}^{(11)}$ & $\mathrm{C}^{(12)}$ & $\mathrm{C}^{(13)}$ & $-16.2(5)$ & $\mathrm{C}^{(15)}$ & $\mathrm{C}^{(16)}$ & $\mathrm{C}^{(17)}$ & $\mathrm{C}^{(12)}$ & $-1.0(7)$ \\
\hline $\mathrm{C}^{(1)}$ & $\mathrm{C}^{(11)}$ & $\mathrm{C}^{(12)}$ & $\mathrm{C}^{(17)}$ & $167.0(3)$ & $\mathrm{C}^{(17)}$ & $\mathrm{C}^{(12)}$ & $\mathrm{C}^{(13)}$ & $\mathrm{C}^{(14)}$ & $-0.4(5)$ \\
\hline
\end{tabular}

Table S7 Hydrogen Atom Coordinates $\left(\AA \times 10^{4}\right)$ and Isotropic Displacement

Parameters $\left(\AA^{2} \times 10^{3}\right)$ for Compound 3g.

\begin{tabular}{|l|l|l|l|l|}
\hline Atom & $\mathrm{x}$ & $\mathrm{y}$ & $\mathrm{z}$ & $\mathrm{U}(\mathrm{eq})$ \\
\hline $\mathrm{H}^{(2)}$ & 12266.09 & -1640.13 & 1554.53 & 54 \\
\hline $\mathrm{H}^{(5)}$ & 8479.08 & 2069.26 & 1833.27 & 61 \\
\hline $\mathrm{H}^{(6)}$ & 7114.62 & 4439.93 & 1891.31 & 68 \\
\hline $\mathrm{H}^{(8)}$ & 9519.2 & 6957.53 & 671.76 & 62 \\
\hline $\mathrm{H}^{(9)}$ & 10933.31 & 4606.47 & 641.71 & 56 \\
\hline $\mathrm{H}^{(10)}$ & 11987.97 & -784.48 & -309.66 & 57 \\
\hline $\mathrm{H}^{(13)}$ & 10255.59 & -2334.29 & 2160.62 & 61 \\
\hline $\mathrm{H}^{(14)}$ & 9839.71 & -4505.64 & 2898.16 & 76 \\
\hline $\mathrm{H}^{(15)}$ & 10101.16 & -4044.04 & 4015.47 & 86 \\
\hline $\mathrm{H}^{(16)}$ & 10815.06 & -1430.79 & 4408.55 & 89 \\
\hline $\mathrm{H}^{(17)}$ & 11216.3 & 769.94 & 3681.72 & 71 \\
\hline
\end{tabular}

